g(2.) Basic Education for Afghan Refugees

\title{
GTZ - COPE
}

Schools Annual Examination Results and Analysis 1996-97

Prepared By:

Monitoring and Evaluation Section of GTZ - BEFARe 


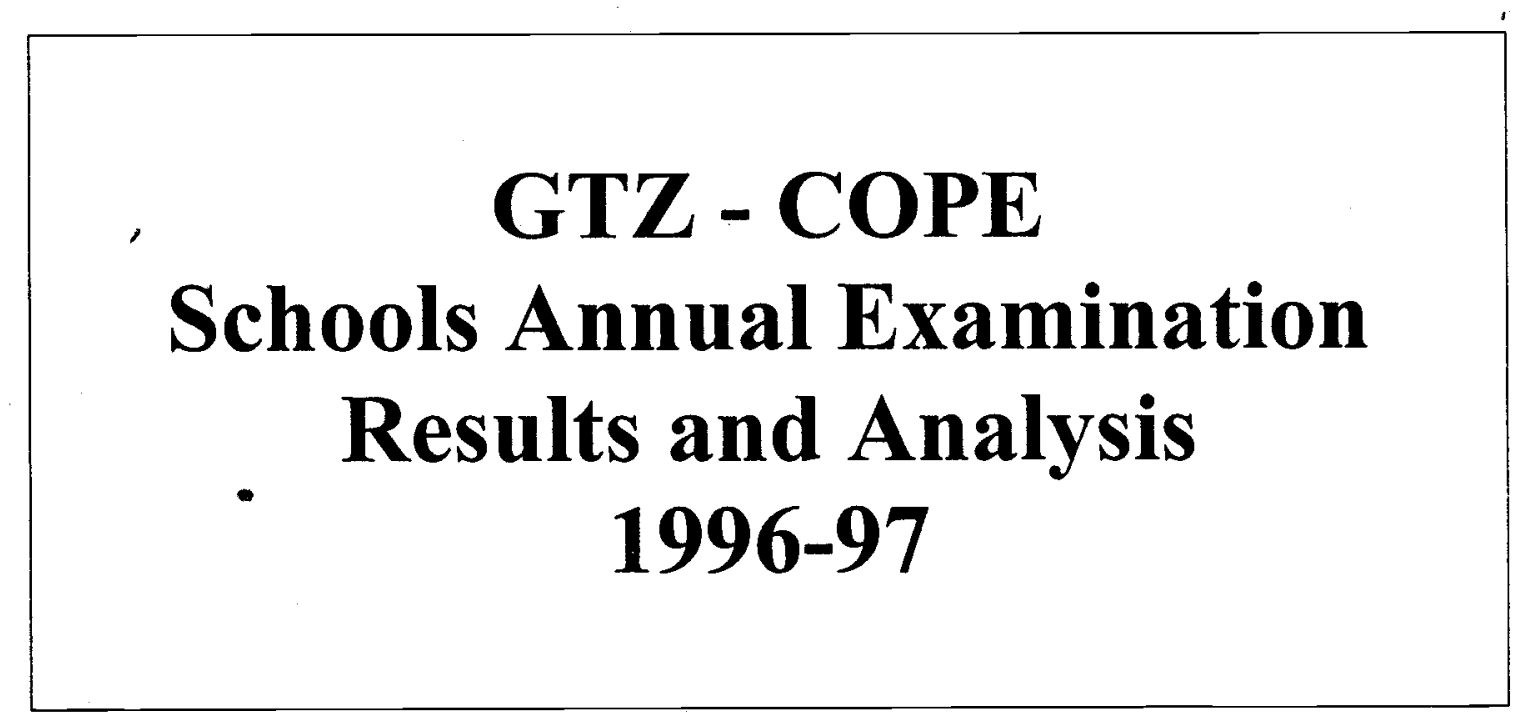

Prepared By:

Monitoring and Evaluation Section of GTZ - BEFARe 


\section{Table of Contents}

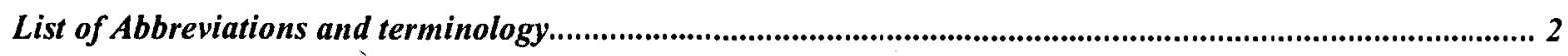

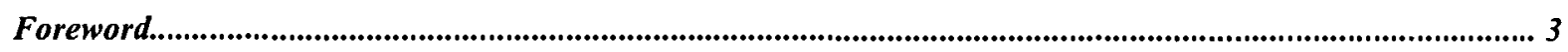

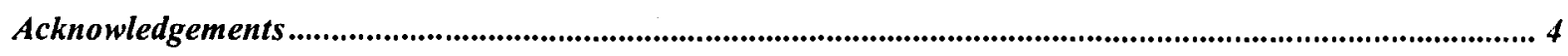

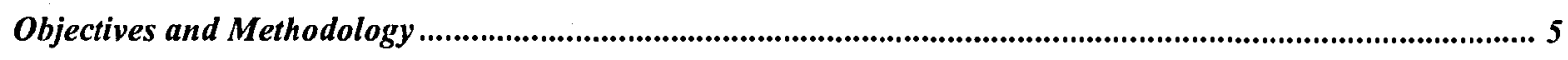

Table: 1 Annual results for 1996-97 Sub-Centre Level............................................................................ 6

Table: 2 Annual results for 1996-97 (Grade Level for All Sub-Centres).................................................... 7

Table: 3 Abbotabad - Grade Wise Annual results and Percentage Analysis ............................................ 8

Table: 4 Bannu - Grade Wise Annual results and Percentage Analysis ...................................................9

Table: 5 Female - Grade Wise Annual results and Percentage Analysis ............................................ 10

Table: 6 Peshawar - Grade Wise Annual results and Percentage Analysis......................................... 12

Table: 7 Thall - Grade Wise Annual results and Percentage Analysis ................................................ 13

Table: $8^{\prime}$ Timargara - Grade Wise Annual results and Percentage Analysis........................................ 14

Table: 9 Annual results for 1996-97 and Percentage Analysis for all Grades (Sub-Centre Level) .... 15

Table: 10 Annual results for 1996-97 and Percentage Analysis for Grade One (Sub-Centre Level)... 16

Table: 11 Annual results for 1996-97 and Percentage Analysis for Grade Two (Sub-Centre Level) .. 17

Table: 12 Annual results for 1996-97 and Percentage Analysis for Grade Three (Sub-Centre Level) 17

Table: 13 Annual results for 1996-97 and Percentage Analysis for Grade Four (Sub-Centre Level).. 18

Table: 14 Annual results for 1996-97 and Percentage Analysis for Grade Five (Sub-Centre Level)... 18

Table: 15 Annual results for 1996-97 and Percentage Analysis for Grade Six (Sub-Centre Level).... 19

Table: 16 Annual results for 1996-97, Gender Distribution for Boys (Sub-Centre Level) ..................... 19

Table: 17 Annual results for 1996-97, Gender Distribution for Girls (Sub-Centre Level) .................... 20

Table: 18 Annual results for 1996-97, Comparative Gender Performance............................................... 20

Table: 19 Enrolment per school Sub-Centre Level ............................................................................ 20

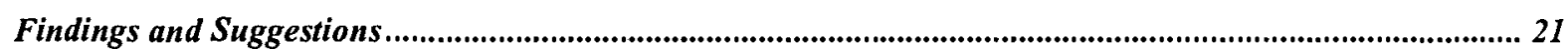

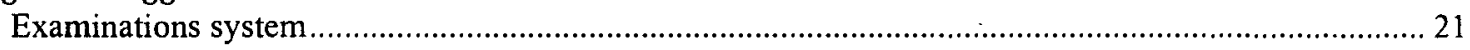

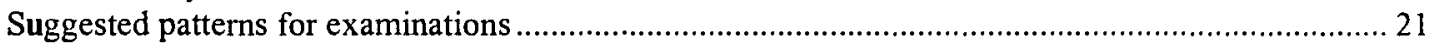

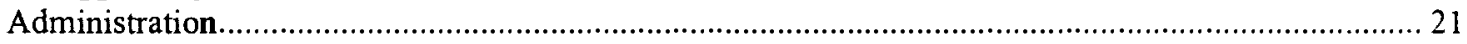

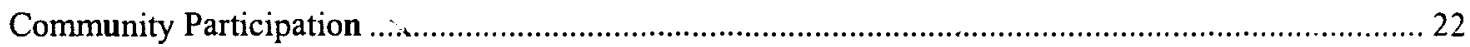

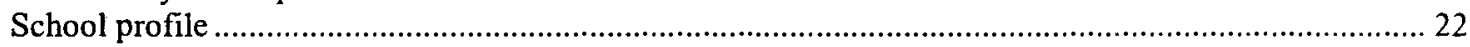

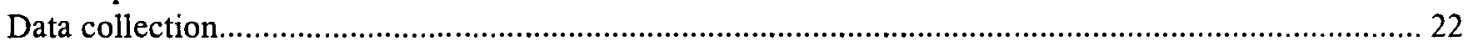

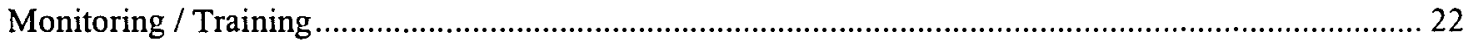

Data Collection form for COPE School Annual Examination Results........................................................ 22

COPE Schools Annual results 1996-97 Graphical Presentations............................................................... 22

Detailed School Based Annual results for 1996-97 …...................................................................................... 22 


\section{List of Abbreviations and terminology}

\begin{tabular}{|ll|}
\hline $\begin{array}{l}\text { Abbreviation or } \\
\text { Terminology }\end{array}$ & Description \\
\hline Appeared & Students participated in annual examination \\
\hline ARGPS & Afghan Refugee Girls Primary School \\
\hline ARPS & Afghan Refugee Primary School \\
\hline BEFARe & Basic Education for Afghan Refugees \\
\hline CM & Community Motivator \\
\hline COPE & Community Oriented Primary Education \\
\hline CP & Community Participation \\
\hline CSA & COPE School Advisor \\
\hline CTA & Chief Technical Advisor \\
\hline Dropped & $\begin{array}{l}\text { Students dropped/left school before annual examination with or } \\
\text { without proper procedure }\end{array}$ \\
\hline Enrolled & Registration of students in COPE Schools \\
\hline Failed & $\begin{array}{l}\text { Students did not qualify for next class and will remain in same grade } \\
\text { for another year }\end{array}$ \\
\hline FES & Field'Education Supervisor \\
\hline G & Grade \\
\hline GTZ & $\begin{array}{l}\text { Deutsche Gesellschaft fur Technische Zusammenarbeit (German } \\
\text { Agency for Technical Cooperation) }\end{array}$ \\
\hline MT & Master Trainer \\
\hline NWFP & North West Frontier Province \\
\hline Passed & Students qualifies for next grade \\
\hline SC & Sub-Center \\
\hline SMC & School Management Committee \\
\hline UNHCR & United Nations High Commissioner for Refugees \\
\hline ZOPP & Ziel Orientierte Projekt Planung (Goal Oriented Project Planning) \\
\hline
\end{tabular}




\section{Foreword}

One of the main objectives of GTZ's development policy is to support monitoring and evaluation for the achievement of project goals, objectives, results and activities formulated by the project management and its respective sections. To serve this aim, the Monitoring and Evaluation section of GTZ-BEFARe conducted this survey, which is to inform all high level planners and administrators of UNHCR and COPE about the actual status of Afghan refugee's primary education in the NWFP. Moreover, I hope that this document will be used by the implementers as a tool, which puts them into the position to plan, to run and to evaluate their project in the most efficient way.

I am happy to say that since COPE took over the responsibility for the Afghan Refugee Primary Education in NWFP, the dropout rate decreased, the number of students having successfully passed the exams increased and the total enrolment of girls doubled. On the other hand the findings provoke questions which need a careful and detailed analysis regarding pedagogical, social and educational economic aspects.

That much is certain, refugee children have very specific education needs:

The condition of Afghan refugee students is directly rooted in forced displacement, the deprivation of freedom, separation and the loss of family, home, a way of life and perhaps even of identity. Many struggle through many years in refugee camps and far too often, students are subjected to dangerous and harsh conditions. A high percentage is still suffering hunger, illness and violence to themselves and their families.

That is why the commitment of UNHCR in Afghan Refugee Education is highly esteemed. It is due to the strong support and efforts of UNHCR that refugee children are given the chance to get a proper primary education, as prosperity and quality of Afghanistan will be i.a. determined by the these children and their ability to solve the problems of the future. Education unlocks this ability and the investment in the teaching and learning of Afghan refugee children is a most important precondition for the reconstruction of Afghanistan and his economic development.

Dr. Michael Hirth

Project Manager

GTZ-BEFARe and COPE 


\section{Acknowledgements}

The nature, situation and variety of the activities of the primary education which are administered all over the North West Frontier Province by the GTZ - Basic Education for Afghan Refugees (BEFARe) required the cooperation, collaboration and coordination of all the experts as well as the auxiliary staff of the project in the collection of the data, transmission, recording and it's subsequent analysis.

This exercise was jointly designed and implemented by the M\&E and Formal Education Sections of GTZ - BEFARe.

I would like to express my very special gratitude to Dr. Michael Hirth, Chief Technical Advisor of GTZ - BEFARe, who identified the need for this document. It was his vision, support, encouragement and confidence that enabled M\&E section to fulfil this task.

I would also like to acknowledge the contribution of Mr. Taj Mohammad, the Co-ordinator of Formal Education, who organized the field activities for this exercise.

Special thanks to Mr. Mobeen Azizi, who has put in lots of hours in feeding the data into the computer which enabled the M\&E section to complete the data processing and its final analysis.

Mr. Askar Azam, Co-ordinator Community Participation, was always available and gave very useful technical guidance and comments. I am indebted for his time.

A very special thanks to Mr. Syed Junaid Shah, who extended his services to M\&E section in the formulation and editing of this document.

GTZ - BEFARe would like to express special thanks to all those who contributed in one way or another in the preparation of this document.

It is hoped that this document will help the management as well as the others in planning, implementing and further enhancing the educational activities for Afghan refugees.

Mohammad Rehan Awan

Co-ordinator Data Processing,

GTZ - BEFARe 


\section{Objectives and Methodology}

The main Objective for the compilation of this document has been to inform the Project Management about the status of the education among Afghan refugee children in the refugee camps of NWFP. The analysis contained in this document may assist the executors, at various levels, as to what necessary steps to take in order to achieve the Project Objectives. Secondly, it can also be used to prepare a baseline for the next year comparative analysis.

In the middle of March 1997, it was decided by the project that the annual results should be collected, on one standard pattern, from all the 265 COPE schools (see Annex - I). This standard form was designed in consultation with the Formal Education section and was later finalised and approved by the Project Director. In March 1997, the forms were distributed to all the schools through the Master Trainers and the group of 40 Field Education Supervisors/COPE School Advisors. The Data collection process was initiated slowly but gradually in April 1997. The figures in the forms were filled in by the teachers of the respective grades and were counter-signed by the Head Teachers and FESs/CSAs. The whole process of collecting data was completed by May 1997. In order to cope and analyse a huge collection of information, a computer based database system was designed by using Microsoft Access for accurate analysis. In June 1997, the initial data entry was completed and a computer printed sheets of the same data was given to the Master trainers for final checking so that any error in the data entry could be eliminated. This exercise was completed July 1997.

After these initial steps, the data was ready for analysis. All the figures were calculated by using Database and Spreadsheet analysis techniques so the accuracy of data could be maintained. Thus for the compilation of these results, percentage techniques of analysis have been used. Wherever necessary the roundup of two (2) decimal has been implemented to bring the figures more closer to reality. All the tables presented in this document are crosschecked with each other and have proven accurate. All the data is sorted in alphabetical order.

For the graphical representations, the data has been used from the same source and with the same accuracy. For this purpose Microsoft Excel Spreadsheet was used to achieve the required results. In most of the tables and especially in table 1 , the percentages for enrolled students are calculated from the total of all sub-centres. In other words the figures are representing their share to the total number of enrolled students.

A two-member team of the M \& E section of GTZ-BEFARe has prepared this document for the perusal of the Project Management and all relevant Sections / Organizations. The results of this analysis are consolidated in the following tables and manuscript of this document. 
Owing mainly to the particularities of culture and being refugees, the level of education in the refugee camps is even lower than the already low national and provincial literacy average. A disparity that is especially critical in the case of females.

However the situation of primary education for Afghans in NWFP since GTZ-COPE took over the responsibilities of implementation is conspicuously identified by the increase in enrollment figures, low dropout rates, reduced absenteeism of teachers and students and the further improvement in quality of teaching and learning.

The figures presented in this document provide us with the basis for future monitoring and evaluations. The results compiled in this document show that a total of 60,804 students were enrolled during the academic year 1996-97. The appearance percentage in the annual examination was $\mathbf{9 2 . 2 1 \%}$ comprising a figure of 56,065 students with a passing percentage of $72.11 \%$. The programme experienced a very minimal dropout of the students during the same year with $7.79 \%$. The tables below provide a comprehensive detail of the annual results of the schools during the academic year 1996-97.

\section{Table: 1 Annual results for 1996-97 Sub-Centre Level}

\begin{tabular}{|c|c|c|c|c|c|c|c|}
\hline & \multicolumn{7}{|c|}{ Number of Students and Percentages } \\
\hline & Abbotabad & Bannu & Female & Peshawar & Thall & Timargara & $\overline{\text { Total }}$ \\
\hline Enrolled & 7,558 & 5,959 & 7,866 & 21,900 & 10,344 & 7,177 & 60,804 \\
\hline \%age & $12.43 \%$ & $9.80 \%$ & $12.94 \%$ & $36.02 \%$ & $17.01 \%$ & $11.80 \%$ & $100.00 \%$ \\
\hline Dropped & 805 & 438 & 480 & 1,584 & 670 & 762 & 4,739 \\
\hline \%age - & $16.99 \%$ & $9.24 \%$ & $10.13 \%$ & $33.42 \%$ & $14.14 \%$ & $16.08 \%$ & $7.79 \%$ \\
\hline Appeared & 6,753 & 5,521 & 7,386 & 20,316 & 9,674 & 6,415 & 56,065 \\
\hline$\%$ age & $12.04 \%$ & $9.85 \%$ & $13.17 \%$ & $36.24 \%$ & $17.25 \%$ & $11.44 \%$ & $92.21 \%$ \\
\hline$\overline{\text { Passed }}$ & 4,951 & 4,156 & 5,293 & 14,049 & 7,463 & 4,519 & $\overline{40,431}$ \\
\hline \%age & $12.25 \%$ & $10.28 \%$ & $13.09 \%$ & $34.75 \%$ & $18.46 \%$ & $11.18 \%$ & $72.11 \%$ \\
\hline Failed & 1,802 & 1,365 & 2,093 & 6,267 & 2,211 & 1,896 & 15,634 \\
\hline \%age & $11.53 \%$ & $8.73 \%$ & $13.39 \%$ & $40.09 \%$ & $14.14 \%$ & $12.13 \%$ & $27.89 \%$ \\
\hline
\end{tabular}

Table: 1 shows over all annual examination results for 1996-97 in all five operational subcentres geographically diversified over N-W.F.P. In this table it can be seen that the percentages of each sub-centre are the share of $100 \%$. Details are as follows: -

A. The numbers of enrolled students in the project were 60804 . The total number of enrolled students in Abbotabad were 7558 , which is $12.43 \%$ of the total enrolled students in the program. In Bannu 5959 (9.80\%) students were enrolled while in the Female sub-centre 7866 girls students were reported to be enrolled which is $12.94 \%$ of the total. Similarly Peshawar had the highest percentage of enrolled figures $(36.02 \%)$ which covers a total figure of 21900 enrolled students. It should be noted that this centre has the maximum strength among all the sub-centres. The sub-centre in Thall had 10344 enrolled students, which is $17.01 \%$ of the total and lasts but not the least Timergara had $7177(11.80 \%)$ enrolled students out of the total 60804 enrolled students.

B. The total number of dropped out students (before examination) was $7.79 \%$ (4738 students). In Abbotabad there were 805 students, which is $16.99 \%$ of the total dropped 
out students in the program. In Bannu 438 (9.24\%) students were enrolled while in the Female sub-centre, 480 girl students were reported to have dropout, which is $10.13 \%$ of the total dropout figures. The dropped out figures for Peshawar were the highest at 1584 students, which is $33.42 \%$ of the total dropped students. Furthermore, in Thall sub-centre, 670 students dropped out comprising $14.14 \%$ of the total. Finally in Timergara 762 students dropped out, which is $16.08 \%$ of the total dropped out figures.

C. The total number of appeared students (in examination) for the academic year 1996 was 56065 students. In Abbotabad 6753 (12.04\%) students appeared in the exams out of which $4951(12.25 \%)$ were successful and the remaining $1802(11.53 \%)$ students could not qualify for next grade. 6753 students $(9.85 \%)$ appeared from Bannu subcentre. From the Female sub-centre, 7386 girl students (13.17\%) appeared in the annual examination. Similarly Peshawar had 20316 students comprising of $36.24 \%$ of the total students appearance, with Thall and Timargara having $9674(17.25 \%)$ and $6415(11.44 \%)$ students appearing in the annual examinations respectively.

D. Out of the total 56065 students who appeared in their annual examinations, 40431 students passed, having a total pass percentage of $72.11 \%$. Of the total pass percentage, Abbotabad had a share of $12.25 \%$ (4951) students, Bannu 10.28\% (4156) students, Female sub-centre had a pass percentage of $13.09 \%$ (5293) students, Péshawar with $34.75 \%$ (14049) students, Thall having $18.46 \%$ (7463) students and Timargara with $11.18 \%$ (4519) students.

E. The percentage of students failing in the annual examination was $27.89 \%(15634$ students) out of which $1802(11.53 \%)$ students were from Abbotabad, Bannu had $1365(8.73 \%)$ failed students, Female sub-centre had $2093(13.09 \%)$ failures. At the same time Peshawar had 6267 students who could not make it to the next grade having a percentage of $40.09 \%$ of the total failed students. Similarly Thall and Timargara had 2211 and 1896 students denoting $14.14 \%$ and $12.13 \%$ of the total students who failed in their annual examinations.

\section{Table: 2 Annual results for 1996-97 (Grade Level for All Sub-Centres)}

\begin{tabular}{|c|c|c|c|c|c|c|c|}
\hline & \multicolumn{7}{|c|}{ Number of Students and Percentages } \\
\hline & Grade 1 & Grade 2 & Grade 3 & Grade 4 & Grade 5 & Grade 6 & Total \\
\hline $\begin{array}{l}\text { Enrolled } \\
\% \text { age }\end{array}$ & $\begin{array}{l}23,676 \\
38.94 \% \\
\end{array}$ & \begin{tabular}{|c|}
13,780 \\
$22.66 \%$
\end{tabular} & 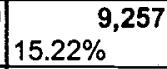 & $\begin{array}{c}\mathbf{6 , 4 6 2} \\
10.63 \%\end{array}$ & $7.78 \%{ }^{4,728}$ & $4.77 \%{ }^{2,901}$ & \begin{tabular}{|l}
$\mathbf{6 0 , 8 0 4}$ \\
$100.00 \%$
\end{tabular} \\
\hline $\begin{array}{l}\text { Dropped } \\
\text { \%age } \\
\end{array}$ & $8.17 \%{ }^{1,935}$ & $6.21 \%{ }^{856}$ & $7.80 \%$ & 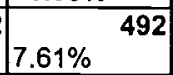 & 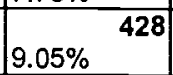 & ${ }_{10.55 \%}^{306}$ & $7.79 \%{ }^{4,739}$ \\
\hline $\begin{array}{l}\text { Appeared } \\
\% \text { age } \\
\end{array}$ & $\begin{array}{c}\mathbf{2 1 , 7 4 1} \\
91.83 \% \\
\end{array}$ & \begin{tabular}{|c|}
12,924 \\
$93.79 \%$ \\
\end{tabular} & $\begin{array}{r}\mathbf{8}^{\mathbf{8 , 5 3 5}} \\
92.20 \%\end{array}$ & \begin{tabular}{|c|}
\multicolumn{2}{c}{$\mathbf{5 , 9 7 0}$} \\
$92.39 \%$
\end{tabular} & $\begin{array}{c}4,300 \\
90.95 \%\end{array}$ & 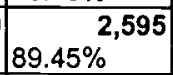 & $\begin{array}{r}\mathbf{5 6 , 0 6 5} \\
92.21 \%\end{array}$ \\
\hline $\begin{array}{l}\text { Passed } \\
\text { \%age }\end{array}$ & $\begin{array}{r}14,019 \\
64.48 \%\end{array}$ & \begin{tabular}{|c|}
$\mathbf{9}, \mathbf{3 6 9}$ \\
$72.49 \%$
\end{tabular} & $\begin{array}{r}6,577 \\
77.06 \%\end{array}$ & $\begin{array}{r}4,768 \\
79.87 \%\end{array}$ & 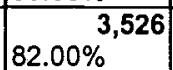 & 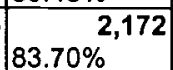 & $\begin{array}{r}\mathbf{4 0 , 4 3 1} \\
72.11 \%\end{array}$ \\
\hline $\begin{array}{l}\text { Failed } \\
\text { \%age } \\
\end{array}$ & $\begin{array}{c}3^{7,722} \\
35.52 \% \\
\end{array}$ & \begin{tabular}{|c|}
$2^{3} 3,555$ \\
$27.51 \%$ \\
\end{tabular} & $22.94 \%$ & $20.13 \%$ & $18.00 \%{ }^{774}$ & $16.30 \%{ }^{423}$ & $\begin{array}{c}15,634 \\
27.89 \% \\
\end{array}$ \\
\hline
\end{tabular}

Table: 2 shows over all annual examination results for 1996-97 in all the five operational subcentres.

A. Over all 60804 students were enrolled in the six grades of the primary schools. The distribution of number of students in the grades 1 to 6 was $23,676,13,780,9257$, 6462,5728 and 2901 students respectively. It is easily noticeable that the figures for 
enrollment of students have gradually descended. As per the figures available only $12.25 \%$ of the Grade 1 students reach Grade 6 .

B. The figures gathered clearly indicate that the dropped out percentage increased with each higher grade. Out of the total 4739 dropped out students, there were 1935 $(8.17 \%)$ in grade one, $856(6.21 \%)$ in grade two, $722(7.80 \%)$ in grade three, 492 $(7.61 \%)$ in grade four, $428(9.05 \%)$ in grade five and $306(10.55 \%)$ in grade six. Thus the dropout percentage increased from $8.71 \%$ in grade 1 to $10.55 \%$ in grade 6 .

C. Similarly figures for the appearance in the annual examination also decreased but the over all percentage of students' appearance in the examinations for all the grades was $92.21 \%$. The highest percentage was for the grade 2 having $93.79 \%$ and the lowest was in grade 6 having a student appearance of $89.45 \%$.

D. The results compiled after the annual examination show a positive passing percentage of $72.11 \%$ of the total 56065 students that appeared in the annual examination., The passing percentage of students from gradel to grade 6 has been $64.48 \%$ (14019 students), $72.49 \%$ (9369 students), $77.06 \%$ (6577 students), $79.87 \%$ (4768 students), $82.00 \%$ (3526 students) and $83.70 \%$ (2172 students) respectively. It is clearly observed that the passing percentage increased with each higher grade, thus if the 'dropout rate can be reduced it is felt that even more students would be able to complete their primary level education.

E. Calculating the percentage of students who were unfortunate to qualify for the next grade, it was observed that the highest percentage of failed students was in grade 1 having $35.52 \%$ (7722 students) of the total percentage of $27.89 \%$ of the 15634 students who failed in their annual examinations. It is noteworthy to state that the percentage of failed students gradually decreased with each higher grade. The break down of the figures for the remaining grades from grade 2 to grade 6 are $27.51 \%$ (3555 students), $22.94 \%$ (1958 students), 20.13\% (1202 students), $18.00 \%$ (774 students) and $16.30 \%$ (423 students) respectively.

\section{Table: 3 Abbotabad - Grade Wise Annual results and Percentage Analysis}

\begin{tabular}{|c|c|c|c|c|c|c|c|}
\hline & \multicolumn{7}{|c|}{ Number of Students and Percentages } \\
\hline & Grade 1 & Grade 2 & Grade 3 & Grade 4 & Grade 5 & Grade 6 & Total \\
\hline Enrolled & 2,640 & 1,765 & 1,190 & 843 & 664 & 456 & 7,558 \\
\hline & $34.93 \%$ & $23.35 \%$ & $15.74 \%$ & $11.15 \%$ & $8.79 \%$ & $6.03 \%$ & $100.00 \%$ \\
\hline Dropped & 253 & 179 & 117 & 89 & 97 & 70 & 805 \\
\hline & $9.58 \%$ & $10.14 \%$ & $9.83 \%$ & $10.56 \%$ & $14.61 \%$ & $15.35 \%$ & $10.65 \%$ \\
\hline Appeared & 2,387 & 1,586 & 1,073 & 754 & 567 & 386 & 6,753 \\
\hline & $90.42 \%$ & $89.86 \%$ & $90.17 \%$ & $89.44 \%$ & $85.39 \%$ & $84.65 \%$ & $89.35 \%$ \\
\hline$\overline{\text { Passed }}$ & 1,517 & 1,186 & 840 & 614 & 455 & 339 & 4,951 \\
\hline & $63.55 \%$ & $74.78 \%$ & $78.29 \%$ & $81.43 \%$ & $80.25 \%$ & $87.82 \%$ & $73.32 \%$ \\
\hline Failed & 870 & 400 & 233 & 140 & 112 & 47 & 1,802 \\
\hline & $36.45 \%$ & $25.22 \%$ & $21.71 \%$ & $18.57 \%$ & $19.75 \%$ & $12.18 \%$ & $26.68 \%$ \\
\hline
\end{tabular}

A. In all 7558 students were enrolled in the six grades of the 30 primary schools coming under the jurisdiction of Abbotabad sub-centre. The distribution of the number of students for the grades 1 to 6 were $2640(34.93 \%), 1765(23.35 \%), 1190(15.74 \%)$, $843(11.15 \%), 664(8.79 \%)$ and $456(6.03 \%)$ students respectively. On the basis of 
figures collected from this centre only $6.03 \%$ of the Grade 1 students reached Grade 6. This is unfortunately, below the program's benchmark, which is $15.25 \%$.

B. The figures collected indicate that the dropped out percentage also increased with each higher grade. Out of the total 805 students that dropped out, there were 253 $(9.58 \%)$ in grade $1,179(10.14 \%)$ in grade $2,117(9.83 \%)$ in grade $3,89(10.561 \%)$ in grade $4,97(14.61 \%)$ in grade 5 and $70(15.35 \%)$ in grade 6 . Thus the dropped out percentage increased from $9.58 \%$ in grade 1 to $15.35 \%$ in grade 6 . The only significance in the dropped out rate was in grade 3, which was less than grade 2.

C. As per the calculation of the figures for the appearance in the annual examinations, there was also a decrease in the students numbers but the over all percentage of students appearing in the examinations for all the grades was $89.35 \%$ (6753 students). The highest percentage was for the grade 3 having $90.17 \%$ (1073 students) and the lowest was in grade 6 having a student appearance rate of $84.65 \%$ (386 students).

D. The results of the annual examination show a positive passing percentage of $73.32 \%$ of the total 4951 students that appeared in the annual examinations. The passing percentage of students from gradel to grade 6 has been $63.45 \%$ (1517 students), $74.78 \%$ (1186 students), $78.29 \%$ (840 students), $81.43 \%$ (614 students), $80.25 \%$ (455 students) and $87.82 \%$ (339 students) respectively.

E. Coming to the percentage of students who could not make it to the next grade, it was observed that the highest percentage of failed students was observed in grade 1 having a percentage of $36.45 \%$ ( 870 students) of the total percentage of $26.68 \%$ (1802 students) of the students who failed in their annual examinations while the lowest was observed in grade 6 have a fail percentage of $12.18 \%$ ( 47 students). The percentage of students who failed in their respective grades is $36.45 \%$ ( 870 students) in grade 1 , $25.22 \%$ (400 students) in grade 2, $21.71 \%$ (233 students) in grade $3,18.57 \%$ (140) in grade $4,19.75 \%$ (112 students) in grade 5 and $12.18 \%$ (47 students) in grade 6.

\section{Table: 4 Bannu - Grade Wise Annual results and Percentage Analysis}

\begin{tabular}{|c|c|c|c|c|c|c|c|}
\hline & \multicolumn{7}{|c|}{ Number of Students and Percentages } \\
\hline & Grade 1 & Grade 2 & Grade 3 & Grade 4 & Grade 5 & Grade 6 & Total \\
\hline Enrolled & $\begin{array}{r}2,253 \\
37.81 \%\end{array}$ & \begin{tabular}{|r|}
1,327 \\
$22.27 \%$
\end{tabular} & $15.74 \%^{938}$ & $10.82 \%{ }^{645}$ & 460 & 336 & $\begin{array}{r}5,959 \\
100.00 \%\end{array}$ \\
\hline Dropped & 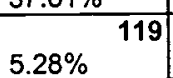 & $7.54 \%$ & 86 & 49 & $8.70 \%$ & $\begin{array}{|ll|}13.10 \% & 44 \\
\end{array}$ & $7.35 \%$ \\
\hline Appeared & $\begin{array}{r}2,134 \\
94.72 \%\end{array}$ & $\begin{array}{c}1,227 \\
92.46 \%\end{array}$ & $90.83 \%{ }^{852}$ & $92.40 \%{ }^{596}$ & $91.30 \%{ }^{420}$ & $86.90 \%{ }^{292}$ & \begin{tabular}{|l}
$\mathbf{S}^{\mathbf{5} 5,521}$ \\
$92.65 \%$
\end{tabular} \\
\hline Passed & 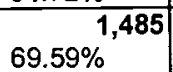 & $7_{78.08 \%}^{958}$ & 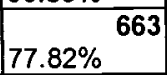 & $83.05 \%{ }^{495}$ & $80.95 \%{ }^{340}$ & 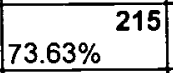 & 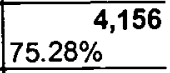 \\
\hline Failed & $30.41 \%$ & $21.92 \%{ }^{269}$ & $22.18 \%{ }^{189}$ & $16.95 \%{ }^{101}$ & $19.05 \%$ & $\begin{array}{|ll|}26.37 \% & 77 \\
\end{array}$ & \begin{tabular}{|l}
1,365 \\
$24.72 \%$
\end{tabular} \\
\hline
\end{tabular}

A. There was a total of 5959 number of students enrolled in the 40 schools being looked after by the Bannu sub-centre. The highest number of enrollment was recorded in grade 1 having a percentage of $37.81 \%$ (2253 students) of the total number of students enrolled in the sub-centre level. The percentage of enrollment for the rest of the grades was $22.27 \%$ (1327 students) for grade 2,grade 3 had $15.75 \%$ (938 students) followed by grade 4 having $10.82 \%$ (645 students). Similarly grade 5 had $7.72 \%$ (460 
students) and grade 6 had 5.64\% (336 students) enrolled during the academic year 1996.

B. The over all dropout rate in 40 schools of Bannu sub-centre was $7.35 \%$ (438 students). The highest percentage of dropout was recorded in grade 6 which was $13.10 \%$ (44 students) and lowest percentage was observed in grade 1 with $5.28 \%$ (119). There were $7.54 \%$ (100 students) in grade $2,9.17 \%$ (86 students) in grade 3 , $7.60 \%$ (49 students) in grade 4 and $8.70 \%$ (40 students) in grade 5.

C. The average appearance rate of the students in the final examinations in the same subcentre was $92.65 \%$ representing student strength of 5521 . The number of students who appeared in their exams in grade 1 was 2134 , which is $94.72 \%$ of the total students enrolled in grade 1. This is followed grade 2 with 1227 students having $92.46 \%$ appearance figures. Grade 3 had 852 students $(90.83 \%$ ), grade 4 had 596 students representing $92.40 \%$, grade 5 having 420 students with $91.30 \%$ and grade 6 with 292 students having a percentage of $86.90 \%$.

D. Bannu sub-centre also showed a positive result with an average of $75.28 \%$ pass percentage. The number of students who successfully passed the $1^{\text {st }}$ grade was $69.59 \%$ (1485 students). Similarly $78.08 \%$ (958 students) in grade $2,77.82 \%$ (663 students) in 'grade 3,83.05\% (495 students) in grade 4, 80.95\% (340 students) in grade 5 and $73.63 \%$ (77 students) were successful in their respective grades.

E. Percentage number of failed students in Bannu sub-centre was recorded to be $24.72 \%$ (1365 students). Grade 1 had the highest percentage of failed students with a percentage of $30.41 \%$ representing student strength of 649 . This is followed by grade 2 with failed percentage of $21.92 \%$ (269 students), grade 3 had $22.18 \%$ (189 students), grade 4 also had a share of failed students with a percentage of $16.95 \%$ (101 students). Grades 5 and 6 had 19.05\% (80 students) and 26.37\% (77 students) respectively.

\section{Table: 5 Female - Grade Wise Annual results and Percentage Analysis}

\begin{tabular}{|c|c|c|c|c|c|c|c|}
\hline & \multicolumn{7}{|c|}{ Number of Students and Percentages } \\
\hline & Grade 1 & Grade 2 & Grade 3 & Grade 4 & Grade 5 & Grade 6 & Total \\
\hline \multirow[t]{2}{*}{ Enrolled } & 3,637 & 2,034 & 1,061 & 617 & 362 & 155 & 7,866 \\
\hline & $46.24 \%$ & $25.86 \%$ & $13.49 \%$ & $7.84 \%$ & $4.60 \%$ & $1.97 \%$ & $100.00 \%$ \\
\hline \multirow[t]{2}{*}{ Dropped } & 330 & 57 & 43 & 13 & 15 & 22 & $\overline{480}$ \\
\hline & $9.07 \%$ & $2.80 \%$ & $4.05 \%$ & $2.11 \%$ & $4.14 \%$ & $14.19 \%$ & $6.10 \%$ \\
\hline \multirow[t]{2}{*}{ Appeared } & 3,307 & 1,977 & 1,018 & 604 & 347 & 133 & 7,386 \\
\hline & $90.93 \%$ & $97.20 \%$ & $95.95 \%$ & $97.89 \%$ & $95.86 \%$ & $85.81 \%$ & $93.90 \%$ \\
\hline \multirow[t]{2}{*}{$\overline{\text { Passed }}$} & 2,273 & 1,332 & 764 & 490 & 308 & 126 & 5,293 \\
\hline & $68.73 \%$ & $67.37 \%$ & $75.05 \%$ & $81.13 \%$ & $88.76 \%$ & $94.74 \%$ & $71.66 \%$ \\
\hline \multirow[t]{2}{*}{ Failed } & 1,034 & 645 & 254 & 114 & 39 & 7 & 2,093 \\
\hline & $31.27 \%$ & $32.63 \%$ & $24.95 \%$ & $18.87 \%$ & $11.24 \%$ & $5.26 \%$ & $28.34 \%$ \\
\hline
\end{tabular}

The project has endeavored to provide education to the girls as well. For the achievement of this aim, it has implemented 40 girl schools all over the province with the exception of Bannu sub-centre. Where due to the cultural restrictions, nor girls' neither co-education school has been established as yet. 
A. The project enrolled 7866 girls for the academic year 1996. This is $12.94 \%$ (refer Table 1) of the total enrollment of the whole program. The percentage of girls dropping out from the formal schooling in different grades stood at $10.13 \%$ (480 girls) (refer Table 1) from the total dropout figures of the program i.e. 4739 students.

B. The results calculated provide a very dismal position of the enrolment of girls in the higher grades. It is clearly observed that from grade 1 onwards, drastic decrease in the enrolment was experienced. 3637 girls were fortunate enough to be allowed to enroll themselves in grade 1 , which is $46.24 \%$ of the total enrolment figures for girls. Grade 2 could only enroll $25.86 \%$, which comes to 2034 girls. Grade 3 experiences a decrease of almost $50 \%$ and the percentage of girls who enroll themselves in this grade is $13.49 \%$ (1061 girls). Grades 4,5 and 6 also show a very steep decline in their enrolment figures with $7.84 \%$ (617 girls), $4.60 \%$ (362 girls) and $1.97 \%$ (155 girls) respectively.

C. An interesting fact that has been observed with regard to the girls schools in general and girls in particular has been their participation in the annual examination. It can be clearly observed that the girls had a far better average of appearing as well as their interest in exams. The percentage of girls who appeared in the $1^{\text {st }}$ grade examination stood at $90.93 \%$ which represents a figure of 3307 number of girls. The percentage of girls who continued and appeared in grade 2 examination was $97.20 \%$ (1977 girls). A total of 1018 girls in grade 3, which is $95.95 \%$ of the girls enrolled, appeared in their final examination. The girls who could make-it to grades 4,5 and 6 had a very impressive participation percentage with $97.89 \%$ (604 girls), 95.86\% (347 girls) and $85.81 \%$ (133 girls) respectively.

D. The pass percentage of girls in grades 1 to 6 has been calculated to be $68.73 \%(2273$ girls) in grade 1, 67.37\% (1332 girls) in grade 2, grade 3 had $75.05 \%$ (764 girls), grade 4 had a pass percentage of $81.13 \%$ (490 girls), $88.75 \%$ (308 girls) was observed in grade 5 and in grade 6,94.74\% (126 girls) successfully passed their annual examination. It is satisfying to note that the passing percentage in grade 6 is among the highest in all the 265 schools that are being implemented by the project.

E. The girls who were unable to qualify to the next grades had almost the same percentage as that of the boys. The total fail average among the girls was recorded to be $28.34 \%$ (2093 girls) of the total enrolled girls i.e. 7866 . The number of girls in grade 1 who could not make it to the next grade was 1034 girls $(31.27 \%)$. Grade 2 had the highest percentage of failed students with $32.63 \%$ (645 girls). Similarly grade 3 had a percentage of $24.95 \%$ (254 girls) who failed the annual examination. Grades 4 and 5 had a fail percentage of $18.87 \%$ (114 girls) and $11.24 \%$ (39 girls). This is followed by grade 6 having only a fail percentage of $5.26 \%$ representing just 7 girls. 


\section{Table: 6 Peshawar - Grade Wise Annual results and Percentage Analysis}

\begin{tabular}{|c|c|c|c|c|c|c|c|}
\hline & \multicolumn{7}{|c|}{ Number of Students and Percentages } \\
\hline & Grade 1 & Grade 2 & Grade 3 & Grade 4 & Grade 5 & Grade 6 & Total \\
\hline \multirow[t]{2}{*}{ Enrolled } & 8,473 & 4,901 & 3,327 & 2,381 & 1,839 & 979 & 21,900 \\
\hline & $38.69 \%$ & $22.38 \%$ & $15.19 \%$ & $10.87 \%$ & $8.40 \%$ & $4.47 \%$ & $100.00 \%$ \\
\hline \multirow[t]{2}{*}{ Dropped } & 658 & 279 & 258 & 177 & 144 & 68 & $1, \overline{584}$ \\
\hline & $7.77 \%$ & $5.69 \%$ & $7.75 \%$ & $7.43 \%$ & $7.83 \%$ & $6.95 \%$ & $7.23 \%$ \\
\hline \multirow[t]{2}{*}{ Appeared } & 7,815 & 4,622 & 3,069 & 2,204 & 1,695 & 911 & 20,316 \\
\hline & $92.23 \%$ & $94.31 \%$ & $92.25 \%$ & $92.57 \%$ & $92.17 \%$ & $93.05 \%$ & $92.77 \%$ \\
\hline \multirow[t]{2}{*}{ Passed } & 4,714 & 3,205 & 2,297 & 1,715 & 1,363 & 755 & 14,049 \\
\hline & $60.32 \%$ & $69.34 \%$ & $74.85 \%$ & $77.81 \%$ & $80.41 \%$ & $82.88 \%$ & $69.15 \%$ \\
\hline \multirow[t]{2}{*}{ Failed } & 3,101 & $\overline{1,417}$ & 772 & 489 & 332 & 156 & 6,267 \\
\hline & $39.68 \%$ & $30.66 \%$ & $25.15 \%$ & $22.19 \%$ & $19.59 \%$ & $17.12 \%$ & $30.85 \%$ \\
\hline
\end{tabular}

Peshawar sub-centre is administrating the largest cluster of schools spread over a large area of Peshawar, greater Peshawar and Mardan districts. The schools that are being looked after by this centre are 67 while most of the camps are located in suburbs of major cities of Peshawar and Mardan. The enrolment rate has been recorded to be an average of 288.16 students per school, which is the highest among all the sub-centres. It has been observed that the registration in the junior grades was very encouraging however the dropout rate in higher grades was at par with the other sub-centres. Therefore the point that is important to be noted is that the number of dropouts recorded (1584 students) is very high considering the urban set-up of majority of the schools coming under the jurisdiction of this centre. It would be appropriate for the project to find out the reasons for such a high number of dropouts in Peshawar sub-centre.

A. A total of 21900 students were enrolled by this sub-centre during the academic year 1996-97. The highest percentage of enrollment was recorded in grade 1 which was $38.69 \%$ (8473 students). This was followed by $22.38 \%$ (4901 students) in grade 2 . The breakdown of enrollment figures for grades $3,4,5$ and 6 were calculated to be $15.19 \%$ (3327 students), $10.87 \%$ (2381 students), $8.40 \%$ (1839 students) and $4.47 \%$ (979 students) respectively.

B. Of the total $7.23 \%$ (1584 students) that dropped out during the same academic year, $7.77 \%$ (658 students) of the total dropped out students belonged to grade 1 . Grade 2 has a dropout rate of $5.69 \%$ (279 students) while grade 3 has a total dropout rate of $7.75 \%$ (258 students). The dropout rate recorded for grade 4 was $7.43 \%$ (177 students). At the same time $7.83 \%$ (144 students) were recorded to have been dropped out in grade 5 . This was followed by grade 6 , which had a dropout rate of $6.95 \%$ (68 students).

C. The Peshawar sub-centre also had an impressive rate of $92.77 \%$ covering a figure of 20316 students who appeared in their annual examinations. The percentage of students in grade 1 who appeared in annual examinations was $92.23 \%(7815$ students). Grade 2 had an appearance rate of $94.31 \%$ (4622 students) while $92.25 \%$ (3069 students) of grade 3 made themselves available for the annual examinations. The appearance rate of grade 4 stood at $92.57 \%$ (2204 students) followed by grade 5 which had an appearance rate of $92.17 \%$ (1695 students). Grade 6 recorded the 
highest percentage of $93.05 \%$ (911 students) of the enrolled students who appeared in their annual examinations.

D. The passed percentage, after the compilation of the annual results, has been recorded to be $69.15 \%$, which represents a passing figure of 14049 students. The percentage of students who passed their grade 1 has been $60.32 \%$ (4714 students). Grade 2 had a passing percentage of $69.34 \%$ (3205 students). Grade 3, 4 and 5 had a passing percentage of $74.85 \%$ (2297 students), $77.81 \%$ (1715 students) and $80.41 \%$ (1363 students) respectively. Grade 6 , as in the other centers also showed a high passing percentage which was recorded to be $82.88 \%$ ( 755 students).

E. The percentage of students who could not make it to the next grade was recorded to be $30.85 \%$ (6267 students) of the total number of students who appeared in the annual examinations. Grade 1 again had the highest percentage of $39.68 \%$ representing a strength of 3101 students who were unfortunate to fail in their annual exam. Grade 2 had the second highest fail percentage of $30.66 \%$ (1417 students). The percentage. of students who failed in their exams decreased with each higher grade, hence grade had $25.15 \%$ (772 students), grade 4 had $22.19 \%$ (489 students) and 19.59\% (332 students) were recorded for grade 5 . In the end grade 6 had only $17.12 \%$ (156 students) who could not make it to the higher grade.

Table: 7 Thall - Grade Wise Annual results and Percentage Analysis

\begin{tabular}{|c|c|c|c|c|c|c|c|}
\hline & \multicolumn{7}{|c|}{ Number of Students and Percentages } \\
\hline & Grade 1 & Grade 2 & Grade 3 & Grade 4 & Grade 5 & Grade 6 & Total \\
\hline \multirow[t]{2}{*}{ Enrolled } & 3,572 & 2,379 & 1,697 & 1,227 & 860 & 609 & 10,344 \\
\hline & $34.53 \%$ & $23.00 \%$ & $16.41 \%$ & $11.86 \%$ & $8.31 \%$ & $5.89 \%$ & $100.00 \%$ \\
\hline \multirow[t]{2}{*}{ Dropped } & 241 & 126 & 103 & 77 & 69 & 54 & 670 \\
\hline & $6.75 \%$ & $5.30 \%$ & $6.07 \%$ & $6.28 \%$ & $8.02 \%$ & $8.87 \%$ & $6.48 \%$ \\
\hline \multirow[t]{2}{*}{ Appeared } & 3,331 & 2,253 & 1,594 & 1,150 & 791 & 555 & 9,674 \\
\hline & $93.25 \%$ & $94.70 \%$ & $93.93 \%$ & $93.72 \%$ & $91.98 \%$ & $91.13 \%$ & $93.52 \%$ \\
\hline \multirow[t]{2}{*}{ Passed } & 2,264 & 1,757 & 1,315 & 933 & 699 & 495 & 7,463 \\
\hline & $67.97 \%$ & $77.98 \%$ & $82.50 \%$ & $81.13 \%$ & $88.37 \%$ & $89.19 \%$ & $77.14 \%$ \\
\hline \multirow[t]{2}{*}{ Failed } & 1,067 & 496 & 279 & 217 & 92 & 60 & 2,211 \\
\hline & $32.03 \%$ & $22.02 \%$ & $17.50 \%$ & $18.87 \%$ & $11.63 \%$ & $10.81 \%$ & $22.86 \%$ \\
\hline
\end{tabular}

A. Thall sub-centre covered a total of 41 schools with a total enrollment figure of 10344 students. The highest percentage of student enrollment was recorded in grade 1 having a percentage figure of $34.53 \%$ covering a student strength of 3572 . This was followed by grade 2 having $23.00 \%$ (2379 students) enrollment strength. A marked reduction in the enrollment figures was experienced for grades $3,4,5$ and 6 having a reduced enrollment percentages of $16.41 \%$ (1697 students), $11.86 \%$ (1227 students), $8.31 \%$ (860 students) and $5.89 \%$ (609 students) respectively.

B. The total average dropout rate recorded for Thall sub-centre has been $6.48 \%(670$ students) in the academic year 1996-97. The lowest dropout rate was recorded for grade 2 having a percentage of 5.30\% (126 students) while the highest dropout rate for any grade was recorded for grade 6 having $8.87 \%$ (54 students) of the total students enrolled these grades. The rest of the grades had a dropout percentage of 
$6.75 \%$ (241 students), 6.07\% (103 students), 6.28\% (77 students) and 8.02\% (69 students) for grades $1,3,4$ and 5 respectively.

C. The respective sub-centre also showed an impressive average rate of students appearing in their annual examinations which stood at $93.52 \%$ (9674 students). The students of grade 2 showed the highest interest in appearing for their annual examinations which was $94.70 \%$ (2253 students) with grade 3 trailing behind with a percentage of $93.93 \%$ (1594 students). Grade 1 and grade 4 had $93.25 \%$ ( 3331 students) and $93.72 \%$ (1150 students) respectively who took their annual examinations seriously. Grade 5 had a slightly lower appearance rate of $91.98 \%$ (791 students) while grade 6 had almost an equal appearance percentage of $91.13 \%$ (555 students).

D. An average pass percentage of $77.14 \%$ ( 7463 students) was recorded against 9674 students who appeared in their annual examination. Of those who passed from their grades, grade 6 had the highest pass percentage of $89.19 \%$ (495 students) out of a total of 555 students. This was followed by grade 5 having a pass percentage of $88.37 \%$ (699 students). It was again observed that grade 1 had the lowest pass percentage which was $67.97 \%$ (2264 students). Grades 2, 3 and 4 had a pass percentage of $77.98 \%$ (1757 students), $82.50 \%$ (1315 students) and $81.13 \%$ (933 students) respectively.

E. Similarly the students failing in their exams has been recorded to the $22.86 \%$ (2211 students) of the total number of students that appeared in their examinations. The students in grade 6 had the lowest fail percentage of $10.81 \%(60)$ students while grade 1 at the highest with $32.03 \%$ (1067 students) who could not make it to the next grade. The students belonging to grade 2 had a fail percentage of $22.02 \%$ (496 students) while $17.50 \%$ (279 students) was recorded for grade 3 . At the same time grade 4 and 5 show a fail percentage of $18.87 \%$ (217 students) and $11.63 \%$ (92 students) respectively.

\section{Table: 8 Timargara - Grade Wise Annual results and Percentage Analysis}

\begin{tabular}{|c|c|c|c|c|c|c|c|}
\hline & \multicolumn{7}{|c|}{ Number of Students and Percentages } \\
\hline & Grade 1 & Grade 2 & Grade 3 & Grade 4 & Grade 5 & Grade 6 & Total \\
\hline \multirow[t]{2}{*}{ Enrolled } & 3,101 & 1,374 & 1,044 & 749 & 543 & 366 & $\overline{7,177}$ \\
\hline & $43.21 \%$ & $19.14 \%$ & $14.55 \%$ & $10.44 \%$ & $7.57 \%$ & $5.10 \%$ & $100.00 \%$ \\
\hline \multirow[t]{2}{*}{ Dropped } & 334 & 115 & 115 & 87 & 63 & 48 & 762 \\
\hline & $10.77 \%$ & $8.37 \%$ & $11.02 \%$ & $11.62 \%$ & $11.60 \%$ & $13.11 \%$ & $10.62 \%$ \\
\hline \multirow[t]{2}{*}{ Appeared } & 2,767 & 1,259 & 929 & 662 & 480 & 318 & $\overline{6,415}$ \\
\hline & $89.23 \%$ & $91.63 \%$ & $88.98 \%$ & $88.38 \%$ & $88.40 \%$ & $86.89 \%$ & $89.38 \%$ \\
\hline \multirow[t]{2}{*}{ Passed } & 1,766 & 931 & 698 & 521 & 361 & 242 & $\overline{4,519}$ \\
\hline & $63.82 \%$ & $73.95 \%$ & $75.13 \%$ & $78.70 \%$ & $75.21 \%$ & $76.10 \%$ & $70.44 \%$ \\
\hline \multirow[t]{2}{*}{ Failed } & 1,001 & 328 & 231 & 141 & 119 & 76 & 1,896 \\
\hline & $36.18 \%$ & $26.05 \%$ & $24.87 \%$ & $21.30 \%$ & $24.79 \%$ & $23.90 \%$ & $29.56 \%$ \\
\hline
\end{tabular}

A. A total of 7177 students were enrolled in the 38 schools that were looked after by the Timargara sub-centre during the academic year 1996-97. The highest percentage of enrolment of students was recorded in grade 1 with $43.21 \%$ (3101 students) while the 
lowest enrolment figures were recorded for grade 6 with $5.10 \%$ (366 students) out of the total enrolment figures. The number of students who got themselves enrolled reduced by each higher grade i.e. in grade 2 the enrolment figures fell down to $19.14 \%$ (1374 students). In grade 3 the percentage was further reduce to $14.55 \%$ (1044 students). Grade 4 had a percentage of $10.44 \%$ ( 749 students) while grade 5 had a total enrolment figure of $7.57 \%$ (543 students).

B. The dropout rate recorded for thi's centre was $10.62 \%$ (762 students). The highest percentage of students from any grade, who dropped out, belonged to grade 6 having $13.11 \%$ (48 students). Similarly grade 1 had $10.77 \%$ (334 students), grade 2 had $8.37 \%$ (115 students), grade 3 had $11.02 \%$ (115 students) grade 4 had $11.62 \%$ (87 students) and grade 5 had $11.60 \%$ (63 students) who dropped out during their course of studies.

C. The average rate of students appearing in their examinations was calculated to be $89.38 \%$ ( 6415 students) out of a total of 7177 students that were enrolled during the academic year 1996-97. The students appearance rate in the examinations for each respective grade has been calculated to be $89.23 \%$ ( 2767 students) in grade $1,91.63 \%$ (1259 students) in grade 2, 88.98\% (929 students) in grade 3, 88.38\% (662 students) in grade $4,88.40 \%$ (480 students) grade 5 and $86.89 \%$ (318 students) in grade 6.

D. The average pass percentage of students after the compilation of the results was $70.44 \%$ (4519 students). The pass percentage of the students increased with each higher grade, thus grade 1 had pass percentage of $63.82 \%$ (1766 students). Grade 2 showed an improvement by having $73.95 \%$ (931 students) of its total enrolled students to move into the next higher grade. Grade 3 had a pass percentage of $75.13 \%$ (698 students) while grade 4 had $78.70 \%$ (521 students) who got a move over to the next grades. Similarly grade 5 had a pass percentage of $75.21 \%$ (361 students) and grade 6 showed a result of $76.10 \%$ (242 students).

E. The respective sub-centre, at the same time, recorded a percentage of $29.56 \%$ students who could not make it to the next grade. The breakdown of grades that recorded failed students was $36.18 \%$ (1001 students) in grade $1,26.05 \%$ (328 students) in grade 2, $24.87 \%$ (231 students) in grade 3, 21.30\% (141 students) in grade 4, 24.79\% (119 students) in grade 5 and $23.90 \%$ ( 76 students) in grade 6.

\section{Table: 9 Annual results for 1996-97 and Percentage Analysis for all Grades (Sub-Centre Level)}

\begin{tabular}{|c|c|c|c|c|c|c|c|c|c|}
\hline & \multicolumn{5}{|c|}{ Number of Students } & \multicolumn{4}{|c|}{ Percent of Students } \\
\hline & Enrolled & Dropped & Appeared & Passed & Failed & Dropped & Appeared & Passed & Failed \\
\hline Abbotabad & 7,558 & 805 & 6,753 & 4,951 & 1,802 & $10.65 \%$ & $89.35 \%$ & $73.32 \%$ & $26.68 \%$ \\
\hline Bannu & 5,959 & 438 & 5,521 & 4,156 & 1,365 & $7.35 \%$ & $92.65 \%$ & $75.28 \%$ & $24.72 \%$ \\
\hline Female & 7,866 & 480 & 7,386 & 5,293 & 2,093 & $6.10 \%$ & $93.90 \%$ & $71.66 \%$ & $28.34 \%$ \\
\hline Peshawar & 21,900 & 1,584 & 20,316 & 14,049 & 6,267 & $7.23 \%$ & $92.77 \%$ & $69.15 \%$ & $30.85 \%$ \\
\hline Thall & 10,344 & 670 & 9,674 & 7,463 & 2,211 & $6.48 \%$ & $93.52 \%$ & $77.14 \%$ & $22.86 \%$ \\
\hline Timargara & 7,177 & 762 & 6,415 & 4,519 & 1,896 & $10.62 \%$ & $89.38 \%$ & $70.44 \%$ & $29.56 \%$ \\
\hline Total & 60,804 & 4,739 & 56,065 & 40,431 & 15,634 & $7.79 \%$ & $92.21 \%$ & $72.11 \%$ & $27.89 \%$ \\
\hline
\end{tabular}


A. Comparing the analysis of the annual result percentage for all the grades on subcentre levels, it is satisfying to know that the average rate of students appearing in their annual examinations was $92.21 \%$ out of which $72.11 \%$ were successful and were admitted in to the next higher grades. The average percentage of students failing in their examinations was $27.89 \%$. Similarly the dropout rate for the whole project was $7.79 \%$ of the total enrolment figures.

B. It can be clearly observed that sub-centre Thall had the highest pass percentage (77.14\%) of students passing in their annual examination while Peshawar sub-centre at the lowest figure of $69.15 \%$. The highest students appearing rate was $93.90 \%$ among the female centre while Abbotabad had the lowest with $89.35 \%$ of the total students that were enrolled during the academic year 1996-97.

C. Analyzing the results of students who failed in the examinations, it is observed that Peshawar sub-centre had the highest rate of failure, which is $30.85 \%$ while sub-center Thall at the lowest failure percentage of $22.86 \%$. Similarly female sub-centre had the lowest dropout rate of $6.10 \%$ followed by sub-center Thall with $6.48 \%$ while Abbotabad sub-centre had the highest dropout figures of $10.65 \%$ and Timargara trailing behind with $10.62 \%$.

\section{Table: 10 Annual results for 1996-97 and Percentage Analysis for Grade} One (Sub-Centre Level)

\begin{tabular}{|c|c|c|c|c|c|c|c|c|c|}
\hline & \multicolumn{5}{|c|}{ Number of Students } & \multicolumn{4}{|c|}{ Percent of Students } \\
\hline & Enrolled & Dropped & Appeared & Passed & Failed & Dropped & Appeared & Passed & Failed \\
\hline Abbotabad & 2,640 & 253 & 2,387 & 1,517 & 870 & $9.58 \%$ & $90.42 \%$ & $63.55 \%$ & $36.45 \%$ \\
\hline Bannu & 2,253 & 119 & 2,134 & 1,485 & 649 & $5.28 \%$ & $94.72 \%$ & $69.59 \%$ & $30.41 \%$ \\
\hline Female & 3,637 & 330 & 3,307 & 2,273 & 1,034 & $9.07 \%$ & $90.93 \%$ & $68.73 \%$ & $31.27 \%$ \\
\hline Peshawar & 8,473 & 658 & 7,815 & 4,714 & 3,101 & $7.77 \%$ & $92.23 \%$ & $60.32 \%$ & $39.68 \%$ \\
\hline Thall & 3,572 & 241 & 3,331 & 2,264 & 1,067 & $6.75 \%$ & $93.25 \%$ & $67.97 \%$ & $32.03 \%$ \\
\hline Timargara & 3,101 & 334 & 2,767 & 1,766 & 1,001 & $10.77 \%$ & $89.23 \%$ & $63.82 \%$ & $36.18 \%$ \\
\hline Total & 23,676 & 1,935 & 21,741 & 14,019 & 7,722 & $8.17 \%$ & $91.83 \%$ & $64.48 \%$ & $35.52 \%$ \\
\hline
\end{tabular}

A. The total percentage of the students that appeared in grade 1 annual examinations from all the sub-centres was $91.83 \%$ with the passing percentage of $64.48 \%$. Bannu sub-centre recorded the highest appearing rate of $94.72 \%$ as well as the highest percentage of students passing their examinations, which was $69.95 \%$ while Timargara had an appearance rate of $89.23 \%$ with $63.82 \%$ has the passing percentage. The overall percentage of students who failed in the grade 1 examination stood at $35.52 \%$ with a dropout rate of $8.17 \%$.

B. The highest percentage of students failing in the examinations of grade 1 was recorded for Peshawar sub-centre with $39.68 \%$ students not making to the next higher grade. Abbotabad and Timargara sub-centres also had a higher fail percentage of $36.45 \%$ and $36.18 \%$ respectively. 
Table: 11 Annual results for 1996-97 and Percentage Analysis for Grade Two (Sub-Centre Level)

\begin{tabular}{lrrrrr|rrrr} 
& \multicolumn{5}{c|}{ Number of Students } & \multicolumn{4}{|c}{ Percent of Students } \\
\cline { 2 - 9 } & Enrolled & Dropped Appeared & Passed & Failed & Dropped & Appeared & Passed & Failed \\
\hline Abbotabad & 1,765 & 179 & 1,586 & 1,186 & $\mathbf{4 0 0}$ & $10.14 \%$ & $89.86 \%$ & $\mathbf{7 4 . 7 8 \%}$ & $\mathbf{2 5 . 2 2 \%}$ \\
Bannu & 1,327 & 100 & 1,227 & 958 & 269 & $7.54 \%$ & $92.46 \%$ & $78.08 \%$ & $21.92 \%$ \\
Female & 2,034 & 57 & 1,977 & 1,332 & 645 & $2.80 \%$ & $97.20 \%$ & $67.37 \%$ & $32.63 \%$ \\
Peshawar & $\mathbf{4 , 9 0 1}$ & 279 & $\mathbf{4 , 6 2 2}$ & 3,205 & 1,417 & $5.69 \%$ & $94.31 \%$ & $69.34 \%$ & $30.66 \%$ \\
Thall & 2,379 & 126 & 2,253 & 1,757 & 496 & $\mathbf{5 . 3 0 \%}$ & $94.70 \%$ & $\mathbf{7 7 . 9 8 \%}$ & $\mathbf{2 2 . 0 2 \%}$ \\
Timargara & 1,374 & 115 & 1,259 & 931 & 328 & $\mathbf{8 . 3 7 \%}$ & $91.63 \%$ & $\mathbf{7 3 . 9 5 \%}$ & $\mathbf{2 6 . 0 5 \%}$ \\
\hline Total & 13,780 & $\mathbf{8 5 6}$ & 12,924 & 9,369 & 3,555 & $6.21 \%$ & $93.79 \%$ & $\mathbf{7 2 . 4 9 \%}$ & $\mathbf{2 7 . 5 1 \%}$ \\
\hline
\end{tabular}

A. The percentage of students appearing in the annual examination for grade 2 on all sub-centre levels was $93.79 \%$ with the passing percentage of $72.49 \%$. The average dropouts' rate from all the sub-centres hence remained $6.21 \%$.

B. The highest appearance rate among the sub-centres was the highest with $97.20 \%$ in the female sub-centre and the lowest in Abbotabad with $89.86 \%$. Bannu sub-centres showed the highest pass percentage for grade 2 at $78.08 \%$ while Peshawar sub-centre could only claim $69.34 \%$ of the total students to have passed their annual examinations.

C. Female sub-centre showed a very small reduction in the dropout rate for grade 2 which was $2.80 \%$ while Abbotabad sub-centre had the highest dropout figures of $10.14 \%$. Similarly female sub-centre with $32.16 \%$ and Peshawar sub-centre with $30.66 \%$ showed the highest percentage of students failing in their final examinations.

\section{Table: 12 Annual results for 1996-97 and Percentage Analysis for Grade Three (Sub-Centre Level)}

\begin{tabular}{|c|c|c|c|c|c|c|c|c|c|}
\hline & \multicolumn{5}{|c|}{ Number of Students } & \multicolumn{4}{|c|}{ Percent of Students } \\
\hline & Enrolled & Dropped & Appeared & Passed & Failed & Dropped & Appeared & Passed & Failed \\
\hline Abbotabad & 1,190 & 117 & 1,073 & 840 & 233 & $9.83 \%$ & $90.17 \%$ & $78.29 \%$ & $21.71 \%$ \\
\hline Bannu & 938 & 86 & 852 & 663 & 189 & $9.17 \%$ & $90.83 \%$ & $77.82 \%$ & $22.18 \%$ \\
\hline Female & 1,061 & 43 & 1,018 & 764 & 254 & $4.05 \%$ & $95.95 \%$ & $75.05 \%$ & $24.95 \%$ \\
\hline Peshawar & 3,327 & 258 & 3,069 & 2,297 & 772 & $7.75 \%$ & $92.25 \%$ & $74.85 \%$ & $25.15 \%$ \\
\hline Thall & 1,697 & 103 & 1,594 & 1,315 & 279 & $6.07 \%$ & $93.93 \%$ & $82.50 \%$ & $17.50 \%$ \\
\hline Timargara & 1,044 & 115 & 929 & 698 & 231 & $11.02 \%$ & $88.98 \%$ & $75.13 \%$ & $24.87 \%$ \\
\hline Total & 9,257 & 722 & 8,535 & 6,577 & 1,958 & $7.80 \%$ & $92.20 \%$ & $77.06 \%$ & $22.94 \%$ \\
\hline
\end{tabular}

A. Analysis of the results for grade 3 in all the sub-centres show an appearance rate of $92.20 \%$ while the pass percentage among all the students in all the sub-centres was $77.06 \%$. The percentage analysis for the students failing as well as dropping out stood at $22.94 \%$ and $7.80 \%$ respectively.

B. Female sub-centre recorded the highest appearance percentage of $95.59 \%$ while the lowest was in Timargara with $88.98 \%$. However Thall sub-centre claimed the highest 
percentage of $82.50 \%$ of students who passed their annual examinations with Peshawar sub-centre having the lowest percentage of $74.85 \%$.

Table: 13 Annual results for 1996-97 and Percentage Analysis for Grade Four (Sub-Centre Level)

\begin{tabular}{lrrrrr|rrrr} 
& \multicolumn{5}{c|}{ Number of Students } & \multicolumn{4}{c}{ Percent of Students } \\
\cline { 2 - 9 } & Enrolled & Dropped & Appeared & Passed & Failed & Dropped & Appeared & Passed & Failed \\
\hline Abbotabad & 843 & 89 & 754 & 614 & 140 & $10.56 \%$ & $89.44 \%$ & $81.43 \%$ & $18.57 \%$ \\
Bannu & 645 & 49 & 596 & 495 & 101 & $7.60 \%$ & $92.40 \%$ & $83.05 \%$ & $16.95 \%$ \\
Female & 617 & 13 & 604 & 490 & 114 & $2.11 \%$ & $97.89 \%$ & $81.13 \%$ & $18.87 \%$ \\
Peshawar & 2,381 & 177 & 2,204 & 1,715 & 489 & $7.43 \%$ & $92.57 \%$ & $77.81 \%$ & $22.19 \%$ \\
Thall & 1,227 & 77 & 1,150 & 933 & 217 & $6.28 \%$ & $93.72 \%$ & $81.13 \%$ & $18.87 \%$ \\
Timargara & 749 & 87 & 662 & 521 & 141 & $11.62 \%$ & $88.38 \%$ & $78.70 \%$ & $21.30 \%$ \\
\hline Total & 6,462 & 492 & 5,970 & 4,768 & 1,202 & $7.61 \%$ & $92.39 \%$ & $79.87 \%$ & $20.13 \%$ \\
\hline
\end{tabular}

A. Results compiled for grade 4 for all the sub-centres indicate an appearance rate of $92.39 \%$ with a passing average of $79.87 \%$. The average percentage of students failing in their examinations for grade 4 has been calculated to be $20.13 \%$. Similarly the average dropout rate for the same grade during the academic year was $7.61 \%$.

B. Female sub-centre recorded the highest appearance rate of $97.89 \%$ and at the same time had the lowest dropout rate of $2.11 \%$. Timargara sub-centre at the lowest appearance rate of $88.38 \%$ including the highest dropout rate of $11.62 \%$.

C. Bannu sub-centre had the highest pass percentage of $83.05 \%$ for grade 4 while Peshawar sub-centre had the lowest with $77.81 \%$.

Table: 14 Annual results for 1996-97 and Percentage Analysis for Grade Five (Sub-Centre Level)

\begin{tabular}{lrrrrr|rrrr} 
& \multicolumn{5}{c|}{ Number of Students } & \multicolumn{4}{c}{ Percent of Students } \\
\cline { 2 - 9 } & Enrolled & Dropped & Appeared & Passed & Failed & Dropped & Appeared & Passed & Failed \\
\hline Abbotabad & 664 & 97 & 567 & 455 & 112 & $14.61 \%$ & $85.39 \%$ & $80.25 \%$ & $19.75 \%$ \\
Bannu & 460 & 40 & 420 & 340 & 80 & $8.70 \%$ & $91.30 \%$ & $80.95 \%$ & $19.05 \%$ \\
Female & 362 & 15 & 347 & 308 & 39 & $4.14 \%$ & $95.86 \%$ & $88.76 \%$ & $11.24 \%$ \\
Peshawar & 1,839 & 144 & 1,695 & 1,363 & 332 & $7.83 \%$ & $92.17 \%$ & $80.41 \%$ & $19.59 \%$ \\
Thall & 860 & 69 & 791 & 699 & 92 & $8.02 \%$ & $91.98 \%$ & $88.37 \%$ & $11.63 \%$ \\
Timargara & 543 & 63 & 480 & 361 & 119 & $11.60 \%$ & $88.40 \%$ & $75.21 \%$ & $24.79 \%$ \\
\hline Total & 4,728 & 428 & 4,300 & 3,526 & 774 & $9.05 \%$ & $90.95 \%$ & $82.00 \%$ & $18.00 \%$ \\
\hline
\end{tabular}

A. The average appearance rate for grade 5 for all the sub-centres has been calculated to be $90.95 \%$ with a pass average of $82.00 \%$. The average dropout rate for all the subcentres came to $9.05 \%$ with a fail percentage of $18.00 \%$ for this grade.

B. Grade 5 of the female sub-centre recorded the highest percentage of appearance rate $95.86 \%$ while the lowest was calculated for Abbotabad sub-centre having 85.39\%. 
C. Female sub-centre again had the highest pass percentage as well as the lowest dropout rate of $88.76 \%$ and $4.14 \%$ respectively. The lowest pass percentage for this grade was recorded for Timargara sub-centre having $75.21 \%$ while Abbotabad had the highest dropout rate of $14.61 \%$.

D. $24.79 \%$ of the students in Timargara sub-centre failed in their annual examinations, which is the highest for this grade while female sub-centre at the lowest failure rate of $11.24 \%$.

Table: 15 Annual results for 1996-97 and Percentage Analysis for Grade Six (Sub-Centre Level)

\begin{tabular}{lrrrrr|rrrr} 
& \multicolumn{5}{c}{ Number of Students } & \multicolumn{4}{c}{ Percent of Students } \\
\cline { 2 - 10 } & Enrolled & Dropped & Appeared & Passed & Failed & Dropped & Appeared & Passed & Failed \\
\hline Abbotabad & $\mathbf{4 5 6}$ & $\mathbf{7 0}$ & $\mathbf{3 8 6}$ & 339 & $\mathbf{4 7}$ & $\mathbf{1 5 . 3 5 \%}$ & $\mathbf{8 4 . 6 5 \%}$ & $\mathbf{8 7 . 8 2 \%}$ & $12.18 \%$ \\
Bannu & 336 & $\mathbf{4 4}$ & 292 & 215 & $\mathbf{7 7}$ & $\mathbf{1 3 . 1 0 \%}$ & $\mathbf{8 6 . 9 0 \%}$ & $\mathbf{7 3 . 6 3 \%}$ & $\mathbf{2 6 . 3 7 \%}$ \\
Female & 155 & 22 & 133 & 126 & 7 & $14.19 \%$ & $\mathbf{8 5 . 8 1 \%}$ & $\mathbf{9 4 . 7 4 \%}$ & $5.26 \%$ \\
Peshawar & 979 & 68 & 911 & 755 & 156 & $6.95 \%$ & $93.05 \%$ & $\mathbf{8 2 . 8 8 \%}$ & $17.12 \%$ \\
Thall & 609 & 54 & 555 & $\mathbf{4 9 5}$ & 60 & $\mathbf{8 . 8 7} \%$ & $91.13 \%$ & $\mathbf{8 9 . 1 9 \%}$ & $10.81 \%$ \\
Timargara & 366 & $\mathbf{4 8}$ & $\mathbf{3 1 8}$ & 242 & $\mathbf{7 6}$ & $13.11 \%$ & $\mathbf{8 6 . 8 9 \%}$ & $\mathbf{7 6 . 1 0 \%}$ & $\mathbf{2 3 . 9 0 \%}$ \\
\hline Total & 2,901 & 306 & 2,595 & $\mathbf{2 , 1 7 2}$ & $\mathbf{4 2 3}$ & $10.55 \%$ & $\mathbf{8 9 . 4 5 \%}$ & $\mathbf{8 3 . 7 0 \%}$ & $16.30 \%$ \\
\hline
\end{tabular}

A. The results and the figures compiled for the academic year 1996-97 for grade 6 on all the sub-centres levels indicate an average appearance rate of $89.45 \%$ with $83.70 \%$ of the students passing their annual examination of grade 6 . The same grade showed a drop rate of $10.55 \%$ of the total enrolled students. $16.30 \%$ of the students were also recorded to have failed in their exams.

B. It is again clearly observed that female sub-centre had the highest pass percentage of $94.74 \%$ while Bannu sub-centre with the lowest could only claim $73.63 \%$ of students to have been successful in the annual examination.

C. Peshawar sub-centre had the lowest dropout rate of $6.95 \%$ for grade 6 while Abbotabad was with the highest percentage of $15.35 \%$. However female sub-centre had the lowest percentage of $5.26 \%$ while the highest was in Bannu with $26.37 \%$.

\section{Table: 16 Annual results for 1996-97, Gender Distribution for Boys (Sub-} Centre Lekel)

\begin{tabular}{lrrrrrrr} 
& \multicolumn{7}{c}{ Number of Boys Students in Boys Schools } \\
\cline { 2 - 8 } & Abbotabad & Bannu & Female & Peshawar & Thall Timergara & Total \\
\hline Enrolled & 7,331 & 5,959 & 0 & 21,243 & 10,344 & 6,962 & 51,839 \\
Dropped & 781 & 438 & 0 & 1,536 & 670 & 739 & 4,164 \\
Appeared & 6,550 & 5,521 & 0 & 19,707 & 9,674 & 6,223 & 47,675 \\
Passed & 4,802 & 4,156 & 0 & 13,628 & 7,463 & 4,383 & 34,432 \\
Failed & 1,748 & 1,365 & 0 & 6,079 & 2,211 & 1,839 & 13,242 \\
\hline
\end{tabular}

Table 16 presents the gender distribution of boys on the sub-centre level. It is to be understood that no boys' students were enrolled in any of the Afghan refugees girls schools which fall under the jurisdiction of the female sub-centre. However table 17 which represents 
the distribution and enrolment of girls in various sub-centres of the project, it is clearly observed that with the exception of Bannu and Thall sub-centres, girls were enrolled in the boys schools of the remaining sub-centres except the schools that were coming under the female sub-centre of the project. The figures compiled for girls for the remaining sub-centres namely Abbotabad, Peshawar and Timargara have been calculated as $3 \%$ of the total of the respective sub-centre.

Table: 17 Annual results for 1996-97, Gender Distribution for Girls (SubCentre Level)

\begin{tabular}{lrrrrrrr} 
& \multicolumn{7}{c}{ Number of Girls Students in Co-education and Girls Schools } \\
\cline { 2 - 8 } & Abbotabad & Bannu & Female & Peshawar & Thall Timargara & Total \\
\hline Enrolled & 227 & 0 & 7,866 & 657 & 0 & 215 & 8,965 \\
Dropped & 24 & 0 & 480 & 48 & 0 & 23 & 575 \\
Appeared & 203 & 0 & 7,386 & 609 & 0 & 192 & 8,390 \\
Passed & 149 & 0 & 5,293 & 421 & 0 & 136 & 5,999 \\
Failed & 54 & 0 & 2,093 & 188 & 0 & 57 & 2,392 \\
\hline
\end{tabular}

\section{Table: 18 Annual results for 1996-97, Comparative Gender Performance}

\begin{tabular}{|c|c|c|c|c|c|c|c|c|c|}
\hline \multirow[t]{2}{*}{$\prime$} & \multicolumn{5}{|c|}{ Number of Students } & \multicolumn{4}{|c|}{ Percent of Students } \\
\hline & Enrolled & Dropped & Appeared & Passed & Failed & Dropped & Appeared & Passed & Failed \\
\hline Boys & 51,839 & 4,164 & 47,675 & 34,432 & 13,242 & $8.03 \%$ & $91.97 \%$ & $72.22 \%$ & $\overline{27.78 \%}$ \\
\hline Girls & 8,965 & 575 & 8,390 & 5,999 & 2,392 & $6.41 \%$ & $93.59 \%$ & $71.50 \%$ & $28.51 \%$ \\
\hline Total & 60,804 & 4,739 & 56,065 & 40,431 & 15,634 & $7.79 \%$ & $92.21 \%$ & $72.11 \%$ & $27.89 \%$ \\
\hline
\end{tabular}

Comparing the gender performance it is observed that the girls had a higher appearance percentage of $93.59 \%$ as comparing to boys which had an average appearance of $91.97 \%$. However the pass percentage is the other way around with boys leading at $72.22 \%$ and the girls trailing at $71.50 \%$ the dropout rate among the boys and girls has been calculated to the average of $8.03 \%$ and $6.41 \%$ respectively.

Table: 19 Enrolment per school Sub-Centre Level

\begin{tabular}{lrrr} 
& \multicolumn{3}{c}{ Number of } \\
\cline { 2 - 4 } Sub-centres & schools & $\begin{array}{r}\text { Enrolled } \\
\text { Students }\end{array}$ & $\begin{array}{r}\text { Students per } \\
\text { school }\end{array}$ \\
\cline { 2 - 4 } Abbotabad & 30 & 7,558 & 252.93 \\
Bannu & 40 & 5,959 & 148.98 \\
Female & 40 & 7,866 & 196.65 \\
Peshawar & 76 & 21,900 & 288.16 \\
Thall & 41 & 10,344 & 252.29 \\
Timargara & 38 & 7,177 & 188.87 \\
\hline Total & 265 & 60,804 & 229.45 \\
\hline
\end{tabular}

The enrolment figures of students per schools at sub-centres level has also been calculated which gives an average figure of 229.45 students per schools. The highest ratio of students per school has been recorded in Peshawar sub-centre having an average of 288.16 students per school while the lowest is in Bannu with 148.98 students per school. 


\section{Findings and Suggestions}

\section{Examinations system}

- It has been persistently observed that the annual examinations were conducted in the respective schools by the head teacher and teachers. Since the teaching staff are not properly trained nor are they expert in designing the examination papers, therefore, no standard criteria was adopted for conducting the examinations, thus the results obtained from different sub-centres as well as various schools have a varying degree of results. Hence the first step suggested is to develop a standardized examination system, including the formulation of the exam papers as well as the mode of checking the answers of the students. This should be adopted and implemented in all 265 COPE schools for the academic year 1997-98.

- It is also suggested that if the exams are centrally designed for grade 6 and are conducted . by the trained staff, then the quality could be further enhanced and the student of grade 6 would be able to easily get admission in higher classes.

\section{Suggested patterns for examinations}

- Four main examinations can be possibly designed for each academic year representing four terms from grade 3 onwards. In the first three term examinations, students can be awarded with a maximum of 20 credits (marks) while the final term examination can have the remaining of 40 credits (marks). This exercise would enable the project to maintain the standard of education and at the same time further increase the pass percentage.

- Similarly for grade 1 and $2,65 \%$ to $75 \%$ of credits (marks) can be awarded on monthly or bi-monthly exercises/tests on the basis of their performance and the remaining $25 \%$ of credits (marks) can be adjusted in their final examinations. Since the students would be familiar with the tests/exams so they would easily adopt the formal examination exercises in the next higher grades. Thus the students in the primary level would know that their promotion to the next higher grades would depend on their regular appearance in the yearly terms.

\section{Administration}

- It has been acutely felt that Peshawar sub-centre covers a large number of schools for which there is one Master Trainer in-charge. The logistics and the number of schools and teachers make it necessary to split this centre into two operational zones. The administrative structure would remain the same but the responsibilities on the operational levels could be distributed for enhanced qualitative results. This sub-centre could be split into Peshawar and Mardan or Peshawar and greater Peshawar with an average of 40 schools each having one MT responsible for each zone without entailing extra financial considerations.

- During the process of Data collection, it was realized that the duties of Master Trainers, Administrators and Community Motivators should be more harmonized so that any future exercise can be more easily conducted. 


\section{Community Participation}

- After the compilation of the results, it was noticed that wherever the School Management Committees had been established the enrolment had increased and helped the project to brought the dropout rate to the minimum. In the Female sub-centre schools, it is highly felt that the community work and participation should be the top priority for further improving girl's education among the Afghan refugees.

- In Bannu sub-centre, it is highly suggested that female schools along with their management committees should also be established as soon as possible since there are no schools available for girls at present.

\section{School profile}

It is also observed that there is no comprehensive computer based school profile. The same should be available for all the COPE schools, this will help in obtaining more agreeable and readily available data for analysis.

\section{$\underline{\text { Data collection }}$}

- On the conclusion of the exercise, the data collection forms were evaluated and the need is felt to improve these forms. Improved forms could be more functional and easy to fill for getting the data of the co-education schools, which were not properly addressed during this exercise.

- Another aspect was the time consumed during data collection, its transmission to the subcentres and onward delivery to the head office. Similarly feeding of data, its analysis and formulation of the document also consumed a lot of time.

\section{Monitoring/Training}

- It is suggested that special modules should also be developed on examinations, which could be utilized during training as well as in regular monitoring of the project's activities.

\section{Data Collection form for COPE School Annual Examination Results}

See Annex-I

COPE Schools Annual results 1996-97 Graphical Presentations

See Annex-II

Detailed School Based Annual results for 1996-97

See Annex-III

$$
\infty
$$




\section{Annex-I}

\section{Data Collection Form}

for

COPE School Annual Examination

Results 1996-97 
COPE School Annual Examination Results for 1996-97

School Code:

\begin{tabular}{|c|c|c|c|c|c|c|c|c|}
\hline \multirow{2}{*}{$\begin{array}{c}\text { Classes I } \\
\text { Grades }\end{array}$} & \multirow{2}{*}{$\begin{array}{l}\text { No. of } \\
\text { Enrolled } \\
\text { Students }\end{array}$} & \multirow{2}{*}{$\begin{array}{c}\text { No. of Drop } \\
\text { out } \\
\text { Students }\end{array}$} & \multirow{2}{*}{$\begin{array}{l}\text { No. of } \\
\text { Students } \\
\text { Appeared } \\
\text { in Exam }\end{array}$} & \multirow[t]{2}{*}{$\begin{array}{c}\text { No. of Pass } \\
\text { Students }\end{array}$} & \multirow{2}{*}{$\begin{array}{c}\text { No. of } \\
\text { Failed } \\
\text { Students }\end{array}$} & \multicolumn{2}{|c|}{ Co-education Classes } & \multirow[t]{2}{*}{$\begin{array}{l}\text { Signature } \\
\text { of Teachers }\end{array}$} \\
\hline & & & & & & Boys & Giris & \\
\hline \multicolumn{9}{|l|}{ Grade $1 \mathrm{~A}$} \\
\hline \multicolumn{9}{|l|}{ Grade 1 B } \\
\hline \multicolumn{9}{|l|}{ Grade $1 \mathrm{C}$} \\
\hline \multicolumn{9}{|l|}{ Grade 2 A } \\
\hline \multicolumn{9}{|l|}{ Grade 2 B } \\
\hline \multicolumn{9}{|l|}{ Grade 3 A } \\
\hline \multicolumn{9}{|l|}{ Grade 3 B } \\
\hline \multicolumn{9}{|l|}{ Grade 4} \\
\hline \multicolumn{9}{|l|}{ Grade 5} \\
\hline Grade 6 & & & & & & & & \\
\hline
\end{tabular}

Name \& Signature

Head Teacher:

FES/CSA: 


\section{Annex-II}

COPE Schools Annual Results

$$
\text { 1996-97 }
$$

Graphical Presentations 
g(2)

Basic Education for Afghan Refugees Monitoring and Evaluation Section

Annex - II

COPE Schools Annual Results 1996-97 Graphical Presentation

Graph 1:

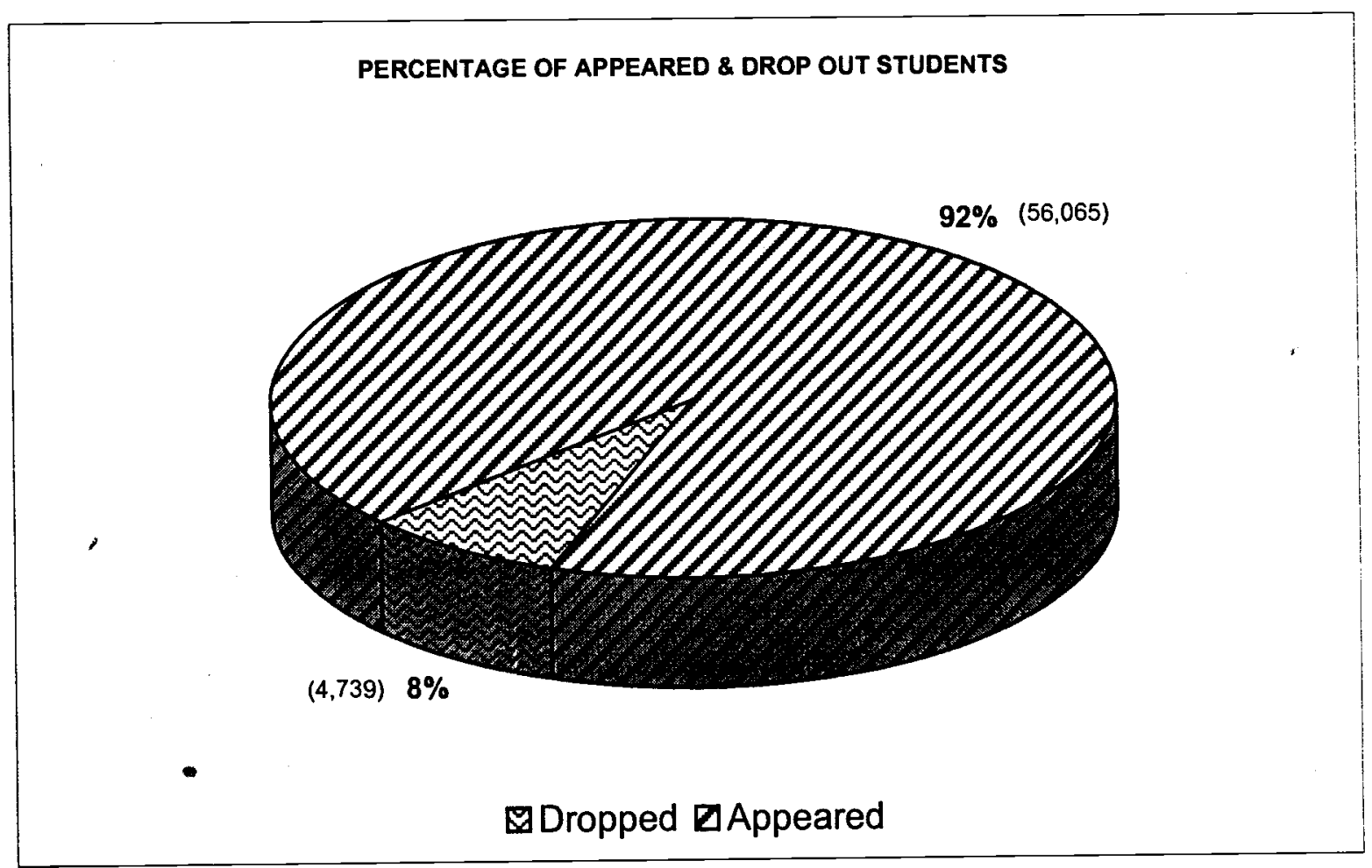

Graph 2:

PERCENTAGE OF PASSED \& FAILED STUDENTS

$(40,431) 72 \%$

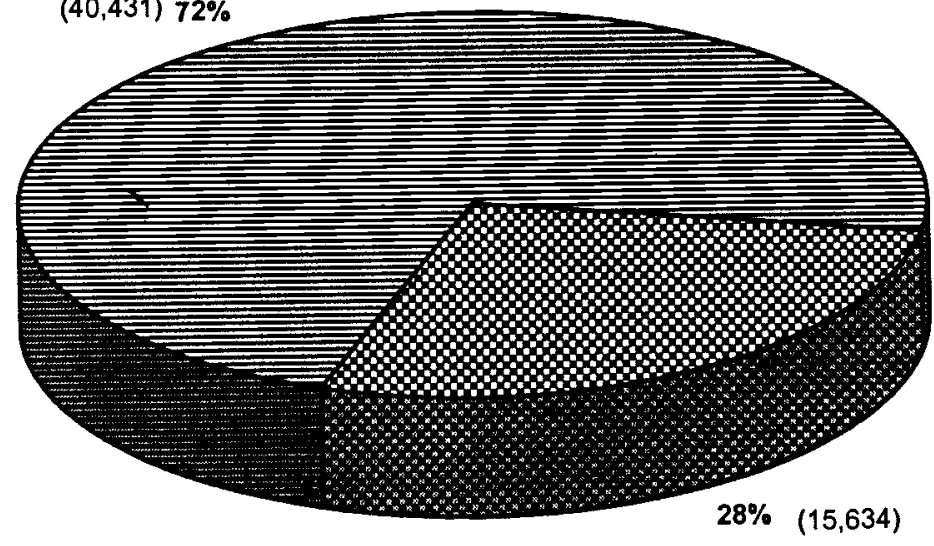

目Passed GFailed 


\section{Graph 3:}

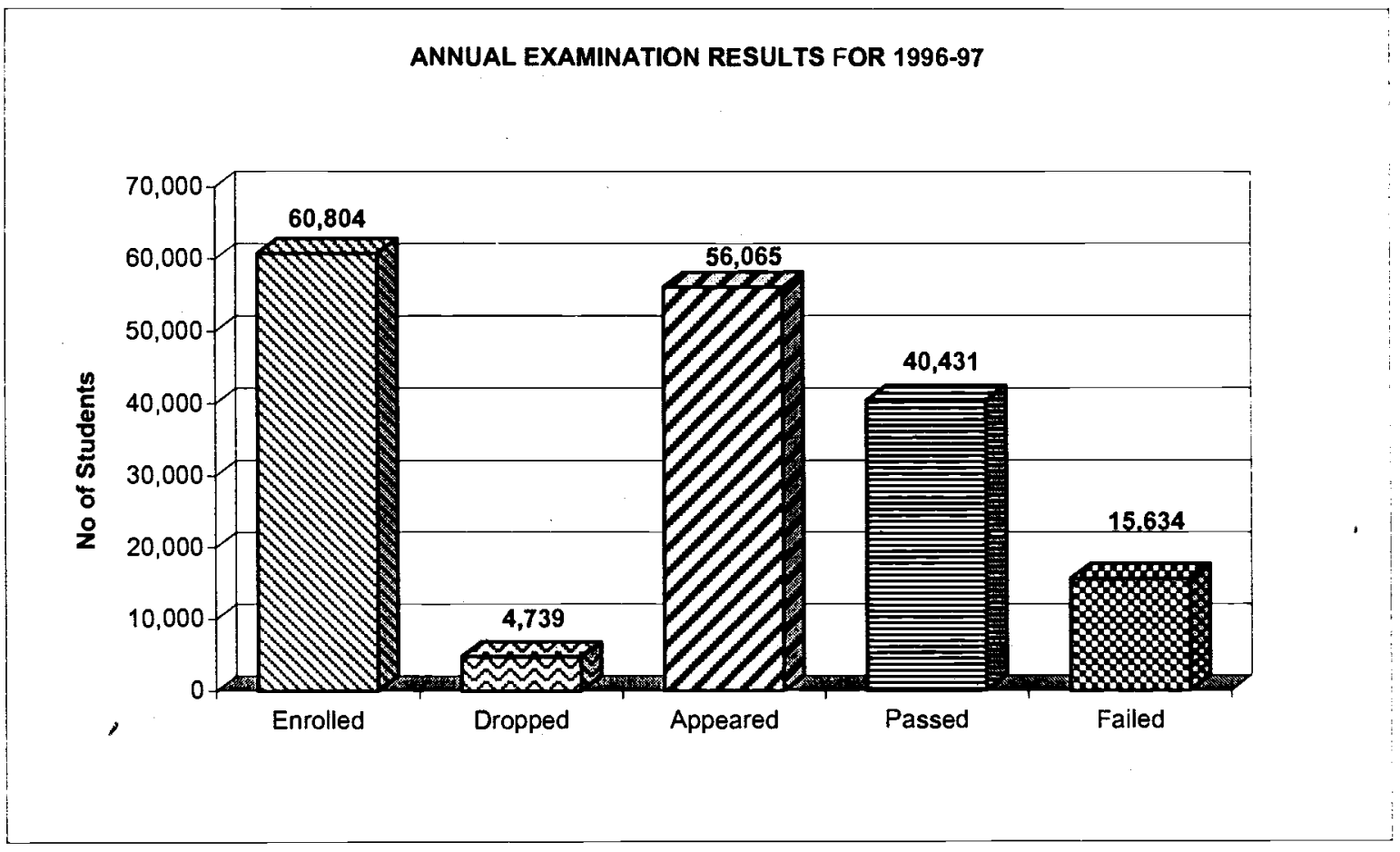

Graph 4:

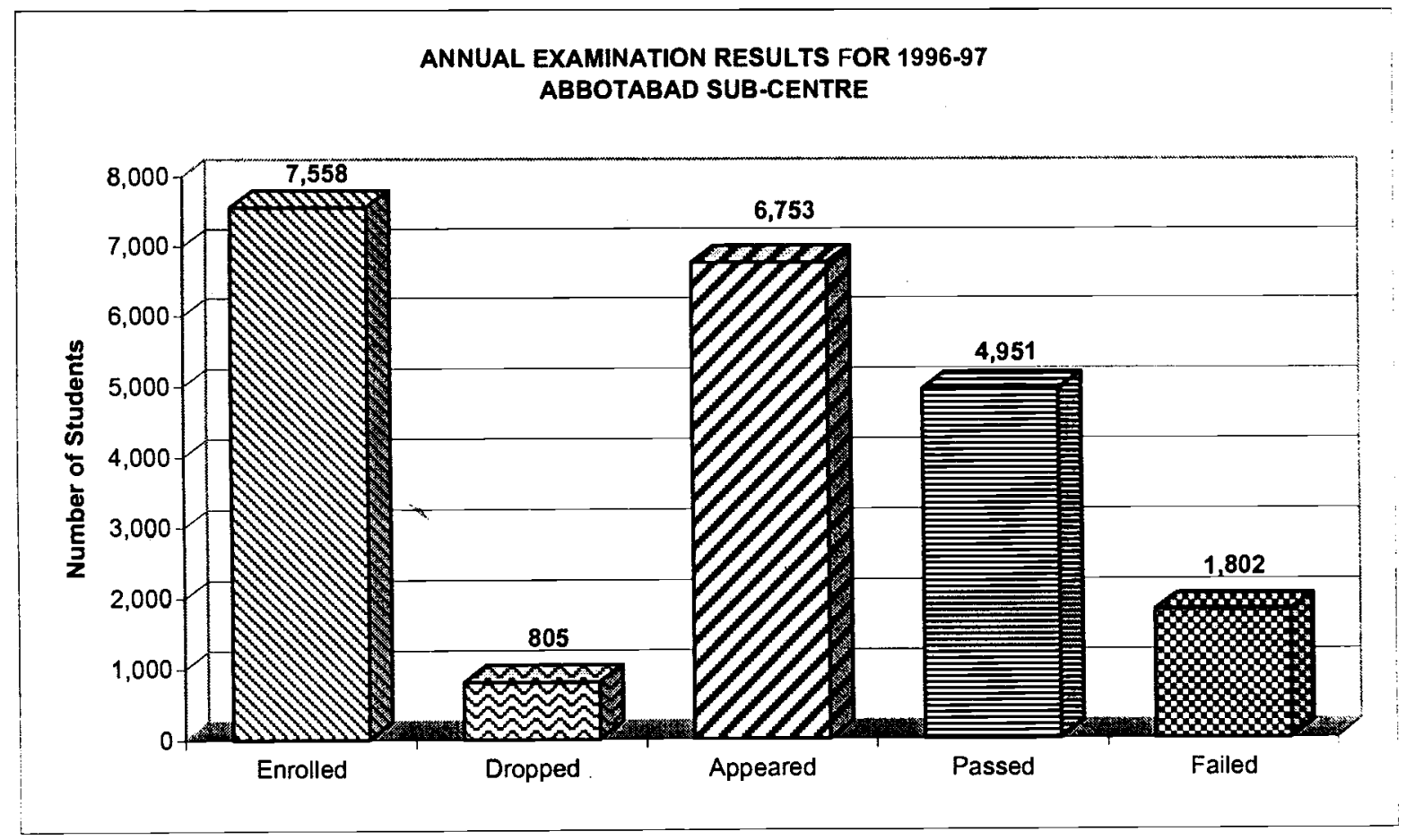




\section{Graph 5:}

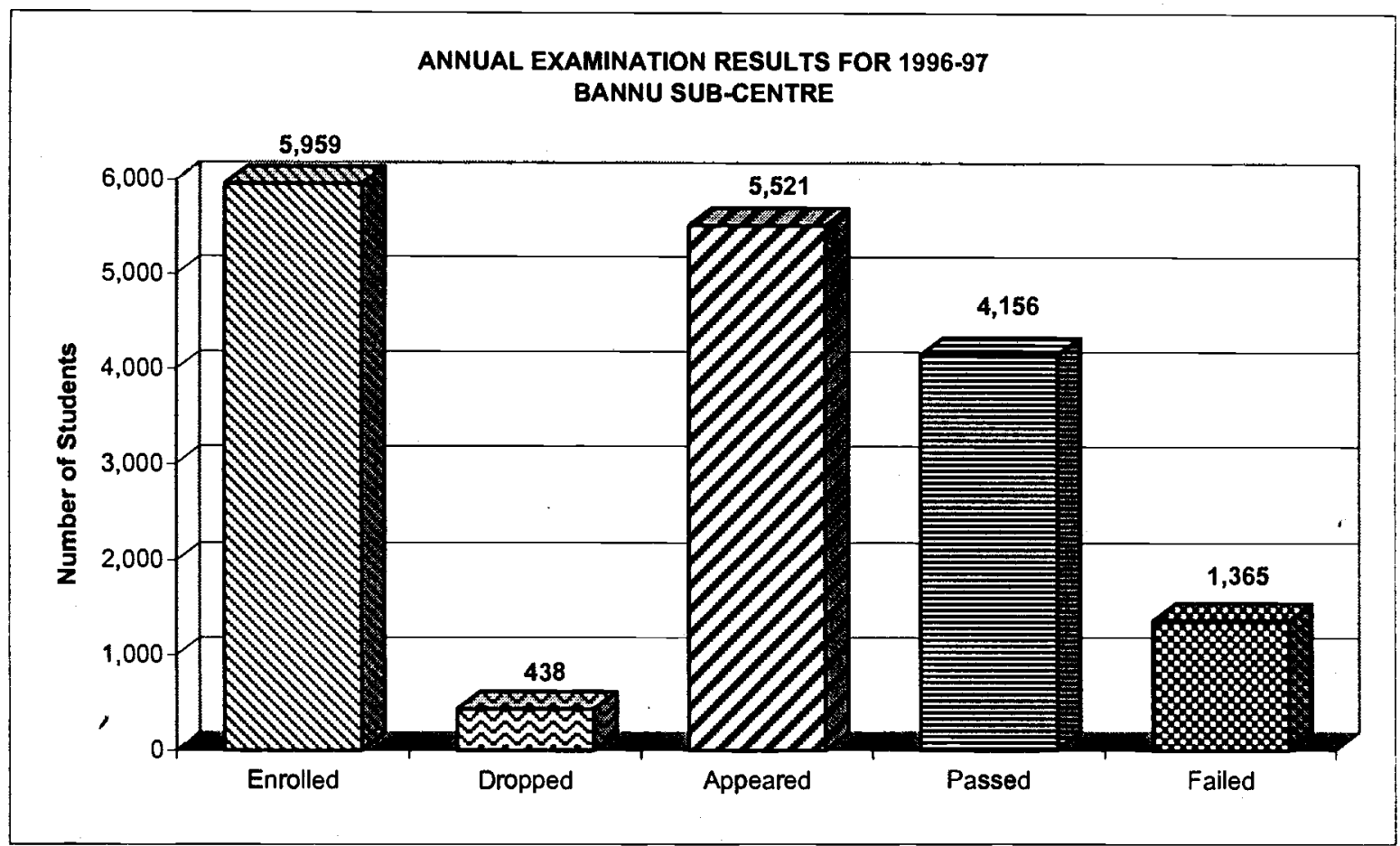

Graph 6:

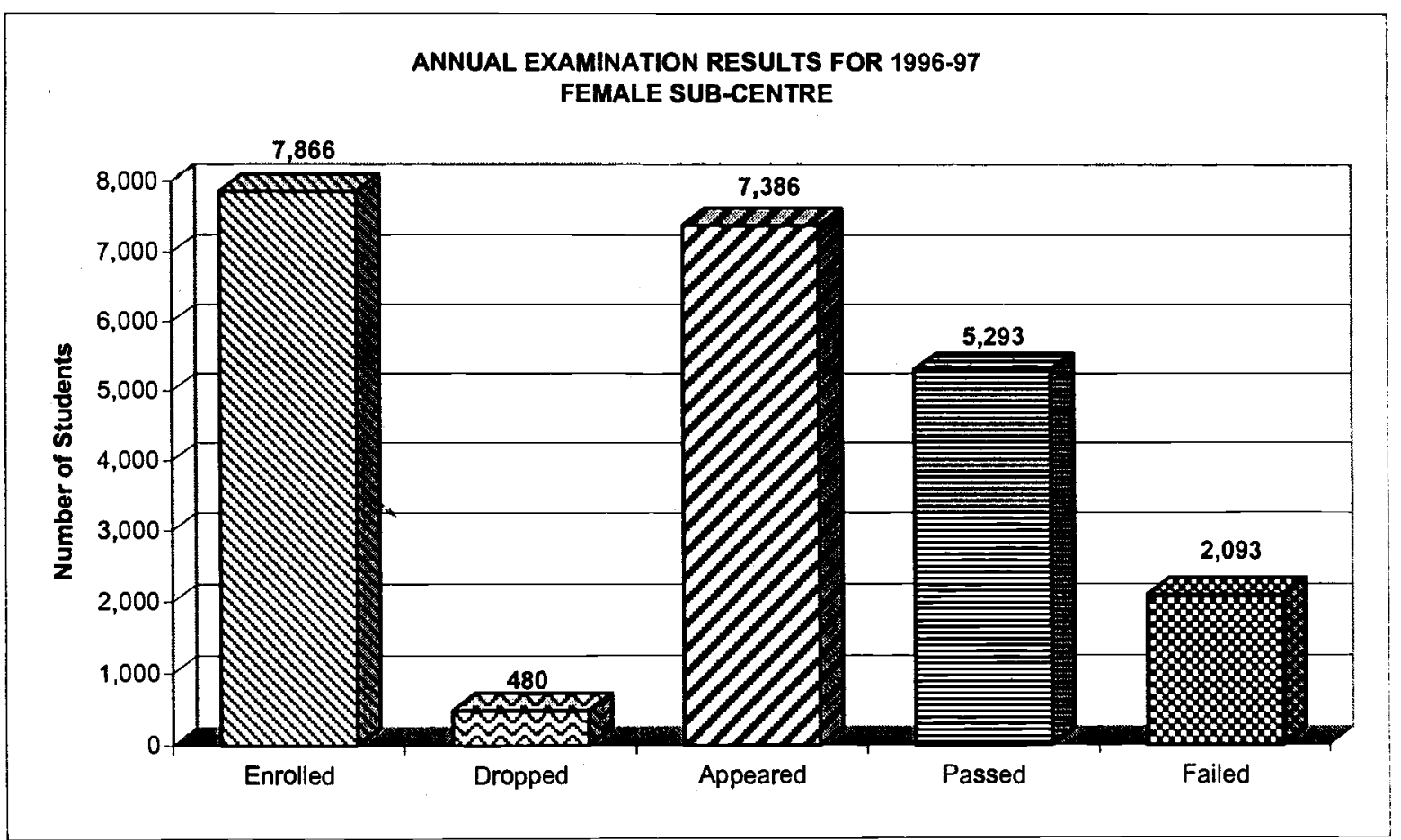




\section{Graph 7:}

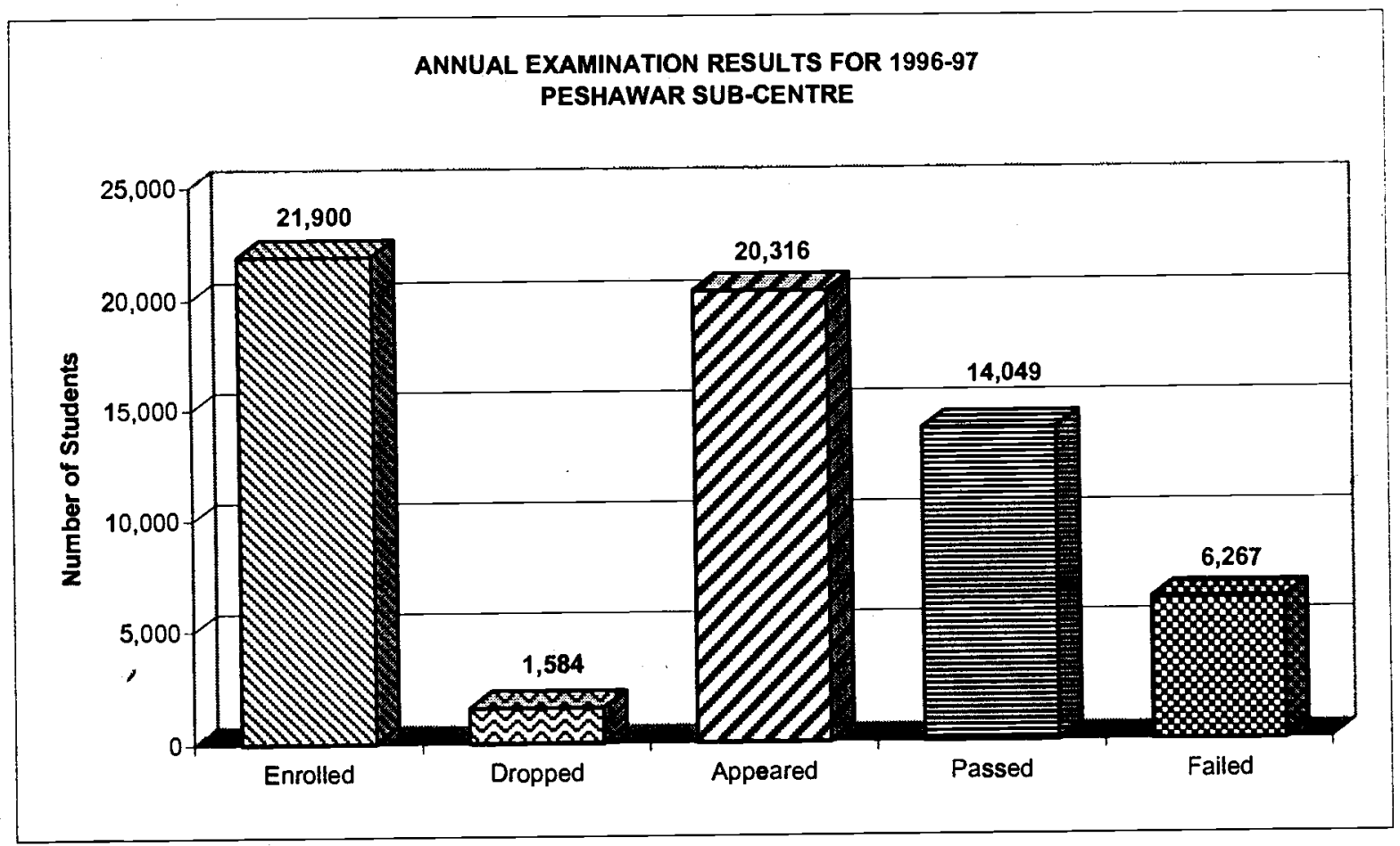

Graph 8:

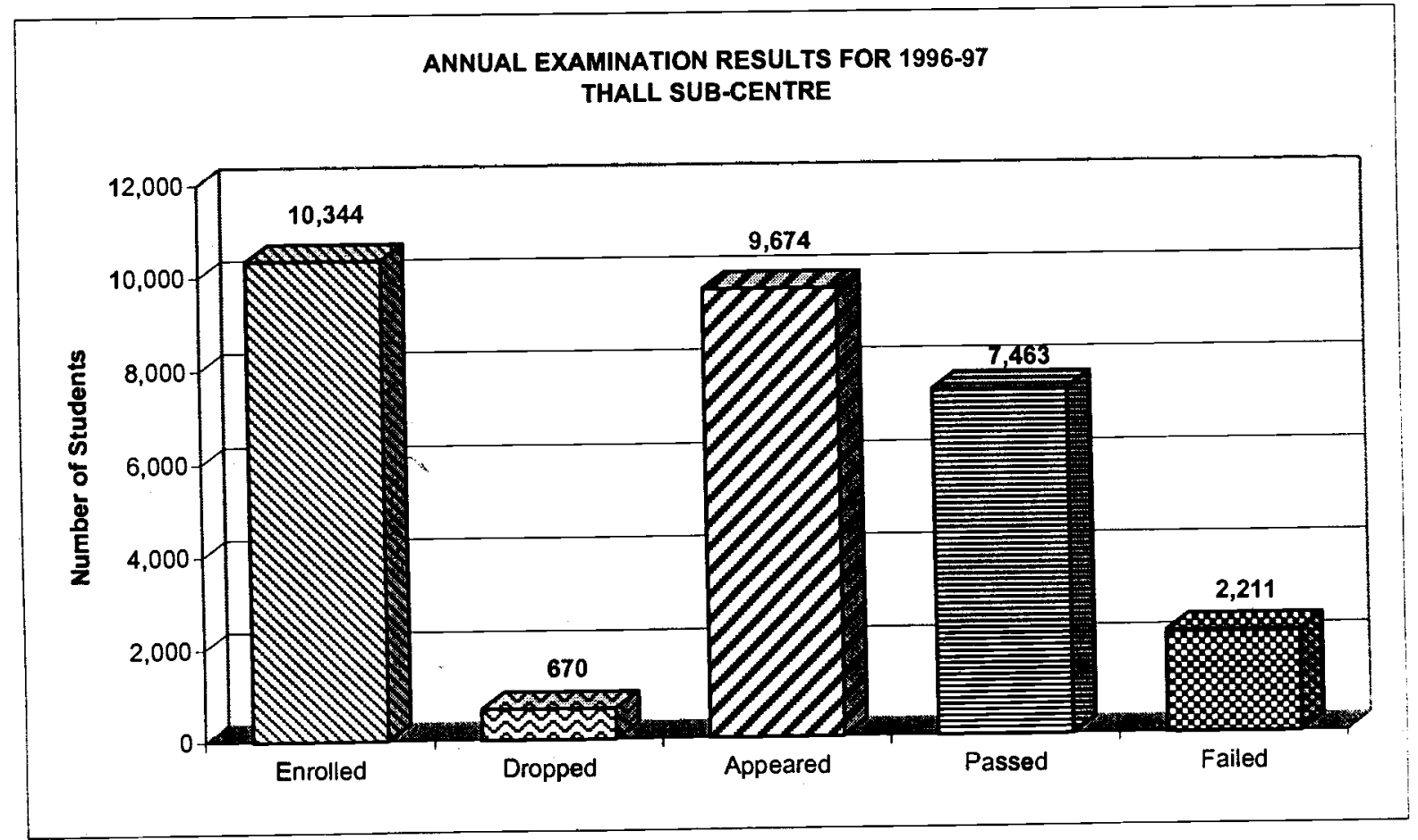


Graph 9:

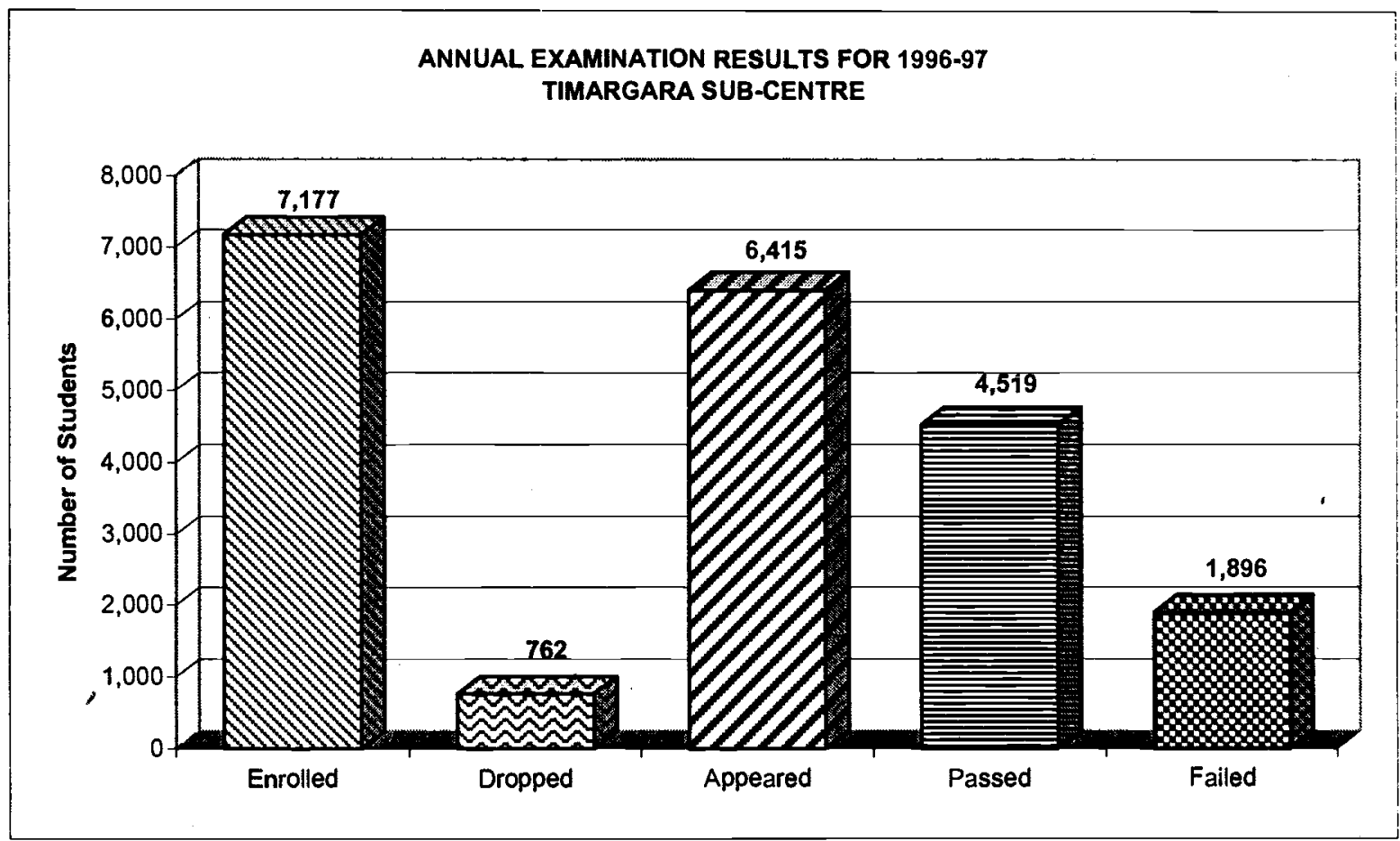

Graph 10:

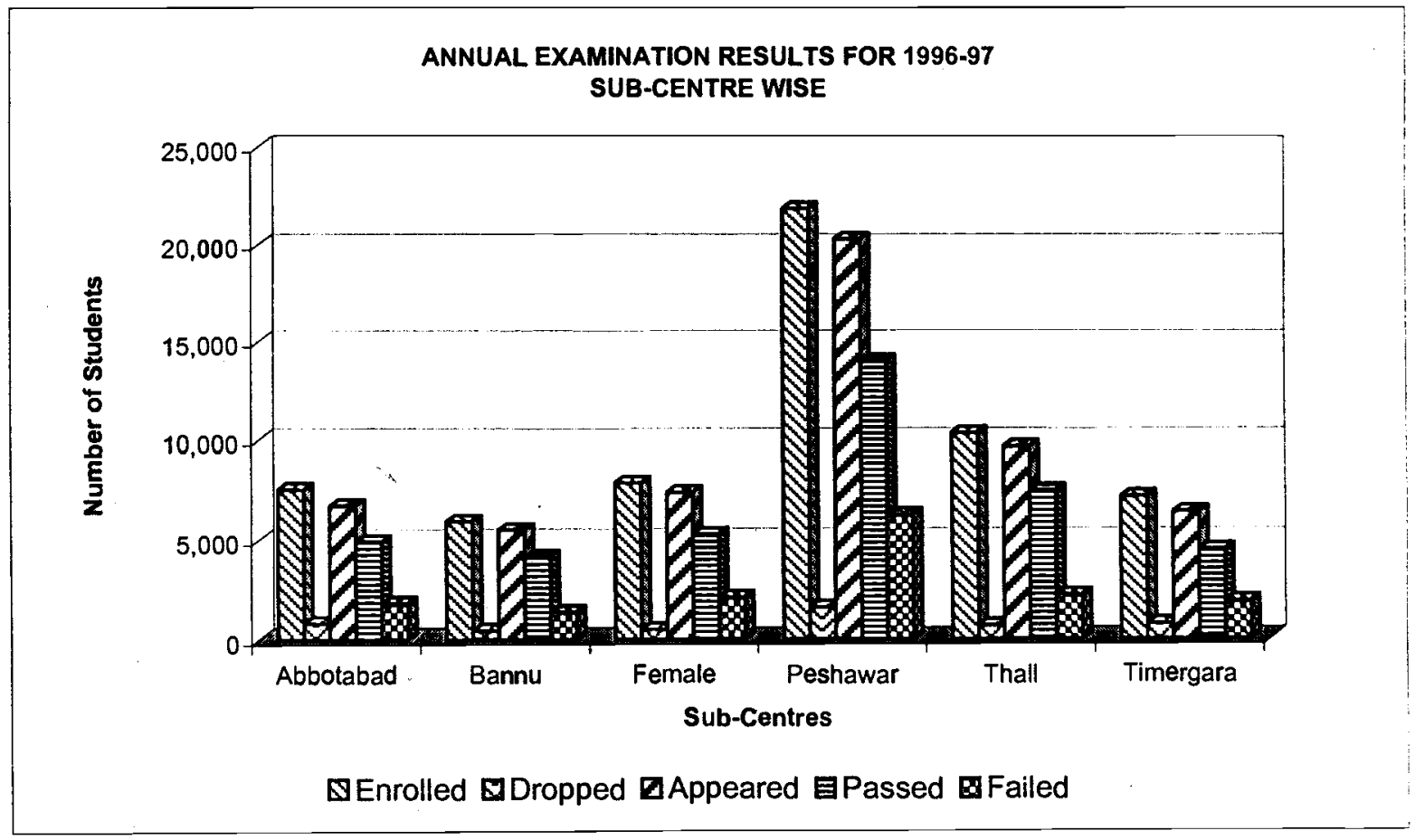


Graph 11:

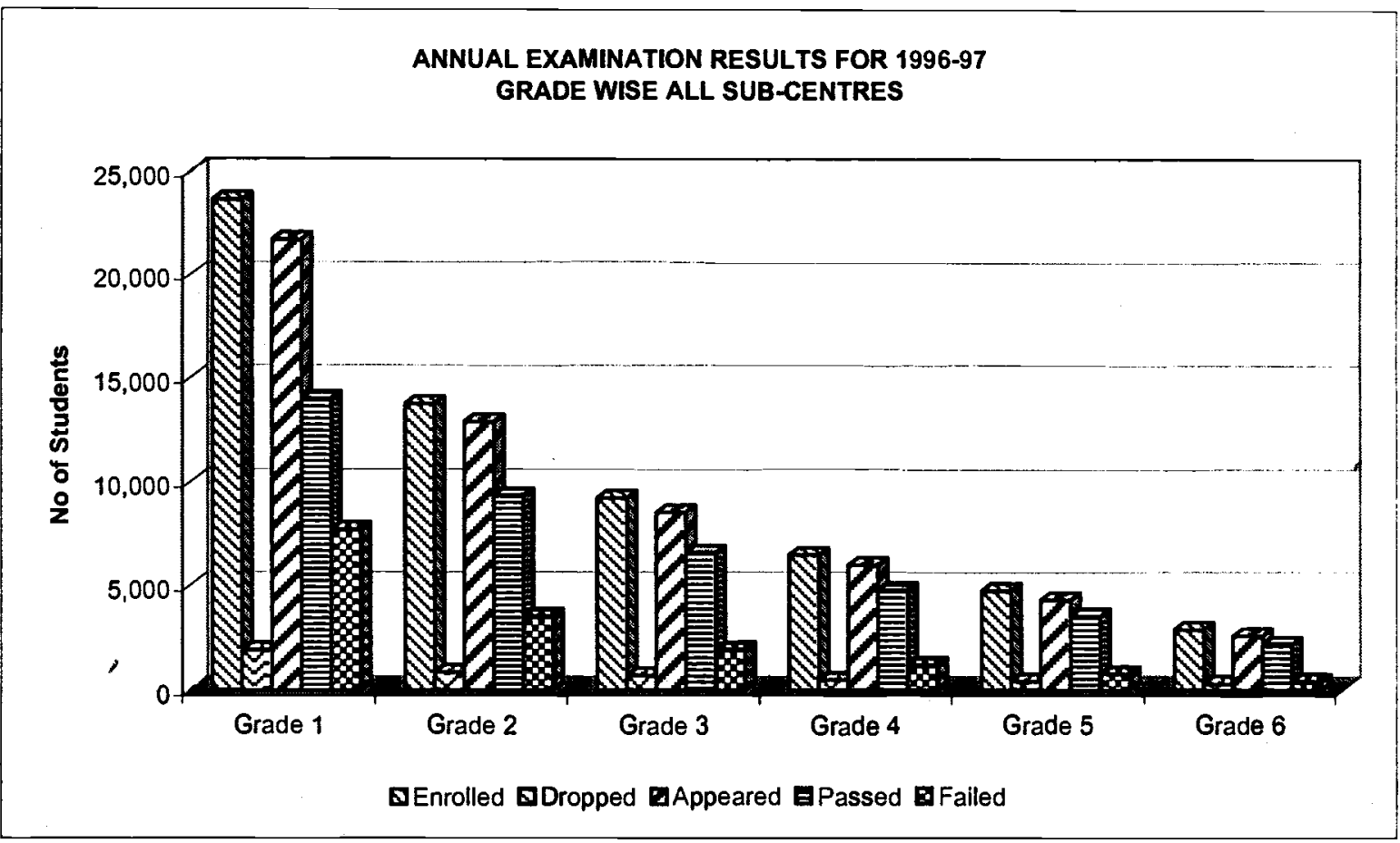

Graph 12:

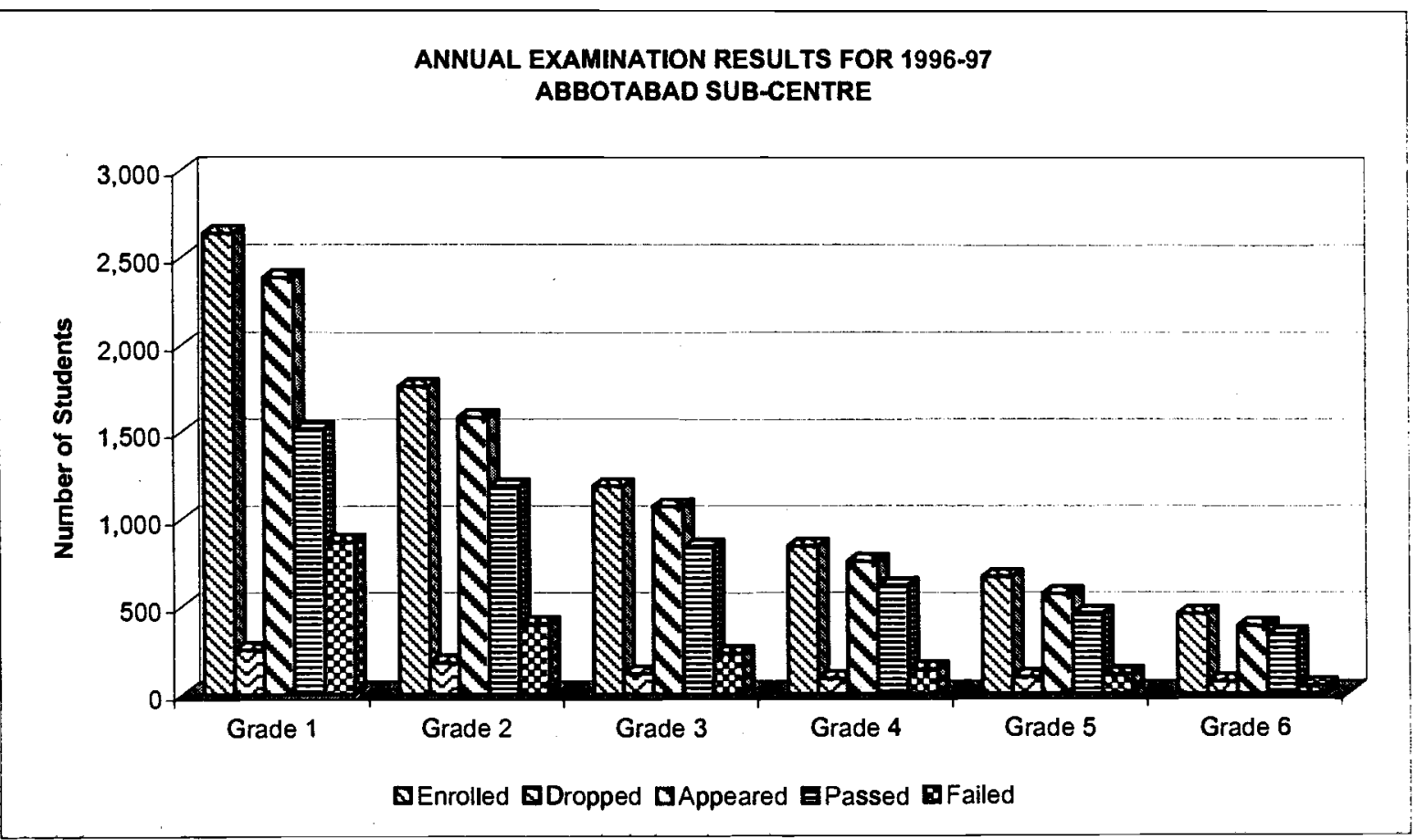


Graph 13:

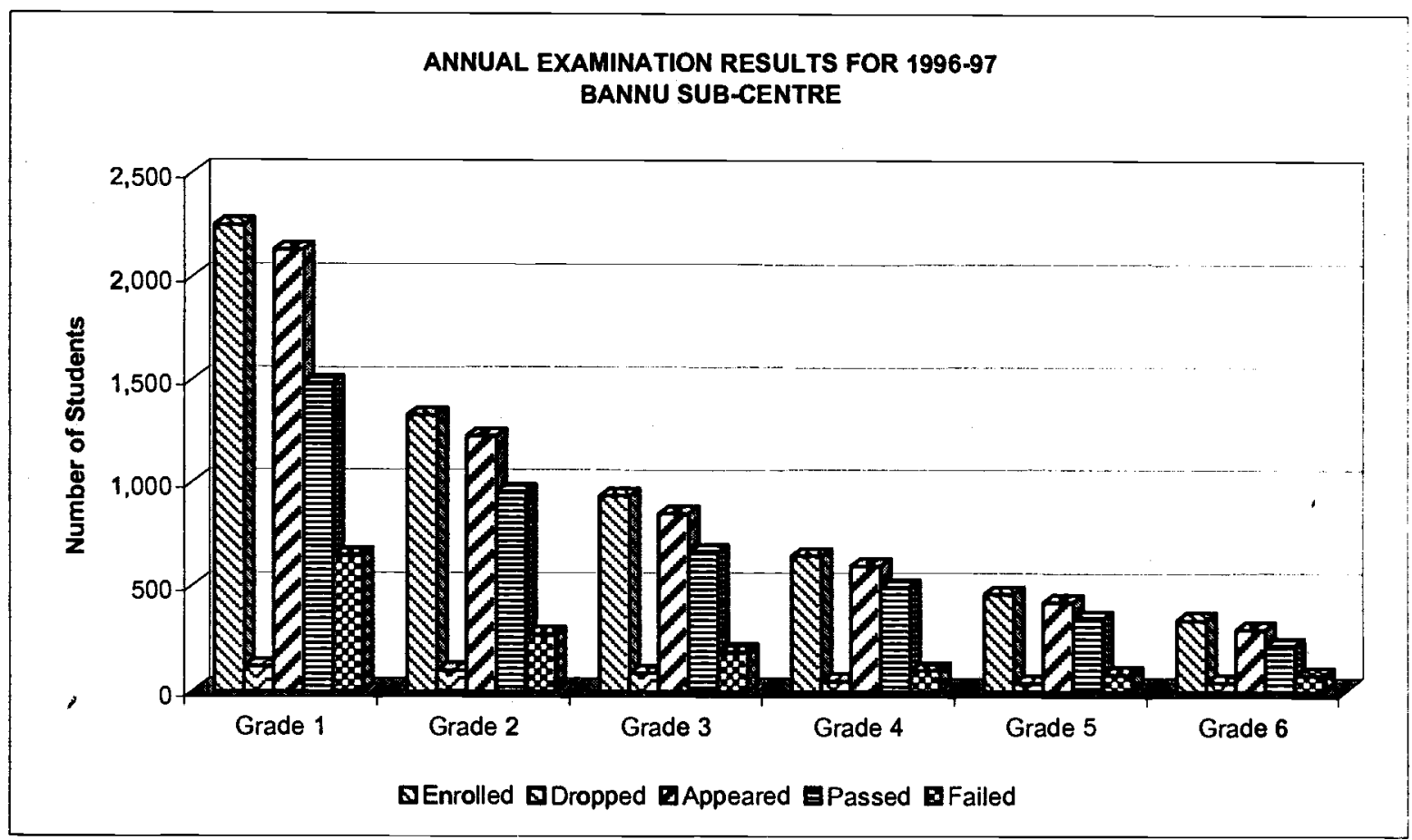

Graph 14:

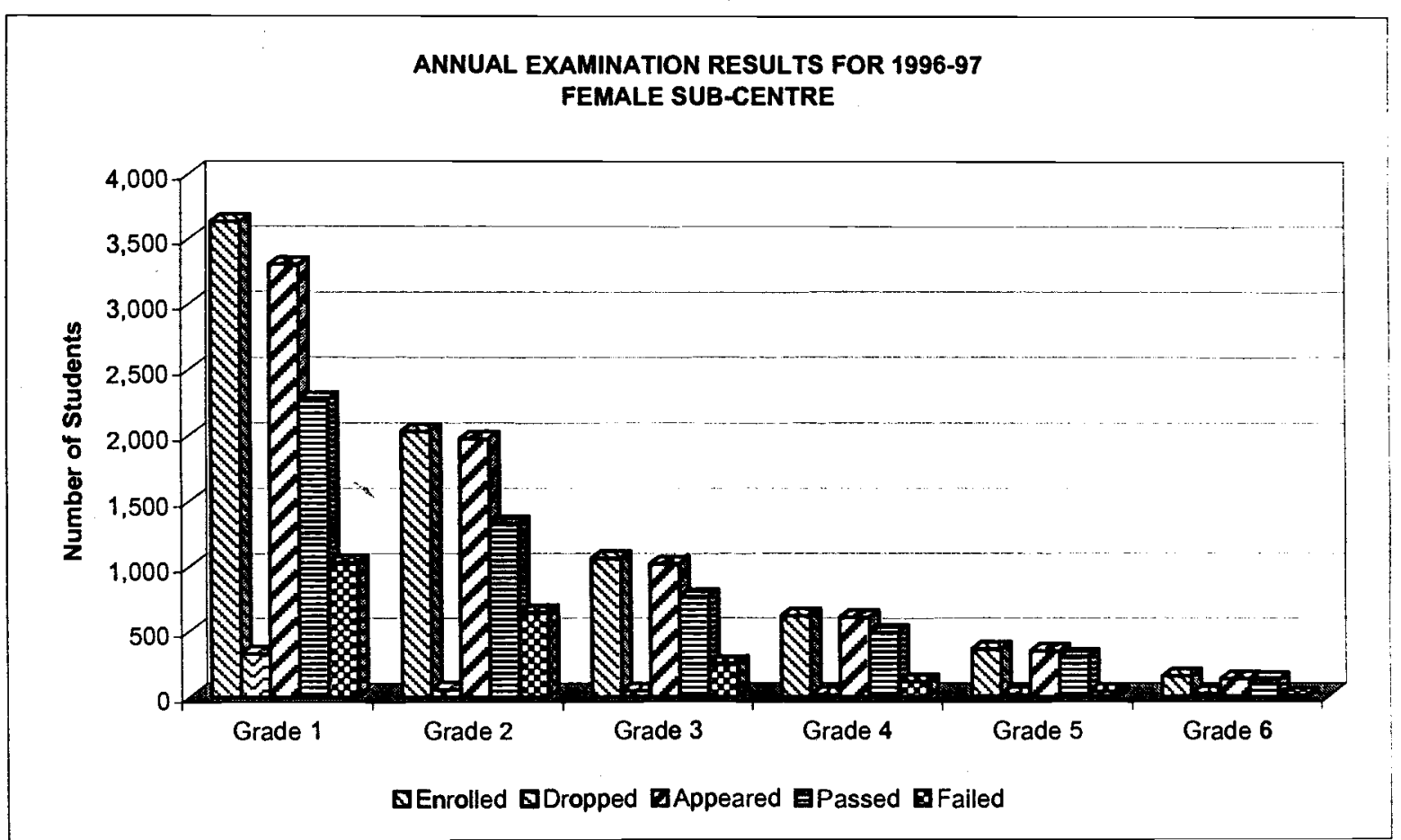




\section{Graph 15:}

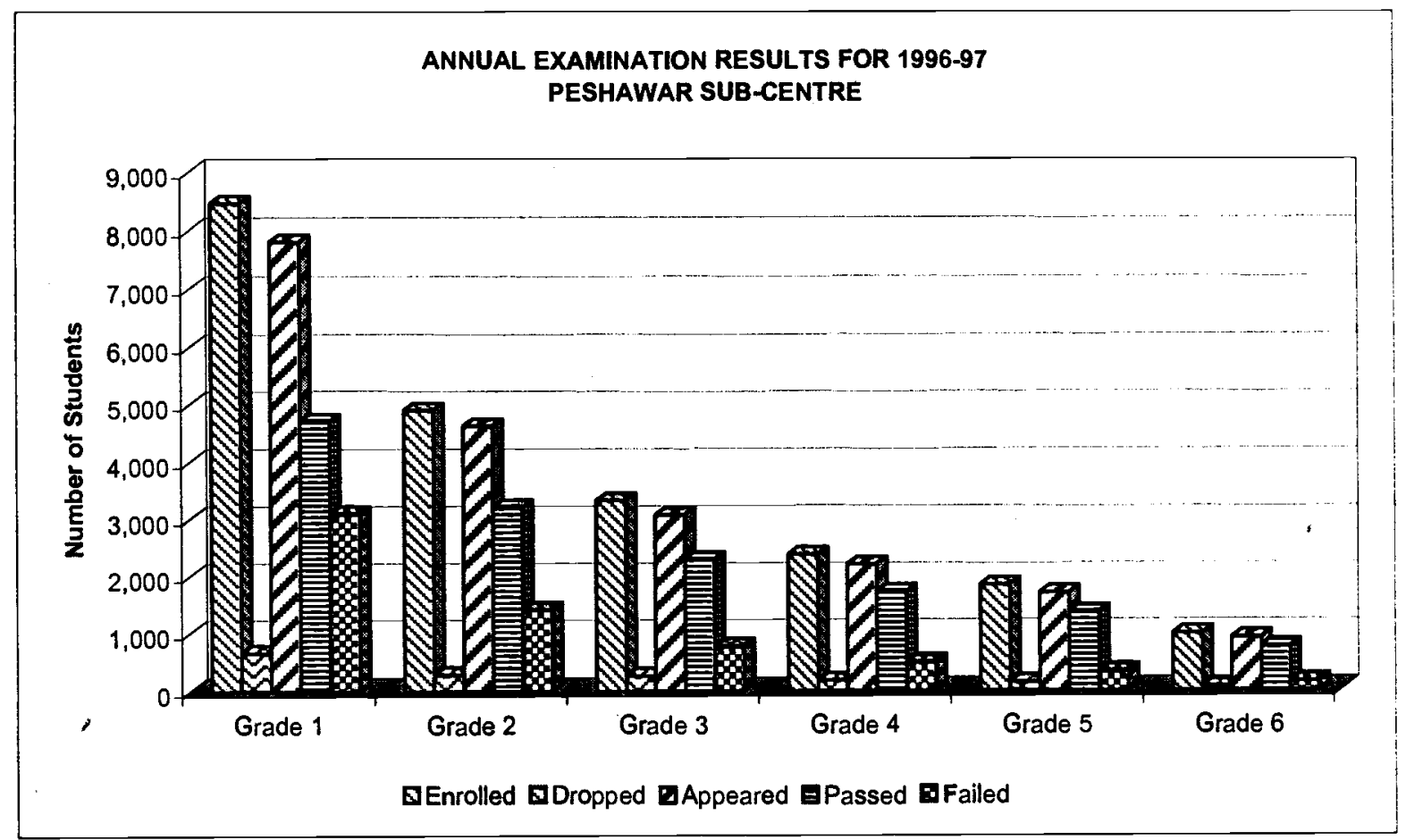

Graph 16: *

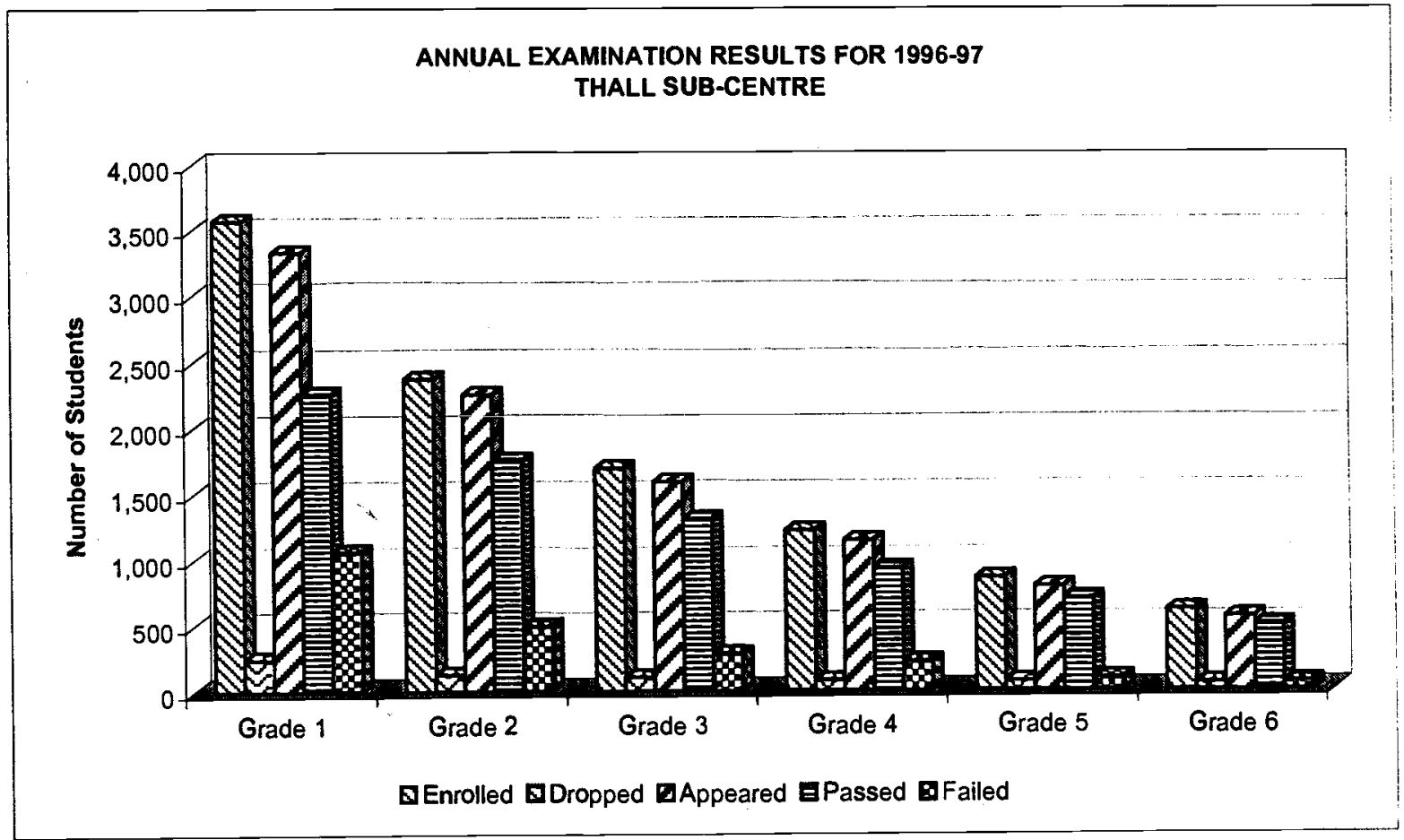




\section{Graph 17:}

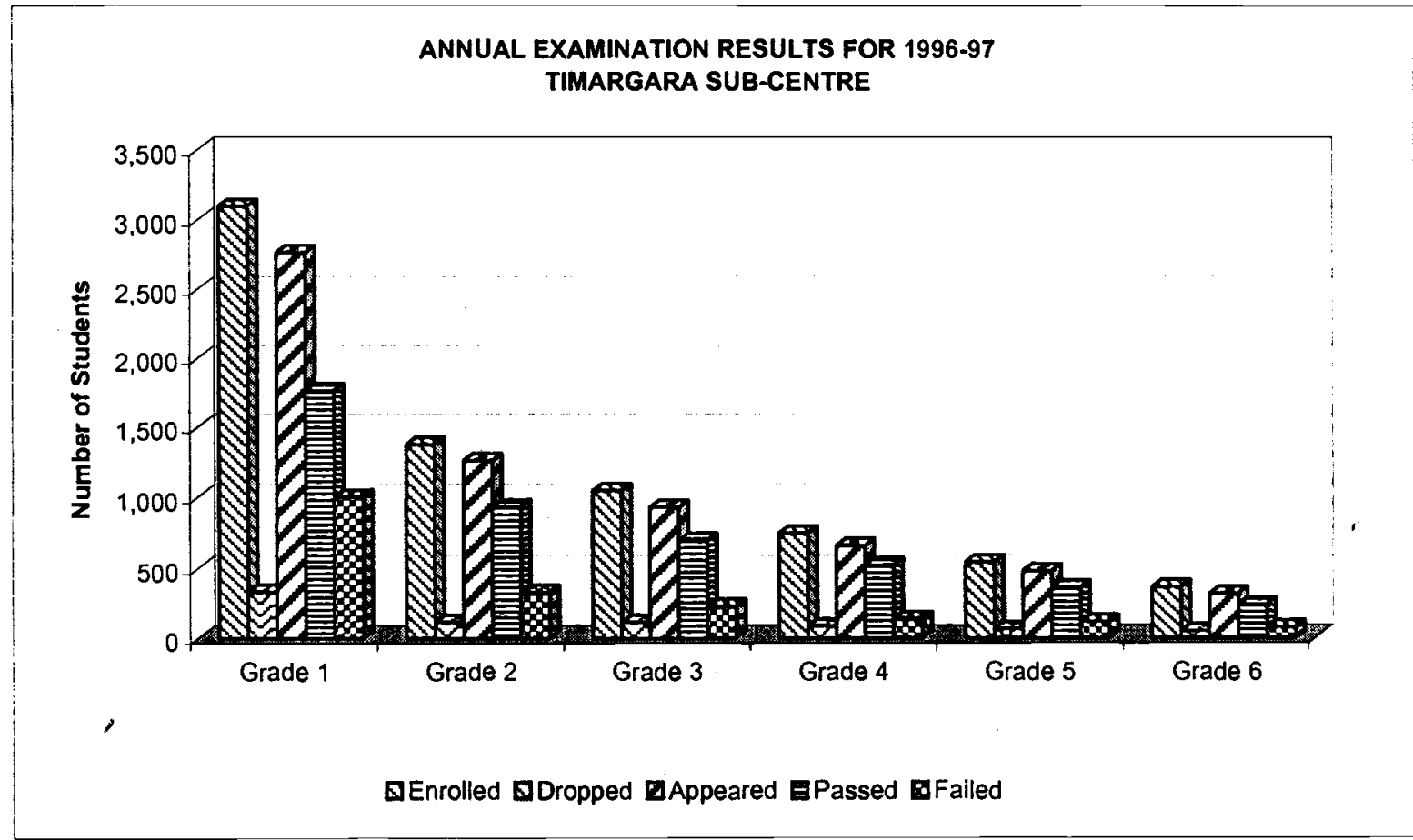

Graph 18: -

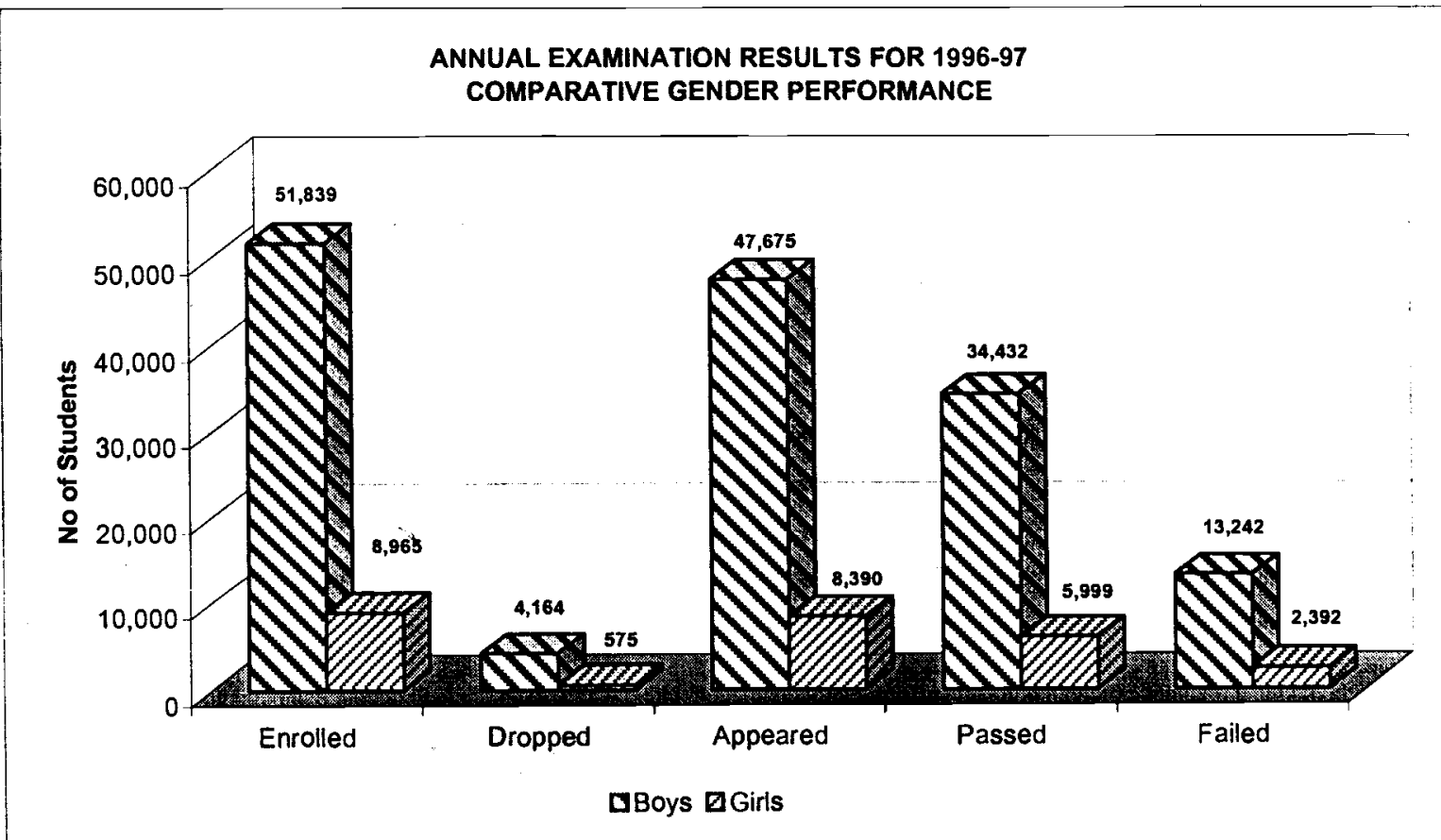


Annex-III

\section{Detailed School Based Annual Results for 1996-97}




\title{
Basic Education for Afghan Refugees
}

\author{
Monitoring and Evaluation Section
}

COPE Schools Based Annual Results for 1996-97 and Percentage Analysis

Sub-Centre: Abbotabad

\begin{tabular}{|c|c|c|c|c|c|c|c|c|c|c|}
\hline \multirow[b]{2}{*}{$\begin{array}{l}\text { Grades / } \\
\text { Sections }\end{array}$} & \multicolumn{5}{|c|}{ - Number of Students- } & \multirow[b]{2}{*}{ Grades } & \multicolumn{4}{|c|}{ Percent of Students } \\
\hline & Enrolled & Dropped & $\begin{array}{l}\text { Appeared } \\
\text { in Exam }\end{array}$ & $\begin{array}{c}\text { Passed in } \\
\text { Exams }\end{array}$ & $\begin{array}{l}\text { Failed in } \\
\text { Exams }\end{array}$ & & Dropped & $\begin{array}{l}\text { Appeared } \\
\text { in Exams }\end{array}$ & $\begin{array}{r}\text { Passed in } \\
\text { Exams }\end{array}$ & $\begin{array}{r}\text { Failed in } \\
\text { Exams }\end{array}$ \\
\hline Grade $1 \mathrm{~A}$ & 60 & 4 & 56 & 40 & 16 & Grade 1 & $6.67 \%$ & $93.33 \%$ & $71.43 \%$ & $28.57 \%$ \\
\hline Grade 1 B & 0 & 0 & 0 & 0 & 0 & Grade 2 & $9.09 \%$ & $90.91 \%$ & $87.50 \%$ & $12.50 \%$ \\
\hline Grade $1 \mathrm{C}$ & 0 & 0 & 0 & 0 & 0 & Grade 3 & $6.25 \%$ & $93.75 \%$ & $90.00 \%$ & $10.00 \%$ \\
\hline Grade $2 \mathrm{~A}$ & 44 & 4 & 40 & 35 & 5 & Grade 4 & $6.25 \%$ & $93.75 \%$ & $93.33 \%$ & $6.67 \%$ \\
\hline Grade 2 B & 0 & 0 & 0 & 0 & 0 & Grade 5 & $14.29 \%$ & $85.71 \%$ & $88.89 \%$ & $11.11 \%$ \\
\hline Grade $3 \mathrm{~A}$ & 32 & 2 & 30 & 27 & 3 & Grade 6 & $0.00 \%$ & $100.00 \%$ & $100.00 \%$ & $0.00 \%$ \\
\hline Grade 3 B & 0 & 0 & 0 & 0 & 0 & & & & & \\
\hline Grade 4 & 32 & 2 & 30 & 28 & 2 & Remarks: & & & & . \\
\hline Grade 5 & 21 & 3 & 18 & 16 & 2 & & & & & \\
\hline Grade 6 & 10 & 0 & 10 & 10 & 0 & & & & & \\
\hline School Total & 199 & 15 & 184 & 156 & 28 & School \%age & $7.54 \%$ & $92.46 \%$ & $84.78 \%$ & $15.22 \%$ \\
\hline
\end{tabular}

\begin{tabular}{|c|c|c|c|c|c|c|c|c|c|c|}
\hline \multirow[b]{2}{*}{$\begin{array}{l}\text { Grades I } \\
\text { Section's }\end{array}$} & \multicolumn{5}{|c|}{ - Number of Students- } & \multirow[b]{2}{*}{ Grades } & \multicolumn{4}{|c|}{$t$ of Stu } \\
\hline & Enrolled & Dropped & $\begin{array}{l}\text { Appeared } \\
\text { in Exam }\end{array}$ & $\begin{array}{c}\text { Passed in } \\
\text { Exams }\end{array}$ & $\begin{array}{l}\text { Failed in } \\
\text { Exams }\end{array}$ & & Dropped & $\begin{array}{l}\text { Appeared } \\
\text { in Exams }\end{array}$ & $\begin{array}{c}\text { Passed in } \\
\text { Exams }\end{array}$ & $\begin{array}{l}\text { Failed in } \\
\text { Exams }\end{array}$ \\
\hline Grade $1 \mathrm{~A}$ & 50 & 2 & 48 & 35 & 13 & Grade 1 & $4.00 \%$ & $96.00 \%$ & $62.50 \%$ & $37.50 \%$ \\
\hline Grade 1 B & 50 & 2 & 48 & 25 & 23 & Grade 2 & $5.26 \%$ & $94.74 \%$ & $77.78 \%$ & $22.22 \%$ \\
\hline Grade $1 \mathrm{C}$ & 0 & 0 & 0 & 0 & 0 & Grade 3 & $6.98 \%$ & $93.02 \%$ & $90.00 \%$ & $10.00 \%$ \\
\hline Grade $2 \mathrm{~A}$ & 50 & 3 & 47 & 39 & 8 & Grade 4 & $6.45 \%$ & $93.55 \%$ & $93.10 \%$ & $6.90 \%$ \\
\hline Grade 2 B & 45 & 2 & 43 & 31 & 12 & Grade 5 & $9.09 \%$ & $90.91 \%$ & $93.33 \%$ & $6.67 \%$ \\
\hline Grade $3 \mathrm{~A}$ & 43 & 3 & 40 & 36 & 4 & Grade 6 & $9.09 \%$ & $90.91 \%$ & $100.00 \%$ & $0.00 \%$ \\
\hline Grade 3 B & 0 & 0 & 0 & 0 & 0 & & & & & \\
\hline Grade 4 & 31 & 2 & 29 & 27 & 2 & Remarks: & & & & \\
\hline Grade 5 & 33 & 3 & 30 & 28 & 2 & & & & & \\
\hline Grade 6 & 22 & 2 & 20 & 20 & 0 & & & & & \\
\hline School Total & 324 & 19 & 305 & 241 & 64 & School \%age & $5.86 \%$ & $94.14 \%$ & $79.02 \%$ & $20.98 \%$ \\
\hline
\end{tabular}

\begin{tabular}{|c|c|c|c|c|c|c|c|c|c|c|}
\hline \multirow[b]{2}{*}{$\begin{array}{l}\text { Grades I } \\
\text { Sections }\end{array}$} & \multicolumn{5}{|c|}{ - Number of Students } & \multirow[b]{2}{*}{ Grades } & \multicolumn{4}{|c|}{ - Percent of Students } \\
\hline & Enrolled & Dropped & $\begin{array}{l}\text { Appeared } \\
\text { iñ Exam }\end{array}$ & $\begin{array}{c}\text { Passed in } \\
\text { Exams }\end{array}$ & $\begin{array}{l}\text { Failed in } \\
\text { Exams }\end{array}$ & & Dropped & $\begin{array}{c}\text { Appeared } \\
\text { in Exams }\end{array}$ & $\begin{array}{l}\text { Passed in } \\
\text { Exams }\end{array}$ & $\begin{array}{l}\text { Falled in } \\
\text { Exams }\end{array}$ \\
\hline Grade $1 \mathrm{~A}$ & 31 & 3 & 28 & 24 & 4 & Grade 1 & $9.68 \%$ & $90.32 \%$ & $85.71 \%$ & $14.29 \%$ \\
\hline Grade 1 B & 0 & 0 & 0 & 0 & 0 & Grade 2 & $28.95 \%$ & $71.05 \%$ & $96.30 \%$ & $3.70 \%$ \\
\hline Grade $1 \mathrm{C}$ & 0 & 0 & 0 & 0 & 0 & Grade 3 & $23.08 \%$ & $76.92 \%$ & $100.00 \%$ & $0.00 \%$ \\
\hline Grade $2 \mathrm{~A}$ & 38 & 11 & 27 & 26 & 1 & Grade 4 & $0.00 \%$ & $100.00 \%$ & $77.78 \%$ & $22.22 \%$ \\
\hline Grade 2 B & 0 & 0 & .0 & 0 & 0 & Grade 5 & \#Num! & \#Num! & \#Num! & \#Num! \\
\hline Grade $3 \mathrm{~A}$ & 26 & 6 & 20 & 20 & 0 & Grade 6 & $36.36 \%$ & $63.64 \%$ & $100.00 \%$ & $0.00 \%$ \\
\hline Grade 3 B & 0 & 0 & 0 & 0 & 0 & & & & & \\
\hline Grade 4 & 18 & 0 & 18 & 14 & 4 & Remarks: & & & & \\
\hline Grade 5 & 0 & 0 & 0 & 0 & 0 & & & & & \\
\hline Grade 6 & 11 & 4 & 7 & 7 & 0 & & & & & \\
\hline School Total & 124 & 24 & 100 & 91 & 9 & School \%age & $19.35 \%$ & $80.65 \%$ & $91.00 \%$ & $9.00 \%$ \\
\hline
\end{tabular}


COPE Schools Based Annual Results for 1996-97 and Percentage Analysis

Sub-Centre: Abbotabad

\begin{tabular}{|c|c|c|c|c|c|c|c|c|c|c|}
\hline \multirow[b]{2}{*}{$\begin{array}{l}\text { Grades I } \\
\text { Sections }\end{array}$} & \multicolumn{5}{|c|}{ - Number of Students- } & \multirow[b]{2}{*}{ Grades } & \multicolumn{4}{|c|}{-Percent of Students- } \\
\hline & Enrolled & Dropped & $\begin{array}{c}\text { Appeared } \\
\text { in Exam }\end{array}$ & $\begin{array}{c}\text { Passed in } \\
\text { Exams }\end{array}$ & $\begin{array}{c}\text { Failed in } \\
\text { Exams }\end{array}$ & & Dropped & $\begin{array}{l}\text { Appeared } \\
\text { in Exams }\end{array}$ & $\begin{array}{c}\text { Passed in } \\
\text { Exams }\end{array}$ & $\begin{array}{l}\text { Falied in } \\
\text { Exams }\end{array}$ \\
\hline Grade $1 \mathrm{~A}$ & 58 & 19 & 39 & 27 & 12 & Grade 1 & $32.76 \%$ & $67.24 \%$ & $69.23 \%$ & $30.77 \%$ \\
\hline Grade 1 B & 0 & 0 & 0 & 0 & 0 & Grade 2 & $12.50 \%$ & $87.50 \%$ & $57.14 \%$ & $42.86 \%$ \\
\hline Grade $1 \mathrm{C}$ & 0 & 0 & 0 & 0 & 0 & Grade 3 & $31.25 \%$ & $68.75 \%$ & $57.58 \%$ & $42.42 \%$ \\
\hline Grade $2 \mathrm{~A}$ & 56 & 7 & 49 & 28 & 21 & Grade 4 & $25.00 \%$ & $75.00 \%$ & $72.22 \%$ & $27.78 \%$ \\
\hline Grade 2 B & 0 & 0 & 0 & 0 & 0 & Grade 5 & $30.77 \%$ & $69.23 \%$ & $50.00 \%$ & $50.00 \%$ \\
\hline Grade $3 \mathrm{~A}$ & 48 & 15 & 33 & 19 & 14 & Grade 6 & $45.00 \%$ & $55.00 \%$ & $63.64 \%$ & $36.36 \%$ \\
\hline Grade 3 B & 0 & 0 & 0 & 0 & 0 & & & & & \\
\hline Grade 4 & 24 & 6 & 18 & 13 & 5 & Remarks: & & & & \\
\hline Grade 5 & 26 & 8 & 18 & 9 & 9 & & & & & \\
\hline Grade 6 & 20 & 9 & 11 & 7 & 4 & & & & & \\
\hline School Total & 232 & 64 & 168 & 103 & 65 & School \%age & $27.59 \%$ & $72.41 \%$ & $61.31 \%$ & $38.69 \%$ \\
\hline
\end{tabular}

\begin{tabular}{|c|c|c|c|c|c|c|c|c|c|c|c|}
\hline \multirow[b]{2}{*}{$\begin{array}{l}\text { Grades I } \\
\text { Sections }\end{array}$} & \multicolumn{6}{|c|}{ —Number of Students- } & \multirow[b]{2}{*}{ Grades } & \multicolumn{4}{|c|}{-Percent of Students- } \\
\hline & 1 & Enrolled & Dropped & $\begin{array}{l}\text { Appeared } \\
\text { in Exam }\end{array}$ & $\begin{array}{l}\text { Passed in } \\
\text { Exams }\end{array}$ & $\begin{array}{l}\text { Failed in } \\
\text { Exams }\end{array}$ & & Dropped & $\begin{array}{l}\text { Appeared } \\
\text { in Exams }\end{array}$ & $\begin{array}{l}\text { Passed in } \\
\text { Exams }\end{array}$ & $\begin{array}{c}\text { Failed in } \\
\text { Exams }\end{array}$ \\
\hline Grade $1 \mathrm{~A}$ & & 50 & 0 & 50 & 39 & 11 & Grade 1 & $0.00 \%$ & $100.00 \%$ & $78.00 \%$ & $22.00 \%$ \\
\hline Grade $1 \mathrm{~B}$ & & 0 & 0 & 0 & 0 & 0 & Grade 2 & $0.00 \%$ & $100.00 \%$ & $87.76 \%$ & $12.24 \%$ \\
\hline Grade $1 \mathrm{C}$ & & 0 & 0 & 0 & 0 & 0 & Grade 3 & $0.00 \%$ & $100.00 \%$ & $83.33 \%$ & $16.67 \%$ \\
\hline Grade $2 \mathrm{~A}$ & & 0 & 0 & 0 & 0 & 0 & Grade 4 & $27.59 \%$ & $72.41 \%$ & $85.71 \%$ & $14.29 \%$ \\
\hline Grade 2 B & & 49 & 0 & 49 & 43 & 6 & Grade 5 & $11.11 \%$ & $88.89 \%$ & $91.67 \%$ & $8.33 \%$ \\
\hline Grade $3 \mathrm{~A}$ & & 36 & 0 & 36 & 30 & 6 & Grade 6 & $22.22 \%$ & $77.78 \%$ & $78.57 \%$ & $21.43 \%$ \\
\hline Grade 3 B & & 0 & 0 & 0 & 0 & 0 & & & & & \\
\hline Grade 4 & & 29 & 8 & 21 & 18 & 3 & Remarks: & & & & \\
\hline Grade 5 & & 27 & 3 & 24 & 22 & 2 & & & & & \\
\hline Grade 6 & & 18 & 4 & 14 & 11 & 3 & & & & & \\
\hline School Total & & 209 & 15 & 194 & 163 & 31 & School \%age & $7.18 \%$ & $92.82 \%$ & $84.02 \%$ & $15.98 \%$ \\
\hline
\end{tabular}

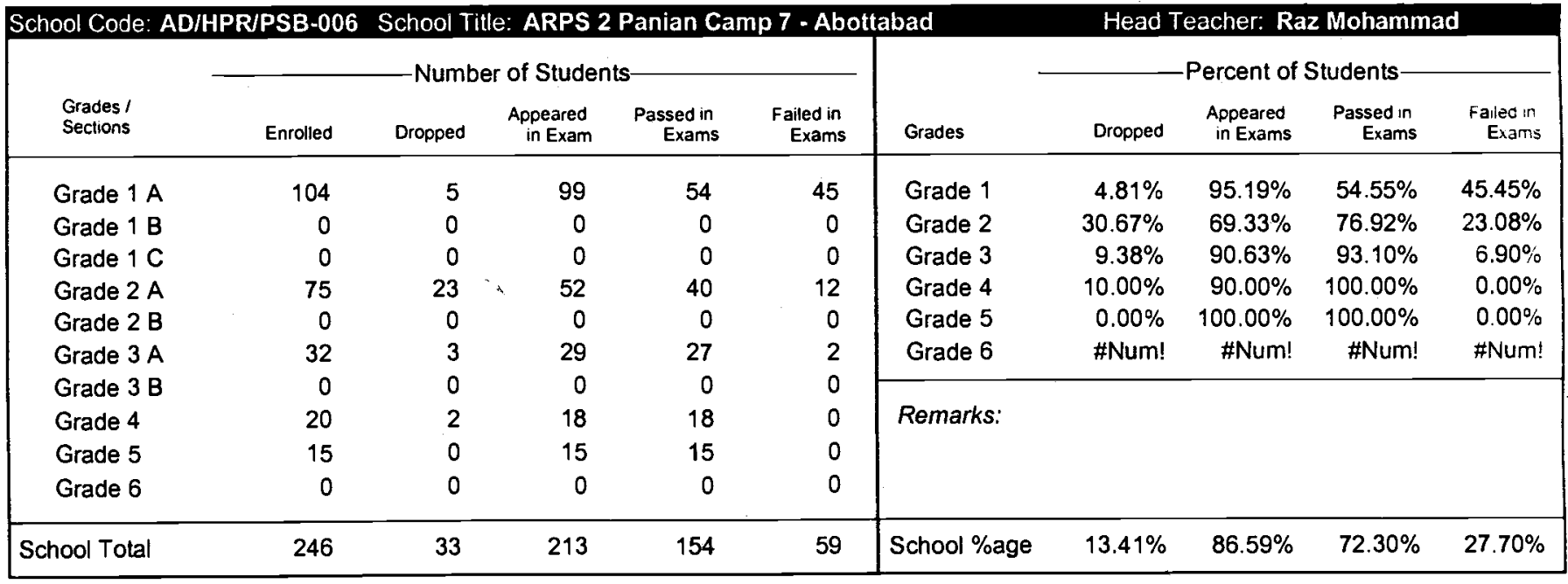


Sub-Centre: Abbotabad

School Code: AD/HPRPSB-007 School Title: ARPS Panian Camp 7 - Aboltabad

\begin{tabular}{|c|c|c|c|c|c|c|c|c|c|c|}
\hline \multirow[b]{2}{*}{$\begin{array}{l}\text { Grades I } \\
\text { Sections }\end{array}$} & \multicolumn{5}{|c|}{-Number of Students- } & \multirow[b]{2}{*}{ Grades } & \multicolumn{4}{|c|}{-Percent of Students } \\
\hline & Enrolled & Dropped & $\begin{array}{l}\text { Appeared } \\
\text { in Exam }\end{array}$ & $\begin{array}{l}\text { Passed in } \\
\text { Exams }\end{array}$ & $\begin{array}{l}\text { Failed in } \\
\text { Exams }\end{array}$ & & Dropped & $\begin{array}{l}\text { Appeared } \\
\text { in Exams }\end{array}$ & $\begin{array}{c}\text { Passed in } \\
\text { Exams }\end{array}$ & $\begin{array}{c}\text { Faled in } \\
\text { Exams }\end{array}$ \\
\hline Grade $1 \mathrm{~A}$ & 46 & 6 & 40 & 29 & 11 & Grade 1 & $13.48 \%$ & $86.52 \%$ & $67.53 \%$ & $32.47 \%$ \\
\hline Grade $1 \mathrm{~B}$ & 43 & 6 & 37 & 23 & 14 & Grade 2 & $8.14 \%$ & $91.86 \%$ & $74.68 \%$ & $25.32 \%$ \\
\hline Grade $1 \mathrm{C}$ & 0 & 0 & 0 & 0 & 0 & Grade 3 & $14.67 \%$ & $85.33 \%$ & $65.63 \%$ & $34.38 \%$ \\
\hline Grade 2 A & 42 & 2 & 40 & 35 & 5 & Grade 4 & $12.50 \%$ & $87.50 \%$ & $71.43 \%$ & $28.57 \%$ \\
\hline Grade 2 B & 44 & 5 & 39 & 24 & 15 & Grade 5 & $17.24 \%$ & $82.76 \%$ & $83.33 \%$ & $16.67 \%$ \\
\hline Grade 3 A & 75 & 11 & 64 & 42 & 22 & Grade 6 & $17.39 \%$ & $82.61 \%$ & $100.00 \%$ & $0.00 \%$ \\
\hline Grade 3 B & 0 & 0 & 0 & 0 & 0 & & & & & \\
\hline Grade 4 & 40 & 5 & 35 & 25 & 10 & Remarks: & & & & \\
\hline Grade 5 & 29 & 5 & 24 & 20 & 4 & & & & & \\
\hline Grade 6 & 23 & 4 & 19 & 19 & 0 & & & & & \\
\hline School Total & 342 & 44 & 298 & 217 & 81 & School \%age & $12.87 \%$ & $87.13 \%$ & $72.82 \%$ & $27.18 \%$ \\
\hline
\end{tabular}

\begin{tabular}{|c|c|c|c|c|c|c|c|c|c|c|c|}
\hline \multirow[b]{2}{*}{$\begin{array}{l}\text { Grades / } \\
\text { Sections }\end{array}$} & \multicolumn{6}{|c|}{ Number of Students____ N__ } & \multirow[b]{2}{*}{ Grades } & \multicolumn{4}{|c|}{ Percent of Students } \\
\hline & ' & Enrolled & Dropped & $\begin{array}{l}\text { Appeared } \\
\text { in Exam }\end{array}$ & $\begin{array}{l}\text { Passed in } \\
\text { Exams }\end{array}$ & $\begin{array}{c}\text { Failed in } \\
\text { Exams }\end{array}$ & & Dropped & $\begin{array}{l}\text { Appeared } \\
\text { in Exams }\end{array}$ & $\begin{array}{l}\text { Passed in } \\
\text { Exams }\end{array}$ & $\begin{array}{l}\text { Falied in } \\
\text { Exams }\end{array}$ \\
\hline Grade $1 \mathrm{~A}$ & & 52 & 3 & 49 & 44 & 5 & Grade 1 & $7.14 \%$ & $92.86 \%$ & $85.31 \%$ & $14.69 \%$ \\
\hline Grade $1 \mathrm{~B}$ & & 50 & 0 & 50 & 43 & 7 & Grade 2 & $0.00 \%$ & $100.00 \%$ & $86.96 \%$ & $13.04^{\mathrm{c}} \circ$ \\
\hline Grade $1 \mathrm{C}$ & & 52 & 8 & 44 & 35 & 9 & Grade 3 & $3.06 \%$ & $96.94 \%$ & $92.63 \%$ & $7.37 \%$ \\
\hline Grade 2 A & & 62 & 0 & 62 & 54 & 8 & Grade 4 & $1.89 \%$ & $98.11 \%$ & $86.54 \%$ & $13.46 \%$ \\
\hline Grade 2 B & & 53 & 0 & 53 & 46 & 7 & Grade 5 & $8.33 \%$ & $91.67 \%$ & $96.36 \%$ & $3.64 \%$ \\
\hline Grade 3 A & & 51 & 3 & 48 & 43 & 5 & Grade 6 & $2.38 \%$ & $97.62 \%$ & $100.00 \%$ & $0.00 \%$ \\
\hline Grade 3 B & & 47 & 0 & 47 & 45 & 2 & & & & & \\
\hline Grade 4 & & 53 & 1 & 52 & 45 & 7 & Remarks: & & & & \\
\hline Grade 5 & & 60 & 5 & 55 & 53 & 2 & & & & & \\
\hline Grade 6 & & 42 & 1 & 41 & 41 & 0 & & & & & \\
\hline School Total & & 522 & 21 & 501 & 449 & 52 & School \%age & $4.02 \%$ & $95.98 \%$ & $89.62 \%$ & $10.38 \%$ \\
\hline
\end{tabular}

\begin{tabular}{|c|c|c|c|c|c|c|c|c|c|c|}
\hline \multirow[b]{2}{*}{$\begin{array}{l}\text { Grades / } \\
\text { Sections }\end{array}$} & \multicolumn{5}{|c|}{ - Number of Students- } & \multirow[b]{2}{*}{ Grades } & \multicolumn{4}{|c|}{-Percent of Students } \\
\hline & Enrolled & Dropped & $\begin{array}{l}\text { Appeared } \\
\text { in Exam }\end{array}$ & $\begin{array}{l}\text { Passed in } \\
\text { Exams }\end{array}$ & $\begin{array}{l}\text { Failed in } \\
\text { Exams }\end{array}$ & & Dropped & $\begin{array}{l}\text { Appeared } \\
\text { in Exams }\end{array}$ & $\begin{array}{l}\text { Passed in } \\
\text { Exams }\end{array}$ & $\begin{array}{l}\text { Failed in } \\
\text { Exams }\end{array}$ \\
\hline Grade $1 \mathrm{~A}$ & 37 & 0 & 37 & 18 & 19 & Grade 1 & $0.00 \%$ & $100.00 \%$ & $46.53 \%$ & $53.47 \%$ \\
\hline Grade $1 \mathrm{~B}$ & 64 & 0 & 64 & 29 & 35 & Grade 2 & $0.00 \%$ & $100.00 \%$ & $59.09 \%$ & $40.91 \%$ \\
\hline Grade $1 \mathrm{C}$ & 0 & 0 & 0 & 0 & 0 & Grade 3 & $0.00 \%$ & $100.00 \%$ & $57.14 \%$ & $42.86 \%$ \\
\hline Grade 2 A & 37 & 0 & 37 & 20 & 17 & Grade 4 & $0.00 \%$ & $100.00 \%$ & $72.22 \%$ & $27.78 \%$ \\
\hline Grade 2 B & 29 & 0 & 29 & 19 & 10 & Grade 5 & $0.00 \%$ & $100.00 \%$ & $100.00 \%$ & $0.00 \%$ \\
\hline Grade 3 A & 20 & 0 & 20 & 12 & 8 & Grade 6 & \#Num! & \#Num! & \#Num! & \#Num! \\
\hline Grade 3 B & 22 & 0 & 22 & 12 & 10 & & & & & \\
\hline Grade 4 & 18 & 0 & 18 & 13 & 5 & Remarks: & & & & \\
\hline Grade 5 & 12 & 0 & 12 & 12 & 0 & & & & & \\
\hline Grade 6 & 0 & 0 & 0 & 0 & 0 & & & & & \\
\hline School Total & 239 & 0 & 239 & 135 & 104 & School \%age & $0.00 \%$ & $100.00 \%$ & $56.49 \%$ & $43.51 \%$ \\
\hline
\end{tabular}

Note:

\#Num! = Enrollment in respective grade(s) is nill therefore calculation is nol applicable
Page 3 of 91

Produced by DP/MIS Section on $3: \cdot$ Jul.:5 
Sub-Centre: Abbotabad

\begin{tabular}{|c|c|c|c|c|c|c|c|c|c|c|}
\hline \multicolumn{6}{|c|}{$\begin{array}{c}\text { School Code: AD/HPR/MSB-010 School Title: ARPS } 1 \text { Panian Camp } 12 \text { - Abc } \\
\text { Number of Students }\end{array}$} & \multirow[b]{2}{*}{ Grades } & \multicolumn{4}{|c|}{-Percent of Students - } \\
\hline $\begin{array}{l}\text { Grades / } \\
\text { Sections }\end{array}$ & Enrolled & Dropped & $\begin{array}{c}\text { Appeared } \\
\text { in Exam }\end{array}$ & $\begin{array}{r}\text { Passed in } \\
\text { Exams }\end{array}$ & $\begin{array}{c}\text { Failed in } \\
\text { Exams }\end{array}$ & & Dropped & $\begin{array}{c}\text { Appeared } \\
\text { in Exams }\end{array}$ & $\begin{array}{r}\text { Passed in } \\
\text { Exams }\end{array}$ & $\begin{array}{l}\text { Failed in } \\
\text { Exams }\end{array}$ \\
\hline Grade $1 \mathrm{~A}$ & 41 & 0 & 41 & 36 & 5 & Grade 1 & $1.30 \%$ & $98.70 \%$ & $70.39 \%$ & $29.61 \%$ \\
\hline Grade 1 B & 48 & 0 & 48 & 31 & 17 & Grade 2 & $5.22 \%$ & $94.78 \%$ & $77.95 \%$ & $22.05 \%$ \\
\hline Grade $1 \mathrm{C}$ & 65 & 2 & 63 & 40 & 23 & Grade 3 & $0.00 \%$ & $100.00 \%$ & $72.86 \%$ & $27.14 \%$ \\
\hline Grade 2 A & 68 & 4 & 64 & 50 & 14 & Grade 4 & $7.27 \%$ & $92.73 \%$ & $80.39 \%$ & $19.61 \%$ \\
\hline Grade 2 B & 66 & 3 & 63 & 49 & 14 & Grade 5 & $2.22 \%$ & $97.78 \%$ & $70.45 \%$ & $29.55 \%$ \\
\hline Grade 3 A & 32 & 0 & 32 & 24 & 8 & Grade 6 & $0.00 \%$ & $100.00 \%$ & $100.00 \%$ & $0.00 \%$ \\
\hline Grade 3 B & 38 & 0 & 38 & 27 & 11 & & & & & \\
\hline Grade 4 & 55 & 4 & 51 & 41 & 10 & Remarks: & & & & \\
\hline Grade 5 & 45 & 1 & 44 & 31 & 13 & & & & & \\
\hline Grade 6 & 27 & 0 & 27 & 27 & 0 & & & & & \\
\hline School Total & 485 & 14 & 471 & 356 & 115 & School \%age & $2.89 \%$ & $97.11 \%$ & $75.58 \%$ & $24.42 \%$ \\
\hline
\end{tabular}

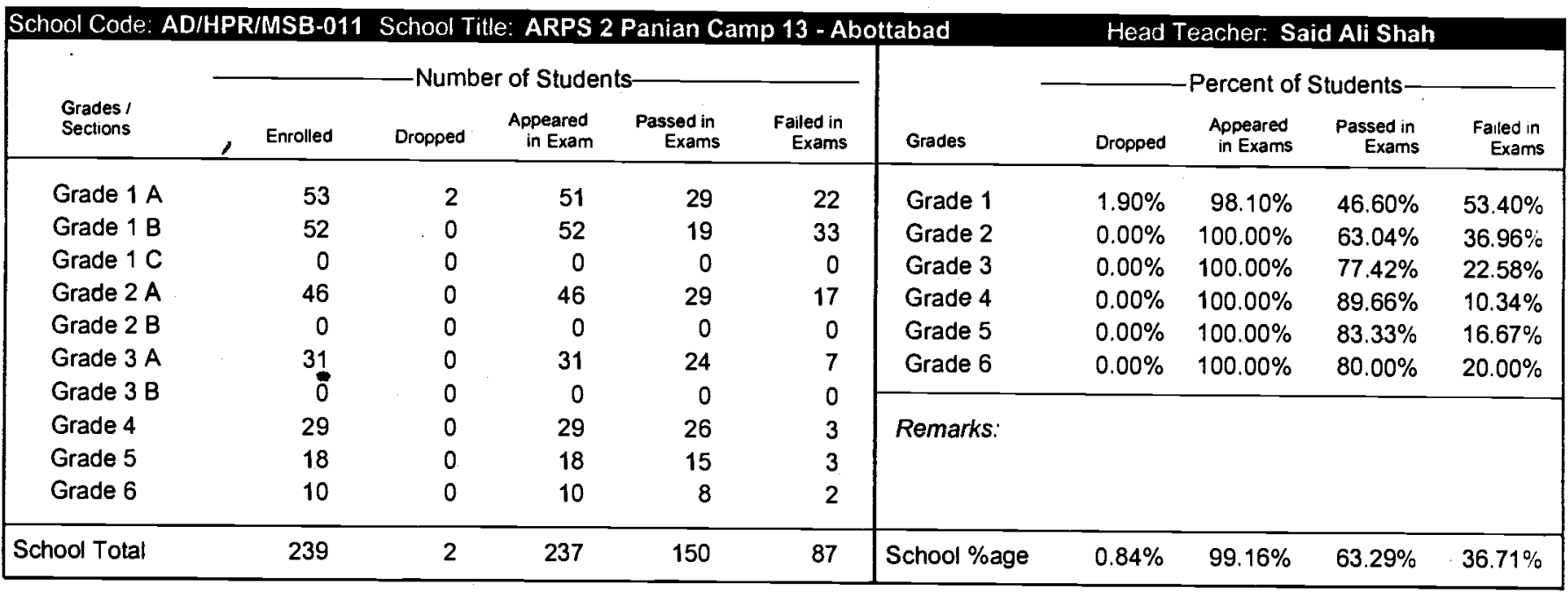

\begin{tabular}{|c|c|c|c|c|c|c|c|c|c|c|}
\hline \multirow[b]{2}{*}{$\begin{array}{l}\text { Grades / } \\
\text { Sections }\end{array}$} & MSB-O & $\begin{array}{l}\text { School T } \\
\text { Numb }\end{array}$ & $\begin{array}{l}\text { ARPS } \\
\text { of Stude }\end{array}$ & anian $\mathrm{C}$ & D $13 \cdot A b$ & \multirow[b]{2}{*}{ Grades } & \multicolumn{4}{|c|}{-Percent of Students } \\
\hline & Enrolied & Dropped & $\begin{array}{l}\text { Appeared } \\
\text { in Exam }\end{array}$ & $\begin{array}{l}\text { Passed in } \\
\text { Exams }\end{array}$ & $\begin{array}{l}\text { Failed in } \\
\text { Exams }\end{array}$ & & Dropped & $\begin{array}{l}\text { Appeared } \\
\text { in Exams }\end{array}$ & $\begin{array}{l}\text { Passed in } \\
\text { Exams }\end{array}$ & $\begin{array}{l}\text { Fatled in } \\
\text { Exams }\end{array}$ \\
\hline Grade $1 \mathrm{~A}$ & 84 & 4 & 80 & 49 & 31 & Grade 1 & $4.26 \%$ & $95.74 \%$ & $57.78 \%$ & $42.22 \%$ \\
\hline Grade $1 \mathrm{~B}$ & 78 & 4 & 74 & 47 & 27 & Grade 2 & $17.54 \%$ & $82.46 \%$ & $78.72 \%$ & $21.28 \%$ \\
\hline Grade $1 \mathrm{C}$ & 73 & 2 & 71 & 34 & 37 & Grade 3 & $6.12 \%$ & $93.88 \%$ & $67.39 \%$ & $32.61 \%$ \\
\hline Grade 2 A & 58 & 8 & 50 & 42 & 8 & Grade 4 & $11.11 \%$ & $88.89 \%$ & $78.13 \%$ & $21.88 \%$ \\
\hline Grade 2 B & 56 & 12 & 44 & 32 & 12 & Grade 5 & $21.74 \%$ & $78.26 \%$ & $75.00 \%$ & $25.00 \%$ \\
\hline Grade 3 A & 49 & 3 & 46 & 31. & 15 & Grade 6 & $13.16 \%$ & $86.84 \%$ & $72.73 \%$ & $27.27 \%$ \\
\hline Grade 3 B & 0 & 0 & 0 & 0 & 0 & & & & & \\
\hline Grade 4 & 36 & 4 & 32 & 25 & 7 & Remarks: & & & & \\
\hline Grade 5 & 46 & 10 & 36 & 27 & 9 & & & & & \\
\hline Grade 6 & 38 & 5 & 33 & 24 & 9 & & & & & \\
\hline School Total & 518 & 52 & 466 & 311 & 155 & School \%age & $10.04 \%$ & $89.96 \%$ & $66.74 \%$ & $33.26 \%$ \\
\hline
\end{tabular}


Sub-Centre: Abbotabad

School Code: AD/HPR/PSC-013 School Title: ARPS 1 Panian Camp 13 - Abottabad

Head Teacher: Mohammad Azim

\begin{tabular}{|c|c|c|c|c|c|c|c|c|c|c|}
\hline \multirow[b]{2}{*}{$\begin{array}{l}\text { Grades / } \\
\text { Sections }\end{array}$} & \multicolumn{5}{|c|}{-Number of Students- } & \multirow[b]{2}{*}{ Grades } & \multicolumn{4}{|c|}{-Percent of Students } \\
\hline & Enrolled & Dropped & $\begin{array}{l}\text { Appeared } \\
\text { in Exam }\end{array}$ & $\begin{array}{l}\text { Passed in } \\
\text { Exams }\end{array}$ & $\begin{array}{c}\text { Failed in } \\
\text { Exams }\end{array}$ & & Dropped & $\begin{array}{l}\text { Appeared } \\
\text { in Exams }\end{array}$ & $\begin{array}{l}\text { Passed in } \\
\text { Exams }\end{array}$ & $\begin{array}{l}\text { Falled in } \\
\text { Exams }\end{array}$ \\
\hline Grade $1 \mathrm{~A}$ & 68 & 0 & 68 & 34 & 34 & Grade 1 & $0.00 \%$ & $100.00 \%$ & $45.00 \%$ & $55.00 \%$ \\
\hline Grade $1 \mathrm{~B}$ & 32 & 0 & 32 & 11 & 21 & Grade 2 & $0.00 \%$ & $100.00 \%$ & $57.14 \%$ & $42.86 \%$ \\
\hline Grade $1 \mathrm{C}$ & 0 & 0 & 0 & 0 & 0 & Grade 3 & $4.35 \%$ & $95.65 \%$ & $68.18 \%$ & $31.82 \%$ \\
\hline Grade $2 \mathrm{~A}$ & 42 & 0 & 42 & 27 & 15 & Grade 4 & $4.76 \%$ & $95.24 \%$ & $75.00 \%$ & $25.00 \%$ \\
\hline Grade 2 B & 21 & 0 & 21 & 9 & 12 & Grade 5 & $0.00 \%$ & $100.00 \%$ & $72.22 \%$ & $27.78 \%$ \\
\hline Grade $3 \mathrm{~A}$ & 23 & 1 & 22 & 15 & 7 & Grade 6 & $0.00 \%$ & $100.00 \%$ & $92.86 \%$ & $7.14 \%$ \\
\hline Grade 3 B & 0 & 0 & 0 & 0 & 0 & & & & & \\
\hline Grade 4 & 21 & 1 & 20 & 15 & 5 & Remarks: & & & & \\
\hline Grade 5 & 18 & 0 & 18 & 13 & 5 & & & & & \\
\hline Grade 6 & 14 & 0 & 14 & 13 & 1 & & & & & \\
\hline School Total & 239 & 2 & 237 & 137 & 100 & School \%age & $0.84 \%$ & $99.16 \%$ & $57.81 \%$ & $42.19 \%$ \\
\hline
\end{tabular}

\begin{tabular}{|c|c|c|c|c|c|c|c|c|c|c|c|}
\hline \multirow[b]{2}{*}{$\begin{array}{l}\text { Grades I } \\
\text { Sections }\end{array}$} & \multicolumn{6}{|c|}{ - Number of Students- } & \multirow[b]{2}{*}{ Grades } & \multicolumn{4}{|c|}{-Percent of Students- } \\
\hline & ' & Enrolled & Dropped & $\begin{array}{l}\text { Appeared } \\
\text { in Exam }\end{array}$ & $\begin{array}{l}\text { Passed in } \\
\text { Exams }\end{array}$ & $\begin{array}{c}\text { Failed in } \\
\text { Exams }\end{array}$ & & Dropped & $\begin{array}{c}\text { Appeared } \\
\text { in Exams }\end{array}$ & $\begin{array}{l}\text { Passed in } \\
\text { Exams }\end{array}$ & $\begin{array}{l}\text { Failed in } \\
\text { Exams }\end{array}$ \\
\hline Grade $1 \mathrm{~A}$ & & 86 & 6 & 80 & 45 & 35 & Grade 1 & $6.98 \%$ & $93.02 \%$ & $56.25 \%$ & $43.75 \%$ \\
\hline Grade 1 B & & 0 & 0 & 0 & 0 & 0 & Grade 2 & $0.00 \%$ & $100.00 \%$ & $88.00 \%$ & $12.00 \%$ \\
\hline Grade $1 \mathrm{C}$ & & 0 & 0 & 0 & 0 & 0 & Grade 3 & $3.23 \%$ & $96.77 \%$ & $100.00 \%$ & $0.00 \%$ \\
\hline Grade 2 A & & 50 & 0 & 50 & 44 & 6 & Grade 4 & $0.00 \%$ & $100.00 \%$ & $80.00 \%$ & $20.00 \%$ \\
\hline Grade 2 B & & 0 & 0 & 0 & 0 & 0 & Grade 5 & $0.00 \%$ & $100.00 \%$ & $83.33 \%$ & $16.67 \%$ \\
\hline Grade $3 \mathrm{~A}$ & & 37 & 1 & 30 & 30 & 0 & Grade 6 & $0.00 \%$ & $100.00 \%$ & $100.00 \%$ & $0.00 \%$ \\
\hline Grade 3 B & & 0 & 0 & 0 & 0 & 0 & & & & & \\
\hline Grade 4 & & 25 & 0 & 25 & 20 & 5 & Remarks: & & & & \\
\hline Grade 5 & & 12 & 0 & 12 & 10 & 2 & & & & & \\
\hline Grade 6 & & 11 & 0 & 11 & 11 & 0 & & & & & \\
\hline School Total & & 215 & 7 & 208 & 160 & 48 & School \%age & $3.26 \%$ & $96.74 \%$ & $76.92 \%$ & $23.08 \%$ \\
\hline
\end{tabular}

\begin{tabular}{|c|c|c|c|c|c|c|c|c|c|c|}
\hline \multirow[b]{2}{*}{$\begin{array}{l}\text { Grades I } \\
\text { Sections }\end{array}$} & \multicolumn{5}{|c|}{ - Number of Students } & \multirow[b]{2}{*}{ Grades } & \multicolumn{4}{|c|}{ Percent of Students- } \\
\hline & Enrolled & Dropped & $\begin{array}{l}\text { Appeared } \\
\text { in Exam }\end{array}$ & $\begin{array}{l}\text { Passed in } \\
\text { Exams }\end{array}$ & $\begin{array}{l}\text { Failed in } \\
\text { Exams }\end{array}$ & & Dropped & $\begin{array}{l}\text { Appeared } \\
\text { in Exams }\end{array}$ & $\begin{array}{l}\text { Passed in } \\
\text { Exams }\end{array}$ & $\begin{array}{l}\text { Failed in } \\
\text { Exams }\end{array}$ \\
\hline Grade $1 \mathrm{~A}$ & 65 & 21 & 44 & 33 & 11 & Grade 1 & $25.87 \%$ & $74.13 \%$ & $64.15 \%$ & $35.85 \%$ \\
\hline Grade $1 \mathrm{~B}$ & 78 & 16 & 62 & 35 & 27 & Grade 2 & $21.82 \%$ & $78.18 \%$ & $90.70 \%$ & $9.30 \%$ \\
\hline Grade $1 \mathrm{C}$ & 0 & 0 & 0 & 0 & 0 & Grade 3 & $29.41 \%$ & $70.59 \%$ & $70.83 \%$ & $29.17 \%$ \\
\hline Grade 2 A & 55 & 12 & 43 & 39 & 4 & Grade 4 & $31.71 \%$ & $68.29 \%$ & $82.14 \%$ & $17.86 \%$ \\
\hline Grade 2 B & 0 & 0 & 0 & 0 & 0 & Grade 5 & $31.25 \%$ & $68.75 \%$ & $77.27 \%$ & $22.73 \%$ \\
\hline Grade $3 \mathrm{~A}$ & 68 & 20 & 48 & 34 & 14 & Grade 6 & $29.63 \%$ & $70.37 \%$ & $84.21 \%$ & $15.79 \%$ \\
\hline Grade 3 B & 0 & 0 & 0 & 0 & 0 & & & & & \\
\hline Grade 4 & 41 & 13 & 28 & 23 & 5 & Remarks: & & & & \\
\hline Grade 5 & 32 & 10 & 22 & 17 & 5 & & & & & \\
\hline Grade 6 & 27 & 8 & 19 & 16 & 3 & & & & & \\
\hline School Total & 366 & 100 & 266 & 197 & 69 & School \%age & $27.32 \%$ & $72.68 \%$ & $74.06 \%$ & $25.94 \%$ \\
\hline
\end{tabular}

Note:

\#Num! = Enrollment in respective grade(s) is nill therefore calculation is not applicable
Page 5 of 91

Produced by DP/MIS Section on $31 \cdot$ Jul -97 
Sub-Centre: Abbotabad

\begin{tabular}{|c|c|c|c|c|c|c|c|c|c|c|}
\hline \multirow[b]{2}{*}{$\begin{array}{l}\text { Grades / } \\
\text { Sections }\end{array}$} & \multicolumn{5}{|c|}{ - Number of Students- } & \multirow[b]{2}{*}{ Grades } & \multicolumn{4}{|c|}{-Percent of Students- } \\
\hline & Enrolled & Dropped & $\begin{array}{c}\text { Appeared } \\
\text { in Exam }\end{array}$ & $\begin{array}{c}\text { Passed in } \\
\text { Exams }\end{array}$ & $\begin{array}{l}\text { Failed in } \\
\text { Exams }\end{array}$ & & Dropped & $\begin{array}{l}\text { Appeared } \\
\text { in Exams }\end{array}$ & $\begin{array}{c}\text { Passed in } \\
\text { Exams }\end{array}$ & $\begin{array}{l}\text { Faled in } \\
\text { Exams }\end{array}$ \\
\hline Grade $1 \mathrm{~A}$ & 84 & 9 & 75 & 42 & 33 & Grade 1 & $10.71 \%$ & $89.29 \%$ & $56.00 \%$ & $44.00 \%$ \\
\hline Grade 1 B & 0 & 0 & 0 & 0 & 0 & Grade 2 & $19.57 \%$ & $80.43 \%$ & $86.49 \%$ & $13.51 \%$ \\
\hline Grade $1 \mathrm{C}$ & 0 & 0 & 0 & 0 & 0 & Grade 3 & $9.68 \%$ & $90.32 \%$ & $100.00 \%$ & $0.00 \%$ \\
\hline Grade 2 A & 46 & 9 & 37 & 32 & 5 & Grade 4 & $7.41 \%$ & $92.59 \%$ & $84.00 \%$ & $16.00 \%$ \\
\hline Grade 2 B & 0 & 0 & 0 & 0 & 0 & Grade 5 & $20.00 \%$ & $80.00 \%$ & $100.00 \%$ & $0.00 \%$ \\
\hline Grade 3 A & 31 & 3 & 28 & 28 & 0 & Grade 6 & $7.69 \%$ & $92.31 \%$ & $100.00 \%$ & $0.00 \%$ \\
\hline Grade 3 B & 0 & 0 & 0 & 0 & 0 & & & & & \\
\hline Grade 4 & 27 & 2 & 25 & 21 & 4 & Remarks: & & & & \\
\hline Grade 5 & 15 & 3 & 12 & 12 & 0 & & & & & \\
\hline Grade 6 & 13 & 1 & 12 & 12 & 0 & & & & & \\
\hline School Total & 216 & 27 & 189 & 147 & 42 & School \%age & $12.50 \%$ & $87.50 \%$ & $77.78 \%$ & $22.22 \%$ \\
\hline
\end{tabular}

\begin{tabular}{|c|c|c|c|c|c|c|c|c|c|c|c|}
\hline \multirow[b]{2}{*}{$\begin{array}{l}\text { Grades / } \\
\text { Sections }\end{array}$} & \multicolumn{6}{|c|}{ Number of Students } & \multirow[b]{2}{*}{ Grades } & \multicolumn{4}{|c|}{-Percent of Students - } \\
\hline & ' & Enrolled & Dropped & $\begin{array}{l}\text { Appeared } \\
\text { in Exam }\end{array}$ & $\begin{array}{l}\text { Passed in } \\
\text { Exams }\end{array}$ & $\begin{array}{l}\text { Failed in } \\
\text { Exams }\end{array}$ & & Dropped & $\begin{array}{l}\text { Appeared } \\
\text { in Exams }\end{array}$ & $\begin{array}{l}\text { Passed in } \\
\text { Exams }\end{array}$ & $\begin{array}{l}\text { Failed in } \\
\text { Exams }\end{array}$ \\
\hline Grade $1 \mathrm{~A}$ & & 71 & 0 & 71 & 50 & 21 & Grade 1 & $3.03 \%$ & $96.97 \%$ & $75.00 \%$ & $25.00 \%$ \\
\hline Grade $1 \mathrm{~B}$ & & 28 & 3 & 25 & 22 & 3 & Grade 2 & $2.94 \%$ & $97.06 \%$ & $93.94 \%$ & $6.06 \%$ \\
\hline Grade $1 \mathrm{C}$ & & 0 & 0 & 0 & 0 & 0 & Grade 3 & $0.00 \%$ & $100.00 \%$ & $81.48 \%$ & $18.52 \%$ \\
\hline Grade 2 A & & 34 & 1 & 33 & 31 & 2 & Grade 4 & $0.00 \%$ & $100.00 \%$ & $84.62 \%$ & $15.38 \%$ \\
\hline Grade 2 B & & 0 & 0 & 0 & 0 & 0 & Grade 5 & $0.00 \%$ & $100.00 \%$ & $100.00 \%$ & $0.00 \%$ \\
\hline Grade $3 \mathrm{~A}$ & & 27 & 0 & 27 & 22 & 5 & Grade 6 & $0.00 \%$ & $100.00 \%$ & $85.71 \%$ & $14.29 \%$ \\
\hline Grade 3 B & & 0 & 0 & 0 & 0 & 0 & & & & & \\
\hline Grade 4 & & 26 & 0 & 26 & 22 & 4 & Remarks: & & & & \\
\hline Grade 5 & & 10 & 0 & 10 & 10 & 0 & & & & & \\
\hline Grade 6 & & 7 & 0 & 7 & 6 & 1 & & & & & \\
\hline School Total & & 203 & 4 & 199 & 163 & 36 & School \%age & $1.97 \%$ & $98.03 \%$ & $81.91 \%$ & $18.09 \%$ \\
\hline
\end{tabular}

\begin{tabular}{|c|c|c|c|c|c|c|c|c|c|c|}
\hline \multirow[b]{2}{*}{$\begin{array}{l}\text { Grades I } \\
\text { Sections }\end{array}$} & \multicolumn{5}{|c|}{-Number of Students- } & \multirow[b]{2}{*}{ Grades } & \multicolumn{4}{|c|}{-Percent of Students- } \\
\hline & Enrolled & Dropped & $\begin{array}{l}\text { Appeared } \\
\text { in Exam }\end{array}$ & $\begin{array}{c}\text { Passed in } \\
\text { Exams }\end{array}$ & $\begin{array}{c}\text { Failed in } \\
\text { Exams }\end{array}$ & & Dropped & $\begin{array}{c}\text { Appeared } \\
\text { in Exams }\end{array}$ & $\begin{array}{c}\text { Passed in } \\
\text { Exams } \\
\end{array}$ & $\begin{array}{c}\text { Falled in } \\
\text { Exams }\end{array}$ \\
\hline Grade $1 \mathrm{~A}$ & 65 & 6 & 59 & 41 & 18 & Grade 1 & $9.23 \%$ & $90.77 \%$ & $69.49 \%$ & $30.51 \%$ \\
\hline Grade 1 B & 0 & 0 & 0 & 0 & 0 & Grade 2 & $8.62 \%$ & $91.38 \%$ & $66.04 \%$ & $33.96 \%$ \\
\hline Grade $1 \mathrm{C}$ & 0 & 0 & 0 & 0 & 0 & Grade 3 & $12.31 \%$ & $87.69 \%$ & $84.21 \%$ & $15.79 \%$ \\
\hline Grade $2 \mathrm{~A}$ & 58 & 5 & 53 & 35 & 18 & Grade 4 & $6.98 \%$ & $93.02 \%$ & $82.50 \%$ & $17.50 \%$ \\
\hline Grade 2 B & 0 & 0 & 0 & 0 & 0 & Grade 5 & $10.00 \%$ & $90.00 \%$ & $85.19 \%$ & $14.81 \%$ \\
\hline Grade 3 A & 65 & 8 & 57 & 48 & 9 & Grade 6 & $14.29 \%$ & $85.71 \%$ & $83.33 \%$ & $16.67 \%$ \\
\hline Grade 3 B & 0 & 0 & 0 & 0 & 0 & & & & & \\
\hline Grade 4 & 43 & 3 & 40 & 33 & 7 & Remarks: & & & & \\
\hline Grade 5 & 30 & 3 & 27 & 23 & 4 & & & & & \\
\hline Grade 6 & 35 & 5 & 30 & 25 & 5 & & & & & \\
\hline School Total & 296 & 30 & 266 & 205 & 61 & School \%age & $10.14 \%$ & $89.86 \%$ & $77.07 \%$ & $22.93 \%$ \\
\hline
\end{tabular}

Note: 
Sub-Centre: Abbotabad

School Code: AD/MNS/PSB-019 School Title: ARPS 2 Brari Camp 2 - Mansehra Head Teacher: Daud Shah

\begin{tabular}{|c|c|c|c|c|c|c|c|c|c|c|}
\hline \multirow[b]{2}{*}{$\begin{array}{l}\text { Grades / } \\
\text { Sections }\end{array}$} & \multicolumn{5}{|c|}{ - Number of Students- } & \multirow[b]{2}{*}{ Grades } & \multicolumn{4}{|c|}{-Percent of Students- } \\
\hline & Enrolled & Dropped & $\begin{array}{l}\text { Appeared } \\
\text { in Exam }\end{array}$ & $\begin{array}{l}\text { Passed in } \\
\text { Exams }\end{array}$ & $\begin{array}{l}\text { Failed in } \\
\text { Exams }\end{array}$ & & Dropped & $\begin{array}{c}\text { Appeared } \\
\text { in Exams }\end{array}$ & $\begin{array}{l}\text { Passed in } \\
\text { Exams }\end{array}$ & $\begin{array}{r}\text { Falled in } \\
\text { Exams }\end{array}$ \\
\hline Grade $1 \mathrm{~A}$ & 50 & 0 & 50 & 34 & 16 & Grade 1 & $0.00 \%$ & $100.00 \%$ & $68.00 \%$ & $32.00 \%$ \\
\hline Grade $1 \mathrm{~B}$ & 0 & 0 & 0 & 0 & 0 & Grade 2 & $0.00 \%$ & $100.00 \%$ & $90.24 \%$ & $9.76 \%$ \\
\hline Grade $1 \mathrm{C}$ & 0 & 0 & 0 & 0 & 0 & Grade 3 & $0.00 \%$ & $100.00 \%$ & $86.96 \%$ & $13.04 \%$ \\
\hline Grade $2 \mathrm{~A}$ & 41 & 0 & 41 & 37 & 4 & Grade 4 & $0.00 \%$ & $100.00 \%$ & $88.89 \%$ & $11.11 \%$ \\
\hline Grade 2 B & 0 & 0 & 0 & 0 & 0 & Grade 5 & $0.00 \%$ & $100.00 \%$ & $64.29 \%$ & $35.71 \%$ \\
\hline Grade $3 \mathrm{~A}$ & 23 & 0 & 23 & 20 & 3 & Grade 6 & \#Num! & \#Num! & \#Num! & \#Num! \\
\hline Grade 3 B & 0 & 0 & 0 & 0 & 0 & & & & & \\
\hline Grade 4 & 18 & 0 & 18 & 16 & 2 & Remarks: & & & & \\
\hline Grade 5 & 14 & 0 & 14 & 9 & 5 & & & & & \\
\hline Grade 6 & 0 & 0 & 0 & 0 & 0 & & & & & \\
\hline chool Total & 146 & 0 & 146 & 116 & 30 & School \%age & $0.00 \%$ & $100.00 \%$ & $79.45 \%$ & $20.55 \%$ \\
\hline
\end{tabular}

\begin{tabular}{|c|c|c|c|c|c|c|c|c|c|c|c|}
\hline \multirow[b]{2}{*}{$\begin{array}{l}\text { Grades / } \\
\text { Sections }\end{array}$} & \multicolumn{6}{|c|}{ - Number of Students- } & \multirow[b]{2}{*}{ Grades } & \multicolumn{4}{|c|}{-Percent of Students } \\
\hline & , & Enrolled & Dropped & $\begin{array}{l}\text { Appeared } \\
\text { in Exam }\end{array}$ & $\begin{array}{r}\text { Passed in } \\
\text { Exams }\end{array}$ & $\begin{array}{c}\text { Failed in } \\
\text { Exams }\end{array}$ & & Dropped & $\begin{array}{l}\text { Appeared } \\
\text { in Exams }\end{array}$ & $\begin{array}{l}\text { Passed in } \\
\text { Exams }\end{array}$ & $\begin{array}{l}\text { Faled in } \\
\text { Exams }\end{array}$ \\
\hline Grade $1 \mathrm{~A}$ & & 30 & 0 & 30 & 20 & 10 & Grade 1 & $0.00 \%$ & $100.00 \%$ & $66.67 \%$ & $33.33 \%$ \\
\hline Grade 1 B & & 0 & 0 & 0 & 0 & 0 & Grade 2 & $0.00 \%$ & $100.00 \%$ & $72.00 \%$ & $28.00 \%$ \\
\hline Grade $1 \mathrm{C}$ & & 0 & 0 & 0 & 0 & 0 & Grade 3 & $0.00 \%$ & $100.00 \%$ & $76.47 \%$ & $23.53 \%$ \\
\hline Grade 2 A & & 25 & 0 & 25 & 18 & 7 & Grade 4 & $0.00 \%$ & $100.00 \%$ & $78.95 \%$ & $21.05 \%$ \\
\hline Grade 2 B & & 0 & 0 & 0 & 0 & 0 & Grade 5 & $0.00 \%$ & $100.00 \%$ & $61.54 \%$ & $38.46 \%$ \\
\hline Grade 3 A & & 17 & 0 & 17 & 13 & 4 & Grade 6 & \#Num! & \#Num! & \#Num! & \#Num! \\
\hline Grade 3 B & & 0 & 0 & 0 & 0 & 0 & & & & & \\
\hline Grade 4 & & 19 & 0 & 19 & 15 & 4 & Remarks: & & & & \\
\hline Grade 5 & & 13 & 0 & 13 & 8 & 5 & & & & & \\
\hline Grade 6 & & 0 & 0 & 0 & 0 & 0 & & & & & \\
\hline School Total & & 104 & 0 & 104 & 74 & 30 & School \%age & $0.00 \%$ & $100.00 \%$ & $71.15 \%$ & $28.85 \%$ \\
\hline
\end{tabular}

\begin{tabular}{|c|c|c|c|c|c|c|c|c|c|c|}
\hline \multirow[b]{2}{*}{$\begin{array}{l}\text { Grades I } \\
\text { Sections }\end{array}$} & \multicolumn{5}{|c|}{ - Number of Students- } & \multirow[b]{2}{*}{ Grades } & \multicolumn{4}{|c|}{ Percent of Students } \\
\hline & Enrolled & Dropped & $\begin{array}{l}\text { Appeared } \\
\text { in Exam }\end{array}$ & $\begin{array}{l}\text { Passed in } \\
\text { Exams }\end{array}$ & $\begin{array}{c}\text { Failed in } \\
\text { Exams }\end{array}$ & & Dropped & $\begin{array}{l}\text { Appeared } \\
\text { in Exams }\end{array}$ & $\begin{array}{c}\text { Passed in } \\
\text { Exams }\end{array}$ & $\begin{array}{c}\text { Falled in } \\
\text { Exams }\end{array}$ \\
\hline Grade $1 \mathrm{~A}$ & 53 & 1 & 52 & 40 & 12 & Grade 1 & $4.17 \%$ & $95.83 \%$ & $77.17 \%$ & $22.83 \%$ \\
\hline Grade 1 B & 43 & 3 & 40 & 31 & 9 & Grade 2 & $0.00 \%$ & $100.00 \%$ & $74.00 \%$ & $26.00 \%$ \\
\hline Grade $1 \mathrm{C}$ & 0 & 0 & 0 & 0 & 0 & Grade 3 & $3.23 \%$ & $96.77 \%$ & $66.67 \%$ & $33.33 \%$ \\
\hline Grade 2 A & 50 & 0 & 50 & 37 & 13 & Grade 4 & $8.33 \%$ & $91.67 \%$ & $72.73 \%$ & $27.27 \%$ \\
\hline Grade 2 B & 0 & 0 & 0 & 0 & 0 & Grade 5 & $0.00 \%$ & $100.00 \%$ & $88.24 \%$ & $11.76 \%$ \\
\hline Grade 3 A & 31 & 1 & 30 & 20 & 10 & Grade 6 & $10.00 \%$ & $90.00 \%$ & $77.78 \%$ & $22.22 \%$ \\
\hline Grade 3 B & 0 & 0 & 0 & 0 & 0 & & & & & \\
\hline Grade 4 & 24 & 2 & 22 & 16 & 6 & Remarks: & & & & \\
\hline Grade 5 & 17 & 0 & 17 & 15 & 2 & & & & & \\
\hline Grade 6 & 10 & 1 & 9 & 7 & 2 & & & & & \\
\hline School Total & 228 & 8 & 220 & 166 & 54 & School \%age & $3.51 \%$ & $96.49 \%$ & $75.45 \%$ & $24.55 \%$ \\
\hline
\end{tabular}

Note: 
Sub-Centre: Abbotabad

\begin{tabular}{|c|c|c|c|c|c|c|c|c|c|c|}
\hline \multirow[b]{2}{*}{$\begin{array}{l}\text { Grades } 1 \\
\text { Sections }\end{array}$} & \multicolumn{5}{|c|}{ Number of Students } & \multirow[b]{2}{*}{ Grades } & \multicolumn{4}{|c|}{-Percent of Students- } \\
\hline & Enrolled & Dropped & $\begin{array}{l}\text { Appeared } \\
\text { in Exam }\end{array}$ & $\begin{array}{r}\text { Passed in } \\
\text { Exams }\end{array}$ & $\begin{array}{l}\text { Failed in } \\
\text { Exams }\end{array}$ & & Dropped & $\begin{array}{l}\text { Appeared } \\
\text { in Exarns }\end{array}$ & $\begin{array}{l}\text { Passed in } \\
\text { Exams }\end{array}$ & $\begin{array}{l}\text { Fallec in } \\
\text { Exams }\end{array}$ \\
\hline Grade $1 \mathrm{~A}$ & 52 & 0 & 52 & 24 & 28 & Grade 1 & $0.00 \%$ & $100.00 \%$ & $46.15 \%$ & $53.85 \%$ \\
\hline Grade 1 B & 0 & 0 & 0 & 0 & 0 & Grade 2 & $0.00 \%$ & $100.00 \%$ & $48.65 \%$ & $51.35 \%$ \\
\hline Grade $1 \mathrm{C}$ & 0 & 0 & 0 & 0 & 0 & Grade 3 & $13.04 \%$ & $86.96 \%$ & $65.00 \%$ & $35.00 \%$ \\
\hline Grade 2 A & 37 & 0 & 37 & 18 & 19 & Grade 4 & $6.67 \%$ & $93.33 \%$ & $64.29 \%$ & $35.71 \%$ \\
\hline Grade 2 B & 0 & 0 & 0 & 0 & 0 & Grade 5 & $10.00 \%$ & $90.00 \%$ & $55.56 \%$ & $44.44 \%$ \\
\hline Grade $3 \mathrm{~A}$ & 23 & 3 & 20 & 13 & 7 & Grade 6 & $0.00 \%$ & $100.00 \%$ & $57.14 \%$ & $42.86 \%$ \\
\hline Grade 3 B & 0 & 0 & 0 & 0 & 0 & & & & & \\
\hline Grade 4 & 15 & 1 & 14 & 9 & 5 & Remarks: & & & & \\
\hline Grade 5 & 10 & 1 & 9 & 5 & 4 & & & & & \\
\hline Grade 6 & 7 & 0 & 7 & 4 & 3 & & & & & \\
\hline School Total & 144 & 5 & 139 & 73 & 66 & School \%age & $3.47 \%$ & $96.53 \%$ & $52.52 \%$ & $47.48 \%$ \\
\hline
\end{tabular}

School Code: AD/MNS/PSB-023 School Title: ARPS H. Maira Camp 6 - Mansehra

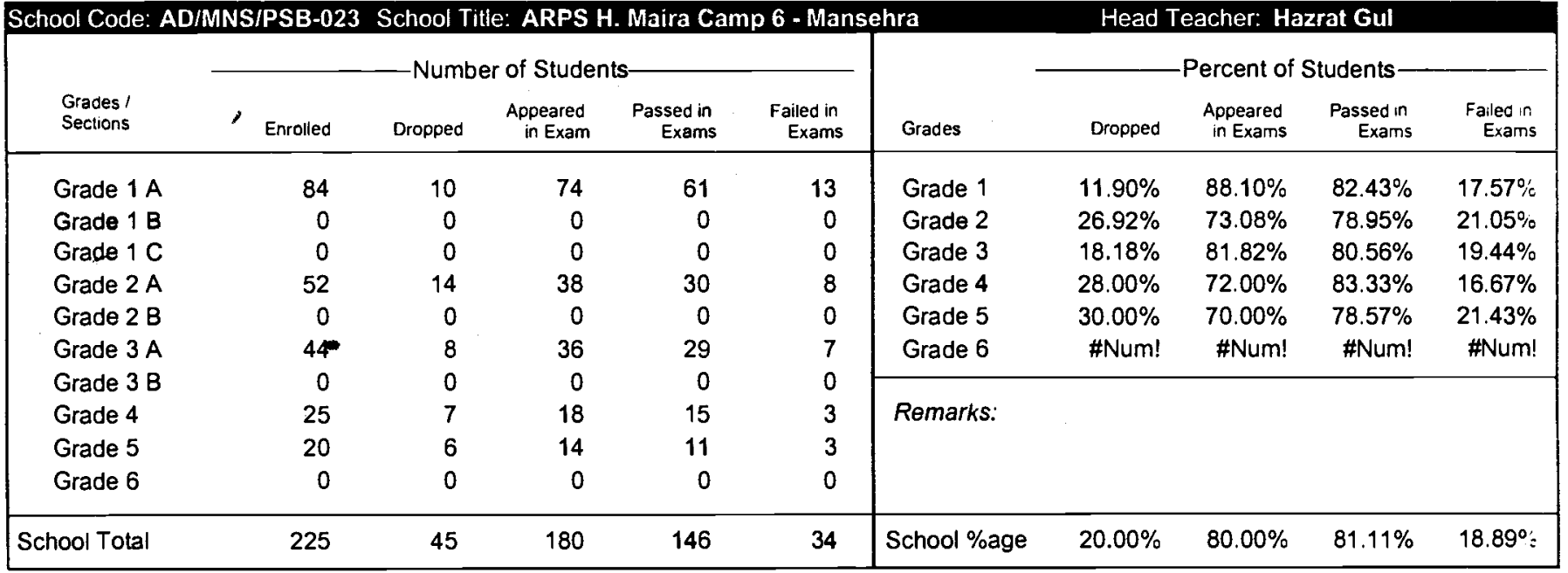

\begin{tabular}{|c|c|c|c|c|c|c|c|c|c|c|}
\hline \multirow[b]{2}{*}{$\begin{array}{l}\text { Grades I } \\
\text { Sections }\end{array}$} & \multicolumn{5}{|c|}{ Number of Students } & \multirow[b]{2}{*}{ Grades } & \multicolumn{4}{|c|}{-Percent of Students - } \\
\hline & Enrolled & Dropped & $\begin{array}{l}\text { Appeared } \\
\text { in Exam }\end{array}$ & $\begin{array}{l}\text { Passed in } \\
\text { Exams }\end{array}$ & $\begin{array}{l}\text { Failed in } \\
\text { Exams }\end{array}$ & & Dropped & $\begin{array}{l}\text { Appeared } \\
\text { in Exams }\end{array}$ & $\begin{array}{l}\text { Passed in } \\
\text { Exams }\end{array}$ & $\begin{array}{l}\text { Fasled in } \\
\text { Exams }\end{array}$ \\
\hline Grade 1 A & 73 & 18 & 55 & 35 & 20 & Grade 1 & $24.66 \%$ & $75.34 \%$ & $63.64 \%$ & $36.36 \%$ \\
\hline Grade 1 B & 0 & 0 & 0 & 0 & 0 & Grade 2 & $25.53 \%$ & $74.47 \%$ & $68.57 \%$ & $31.43 \%$ \\
\hline Grade $1 \mathrm{C}$ & 0 & 0 & 0 & 0 & 0 & Grade 3 & $20.69 \%$ & $79.31 \%$ & $56.52 \%$ & $43.48 \%$ \\
\hline Grade 2 A & 47 & 12 & 35 & 24 & 11 & Grade 4 & $40.00 \%$ & $60.00 \%$ & $53.33 \%$ & $46.67 \%$ \\
\hline Grade 2 B & 0 & 0 & 0 & 0 & 0 & Grade 5 & $32.14 \%$ & $67.86 \%$ & $31.58 \%$ & $68.42 \%$ \\
\hline Grade 3 A & 29 & 6 & 23 & 13 & 10 & Grade 6 & $26.83 \%$ & $73.17 \%$ & $60.00 \%$ & $40.00 \%$ \\
\hline Grade 3 B & 0 & 0 & 0 & 0 & 0 & & & & & \\
\hline Grade 4 & 25 & 10 & 15 & 8 & 7 & Remarks: & & & & \\
\hline Grade 5 & 28 & 9 & 19 & 6 & 13 & & & & & \\
\hline Grade 6 & 41 & 11 & 30 & 18 & 12 & & & & & \\
\hline School Total & 243 & 66 & 177 & 104 & 73 & School \%age & $27.16 \%$ & $72.84 \%$ & $58.76 \%$ & $41.24 \%$ \\
\hline
\end{tabular}

Note:

\#Num! = Enroliment in respective grade(s) is nill therefore calculation is not applicable
Page 8 of 91 Produced by DP/MIS Section on 31-Jul-97 
Sub-Centre: Abbotabad

\begin{tabular}{|c|c|c|c|c|c|c|c|c|c|c|}
\hline \multirow[b]{2}{*}{$\begin{array}{l}\text { Grades I } \\
\text { Sections }\end{array}$} & \multicolumn{5}{|c|}{ - Number of Students- } & \multirow[b]{2}{*}{ Grades } & \multicolumn{4}{|c|}{ Percent of Students } \\
\hline & Enrolled & Dropped & $\begin{array}{l}\text { Appeared } \\
\text { in Exam }\end{array}$ & $\begin{array}{l}\text { Passed in } \\
\text { Exams }\end{array}$ & $\begin{array}{l}\text { Failed in } \\
\text { Exams }\end{array}$ & & Dropped & $\begin{array}{l}\text { Appeared } \\
\text { in Exams }\end{array}$ & $\begin{array}{l}\text { Passed in } \\
\text { Exams }\end{array}$ & $\begin{array}{l}\text { Faled in } \\
\text { Exams }\end{array}$ \\
\hline Grade $1 \mathrm{~A}$ & 51 & 13 & 38 & 22 & 16 & Grade 1 & $25.49 \%$ & $74.51 \%$ & $57.89 \%$ & $42.11 \%$ \\
\hline Grade $1 \mathrm{~B}$ & 0 & 0 & 0 & 0 & 0 & Grade 2 & $4.44 \%$ & $95.56 \%$ & $60.47 \%$ & $39.53 \%$ \\
\hline Grade $1 \mathrm{C}$ & 0 & 0 & 0 & 0 & 0 & Grade 3 & $17.14 \%$ & $82.86 \%$ & $55.17 \%$ & $44.83 \%$ \\
\hline Grade 2 A & 45 & 2 & 43 & 26 & 17 & Grade 4 & $23.81 \%$ & $76.19 \%$ & $87.50 \%$ & $12.50 \%$ \\
\hline Grade 2 B & 0 & 0 & 0 & 0 & 0 & Grade 5 & $25.00 \%$ & $75.00 \%$ & $86.67 \%$ & $13.33 \%$ \\
\hline Grade 3 A & 35 & 6 & 29 & 16 & 13 & Grade 6 & \#Num! & \#Num! & \#Num! & \#Num! \\
\hline Grade 3 B & 0 & 0 & 0 & 0 & 0 & & & & & \\
\hline Grade 4 & 21 & 5 & 16 & 14 & 2 & Remarks: & & & & \\
\hline Grade 5 & 20 & 5 & 15 & 13 & 2 & & & & & \\
\hline Grade 6 & 0 & 0 & 0 & 0 & 0 & & & & & \\
\hline School Total & 172 & 31 & 141 & 91 & 50 & School \%age & $18.02 \%$ & $81.98 \%$ & $64.54 \%$ & $35.46 \%$ \\
\hline
\end{tabular}

\begin{tabular}{|c|c|c|c|c|c|c|c|c|c|c|}
\hline \multicolumn{11}{|c|}{ School Code: AD/MNS/PSB-026 School Title: ARPS Khaki Camp 6 - Mansehra } \\
\hline $\begin{array}{l}\text { Grades I } \\
\text { Sections }\end{array}$ & ' Enrolled & Dropped & $\begin{array}{c}\text { Appeared } \\
\text { in Exam }\end{array}$ & $\begin{array}{r}\text { Passed in } \\
\text { Exams }\end{array}$ & $\begin{array}{l}\text { Failed in } \\
\text { Exams }\end{array}$ & Grades & Dropped & $\begin{array}{c}\text { Appeared } \\
\text { in Exams }\end{array}$ & $\begin{array}{l}\text { Passed in } \\
\text { Exams }\end{array}$ & $\begin{array}{l}\text { Faled in } \\
\text { Exams }\end{array}$ \\
\hline Grade $1 \mathrm{~A}$ & 88 & 16 & 72 & 46 & 26 & Grade 1 & $18.18 \%$ & $81.82 \%$ & $63.89 \%$ & $36.11 \%$ \\
\hline Grade $1 \mathrm{~B}$ & 0 & 0 & 0 & 0 & 0 & Grade 2 & $20.31 \%$ & $79.69 \%$ & $62.75 \%$ & $37.25 \%$ \\
\hline Grade $1 \mathrm{C}$ & 0 & 0 & 0 & 0 & 0 & Grade 3 & $12.82 \%$ & $87.18 \%$ & $64.71 \%$ & $35.29 \%$ \\
\hline Grade 2 A & 64 & 13 & 51 & 32 & 19 & Grade 4 & $14.71 \%$ & $85.29 \%$ & $58.62 \%$ & $41.38 \%$ \\
\hline Grade 2 B & 0 & 0 & 0 & 0 & 0 & Grade 5 & $25.00 \%$ & $75.00 \%$ & $55.56 \%$ & $44.44 \%$ \\
\hline Grade 3 A & 39 & 5 & 34 & 22 & 12 & Grade 6 & $21.05 \%$ & $78.95 \%$ & $100.00 \%$ & $0.00 \%$ \\
\hline Grade 3 B & 0 & 0 & 0 & 0 & 0 & & & & & \\
\hline Grade 4 & 34 & 5 & 29 & 17 & 12 & Remarks: & & & & \\
\hline Grade 5 & 24 & 6 & 18 & 10 & 8 & & & & & \\
\hline Grade 6 & 19 & 4 & 15 & 15 & 0 & & & & & \\
\hline School Total & 268 & 49 & 219 & 142 & 77 & School \%age & $18.28 \%$ & $81.72 \%$ & $64.84 \%$ & $35.16 \%$ \\
\hline
\end{tabular}

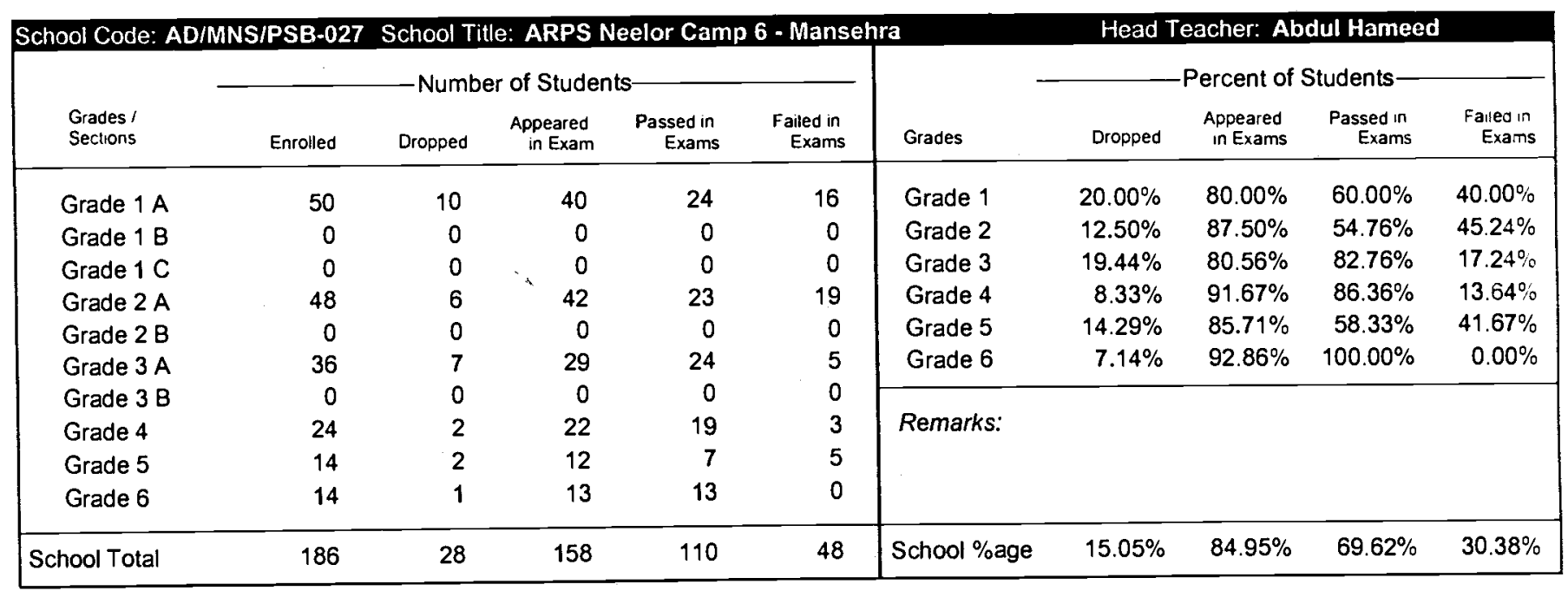

Note:

\#Num! = Enrollment in respective grade(s) is nill therefore calculation is not applicable
Page 9 of 91

Produced by DP/MIS Section on 31-Jul-97 
Sub-Centre: Abbotabad

\begin{tabular}{|c|c|c|c|c|c|c|c|c|c|c|}
\hline \multirow[b]{2}{*}{$\begin{array}{l}\text { Grades I } \\
\text { Sections }\end{array}$} & \multicolumn{5}{|c|}{ _Number of Students- } & \multirow[b]{2}{*}{ Grades } & \multicolumn{4}{|c|}{ Percent of Students } \\
\hline & Enrolled & Dropped & $\begin{array}{l}\text { Appeared } \\
\text { in Exam }\end{array}$ & $\begin{array}{r}\text { Passed in } \\
\text { Exams }\end{array}$ & $\begin{array}{l}\text { Failed in } \\
\text { Exams }\end{array}$ & & Dropped & $\begin{array}{l}\text { Appeared } \\
\text { in Exams }\end{array}$ & $\begin{array}{r}\text { Passed in } \\
\text { Exams }\end{array}$ & $\begin{array}{l}\text { Failed in } \\
\text { Exams }\end{array}$ \\
\hline Grade $1 \mathrm{~A}$ & 125 & 45 & 80 & 47 & 33 & Grade 1 & $36.00 \%$ & $64.00 \%$ & $58.75 \%$ & $41.25 \%$ \\
\hline Grade 1 B & 0 & 0 & 0 & 0 & 0 & Grade 2 & $32.14 \%$ & $67.86 \%$ & $73.68 \%$ & $26.32 \%$ \\
\hline Grade $1 \mathrm{C}$ & 0 & 0 & 0 & 0 & 0 & Grade 3 & $2.78 \%$ & $97.22 \%$ & $82.86 \%$ & $17.14 \%$ \\
\hline Grade $2 \mathrm{~A}$ & 56 & 18 & 38 & 28 & 10 & Grade 4 & $4.00 \%$ & $96.00 \%$ & $79.17 \%$ & $20.83 \%$ \\
\hline Grade 2 B & 0 & 0 & 0 & 0 & 0 & Grade 5 & $28.57 \%$ & $71.43 \%$ & $100.00 \%$ & $0.00 \%$ \\
\hline Grade $3 \mathrm{~A}$ & 36 & 1 & 35 & 29 & 6 & Grade 6 & $38.10 \%$ & $61.90 \%$ & $84.62 \%$ & $15.38 \%$ \\
\hline Grade 3 B & 0 & 0 & 0 & 0 & 0 & & & & & \\
\hline Grade 4 & 25 & 1 & 24 & 19 & 5 & Remarks: & & & & \\
\hline Grade 5 & 28 & 8 & 20 & 20 & 0 & & & & & \\
\hline Grade 6 & 21 & 8 & 13 & 11 & 2 & & & & & \\
\hline School Total & 291 & 81 & 210 & 154 & 56 & School \%age & $27.84 \%$ & $72.16 \%$ & $73.33 \%$ & $26.67 \%$ \\
\hline
\end{tabular}

\begin{tabular}{|c|c|c|c|c|c|c|c|c|c|c|c|}
\hline \multirow[b]{2}{*}{$\begin{array}{l}\text { Grades / } \\
\text { Sections }\end{array}$} & \multicolumn{6}{|c|}{-Number of Students } & \multirow[b]{2}{*}{ Grades } & \multicolumn{4}{|c|}{-Percent of Students- } \\
\hline & ' & Enrolled & Dropped & $\begin{array}{l}\text { Appeared } \\
\text { in Exam }\end{array}$ & $\begin{array}{r}\text { Passed in } \\
\text { Exams }\end{array}$ & $\begin{array}{l}\text { Failed in } \\
\text { Exams }\end{array}$ & & Dropped & $\begin{array}{l}\text { Appeared } \\
\text { in Exams }\end{array}$ & $\begin{array}{l}\text { Passed in } \\
\text { Exams }\end{array}$ & $\begin{array}{c}\text { Failed in } \\
\text { Exams }\end{array}$ \\
\hline Grade $1 \mathrm{~A}$ & & 71 & 0 & 71 & 38 & 33 & Grade 1 & $0.00 \%$ & $100.00 \%$ & $53.52 \%$ & $46.48 \%$ \\
\hline Grade $1 \mathrm{~B}$ & & 0 & 0 & 0 & 0 & 0 & Grade 2 & $0.00 \%$ & $100.00 \%$ & $74.42 \%$ & $25.58 \% \mathrm{c}$ \\
\hline Grade $1 \mathrm{C}$ & & 0 & 0 & 0 & 0 & 0 & Grade 3 & $0.00 \%$ & $100.00 \%$ & $88.46 \%$ & $11.54 \%$ \\
\hline Gräde $2 \mathrm{~A}$ & & 43 & 0 & 43 & 32 & 11 & Grade 4 & $0.00 \%$ & $100.00 \%$ & $96.00 \%$ & $4.00 \%$ \\
\hline Grade 2 B & & 0 & 0 & 0 & 0 & 0 & Grade 5 & $6.67 \%$ & $93.33 \%$ & $78.57 \%$ & $21.43 \%$ \\
\hline Grade $3 \mathrm{~A}$ & & 26 & 0 & 26 & 23 & 3 & Grade 6 & $0.00 \%$ & $100.00 \%$ & $100.00 \%$ & $0.00 \%$ \\
\hline Grade 3 B & & 0 & 0 & 0 & 0 & 0 & & & & & \\
\hline Grade 4 & & 25 & 0 & 25 & 24 & 1 & Remarks: & & & & \\
\hline Grade 5 & & 15 & 1 & 14 & 11 & 3 & & & & & \\
\hline Grade 6 & & 8 & 0 & 8 & 8 & 0 & & & & & \\
\hline School Total & & 188 & 1 & 187 & 136 & 51 & School \%age & $0.53 \%$ & $99.47 \%$ & $72.73 \%$ & $27.27 \%$ \\
\hline
\end{tabular}

\begin{tabular}{|c|c|c|c|c|c|c|c|c|c|c|}
\hline \multirow[b]{2}{*}{$\begin{array}{l}\text { Grades I } \\
\text { Sections }\end{array}$} & \multicolumn{5}{|c|}{-Number of Students- } & \multirow[b]{2}{*}{ Grades } & \multicolumn{4}{|c|}{-Percent of Students } \\
\hline & Enrolled & Dropped & $\begin{array}{l}\text { Appeared } \\
\text { in Exam }\end{array}$ & $\begin{array}{c}\text { Passed in } \\
\text { Exams }\end{array}$ & $\begin{array}{l}\text { Failed in } \\
\text { Exams }\end{array}$ & & Dropped & $\begin{array}{l}\text { Appeared } \\
\text { in Exams }\end{array}$ & $\begin{array}{l}\text { Passed in } \\
\text { Exams }\end{array}$ & $\begin{array}{r}\text { Falled in } \\
\text { Exams }\end{array}$ \\
\hline Grade $1 \mathrm{~A}$ & 52 & 4 & 48 & 32 & 16 & Grade 1 & $7.69 \%$ & $92.31 \%$ & $66.67 \%$ & $33.33 \%$ \\
\hline Grade 1 B & 0 & 0 & 0 & 0 & 0 & Grade 2 & $9.09 \%$ & $90.91 \%$ & $73.33 \%$ & $26.67 \%$ \\
\hline Grade $1 \mathrm{C}$ & 0 & 0 & 0 & 0 & 0 & Grade 3 & $4.17 \%$ & $95.83 \%$ & $95.65 \%$ & $4.35 \%$ \\
\hline Grade $2 \mathrm{~A}$ & 33 & . 3 & 30 & 22 & 8 & Grade 4 & $15.00 \%$ & $85.00 \%$ & $88.24 \%$ & $11.76 \%$ \\
\hline Grade 2 B & 0 & 0 & 0 & 0 & 0 & Grade 5 & $41.67 \%$ & $58.33 \%$ & $100.00 \%$ & $0.00 \%$ \\
\hline Grade $3 \mathrm{~A}$ & 24 & 1 & 23 & 22 & 1 & Grade 6 & $25.00 \%$ & $75.00 \%$ & $100.00 \%$ & $0.00 \%$ \\
\hline Grade 3 B & 0 & 0 & 0 & 0 & 0 & & & & & \\
\hline Grade 4 & 20 & 3 & 17 & 15 & 2 & Remarks: & & & & \\
\hline Grade 5 & 12 & 5 & 7 & 7 & 0 & & & & & \\
\hline Grade 6 & 8 & 2 & 6 & 6 & 0 & & & & & \\
\hline School Total & 149 & 18 & 131 & 104 & 27 & School \%age & $12.08 \%$ & $87.92 \%$ & $79.39 \%$ & $20.61 \%$ \\
\hline
\end{tabular}

\begin{tabular}{|c|c|c|c|c|c|c|c|c|c|c|}
\hline Sub-Centre Total & 7,558 & 805 & 6,753 & 4,951 & 1,802 & Sub-Centre \%age & $10.65 \%$ & $89.35 \%$ & $73.32 \%$ & $26.68 \%$ \\
\hline
\end{tabular}

Note: 
Sub-Centre: Bannu

\begin{tabular}{|c|c|c|c|c|c|c|c|c|c|c|}
\hline \multirow[b]{2}{*}{$\begin{array}{l}\text { Grades / } \\
\text { Sections }\end{array}$} & \multicolumn{5}{|c|}{ - Number of Students } & \multirow[b]{2}{*}{ Grades } & \multicolumn{4}{|c|}{ Percent of Students- } \\
\hline & Enrolled & Dropped & $\begin{array}{l}\text { Appeared } \\
\text { in Exam }\end{array}$ & $\begin{array}{c}\text { Passed in } \\
\text { Exams }\end{array}$ & $\begin{array}{l}\text { Failed in } \\
\text { Exams }\end{array}$ & & Dropped & $\begin{array}{l}\text { Appeared } \\
\text { in Exams }\end{array}$ & $\begin{array}{l}\text { Passed in } \\
\text { Exams }\end{array}$ & $\begin{array}{l}\text { Falled in } \\
\text { Exams }\end{array}$ \\
\hline Grade $1 \mathrm{~A}$ & 56 & 0 & 56 & 35 & 21 & Grade 1 & $0.00 \%$ & $100.00 \%$ & $62.50 \%$ & $37.50 \%$ \\
\hline Grade 1 B & 0 & 0 & 0 & 0 & 0 & Grade 2 & $8.70 \%$ & $91.30 \%$ & $85.71 \%$ & $14.29 \%$ \\
\hline Grade $1 \mathrm{C}$ & 0 & 0 & 0 & 0 & 0 & Grade 3 & $9.68 \%$ & $90.32 \%$ & $60.71 \%$ & $39.29 \%$ \\
\hline Grade $2 \mathrm{~A}$ & 23 & 2 & 21 & 18 & 3 & Grade 4 & $13.33 \%$ & $86.67 \%$ & $84.62 \%$ & $15.38 \%$ \\
\hline Grade 2 B & 0 & 0 & 0 & 0 & 0 & Grade 5 & $0.00 \%$ & $100.00 \%$ & $80.00 \%$ & $20.00 \%$ \\
\hline Grade 3 A & 31 & 3 & 28 & 17 & 11 & Grade 6 & $14.29 \%$ & $85.71 \%$ & $83.33 \%$ & $16.67 \%$ \\
\hline Grade 3 B & 0 & 0 & 0 & 0 & 0 & & & & & \\
\hline Grade 4 & 15 & 2 & 13 & 11 & 2 & Remarks: & & & & \\
\hline Grade 5 & 10 & 0 & 10 & 8 & 2 & & & & & \\
\hline Grade 6 & 7 & 1 & 6 & 5 & 1 & & & & & \\
\hline School Total & 142 & 8 & 134 & 94 & 40 & School \%age & $5.63 \%$ & $94.37 \%$ & $70.15 \%$ & $29.85 \%$ \\
\hline
\end{tabular}

\begin{tabular}{|c|c|c|c|c|c|c|c|c|c|c|}
\hline \multicolumn{11}{|c|}{ School Code: BN/BNU/PSB-032 School Title: ARPS Gambela - Bannu } \\
\hline \multirow[b]{2}{*}{$\begin{array}{l}\text { Grades I } \\
\text { Sections }\end{array}$} & \multicolumn{5}{|c|}{ - Number of Students } & \multirow[b]{2}{*}{ Grades } & \multicolumn{4}{|c|}{-Percent of Students- } \\
\hline & ' Enrolled & Dropped & $\begin{array}{l}\text { Appeared } \\
\text { in Exam }\end{array}$ & $\begin{array}{l}\text { Passed in } \\
\text { Exams }\end{array}$ & $\begin{array}{l}\text { Failed in } \\
\text { Exams }\end{array}$ & & Dropped & $\begin{array}{l}\text { Appeared } \\
\text { in Exams }\end{array}$ & $\begin{array}{l}\text { Passed in } \\
\text { Exams }\end{array}$ & $\begin{array}{c}\text { Falled in } \\
\text { Exams }\end{array}$ \\
\hline Grade $1 \mathrm{~A}$ & 50 & 0 & 50 & 48 & 2 & Grade 1 & $0.00 \%$ & $100.00 \%$ & $96.00 \%$ & $4.00 \%$ \\
\hline Grade 1 B & 0 & 0 & 0 & 0 & 0 & Grade 2 & $34.62 \%$ & $65.38 \%$ & $100.00 \%$ & $0.00 \%$ \\
\hline Grade $1 \mathrm{C}$ & 0 & 0 & 0 & 0 & 0 & Grade 3 & $18.18 \%$ & $81.82 \%$ & $100.00 \%$ & $0.00 \%$ \\
\hline Grade 2 A & 26 & 9 & 17 & 17 & 0 & Grade 4 & $41.18 \%$ & $58.82 \%$ & $100.00 \%$ & $0.00 \%$ \\
\hline Grade 2 B & 0 & 0 & 0 & 0 & 0 & Grade 5 & $27.27 \%$ & $72.73 \%$ & $100.00 \%$ & $0.00 \%$ \\
\hline Grade $3 \mathrm{~A}$ & $22 *$ & 4 & 18 & 18 & 0 & Grade 6 & $28.57 \%$ & $71.43 \%$ & $100.00 \%$ & $0.00 \%$ \\
\hline Grade 3 B & 0 & 0 & 0 & 0 & 0 & & & & & \\
\hline Grade 4 & 17 & 7 & 10 & 10 & 0 & Remarks: & & & & \\
\hline Grade 5 & 11 & 3 & 8 & 8 & 0 & & & & & \\
\hline Grade 6 & 14 & 4 & 10 & 10 & 0 & & & & & \\
\hline School Total & 140 & 27 & 113 & 111 & 2 & School \%age & $19.29 \%$ & $80.71 \%$ & $98.23 \%$ & $1.77 \%$ \\
\hline
\end{tabular}

\begin{tabular}{|c|c|c|c|c|c|c|c|c|c|c|}
\hline \multirow[b]{2}{*}{$\begin{array}{l}\text { Grades I } \\
\text { Sections }\end{array}$} & \multicolumn{5}{|c|}{ - Number of Students } & \multirow[b]{2}{*}{ Grades } & \multicolumn{4}{|c|}{ Percent of Students - } \\
\hline & Enrolled & Dropped & $\begin{array}{l}\text { Appeared } \\
\text { in Exam }\end{array}$ & $\begin{array}{l}\text { Passed in } \\
\text { Exams }\end{array}$ & $\begin{array}{c}\text { Failed in } \\
\text { Exams }\end{array}$ & & Dropped & $\begin{array}{l}\text { Appeared } \\
\text { in Exams }\end{array}$ & $\begin{array}{c}\text { Passed in } \\
\text { Exams }\end{array}$ & $\begin{array}{c}\text { Falled in } \\
\text { Exams }\end{array}$ \\
\hline Grade $1 \mathrm{~A}$ & 37 & 9 & 28 & 24 & 4 & Grade 1 & $24.32 \%$ & $75.68 \%$ & $85.71 \%$ & $14.29 \%$ \\
\hline Grade $1 \mathrm{~B}$ & 0 & 0 & 0 & 0 & 0 & Grade 2 & $19.35 \%$ & $80.65 \%$ & $72.00 \%$ & $28.00 \%$ \\
\hline Grade $1 \mathrm{C}$ & 0 & 0 & 0 & 0 & 0 & Grade 3 & $42.86 \%$ & $57.14 \%$ & $75.00 \%$ & $25.00 \%$ \\
\hline Grade $2 \mathrm{~A}$ & 31 & 6 & 25 & 18 & 7 & Grade 4 & $0.00 \%$ & $100.00 \%$ & $85.71 \%$ & $14.29 \%$ \\
\hline Grade 2 B & 0 & 0 & 0 & 0 & 0 & Grade 5 & $47.62 \%$ & $52.38 \%$ & $72.73 \%$ & $27.27 \%$ \\
\hline Grade $3 \mathrm{~A}$ & 21 & 9 & 12 & 9 & 3 & Grade 6 & $42.31 \%$ & $57.69 \%$ & $60.00 \%$ & $40.00 \%$ \\
\hline Grade 3 B & 0 & 0 & 0 & 0 & 0 & & & & & \\
\hline Grade 4 & 14 & 0 & 14 & 12 & 2 & Remarks: & & & & \\
\hline Grade 5 & 21 & 10 & 11 & 8 & 3 & & & & & \\
\hline Grade 6 & 26 & 11 & 15 & 9 & 6 & & & & & \\
\hline School Total & 150 & 45 & 105 & 80 & 25 & School \%age & $30.00 \%$ & $70.00 \%$ & $76.19 \%$ & $23.81 \%$ \\
\hline
\end{tabular}

Note:

\#Num! = Enrollment in respective grade(s) is nill therefore calculation is not applicable
Page 11 of 91

Produced by DP/MIS Section on 31-Jul.97 
Sub-Centre: Bannu

\begin{tabular}{|c|c|c|c|c|c|c|c|c|c|c|}
\hline \multirow[b]{2}{*}{$\begin{array}{l}\text { Grades / } \\
\text { Sections }\end{array}$} & \multicolumn{5}{|c|}{-Number of Students- } & \multirow[b]{2}{*}{ Grades } & \multicolumn{4}{|c|}{-Percent of Students } \\
\hline & Enrolled & Dropped & $\begin{array}{l}\text { Appeared } \\
\text { in Exam }\end{array}$ & $\begin{array}{l}\text { Passed in } \\
\text { Exams }\end{array}$ & $\begin{array}{l}\text { Failed in } \\
\text { Exams }\end{array}$ & & Dropped & $\begin{array}{l}\text { Appeared } \\
\text { in Exams }\end{array}$ & $\begin{array}{l}\text { Passed in } \\
\text { Exams }\end{array}$ & $\begin{array}{l}\text { Fasled in } \\
\text { Exams }\end{array}$ \\
\hline Grade $1 \mathrm{~A}$ & 61 & 0 & 61 & 38 & 23 & Grade 1 & $0.00 \%$ & $100.00 \%$ & $62.30 \%$ & $37.70 \%$ \\
\hline Grade $1 \mathrm{~B}$ & 0 & 0 & 0 & 0 & 0 & Grade 2 & $0.00 \%$ & $100.00 \%$ & $73.33 \%$ & $26.67 \%$ \\
\hline Grade $1 \mathrm{C}$ & 0 & 0 & 0 & 0 & 0 & Grade 3 & $4.55 \%$ & $95.45 \%$ & $100.00 \%$ & $0.00 \%$ \\
\hline Grade $2 \mathrm{~A}$ & 30 & 0 & 30 & 22 & 8 & Grade 4 & $0.00 \%$ & $100.00 \%$ & $93.75 \%$ & $6.25 \%$ \\
\hline Grade 2 B & 0 & 0 & 0 & 0 & 0 & Grade 5 & $7.14 \%$ & $92.86 \%$ & $100.00 \%$ & $0.00 \%$ \\
\hline Grade $3 \mathrm{~A}$ & 22 & 1 & 21 & 21 & 0 & Grade 6 & $0.00 \%$ & $100.00 \%$ & $86.67 \%$ & $13.33 \%$ \\
\hline Grade 3 B & 0 & 0 & 0 & 0 & 0 & & & & & \\
\hline Grade 4 & 16 & 0 & 16 & 15 & 1 & Remarks: & & & & \\
\hline Grade 5 & 14 & 1 & 13 & 13 & 0 & & & & & \\
\hline Grade 6 & 15 & 0 & 15 & 13 & 2 & & & & & \\
\hline School Total & 158 & 2 & 156 & 122 & 34 & School \%age & $1.27 \%$ & $98.73 \%$ & $78.21 \%$ & $21.79 \%$ \\
\hline
\end{tabular}

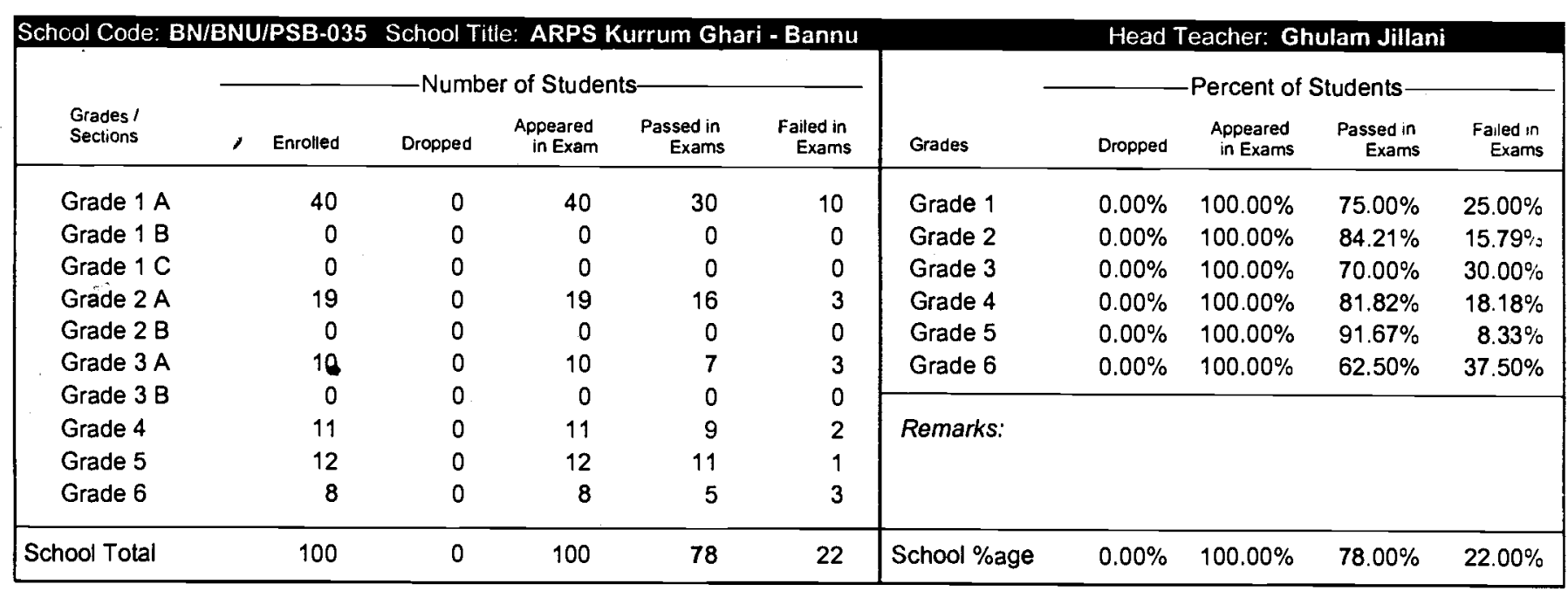

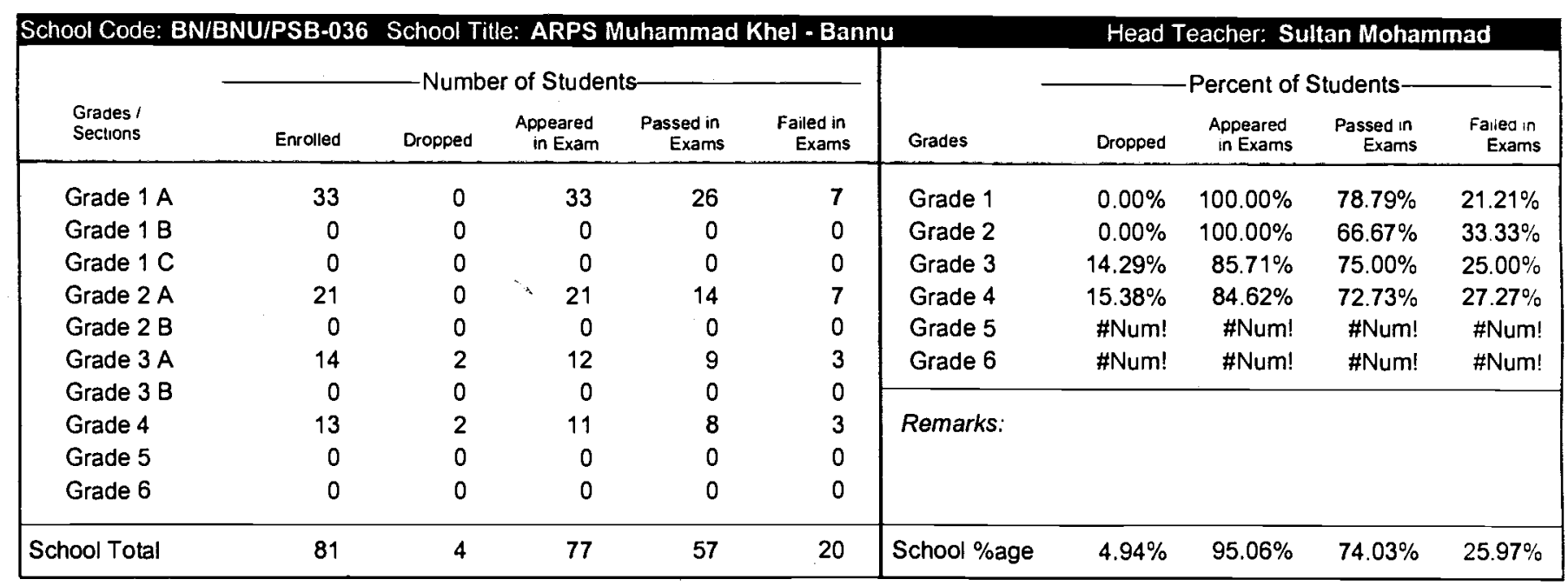

Note:

\#Num! = Enrollmenl in respective grade(s) is nill therefore calculation is not applicable
Page 12 of 91

Produced by DP/MIS Secion on 31.Jul.97 
Sub-Centre: Bannu

\begin{tabular}{|c|c|c|c|c|c|c|c|c|c|c|}
\hline \multirow[b]{2}{*}{$\begin{array}{l}\text { Grades / } \\
\text { Sections }\end{array}$} & \multicolumn{5}{|c|}{-Number of Students } & \multirow[b]{2}{*}{ Grades } & \multicolumn{4}{|c|}{ Percent of Students } \\
\hline & Enrolled & Dropped & $\begin{array}{l}\text { Appeared } \\
\text { in Exarn }\end{array}$ & $\begin{array}{l}\text { Passed in } \\
\text { Exams }\end{array}$ & $\begin{array}{l}\text { Failed in } \\
\text { Exams }\end{array}$ & & Dropped & $\begin{array}{l}\text { Appeared } \\
\text { in Exams }\end{array}$ & $\begin{array}{l}\text { Passed in } \\
\text { Exams }\end{array}$ & $\begin{array}{l}\text { Falled in } \\
\text { Exams }\end{array}$ \\
\hline Grade $1 \mathrm{~A}$ & 40 & 5 & 35 & 27 & 8 & Grade 1 & $12.50 \%$ & $87.50 \%$ & $77.14 \%$ & $22.86 \%$ \\
\hline Grade 1 B & 0 & 0 & 0 & 0 & 0 & Grade 2 & $30.77 \%$ & $69.23 \%$ & $55.56 \%$ & $44.44 \%$ \\
\hline Grade $1 \mathrm{C}$ & 0 & 0 & 0 & 0 & 0 & Grade 3 & $23.53 \%$ & $76.47 \%$ & $84.62 \%$ & $15.38 \%$ \\
\hline Grade 2 A & 13 & 4 & 9 & 5 & 4 & Grade 4 & $20.00 \%$ & $80.00 \%$ & $83.33 \%$ & $16.67 \%$ \\
\hline Grade 2 B & 0 & 0 & 0 & 0 & 0 & Grade 5 & $22.22 \%$ & $77.78 \%$ & $57.14 \%$ & $42.86 \%$ \\
\hline Grade 3 A & 17 & 4 & 13 & 11 & 2 & Grade 6 & $33.33 \%$ & $66.67 \%$ & $66.67 \%$ & $33.33 \%$ \\
\hline Grade 3 B & 0 & 0 & 0 & 0 & 0 & & & & & \\
\hline Grade 4 & 15 & 3 & 12 & 10 & 2 & Remarks: & & & & \\
\hline Grade 5 & 9 & 2 & 7 & 4 & 3 & & & & & \\
\hline Grade 6 & 9 & 3 & 6 & 4 & 2 & & & & & \\
\hline School Total & 103 & 21 & 82 & 61 & 21 & School \%age & $20.39 \%$ & $79.61 \%$ & $74.39 \%$ & $25.61 \%$ \\
\hline
\end{tabular}

\begin{tabular}{|c|c|c|c|c|c|c|c|c|c|c|}
\hline \multicolumn{6}{|c|}{$\begin{array}{l}\text { School Code: BN/BNU/PSB-038 School Title: ARPS } 2 \text { Naurang - Bannu } \\
\text { Number of Students- }\end{array}$} & \multicolumn{5}{|c|}{ Head Teacher: Yousaf Khan } \\
\hline $\begin{array}{l}\text { Grades / } \\
\text { Sections }\end{array}$ & 'Enrolled & Dropped & $\begin{array}{l}\text { Appeared } \\
\text { in Exam }\end{array}$ & $\begin{array}{l}\text { Passed in } \\
\text { Exams }\end{array}$ & $\begin{array}{l}\text { Failed in } \\
\text { Exams }\end{array}$ & Grades & Dropped & $\begin{array}{l}\text { Appeared } \\
\text { in Exams }\end{array}$ & $\begin{array}{l}\text { Passed in } \\
\text { Exams }\end{array}$ & $\begin{array}{l}\text { Failed in } \\
\text { Exams }\end{array}$ \\
\hline Grade $1 \mathrm{~A}$ & 72 & 1 & 71 & 50 & 21 & Grade 1 & $1.39 \%$ & $98.61 \%$ & $70.42 \%$ & $29.58 \%$ \\
\hline Grade $1 \mathrm{~B}$ & 0 & 0 & 0 & 0 & 0 & Grade 2 & $22.22 \%$ & $77.78 \%$ & $80.00 \%$ & $20.00 \%$ \\
\hline Grade-1 C & 0 & 0 & 0 & 0 & 0 & Grade 3 & $11.76 \%$ & $88.24 \%$ & $83.33 \%$ & $16.67 \%$ \\
\hline Grade 2 A & 45 & 10 & 35 & 28 & 7 & Grade 4 & $25.00 \%$ & $75.00 \%$ & $100.00 \%$ & $0.00 \%$ \\
\hline Grade 2 B & 0 & 0 & 0 & 0 & 0 & Grade 5 & $26.67 \%$ & $73.33 \%$ & $81.82 \%$ & $18.18 \%$ \\
\hline Grade 3 A & $34 *$ & 4 & 30 & 25 & 5 & Grade 6 & $27.78 \%$ & $72.22 \%$ & $61.54 \%$ & $38.46 \%$ \\
\hline Grade $3 \mathrm{~B}$ & 0 & 0 & 0 & 0 & 0 & & & & & \\
\hline Grade 4 & 16 & 4 & 12 & 12 & 0 & Remarks: & & & & \\
\hline Grade 5 & 15 & 4 & 11 & 9 & 2 & & & & & \\
\hline Grade 6 & 18 & 5 & 13 & 8 & 5 & & & & & \\
\hline School Total & 200 & 28 & 172 & 132 & 40 & School \%age & $14.00 \%$ & $86.00 \%$ & $76.74 \%$ & $23.26 \%$ \\
\hline
\end{tabular}

\begin{tabular}{|c|c|c|c|c|c|c|c|c|c|c|}
\hline \multicolumn{11}{|c|}{ School Code: BN/DIKJPSB-039 School Title: ARPS 1 Dabara - D. I. Khan } \\
\hline \multirow[b]{2}{*}{$\begin{array}{l}\text { Grades I } \\
\text { Sections }\end{array}$} & \multicolumn{5}{|c|}{ Number of Students } & \multirow[b]{2}{*}{ Grades } & \multicolumn{4}{|c|}{ Percent of Students- } \\
\hline & Enrolled & Dropped & $\begin{array}{l}\text { Appeared } \\
\text { in Exam }\end{array}$ & $\begin{array}{l}\text { Passed in } \\
\text { Exams }\end{array}$ & $\begin{array}{l}\text { Failed in } \\
\text { Exams }\end{array}$ & & Dropped & $\begin{array}{l}\text { Appeared } \\
\text { in Exams }\end{array}$ & $\begin{array}{l}\text { Passed in } \\
\text { Exams }\end{array}$ & $\begin{array}{l}\text { Falled in } \\
\text { Exams }\end{array}$ \\
\hline Grade $1 \mathrm{~A}$ & 40 & 0 & 40 & 23 & 17 & Grade 1 & $0.00 \%$ & $100.00 \%$ & $57.50 \%$ & $42.50 \%$ \\
\hline Grade $1 \mathrm{~B}$ & 0 & 0 & 0 & 0 & 0 & Grade 2 & $0.00 \%$ & $100.00 \%$ & $66.67 \%$ & $33.33 \%$ \\
\hline Grade $1 \mathrm{C}$ & 0 & 0 & 0 & 0 & 0 & Grade 3 & $0.00 \%$ & $100.00 \%$ & $66.67 \%$ & $33.33 \%$ \\
\hline Grade 2 A & 15 & 0 & 15 & 10 & 5 & Grade 4 & $0.00 \%$ & $100.00 \%$ & $88.89 \%$ & $11.11 \%$ \\
\hline Grade 2 B & 0 & 0 & 0 & 0 & 0 & Grade 5 & $0.00 \%$ & $100.00 \%$ & $90.00 \%$ & $10.00 \%$ \\
\hline Grade 3 A & 9 & 0 & 9 & 6 & 3 & Grade 6 & \#Num! & \#Num! & \#Num! & \#Num! \\
\hline Grade 3 B & 0 & 0 & 0 & 0 & 0 & & & & & \\
\hline Grade 4 & 9 & 0 & 9 & 8 & 1 & Remarks: & & & & \\
\hline Grade 5 & 10 & 0 & 10 & 9 & 1 & & & & & \\
\hline Grade 6 & 0 & 0 & 0 & 0 & 0 & & & & & \\
\hline School Total & 83 & 0 & 83 & 56 & 27 & School \%age & $0.00 \%$ & $100.00 \%$ & $67.47 \%$ & $32.53 \%$ \\
\hline
\end{tabular}

Note: 
Sub-Centre: Bannu

\begin{tabular}{|c|c|c|c|c|c|c|c|c|c|c|}
\hline \multirow[b]{2}{*}{$\begin{array}{l}\text { Grades ' } \\
\text { Sections }\end{array}$} & \multicolumn{5}{|c|}{-Number of Students- } & \multirow[b]{2}{*}{ Grades } & \multicolumn{4}{|c|}{-Percent of Students } \\
\hline & Enrolled & Dropped & $\begin{array}{l}\text { Appeared } \\
\text { in Exam }\end{array}$ & $\begin{array}{r}\text { Passed in } \\
\text { Exams }\end{array}$ & $\begin{array}{l}\text { Failed in } \\
\text { Exams }\end{array}$ & & Dropped & $\begin{array}{c}\text { Appeared } \\
\text { in Exams }\end{array}$ & $\begin{array}{c}\text { Passed in } \\
\text { Exams }\end{array}$ & $\begin{array}{c}\text { Falled in } \\
\text { Exams }\end{array}$ \\
\hline Grade $1 \mathrm{~A}$ & 42 & 0 & 42 & 32 & 10 & Grade 1 & $0.00 \%$ & $100.00 \%$ & $76.19 \%$ & $23.81 \%$ \\
\hline Grade 1 B & 0 & 0 & 0 & 0 & 0 & Grade 2 & $0.00 \%$ & $100.00 \%$ & $77.50 \%$ & $22.50 \%$ \\
\hline Grade $1 \mathrm{C}$ & 0 & 0 & 0 & 0 & 0 & Grade 3 & $0.00 \%$ & $100.00 \%$ & $78.57 \%$ & $21.43 \%$ \\
\hline Grade 2 A & 40 & 0 & 40 & 31 & 9 & Grade 4 & $0.00 \%$ & $100.00 \%$ & $80.00 \%$ & $20.00 \%$ \\
\hline Grade 2 B & 0 & 0 & 0 & 0 & 0 & Grade 5 & $0.00 \%$ & $100.00 \%$ & $75.00 \%$ & $25.00 \%$ \\
\hline Grade $3 \mathrm{~A}$ & 14 & 0 & 14 & 11 & 3 & Grade 6 & $0.00 \%$ & $100.00 \%$ & $28.57 \%$ & $71.43 \%$ \\
\hline Grade 3 B & 0 & 0 & 0 & 0 & 0 & & & & & \\
\hline Grade 4 & 10 & 0 & 10 & 8 & 2 & Remarks: & & & & \\
\hline Grade 5 & 12 & 0 & 12 & 9 & 3 & & & & & \\
\hline Grade 6 & 7 & 0 & 7 & 2 & 5 & & & & & \\
\hline School Total & 125 & 0 & 125 & 93 & 32 & School \%age & $0.00 \%$ & $100.00 \%$ & $74.40 \%$ & $25.60 \%$ \\
\hline
\end{tabular}

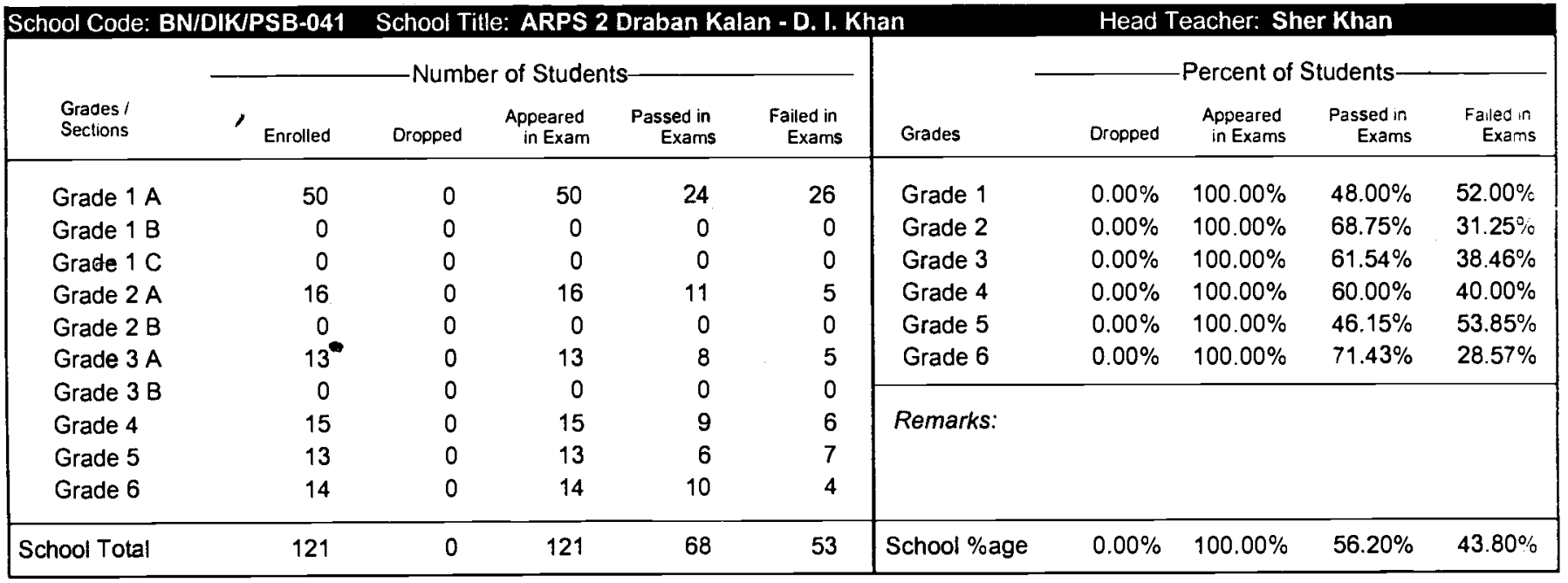

\begin{tabular}{|c|c|c|c|c|c|c|c|c|c|c|}
\hline \multirow[b]{2}{*}{$\begin{array}{l}\text { Grades / } \\
\text { Seclions }\end{array}$} & \multicolumn{5}{|c|}{-Number of Students } & \multirow[b]{2}{*}{ Grades } & \multicolumn{4}{|c|}{ Percent of Students } \\
\hline & Enrolled & Dropped & $\begin{array}{l}\text { Appeared } \\
\text { in Exam }\end{array}$ & $\begin{array}{c}\text { Passed in } \\
\text { Exams }\end{array}$ & $\begin{array}{l}\text { Failed in } \\
\text { Exams }\end{array}$ & & Dropped & $\begin{array}{c}\text { Appeared } \\
\text { in Exams }\end{array}$ & $\begin{array}{l}\text { Passed in } \\
\text { Exams }\end{array}$ & $\begin{array}{c}\text { Falied in } \\
\text { Exams }\end{array}$ \\
\hline Grade $1 \mathrm{~A}$ & 80 & 11 & 69 & 23 & 46 & Grade 1 & $13.75 \%$ & $86.25 \%$ & $33.33 \%$ & $66.67 \%$ \\
\hline Grade $1 \mathrm{~B}$ & 0 & 0 & 0 & 0 & 0 & Grade 2 & $0.00 \%$ & $100.00 \%$ & $52.38 \%$ & $47.62 \%$ \\
\hline Grade $1 \mathrm{C}$ & 0 & 0 & 0 & 0 & 0 & Grade 3 & $12.50 \%$ & $87.50 \%$ & $71.43 \%$ & $28.57 \%$ \\
\hline Grade $2 \mathrm{~A}$ & 21 & 0 & 21 & 11 & 10 & Grade 4 & $0.00 \%$ & $100.00 \%$ & $90.00 \%$ & $10.00 \%$ \\
\hline Grade 2 B & 0 & 0 & 0 & 0 & 0 & Grade 5 & $0.00 \%$ & $100.00 \%$ & $100.00 \%$ & $0.00 \%$ \\
\hline Grade $3 \mathrm{~A}$ & 16 & 2 & 14 & 10 & 4 & Grade 6 & $16.67 \%$ & $83.33 \%$ & $90.00 \%$ & $10.00 \%$ \\
\hline Grade 3 B & 0 & 0 & 0 & 0 & 0 & & & & & \\
\hline Grade 4 & 10 & 0 & 10 & 9 & 1 & Remarks: & & & & \\
\hline Grade 5 & 8 & 0 & 8 & 8 & 0 & & & & & \\
\hline Grade 6 & 12 & 2 & 10 & 9 & 1 & & & & & \\
\hline School Total & 147 & 15 & 132 & 70 & 62 & School \%age & $10.20 \%$ & $89.80 \%$ & $53.03 \%$ & $46.97 \%$ \\
\hline
\end{tabular}


Sub-Centre: Bannu

School Code: BN/DIK/PSB-043 School Title: ARPS Kokar - D. I. Khan Head Teacher: Ghulam Mahboob Shah

\begin{tabular}{|c|c|c|c|c|c|c|c|c|c|c|}
\hline \multirow[b]{2}{*}{$\begin{array}{l}\text { Grades I } \\
\text { Sections }\end{array}$} & \multicolumn{5}{|c|}{ Number of Students } & \multirow[b]{2}{*}{ Grades } & \multicolumn{4}{|c|}{-Percent of Students } \\
\hline & Enrolled & Dropped & $\begin{array}{l}\text { Appeared } \\
\text { in Exam }\end{array}$ & $\begin{array}{l}\text { Passed in } \\
\text { Exams }\end{array}$ & $\begin{array}{l}\text { Failed in } \\
\text { Exams }\end{array}$ & & Dropped & $\begin{array}{l}\text { Appeared } \\
\text { in Exams }\end{array}$ & $\begin{array}{l}\text { Passed in } \\
\text { Exams }\end{array}$ & $\begin{array}{l}\text { Falied in } \\
\text { Exams }\end{array}$ \\
\hline Grade $1 \mathrm{~A}$ & 80 & 0 & 80 & 54 & 26 & Grade 1 & $0.00 \%$ & $100.00 \%$ & $67.50 \%$ & $32.50 \%$ \\
\hline Grade 1 B & 0 & 0 & 0 & 0 & 0 & Grade 2 & $7.69 \%$ & $92.31 \%$ & $70.83 \%$ & $29.17 \%$ \\
\hline Grade $1 \mathrm{C}$ & 0 & 0 & 0 & 0 & 0 & Grade 3 & $10.00 \%$ & $90.00 \%$ & $94.44 \%$ & $5.56 \%$ \\
\hline Grade 2 A & 26 & 2 & 24 & 17 & 7 & Grade 4 & $0.00 \%$ & $100.00 \%$ & $100.00 \%$ & $0.00 \%$ \\
\hline Grade 2 B & 0 & 0 & 0 & 0 & 0 & Grade 5 & $0.00 \%$ & $100.00 \%$ & $100.00 \%$ & $0.00 \%$ \\
\hline Grade 3 A & 20 & 2 & 18 & 17 & 1 & Grade 6 & $0.00 \%$ & $100.00 \%$ & $100.00 \%$ & $0.00 \%$ \\
\hline Grade 3 B & 0 & 0 & 0 & 0 & 0 & & & & & \\
\hline Grade 4 & 13 & 0 & 13 & 13 & 0 & Remarks: & & & & \\
\hline Grade 5 & 8 & 0 & 8 & 8 & 0 & & & & & \\
\hline Grade 6 & 8 & 0 & 8 & 8 & 0 & & & & & \\
\hline chool Total & 155 & 4 & 151 & 117 & 34 & School \%age & $2.58 \%$ & $97.42 \%$ & $77.48 \%$ & $22.52 \%$ \\
\hline
\end{tabular}

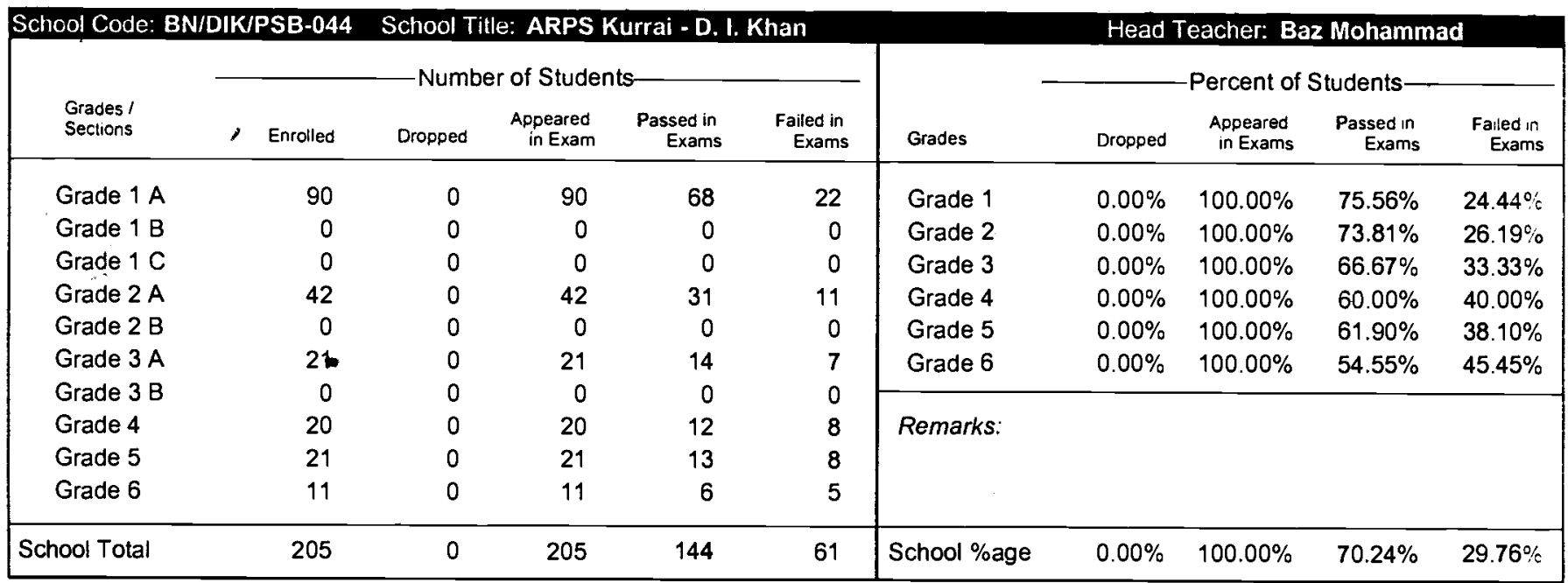

\begin{tabular}{|c|c|c|c|c|c|c|c|c|c|c|}
\hline \multirow[t]{2}{*}{ School Code: B } & \multicolumn{5}{|c|}{$\begin{array}{l}\text { School Title: ARPS Pashapul - D. I. Khan } \\
\text { Number of Students- }\end{array}$} & \multicolumn{5}{|c|}{$\begin{array}{r}\text { Head Teacher: Khair Ullah } \\
\text {-Percent of Students- }\end{array}$} \\
\hline & Enrolled & Dropped & $\begin{array}{l}\text { Appeared } \\
\text { in Exam }\end{array}$ & $\begin{array}{l}\text { Passed in } \\
\text { Exams }\end{array}$ & $\begin{array}{l}\text { Failed in } \\
\text { Exams }\end{array}$ & Grades & Dropped & $\begin{array}{l}\text { Appeared } \\
\text { in Exams }\end{array}$ & $\begin{array}{c}\text { Passed in } \\
\text { Exams }\end{array}$ & $\begin{array}{l}\text { Falled in } \\
\text { Exams }\end{array}$ \\
\hline Grade $1 \mathrm{~A}$ & 47 & 7 & 40 & 30 & 10 & Grade 1 & $14.89 \%$ & $85.11 \%$ & $75.00 \%$ & $25.00 \%$ \\
\hline Grade 1 B & 0 & 0 & 0 & 0 & 0 & Grade 2 & $13.33 \%$ & $86.67 \%$ & $100.00 \%$ & $0.00 \%$ \\
\hline Grade $1 \mathrm{C}$ & 0 & 0 & 0 & 0 & 0 & Grade 3 & $0.00 \%$ & $100.00 \%$ & $100.00 \%$ & $0.00 \%$ \\
\hline Grade $2 \mathrm{~A}$ & 15 & 2 & 13 & 13 & 0 & Grade 4 & \#Num! & \#Num! & \#Num! & \#Num! \\
\hline Grade 2 B & 0 & 0 & 0 & 0 & 0 & Grade 5 & $0.00 \%$ & $100.00 \%$ & $88.89 \%$ & $11.11 \%$ \\
\hline Grade $3 \mathrm{~A}$ & 6 & 0 & 6 & 6 & 0 & Grade 6 & \#Num! & \#Num! & \#Num! & \#Num! \\
\hline Grade 3 B & 0 & 0 & 0 & 0 & 0 & & & & & \\
\hline Grade 4 & 0 & 0 & 0 & 0 & 0 & Remarks: & & & & \\
\hline Grade 5 & 9 & 0 & 9 & 8 & 1 & & & & & \\
\hline Grade 6 & 0 & 0 & 0 & 0 & 0 & & & & & \\
\hline School Total & 77 & 9 & 68 & 57 & 11 & School \%age & $11.69 \%$ & $88.31 \%$ & $83.82 \%$ & $16.18 \%$ \\
\hline
\end{tabular}


Sub-Centre: Bannu

\begin{tabular}{|c|c|c|c|c|c|c|c|c|c|c|}
\hline \multicolumn{11}{|c|}{ School Code: BN/DIK/PSB-046 School Title: ARPS Rak Zandani - D. I. Khan } \\
\hline \multirow[b]{2}{*}{$\begin{array}{l}\text { Grades / } \\
\text { Sections }\end{array}$} & & Num & of Stude & & & \multirow[b]{2}{*}{ Grades } & \multicolumn{4}{|c|}{-Percent of Students } \\
\hline & Enrolled & Dropped & $\begin{array}{l}\text { Appeared } \\
\text { in Exam }\end{array}$ & $\begin{array}{l}\text { Passed in } \\
\text { Exams }\end{array}$ & $\begin{array}{l}\text { Failed in } \\
\text { Exams }\end{array}$ & & Dropped & $\begin{array}{l}\text { Appeared } \\
\text { in Exams }\end{array}$ & $\begin{array}{l}\text { Passed in } \\
\text { Exams }\end{array}$ & $\begin{array}{l}\text { Fa:led in } \\
\text { Exams }\end{array}$ \\
\hline Grade $1 \mathrm{~A}$ & 30 & 0 & 30 & 20 & 10 & Grade 1 & $0.00 \%$ & $100.00 \%$ & $66.67 \%$ & $33.33 \%$ \\
\hline Grade $1 \mathrm{~B}$ & 0 & 0 & 0 & 0 & 0 & Grade 2 & $0.00 \%$ & $100.00 \%$ & $76.92 \%$ & $23.08 \%$ \\
\hline Grade $1 \mathrm{C}$ & 0 & 0 & 0 & 0 & 0 & Grade 3 & $0.00 \%$ & $100.00 \%$ & $100.00 \%$ & $0.00 \%$ \\
\hline Grade $2 \mathrm{~A}$ & 13 & 0 & 13 & 10 & 3 & Grade 4 & $0.00 \%$ & $100.00 \%$ & $85.71 \%$ & $14.29 \%$ \\
\hline Grade 2 B & 0 & 0 & 0 & 0 & 0 & Grade 5 & $0.00 \%$ & $100.00 \%$ & $100.00 \%$ & $0.00 \%$ \\
\hline Grade 3 A & 11 & 0 & 11 & 11 & 0 & Grade 6 & \#Num! & \#Num! & \#Num! & \#Num! \\
\hline Grade 3 B & 0 & 0 & 0 & 0 & 0 & & & & & \\
\hline Grade 4 & 7 & 0 & 7 & 6 & 1 & Remarks: & & & & \\
\hline Grade 5 & 5 & 0 & 5 & 5 & 0 & & & & & \\
\hline Grade 6 & 0 & 0 & 0 & 0 & 0 & & & & & \\
\hline School Total & 66 & 0 & 66 & 52 & 14 & School \%age & $0.00 \%$ & $100.00 \%$ & $78.79 \%$ & $21.21 \%$ \\
\hline
\end{tabular}

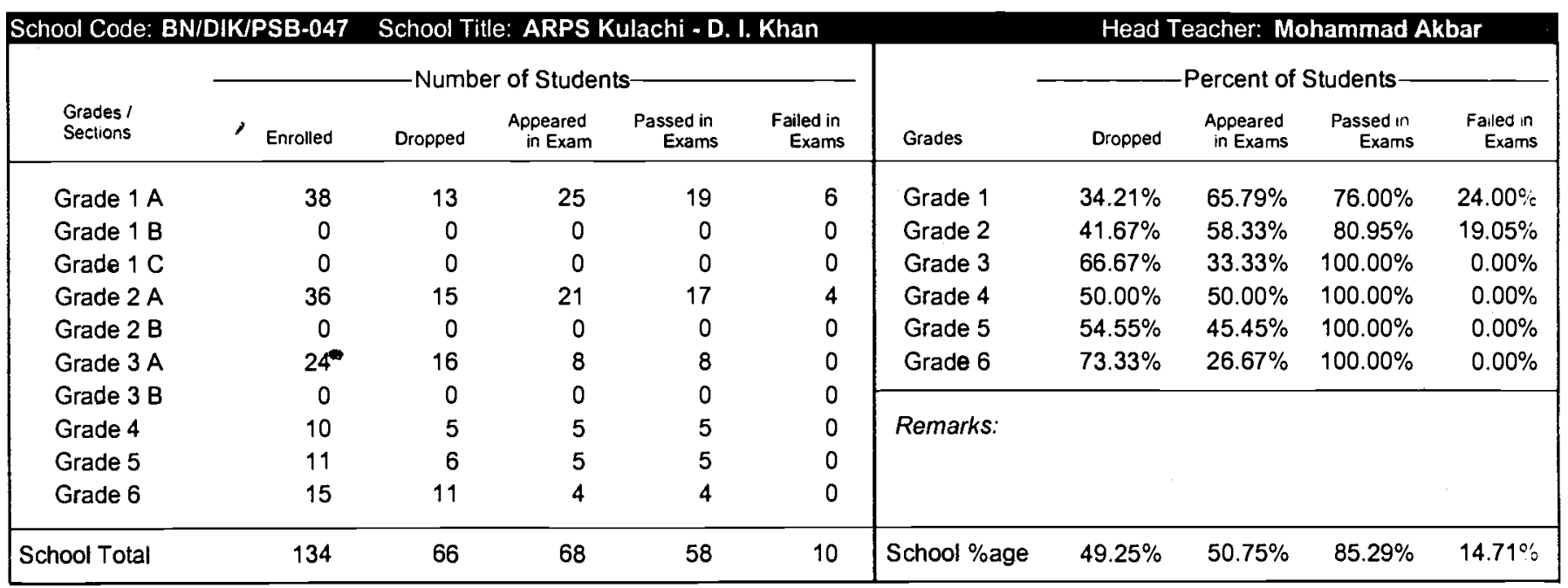

\begin{tabular}{|c|c|c|c|c|c|c|c|c|c|c|}
\hline \multicolumn{11}{|c|}{ School Code: BN/DIKJPSB-048 } \\
\hline \multirow[b]{2}{*}{$\begin{array}{l}\text { Grades / } \\
\text { Sections }\end{array}$} & \multicolumn{5}{|c|}{ - Number of Students- } & \multirow[b]{2}{*}{ Grades } & \multicolumn{4}{|c|}{ Percent of Students- } \\
\hline & Enrolled & Dropped & $\begin{array}{l}\text { Appeared } \\
\text { in Exam }\end{array}$ & $\begin{array}{c}\text { Passed in } \\
\text { Exams }\end{array}$ & $\begin{array}{l}\text { Failed in } \\
\text { Exams }\end{array}$ & & Dropped & $\begin{array}{c}\text { Appeared } \\
\text { in Exams }\end{array}$ & $\begin{array}{l}\text { Passed in } \\
\text { Exams }\end{array}$ & $\begin{array}{c}\text { Falied in } \\
\text { Exams }\end{array}$ \\
\hline Grade $1 \mathrm{~A}$ & 48 & 0 & 48 & 32 & 16 & Grade 1 & $0.00 \%$ & $100.00 \%$ & $66.67 \%$ & $33.33 \%$ \\
\hline Grade 1 B & 0 & 0 & 0 & 0 & 0 & Grade 2 & $0.00 \%$ & $100.00 \%$ & $83.33 \%$ & $16.67 \%$ \\
\hline Grade $1 \mathrm{C}$ & 0 & 0 & 0 & 0 & 0 & Grade 3 & $0.00 \%$ & $100.00 \%$ & $71.43 \%$ & $28.57 \%$ \\
\hline Grade $2 \mathrm{~A}$ & 18 & 0 & 18 & 15 & 3 & Grade 4 & $0.00 \%$ & $100.00 \%$ & $88.89 \%$ & $11.11 \%$ \\
\hline Grade 2 B & 0 & 0 & 0 & 0 & 0 & Grade 5 & \#Num! & \#Num! & \#Num! & \#Num! \\
\hline Grade $3 \mathrm{~A}$ & 7 & 0 & 7 & 5 & 2 & Grade 6 & \#Num! & \#Num! & \#Num! & \#Num! \\
\hline Grade 3 B & 0 & 0 & 0 & 0 & 0 & & & & & \\
\hline Grade 4 & 9 & 0 & 9 & 8 & 1 & Remarks: & & & & \\
\hline Grade 5 & 0 & 0 & 0 & 0 & 0 & & & & & \\
\hline Grade 6 & 0 & 0 & 0 & 0 & 0 & & & & & \\
\hline School Total & 82 & 0 & 82 & 60 & 22 & School \%age & $0.00 \%$ & $100.00 \%$ & $73.17 \%$ & $26.83 \%$ \\
\hline
\end{tabular}

Note:

\#Num! = Enrollment in respective grade(s) is nill lherefore calculation is nol applicable
Page 16 of 91

Produced by DP/MIS Section on 31-Jul-97 
Sub-Centre: Bannu

School Code: BN/MSH/PSB-049 School Tille: ARPS Bhatti Camp 1-Miranshah

\begin{tabular}{|c|c|c|c|c|c|c|c|c|c|c|}
\hline \multirow[b]{2}{*}{$\begin{array}{l}\text { Grades I } \\
\text { Sections }\end{array}$} & \multicolumn{5}{|c|}{ - Number of Students } & \multirow[b]{2}{*}{ Grades } & \multicolumn{4}{|c|}{ Percent of Students - } \\
\hline & Enrolled & Dropped & $\begin{array}{c}\text { Appeared } \\
\text { in Exam }\end{array}$ & $\begin{array}{c}\text { Passed in } \\
\text { Exams } \\
\end{array}$ & $\begin{array}{c}\text { Failed in } \\
\text { Exams }\end{array}$ & & Dropped & $\begin{array}{c}\text { Appeared } \\
\text { in Exams }\end{array}$ & $\begin{array}{c}\text { Passed in } \\
\text { Exams }\end{array}$ & $\begin{array}{l}\text { Faled in } \\
\text { Exams }\end{array}$ \\
\hline Grade $1 \mathrm{~A}$ & 53 & 0 & 53 & 33 & 20 & Grade 1 & $0.00 \%$ & $100.00 \%$ & $57.14 \%$ & $42.86 \%$ \\
\hline Grade 1 B & 52 & 0 & 52 & 27 & 25 & Grade 2 & $10.84 \%$ & $89.16 \%$ & $85.14 \%$ & $14.86 \%$ \\
\hline Grade $1 \mathrm{C}$ & 0 & 0 & 0 & 0 & 0 & Grade 3 & $0.00 \%$ & $100.00 \%$ & $66.67 \%$ & $33.33 \%$ \\
\hline Grade $2 \mathrm{~A}$ & 83 & 9 & 74 & 63 & 11 & Grade 4 & $0.00 \%$ & $100.00 \%$ & $71.43 \%$ & $28.57 \%$ \\
\hline Grade $2 \mathrm{~B}$ & 0 & 0 & 0 & 0 & 0 & Grade 5 & $0.00 \%$ & $100.00 \%$ & $57.69 \%$ & $42.31 \%$ \\
\hline Grade 3 A & 63 & 0 & 63 & 42 & 21 & Grade 6 & $0.00 \%$ & $100.00 \%$ & $50.00 \%$ & $50.00 \%$ \\
\hline Grade 3 B & 0 & 0 & 0 & 0 & 0 & & & & & \\
\hline Grade 4 & 35 & 0 & 35 & 25 & 10 & Remarks: & & & & \\
\hline Grade 5 & 26 & 0 & 26 & 15 & 11 & & & & & \\
\hline Grade 6 & 20 & 0 & 20 & 10 & 10 & & & & & \\
\hline School Total & 332 & 9 & 323 & 215 & 108 & School \%age & $2.71 \%$ & $97.29 \%$ & $66.56 \%$ & $33.44 \%$ \\
\hline
\end{tabular}

\begin{tabular}{|c|c|c|c|c|c|c|c|c|c|c|}
\hline \multirow[b]{2}{*}{$\begin{array}{l}\text { Grades I } \\
\text { Sections }\end{array}$} & \multicolumn{5}{|c|}{ - Number of Students- } & \multirow[b]{2}{*}{ Grades } & \multicolumn{4}{|c|}{ Percent of Students } \\
\hline & Enrolled & Dropped & $\begin{array}{c}\text { Appeared } \\
\text { in Exam }\end{array}$ & $\begin{array}{c}\text { Passed in } \\
\text { Exams }\end{array}$ & $\begin{array}{c}\text { Failed in } \\
\text { Exams }\end{array}$ & & Dropped & $\begin{array}{l}\text { Appeared } \\
\text { in Exams }\end{array}$ & $\begin{array}{c}\text { Passed in } \\
\text { Exams }\end{array}$ & $\begin{array}{l}\text { Falled in } \\
\text { Exams }\end{array}$ \\
\hline Grade $1 \mathrm{~A}$ & 34 & 0 & 34 & 18 & 16 & Grade 1 & $0.00 \%$ & $100.00 \%$ & $52.94 \%$ & $47.06 \%$ \\
\hline Grade $1 \mathrm{~B}$ & 0 & 0 & 0 & 0 & 0 & Grade 2 & $2.78 \%$ & $97.22 \%$ & $65.71 \%$ & $34.29 \%$ \\
\hline Grade $1 \mathrm{C}$ & 0 & 0 & 0 & 0 & 0 & Grade 3 & $25.93 \%$ & $74.07 \%$ & $75.00 \%$ & $25.00 \%$ \\
\hline Grade $2 \mathrm{~A}$ & 36 & 1 & 35 & 23 & 12 & Grade 4 & $10.00 \%$ & $90.00 \%$ & $72.22 \%$ & $27.78 \%$ \\
\hline Grade 2 B & 0 & 0 & 0 & 0 & 0 & Grade 5 & $22.22 \%$ & $77.78 \%$ & $71.43 \%$ & $28.57 \%$ \\
\hline Grade $3 \mathrm{~A}$ & 27 & 7 & 20 & 15 & 5 & Grade 6 & $0.00 \%$ & $100.00 \%$ & $63.64 \%$ & $36.36 \%$ \\
\hline Grade 3 B & 0 & 0 & 0 & 0 & 0 & & & & & \\
\hline Grade 4 & 20 & 2 & 18 & 13 & 5 & Remarks: & & & & \\
\hline Grade 5 & 9 & 2 & 7 & 5 & 2 & & & & & \\
\hline Grade 6 & 11 & 0 & 11 & 7 & 4 & & & & & \\
\hline School Total & 137 & 12 & 125 & 81 & 44 & School \%age & $8.76 \%$ & $91.24 \%$ & $64.80 \%$ & $35.20 \%$ \\
\hline
\end{tabular}

\begin{tabular}{|c|c|c|c|c|c|c|c|c|c|c|}
\hline \multirow[b]{2}{*}{$\begin{array}{l}\text { Grades I } \\
\text { Sections }\end{array}$} & \multicolumn{5}{|c|}{ - Number of Students } & \multirow[b]{2}{*}{ Grades } & \multicolumn{4}{|c|}{ Percent of Students- } \\
\hline & Enrolled & Dropped & $\begin{array}{c}\text { Appeared } \\
\text { in Exam }\end{array}$ & $\begin{array}{c}\text { Passed in } \\
\text { Exams }\end{array}$ & $\begin{array}{c}\text { Failed in } \\
\text { Exams }\end{array}$ & & Dropped & $\begin{array}{l}\text { Appeared } \\
\text { in Exams }\end{array}$ & $\begin{array}{l}\text { Passed in } \\
\text { Exams }\end{array}$ & $\begin{array}{l}\text { Falled in } \\
\text { Exams }\end{array}$ \\
\hline Grade $1 \mathrm{~A}$ & 60 & 0 & 60 & 54 & 6 & Grade 1 & $0.00 \%$ & $100.00 \%$ & $90.00 \%$ & $10.00 \%$ \\
\hline Grade $1 \mathrm{~B}$ & 0 & 0 & 0 & 0 & 0 & Grade 2 & $23.53 \%$ & $76.47 \%$ & $92.31 \%$ & $7.69 \%$ \\
\hline Grade $1 \mathrm{C}$ & 0 & 0 & 0 & 0 & 0 & Grade 3 & $44.00 \%$ & $56.00 \%$ & $85.71 \%$ & $14.29 \%$ \\
\hline Grade $2 \mathrm{~A}$ & 34 & 8 & 26 & 24 & 2 & Grade 4 & $45.00 \%$ & $55.00 \%$ & $100.00 \%$ & $0.00 \%$ \\
\hline Grade 2 B & 0 & 0 & 0 & 0 & 0 & Grade 5 & \#Num! & \#Num! & \#Num! & \#Num! \\
\hline Grade $3 \mathrm{~A}$ & 0 & 0 & 0 & 0 & 0 & Grade 6 & \#Num! & \#Num! & \#Num! & \#Num! \\
\hline Grade 3 B & 25 & 11 & 14 & 12 & 2 & & & & & \\
\hline Grade 4 & 20 & 9 & 11 & 11 & 0 & Remarks: & & & & \\
\hline Grade 5 & 0 & 0 & 0 & 0 & 0 & & & & & \\
\hline Grade 6 & 0 & 0 & 0 & 0 & 0 & & & & & \\
\hline School Total & 139 & 28 & 111 & 101 & 10 & School \%age & $20.14 \%$ & $79.86 \%$ & $90.99 \%$ & $9.01 \%$ \\
\hline
\end{tabular}


Sub-Centre: Bannu

\begin{tabular}{|c|c|c|c|c|c|c|c|c|c|c|}
\hline \multirow[b]{2}{*}{$\begin{array}{l}\text { Grades / } \\
\text { Sections }\end{array}$} & \multicolumn{5}{|c|}{ - Number of Students- } & \multirow[b]{2}{*}{ Grades } & \multicolumn{4}{|c|}{ Percent of Students } \\
\hline & Enrolled & Dropped & $\begin{array}{l}\text { Appeared } \\
\text { in Exam }\end{array}$ & $\begin{array}{l}\text { Passed in } \\
\text { Exams }\end{array}$ & $\begin{array}{l}\text { Failed in } \\
\text { Exams }\end{array}$ & & Dropped & $\begin{array}{l}\text { Appeared } \\
\text { in Exams }\end{array}$ & $\begin{array}{l}\text { Passed in } \\
\text { Exams }\end{array}$ & $\begin{array}{l}\text { Falled in } \\
\text { Exams }\end{array}$ \\
\hline Grade $1 \mathrm{~A}$ & 71 & 0 & 71 & 50 & 21 & Grade 1 & $0.00 \%$ & $100.00 \%$ & $70.42 \%$ & $29.58 \%$ \\
\hline Grade 1 B & 0 & 0 & 0 & 0 & 0 & Grade 2 & $0.00 \%$ & $100.00 \%$ & $80.00 \%$ & $20.00 \%$ \\
\hline Grade $1 \mathrm{C}$ & 0 & 0 & 0 & 0 & 0 & Grade 3 & $0.00 \%$ & $100.00 \%$ & $78.95 \%$ & $21.05 \%$ \\
\hline Grade 2 A & 40 & 0 & 40 & 32 & 8 & Grade 4 & $5.26 \%$ & $94.74 \%$ & $66.67 \%$ & $33.33 \%$ \\
\hline Grade 2 B & 0 & 0 & 0 & 0 & 0 & Grade 5 & $6.25 \%$ & $93.75 \%$ & $80.00 \%$ & $20.00 \%$ \\
\hline Grade 3 A & 38 & 0 & 38 & 30 & 8 & Grade 6 & \#Num! & \#Num! & \#Num! & \#Num! \\
\hline Grade 3 B & 0 & 0 & 0 & 0 & 0 & & & & & \\
\hline Grade 4 & 19 & 1 & 18 & 12 & 6 & Remarks: & & & & \\
\hline Grade 5 & 16 & 1 & 15 & 12 & 3 & & & & & \\
\hline Grade 6 & 0 & 0 & 0 & 0 & 0 & & & & & \\
\hline School Total & 184 & 2 & 182 & 136 & 46 & School \%age & $1.09 \%$ & $98.91 \%$ & $74.73 \%$ & $25.27 \%$ \\
\hline
\end{tabular}

\begin{tabular}{|c|c|c|c|c|c|c|c|c|c|c|c|}
\hline \multirow[b]{2}{*}{$\begin{array}{l}\text { Grades / } \\
\text { Sections }\end{array}$} & \multicolumn{6}{|c|}{-Number of Students } & \multirow[b]{2}{*}{ Grades } & \multicolumn{4}{|c|}{ Percent of Students } \\
\hline & $y$ & Enrolled & Dropped & $\begin{array}{l}\text { Appeared } \\
\text { in Exam }\end{array}$ & $\begin{array}{l}\text { Passed in } \\
\text { Exams }\end{array}$ & $\begin{array}{c}\text { Failed in } \\
\text { Exams }\end{array}$ & & Dropped & $\begin{array}{l}\text { Appeared } \\
\text { in Exams }\end{array}$ & $\begin{array}{l}\text { Passed in } \\
\text { Exams }\end{array}$ & $\begin{array}{l}\text { Failed in } \\
\text { Exams }\end{array}$ \\
\hline Grade $1 \mathrm{~A}$ & & 57 & 0 & 57 & 35 & 22 & Grade 1 & $0.00 \%$ & $100.00 \%$ & $61.40 \%$ & $38.60 \%$ \\
\hline Grade $1 \mathrm{~B}$ & & 0 & 0 & 0 & 0 & 0 & Grade 2 & $0.00 \%$ & $100.00 \%$ & $80.00 \%$ & $20.00^{c i c}$ \\
\hline Grade $1 \mathrm{C}$ & & 0 & 0 & 0 & 0 & 0 & Grade 3 & $0.00 \%$ & $100.00 \%$ & $78.57 \%$ & $21.43 \%$ \\
\hline Grade 2 A & & 0 & 0 & 0 & 0 & 0 & Grade 4 & $0.00 \%$ & $100.00 \%$ & $75.00 \%$ & $25.00 \%$ \\
\hline Grade 2 B & & 35 & 0 & 35 & 28 & 7 & Grade 5 & $0.00 \%$ & $100.00 \%$ & $75.00 \%$ & $25.00 \%$ \\
\hline Grade $3 \mathrm{~A}$ & & 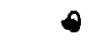 & 0 & 0 & 0 & 0 & Grade 6 & \#Num! & \#Num! & \#Num! & \#Num! \\
\hline Grade 3 B & & 28 & 0 & 28 & 22 & 6 & & & & & \\
\hline Grade 4 & & 20 & 0 & 20 & 15 & 5 & Remarks: & & & & \\
\hline Grade 5 & & 28 & 0 & 28 & 21 & 7 & & & & & \\
\hline Grade 6 & & 0 & 0 & 0 & 0 & 0 & & & & & \\
\hline School Total & & 168 & 0 & 168 & 121 & 47 & School \%age & $0.00 \%$ & $100.00 \%$ & $72.02 \%$ & $27.98 \%$ \\
\hline
\end{tabular}

\begin{tabular}{|c|c|c|c|c|c|c|c|c|c|c|}
\hline \multirow[b]{2}{*}{$\begin{array}{l}\text { Grades I } \\
\text { Sections }\end{array}$} & \multicolumn{5}{|c|}{ - Number of Students } & \multirow[b]{2}{*}{ Grades } & \multicolumn{4}{|c|}{ Percent of Students } \\
\hline & Enrolled & Dropped & $\begin{array}{l}\text { Appeared } \\
\text { in Exam }\end{array}$ & $\begin{array}{l}\text { Passed in } \\
\text { Exams }\end{array}$ & $\begin{array}{l}\text { Failed in } \\
\text { Exams }\end{array}$ & & Dropped & $\begin{array}{l}\text { Appeared } \\
\text { in Exams }\end{array}$ & $\begin{array}{l}\text { Passed in } \\
\text { Exams }\end{array}$ & $\begin{array}{l}\text { Faied in: } \\
\text { Exams }\end{array}$ \\
\hline Grade $1 \mathrm{~A}$ & 44 & 16 & 28 & 20 & 8 & Grade 1 & $36.36 \%$ & $63.64 \%$ & $71.43 \%$ & $28.57 \%$ \\
\hline Grade 1 B & 0 & 0 & 0 & 0 & 0 & Grade 2 & $0.00 \%$ & $100.00 \%$ & $86.96 \%$ & $13.04 \%$ \\
\hline Grade $1 \mathrm{C}$ & 0 & 0 & 0 & 0 & 0 & Grade 3 & $6.67 \%$ & $93.33 \%$ & $89.29 \%$ & $10.71 \%$ \\
\hline Grade 2 A & 23 & 0 & 23 & 20 & 3 & Grade 4 & $5.88 \%$ & $94.12 \%$ & $87.50 \%$ & $12.50 \%$ \\
\hline Grade 2 B & 0 & 0 & 0 & 0 & 0 & Grade 5 & $22.22 \%$ & $77.78 \%$ & $100.00 \%$ & $0.00 \%$ \\
\hline Grade 3 A & 30 & 2 & 28 & 25 & 3 & Grade 6 & $25.00 \%$ & $75.00 \%$ & $66.67 \%$ & $33.33 \%$ \\
\hline Grade 3 B & 0 & 0 & 0 & 0 & 0 & & & & & \\
\hline Grade 4 & 17 & 1 & 16 & 14 & 2 & Remarks: & & & & \\
\hline Grade 5 & 9 & 2 & 7 & 7 & 0 & & & & & \\
\hline Grade 6 & 16 & 4 & 12 & 8 & 4 & & & & & \\
\hline School Total & 139 & 25 & 114 & 94 & 20 & School \%age & $17.99 \%$ & $82.01 \%$ & $82.46 \%$ & $17.54 \%$ \\
\hline
\end{tabular}


Sub-Centre: Bannu

School Code: BN/MSH/PSB-055 School Title: ARPS Shuga Datta Khel - Miranshah Head Teacher: Mohammad Alam

\begin{tabular}{|c|c|c|c|c|c|c|c|c|c|c|}
\hline \multirow[b]{2}{*}{$\begin{array}{l}\text { Grades I } \\
\text { Sections }\end{array}$} & \multicolumn{5}{|c|}{-Number of Students- } & \multirow[b]{2}{*}{ Grades } & \multicolumn{4}{|c|}{-Percent of Students } \\
\hline & Enrolled & Dropped & $\begin{array}{l}\text { Appeared } \\
\text { in Exam }\end{array}$ & $\begin{array}{l}\text { Passed in } \\
\text { Exams }\end{array}$ & $\begin{array}{l}\text { Failed in } \\
\text { Exams }\end{array}$ & & Dropped & $\begin{array}{l}\text { Appeared } \\
\text { in Exams }\end{array}$ & $\begin{array}{l}\text { Passed in } \\
\text { Exams }\end{array}$ & $\begin{array}{c}\text { Faled in } \\
\text { Exams }\end{array}$ \\
\hline Grade $1 \mathrm{~A}$ & 50 & 0 & 50 & 40 & 10 & Grade 1 & $0.00 \%$ & $100.00 \%$ & $80.00 \%$ & $20.00 \%$ \\
\hline Grade 1 B & 0 & 0 & 0 & 0 & 0 & Grade 2 & $0.00 \%$ & $100.00 \%$ & $100.00 \%$ & $0.00 \%$ \\
\hline Grade $1 \mathrm{C}$ & 0 & 0 & 0 & 0 & 0 & Grade 3 & $0.00 \%$ & $100.00 \%$ & $100.00 \%$ & $0.00 \%$ \\
\hline Grade 2 A & 30 & 0 & 30 & 30 & 0 & Grade 4 & $0.00 \%$ & $100.00 \%$ & $100.00 \%$ & $0.00 \%$ \\
\hline Grade 2 B & 0 & 0 & 0 & 0 & 0 & Grade 5 & $0.00 \%$ & $100.00 \%$ & $100.00 \%$ & $0.00 \%$ \\
\hline Grade 3 A & 18 & 0 & 18 & 18 & 0 & Grade 6 & \#Num! & \#Num! & \#Num! & \#Num! \\
\hline Grade 3 B & 0 & 0 & 0 & 0 & 0 & & & & & \\
\hline Grade 4 & 16 & 0 & 16 & 16 & 0 & Remarks: & & & & \\
\hline Grade 5 & 15 & 0 & 15 & 15 & 0 & & & & & \\
\hline Grade 6 & 0 & 0 & 0 & 0 & 0 & & & & & \\
\hline School Total & 129 & 0 & 129 & 119 & 10 & School \%age & $0.00 \%$ & $100.00 \%$ & $92.25 \%$ & $7.75 \%$ \\
\hline
\end{tabular}

\begin{tabular}{|c|c|c|c|c|c|c|c|c|c|c|}
\hline \multirow[b]{2}{*}{$\begin{array}{l}\text { Grades / } \\
\text { Sections }\end{array}$} & \multicolumn{5}{|c|}{ - Number of Students } & \multirow[b]{2}{*}{ Grades } & \multicolumn{4}{|c|}{-Percent of Students } \\
\hline & Enrolled & Dropped & $\begin{array}{l}\text { Appeared } \\
\text { in Exam }\end{array}$ & $\begin{array}{c}\text { Passed in } \\
\text { Exams }\end{array}$ & $\begin{array}{l}\text { Failed in } \\
\text { Exams }\end{array}$ & & Dropped & $\begin{array}{l}\text { Appeared } \\
\text { in Exams }\end{array}$ & $\begin{array}{l}\text { Passed in } \\
\text { Exams }\end{array}$ & $\begin{array}{l}\text { Faled in } \\
\text { Exams }\end{array}$ \\
\hline Grade $1 \mathrm{~A}$ & 55 & 6 & 49 & 40 & 9 & Grade 1 & $5.56 \%$ & $94.44 \%$ & $68.91 \%$ & $31.09 \%$ \\
\hline Grade $1 \mathrm{~B}$ & 71 & 1 & 70 & 42 & 28 & Grade 2 & $5.19 \%$ & $94.81 \%$ & $58.90 \%$ & $41.10 \%$ \\
\hline Grade $1 \mathrm{C}$ & 0 & 0 & 0 & 0 & 0 & Grade 3 & $6.78 \%$ & $93.22 \%$ & $56.36 \%$ & $43.64 \%$ \\
\hline Grade 2 A & 77 & 4 & 73 & 43 & 30 & Grade 4 & $10.34 \%$ & $89.66 \%$ & $73.08 \%$ & $26.92 \%$ \\
\hline Grade 2 B & 0 & 0 & 0 & 0 & 0 & Grade 5 & $10.53 \%$ & $89.47 \%$ & $88.24 \%$ & $11.76 \%$ \\
\hline Grade $3 \mathrm{~A}$ & 59 & 4 & 55 & 31 & 24 & Grade 6 & $4.00 \%$ & $96.00 \%$ & $79.17 \%$ & $20.83 \%$ \\
\hline Grade 3 B & 0 & 0 & 0 & 0 & 0 & & & & & \\
\hline Grade 4 & 29 & 3 & 26 & 19 & 7 & Remarks: & & & & \\
\hline Grade 5 & 19 & 2 & 17 & 15 & 2 & & & & & \\
\hline Grade 6 & 25 & 1 & 24 & 19 & 5 & & & & & \\
\hline School Total & 335 & 21 & 314 & 209 & 105 & School \%age & $6.27 \%$ & $93.73 \%$ & $66.56 \%$ & $33.44 \%$ \\
\hline
\end{tabular}

\begin{tabular}{|c|c|c|c|c|c|c|c|c|c|c|}
\hline \multicolumn{11}{|c|}{ School Code: BN/MSHIPSB-057 School Title: ARPS Mir Ali - Miranshah } \\
\hline \multirow[b]{2}{*}{$\begin{array}{l}\text { Grades I } \\
\text { Sections }\end{array}$} & \multicolumn{5}{|c|}{-Number of Students- } & \multirow[b]{2}{*}{ Grades } & \multicolumn{4}{|c|}{ Percent of Students - } \\
\hline & Enrolled & Dropped & $\begin{array}{l}\text { Appeared } \\
\text { in Exam }\end{array}$ & $\begin{array}{l}\text { Passed in } \\
\text { Exams }\end{array}$ & $\begin{array}{l}\text { Failed in } \\
\text { Exams }\end{array}$ & & Dropped & $\begin{array}{l}\text { Appeared } \\
\text { in Exams }\end{array}$ & $\begin{array}{l}\text { Passed in } \\
\text { Exams }\end{array}$ & $\begin{array}{l}\text { Falled in } \\
\text { Exams }\end{array}$ \\
\hline Grade $1 \mathrm{~A}$ & 60 & 4 & 56 & 34 & 22 & Grade 1 & $6.67 \%$ & $93.33 \%$ & $60.71 \%$ & $39.29 \%$ \\
\hline Grade 1 B & 0 & 0 & 0 & 0 & 0 & Grade 2 & $4.00 \%$ & $96.00 \%$ & $83.33 \%$ & $16.67 \%$ \\
\hline Grade $1 \mathrm{C}$ & 0 & 0 & 0 & 0 & 0 & Grade 3 & $12.50 \%$ & $87.50 \%$ & $100.00 \%$ & $0.00 \%$ \\
\hline Grade 2 A & 25 & 1 & 24 & 20 & 4 & Grade 4 & $7.14 \%$ & $92.86 \%$ & $100.00 \%$ & $0.00 \%$ \\
\hline Grade 2 B & 0 & 0 & 0 & 0 & 0 & Grade 5 & $0.00 \%$ & $100.00 \%$ & $100.00 \%$ & $0.00 \%$ \\
\hline Grade 3 A & 16 & 2 & 14 & 14 & 0 & Grade 6 & \#Num! & \#Num! & \#Num! & \#Num! \\
\hline Grade 3 B & 0 & 0 & 0 & 0 & 0 & & & & & \\
\hline Grade 4 & 14 & 1 & 13 & 13 & 0 & Remarks: & & & & \\
\hline Grade 5 & 9 & 0 & 9 & 9 & 0 & & & & & \\
\hline Grade 6 & 0 & 0 & 0 & 0 & 0 & & & & & \\
\hline School Total & 124 & 8 & 116 & 90 & 26 & School \%age & $6.45 \%$ & $93.55 \%$ & $77.59 \%$ & $22.41 \%$ \\
\hline
\end{tabular}


Sub-Centre: Bannu

\begin{tabular}{|c|c|c|c|c|c|c|c|c|c|c|}
\hline \multicolumn{11}{|c|}{ School Code: BN/MSH/PSB-058 School Title: ARPS 1 Muhammad Khel - Miranshah } \\
\hline \multirow[b]{2}{*}{$\begin{array}{l}\text { Grades I } \\
\text { Sections }\end{array}$} & \multicolumn{5}{|c|}{-Number of Students- } & \multirow[b]{2}{*}{ Grades } & \multicolumn{4}{|c|}{-Percent of Students- } \\
\hline & Enrolied & Dropped & $\begin{array}{l}\text { Appeared } \\
\text { in Exam }\end{array}$ & $\begin{array}{c}\text { Passed in } \\
\text { Exams }\end{array}$ & $\begin{array}{c}\text { Failed in } \\
\text { Exams }\end{array}$ & & Dropped & $\begin{array}{l}\text { Appeared } \\
\text { in Exams }\end{array}$ & $\begin{array}{c}\text { Passed in } \\
\text { Exams }\end{array}$ & $\begin{array}{l}\text { Falled in } \\
\text { Exams }\end{array}$ \\
\hline Grade $1 \mathrm{~A}$ & 36 & 3 & 33 & 20 & 13 & Grade 1 & $8.33 \%$ & $91.67 \%$ & $60.61 \%$ & $39.39 \%$ \\
\hline Grade 1 B & 0 & 0 & 0 & 0 & 0 & Grade 2 & $4.35 \%$ & $95.65 \%$ & $77.27 \%$ & $22.73 \%$ \\
\hline Grade $1 \mathrm{C}$ & 0 & 0 & 0 & 0 & 0 & Grade 3 & $0.00 \%$ & $100.00 \%$ & $78.95 \%$ & $21.05 \%$ \\
\hline Grade 2 A & 23 & 1 & 22 & 17 & 5 & Grade 4 & $0.00 \%$ & $100.00 \%$ & $100.00 \%$ & $0.00 \%$ \\
\hline Grade 2 B & 0 & 0 & 0 & 0 & 0 & Grade 5 & $0.00 \%$ & $100.00 \%$ & $66.67 \%$ & $33.33 \%$ \\
\hline Grade $3 \mathrm{~A}$ & 19 & 0 & 19 & 15 & 4 & Grade 6 & $0.00 \%$ & $100.00 \%$ & $66.67 \%$ & $33.33 \%$ \\
\hline Grade 3 B & 0 & 0 & 0 & 0 & 0 & & & & & \\
\hline Grade 4 & 10 & 0 & 10 & 10 & 0 & Remarks: & & & & \\
\hline Grade 5 & 12 & 0 & 12 & 8 & 4 & & & & & \\
\hline Grade 6 & 12 & 0 & 12 & 8 & 4 & & & & & \\
\hline School Total & 112 & 4 & 108 & 78 & 30 & School \%age & $3.57 \%$ & $96.43 \%$ & $72.22 \%$ & $27.78 \%$ \\
\hline
\end{tabular}

\begin{tabular}{|c|c|c|c|c|c|c|c|c|c|c|}
\hline \multirow[b]{2}{*}{$\begin{array}{l}\text { Grades I } \\
\text { Sections }\end{array}$} & \multicolumn{5}{|c|}{ Number of Students- } & \multirow[b]{2}{*}{ Grades } & \multicolumn{4}{|c|}{-Percent of Students } \\
\hline & / Enrolled & Dropped & $\begin{array}{l}\text { Appeared } \\
\text { in Exam }\end{array}$ & $\begin{array}{c}\text { Passed in } \\
\text { Exams }\end{array}$ & $\begin{array}{l}\text { Failed in } \\
\text { Exams }\end{array}$ & & Dropped & $\begin{array}{l}\text { Appeared } \\
\text { in Exams }\end{array}$ & $\begin{array}{c}\text { Passed in } \\
\text { Exams }\end{array}$ & $\begin{array}{l}\text { Falled in } \\
\text { Exams }\end{array}$ \\
\hline Grade $1 \mathrm{~A}$ & 52 & 11 & 41 & 32 & 9 & Grade 1 & $21.15 \%$ & $78.85 \%$ & $78.05 \%$ & $21.95 \%$ \\
\hline Grade 1 B & 0 & 0 & 0 & 0 & 0 & Grade 2 & $10.00 \%$ & $90.00 \%$ & $77.78 \%$ & $22.22 \%$ \\
\hline Grade $1 \mathrm{C}$ & 0 & 0 & 0 & 0 & 0 & Grade 3 & $0.00 \%$ & $100.00 \%$ & $75.00 \%$ & $25.00 \%$ \\
\hline Grade 2 A & 20 & 2 & 18 & 14 & 4 & Grade 4 & $12.50 \%$ & $87.50 \%$ & $71.43 \%$ & $28.57 \%$ \\
\hline Grade 2 B & 0 & 0 & 0 & 0 & 0 & Grade 5 & $0.00 \%$ & $100.00 \%$ & $70.00 \%$ & $30.00 \%$ \\
\hline Grade $3 \mathrm{~A}$ & 20 & 0 & 20 & 15 & 5 & Grade 6 & \#Num! & \#Num! & \#Num! & \#Num! \\
\hline Grade 3 B & 0 & 0 & 0 & 0 & 0 & & & & & \\
\hline Grade 4 & 8 & 1 & 7 & 5 & 2 & Remarks: & & & & \\
\hline Grade 5 & 10 & 0 & 10 & 7 & 3 & & & & & \\
\hline Grade 6 & 0 & 0 & 0 & 0 & 0 & & & & & \\
\hline School Total & 110 & 14 & 96 & 73 & 23 & School \%age & $12.73 \%$ & $87.27 \%$ & $76.04 \%$ & $23.96 \%$ \\
\hline
\end{tabular}

\begin{tabular}{|c|c|c|c|c|c|c|c|c|c|c|}
\hline \multirow[b]{2}{*}{$\begin{array}{l}\text { Grades / } \\
\text { Sections }\end{array}$} & \multicolumn{5}{|c|}{ - Number of Students- } & \multirow[b]{2}{*}{ Grades } & \multicolumn{4}{|c|}{ Percent of Students } \\
\hline & Enrolled & Dropped & $\begin{array}{l}\text { Appeared } \\
\text { in Exam }\end{array}$ & $\begin{array}{c}\text { Passed in } \\
\text { Exams }\end{array}$ & $\begin{array}{l}\text { Failed in } \\
\text { Exams }\end{array}$ & & Dropped & $\begin{array}{l}\text { Appeared } \\
\text { in Exams }\end{array}$ & $\begin{array}{c}\text { Passed in } \\
\text { Exams }\end{array}$ & $\begin{array}{l}\text { Falled in } \\
\text { Exams }\end{array}$ \\
\hline Grade $1 \mathrm{~A}$ & 0 & 0 & 0 & 0 & 0 & Grade 1 & \#Num! & \#Num! & \#Num! & \#Num! \\
\hline Grade 1 B & 0 & 0 & 0 & 0 & 0 & Grade 2 & $5.56 \%$ & $94.44 \%$ & $88.24 \%$ & $11.76 \%$ \\
\hline Grade $1 \mathrm{C}$ & 0 & 0 & 0 & 0 & 0 & Grade 3 & $3.70 \%$ & $96.30 \%$ & $88.46 \%$ & $11.54 \%$ \\
\hline Grade 2 A & 36 & 2 & 34 & 30 & 4 & Grade 4 & $4.76 \%$ & $95.24 \%$ & $90.00 \%$ & $10.00 \%$ \\
\hline Grade 2 B & 0 & 0 & 0 & 0 & 0 & Grade 5 & $0.00 \%$ & $100.00 \%$ & $81.25 \%$ & $18.75 \%$ \\
\hline Grade $3 \mathrm{~A}$ & 27 & 1 & 26 & 23 & 3 & Grade 6 & $0.00 \%$ & $100.00 \%$ & $72.73 \%$ & $27.27 \%$ \\
\hline Grade 3 B & 0 & 0 & 0 & 0 & 0 & & & & & \\
\hline Grade 4 & 21 & 1 & 20 & 18 & 2 & Remarks: & & & & \\
\hline Grade 5 & 16 & 0 & 16 & 13 & 3 & & & & & \\
\hline Grade 6 & 11 & 0 & 11 & 8 & 3 & & & & & \\
\hline School Total & 111 & 4 & 107 & 92 & 15 & School \%age & $3.60 \%$ & $96.40 \%$ & $85.98 \%$ & $14.02 \%$ \\
\hline
\end{tabular}


Sub-Centre: Bannu

School Code: BN/MSH/PSB-061 School Title: ARPS Stanya - Miranshah Head Teacher: Mohammad Siddique

\begin{tabular}{|c|c|c|c|c|c|c|c|c|c|c|}
\hline \multirow[b]{2}{*}{$\begin{array}{l}\text { Grades / } \\
\text { Sections }\end{array}$} & \multicolumn{5}{|c|}{-Number of Students- } & \multirow[b]{2}{*}{ Grades } & \multicolumn{4}{|c|}{ Percent of Students } \\
\hline & Enrolled & Dropped & $\begin{array}{l}\text { Appeared } \\
\text { in Exam }\end{array}$ & $\begin{array}{c}\text { Passed in } \\
\text { Exams }\end{array}$ & $\begin{array}{l}\text { Failed in } \\
\text { Exams }\end{array}$ & & Dropped & $\begin{array}{l}\text { Appeared } \\
\text { in Exams }\end{array}$ & $\begin{array}{c}\text { Passed in } \\
\text { Exams }\end{array}$ & $\begin{array}{l}\text { Fatled in } \\
\text { Exams }\end{array}$ \\
\hline Grade $1 \mathrm{~A}$ & 49 & 0 & 49 & 23 & 26 & Grade 1 & $0.00 \%$ & $100.00 \%$ & $46.94 \%$ & $53.06 \%$ \\
\hline Grade $1 \mathrm{~B}$ & 0 & 0 & 0 & 0 & 0 & Grade 2 & $4.55 \%$ & $95.45 \%$ & $64.29 \%$ & $35.71 \%$ \\
\hline Grade $1 \mathrm{C}$ & 0 & 0 & 0 & 0 & 0 & Grade 3 & $18.18 \%$ & $81.82 \%$ & $83.33 \%$ & $16.67 \%$ \\
\hline Grade $2 \mathrm{~A}$ & 44 & 2 & 42 & 27 & 15 & Grade 4 & $0.00 \%$ & $100.00 \%$ & $100.00 \%$ & $0.00 \%$ \\
\hline Grade 2 B & 0 & 0 & 0 & 0 & 0 & Grade 5 & $5.56 \%$ & $94.44 \%$ & $82.35 \%$ & $17.65 \%$ \\
\hline Grade $3 \mathrm{~A}$ & 22 & 4 & 18 & 15 & 3 & Grade 6 & $16.67 \%$ & $83.33 \%$ & $80.00 \%$ & $20.00 \%$ \\
\hline Grade 3 B & 0 & 0 & 0 & 0 & 0 & & & & & \\
\hline Grade 4 & 14 & 0 & 14 & 14 & 0 & Remarks: & & & & \\
\hline Grade 5 & 18 & 1 & 17 & 14 & 3 & & & & & \\
\hline Grade 6 & 12 & 2 & 10 & 8 & 2 & & & & & \\
\hline ool Total & 159 & 9 & 150 & 101 & 49 & School \%age & $5.66 \%$ & $94.34 \%$ & $67.33 \%$ & $32.67 \%$ \\
\hline
\end{tabular}

\begin{tabular}{|c|c|c|c|c|c|c|c|c|c|c|}
\hline \multirow[b]{2}{*}{$\begin{array}{l}\text { Grades I } \\
\text { Sections }\end{array}$} & \multicolumn{5}{|c|}{-Number of Students } & \multirow[b]{2}{*}{ Grades } & \multicolumn{4}{|c|}{-Percent of Students } \\
\hline & 'Enrolled & Dropped & $\begin{array}{l}\text { Appeared } \\
\text { in Exam }\end{array}$ & $\begin{array}{l}\text { Passed in } \\
\text { Exams }\end{array}$ & $\begin{array}{l}\text { Failed in } \\
\text { Exams }\end{array}$ & & Dropped & $\begin{array}{l}\text { Appeared } \\
\text { in Exams }\end{array}$ & $\begin{array}{c}\text { Passed in } \\
\text { Exams }\end{array}$ & $\begin{array}{c}\text { Falled in } \\
\text { Exams }\end{array}$ \\
\hline Grade 1 A & 60 & 8 & 52 & 35 & 17 & Grade 1 & $13.33 \%$ & $86.67 \%$ & $67.31 \%$ & $32.69 \%$ \\
\hline Grade 1 B & 0 & 0 & 0 & 0 & 0 & Grade 2 & $15.00 \%$ & $85.00 \%$ & $94.12 \%$ & $5.88 \%$ \\
\hline Grade $1 \mathrm{C}$ & 0 & 0 & 0 & 0 & 0 & Grade 3 & $23.08 \%$ & $76.92 \%$ & $90.00 \%$ & $10.00 \%$ \\
\hline Grade 2 A & 20 & 3 & 17 & 16 & 1 & Grade 4 & $0.00 \%$ & $100.00 \%$ & $75.00 \%$ & $25.00 \%$ \\
\hline Grade 2 B & 0 & 0 & 0 & 0 & 0 & Grade 5 & \#Num! & \#Num! & \#Num! & \#Num! \\
\hline Grade $3 \mathrm{~A}$ & 0 . & 0 & 0 & 0 & 0 & Grade 6 & \#Num! & \#Num! & \#Num! & \#Num! \\
\hline Grade 3 B & 13 & 3 & 10 & 9 & 1 & & & & & \\
\hline Grade 4 & 8 & 0 & 8 & 6 & 2 & Remarks: & & & & \\
\hline Grade 5 & 0 & 0 & 0 & 0 & 0 & & & & & \\
\hline Grade 6 & 0 & 0 & 0 & 0 & 0 & & & & & \\
\hline School Total & 101 & 14 & 87 & 66 & 21 & School \%age & $13.86 \%$ & $86.14 \%$ & $75.86 \%$ & $24.14 \%$ \\
\hline
\end{tabular}

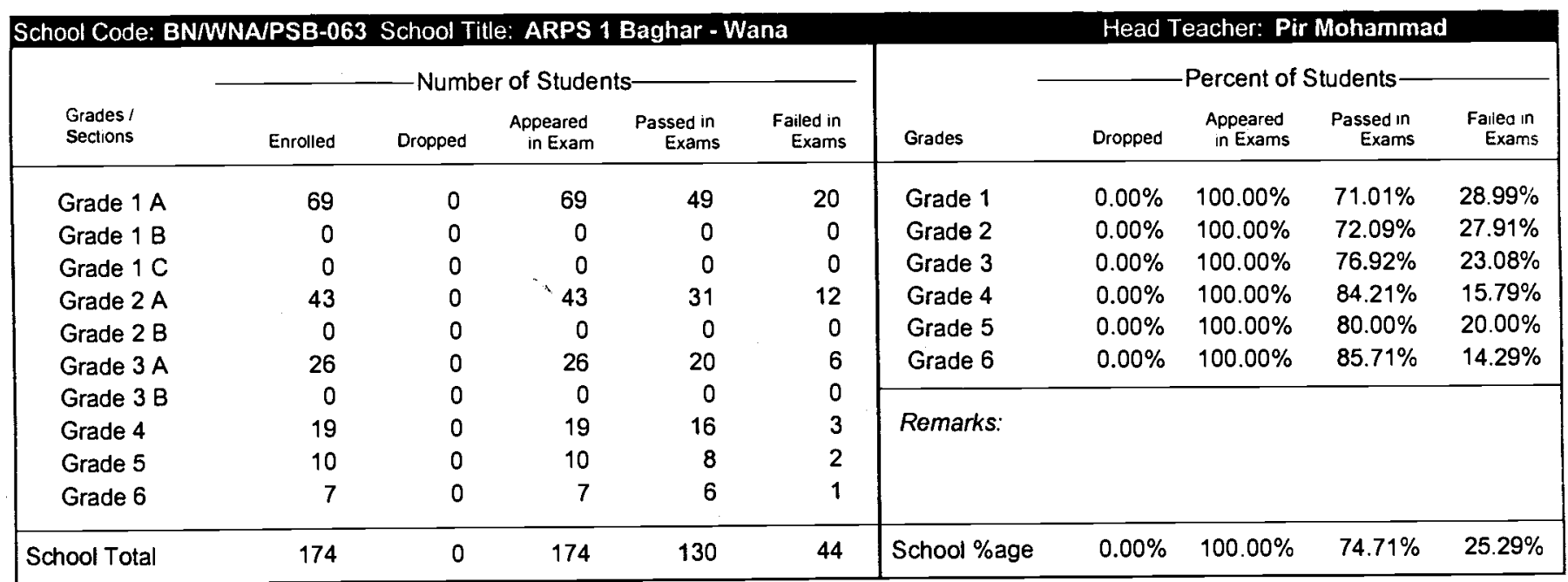


Sub-Centre: Bannu

\begin{tabular}{|c|c|c|c|c|c|c|c|c|c|c|}
\hline \multicolumn{11}{|c|}{ School Code: BNWWAPSB-064 School Title: ARPS 1 Zari Noor - Camp 1 Wana } \\
\hline \multirow[b]{2}{*}{$\begin{array}{l}\text { Grades I } \\
\text { Sections }\end{array}$} & \multicolumn{5}{|c|}{ —Number of Students- } & \multirow[b]{2}{*}{ Grades } & \multicolumn{4}{|c|}{-Percent of Students- } \\
\hline & Enrolled & Dropped & $\begin{array}{l}\text { Appeared } \\
\text { in Exam }\end{array}$ & $\begin{array}{l}\text { Passed in } \\
\text { Exams }\end{array}$ & $\begin{array}{l}\text { Failed in } \\
\text { Exams }\end{array}$ & & Dropped & $\begin{array}{l}\text { Appeared } \\
\text { in Exams }\end{array}$ & $\begin{array}{l}\text { Passed in } \\
\text { Exams }\end{array}$ & $\begin{array}{l}\text { Falled in } \\
\text { Exams }\end{array}$ \\
\hline Grade $1 \mathrm{~A}$ & 44 & 9 & 35 & 22 & 13 & Grade 1 & $20.45 \%$ & $79.55 \%$ & $62.86 \%$ & $37.14 \%$ \\
\hline Grade $1 \mathrm{~B}$ & 0 & 0 & 0 & 0 & 0 & Grade 2 & $22.73 \%$ & $77.27 \%$ & $88.24 \%$ & $11.76 \%$ \\
\hline Grade $1 \mathrm{C}$ & 0 & 0 & 0 & 0 & 0 & Grade 3 & $7.69 \%$ & $92.31 \%$ & $50.00 \%$ & $50.00 \%$ \\
\hline Grade $2 \mathrm{~A}$ & 22 & 5 & 17 & 15 & 2 & Grade 4 & $10.00 \%$ & $90.00 \%$ & $44.44 \%$ & $55.56 \%$ \\
\hline Grade 2 B & 0 & 0 & 0 & 0 & 0 & Grade 5 & \#Num! & \#Num! & \#Num! & \#Num! \\
\hline Grade $3 \mathrm{~A}$ & 13 & 1 & 12 & 6 & 6 & Grade 6 & \#Num! & \#Num! & \#Num! & \#um! \\
\hline Grade 3 B & 0 & 0 & 0 & 0 & 0 & & & & & \\
\hline Grade 4 & 10 & 1 & 9 & 4 & 5 & Remarks: & & & & \\
\hline Grade 5 & 0 & 0 & 0 & 0 & 0 & & & & & \\
\hline Grade 6 & 0 & 0 & 0 & 0 & 0 & & & & & \\
\hline School Total & 89 & 16 & 73 & 47 & 26 & School \%age & $17.98 \%$ & $82.02 \%$ & $64.38 \%$ & $35.62 \%$ \\
\hline
\end{tabular}

\begin{tabular}{|c|c|c|c|c|c|c|c|c|c|c|}
\hline \multirow[b]{2}{*}{$\begin{array}{l}\text { Grades I } \\
\text { Sections }\end{array}$} & \multicolumn{5}{|c|}{ Number of Students- } & \multirow[b]{2}{*}{ Grades } & \multicolumn{4}{|c|}{-Percent of Students } \\
\hline & ,Enrolled & Dropped & $\begin{array}{l}\text { Appeared } \\
\text { in Exam }\end{array}$ & $\begin{array}{l}\text { Passed in } \\
\text { Exams }\end{array}$ & $\begin{array}{l}\text { Faited in } \\
\text { Exams }\end{array}$ & & Dropped & $\begin{array}{c}\text { Appeared } \\
\text { in Exams }\end{array}$ & $\begin{array}{l}\text { Passed in } \\
\text { Exams }\end{array}$ & $\begin{array}{c}\text { Falled in } \\
\text { Exams }\end{array}$ \\
\hline Grade $1 \mathrm{~A}$ & 62 & 0 & 62 & 35 & 27 & Grade 1 & $0.00 \%$ & $100.00 \%$ & $56.45 \%$ & $43.55 \%$ \\
\hline Grade $1 \mathrm{~B}$ & 0 & 0 & 0 & 0 & 0 & Grade 2 & $0.00 \%$ & $100.00 \%$ & $68.00 \%$ & $32.00 \%$ \\
\hline Grade $1 \mathrm{C}$ & 0 & 0 & 0 & 0 & 0 & Grade 3 & $0.00 \%$ & $100.00 \%$ & $79.17 \%$ & $20.83 \%$ \\
\hline Grade $2 \mathrm{~A}$ & 50 & 0 & 50 & 34 & 16 & Grade 4 & $0.00 \%$ & $100.00 \%$ & $61.11 \%$ & $38.89 \%$ \\
\hline Grade 2 B & 0 & 0 & 0 & 0 & 0 & Grade 5 & $0.00 \%$ & $100.00 \%$ & $72.73 \%$ & $27.27 \%$ \\
\hline Grade $3 \mathrm{~A}$ & 24 & 0 & 24 & 19 & 5 & Grade 6 & $0.00 \%$ & $100.00 \%$ & $100.00 \%$ & $0.00 \%$ \\
\hline Grade 3 B & 0 & 0 & 0 & 0 & 0 & & & & & \\
\hline Grade 4 & 18 & 0 & 18 & 11 & 7 & Remarks: & & & & \\
\hline Grade 5 & 11 & 0 & 11 & 8 & 3 & & & & & \\
\hline Grade 6 & 8 & 0 & 8 & 8 & 0 & & & & & \\
\hline School Total & 173 & 0 & 173 & 115 & 58 & School \%age & $0.00 \%$ & $100.00 \%$ & $66.47 \%$ & $33.53 \%$ \\
\hline
\end{tabular}

\begin{tabular}{|c|c|c|c|c|c|c|c|c|c|c|}
\hline \multirow[b]{2}{*}{$\begin{array}{l}\text { Grades I } \\
\text { Sections }\end{array}$} & \multicolumn{5}{|c|}{-Number of Students } & \multirow[b]{2}{*}{ Grades } & \multicolumn{4}{|c|}{-Percent of Students- } \\
\hline & Enrolled & Dropped & $\begin{array}{l}\text { Appeared } \\
\text { in Exam }\end{array}$ & $\begin{array}{l}\text { Passed in } \\
\text { Exams }\end{array}$ & $\begin{array}{l}\text { Failed in } \\
\text { Exams }\end{array}$ & & Dropped & $\begin{array}{l}\text { Appeared } \\
\text { in Exams }\end{array}$ & $\begin{array}{l}\text { Passed in } \\
\text { Exams }\end{array}$ & $\begin{array}{c}\text { Falled in } \\
\text { Exams }\end{array}$ \\
\hline Grade $1 \mathrm{~A}$ & 52 & 0 & 52 & 44 & 8 & Grade 1 & $0.00 \%$ & $100.00 \%$ & $83.78 \%$ & $16.22 \%$ \\
\hline Grade 1 B & 59 & 0 & 59 & 49 & 10 & Grade 2 & $0.00 \%$ & $100.00 \%$ & $81.48 \%$ & $18.52 \%$ \\
\hline Grade $1 \mathrm{C}$ & 0 & 0 & 0 & 0 & 0 & Grade 3 & $0.00 \%$ & $100.00 \%$ & $80.00 \%$ & $20.00 \%$ \\
\hline Grade 2 A & 81 & 0 & 81 & 66 & 15 & Grade 4 & $0.00 \%$ & $100.00 \%$ & $87.50 \%$ & $12.50 \%$ \\
\hline Grade 2 B & 0 & 0 & 0 & 0 & 0 & Grade 5 & $0.00 \%$ & $100.00 \%$ & $90.91 \%$ & $9.09 \%$ \\
\hline Grade 3 A & 30 & 0 & 30 & 24 & 6 & Grade 6 & $0.00 \%$ & $100.00 \%$ & $90.00 \%$ & $10.00 \%$ \\
\hline Grade 3 B & 0 & 0 & 0 & 0 & 0 & & & & & \\
\hline Grade 4 & 24 & 0 & 24 & 21 & 3 & Remarks: & & & & \\
\hline Grade 5 & 11 & 0 & 11 & 10 & 1 & & & & & \\
\hline Grade 6 & 10 & 0 & 10 & 9 & 1 & & & & & \\
\hline School Total & 267 & 0 & 267 & 223 & 44 & School \%age & $0.00 \%$ & $100.00 \%$ & $83.52 \%$ & $16.48 \%$ \\
\hline
\end{tabular}


Sub-Centre: Bannu

\begin{tabular}{|c|c|c|c|c|c|c|c|c|c|c|}
\hline \multirow[b]{2}{*}{$\begin{array}{l}\text { Grades / } \\
\text { Sections }\end{array}$} & \multicolumn{5}{|c|}{-Number of Students- } & \multirow[b]{2}{*}{ Grades } & \multicolumn{4}{|c|}{-Percent of Students- } \\
\hline & Enrolled & Dropped & $\begin{array}{l}\text { Appeared } \\
\text { in Exam }\end{array}$ & $\begin{array}{r}\text { Passed in } \\
\text { Exams }\end{array}$ & $\begin{array}{l}\text { Failed in } \\
\text { Exams }\end{array}$ & & Dropped & $\begin{array}{l}\text { Appeared } \\
\text { in Exams }\end{array}$ & $\begin{array}{r}\text { Passed in } \\
\text { Exams }\end{array}$ & $\begin{array}{l}\text { Falled in } \\
\text { Exams }\end{array}$ \\
\hline Grade $1 \mathrm{~A}$ & 52 & 3 & 49 & 44 & 5 & Grade 1 & $6.19 \%$ & $93.81 \%$ & $92.31 \%$ & $7.69 \%$ \\
\hline Grade $1 \mathrm{~B}$ & 45 & 3 & 42 & 40 & 2 & Grade 2 & $11.94 \%$ & $88.06 \%$ & $94.92 \%$ & $5.08 \%$ \\
\hline Grade $1 \mathrm{C}$ & 0 & 0 & 0 & 0 & 0 & Grade 3 & $5.88 \%$ & $94.12 \%$ & $93.75 \%$ & $6.25 \%$ \\
\hline Grade 2 A & 67 & 8 & 59 & 56 & 3 & Grade 4 & $5.41 \%$ & $94.59 \%$ & $88.57 \%$ & $11.43 \%$ \\
\hline Grade 2 B & 0 & 0 & 0 & 0 & 0 & Grade 5 & $10.53 \%$ & $89.47 \%$ & $88.24 \%$ & $11.76 \%$ \\
\hline Grade 3 A & 0 & 0 & 0 & 0 & 0 & Grade 6 & $0.00 \%$ & $100.00 \%$ & $80.00 \%$ & $20.00 \%$ \\
\hline Grade 3 B & 34 & 2 & 32 & 30 & 2 & & & & & \\
\hline Grade 4 & 37 & 2 & 35 & 31 & 4 & Remarks: & & & & \\
\hline Grade 5 & 19 & 2 & 17 & 15 & 2 & & & & & \\
\hline Grade 6 & 20 & 0 & 20 & 16 & 4 & & & & & \\
\hline School Total & 274 & 20 & 254 & 232 & 22 & School \%age & $7.30 \%$ & $92.70 \%$ & $91.34 \%$ & $8.66 \%$ \\
\hline
\end{tabular}

\begin{tabular}{|c|c|c|c|c|c|c|c|c|c|c|c|}
\hline \multirow[b]{2}{*}{$\begin{array}{l}\text { Grades I } \\
\text { Sections }\end{array}$} & \multicolumn{6}{|c|}{-Number of Students- } & \multirow[b]{2}{*}{ Grades } & \multicolumn{4}{|c|}{-Percent of Students } \\
\hline & , & Enrolled & Dropped & $\begin{array}{l}\text { Appeared } \\
\text { in Exam }\end{array}$ & $\begin{array}{l}\text { Passed in } \\
\text { Exams }\end{array}$ & $\begin{array}{l}\text { Failed in } \\
\text { Exams }\end{array}$ & & Dropped & $\begin{array}{l}\text { Appeared } \\
\text { in Exams }\end{array}$ & $\begin{array}{r}\text { Passed in } \\
\text { Exams }\end{array}$ & $\begin{array}{l}\text { Failed in } \\
\text { Exams }\end{array}$ \\
\hline Grade $1 \mathrm{~A}$ & & 23 & 0 & 23 & 20 & 3 & Grade 1 & $0.00 \%$ & $100.00 \%$ & $86.96 \%$ & $13.04 \%$ \\
\hline Grade 1 B & & 0 & 0 & 0 & 0 & 0 & Grade 2 & $0.00 \%$ & $100.00 \%$ & $90.91 \%$ & $9.09 \%$ \\
\hline Grade $1 \mathrm{C}$ & & 0 & 0 & 0 & 0 & 0 & Grade 3 & $0.00 \%$ & $100.00 \%$ & $79.17 \%$ & $20.83 \%$ \\
\hline Grade 2 A & & 22 & 0 & 22 & 20 & 2 & Grade 4 & $0.00 \%$ & $100.00 \%$ & $95.24 \%$ & $4.76 \%$ \\
\hline Grade 2 B & & 0 & 0 & 0 & 0 & 0 & Grade 5 & \#Num! & \#Num! & \#Num! & \#Num! \\
\hline Grade 3 A & & 24. & 0 & 24 & 19 & 5 & Grade 6 & \#Num! & \#Num! & \#Num! & \#Num! \\
\hline Grade 3 B & & 0 & 0 & 0 & 0 & 0 & & & & & \\
\hline Grade 4 & & 21 & 0 & 21 & 20 & 1 & Remarks: & & & & \\
\hline Grade 5 & & 0 & 0 & 0 & 0 & 0 & & & & & \\
\hline Grade 6 & & 0 & 0 & 0 & 0 & 0 & & & & & \\
\hline School Total & & 90 & 0 & 90 & 79 & 11 & School \%age & $0.00 \%$ & $100.00 \%$ & $87.78 \%$ & $12.22 \%$ \\
\hline
\end{tabular}

\begin{tabular}{|c|c|c|c|c|c|c|c|c|c|c|}
\hline \multirow[b]{2}{*}{$\begin{array}{l}\text { Grades / } \\
\text { Sections }\end{array}$} & \multicolumn{5}{|c|}{ _Number of Students } & \multirow[b]{2}{*}{ Grades } & \multicolumn{4}{|c|}{-Percent of Students } \\
\hline & Enrolled & Dropped & $\begin{array}{l}\text { Appeared } \\
\text { in Exam }\end{array}$ & $\begin{array}{c}\text { Passed in } \\
\text { Exams }\end{array}$ & $\begin{array}{c}\text { Failed in } \\
\text { Exams }\end{array}$ & & Dropped & $\begin{array}{l}\text { Appeared } \\
\text { in Exams }\end{array}$ & $\begin{array}{r}\text { Passed in } \\
\text { Exams }\end{array}$ & $\begin{array}{r}\text { Failed in } \\
\text { Exams }\end{array}$ \\
\hline Grade $1 \mathrm{~A}$ & 67 & 0 & 67 & 51 & 16 & Grade 1 & $0.00 \%$ & $100.00 \%$ & $76.12 \%$ & $23.88 \%$ \\
\hline Grade 1 B & 0 & 0 & 0 & 0 & 0 & Grade 2 & $0.00 \%$ & $100.00 \%$ & $80.95 \%$ & $19.05 \%$ \\
\hline Grade $1 \mathrm{C}$ & 0 & 0 & 0 & 0 & 0 & Grade 3 & $0.00 \%$ & $100.00 \%$ & $67.31 \%$ & $32.69 \%$ \\
\hline Grade 2 A & 42 & 0 & 42 & 34 & 8 & Grade 4 & $0.00 \%$ & $100.00 \%$ & $86.21 \%$ & $13.79 \%$ \\
\hline Grade 2 B & 0 & 0 & 0 & 0 & 0 & Grade 5 & $0.00 \%$ & $100.00 \%$ & $86.96 \%$ & $13.04 \%$ \\
\hline Grade 3 A & 52 & 0 & 52 & 35 & 17 & Grade 6 & $0.00 \%$ & $100.00 \%$ & $75.00 \%$ & $25.00 \%$ \\
\hline Grade 3 B & 0 & 0 & 0 & 0 & 0 & & & & & \\
\hline Grade 4 & 29 & 0 & 29 & 25 & 4 & Remarks: & & & & \\
\hline Grade 5 & 23 & 0 & 23 & 20 & 3 & & & & & \\
\hline Grade 6 & 20 & 0 & 20 & 15 & 5 & & & & & \\
\hline School Total & 233 & 0 & 233 & 180 & 53 & School \%age & $0.00 \%$ & $100.00 \%$ & $77.25 \%$ & $22.75 \%$ \\
\hline
\end{tabular}

Note:

\#Num! = Enroliment in respective grade(s) is nill therefore calculation is not applicable
Page 23 of 91

Produced by DP/MIS Section on $31 \cdot$ Jul-97 
COPE Schools Based Annual Results for 1996-97 and Percentage Analysis

Sub-Centre: Bannu

School Code: BN/WNAPSB-070 School Title: ARPS 3 Zari Noor -Camp 2 Wana Head Teacher: Mohammad Salim

\begin{tabular}{|c|c|c|c|c|c|c|c|c|c|c|}
\hline \multirow{2}{*}{$\begin{array}{l}\text { Grades / } \\
\text { Sections }\end{array}$} & \multicolumn{5}{|c|}{ - Number of Students } & \multirow[b]{2}{*}{ Grades } & \multicolumn{4}{|c|}{ Percent of Students - } \\
\hline & Enrolled & Dropped & $\begin{array}{l}\text { Appeared } \\
\text { in Exam }\end{array}$ & $\begin{array}{l}\text { Passed in } \\
\text { Exams }\end{array}$ & $\begin{array}{l}\text { Failed in } \\
\text { Exams }\end{array}$ & & Dropped & $\begin{array}{l}\text { Appeared } \\
\text { in Exams }\end{array}$ & $\begin{array}{c}\text { Passed in } \\
\text { Exams }\end{array}$ & $\begin{array}{l}\text { Falled in } \\
\text { Exams }\end{array}$ \\
\hline Grade $1 \mathrm{~A}$ & 42 & 9 & 33 & 25 & 8 & Grade 1 & $21.43 \%$ & $78.57 \%$ & $75.76 \%$ & $24.24 \%$ \\
\hline Grade 1 B & 0 & 0 & 0 & 0 & 0 & Grade 2 & $16.67 \%$ & $83.33 \%$ & $55.00 \%$ & $45.00 \%$ \\
\hline Grade $1 \mathrm{C}$ & 0 & 0 & 0 & 0 & 0 & Grade 3 & $11.11 \%$ & $88.89 \%$ & $68.75 \%$ & $31.25 \%$ \\
\hline Grade 2 A & 24 & 4 & 20 & 11 & 9 & Grade 4 & $25.00 \%$ & $75.00 \%$ & $91.67 \%$ & $8.33 \%$ \\
\hline Grade 2 B & 0 & 0 & 0 & 0 & 0 & Grade 5 & $40.00 \%$ & $60.00 \%$ & $100.00 \%$ & $0.00 \%$ \\
\hline Grade 3 A & 18 & 2 & 16 & 11 & 5 & Grade 6 & \#Num! & \#Num! & \#Num! & \#Num! \\
\hline Grade 3 B & 0 & 0 & 0 & 0 & 0 & & & & & \\
\hline Grade 4 & 16 & 4 & 12 & 11 & 1 & Remarks: & & & & \\
\hline Grade 5 & 10 & 4 & 6 & 6 & 0 & & & & & \\
\hline Grade 6 & 0 & 0 & 0 & 0 & 0 & & & & & \\
\hline School Total & 110 & 23 & 87 & 64 & 23 & School \%age & $20.91 \%$ & $79.09 \%$ & $73.56 \%$ & $26.44 \%$ \\
\hline
\end{tabular}

\begin{tabular}{|c|c|c|c|c|c|c|c|c|c|c|}
\hline Sub-Centre Total & 5,959 & 438 & 5,521 & 4,156 & 1,365 & Sub-Centre \%age & $7.35 \%$ & $92.65 \%$ & $75.28 \%$ & $24.72 \%$ \\
\hline
\end{tabular}


Sub-Centre: Female

\begin{tabular}{|c|c|c|c|c|c|c|c|c|c|c|}
\hline \multirow[b]{2}{*}{$\begin{array}{l}\text { Grades / } \\
\text { Sections }\end{array}$} & \multicolumn{5}{|c|}{ - Number of Students- } & \multirow[b]{2}{*}{ Grades } & \multicolumn{4}{|c|}{ Percent of Students- } \\
\hline & Enrolled & Dropped & $\begin{array}{l}\text { Appeared } \\
\text { in Exam }\end{array}$ & $\begin{array}{c}\text { Passed in } \\
\text { Exams }\end{array}$ & $\begin{array}{l}\text { Failed in } \\
\text { Exams }\end{array}$ & & Dropped & $\begin{array}{l}\text { Appeared } \\
\text { in Exams }\end{array}$ & $\begin{array}{l}\text { Passed in } \\
\text { Exams }\end{array}$ & $\begin{array}{l}\text { Faled in } \\
\text { Exams }\end{array}$ \\
\hline Grade $1 \mathrm{~A}$ & 59 & 11 & 48 & 24 & 24 & Grade 1 & $20.62 \%$ & $79.37 \%$ & $52.76 \%$ & $47.24 \%$ \\
\hline Grade 1 B & 52 & 22 & 30 & 22 & 8 & Grade 2 & $0.00 \%$ & $100.00 \%$ & $46.28 \%$ & $53.72 \%$ \\
\hline Grade $1 \mathrm{C}$ & 49 & 0 & 49 & 21 & 28 & Grade 3 & $1.52 \%$ & $98.48 \%$ & $73.85 \%$ & $26.15 \%$ \\
\hline Grade $2 \mathrm{~A}$ & 94 & 0 & 94 & 57 & 37 & Grade 4 & $0.00 \%$ & $100.00 \%$ & $51.16 \%$ & $48.84 \%$ \\
\hline Grade 2 B & 94 & 0 & 94 & 30 & 64 & Grade 5 & \#Num! & \#Num! & \#Num! & \#Num! \\
\hline Grade $3 \mathrm{~A}$ & 66 & 1 & 65 & 48 & 17 & Grade 6 & \#Num! & \#Num! & \#Num! & \#Num! \\
\hline Grade 3 B & 0 & 0 & 0 & 0 & 0 & & & & & \\
\hline Grade 4 & 43 & 0 & 43 & 22 & 21 & Remarks: & & & & \\
\hline Grade 5 & 0 & 0 & 0 & 0 & 0 & & & & & \\
\hline Grade 6 & 0 & 0 & 0 & 0 & 0 & & & & & \\
\hline School Total & 457 & 34 & 423 & 224 & 199 & School \%age & $7.44 \%$ & $92.56 \%$ & $52.96 \%$ & $47.04 \%$ \\
\hline
\end{tabular}

\begin{tabular}{|c|c|c|c|c|c|c|c|c|c|c|}
\hline \multirow[b]{2}{*}{$\begin{array}{l}\text { Grades I } \\
\text { Sections }\end{array}$} & \multicolumn{5}{|c|}{ - Number of Students- } & \multirow[b]{2}{*}{ Grades } & \multicolumn{4}{|c|}{-Percent of Students- } \\
\hline & ' Enrolled & Dropped & $\begin{array}{c}\text { Appeared } \\
\text { in Exam }\end{array}$ & $\begin{array}{l}\text { Passed in } \\
\text { Exams }\end{array}$ & $\begin{array}{l}\text { Failed in } \\
\text { Exams }\end{array}$ & & Dropped & $\begin{array}{c}\text { Appeared } \\
\text { in Exams }\end{array}$ & $\begin{array}{l}\text { Passed in } \\
\text { Exams }\end{array}$ & $\begin{array}{l}\text { Faled in } \\
\text { Exams }\end{array}$ \\
\hline Grade $1 \mathrm{~A}$ & 113 & 0 & 113 & 93 & 20 & Grade 1 & $0.00 \%$ & $100.00 \%$ & $82.30 \%$ & $17.70 \%$ \\
\hline Grade 1 B & 0 & 0 & 0 & 0 & 0 & Grade 2 & $1.89 \%$ & $98.11 \%$ & $92.31 \%$ & $7.69 \%$ \\
\hline Grade $1 \mathrm{C}$ & 0 & 0 & 0 & 0 & 0 & Grade 3 & $0.00 \%$ & $100.00 \%$ & $70.37 \%$ & $29.63 \%$ \\
\hline Grade $2 \mathrm{~A}$ & 53 & 1 & 52 & 48 & 4 & Grade 4 & $0.00 \%$ & $100.00 \%$ & $57.14 \%$ & $42.86 \%$ \\
\hline Grade 2 B & 0 & 0 & 0 & 0 & 0 & Grade 5 & $0.00 \%$ & $100.00 \%$ & $75.00 \%$ & $25.00 \%$ \\
\hline Grade $3 \mathrm{~A}$ & $27^{*}$ & 0 & 27 & 19 & 8 & Grade 6 & \#Num! & \#Num! & \#Num! & \#Num! \\
\hline Grade 3 B & 0 & 0 & 0 & 0 & 0 & & & & & \\
\hline Grade 4 & 14 & 0 & 14 & 8 & 6 & Remarks: & & & & \\
\hline Grade 5 & 8 & 0 & 8 & 6 & 2 & & & & & \\
\hline Grade 6 & 0 & 0 & 0 & 0 & 0 & & & & & \\
\hline School Total & 215 & 1 & 214 & 174 & 40 & School \%age & $0.47 \%$ & $99.53 \%$ & $81.31 \%$ & $18.69 \%$ \\
\hline
\end{tabular}

\begin{tabular}{|c|c|c|c|c|c|c|c|c|c|c|}
\hline \multicolumn{6}{|c|}{ School Code: FE/BJR/PSG-073 School Title: ARPS Umari } & \multicolumn{5}{|c|}{ Head Teacher: Salma } \\
\hline \multirow[b]{2}{*}{$\begin{array}{l}\text { Grades / } \\
\text { Sections }\end{array}$} & \multicolumn{5}{|c|}{ Number of Students } & \multirow[b]{2}{*}{ Grades } & \multicolumn{4}{|c|}{-Percent of Students } \\
\hline & Enrolled & Dropped & $\begin{array}{l}\text { Appeared } \\
\text { in Exam }\end{array}$ & $\begin{array}{l}\text { Passed in } \\
\text { Exams }\end{array}$ & $\begin{array}{l}\text { Failed in } \\
\text { Exams }\end{array}$ & & Dropped & $\begin{array}{l}\text { Appeared } \\
\text { in Exams }\end{array}$ & $\begin{array}{l}\text { Passed in } \\
\text { Exams }\end{array}$ & $\begin{array}{l}\text { Falled in } \\
\text { Exams }\end{array}$ \\
\hline Grade $1 \mathrm{~A}$ & 101 & 0 & 101 & 34 & 67 & Grade 1 & $0.00 \%$ & $100.00 \%$ & $33.66 \%$ & $66.34 \%$ \\
\hline Grade 1 B & 0 & 0 & 0 & 0 & 0 & Gra'de 2 & $0.00 \%$ & $100.00 \%$ & $33.33 \%$ & $66.67 \%$ \\
\hline Grade $1 \mathrm{C}$ & 0 & 0 & 0 & 0 & 0 & Grade 3 & $0.00 \%$ & $100.00 \%$ & $63.89 \%$ & $36.11 \%$ \\
\hline Grade 2 A & 75 & 0 & 75 & 25 & 50 & Grade 4 & $0.00 \%$ & $100.00 \%$ & $93.75 \%$ & $6.25 \%$ \\
\hline Grade 2 B & 0 & 0 & 0 & 0 & 0 & Grade 5 & $9.09 \%$ & $90.91 \%$ & $80.00 \%$ & $20.00 \%$ \\
\hline Grade $3 \mathrm{~A}$ & 36 & 0 & 36 & 23 & 13 & Grade 6 & \#Num! & \#Num! & \#Num! & \#Num! \\
\hline Grade 3 B & 0 & 0 & 0 & 0 & 0 & & & & & \\
\hline Grade 4 & 16 & 0 & 16 & 15 & 1 & Remarks: & & & & \\
\hline Grade 5 & 11 & 1 & 10 & 8 & 2 & & & & & \\
\hline Grade 6 & 0 & 0 & 0 & 0 & 0 & & & & & \\
\hline School Total & 239 & 1 & 238 & 105 & 133 & School \%age & $0.42 \%$ & $99.58 \%$ & $44.12 \%$ & $55.88 \%$ \\
\hline
\end{tabular}


Sub-Centre: Female

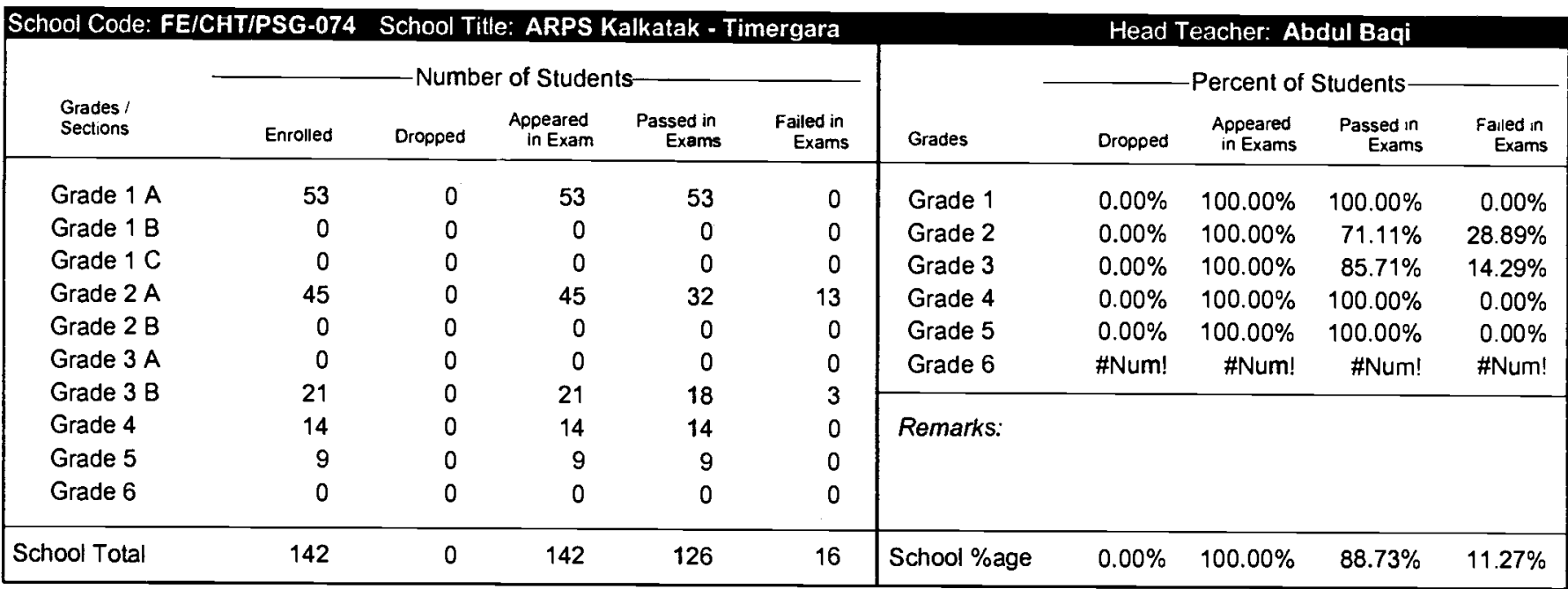

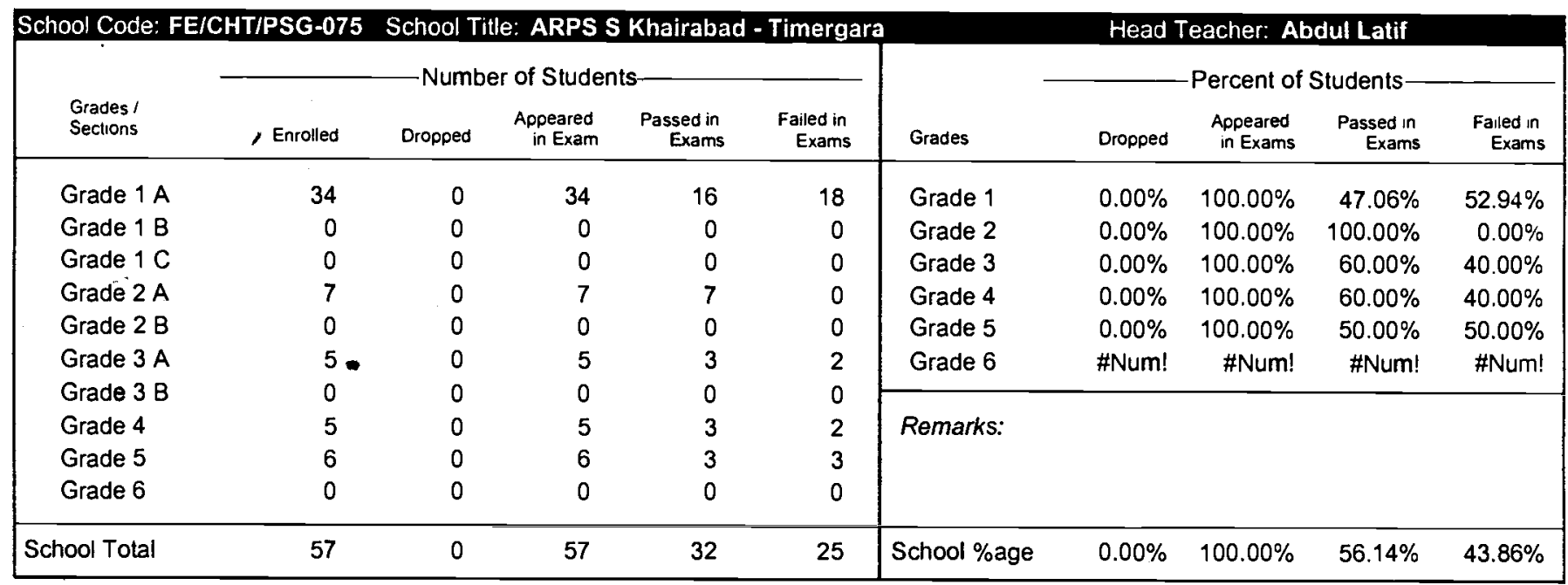

\begin{tabular}{|c|c|c|c|c|c|c|c|c|c|c|}
\hline \multirow[b]{2}{*}{$\begin{array}{l}\text { Grades / } \\
\text { Sections }\end{array}$} & \multicolumn{5}{|c|}{ School Title: ARPS Berarai } & \multicolumn{5}{|c|}{ Head Teacher: Zaitoon } \\
\hline & Enrolled & Dropped & $\begin{array}{l}\text { Appeared } \\
\text { in Exam }\end{array}$ & $\begin{array}{l}\text { Passed in } \\
\text { Exams }\end{array}$ & $\begin{array}{l}\text { Failed in } \\
\text { Exams }\end{array}$ & Grades & Dropped & $\begin{array}{l}\text { Appeared } \\
\text { in Exams }\end{array}$ & $\begin{array}{l}\text { Passed in } \\
\text { Exams }\end{array}$ & $\begin{array}{l}\text { Falled in } \\
\text { Exams }\end{array}$ \\
\hline Grade $1 \mathrm{~A}$ & 39 & 0 & 39 & 30 & 9 & Grade 1 & $0.00 \%$ & $100.00 \%$ & $76.92 \%$ & $23.08 \%$ \\
\hline Grade 1 B & 0 & 0 & 0 & 0 & 0 & Grade 2 & $0.00 \%$ & $100.00 \%$ & $67.65 \%$ & $32.35 \%$ \\
\hline Grade $1 \mathrm{C}$ & 0 & 0 & 0 & 0 & 0 & Grade 3 & $0.00 \%$ & $100.00 \%$ & $83.33 \%$ & $16.67 \%$ \\
\hline Grade 2 A & 34 & 0 & 34 & 23 & 11 & Grade 4 & $0.00 \%$ & $100.00 \%$ & $100.00 \%$ & $0.00 \%$ \\
\hline Grade 2 B & 0 & 0 & 0 & 0 & 0 & Grade 5 & $0.00 \%$ & $100.00 \%$ & $100.00 \%$ & $0.00 \%$ \\
\hline Grade 3 A & 12 & 0 & 12 & 10 & 2 & Grade 6 & $0.00 \%$ & $100.00 \%$ & $100.00 \%$ & $0.00 \%$ \\
\hline Grade 3 B & 0 & 0 & 0 & 0 & 0 & & & & & \\
\hline Grade 4 & 11 & 0 & 11 & 11 & 0 & Remarks: & & & & \\
\hline Grade 5 & 8 & 0 & 8 & 8 & 0 & & & & & \\
\hline Grade 6 & 13 & 0 & 13 & 13 & 0 & & & & & \\
\hline School Total & 117 & 0 & 117 & 95 & 22 & School \%age & $0.00 \%$ & $100.00 \%$ & $81.20 \%$ & $18.80 \%$ \\
\hline
\end{tabular}


Sub-Centre: Female

\begin{tabular}{|c|c|c|c|c|c|c|c|c|c|c|}
\hline \multirow[b]{2}{*}{$\begin{array}{l}\text { Grades I } \\
\text { Sections }\end{array}$} & \multicolumn{5}{|c|}{ School Title: ARPS 1 Chakdada - Bajour } & \multicolumn{5}{|c|}{ Head Teacher: Riffat } \\
\hline & Enrolled & Dropped & $\begin{array}{c}\text { Appeared } \\
\text { in Exam }\end{array}$ & $\begin{array}{l}\text { Passed in } \\
\text { Exams }\end{array}$ & $\begin{array}{c}\text { Failed in } \\
\text { Exams }\end{array}$ & Grades & Dropped & $\begin{array}{l}\text { Appeared } \\
\text { in Exams }\end{array}$ & $\begin{array}{l}\text { Passed in } \\
\text { Exams }\end{array}$ & $\begin{array}{l}\text { Failed in } \\
\text { Exams }\end{array}$ \\
\hline Grade $1 \mathrm{~A}$ & 42 & 0 & 42 & 39 & 3 & Grade 1 & $0.00 \%$ & $100.00 \%$ & $92.86 \%$ & $7.14 \%$ \\
\hline Grade 1 B & 0 & 0 & 0 & 0 & 0 & Grade 2 & $0.00 \%$ & $100.00 \%$ & $79.17 \%$ & $20.83 \%$ \\
\hline Grade $1 \mathrm{C}$ & 0 & 0 & 0 & 0 & 0 & Grade 3 & $0.00 \%$ & $100.00 \%$ & $64.29 \%$ & $35.71 \%$ \\
\hline Grade 2 A & 24 & 0 & 24 & 19 & 5 & Grade 4 & $0.00 \%$ & $100.00 \%$ & $76.19 \%$ & $23.81 \%$ \\
\hline Grade 2 B & 0 & 0 & 0 & 0 & 0 & Grade 5 & $0.00 \%$ & $100.00 \%$ & $100.00 \%$ & $0.00 \%$ \\
\hline Grade 3 A & 28 & 0 & 28 & 18 & 10 & Grade 6 & \# Num! & \#um! & \#Num! & \#Uum! \\
\hline Grade 3 B & 0 & 0 & 0 & 0 & 0 & & & & & \\
\hline Grade 4 & 21 & 0 & 21 & 16 & 5 & Remarks: & & & & \\
\hline Grade 5 & 12 & 0 & 12 & 12 & 0 & & & & & \\
\hline Grade 6 & 0 & 0 & 0 & 0 & 0 & & & & & \\
\hline School Total & 127 & 0 & 127 & 104 & 23 & School \%age & $0.00 \%$ & $100.00 \%$ & $81.89 \%$ & $18.11 \%$ \\
\hline
\end{tabular}

\begin{tabular}{|c|c|c|c|c|c|c|c|c|c|c|}
\hline \multirow[b]{2}{*}{$\begin{array}{l}\text { Grades / } \\
\text { Sections }\end{array}$} & \multicolumn{5}{|c|}{ - Number of Students- } & \multirow[b]{2}{*}{ Grades } & \multicolumn{4}{|c|}{-Percent of Students } \\
\hline & , Enrolled & Dropped & $\begin{array}{l}\text { Appeared } \\
\text { in Exam }\end{array}$ & $\begin{array}{l}\text { Passed in } \\
\text { Exams }\end{array}$ & $\begin{array}{l}\text { Failed in } \\
\text { Exams }\end{array}$ & & Dropped & $\begin{array}{l}\text { Appeared } \\
\text { in Exams }\end{array}$ & $\begin{array}{l}\text { Passed in } \\
\text { Exams }\end{array}$ & $\begin{array}{c}\text { Falled in } \\
\text { Exams }\end{array}$ \\
\hline Grade $1 \mathrm{~A}$ & 71 & 0 & 71 & 43 & 28 & Grade 1 & $0.00 \%$ & $100.00 \%$ & $60.56 \%$ & $39.44 \%$ \\
\hline Grade 18 & 0 & 0 & 0 & 0 & 0 & Grade 2 & $0.00 \%$ & $100.00 \%$ & $51.16 \%$ & $48.84 \%$ \\
\hline Grade $1 \mathrm{C}$ & 0 & 0 & 0 & 0 & 0 & Grade 3 & $0.00 \%$ & $100.00 \%$ & $55.00 \%$ & $45.00 \%$ \\
\hline Grade 2 A & 43 & 0 & 43 & 22 & 21 & Grade 4 & $0.00 \%$ & $100.00 \%$ & $61.11 \%$ & $38.89 \%$ \\
\hline Grade 2 B & 0 & 0 & 0 & 0 & 0 & Grade 5 & $0.00 \%$ & $100.00 \%$ & $100.00 \%$ & $0.00 \%$ \\
\hline Grade $3 \mathrm{~A}$ & 20 & 0 & 20 & 11 & 9 & Grade 6 & \#Num! & \#Num! & \#Num! & \#Num \\
\hline Grade 3 B & 0 & 0 & 0 & 0 & 0 & & & & & \\
\hline Grade 4 & 18 & 0 & 18 & 11 & 7 & Remarks: & & & & \\
\hline Grade 5 & 13 & 0 & 13 & 13 & 0 & & & & & \\
\hline Grade 6 & 0 & 0 & 0 & 0 & 0 & & & & & \\
\hline School Total & 165 & 0 & 165 & 100 & 65 & School \%age & $0.00 \%$ & $100.00 \%$ & $60.61 \%$ & $39.39 \%$ \\
\hline
\end{tabular}

\begin{tabular}{|c|c|c|c|c|c|c|c|c|c|c|}
\hline \multirow[b]{2}{*}{$\begin{array}{l}\text { Grades / } \\
\text { Sections }\end{array}$} & \multicolumn{5}{|c|}{ - Number of Students- } & \multirow[b]{2}{*}{ Grades } & \multicolumn{4}{|c|}{-Percent of Students } \\
\hline & Enrolled & Dropped & $\begin{array}{l}\text { Appeared } \\
\text { in Exam }\end{array}$ & $\begin{array}{l}\text { Passed in } \\
\text { Exams }\end{array}$ & $\begin{array}{l}\text { Failed in } \\
\text { Exams }\end{array}$ & & Dropped & $\begin{array}{l}\text { Appeared } \\
\text { in Exams }\end{array}$ & $\begin{array}{c}\text { Passed in } \\
\text { Exams }\end{array}$ & $\begin{array}{l}\text { Falled in } \\
\text { Exams }\end{array}$ \\
\hline Grade $1 \mathrm{~A}$ & 100 & 0 & 100 & 90 & 10 & Grade 1 & $0.00 \%$ & $100.00 \%$ & $87.50 \%$ & $12.50 \%$ \\
\hline Grade $1 \mathrm{~B}$ & 100 & 0 & 100 & 85 & 15 & Grade 2 & $0.00 \%$ & $100.00 \%$ & $46.67 \%$ & $53.33 \%$ \\
\hline Grade $1 \mathrm{C}$ & 0 & 0 & 0 & 0 & 0 & Grade 3 & $0.00 \%$ & $100.00 \%$ & $65.52 \%$ & $34.48 \%$ \\
\hline Grade 2 A & 105 & 0 & 105 & 49 & 56 & Grade 4 & $0.00 \%$ & $100.00 \%$ & $80.00 \%$ & $20.00 \%$ \\
\hline Grade 2 B & 0 & 0 & 0 & 0 & 0 & Grade 5 & $0.00 \%$ & $100.00 \%$ & $100.00 \%$ & $0.00 \%$ \\
\hline Grade $3 \mathrm{~A}$ & 58 & 0 & 58 & 38 & 20 & Grade 6 & $0.00 \%$ & $100.00 \%$ & $100.00 \%$ & $0.00 \%$ \\
\hline Grade 3 B & 0 & 0 & 0 & 0 & 0 & & & & & \\
\hline Grade 4 & 15 & 0 & 15 & 12 & 3 & Remarks: & & & & \\
\hline Grade 5 & 5 & 0 & 5 & 5 & 0 & & & & & \\
\hline Grade 6 & 5 & 0 & 5 & 5 & 0 & & & & & \\
\hline School Total & 388 & 0 & 388 & 284 & 104 & School \%age & $0.00 \%$ & $100.00 \%$ & $73.20 \%$ & $26.80 \%$ \\
\hline
\end{tabular}


Sub-Centre: Female

\begin{tabular}{|c|c|c|c|c|c|c|c|c|c|c|}
\hline \multicolumn{6}{|c|}{ School Code: FE/DIR/PSG.080 } & \multicolumn{5}{|c|}{$\begin{array}{l}\text { Head Teacher: Zeba } \\
\text { Percent of Students }\end{array}$} \\
\hline $\begin{array}{l}\text { Grades I } \\
\text { Sections }\end{array}$ & Enrolled & Dropped & $\begin{array}{l}\text { Appeared } \\
\text { in Exam }\end{array}$ & $\begin{array}{l}\text { Passed in } \\
\text { Exams }\end{array}$ & $\begin{array}{l}\text { Failed in } \\
\text { Exams }\end{array}$ & Grades & Dropped & $\begin{array}{l}\text { Appeared } \\
\text { in Exams }\end{array}$ & $\begin{array}{r}\text { Passed in } \\
\text { Exams } \\
\end{array}$ & $\begin{array}{c}\text { Falled in } \\
\text { Exams }\end{array}$ \\
\hline Grade $1 \mathrm{~A}$ & 64 & 3 & 61 & 36 & 25 & Grade 1 & $4.69 \%$ & $95.31 \%$ & $59.02 \%$ & $40.98 \%$ \\
\hline Grade $1 \mathrm{~B}$ & 0 & 0 & 0 & 0 & 0 & Grade 2 & $0.00 \%$ & $100.00 \%$ & $45.45 \%$ & $54.55 \%$ \\
\hline Grade $1 \mathrm{C}$ & 0 & 0 & 0 & 0 & 0 & Grade 3 & $0.00 \%$ & $100.00 \%$ & $80.00 \%$ & $20.00 \%$ \\
\hline Grade $2 \mathrm{~A}$ & 44 & 0 & 44 & 20 & 24 & Grade 4 & $0.00 \%$ & $100.00 \%$ & $70.00 \%$ & $30.00 \%$ \\
\hline Grade 2 B & 0 & 0 & 0 & 0 & 0 & Grade 5 & $0.00 \%$ & $100.00 \%$ & $76.92 \%$ & $23.08 \%$ \\
\hline Grade $3 \mathrm{~A}$ & 20 & 0 & 20 & 16 & 4 & Grade 6 & \#Num! & \#Num! & \#Num! & \#Num! \\
\hline Grade 3 B & 0 & 0 & 0 & 0 & 0 & & & & & \\
\hline Grade 4 & 10 & 0 & 10 & 7 & 3 & Remarks: & & & & \\
\hline Grade 5 & 13 & 0 & 13 & 10 & 3 & & & & & \\
\hline Grade 6 & 0 & 0 & 0 & 0 & 0 & & & & & \\
\hline School Total & 151 & 3 & 148 & 89 & 59 & School \%age & $1.99 \%$ & $98.01 \%$ & $60.14 \%$ & $39.86 \%$ \\
\hline
\end{tabular}

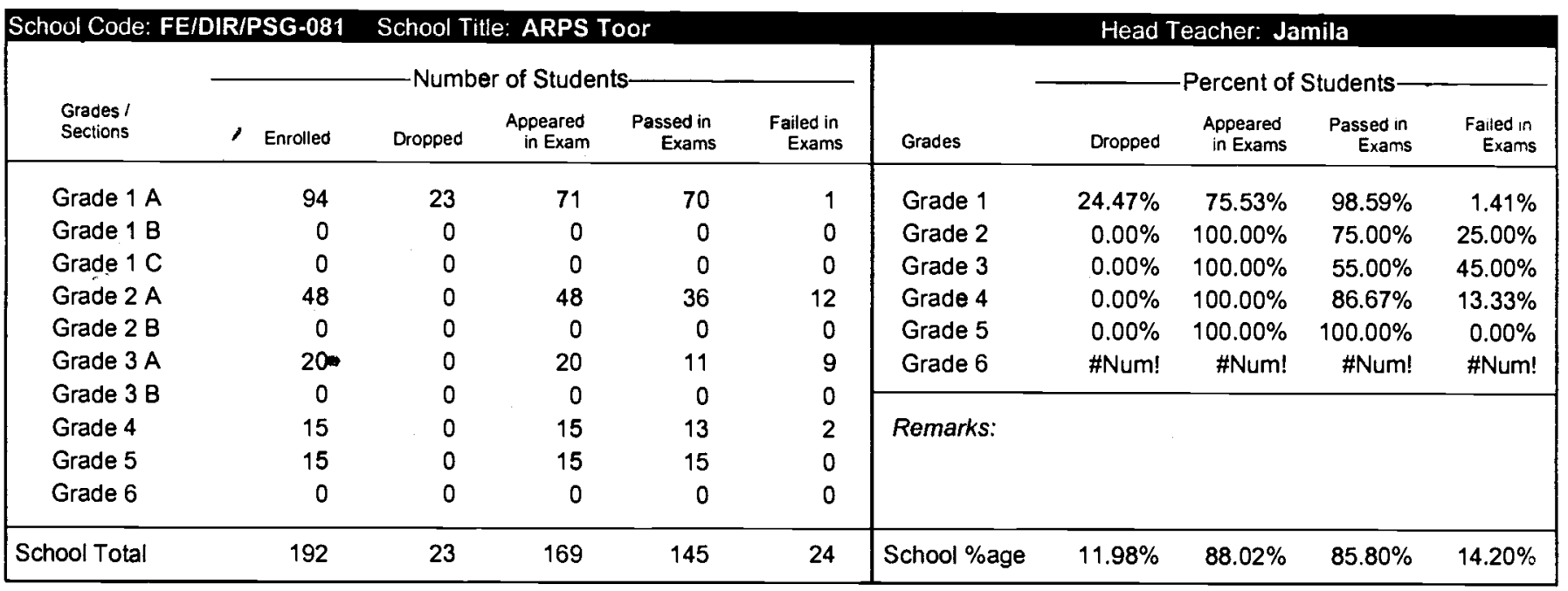

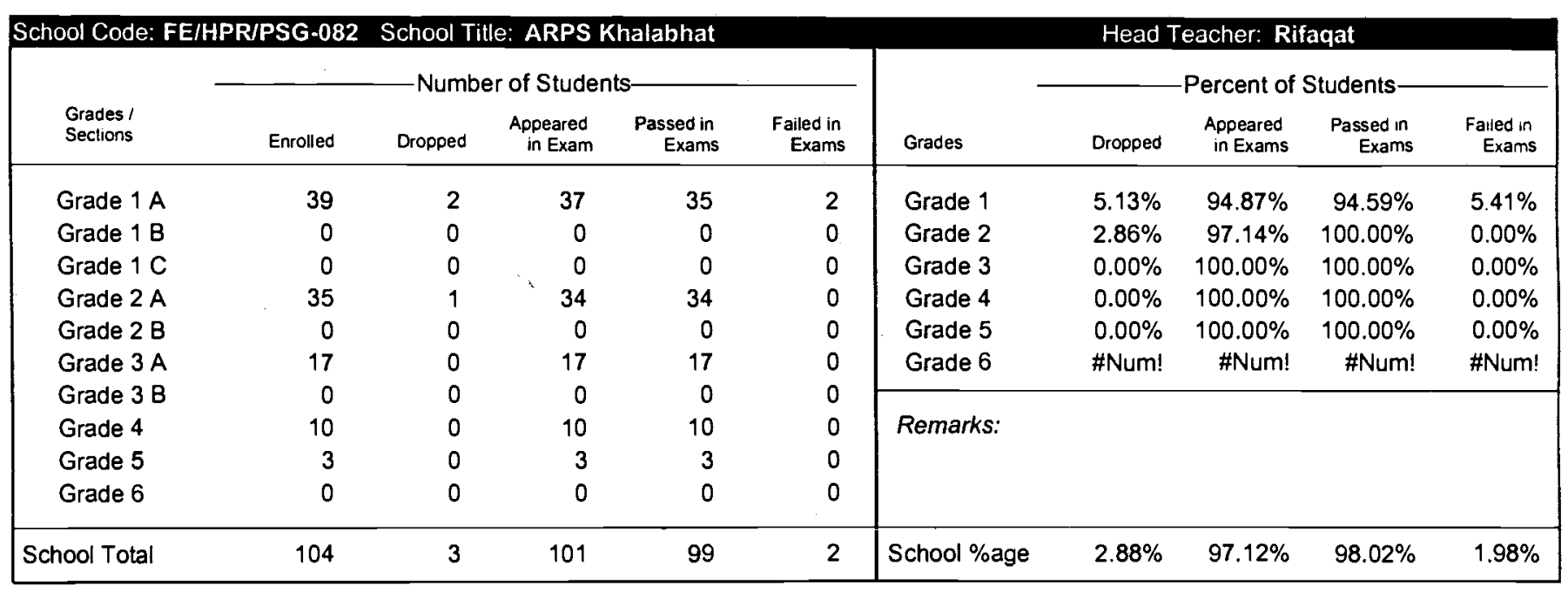


Sub-Centre: Female

\begin{tabular}{|c|c|c|c|c|c|c|c|c|c|c|}
\hline \multirow[b]{2}{*}{$\begin{array}{l}\text { Grades / } \\
\text { Sections }\end{array}$} & \multicolumn{5}{|c|}{ - Number of Students- } & \multirow[b]{2}{*}{ Grades } & \multicolumn{4}{|c|}{-Percent of Students } \\
\hline & Enrolled & Dropped & $\begin{array}{l}\text { Appeared } \\
\text { in Exam }\end{array}$ & $\begin{array}{l}\text { Passed in } \\
\text { Exams }\end{array}$ & $\begin{array}{l}\text { Failed in } \\
\text { Exams }\end{array}$ & & Dropped & $\begin{array}{c}\text { Appeared } \\
\text { in Exams }\end{array}$ & $\begin{array}{c}\text { Passed in } \\
\text { Exams }\end{array}$ & $\begin{array}{l}\text { Failed in } \\
\text { Exams }\end{array}$ \\
\hline Grade $1 \mathrm{~A}$ & 62 & 20 & 42 & 39 & 3 & Grade 1 & $32.26 \%$ & $67.74 \%$ & $92.86 \%$ & $7.14 \%$ \\
\hline Grade $1 \mathrm{~B}$ & 0 & 0 & 0 & 0 & 0 & Grade 2 & $20.00 \%$ & $80.00 \%$ & $85.00 \%$ & $15.00 \%$ \\
\hline Grade $1 \mathrm{C}$ & 0 & 0 & 0 & 0 & 0 & Grade 3 & $0.00 \%$ & $100.00 \%$ & $100.00 \%$ & $0.00 \%$ \\
\hline Grade 2 A & 25 & 5 & 20 & 17 & 3 & Grade 4 & $0.00 \%$ & $100.00 \%$ & $100.00 \%$ & $0.00 \%$ \\
\hline Grade 2 B & 0 & 0 & 0 & 0 & 0 & Grade 5 & $14.29 \%$ & $85.71 \%$ & $100.00 \%$ & $0.00 \%$ \\
\hline Grade $3 \mathrm{~A}$ & 6 & 0 & 6 & 6 & 0 & Grade 6 & \#Num! & $\#$ \#um! & \#Num! & \#Num! \\
\hline Grade 3 B & 0 & 0 & 0 & 0 & 0 & & & & & \\
\hline Grade 4 & 4 & 0 & 4 & 4 & 0 & Remarks: & & & & \\
\hline Grade 5 & 7 & 1 & 6 & 6 & 0 & & & & & \\
\hline Grade 6 & 0 & 0 & 0 & 0 & 0 & & & & & \\
\hline School Total & 104 & 26 & 78 & 72 & 6 & School \%age & $25.00 \%$ & $75.00 \%$ & $92.31 \%$ & $7.69 \%$ \\
\hline
\end{tabular}

\begin{tabular}{|c|c|c|c|c|c|c|c|c|c|c|c|}
\hline \multirow[b]{2}{*}{$\begin{array}{l}\text { Grades I } \\
\text { Sections }\end{array}$} & \multicolumn{6}{|c|}{ — Number of Students } & \multirow[b]{2}{*}{ Grades } & \multicolumn{4}{|c|}{-Percent of Students- } \\
\hline & , & Enrolled & Dropped & $\begin{array}{l}\text { Appeared } \\
\text { in Exam }\end{array}$ & $\begin{array}{l}\text { Passed in } \\
\text { Exams }\end{array}$ & $\begin{array}{l}\text { Failed in } \\
\text { Exams }\end{array}$ & & Dropped & $\begin{array}{l}\text { Appeared } \\
\text { in Exams }\end{array}$ & $\begin{array}{l}\text { Passed in } \\
\text { Exams }\end{array}$ & $\begin{array}{l}\text { Falled in } \\
\text { Exams }\end{array}$ \\
\hline Grade $1 \mathrm{~A}$ & & 88 & 6 & 82 & 64 & 18 & Grade 1 & $6.82 \%$ & $93.18 \%$ & $78.05 \%$ & $21.95 \%$ \\
\hline Grade $1 \mathrm{~B}$ & & 0 & 0 & 0 & 0 & 0 & Grade 2 & $0.00 \%$ & $100.00 \%$ & $86.76 \%$ & $13.24 \%$ \\
\hline Grade $1 \mathrm{C}$ & & 0 & 0 & 0 & 0 & 0 & Grade 3 & $0.00 \%$ & $100.00 \%$ & $92.00 \%$ & $8.00 \%$ \\
\hline Grade 2 A & & 68 & 0 & 68 & 59 & 9 & Grade 4 & $0.00 \%$ & $100.00 \%$ & $100.00 \%$ & $0.00 \%$ \\
\hline Grade 2 B & & 0 & 0 & 0 & 0 & 0 & Grade 5 & $0.00 \%$ & $100.00 \%$ & $100.00 \%$ & $0.00 \%$ \\
\hline Grade $3 \mathrm{~A}$ & & 26 & 0 & 25 & 23 & 2 & Grade 6 & \#Num! & \#Num! & \#Num! & \#Num! \\
\hline Grade 3 B & & 0 & 0 & 0 & 0 & 0 & & & & & \\
\hline Grade 4 & & 17 & 0 & 17 & 17 & 0 & Remarks: & & & & \\
\hline Grade 5 & & 12 & 0 & 12 & 12 & 0 & & & & & \\
\hline Grade 6 & & 0 & 0 & 0 & 0 & 0 & & & & & \\
\hline School Total & & 210 & 6 & 204 & 175 & 29 & School \%age & $2.86 \%$ & $97.14 \%$ & $85.78 \%$ & $14.22 \%$ \\
\hline
\end{tabular}

\begin{tabular}{|c|c|c|c|c|c|c|c|c|c|c|}
\hline \multirow[b]{2}{*}{$\begin{array}{l}\text { Grades I } \\
\text { Sections }\end{array}$} & \multicolumn{5}{|c|}{ - Number of Students- } & \multirow[b]{2}{*}{ Grades } & \multicolumn{4}{|c|}{-Percent of Students- } \\
\hline & Enrolled & Dropped & $\begin{array}{l}\text { Appeared } \\
\text { in Exam }\end{array}$ & $\begin{array}{l}\text { Passed in } \\
\text { Exams }\end{array}$ & $\begin{array}{l}\text { Failed in } \\
\text { Exams }\end{array}$ & & Dropped & $\begin{array}{c}\text { Appeared } \\
\text { in Exams }\end{array}$ & $\begin{array}{l}\text { Passed in } \\
\text { Exams }\end{array}$ & $\begin{array}{c}\text { Failed in } \\
\text { Exams }\end{array}$ \\
\hline Grade $1 \mathrm{~A}$ & 31 & 0 & 31 & 22 & 9 & Grade 1 & $0.00 \%$ & $100.00 \%$ & $70.97 \%$ & $29.03 \%$ \\
\hline Grade $1 \mathrm{~B}$ & 0 & 0 & 0 & 0 & 0 & Grade 2 & $0.00 \%$ & $100.00 \%$ & $75.00 \%$ & $25.00 \%$ \\
\hline Grade $1 \mathrm{C}$ & 0 & 0 & 0 & 0 & 0 & Grade 3 & $0.00 \%$ & $100.00 \%$ & $65.38 \%$ & $34.62 \%$ \\
\hline Grade 2 A & 28 & 0 & 28 & 21 & 7 & Grade 4 & $0.00 \%$ & $100.00 \%$ & $66.67 \%$ & $33.33 \%$ \\
\hline Grade 2 B & 0 & 0 & 0 & 0 & 0 & Grade 5 & $0.00 \%$ & $100.00 \%$ & $45.45 \%$ & $54.55 \%$ \\
\hline Grade 3 A & 26 & 0 & 26 & 17 & 9 & Grade 6 & \#Num! & \#Num! & \#Num! & \#Num! \\
\hline Grade 3 B & 0 & 0 & 0 & 0 & 0 & & & & & \\
\hline Grade 4 & 9 & 0 & 9 & 6 & 3 & Remarks: & & & & \\
\hline Grade 5 & 11 & 0 & 11 & 5 & 6 & & & & & \\
\hline Grade 6 & 0 & 0 & 0 & 0 & 0 & & & & & \\
\hline School Total & 105 & 0 & 105 & 71 & 34 & School \%age & $0.00 \%$ & $100.00 \%$ & $67.62 \%$ & $32.38 \%$ \\
\hline
\end{tabular}


Sub-Centre: Female

\begin{tabular}{|c|c|c|c|c|c|c|c|c|c|c|}
\hline \multicolumn{6}{|c|}{ School Code: FE/KHT/PSG-086 School Title: ARPGS Thall } & \multicolumn{5}{|c|}{ Head Teacher: Rizwana } \\
\hline \multirow[b]{2}{*}{$\begin{array}{l}\text { Grades I } \\
\text { Sections }\end{array}$} & & -Numb & of Stude & & & \multirow[b]{2}{*}{ Grades } & \multicolumn{4}{|c|}{-Percent of Students- } \\
\hline & Enrolled & Dropped & $\begin{array}{l}\text { Appeared } \\
\text { in Exam }\end{array}$ & $\begin{array}{l}\text { Passed in } \\
\text { Exams }\end{array}$ & $\begin{array}{c}\text { Failed in } \\
\text { Exams }\end{array}$ & & Oropped & $\begin{array}{l}\text { Appeared } \\
\text { in Exams }\end{array}$ & $\begin{array}{c}\text { Passed in } \\
\text { Exams }\end{array}$ & $\begin{array}{c}\text { Failed in } \\
\text { Exams }\end{array}$ \\
\hline Grade $1 \mathrm{~A}$ & 80 & 10 & 70 & 50 & 20 & Grade 1 & $12.50 \%$ & $87.50 \%$ & $71.43 \%$ & $28.57 \%$ \\
\hline Grade 1 B & 0 & 0 & 0 & 0 & 0 & Grade 2 & $6.67 \%$ & $93.33 \%$ & $83.33 \%$ & $16.67 \%$ \\
\hline Grade $1 \mathrm{C}$ & 0 & 0 & 0 & 0 & 0 & Grade 3 & $10.53 \%$ & $89.47 \%$ & $70.59 \%$ & $29.41 \%$ \\
\hline Grade 2 A & 45 & 3 & 42 & 35 & 7 & Grade 4 & $0.00 \%$ & $100.00 \%$ & $60.00 \%$ & $40.00 \%$ \\
\hline Grade 2 B & 0 & 0 & 0 & 0 & 0 & Grade 5 & $0.00 \%$ & $100.00 \%$ & $100.00 \%$ & $0.00 \%$ \\
\hline Grade 3 A & 19 & 2 & 17 & 12 & 5 & Grade 6 & \#Num! & \#Num! & \#Num! & \#Num! \\
\hline Grade 3 B & 0 & 0 & 0 & 0 & 0 & & & & & \\
\hline Grade 4 & 5 & 0 & 5 & 3 & 2 & Remarks: & & & & \\
\hline Grade 5 & 2 & 0 & 2 & 2 & 0 & & & & & \\
\hline Grade 6 & 0 & 0 & 0 & 0 & 0 & & & & & \\
\hline School Total & 151 & 15 & 136 & 102 & 34 & School \%age & $9.93 \%$ & $90.07 \%$ & $75.00 \%$ & $25.00 \%$ \\
\hline
\end{tabular}

\begin{tabular}{|c|c|c|c|c|c|c|c|c|c|c|}
\hline \multicolumn{11}{|c|}{ School Code: FE/KRM/PSG-087 School Title: ARPS S - Baggan - Kurrum } \\
\hline \multirow[b]{2}{*}{$\begin{array}{l}\text { Grades I } \\
\text { Sections }\end{array}$} & \multicolumn{5}{|c|}{-Number of Students- } & \multirow[b]{2}{*}{ Grades } & \multicolumn{4}{|c|}{ Percent of Students- } \\
\hline & Enrolled & Dropped & $\begin{array}{c}\text { Appeared } \\
\text { in Exam }\end{array}$ & $\begin{array}{c}\text { Passed in } \\
\text { Exams }\end{array}$ & $\begin{array}{l}\text { Failed in } \\
\text { Exams }\end{array}$ & & Dropped & $\begin{array}{l}\text { Appeared } \\
\text { in Exams }\end{array}$ & $\begin{array}{l}\text { Passed in } \\
\text { Exams }\end{array}$ & $\begin{array}{l}\text { Falled in } \\
\text { Exams }\end{array}$ \\
\hline Grade $1 \mathrm{~A}$ & 61 & 6 & 55 & 42 & 13 & Grade 1 & $9.84 \%$ & $90.16 \%$ & $76.36 \%$ & $23.64 \%$ \\
\hline Grade $1 \mathrm{~B}$ & 0 & 0 & 0 & 0 & 0 & Grade 2 & $8.33 \%$ & $91.67 \%$ & $75.76 \%$ & $24.24 \%$ \\
\hline Grade $1 \mathrm{C}$ & 0 & 0 & 0 & 0 & 0 & Grade 3 & $0.00 \%$ & $100.00 \%$ & $70.59 \%$ & $29.41 \%$ \\
\hline Grade 2 A & 36 & 3 & 33 & 25 & 8 & Grade 4 & $0.00 \%$ & $100.00 \%$ & $100.00 \%$ & $0.00 \%$ \\
\hline Grade 2 B & 0 & 0 & 0 & 0 & 0 & Grade 5 & $0.00 \%$ & $100.00 \%$ & $87.50 \%$ & $12.50 \%$ \\
\hline Grade $3 \mathrm{~A}$ & 17 & 0 & 17 & 12 & 5 & Grade 6 & \#Num! & \#Num! & \#Num! & \#Num! \\
\hline Grade 3 B & 0 & 0 & 0 & 0 & 0 & & & & & \\
\hline Grade 4 & 8 & 0 & 8 & 8 & 0 & Remarks: & & & & \\
\hline Grade 5 & 8 & 0 & 8 & 7 & 1 & & & & & \\
\hline Grade 6 & 0 & 0 & 0 & 0 & 0 & & & & & \\
\hline School Total & 130 & 9 & 121 & 94 & 27 & School \%age & $6.92 \%$ & $93.08 \%$ & $77.69 \%$ & $22.31 \%$ \\
\hline
\end{tabular}

\begin{tabular}{|c|c|c|c|c|c|c|c|c|c|c|}
\hline \multicolumn{7}{|c|}{ School Code: FE/KRMIPSG-088 School Tille: ARPS Boshera } & \multicolumn{4}{|c|}{ Head Teacher: Munera Jana } \\
\hline \multirow[b]{2}{*}{$\begin{array}{l}\text { Grades I } \\
\text { Sections }\end{array}$} & \multicolumn{5}{|c|}{-Number of Students- } & \multirow[b]{2}{*}{ Grades } & \multicolumn{4}{|c|}{ Percent of Students- } \\
\hline & Enrolled & Oropped & $\begin{array}{l}\text { Appeared } \\
\text { in Exam }\end{array}$ & $\begin{array}{c}\text { Passed in } \\
\text { Exams }\end{array}$ & $\begin{array}{l}\text { Failed in } \\
\text { Exams }\end{array}$ & & Dropped & $\begin{array}{c}\text { Appeared } \\
\text { in Exams }\end{array}$ & $\begin{array}{r}\text { Passed in } \\
\text { Exams }\end{array}$ & $\begin{array}{l}\text { Faled in } \\
\text { Exams }\end{array}$ \\
\hline Grade $1 \mathrm{~A}$ & 38 & 0 & 38 & 26 & 12 & Grade 1 & $0.00 \%$ & $100.00 \%$ & $68.42 \%$ & $31.58 \%$ \\
\hline Grade 1 B & 0 & 0 & 0 & 0 & 0 & Grade 2 & $0.00 \%$ & $100.00 \%$ & $55.56 \%$ & $44.44 \%$ \\
\hline Grade $1 \mathrm{C}$ & 0 & 0 & 0 & 0 & 0 & Grade 3 & $0.00 \%$ & $100.00 \%$ & $44.44 \%$ & $55.56 \%$ \\
\hline Grade $2 \mathrm{~A}$ & 36 & 0 & 36 & 20 & 16 & Grade 4 & $0.00 \%$ & $100.00 \%$ & $0.00 \%$ & $100.00 \%$ \\
\hline Grade 2 B & 0 & 0 & 0 & 0 & 0 & Grade 5 & $0.00 \%$ & $100.00 \%$ & $0.00 \%$ & $100.00 \%$ \\
\hline Grade $3 \mathrm{~A}$ & 18 & 0 & 18 & 8 & 10 & Grade 6 & $0.00 \%$ & $100.00 \%$ & $80.00 \%$ & $20.00 \%$ \\
\hline Grade 3 B & 0 & 0 & 0 & 0 & 0 & & & & & \\
\hline Grade 4 & 5 & 0 & 5 & 0 & 5 & Remarks: & & & & \\
\hline Grade 5 & 5 & 0 & 5 & 0 & 5 & & & & & \\
\hline Grade 6 & 5 & 0 & 5 & 4 & 1 & & & & & \\
\hline School Total & 107 & 0 & 107 & 58 & 49 & School \%age & $0.00 \%$ & $100.00 \%$ & $54.21 \%$ & $45.79 \%$ \\
\hline
\end{tabular}


Sub-Centre: Female

\begin{tabular}{|c|c|c|c|c|c|c|c|c|c|c|}
\hline & & -Numb & of Stude & & & & & Percent of & Students- & \\
\hline $\begin{array}{l}\text { Grades I } \\
\text { Sections }\end{array}$ & Enrolled & Dropped & $\begin{array}{c}\text { Appeared } \\
\text { in Exam }\end{array}$ & $\begin{array}{l}\text { Passed in } \\
\text { Exams }\end{array}$ & $\begin{array}{l}\text { Failed in } \\
\text { Exams }\end{array}$ & Grades & Dropped & $\begin{array}{l}\text { Appeared } \\
\text { in Exams }\end{array}$ & $\begin{array}{l}\text { Passed in } \\
\text { Exams }\end{array}$ & $\begin{array}{c}\text { Falec in } \\
\text { Exams }\end{array}$ \\
\hline Grade $1 \mathrm{~A}$ & 95 & 30 & 65 & 39 & 26 & Grade 1 & $31.58 \%$ & $68.42 \%$ & $60.00 \%$ & $40.00 \%$ \\
\hline Grade 1 B & 0 & 0 & 0 & 0 & 0 & Grade 2 & $9.80 \%$ & $90.20 \%$ & $54.35 \%$ & $45.65 \%$ \\
\hline Grade $1 \mathrm{C}$ & 0 & 0 & 0 & 0 & 0 & Grade 3 & $0.00 \%$ & $100.00 \%$ & $100.00 \%$ & $0.00 \%$ \\
\hline Grade 2 A & 51 & 5 & 46 & 25 & 21 & Grade 4 & $0.00 \%$ & $100.00 \%$ & $95.24 \%$ & $4.76 \%$ \\
\hline Grade 2 B & 0 & 0 & 0 & 0 & 0 & Grade 5 & $0.00 \%$ & $100.00 \%$ & $100.00 \%$ & $0.00 \%$ \\
\hline Grade 3 A & 20 & 0 & 20 & 20 & 0 & Grade 6 & $10.00 \%$ & $90.00 \%$ & $100.00 \%$ & $0.00 \%$ \\
\hline Grade 3 B & 0 & 0 & 0 & 0 & 0 & & & & & \\
\hline Grade 4 & 21 & 0 & 21 & 20 & 1 & Remarks: & & & & \\
\hline Grade 5 & 16 & 0 & 16 & 16 & 0 & & & & & \\
\hline Grade 6 & 10 & 1 & 9 & 9 & 0 & & & & & \\
\hline School Total & 213 & 36 & 177 & 129 & 48 & School \%age & $16.90 \%$ & $83.10 \%$ & $72.88 \%$ & $27.12 \%$ \\
\hline
\end{tabular}

\begin{tabular}{|c|c|c|c|c|c|c|c|c|c|c|}
\hline \multirow{2}{*}{$\begin{array}{l}. \\
\text { Grades I } \\
\text { Sections }\end{array}$} & \multicolumn{5}{|c|}{ - Number of Students- } & \multirow[b]{2}{*}{ Grades } & \multicolumn{4}{|c|}{-Percent of Students } \\
\hline & Enrolled & Dropped & $\begin{array}{c}\text { Appeared } \\
\text { in Exam }\end{array}$ & $\begin{array}{r}\text { Passed in } \\
\text { Exams }\end{array}$ & $\begin{array}{l}\text { Failed in } \\
\text { Exams }\end{array}$ & & Dropped & $\begin{array}{l}\text { Appeared } \\
\text { in Exams }\end{array}$ & $\begin{array}{l}\text { Passed in } \\
\text { Exams }\end{array}$ & $\begin{array}{l}\text { Falles in } \\
\text { Exams }\end{array}$ \\
\hline Grade $1 \mathrm{~A}$ & 60 & 0 & 60 & 31 & 29 & Grade 1 & $0.00 \%$ & $100.00 \%$ & $51.67 \%$ & $48.33 \%$ \\
\hline Grade $1 \mathrm{~B}$ & 0 & 0 & 0 & 0 & 0 & Grade 2 & $10.00 \%$ & $90.00 \%$ & $63.89 \%$ & $36.11 \%$ \\
\hline Grade $1 \mathrm{C}$ & 0 & 0 & 0 & 0 & 0 & Grade 3 & $0.00 \%$ & $100.00 \%$ & $78.57 \%$ & $21.43 \%$ \\
\hline Grade $2 \mathrm{~A}$ & 40 & 4 & 36 & 23 & 13 & Grade 4 & $0.00 \%$ & $100.00 \%$ & $90.00 \%$ & $10.00 \%$ \\
\hline Grade 28 & 0 & 0 & 0 & 0 & 0 & Grade 5 & $0.00 \%$ & $100.00 \%$ & $100.00 \%$ & $0.00 \%$ \\
\hline Grade $3 \mathrm{~A}$ & 14 & 0 & 14 & 11 & 3 & Grade 6 & $28.57 \%$ & $71.43 \%$ & $100.00 \%$ & $0.00 \%$ \\
\hline Grade 3 B & 0. & 0 & 0 & 0 & 0 & & & & & \\
\hline Grade 4 & 10 & 0 & 10 & 9 & 1 & Remarks: & & & & \\
\hline Grade 5 & 4 & 0 & 4 & 4 & 0 & & & & & \\
\hline Grade 6 & 7 & 2 & 5 & 5 & 0 & & & & & \\
\hline School Total & 135 & 6 & 129 & 83 & 46 & School \%age & $4.44 \%$ & $95.56 \%$ & $64.34 \%$ & $35.66 \%$ \\
\hline
\end{tabular}

\begin{tabular}{|c|c|c|c|c|c|c|c|c|c|c|}
\hline \multirow[b]{2}{*}{$\begin{array}{l}\text { Grades I } \\
\text { Sections }\end{array}$} & \multicolumn{5}{|c|}{-Number of Students- } & \multirow[b]{2}{*}{ Grades } & \multicolumn{4}{|c|}{-Percent of Students } \\
\hline & Enrolled & Dropped & $\begin{array}{l}\text { Appeared } \\
\text { in Exam }\end{array}$ & $\begin{array}{c}\text { Passed in } \\
\text { Exams }\end{array}$ & $\begin{array}{l}\text { Failed in } \\
\text { Exams }\end{array}$ & & Dropped & $\begin{array}{l}\text { Appeared } \\
\text { in Exams }\end{array}$ & $\begin{array}{c}\text { Passed in } \\
\text { Exams }\end{array}$ & $\begin{array}{l}\text { Falled in } \\
\text { Exams }\end{array}$ \\
\hline Grade $1 \mathrm{~A}$ & 70 & 2 & 68 & 42 & 26 & Grade 1 & $2.86 \%$ & $97.14 \%$ & $61.76 \%$ & $38.24 \%$ \\
\hline Grade 18 & 0 & 0 & 0 & 0 & 0 & Grade 2 & $5.00 \%$ & $95.00 \%$ & $71.05 \%$ & $28.95 \%$ \\
\hline Grade $1 \mathrm{C}$ & 0 & 0 & 0 & 0 & 0 & Grade 3 & $18.75 \%$ & $81.25 \%$ & $73.08 \%$ & $26.92 \%$ \\
\hline Grade $2 \mathrm{~A}$ & 40 & 2 & 38 & 27 & 11 & Grade 4 & $20.00 \%$ & $80.00 \%$ & $81.25 \%$ & $18.75 \%$ \\
\hline Grade 2 B & 0 & 0 & 0 & 0 & 0 & Grade 5 & $16.67 \%$ & $83.33 \%$ & $80.00 \%$ & $20.00 \%$ \\
\hline Grade $3 \mathrm{~A}$ & 32 & 6 & 26 & 19 & 7 & Grade 6 & $50.00 \%$ & $50.00 \%$ & $100.00 \%$ & $0.00 \%$ \\
\hline Grade 38 & 0 & 0 & 0 & 0 & 0 & & & & & \\
\hline Grade 4 & 20 & 4 & 16 & 13 & 3 & Remarks: & & & & \\
\hline Grade 5 & 18 & 3 & 15 & 12 & 3 & & & & & \\
\hline Grade 6 & 8 & 4 & 4 & 4 & 0 & & & & & \\
\hline School Total & 188 & 21 & 167 & 117 & 50 & School \%age & $11.17 \%$ & $88.83 \%$ & $70.06 \%$ & $29.94 \%$ \\
\hline
\end{tabular}


Sub-Centre: Female

\begin{tabular}{|c|c|c|c|c|c|c|c|c|c|c|}
\hline \multirow[b]{2}{*}{$\begin{array}{l}\text { Grades I } \\
\text { Sections }\end{array}$} & \multicolumn{5}{|c|}{ Number of Students } & \multirow[b]{2}{*}{ Grades } & \multicolumn{4}{|c|}{-Percent of Students- } \\
\hline & Enrolled & Dropped & $\begin{array}{l}\text { Appeared } \\
\text { in Exam }\end{array}$ & $\begin{array}{l}\text { Passed in } \\
\text { Exams }\end{array}$ & $\begin{array}{l}\text { Failed in } \\
\text { Exams }\end{array}$ & & Dropped & $\begin{array}{l}\text { Appeared } \\
\text { in Exams }\end{array}$ & $\begin{array}{l}\text { Passed in } \\
\text { Exams }\end{array}$ & $\begin{array}{l}\text { Failed in } \\
\text { Exams }\end{array}$ \\
\hline Grade $1 \mathrm{~A}$ & 95 & 30 & 65 & 39 & 26 & Grade 1 & $31.58 \%$ & $68.42 \%$ & $60.00 \%$ & $40.00 \%$ \\
\hline Grade $1 \mathrm{~B}$ & 0 & 0 & 0 & 0 & 0 & Grade 2 & $9.80 \%$ & $90.20 \%$ & $54.35 \%$ & $45.65 \%$ \\
\hline Grade $1 \mathrm{C}$ & 0 & 0 & 0 & 0 & 0 & Grade 3 & $0.00 \%$ & $100.00 \%$ & $100.00 \%$ & $0.00 \%$ \\
\hline Grade 2 A & 51 & 5 & 46 & 25 & 21 & Grade 4 & $0.00 \%$ & $100.00 \%$ & $95.24 \%$ & $4.76 \%$ \\
\hline Grade 2 B & 0 & 0 & 0 & 0 & 0 & Grade 5 & $0.00 \%$ & $100.00 \%$ & $100.00 \%$ & $0.00 \%$ \\
\hline Grade $3 \mathrm{~A}$ & 20 & 0 & 20 & 20 & 0 & Grade 6 & $10.00 \%$ & $90.00 \%$ & $100.00 \%$ & $0.00 \%$ \\
\hline Grade 3 B & 0 & 0 & 0 & 0 & 0 & & & & & \\
\hline Grade 4 & 21 & 0 & 21 & 20 & 1 & Remarks: & & & & \\
\hline Grade 5 & 16 & 0 & 16 & 16 & 0 & & & & & \\
\hline Grade 6 & 10 & 1 & 9 & 9 & 0 & & & & & \\
\hline School Total & 213 & 36 & 177 & 129 & 48 & School \%age & $16.90 \%$ & $83.10 \%$ & $72.88 \%$ & $27.12 \%$ \\
\hline
\end{tabular}

\begin{tabular}{|c|c|c|c|c|c|c|c|c|c|c|}
\hline \multirow[b]{2}{*}{$\begin{array}{l}\text { Grades / } \\
\text { Sections }\end{array}$} & \multicolumn{5}{|c|}{ - Number of Students } & \multirow[b]{2}{*}{ Grades } & \multicolumn{4}{|c|}{-Percent of Students } \\
\hline & , Enrolled & Dropped & $\begin{array}{l}\text { Appeared } \\
\text { in Exam } \\
\end{array}$ & $\begin{array}{c}\text { Passed in } \\
\text { Exams }\end{array}$ & $\begin{array}{l}\text { Failed in } \\
\text { Exams }\end{array}$ & & Dropped & $\begin{array}{c}\text { Appeared } \\
\text { in Exams }\end{array}$ & $\begin{array}{l}\text { Passed in } \\
\text { Exams }\end{array}$ & $\begin{array}{l}\text { Falled in } \\
\text { Exams }\end{array}$ \\
\hline Grade $1 \mathrm{~A}$ & 60 & 0 & 60 & 31 & 29 & Grade 1 & $0.00 \%$ & $100.00 \%$ & $51.67 \%$ & $48.33 \%$ \\
\hline Grade 1 B & 0 & 0 & 0 & 0 & 0 & Grade 2 & $10.00 \%$ & $90.00 \%$ & $63.89 \%$ & $36.11 \%$ \\
\hline Grade $1 \mathrm{C}$ & 0 & 0 & 0 & 0 & 0 & Grade 3 & $0.00 \%$ & $100.00 \%$ & $78.57 \%$ & $21.43 \%$ \\
\hline Grade $2 \mathrm{~A}$ & 40 & 4 & 36 & 23 & 13 & Grade 4 & $0.00 \%$ & $100.00 \%$ & $90.00 \%$ & $10.00 \%$ \\
\hline Grade 2 B & 0 & 0 & 0 & 0 & 0 & Grade 5 & $0.00 \%$ & $100.00 \%$ & $100.00 \%$ & $0.00 \%$ \\
\hline Grade $3 \mathrm{~A}$ & 14 & 0 & 14 & 11 & 3 & Grade 6 & $28.57 \%$ & $71.43 \%$ & $100.00 \%$ & $0.00 \%$ \\
\hline $\begin{array}{l}\text { Grade } 3 \text { B } \\
\text { Grade } 4\end{array}$ & 0 & 0 & 0 & 0 & 0 & & & & & \\
\hline $\begin{array}{l}\text { Grade } 4 \\
\text { Grade } 5\end{array}$ & $\begin{array}{r}10 \\
4\end{array}$ & $\begin{array}{l}0 \\
0\end{array}$ & $\begin{array}{r}10 \\
4\end{array}$ & $\begin{array}{l}9 \\
4\end{array}$ & $\begin{array}{l}1 \\
0\end{array}$ & Remarks: & & & & \\
\hline Grade 6 & 7 & 2 & 5 & 5 & 0 & & & & & \\
\hline School Total & 135 & 6 & 129 & 83 & 46 & School \%age & $4.44 \%$ & $95.56 \%$ & $64.34 \%$ & $35.66 \%$ \\
\hline
\end{tabular}

\begin{tabular}{|c|c|c|c|c|c|c|c|c|c|c|}
\hline \multicolumn{6}{|c|}{ School Code: FE/MRD/PSG-091 School Title: ARPS Gandaf } & \multicolumn{5}{|c|}{ Head Teacher: Mumlikat } \\
\hline \multirow[b]{2}{*}{$\begin{array}{l}\text { Grades I } \\
\text { Sections }\end{array}$} & & -Numb & of Studer & & & \multirow[b]{2}{*}{ Grades } & \multicolumn{4}{|c|}{-Percent of Students- } \\
\hline & Enrolled & Dropped & $\begin{array}{l}\text { Appeared } \\
\text { in Exam }\end{array}$ & $\begin{array}{l}\text { Passed in } \\
\text { Exams }\end{array}$ & $\begin{array}{l}\text { Failed in } \\
\text { Exams }\end{array}$ & & Dropped & $\begin{array}{l}\text { Appeared } \\
\text { in Exams }\end{array}$ & $\begin{array}{l}\text { Passed in } \\
\text { Exams }\end{array}$ & $\begin{array}{l}\text { Failed in } \\
\text { Exams }\end{array}$ \\
\hline Grade $1 \mathrm{~A}$ & 70 & 2 & 68 & 42 & 26 & Grade 1 & $2.86 \%$ & $97.14 \%$ & $61.76 \%$ & $38.24 \%$ \\
\hline Grade 1 B & 0 & 0 & 0 & 0 & 0 & Grade 2 & $5.00 \%$ & $95.00 \%$ & $71.05 \%$ & $28.95 \%$ \\
\hline Grade $1 \mathrm{C}$ & 0 & 0 & 0 & 0 & 0 & Grade 3 & $18.75 \%$ & $81.25 \%$ & $73.08 \%$ & $26.92 \%$ \\
\hline Grade 2 A & 40 & 2 & $\begin{array}{l}38 \\
+\end{array}$ & 27 & 11 & Grade 4 & $20.00 \%$ & $80.00 \%$ & $81.25 \%$ & $18.75 \%$ \\
\hline Grade 2 B & 0 & 0 & 0 & 0 & 0 & Grade 5 & $16.67 \%$ & $83.33 \%$ & $80.00 \%$ & $20.00 \%$ \\
\hline Grade $3 \mathrm{~A}$ & 32 & 6 & 26 & 19 & 7 & Grade 6 & $50.00 \%$ & $50.00 \%$ & $100.00 \%$ & $0.00 \%$ \\
\hline Grade 3 B & 0 & 0 & 0 & 0 & 0 & & & & & \\
\hline Grade 4 & 20 & 4 & 16 & 13 & 3 & Remarks: & & & & \\
\hline Grade 5 & 18 & 3 & 15 & 12 & 3 & & & & & \\
\hline Grade 6 & 8 & 4 & 4 & 4 & 0 & & & & & \\
\hline School Total & 188 & 21 & 167 & 117 & 50 & School \%age & $11.17 \%$ & $88.83 \%$ & $70.06 \%$ & $29.94 \%$ \\
\hline
\end{tabular}


Sub-Centre: Female

\begin{tabular}{|c|c|c|c|c|c|c|c|c|c|c|}
\hline \multirow[b]{2}{*}{$\begin{array}{l}\text { Grades / } \\
\text { Sections }\end{array}$} & \multicolumn{5}{|c|}{ - Number of Students } & \multirow[b]{2}{*}{ Grades } & \multicolumn{4}{|c|}{ Percent of Students - } \\
\hline & Enrolled & Dropped & $\begin{array}{l}\text { Appeared } \\
\text { in Exam }\end{array}$ & $\begin{array}{l}\text { Passed in } \\
\text { Exams }\end{array}$ & $\begin{array}{l}\text { Failed in } \\
\text { Exams }\end{array}$ & & Dropped & $\begin{array}{l}\text { Appeared } \\
\text { in Exams }\end{array}$ & $\begin{array}{l}\text { Passed in } \\
\text { Exams }\end{array}$ & $\begin{array}{l}\text { Failed in } \\
\text { Exams }\end{array}$ \\
\hline Grade $1 \mathrm{~A}$ & 54 & 0 & 54 & 49 & 5 & Grade 1 & $0.00 \%$ & $100.00 \%$ & $94.12 \%$ & $5.88 \%$ \\
\hline Grade 1 B & 31 & 0 & 31 & 31 & 0 & Grade 2 & $0.00 \%$ & $100.00 \%$ & $79.03 \%$ & $20.97 \%$ \\
\hline Grade $1 \mathrm{C}$ & 0 & 0 & 0 & 0 & 0 & Grade 3 & $0.00 \%$ & $100.00 \%$ & $83.33 \%$ & $16.67 \%$ \\
\hline Grade 2 A & 62 & 0 & 62 & 49 & 13 & Grade 4 & $0.00 \%$ & $100.00 \%$ & $83.33 \%$ & $16.67 \%$ \\
\hline Grade 2 B & 0 & 0 & 0 & 0 & 0 & Grade 5 & $0.00 \%$ & $100.00 \%$ & $85.71 \%$ & $14.29 \%$ \\
\hline Grade 3 A & 24 & 0 & 24 & 20 & 4 & Grade 6 & \#Num! & \#Num! & \#Num! & \#Num! \\
\hline Grade 3 B & 0 & 0 & 0 & 0 & 0 & & & & & \\
\hline Grade 4 & 18 & 0 & 18 & 15 & 3 & Remarks: & & & & \\
\hline Grade 5 & 14 & 0 & 14 & 12 & 2 & & & & & \\
\hline Grade 6 & 0 & 0 & 0 & 0 & 0 & & & & & \\
\hline School Total & 203 & 0 & 203 & 176 & 27 & School \%age & $0.00 \%$ & $100.00 \%$ & $86.70 \%$ & $13.30 \%$ \\
\hline
\end{tabular}

\begin{tabular}{|c|c|c|c|c|c|c|c|c|c|c|c|}
\hline \multirow[b]{2}{*}{$\begin{array}{l}\text { Grades I } \\
\text { Sections }\end{array}$} & \multicolumn{6}{|c|}{-Number of Students } & \multirow[b]{2}{*}{ Grades } & \multicolumn{4}{|c|}{ Percent of Students- } \\
\hline & ' & Enrolled & Dropped & $\begin{array}{l}\text { Appeared } \\
\text { in Exam }\end{array}$ & $\begin{array}{c}\text { Passed in } \\
\text { Exams }\end{array}$ & $\begin{array}{l}\text { Failed in } \\
\text { Exams }\end{array}$ & & Dropped & $\begin{array}{l}\text { Appeared } \\
\text { in Exams }\end{array}$ & $\begin{array}{l}\text { Passed in } \\
\text { Exams }\end{array}$ & $\begin{array}{l}\text { Falled in } \\
\text { Exams }\end{array}$ \\
\hline Grade $1 \mathrm{~A}$ & & 56 & 15 & 41 & 21 & 20 & Grade 1 & $26.79 \%$ & $73.21 \%$ & $51.22 \%$ & $48.78 \%$ \\
\hline Grade $1 \mathrm{~B}$ & & 0 & 0 & 0 & 0 & 0 & Grade 2 & $30.00 \%$ & $70.00 \%$ & $78.57 \%$ & $21.43 \%$ \\
\hline Grade $1 \mathrm{C}$ & & 0 & 0 & 0 & 0 & 0 & Grade 3 & $14.29 \%$ & $85.71 \%$ & $100.00 \%$ & $0.00 \%$ \\
\hline Grade 2 A & & 20 & 6 & 14 & 11 & 3 & Grade 4 & $14.29 \%$ & $85.71 \%$ & $100.00 \%$ & $0.00 \%$ \\
\hline Grade 2 B & & 0 & 0 & 0 & 0 & 0 & Grade 5 & $50.00 \%$ & $50.00 \%$ & $100.00 \%$ & $0.00 \%$ \\
\hline Grade $3 \mathrm{~A}$ & & $z$ & 1 & 6 & 6 & 0 & Grade 6 & \#Num! & \#Num! & \#Num! & \#Num! \\
\hline Grade 3 B & & 0 & 0 & 0 & 0 & 0 & & & & & \\
\hline Grade 4 & & 7 & 1 & 6 & 6 & 0 & Remarks: & & & & \\
\hline Grade 5 & & 8 & 4 & 4 & 4 & 0 & & & & & \\
\hline Grade 6 & & 0 & 0 & 0 & 0 & 0 & & & & & \\
\hline School Total & & 98 & 27 & 71 & 48 & 23 & School \%age & $27.55 \%$ & $72.45 \%$ & $67.61 \%$ & $32.39 \%$ \\
\hline
\end{tabular}

\begin{tabular}{|c|c|c|c|c|c|c|c|c|c|c|}
\hline \multirow[b]{2}{*}{$\begin{array}{l}\text { Grades I } \\
\text { Sections }\end{array}$} & \multicolumn{5}{|c|}{ - Number of Students- } & \multirow[b]{2}{*}{ Grades } & \multicolumn{4}{|c|}{-Percent of Students } \\
\hline & Enrolled & Dropped & $\begin{array}{l}\text { Appeared } \\
\text { in Exam }\end{array}$ & $\begin{array}{c}\text { Passed in } \\
\text { Exams }\end{array}$ & $\begin{array}{c}\text { Failed in } \\
\text { Exams }\end{array}$ & & Dropped & $\begin{array}{c}\text { Appeared } \\
\text { in Exams }\end{array}$ & $\begin{array}{c}\text { Passed in } \\
\text { Exams }\end{array}$ & $\begin{array}{c}\text { Falled in } \\
\text { Exams }\end{array}$ \\
\hline Grade $1 \mathrm{~A}$ & 48 & 11 & 37 & 26 & 11 & Grade 1 & $15.69 \%$ & $84.31 \%$ & $83.72 \%$ & $16.28 \%$ \\
\hline Grade $1 \mathrm{~B}$ & 50 & 8 & 42 & 32 & 10 & Grade 2 & $13.41 \%$ & $86.59 \%$ & $70.42 \%$ & $29.58 \%$ \\
\hline Grade $1 \mathrm{C}$ & 55 & 5 & 50 & 50 & 0 & Grade 3 & $0.00 \%$ & $100.00 \%$ & $53.85 \%$ & $46.15 \%$ \\
\hline Grade $2 \mathrm{~A}$ & 51 & 5 & 46 & 29 & 17 & Grade 4 & $3.33 \%$ & $96.67 \%$ & $44.83 \%$ & $55.17 \%$ \\
\hline Grade 2 B & 31 & 6 & 25 & 21 & 4 & Grade 5 & $0.00 \%$ & $100.00 \%$ & $90.91 \%$ & $9.09 \%$ \\
\hline Grade $3 \mathrm{~A}$ & 39 & 0 & 39 & 21 & 18 & Grade 6 & \#um! & \#Num! & \#Num! & \#Num! \\
\hline Grade 3 B & 0 & 0 & 0 & 0 & 0 & & & & & \\
\hline Grade 4 & 30 & 1 & 29 & 13 & 16 & Remarks: & & & & \\
\hline Grade 5 & 11 & 0 & 11 & 10 & 1 & & & & & \\
\hline Grade 6 & 0 & 0 & 0 & 0 & 0 & & & & & \\
\hline School Total & 315 & 36 & 279 & 202 & 77 & School \%age & $11.43 \%$ & $88.57 \%$ & $72.40 \%$ & $27.60 \%$ \\
\hline
\end{tabular}


Sub-Centre: Female

\begin{tabular}{|c|c|c|c|c|c|c|c|c|c|c|}
\hline \multirow[b]{2}{*}{$\begin{array}{l}\text { Grades / } \\
\text { Sections }\end{array}$} & \multicolumn{5}{|c|}{-Number of Students- } & \multirow[b]{2}{*}{ Grades } & \multicolumn{4}{|c|}{ Percent of Students- } \\
\hline & Enrolled & Dropped & $\begin{array}{l}\text { Appeared } \\
\text { in Exam }\end{array}$ & $\begin{array}{c}\text { Passed in } \\
\text { Exams }\end{array}$ & $\begin{array}{l}\text { Failed in } \\
\text { Exams }\end{array}$ & & Dropped & $\begin{array}{l}\text { Appeared } \\
\text { in Exams }\end{array}$ & $\begin{array}{c}\text { Passed in } \\
\text { Exams }\end{array}$ & $\begin{array}{l}\text { Falled in } \\
\text { Exams }\end{array}$ \\
\hline Grade $1 \mathrm{~A}$ & 42 & 2 & 40 & 28 & 12 & Grade 1 & $4.76 \%$ & $95.24 \%$ & $70.00 \%$ & $30.00 \%$ \\
\hline Grade 1 B & 0 & 0 & 0 & 0 & 0 & Grade 2 & $5.56 \%$ & $94.44 \%$ & $58.82 \%$ & $41.18 \%$ \\
\hline Grade $1 \mathrm{C}$ & 0 & 0 & 0 & 0 & 0 & Grade 3 & $0.00 \%$ & $100.00 \%$ & $43.75 \%$ & $56.25 \%$ \\
\hline Grade 2 A & 18 & 1 & 17 & 10 & 7 & Grade 4 & $0.00 \%$ & $100.00 \%$ & $100.00 \%$ & $0.00 \%$ \\
\hline Grade 2 B & 0 & 0 & 0 & 0 & 0 & Grade 5 & $0.00 \%$ & $100.00 \%$ & $100.00 \%$ & $0.00 \%$ \\
\hline Grade $3 \mathrm{~A}$ & 16 & 0 & 16 & 7 & 9 & Grade 6 & \#Num! & \#Num! & \#Num! & \#Num! \\
\hline Grade 3 B & 0 & 0 & 0 & 0 & 0 & & & & & \\
\hline Grade 4 & 4 & 0 & 4 & 4 & 0 & Remarks: & & & & \\
\hline Grade 5 & 3 & 0 & 3 & 3 & 0 & & & & & \\
\hline Grade 6 & 0 & 0 & 0 & 0 & 0 & & & & & \\
\hline School Total & 83 & 3 & 80 & 52 & 28 & School \%age & $3.61 \%$ & $96.39 \%$ & $65.00 \%$ & $35.00 \%$ \\
\hline
\end{tabular}

\begin{tabular}{|c|c|c|c|c|c|c|c|c|c|c|c|}
\hline \multirow[b]{2}{*}{$\begin{array}{l}\text { Grades I } \\
\text { Sections }\end{array}$} & \multicolumn{6}{|c|}{ - Number of Students } & \multirow[b]{2}{*}{ Grades } & \multicolumn{4}{|c|}{-Percent of Students- } \\
\hline & $\prime$ & Enrolied & Dropped & $\begin{array}{c}\text { Appeared } \\
\text { in Exam }\end{array}$ & $\begin{array}{l}\text { Passed in } \\
\text { Exams }\end{array}$ & $\begin{array}{l}\text { Failed in } \\
\text { Exams }\end{array}$ & & Dropped & $\begin{array}{l}\text { Appeared } \\
\text { in Exams }\end{array}$ & $\begin{array}{r}\text { Passed in } \\
\text { Exams }\end{array}$ & $\begin{array}{l}\text { Falled in } \\
\text { Exams }\end{array}$ \\
\hline Grade $1 \mathrm{~A}$ & & 71 & 0 & 71 & 17 & 54 & Grade 1 & $0.00 \%$ & $100.00 \%$ & $23.94 \%$ & $76.06 \%$ \\
\hline Grade $1 \mathrm{~B}$ & & 0 & 0 & 0 & 0 & 0 & Grade 2 & $11.54 \%$ & $88.46 \%$ & $52.17 \%$ & $47.83 \%$ \\
\hline Grade $1 \mathrm{C}$ & & 0 & 0 & 0 & 0 & 0 & Grade 3 & $0.00 \%$ & $100.00 \%$ & $100.00 \%$ & $0.00 \%$ \\
\hline Grade $2 \mathrm{~A}$ & & 52 & 6 & 46 & 24 & 22 & Grade 4 & $0.00 \%$ & $100.00 \%$ & $87.50 \%$ & $12.50 \%$ \\
\hline Grade 2 B & & 0 & 0 & 0 & 0 & 0 & Grade 5 & $0.00 \%$ & $100.00 \%$ & $100.00 \%$ & $0.00 \%$ \\
\hline Grade $3 \mathrm{~A}$ & & 12 & 0 & 12 & 12 & 0 & Grade 6 & \#Num! & \#Num! & \#Num! & \#Num! \\
\hline Grade 3 B & & 0 & 0 & 0 & 0 & 0 & & & & & \\
\hline Grade 4 & & 8 & 0 & 8 & 7 & 1 & Remarks: & & & & \\
\hline Grade 5 & & 9 & 0 & 9 & 9 & 0 & & & & & \\
\hline Grade 6 & & 0 & 0 & 0 & 0 & 0 & & & & & \\
\hline School Total & & 152 & 6 & 146 & 69 & 77 & School \%age & $3.95 \%$ & $96.05 \%$ & $47.26 \%$ & $52.74 \%$ \\
\hline
\end{tabular}

\begin{tabular}{|c|c|c|c|c|c|c|c|c|c|c|}
\hline \multirow[b]{2}{*}{$\begin{array}{l}\text { Grades / } \\
\text { Sections }\end{array}$} & \multicolumn{5}{|c|}{ - Number of Students- } & \multirow[b]{2}{*}{ Grades } & \multicolumn{4}{|c|}{-Percent of Students } \\
\hline & Enrolled & Dropped & $\begin{array}{l}\text { Appeared } \\
\text { in Exam }\end{array}$ & $\begin{array}{l}\text { Passed in } \\
\text { Exams }\end{array}$ & $\begin{array}{l}\text { Failed in } \\
\text { Exams }\end{array}$ & & Dropped & $\begin{array}{l}\text { Appeared } \\
\text { in Exams }\end{array}$ & $\begin{array}{c}\text { Passed in } \\
\text { Exams } \\
\end{array}$ & $\begin{array}{r}\text { Faled in } \\
\text { Exams } \\
\end{array}$ \\
\hline Grade $1 \mathrm{~A}$ & 97 & 35 & 62 & 59 & 3 & Grade 1 & $21.70 \%$ & $78.30 \%$ & $72.29 \%$ & $27.71 \%$ \\
\hline Grade 1 B & 115 & 11 & 104 & 61 & 43 & Grade 2 & $0.00 \%$ & $100.00 \%$ & $73.85 \%$ & $26.15 \%$ \\
\hline Grade $1 \mathrm{C}$ & 0 & 0 & 0 & 0 & 0 & Grade 3 & $15.56 \%$ & $84.44 \%$ & $78.95 \%$ & $21.05 \%$ \\
\hline Grade 2 A & 65 & 0 & 65 & 48 & 17 & Grade 4 & $0.00 \%$ & $100.00 \%$ & $94.29 \%$ & $5.71 \%$ \\
\hline Grade 2 B & 0 & 0 & 0 & 0 & 0 & Grade 5 & $0.00 \%$ & $100.00 \%$ & $85.71 \%$ & $14.29 \%$ \\
\hline Grade $3 \mathrm{~A}$ & 45 & 7 & 38 & 30 & 8 & Grade 6 & $0.00 \%$ & $100.00 \%$ & $100.00 \%$ & $0.00 \%$ \\
\hline Grade 3 B & 0 & 0 & 0 & 0 & 0 & & & & & \\
\hline Grade 4 & 35 & 0 & 35 & 33 & 2 & Remarks: & & & & \\
\hline Grade 5 & 14 & 0 & 14 & 12 & 2 & & & & & \\
\hline Grade 6 & 8 & 0 & 8 & 8 & 0 & & & & & \\
\hline School Total & 379 & 53 & 326 & 251 & 75 & School \%age & $13.98 \%$ & $86.02 \%$ & $76.99 \%$ & $23.01 \%$ \\
\hline
\end{tabular}


Sub-Centre: Female

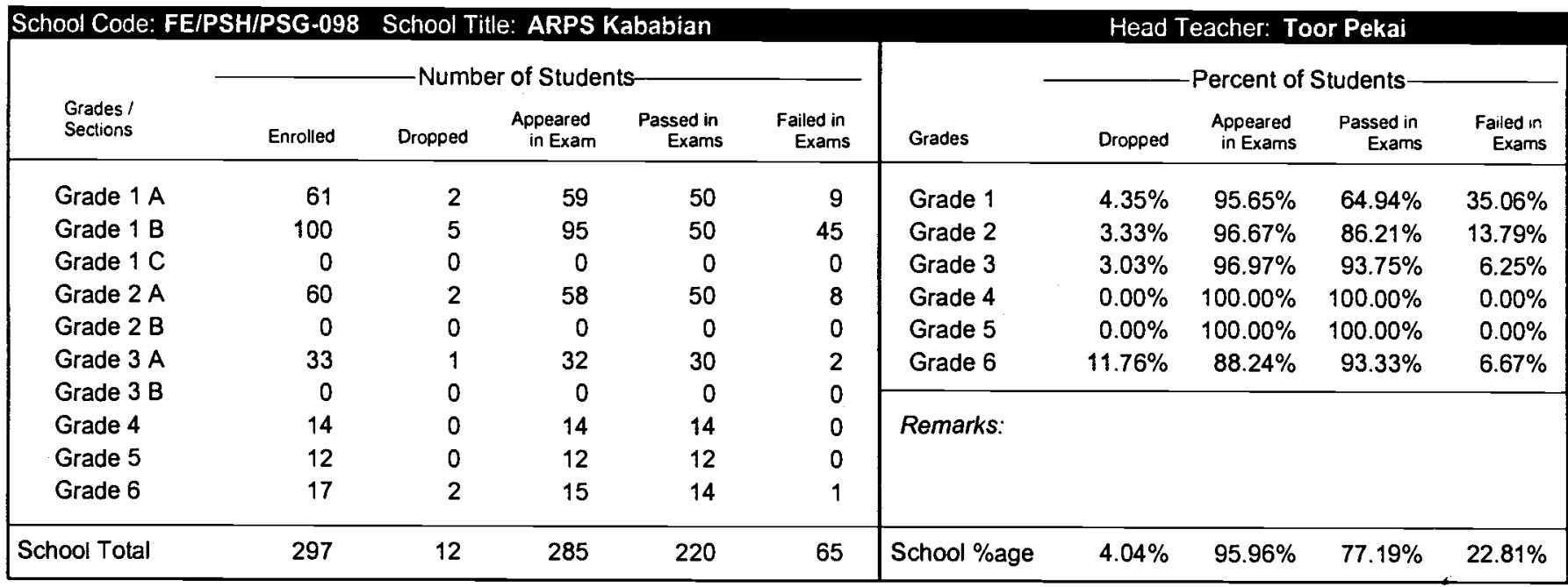

\begin{tabular}{|c|c|c|c|c|c|c|c|c|c|c|}
\hline \multirow[b]{2}{*}{$\begin{array}{l}\text { Grades / } \\
\text { Sections }\end{array}$} & \multicolumn{5}{|c|}{ — Number of Students- } & \multirow[b]{2}{*}{ Grades } & \multicolumn{4}{|c|}{-Percent of Students- } \\
\hline & Enrolled & Dropped & $\begin{array}{l}\text { Appeared } \\
\text { in Exam }\end{array}$ & $\begin{array}{c}\text { Passed in } \\
\text { Exams }\end{array}$ & $\begin{array}{l}\text { Failed in } \\
\text { Exams }\end{array}$ & & Dropped & $\begin{array}{l}\text { Appeared } \\
\text { in Exams }\end{array}$ & $\begin{array}{l}\text { Passed in } \\
\text { Exams }\end{array}$ & $\begin{array}{l}\text { Failed in } \\
\text { Exams }\end{array}$ \\
\hline Grade $1 \mathrm{~A}$ & 70 & 25 & 45 & 32 & 13 & Grade 1 & $30.57 \%$ & $69.43 \%$ & $74.31 \%$ & $25.69 \%$ \\
\hline Grade 1 B & 87 & 23 & 64 & 49 & 15 & Grade 2 & $11.11 \%$ & $88.89 \%$ & $64.29 \%$ & $35.71 \%$ \\
\hline Grade $1 \mathrm{C}$ & 0 & 0 & 0 & 0 & 0 & Grade 3 & $26.00 \%$ & $74.00 \%$ & $70.27 \%$ & $29.73 \%$ \\
\hline Grade 2 A & 63 & 7 & 56 & 36 & 20 & Grade 4 & $0.00 \%$ & $100.00 \%$ & $95.24 \%$ & $4.76 \%$ \\
\hline Grade 2 B & 0 & 0 & 0 & 0 & 0 & Grade 5 & $0.00 \%$ & $100.00 \%$ & $100.00 \%$ & $0.00 \%$ \\
\hline Grade $3 \mathrm{~A}$ & $50^{\circ}$ & 13 & 37 & 26 & 11 & Grade 6 & $0.00 \%$ & $100.00 \%$ & $100.00 \%$ & $0.00 \%$ \\
\hline Grade 3 B & 0 & 0 & 0 & 0 & 0 & & & & & \\
\hline Grade 4 & 21 & 0 & 21 & 20 & 1 & Remarks: & & & & \\
\hline Grade 5 & 23 & 0 & 23 & 23 & 0 & & & & & \\
\hline Grade 6 & 17 & 0 & 17 & 17 & 0 & & & & & \\
\hline School Total & 331 & 68 & 263 & 203 & 60 & School \%age & $20.54 \%$ & $79.46 \%$ & $77.19 \%$ & $22.81 \%$ \\
\hline
\end{tabular}

\begin{tabular}{|c|c|c|c|c|c|c|c|c|c|c|}
\hline \multirow[b]{2}{*}{$\begin{array}{l}\text { Grades / } \\
\text { Sections }\end{array}$} & \multicolumn{5}{|c|}{-Number of Students } & \multirow[b]{2}{*}{ Grades } & \multicolumn{4}{|c|}{-Percent of Students } \\
\hline & Enrolled & Dropped & $\begin{array}{l}\text { Appeared } \\
\text { in Exam }\end{array}$ & $\begin{array}{l}\text { Passed in } \\
\text { Exams }\end{array}$ & $\begin{array}{l}\text { Failed in } \\
\text { Exams }\end{array}$ & & Dropped & $\begin{array}{l}\text { Appeared } \\
\text { in Exams }\end{array}$ & $\begin{array}{l}\text { Passed in } \\
\text { Exams }\end{array}$ & $\begin{array}{l}\text { Faled in } \\
\text { Exams }\end{array}$ \\
\hline Grade $1 \mathrm{~A}$ & 47 & 7 & 40 & 30 & 10 & Grade 1 & $10.00 \%$ & $90.00 \%$ & $65.87 \%$ & $34.13 \%$ \\
\hline Grade 1 B & 47 & 7 & 40 & 20 & 20 & Grade 2 & $0.00 \%$ & $100.00 \%$ & $72.92 \%$ & $27.08 \%$ \\
\hline Grade $1 \mathrm{C}$ & 46 & 0 & 46 & 33 & 13 & Grade 3 & $2.17 \%$ & $97.83 \%$ & $82.22 \%$ & $17.78 \%$ \\
\hline Grade $2 \mathrm{~A}$ & 48 & 0 & 48 & 35 & 13 & Grade 4 & $9.09 \%$ & $90.91 \%$ & $85.00 \%$ & $15.00 \%$ \\
\hline Grade 2 B & 0 & 0 & 0 & 0 & 0 & Grade 5 & $0.00 \%$ & $100.00 \%$ & $86.67 \%$ & $13.33 \%$ \\
\hline Grade $3 \mathrm{~A}$ & 46 & 1 & 45 & 37 & 8 & Grade 6 & $0.00 \%$ & $100.00 \%$ & $100.00 \%$ & $0.00 \%$ \\
\hline Grade 3 B & 0 & 0 & 0 & 0 & 0 & & & & & \\
\hline Grade 4 & 22 & 2 & 20 & 17 & 3 & Remarks: & & & & \\
\hline Grade 5 & 15 & 0 & 15 & 13 & 2 & & & & & \\
\hline Grade 6 & 7 & 0 & 7 & 7 & 0 & & & & & \\
\hline School Total & 278 & 17 & 261 & 192 & 69 & School \%age & $6.12 \%$ & $93.88 \%$ & $73.56 \%$ & $26.44 \%$ \\
\hline
\end{tabular}


Sub-Centre: Female

\begin{tabular}{|c|c|c|c|c|c|c|c|c|c|c|}
\hline \multicolumn{11}{|c|}{ School Code: FE/PSH/PSG-101 School Title: ARPS 2 Kacha Gari Camp 4} \\
\hline \multirow[b]{2}{*}{$\begin{array}{l}\text { Grades / } \\
\text { Sections }\end{array}$} & \multicolumn{5}{|c|}{ - Number of Students } & \multirow[b]{2}{*}{ Grades } & \multicolumn{4}{|c|}{ Percent of Students- } \\
\hline & Enrolled & Dropped & $\begin{array}{l}\text { Appeared } \\
\text { in Exam }\end{array}$ & $\begin{array}{l}\text { Passed in } \\
\text { Exams }\end{array}$ & $\begin{array}{l}\text { Failed in } \\
\text { Exams }\end{array}$ & & Dropped & $\begin{array}{l}\text { Appeared } \\
\text { in Exams }\end{array}$ & $\begin{array}{l}\text { Passed in } \\
\text { Exams }\end{array}$ & $\begin{array}{l}\text { Falled in } \\
\text { Exams }\end{array}$ \\
\hline Grade $1 \mathrm{~A}$ & 52 & 0 & 52 & 48 & 4 & Grade 1 & $1.94 \%$ & $98.06 \%$ & $79.61 \%$ & $20.39 \%$ \\
\hline Grade 1 B & 51 & 0 & 51 & 47 & 4 & Grade 2 & $0.00 \%$ & $100.00 \%$ & $69.74 \%$ & $30.26 \%$ \\
\hline Grade $1 \mathrm{C}$ & 52 & 3 & 49 & 26 & 23 & Grade 3 & $2.13 \%$ & $97.87 \%$ & $78.26 \%$ & $21.74 \%$ \\
\hline Grade 2 A & 76 & 0 & 76 & 53 & 23 & Grade 4 & $0.00 \%$ & $100.00 \%$ & $94.12 \%$ & $5.88 \%$ \\
\hline Grade 2 B & 0 & 0 & 0 & 0 & 0 & Grade 5 & $0.00 \%$ & $100.00 \%$ & $100.00 \%$ & $0.00 \%$ \\
\hline Grade $3 \mathrm{~A}$ & 47 & 1 & 46 & 36 & 10 & Grade 6 & $0.00 \%$ & $100.00 \%$ & $100.00 \%$ & $0.00 \%$ \\
\hline Grade 3 B & 0 & 0 & 0 & 0 & 0 & & & & & \\
\hline Grade 4 & 17 & 0 & 17 & 16 & 1 & Remarks: & & & & \\
\hline Grade 5 & 9 & 0 & 9 & 9 & 0 & & & & & \\
\hline Grade 6 & 8 & 0 & 8 & 8 & 0 & & & & & \\
\hline School Total & 312 & 4 & 308 & 243 & 65 & School \%age & $1.28 \%$ & $98.72 \%$ & $78.90 \%$ & $21.10 \%$ \\
\hline
\end{tabular}

\begin{tabular}{|c|c|c|c|c|c|c|c|c|c|c|c|}
\hline School Code: F & & PSG-10 & ichool T & ARP: & hki & & & Head 7 & eacher: Sh & hzada Kh & am \\
\hline & & & -Numb & of Studer & & & & & Percent of & tudents- & \\
\hline $\begin{array}{l}\text { Grades I } \\
\text { Sections }\end{array}$ & ' & Enrolled & Dropped & $\begin{array}{l}\text { Appeared } \\
\text { in Exam }\end{array}$ & $\begin{array}{l}\text { Passed in } \\
\text { Exams }\end{array}$ & $\begin{array}{c}\text { Failed in } \\
\text { Exams }\end{array}$ & Grades & Dropped & $\begin{array}{c}\text { Appeared } \\
\text { in Exams }\end{array}$ & $\begin{array}{c}\text { Passed in } \\
\text { Exams }\end{array}$ & $\begin{array}{c}\text { Failed in } \\
\text { Exams }\end{array}$ \\
\hline Grade $1 \mathrm{~A}$ & & 30 & 0 & 30 & 23 & 7 & Grade 1 & $0.00 \%$ & $100.00 \%$ & $76.67 \%$ & $23.33 \%$ \\
\hline Grade 1 B & & 0 & 0 & 0 & 0 & 0 & Grade 2 & $0.00 \%$ & $100.00 \%$ & $83.33 \%$ & $16.67 \%$ \\
\hline Grade $1 \mathrm{C}$ & & 0 & 0 & 0 & 0 & 0 & Grade 3 & $0.00 \%$ & $100.00 \%$ & $100.00 \%$ & $0.00 \%$ \\
\hline Grade $2 \mathrm{~A}$ & & 24 & 0 & 24 & 20 & 4 & Grade 4 & $0.00 \%$ & $100.00 \%$ & $100.00 \%$ & $0.00 \%$ \\
\hline Grade 2 B & & 0 & 0 & 0 & 0 & 0 & Grade 5 & \#Num! & \#Num! & \#Num! & \#Num! \\
\hline Grade $3 \mathrm{~A}$ & & 10 & 0 & 10 & 10 & 0 & Grade 6 & \#Num! & \#Num! & \#Num! & \#Num! \\
\hline Grade 3 B & & 0 & 0 & 0 & 0 & 0 & & & & & \\
\hline Grade 4 & & 11 & 0 & 11 & 11 & 0 & Remarks.' & & & & \\
\hline Grade 5 & & 0 & 0 & 0 & 0 & 0 & & & & & \\
\hline Grade 6 & & 0 & 0 & 0 & 0 & 0 & & & & & \\
\hline School Total & & 75 & 0 & 75 & 64 & 11 & School \%age & $0.00 \%$ & $100.00 \%$ & $85.33 \%$ & $14.67 \%$ \\
\hline
\end{tabular}

\begin{tabular}{|c|c|c|c|c|c|c|c|c|c|c|}
\hline \multirow[b]{2}{*}{$\begin{array}{l}\text { Grades I } \\
\text { Sections }\end{array}$} & \multicolumn{5}{|c|}{ - Number of Students- } & \multirow[b]{2}{*}{ Grades } & \multicolumn{4}{|c|}{-Percent of Students } \\
\hline & Enrolled & Dropped & $\begin{array}{l}\text { Appeared } \\
\text { in Exam }\end{array}$ & $\begin{array}{l}\text { Passed in } \\
\text { Exams }\end{array}$ & $\begin{array}{l}\text { Failed in } \\
\text { Exams }\end{array}$ & & Dropped & $\begin{array}{l}\text { Appeared } \\
\text { in Exams }\end{array}$ & $\begin{array}{l}\text { Passed in } \\
\text { Exams }\end{array}$ & $\begin{array}{l}\text { Failed in } \\
\text { Exams }\end{array}$ \\
\hline Grade $1 \mathrm{~A}$ & 77 & 7 & 70 & 23 & 47 & Grade 1 & $9.09 \%$ & $90.91 \%$ & $32.86 \%$ & $67.14 \%$ \\
\hline Grade $1 \mathrm{~B}$ & 0 & 0 & 0 & 0 & 0 & Grade 2 & $0.00 \%$ & $100.00 \%$ & $54.55 \%$ & $45.45 \%$ \\
\hline Grade $1 \mathrm{C}$ & 0 & 0 & 0 & 0 & 0 & Grade 3 & $0.00 \%$ & $100.00 \%$ & $81.48 \%$ & $18.52 \%$ \\
\hline Grade 2 A & 55 & 0 & 55 & 30 & 25 & Grade 4 & $0.00 \%$ & $100.00 \%$ & $75.00 \%$ & $25.00 \%$ \\
\hline Grade 2 B & 0 & 0 & 0 & 0 & 0 & Grade 5 & $21.43 \%$ & $78.57 \%$ & $81.82 \%$ & $18.18 \%$ \\
\hline Grade $3 \mathrm{~A}$ & 27 & 0 & 27 & 22 & 5 & Grade 6 & \#Num! & \#Num! & \#Num! & \#Num! \\
\hline Grade 3 B & 0 & 0 & 0 & 0 & 0 & & & & & \\
\hline Grade 4 & 12 & 0 & 12 & 9 & 3 & Remarks: & & & & \\
\hline Grade 5 & 14 & 3 & 11 & 9 & 2 & & & & & \\
\hline Grade 6 & 0 & 0 & 0 & 0 & 0 & & & & & \\
\hline School Total & 185 & 10 & 175 & 93 & 82 & School \%age & $5.41 \%$ & $94.59 \%$ & $53.14 \%$ & $46.86 \%$ \\
\hline
\end{tabular}


Sub-Centre: Female

\begin{tabular}{|c|c|c|c|c|c|c|c|c|c|c|}
\hline \multirow[b]{2}{*}{$\begin{array}{l}\text { Grades I } \\
\text { Sections }\end{array}$} & \multicolumn{5}{|c|}{$\begin{array}{l}\text { School Title: ARPS Monda } \\
\text { _ Number of Students }\end{array}$} & \multirow[b]{2}{*}{ Grades } & \multicolumn{4}{|c|}{-Percent of Students } \\
\hline & Enrolled & Dropped & $\begin{array}{r}\text { Appeared } \\
\text { in Exam }\end{array}$ & $\begin{array}{c}\text { Passed in } \\
\text { Exams }\end{array}$ & $\begin{array}{l}\text { Failed in } \\
\text { Exams }\end{array}$ & & Dropped & $\begin{array}{l}\text { Appeared } \\
\text { in Exams }\end{array}$ & $\begin{array}{r}\text { Passed in } \\
\text { Exams } \\
\end{array}$ & $\begin{array}{c}\text { Faled in } \\
\text { Exams }\end{array}$ \\
\hline Grade $1 \mathrm{~A}$ & 30 & 0 & 30 & 16 & 14 & Grade 1 & $0.00 \%$ & $100.00 \%$ & $53.33 \%$ & $46.67 \%$ \\
\hline Grade 1 B & 0 & 0 & 0 & 0 & 0 & Grade 2 & $0.00 \%$ & $100.00 \%$ & $76.92 \%$ & $23.08 \%$ \\
\hline Grade $1 \mathrm{C}$ & 0 & 0 & 0 & 0 & 0 & Grade 3 & $0.00 \%$ & $100.00 \%$ & $81.82 \%$ & $18.18 \%$ \\
\hline Grade $2 \mathrm{~A}$ & 0 & 0 & 0 & 0 & 0 & Grade 4 & $0.00 \%$ & $100.00 \%$ & $83.33 \%$ & $16.67 \%$ \\
\hline Grade 2 B & 13 & 0 & 13 & 10 & 3 & Grade 5 & \#Num! & \#Num! & \#Num! & \#Num! \\
\hline Grade 3 A & 11 & 0 & 11 & 9 & 2 & Grade 6 & \#Num! & \#um! & \#Num! & \#Num! \\
\hline Grade 3 B & 0 & 0 & 0 & 0 & 0 & & & & & \\
\hline Grade 4 & 6 & 0 & 6 & 5 & 1 & Remarks: & & & & \\
\hline Grade 5 & 0 & 0 & 0 & 0 & 0 & & & & & \\
\hline Grade 6 & 0 & 0 & 0 & 0 & 0 & & & & & \\
\hline School Total & 60 & 0 & 60 & 40 & 20 & School \%age & $0.00 \%$ & $100.00 \%$ & $66.67 \%$ & $33.33 \%$ \\
\hline
\end{tabular}

\begin{tabular}{|c|c|c|c|c|c|c|c|c|c|c|}
\hline \multirow[b]{2}{*}{$\begin{array}{l}\text { Grades I } \\
\text { Sections }\end{array}$} & \multicolumn{5}{|c|}{ - Number of Students- } & \multirow[b]{2}{*}{ Grades } & \multicolumn{4}{|c|}{-Percent of Students } \\
\hline & Enrolled & Dropped & $\begin{array}{l}\text { Appeared } \\
\text { in Exam }\end{array}$ & $\begin{array}{l}\text { Passed in } \\
\text { Exams }\end{array}$ & $\begin{array}{c}\text { Failed in } \\
\text { Exams }\end{array}$ & & Dropped & $\begin{array}{l}\text { Appeared } \\
\text { in Exams }\end{array}$ & $\begin{array}{l}\text { Passed in } \\
\text { Exams }\end{array}$ & $\begin{array}{c}\text { Falled in } \\
\text { Exams }\end{array}$ \\
\hline Grade $1 \mathrm{~A}$ & 80 & 5 & 75 & 25 & 50 & Grade 1 & $6.25 \%$ & $93.75 \%$ & $33.33 \%$ & $66.67 \%$ \\
\hline Grade $1 \mathrm{~B}$ & 0 & 0 & 0 & 0 & 0 & Grade 2 & $0.00 \%$ & $100.00 \%$ & $48.84 \%$ & $51.16 \%$ \\
\hline Grade $1 \mathrm{C}$ & 0 & 0 & 0 & 0 & 0 & Grade 3 & $4.55 \%$ & $95.45 \%$ & $76.19 \%$ & $23.81 \%$ \\
\hline Grade $2 \mathrm{~A}$ & 43 & 0 & 43 & 21 & 22 & Grade 4 & $8.33 \%$ & $91.67 \%$ & $100.00 \%$ & $0.00 \%$ \\
\hline Grade 2 B & 0 & 0 & 0 & 0 & 0 & Grade 5 & $30.00 \%$ & $70.00 \%$ & $100.00 \%$ & $0.00 \%$ \\
\hline Grade $3 \mathrm{~A}$ & 22 & 1 & 21 & 16 & 5 & Grade 6 & $60.00 \%$ & $40.00 \%$ & $100.00 \%$ & $0.00 \%$ \\
\hline Grade 3 B & 0 & 0 & 0 & 0 & 0 & & & & & \\
\hline Grade 4 & 12 & 1 & 11 & 11 & 0 & Remarks: & & & & \\
\hline Grade 5 & 10 & 3 & 7 & 7 & 0 & & & & & \\
\hline Grade 6 & 10 & 6 & 4 & 4 & 0 & & & & & \\
\hline School Total & 177 & 16 & 161 & 84 & 77 & School \%age & $9.04 \%$ & $90.96 \%$ & $52.17 \%$ & $47.83 \%$ \\
\hline
\end{tabular}

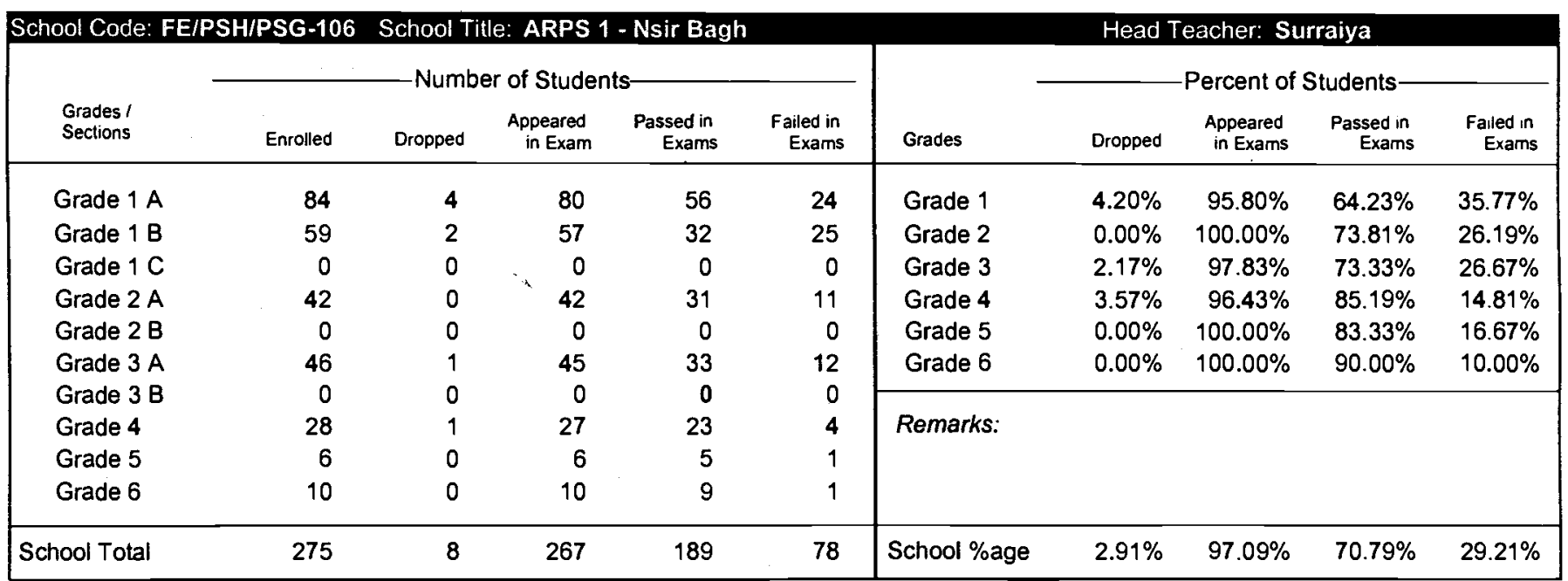


Sub-Centre: Female

\begin{tabular}{|c|c|c|c|c|c|c|c|c|c|c|}
\hline \multirow[b]{2}{*}{$\begin{array}{l}\text { Grades I } \\
\text { Sections }\end{array}$} & \multicolumn{5}{|c|}{ Number of Students } & \multirow[b]{2}{*}{ Grades } & \multicolumn{4}{|c|}{-Percent of Students } \\
\hline & Enrolled & Dropped & $\begin{array}{l}\text { Appeared } \\
\text { in Exam }\end{array}$ & $\begin{array}{l}\text { Passed in } \\
\text { Exams }\end{array}$ & $\begin{array}{l}\text { Failed in } \\
\text { Exams }\end{array}$ & & Dropped & $\begin{array}{l}\text { Appeared } \\
\text { in Exarns }\end{array}$ & $\begin{array}{r}\text { Passed in } \\
\text { Exams }\end{array}$ & $\begin{array}{l}\text { Failed in } \\
\text { Exams }\end{array}$ \\
\hline Grade $1 \mathrm{~A}$ & 90 & 9 & 81 & 67 & 14 & Grade 1 & $6.92 \%$ & $93.08 \%$ & $72.30 \%$ & $27.70 \%$ \\
\hline Grade $1 \mathrm{~B}$ & 69 & 2 & 67 & 40 & 27 & Grade 2 & $0.00 \%$ & $100.00 \%$ & $66.67 \%$ & $33.33 \%$ \\
\hline Grade $1 \mathrm{C}$ & 0 & 0 & 0 & 0 & 0 & Grade 3 & $17.95 \%$ & $82.05 \%$ & $71.88 \%$ & $28.13 \%$ \\
\hline Grade $2 \mathrm{~A}$ & 60 & 0 & 60 & 40 & 20 & Grade 4 & $0.00 \%$ & $100.00 \%$ & $71.43 \%$ & $28.57 \%$ \\
\hline Grade 2 B & 0 & 0 & 0 & 0 & 0 & Grade 5 & $0.00 \%$ & $100.00 \%$ & $72.73 \%$ & $27.27 \%$ \\
\hline Grade $3 \mathrm{~A}$ & 39 & 7 & 32 & 23 & 9 & Grade 6 & $0.00 \%$ & $100.00 \%$ & $90.00 \%$ & $10.00 \%$ \\
\hline Grade 3 B & 0 & 0 & 0 & 0 & 0 & & & & & \\
\hline Grade 4 & 28 & 0 & 28 & 20 & 8 & Remarks: & & & & \\
\hline Grade 5 & 11 & 0 & 11 & 8 & 3 & & & & & \\
\hline Grade 6 & 10 & 0 & 10 & 9 & 1 & & & & & \\
\hline School Total & 307 & 18 & 289 & 207 & 82 & School \%age & $5.86 \%$ & $94.14 \%$ & $71.63 \%$ & $28.37 \%$ \\
\hline
\end{tabular}

School Code: FE/PSH/PSG-108 School Title: ARPS Camp 4 - Nsir Bagh

\begin{tabular}{|c|c|c|c|c|c|c|c|c|c|c|}
\hline \multirow[b]{2}{*}{$\begin{array}{l}\text { Grades I } \\
\text { Sections }\end{array}$} & \multicolumn{5}{|c|}{ - Number of Students- } & \multirow[b]{2}{*}{ Grades } & \multicolumn{4}{|c|}{ Percent of Students- } \\
\hline & Enrolled & Dropped & $\begin{array}{l}\text { Appeared } \\
\text { in Exam }\end{array}$ & $\begin{array}{l}\text { Passed in } \\
\text { Exarns }\end{array}$ & $\begin{array}{l}\text { Falled in } \\
\text { Exams }\end{array}$ & & Dropped & $\begin{array}{l}\text { Appeared } \\
\text { in Exams }\end{array}$ & $\begin{array}{l}\text { Passed in } \\
\text { Exams }\end{array}$ & $\begin{array}{l}\text { Falled in } \\
\text { Exams }\end{array}$ \\
\hline Grade $1 \mathrm{~A}$ & 66 & 7 & 59 & 42 & 17 & Grade 1 & $5.56 \%$ & $94.44 \%$ & $80.67 \%$ & $19.33 \%$ \\
\hline Grade 1 B & 60 & 0 & 60 & 54 & 6 & Grade 2 & $0.00 \%$ & $100.00 \%$ & $83.33 \%$ & $16.67 \%$ \\
\hline Grade $1 \mathrm{C}$ & 0 & 0 & 0 & 0 & 0 & Grade 3 & $2.78 \%$ & $97.22 \%$ & $62.86 \%$ & $37.14 \%$ \\
\hline Grade 2 A & 60 & 0 & 60 & 50 & 10 & Grade 4 & $12.00 \%$ & $88.00 \%$ & $77.27 \%$ & $22.73 \%$ \\
\hline Grade 2 B & 54 & 0 & 54 & 45 & 9 & Grade 5 & \#Num! & \#Num! & \#Num! & \#Num! \\
\hline Grade $3 \mathrm{~A}$ & 36 & 1 & 35 & 22 & 13 & Grade 6 & $35.00 \%$ & $65.00 \%$ & $76.92 \%$ & $23.08 \%$ \\
\hline Grade 3 B & 0 & 0 & 0 & 0 & 0 & & & & & \\
\hline Grade 4 & 25 & 3 & 22 & 17 & 5 & Remarks: & & & & \\
\hline Grade 5 & 0 & 0 & 0 & 0 & 0 & & & & & \\
\hline Grade 6 & 20 & 7 & 13 & 10 & 3 & & & & & \\
\hline School Total & 321 & 18 & 303 & 240 & 63 & School \%age & $5.61 \%$ & $94.39 \%$ & $79.21 \%$ & $20.79 \%$ \\
\hline
\end{tabular}

School Code: FE/PSH/PSG-109 School Title: ARPS Utmanzai

\begin{tabular}{|c|c|c|c|c|c|c|c|c|c|c|}
\hline School Code: F & PSG-10 & School T & : ARPS & manzai & & & Head & eacher: $\mathbf{s}$ & $\mathrm{amim}$ & \\
\hline & & -Numt & of Stude & & & & & Percent of & Students- & - \\
\hline $\begin{array}{l}\text { Grades / } \\
\text { Sections }\end{array}$ & Enrolled & Dropped & $\begin{array}{l}\text { Appeared } \\
\text { in Exam }\end{array}$ & $\begin{array}{c}\text { Passed in, } \\
\text { Exarns }\end{array}$ & $\begin{array}{l}\text { Failed in } \\
\text { Exams }\end{array}$ & Grades & Dropped & $\begin{array}{l}\text { Appeared } \\
\text { in Exarns }\end{array}$ & $\begin{array}{c}\text { Passed in } \\
\text { Exams }\end{array}$ & $\begin{array}{l}\text { Failed ir, } \\
\text { Exams }\end{array}$ \\
\hline Grade $1 \mathrm{~A}$ & 65 & 0 & 65 & 45 & 20 & Grade 1 & $0.00 \%$ & $100.00 \%$ & $69.23 \%$ & $30.77 \%$ \\
\hline Grade 1 B & 0 & 0 & 0 & 0 & 0 & Grade 2 & $0.00 \%$ & $100.00 \%$ & $100.00 \%$ & $0.00 \%$ \\
\hline Grade $1 \mathrm{C}$ & 0 & 0 & 0 & 0 & 0 & Grade 3 & $0.00 \%$ & $100.00 \%$ & $100.00 \%$ & $0.00 \%$ \\
\hline Grade 2 A & 40 & 0 &, 40 & 40 & 0 & Grade 4 & $0.00 \%$ & $100.00 \%$ & $100.00 \%$ & $0.00 \%$ \\
\hline Grade 2 B & 0 & 0 & 0 & 0 & 0 & Grade 5 & $0.00 \%$ & $100.00 \%$ & $100.00 \%$ & $0.00 \%$ \\
\hline Grade $3 \mathrm{~A}$ & 26 & 0 & 26 & 26 & 0 & Grade 6 & \#Num! & \#Num! & \#Num! & \#Num! \\
\hline Grade 3 B & 0 & 0 & 0 & 0 & 0 & & & & & \\
\hline Grade 4 & 16 & 0 & 16 & 16 & 0 & Remarks: & & & & \\
\hline Grade 5 & 9 & 0 & 9 & 9 & 0 & & & & & \\
\hline Grade 6 & 0 & 0 & 0 & 0 & 0 & & & & & \\
\hline School Total & 156 & 0 & 156 & 136 & 20 & School \%age & $0.00 \%$ & $100.00 \%$ & $87.18 \%$ & $12.82 \%$ \\
\hline
\end{tabular}

Note:

\#Num! = Enrollinent in respective grade(s) is nill therefore calculation is not applicable
Page 37 of 91

Produced by DP/MIS Section on 31.Jul.97 
COPE Schools Based Annual Results for 1996-97 and Percentage Analysis

Sub-Centre: Female

\begin{tabular}{|c|c|c|c|c|c|c|c|c|c|c|}
\hline \multirow[b]{2}{*}{$\begin{array}{l}\text { Grades / } \\
\text { Sections }\end{array}$} & \multicolumn{5}{|c|}{ — Number of Students- } & \multirow[b]{2}{*}{ Grades } & \multicolumn{4}{|c|}{-Percent of Students- } \\
\hline & Enrolled & Dropped & $\begin{array}{l}\text { Appeared } \\
\text { in Exam }\end{array}$ & $\begin{array}{l}\text { Passed in } \\
\text { Exams }\end{array}$ & $\begin{array}{c}\text { Failed in } \\
\text { Exams }\end{array}$ & & Dropped & $\begin{array}{l}\text { Appeared } \\
\text { in Exams }\end{array}$ & $\begin{array}{l}\text { Passed in } \\
\text { Exams }\end{array}$ & $\begin{array}{c}\text { Faled in } \\
\text { Exams }\end{array}$ \\
\hline Grade $1 \mathrm{~A}$ & 100 & 0 & 100 & 45 & 55 & Grade 1 & $0.00 \%$ & $100.00 \%$ & $45.00 \%$ & $55.00 \%$ \\
\hline Grade 1 B & 0 & 0 & 0 & 0 & 0 & Grade 2 & $0.00 \%$ & $100.00 \%$ & $92.59 \%$ & $7.41 \%$ \\
\hline Grade $1 \mathrm{C}$ & 0 & 0 & 0 & 0 & 0 & Grade 3 & $0.00 \%$ & $100.00 \%$ & $100.00 \%$ & $0.00 \%$ \\
\hline Grade 2 A & 27 & 0 & 27 & 25 & 2 & Grade 4 & $0.00 \%$ & $100.00 \%$ & $91.67 \%$ & $8.33 \%$ \\
\hline Grade 2 B & 0 & 0 & 0 & 0 & 0 & Grade 5 & $0.00 \%$ & $100.00 \%$ & $87.50 \%$ & $12.50 \%$ \\
\hline Grade $3 \mathrm{~A}$ & 18 & 0 & 18 & 18 & 0 & Grade 6 & \#Num! & \#Num! & \#um! & \#Num! \\
\hline Grade 3 B & 0 & 0 & 0 & 0 & 0 & & & & & \\
\hline Grade 4 & 12 & 0 & 12 & 11 & 1 & Remarks: & & & & \\
\hline Grade 5 & 8 & 0 & 8 & 7 & 1 & & & & & \\
\hline Grade 6 & 0 & 0 & 0 & 0 & 0 & & & & & \\
\hline School Total & 165 & 0 & 165 & 106 & 59 & School \%age & $0.00 \%$ & $100.00 \%$ & $64.24 \%$ & $35.76 \%$ \\
\hline
\end{tabular}

\begin{tabular}{llllll|lllll}
\hline Sub-Centre Total & 7,866 & 480 & 7,386 & 5,293 & 2,093 & Sub-Centre $\% a g e$ & $6.10 \%$ & $93.90 \%$ & $71.66 \%$ & $28.34 \%$ \\
\hline
\end{tabular}


Sub-Centre: Peshawar

\begin{tabular}{|c|c|c|c|c|c|c|c|c|c|c|}
\hline \multirow[b]{2}{*}{$\begin{array}{l}\text { Grades I } \\
\text { Sections }\end{array}$} & \multicolumn{5}{|c|}{ - Number of Students } & \multirow[b]{2}{*}{ Grades } & \multicolumn{4}{|c|}{-Percent of Students } \\
\hline & Enrolled & Dropped & $\begin{array}{l}\text { Appeared } \\
\text { in Exam }\end{array}$ & $\begin{array}{c}\text { Passed in } \\
\text { Exams }\end{array}$ & $\begin{array}{c}\text { Failed in } \\
\text { Exams }\end{array}$ & & Dropped & $\begin{array}{l}\text { Appeared } \\
\text { in Exams }\end{array}$ & $\begin{array}{r}\text { Passed in } \\
\text { Exams }\end{array}$ & $\begin{array}{l}\text { Failed in } \\
\text { Exams }\end{array}$ \\
\hline Grade $1 \mathrm{~A}$ & 70 & 12 & 58 & 21 & 37 & Grade 1 & $10.71 \%$ & $89.29 \%$ & $39.00 \%$ & $61.00 \%$ \\
\hline Grade 1 B & 42 & 0 & 42 & 18 & 24 & Grade 2 & $7.89 \%$ & $92.11 \%$ & $60.00 \%$ & $40.00 \%$ \\
\hline Grade $1 \mathrm{C}$ & 0 & 0 & 0 & 0 & 0 & Grade 3 & $25.00 \%$ & $75.00 \%$ & $86.67 \%$ & $13.33 \%$ \\
\hline Grade $2 \mathrm{~A}$ & 38 & 3 & 35 & 21 & 14 & Grade 4 & $12.50 \%$ & $87.50 \%$ & $90.48 \%$ & $9.52 \%$ \\
\hline Grade 2 B & 0 & 0 & 0 & 0 & 0 & Grade 5 & $0.00 \%$ & $100.00 \%$ & $76.92 \%$ & $23.08 \%$ \\
\hline Grade $3 \mathrm{~A}$ & 20 & 5 & 15 & 13 & 2 & Grade 6 & \#Num! & \#Num! & \#Num! & \#Num! \\
\hline Grade 3 B & 0 & 0 & 0 & 0 & 0 & & & & & \\
\hline Grade 4 & 24 & 3 & 21 & 19 & 2 & Remarks: & & & & \\
\hline Grade 5 & 13 & 0 & 13 & 10 & 3 & & & & & \\
\hline Grade 6 & 0 & 0 & 0 & 0 & 0 & & . & & & \\
\hline School Total & 207 & 23 & 184 & 102 & 82 & School \%age & $11.11 \%$ & $88.89 \%$ & $55.43 \%$ & $44.57 \%$ \\
\hline
\end{tabular}

\begin{tabular}{|c|c|c|c|c|c|c|c|c|c|c|c|}
\hline \multirow[b]{2}{*}{$\begin{array}{l}\text { Grades I } \\
\text { Sections }\end{array}$} & \multicolumn{6}{|c|}{-Number of Students- } & \multirow[b]{2}{*}{ Grades } & \multicolumn{4}{|c|}{-Percent of Students } \\
\hline & ' & Enrolled & Dropped & $\begin{array}{l}\text { Appeared } \\
\text { in Exam }\end{array}$ & $\begin{array}{c}\text { Passed in } \\
\text { Exams }\end{array}$ & $\begin{array}{l}\text { Failed in } \\
\text { Exams }\end{array}$ & & Dropped & $\begin{array}{l}\text { Appeared } \\
\text { in Exams }\end{array}$ & $\begin{array}{c}\text { Passed in } \\
\text { Exams }\end{array}$ & $\begin{array}{c}\text { Failed in } \\
\text { Exams }\end{array}$ \\
\hline Grade $1 \mathrm{~A}$ & & 61 & 0 & 61 & 18 & 43 & Grade 1 & $0.00 \%$ & $100.00 \%$ & $31.90 \%$ & $68.10 \%$ \\
\hline Grade 1 B & & 55 & 0 & 55 & 19 & 36 & Grade 2 & $1.79 \%$ & $98.21 \%$ & $60.00 \%$ & $40.00 \%$ \\
\hline Grade $1 \mathrm{C}$ & & 0 & 0 & 0 & 0 & 0 & Grade 3 & $0.00 \%$ & $100.00 \%$ & $42.22 \%$ & $57.78 \%$ \\
\hline Grade 2 A & & 56 & 1 & 55 & 33 & 22 & Grade 4 & $8.57 \%$ & $91.43 \%$ & $71.88 \%$ & $28.13 \%$ \\
\hline Grade 2 B & & 0 & 0 & 0 & 0 & 0 & Grade 5 & $0.00 \%$ & $100.00 \%$ & $53.33 \%$ & $46.67 \%$ \\
\hline Grade $3 \mathrm{~A}$ & & 45 & 0 & 45 & 19 & 26 & Grade 6 & \#Num! & \#Num! & \#Num! & \#Num! \\
\hline Grade 3 B & & 0 & 0 & 0 & 0 & 0 & & & & & \\
\hline Grade 4 & & 35 & 3 & 32 & 23 & 9 & Remarks: & & & & \\
\hline Grade 5 & & 15 & 0 & 15 & 8 & 7 & & & & & \\
\hline Grade 6 & & 0 & 0 & 0 & 0 & 0 & & & & & \\
\hline School Total & & 267 & 4 & 263 & 120 & 143 & School \%age & $1.50 \%$ & $98.50 \%$ & $45.63 \%$ & $54.37 \%$ \\
\hline
\end{tabular}

\begin{tabular}{|c|c|c|c|c|c|c|c|c|c|c|}
\hline \multirow[b]{2}{*}{$\begin{array}{l}\text { Grades I } \\
\text { Sections }\end{array}$} & \multicolumn{5}{|c|}{-Number of Students } & \multirow[b]{2}{*}{ Grades } & \multicolumn{4}{|c|}{-Percent of Students } \\
\hline & Enrolled & Dropped & $\begin{array}{l}\text { Appeared } \\
\text { in Exam }\end{array}$ & $\begin{array}{l}\text { Passed in } \\
\text { Exams }\end{array}$ & $\begin{array}{c}\text { Failed in } \\
\text { Exams }\end{array}$ & & Dropped & $\begin{array}{c}\text { Appeared } \\
\text { in Exams }\end{array}$ & $\begin{array}{l}\text { Passed in } \\
\text { Exams }\end{array}$ & $\begin{array}{c}\text { Fared in } \\
\text { Exams }\end{array}$ \\
\hline Grade $1 \mathrm{~A}$ & 95 & 9 & 86 & 33 & 53 & Grade 1 & $9.47 \%$ & $90.53 \%$ & $38.37 \%$ & $61.63 \%$ \\
\hline Grade 1 B & 0 & 0 & 0 & 0 & 0 & Grade 2 & $0.00 \%$ & $100.00 \%$ & $88.57 \%$ & $11.43 \%$ \\
\hline Grade $1 \mathrm{C}$ & 0 & 0 & 0 & 0 & 0 & Grade 3 & $6.67 \%$ & $93.33 \%$ & $71.43 \%$ & $28.57 \%$ \\
\hline Grade $2 \mathrm{~A}$ & 35 & 0 & 35 & 31 & 4 & Grade 4 & $0.00 \%$ & $100.00 \%$ & $64.29 \%$ & $35.71 \%$ \\
\hline Grade 2 B & 0 & 0 & 0 & 0 & 0 & Grade 5 & $7.69 \%$ & $92.31 \%$ & $100.00 \%$ & $0.00 \%$ \\
\hline Grade $3 \mathrm{~A}$ & 30 & 2 & 28 & 20 & 8 & Grade 6 & $15.38 \%$ & $84.62 \%$ & $100.00 \%$ & $0.00 \%$ \\
\hline Grade 3 B & 0 & 0 & 0 & 0 & 0 & & & & & \\
\hline Grade 4 & 14 & 0 & 14 & 9 & 5 & Remarks: & & & & \\
\hline Grade 5 & 13 & 1 & 12 & 12 & 0 & & & & & \\
\hline Grade 6 & 13 & 2 & 11 & 11 & 0 & & & & & \\
\hline School Total & 200 & 14 & 186 & 116 & 70 & School \%age & $7.00 \%$ & $93.00 \%$ & $62.37 \%$ & $37.63 \%$ \\
\hline
\end{tabular}


COPE Schools Based Annual Results for 1996-97 and Percentage Analysis

Sub-Centre: Peshawar

\begin{tabular}{|c|c|c|c|c|c|c|c|c|c|c|}
\hline \multirow[b]{2}{*}{$\begin{array}{l}\text { Grades I } \\
\text { Sections }\end{array}$} & \multicolumn{5}{|c|}{ — Number of Students- } & \multirow[b]{2}{*}{ Grades } & \multicolumn{4}{|c|}{-Percent of Students- } \\
\hline & Enrolled & Dropped & $\begin{array}{l}\text { Appeared } \\
\text { in Exam }\end{array}$ & $\begin{array}{c}\text { Passed in } \\
\text { Exams }\end{array}$ & $\begin{array}{l}\text { Failed in } \\
\text { Exams }\end{array}$ & & Dropped & $\begin{array}{l}\text { Appeared } \\
\text { in Exams }\end{array}$ & $\begin{array}{c}\text { Passed in } \\
\text { Exams }\end{array}$ & $\begin{array}{l}\text { Falled in } \\
\text { Exams }\end{array}$ \\
\hline Grade $1 \mathrm{~A}$ & 52 & 0 & 52 & 22 & 30 & Grade 1 & $0.00 \%$ & $100.00 \%$ & $42.31 \%$ & $57.69 \%$ \\
\hline Grade 1 B & 0 & 0 & 0 & 0 & 0 & Grade 2 & $0.00 \%$ & $100.00 \%$ & $66.67 \%$ & $33.33 \%$ \\
\hline Grade $1 \mathrm{C}$ & 0 & 0 & 0 & 0 & 0 & Grade 3 & $0.00 \%$ & $100.00 \%$ & $57.14 \%$ & $42.86 \%$ \\
\hline Grade $2 \mathrm{~A}$ & 30 & 0 & 30 & 20 & 10 & Grade 4 & $0.00 \%$ & $100.00 \%$ & $53.33 \%$ & $46.67 \%$ \\
\hline Grade 2 B & 0 & 0 & 0 & 0 & 0 & Grade 5 & $0.00 \%$ & $100.00 \%$ & $61.54 \%$ & $38.46 \%$ \\
\hline Grade 3 A & 21 & 0 & 21 & 12 & 9 & Grade 6 & \#Num! & \#Num! & \#Num! & \#Num! \\
\hline Grade 3 B & 0 & 0 & 0 & 0 & 0 & & & & & \\
\hline Grade 4 & 15 & 0 & 15 & 8 & 7 & Remarks: & & & & \\
\hline Grade 5 & 13 & 0 & 13 & 8 & 5 & & & & & \\
\hline Grade 6 & 0 & 0 & 0 & 0 & 0 & & & & & \\
\hline School Total & 131 & 0 & 131 & 70 & 61 & School \%age & $0.00 \%$ & $100.00 \%$ & $53.44 \%$ & $46.56 \%$ \\
\hline
\end{tabular}

\begin{tabular}{|c|c|c|c|c|c|c|c|c|c|c|}
\hline \multirow[b]{2}{*}{$\begin{array}{l}\text { Grades I } \\
\text { Sections }\end{array}$} & \multicolumn{5}{|c|}{-Number of Students- } & \multirow[b]{2}{*}{ Grades } & \multicolumn{4}{|c|}{-Percent of Students- } \\
\hline & 'Enrolled & Dropped & $\begin{array}{l}\text { Appeared } \\
\text { in Exam }\end{array}$ & $\begin{array}{l}\text { Passed in } \\
\text { Exams }\end{array}$ & $\begin{array}{c}\text { Falled in } \\
\text { Exams }\end{array}$ & & Dropped & $\begin{array}{l}\text { Appeared } \\
\text { in Exarms }\end{array}$ & $\begin{array}{r}\text { Passed in } \\
\text { Exams }\end{array}$ & $\begin{array}{c}\text { Failed in } \\
\text { Exams }\end{array}$ \\
\hline Grade $1 \mathrm{~A}$ & 73 & 0 & 73 & 36 & 37 & Grade 1 & $0.00 \%$ & $100.00 \%$ & $49.32 \%$ & $50.68 \%$ \\
\hline Grade 1 B & 0 & 0 & 0 & 0 & 0 & Grade 2 & $0.00 \%$ & $100.00 \%$ & $75.61 \%$ & $24.39 \%$ \\
\hline Grade $1 \mathrm{C}$ & 0 & 0 & 0 & 0 & 0 & Grade 3 & $8.57 \%$ & $91.43 \%$ & $90.63 \%$ & $9.38 \%$ \\
\hline Grade 2 A & 41 & 0 & 41 & 31 & 10 & Grade 4 & $0.00 \%$ & $100.00 \%$ & $75.00 \%$ & $25.00 \%$ \\
\hline Grade 2 B & 0. & 0 & 0 & 0 & 0 & Grade 5 & $0.00 \%$ & $100.00 \%$ & $100.00 \%$ & $0.00 \%$ \\
\hline Grade $3 \mathrm{~A}$ & 35 & 3 & 32 & 29 & 3 & Grade 6 & \#Num! & \#Num! & \#Num! & \# Num! \\
\hline Grade 3 B & 0 & 0 & 0 & 0 & 0 & & & & & \\
\hline Grade 4 & 24 & 0 & 24 & 18 & 6 & Remarks: & & & & \\
\hline Grade 5 & 15 & 0 & 15 & 15 & 0 & & & & & \\
\hline Grade 6 & 0 & 0 & 0 & 0 & 0 & & & & & \\
\hline School Total & 188 & 3 & 185 & 129 & 56 & School \%age & $1.60 \%$ & $98.40 \%$ & $69.73 \%$ & $30.27 \%$ \\
\hline
\end{tabular}

\begin{tabular}{|c|c|c|c|c|c|c|c|c|c|c|}
\hline \multirow[b]{2}{*}{$\begin{array}{l}\text { Grades I } \\
\text { Sections }\end{array}$} & \multicolumn{5}{|c|}{-Number of Students- } & \multirow[b]{2}{*}{ Grades } & \multicolumn{4}{|c|}{ Percent of Students } \\
\hline & Enrolled & Dropped & $\begin{array}{l}\text { Appeared } \\
\text { in Exam }\end{array}$ & $\begin{array}{l}\text { Passed in } \\
\text { Exams }\end{array}$ & $\begin{array}{l}\text { Failed in } \\
\text { Exams }\end{array}$ & & Dropped & $\begin{array}{l}\text { Appeared } \\
\text { in Exams }\end{array}$ & $\begin{array}{c}\text { Passed in } \\
\text { Exams }\end{array}$ & $\begin{array}{c}\text { Falled in } \\
\text { Exams }\end{array}$ \\
\hline Grade $1 \mathrm{~A}$ & 41 & 2 & 39 & 30 & 9 & Grade 1 & $4.88 \%$ & $95.12 \%$ & $76.92 \%$ & $23.08 \%$ \\
\hline Grade 1 B & 0 & 0 & 0 & 0 & 0 & Grade 2 & $4.88 \%$ & $95.12 \%$ & $69.23 \%$ & $30.77 \%$ \\
\hline Grade $1 \mathrm{C}$ & 0 & 0 & $\therefore 0$ & 0 & 0 & Grade 3 & $0.00 \%$ & $100.00 \%$ & $57.89 \%$ & $42.11 \%$ \\
\hline Grade 2 A & 41 & 2 & 39 & 27 & 12 & Grade 4 & $4.65 \%$ & $95.35 \%$ & $73.17 \%$ & $26.83 \%$ \\
\hline Grade 2 B & 0 & 0 & 0 & 0 & 0 & Grade 5 & $0.00 \%$ & $100.00 \%$ & $82.61 \%$ & $17.39 \%$ \\
\hline Grade 3 A & 57 & 0 & 57 & 33 & 24 & Grade 6 & $3.03 \%$ & $96.97 \%$ & $81.25 \%$ & $18.75 \%$ \\
\hline Grade 3 B & 0 & 0 & 0 & 0 & 0 & & & & & \\
\hline Grade 4 & 43 & 2 & 41 & 30 & 11 & Remarks: & & & & \\
\hline Grade 5 & 23 & 0 & 23 & 19 & 4 & & & & & \\
\hline Grade 6 & 33 & 1 & 32 & 26 & 6 & & & & & \\
\hline School Total & 238 & 7 & 231 & 165 & 66 & School \%age & $2.94 \%$ & $97.06 \%$ & $71.43 \%$ & $28.57 \%$ \\
\hline
\end{tabular}


Sub-Centre: Peshawar

\begin{tabular}{|c|c|c|c|c|c|c|c|c|c|c|}
\hline \multirow[b]{2}{*}{$\begin{array}{l}\text { Grades I } \\
\text { Sections }\end{array}$} & \multicolumn{5}{|c|}{ - Number of Students- } & \multirow[b]{2}{*}{ Grades } & \multicolumn{4}{|c|}{-Percent of Students } \\
\hline & Enrolled & Dropped & $\begin{array}{l}\text { Appeared } \\
\text { in Exam }\end{array}$ & $\begin{array}{l}\text { Passed in } \\
\text { Exams }\end{array}$ & $\begin{array}{l}\text { Failed in } \\
\text { Exams }\end{array}$ & & Dropped & $\begin{array}{l}\text { Appeared } \\
\text { in Exams }\end{array}$ & $\begin{array}{l}\text { Passed in } \\
\text { Exams }\end{array}$ & $\begin{array}{l}\text { Failed in } \\
\text { Exams }\end{array}$ \\
\hline Grade $1 \mathrm{~A}$ & 54 & 0 & 54 & 27 & 27 & Grade 1 & $0.00 \%$ & $100.00 \%$ & $50.00 \%$ & $50.00 \%$ \\
\hline Grade 1 B & 0 & 0 & 0 & 0 & 0 & Grade 2 & $0.00 \%$ & $100.00 \%$ & $81.25 \%$ & $18.75 \%$ \\
\hline Grade $1 \mathrm{C}$ & 0 & 0 & 0 & 0 & 0 & Grade 3 & $0.00 \%$ & $100.00 \%$ & $85.71 \%$ & $14.29 \%$ \\
\hline Grade 2 A & 32 & 0 & 32 & 26 & 6 & Grade 4 & $7.14 \%$ & $92.86 \%$ & $84.62 \%$ & $15.38 \%$ \\
\hline Grade 2 B & 0 & 0 & 0 & 0 & 0 & Grade 5 & $0.00 \%$ & $100.00 \%$ & $61.11 \%$ & $38.89 \%$ \\
\hline Grade $3 \mathrm{~A}$ & 28 & 0 & 28 & 24 & 4 & Grade 6 & \#um! & \#Num! & \#Num! & \#Num! \\
\hline Grade 3 B & 0 & 0 & 0 & 0 & 0 & & & & & \\
\hline Grade 4 & 28 & 2 & 26 & 22 & 4 & Remarks: & & & & \\
\hline Grade 5 & 18 & 0 & 18 & 11 & 7 & & & & & \\
\hline Grade 6 & 0 & 0 & 0 & 0 & 0 & & & & & \\
\hline School Total & 160 & 2 & 158 & 110 & 48 & School \%age & $1.25 \%$ & $98.75 \%$ & $69.62 \%$ & $30.38 \%$ \\
\hline
\end{tabular}

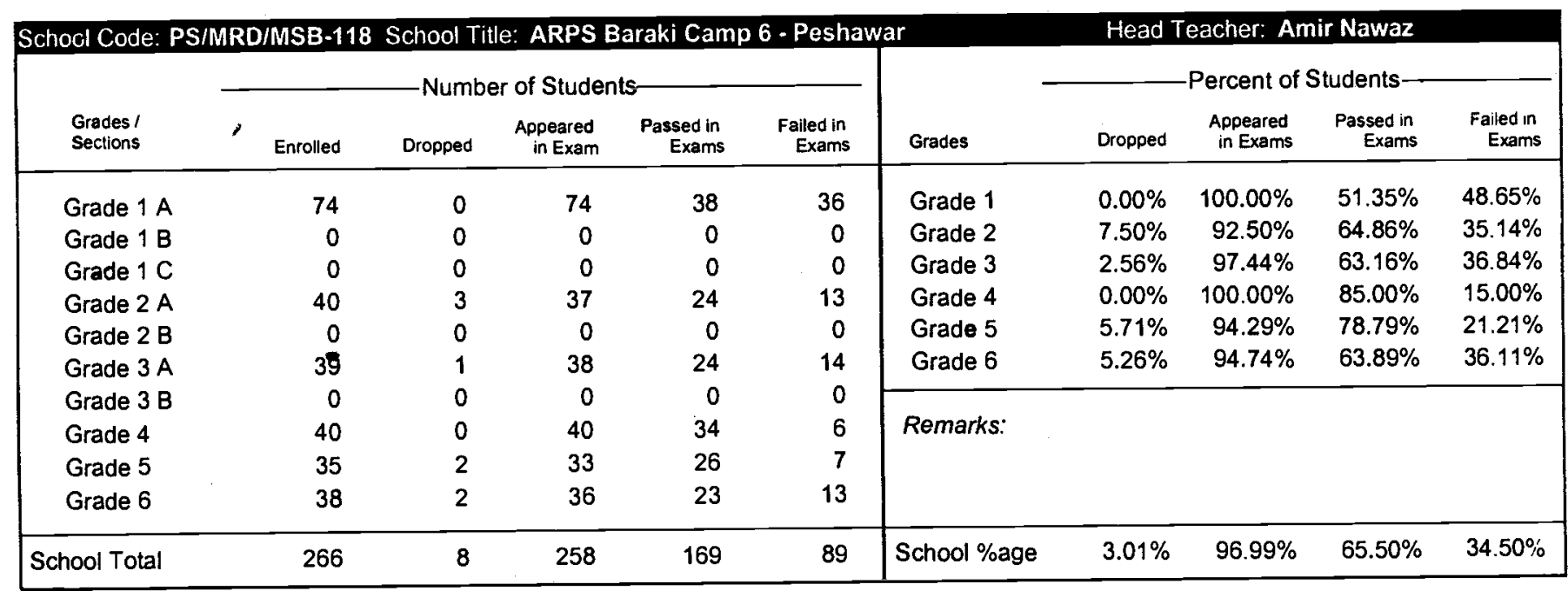

\begin{tabular}{|c|c|c|c|c|c|c|c|c|c|c|}
\hline \multirow[b]{2}{*}{$\begin{array}{l}\text { Grades I } \\
\text { Sections }\end{array}$} & \multicolumn{5}{|c|}{ - Number of Students- } & \multirow[b]{2}{*}{ Grades } & \multicolumn{4}{|c|}{-Percent of Students } \\
\hline & Enrolled & Dropped & $\begin{array}{l}\text { Appeared } \\
\text { in Exam }\end{array}$ & $\begin{array}{c}\text { Passed in } \\
\text { Exams }\end{array}$ & $\begin{array}{l}\text { Failed in } \\
\text { Exams }\end{array}$ & & Dropped & $\begin{array}{c}\text { Appeared } \\
\text { in Exams }\end{array}$ & $\begin{array}{c}\text { Passed in } \\
\text { Exams }\end{array}$ & $\begin{array}{c}\text { Failed in } \\
\text { Exams }\end{array}$ \\
\hline Grade $1 \mathrm{~A}$ & 46 & 6 & 40 & 22 & 18 & Grade 1 & $13.04 \%$ & $86.96 \%$ & $55.00 \%$ & $45.00 \%$ \\
\hline Grade 1 B & 0 & 0 & 0 & 0 & 0 & Grade 2 & $3.13 \%$ & $96.88 \%$ & $70.97 \%$ & $29.03 \%$ \\
\hline Grade $1 \mathrm{C}$ & 0 & 0 & 0 & 0 & 0 & Grade 3 & $3.23 \%$ & $96.77 \%$ & $66.67 \%$ & $33.33 \%$ \\
\hline Grade $2 \mathrm{~A}$ & 32 & 1 & 31 & 22 & 9 & Grade 4 & $4.17 \%$ & $95.83 \%$ & $73.91 \%$ & $26.09 \%$ \\
\hline Grade 2 B & 0 & 0 & 0 & 0 & 0 & Grade 5 & $4.17 \%$ & $95.83 \%$ & $78.26 \%$ & $21.74 \%$ \\
\hline Grade 3 A & 31 & 1 & 30 & 20 & 10 & Grade 6 & \#Num! & \#Num! & \#Num! & \#Num! \\
\hline Grade 3 B & 0 & 0 & 0 & 0 & 0 & & & & & \\
\hline Grade 4 & 24 & 1 & 23 & 17 & 6 & Remarks: & & & & \\
\hline Grade 5 & 24 & 1 & 23 & 18 & 5 & & & & & \\
\hline Grade 6 & 0 & 0 & 0 & 0 & 0 & & & & & \\
\hline School Total & 157 & 10 & 147 & 99 & 48 & School \%age & $6.37 \%$ & $93.63 \%$ & $67.35 \%$ & $32.65 \%$ \\
\hline
\end{tabular}


Sub-Centre: Peshawar

\begin{tabular}{|c|c|c|c|c|c|c|c|c|c|c|}
\hline \multirow[b]{2}{*}{$\begin{array}{l}\text { Grades I } \\
\text { Sections }\end{array}$} & \multicolumn{5}{|c|}{ - Number of Students- } & \multirow[b]{2}{*}{ Grades } & \multicolumn{4}{|c|}{-Percent of Students- } \\
\hline & Enrolled & Dropped & $\begin{array}{l}\text { Appeared } \\
\text { in Exam }\end{array}$ & $\begin{array}{r}\text { Passed in } \\
\text { Exams }\end{array}$ & $\begin{array}{l}\text { Failed in } \\
\text { Exams }\end{array}$ & & Dropped & $\begin{array}{l}\text { Appeared } \\
\text { in Exams }\end{array}$ & $\begin{array}{l}\text { Passed in } \\
\text { Exams }\end{array}$ & $\begin{array}{l}\text { Faled in } \\
\text { Exams }\end{array}$ \\
\hline Grade $1 \mathrm{~A}$ & 55 & 0 & 55 & 22 & 33 & Grade 1 & $0.00 \%$ & $100.00 \%$ & $40.00 \%$ & $60.00 \%$ \\
\hline Grade 1 B & 0 & 0 & 0 & 0 & 0 & Grade 2 & $7.41 \%$ & $92.59 \%$ & $40.00 \%$ & $60.00 \%$ \\
\hline Grade $1 \mathrm{C}$ & 0 & 0 & 0 & 0 & 0 & Grade 3 & $4.55 \%$ & $95.45 \%$ & $47.62 \%$ & $52.38 \%$ \\
\hline Grade $2 \mathrm{~A}$ & 27 & 2 & 25 & 10 & 15 & Grade 4 & $3.70 \%$ & $96.30 \%$ & $50.00 \%$ & $50.00 \%$ \\
\hline Grade 2 B & 0 & 0 & 0 & 0 & 0 & Grade 5 & $0.00 \%$ & $100.00 \%$ & $72.73 \%$ & $27.27 \%$ \\
\hline Grade $3 \mathrm{~A}$ & 22 & 1 & 21 & 10 & 11 & Grade 6 & \#Num! & \#Num! & \#Num! & \#Num! \\
\hline Grade 3 B & 0 & 0 & 0 & 0 & 0 & & & & & \\
\hline Grade 4 & 27 & 1 & 26 & 13 & 13 & Remarks: & & & & \\
\hline Grade 5 & 22 & 0 & 22 & 16 & 6 & & & & & \\
\hline Grade 6 & 0 & 0 & 0 & 0 & 0 & & & & & \\
\hline School Total & 153 & 4 & 149 & 71 & 78 & School \%age & $2.61 \%$ & $97.39 \%$ & $47.65 \%$ & $52.35 \%$ \\
\hline
\end{tabular}

\begin{tabular}{|c|c|c|c|c|c|c|c|c|c|c|}
\hline \multirow[b]{2}{*}{$\begin{array}{l}\text { Grades I } \\
\text { Sections }\end{array}$} & \multicolumn{5}{|c|}{ - Number of Students- } & \multirow[b]{2}{*}{ Grades } & \multicolumn{4}{|c|}{-Percent of Students- } \\
\hline & Enrolled & Dropped & $\begin{array}{l}\text { Appeared } \\
\text { in Exam }\end{array}$ & $\begin{array}{c}\text { Passed in } \\
\text { Exams }\end{array}$ & $\begin{array}{l}\text { Failed in } \\
\text { Exams }\end{array}$ & & Dropped & $\begin{array}{l}\text { Appeared } \\
\text { in Exams }\end{array}$ & $\begin{array}{l}\text { Passed in } \\
\text { Exams }\end{array}$ & $\begin{array}{l}\text { Fariled in } \\
\text { Exams }\end{array}$ \\
\hline Grade $1 \mathrm{~A}$ & 72 & 3 & 69 & 41 & 28 & Grade 1 & $4.17 \%$ & $95.83 \%$ & $59.42 \%$ & $40.58 \%$ \\
\hline Grade 1 B & 0 & 0 & 0 & 0 & 0 & Grade 2 & $3.39 \%$ & $96.61 \%$ & $68.42 \%$ & $31.58 \%$ \\
\hline Grade $1 \mathrm{C}$ & 0 & 0 & 0 & 0 & 0 & Grade 3 & $7.14 \%$ & $92.86 \%$ & $73.08 \%$ & $26.92 \%$ \\
\hline Grade $2 \mathrm{~A}$ & 59 & 2 & 57 & 39 & 18 & Grade 4 & $5.26 \%$ & $94.74 \%$ & $66.67 \%$ & $33.33 \%$ \\
\hline Grade 2 B & 0 & 0 & 0 & 0 & 0 & Grade 5 & $7.14 \%$ & $92.86 \%$ & $69.23 \%$ & $30.77 \%$ \\
\hline Grade 3 A & $28-$ & 2 & 26 & 19 & 7 & Grade 6 & $0.00 \%$ & $100.00 \%$ & $80.95 \%$ & $19.05 \%$ \\
\hline Grade 3 B & 0 & 0 & 0 & 0 & 0 & & & & & \\
\hline Grade 4 & 19 & 1 & 18 & 12 & 6 & Remarks: & & & & \\
\hline Grade 5 & 28 & 2 & 26 & 18 & 8 & & & & & \\
\hline Grade 6 & 21 & 0 & 21 & 17 & 4 & & & & & \\
\hline School Total & 227 & 10 & 217 & 146 & 71 & School \%age & $4.41 \%$ & $95.59 \%$ & $67.28 \%$ & $32.72 \%$ \\
\hline
\end{tabular}

\begin{tabular}{|c|c|c|c|c|c|c|c|c|c|c|}
\hline \multirow[b]{2}{*}{$\begin{array}{l}\text { Grades I } \\
\text { Sections }\end{array}$} & \multicolumn{5}{|c|}{ - Number of Students- } & \multirow[b]{2}{*}{ Grades } & \multicolumn{4}{|c|}{-Percent of Students- } \\
\hline & Enrolled & Dropped & $\begin{array}{l}\text { Appeared } \\
\text { in Exam }\end{array}$ & $\begin{array}{c}\text { Passed in } \\
\text { Exams }\end{array}$ & $\begin{array}{c}\text { Failed in } \\
\text { Exams }\end{array}$ & & Dropped & $\begin{array}{c}\text { Appeared } \\
\text { in Exams }\end{array}$ & $\begin{array}{r}\text { Passed in } \\
\text { Exams }\end{array}$ & $\begin{array}{c}\text { Failed in } \\
\text { Exams }\end{array}$ \\
\hline Grade $1 \mathrm{~A}$ & 54 & 4 & 50 & 31 & 19 & Grade 1 & $7.41 \%$ & $92.59 \%$ & $62.00 \%$ & $38.00 \%$ \\
\hline Grade $1 \mathrm{~B}$ & 0 & 0 & 0 & 0 & 0 & Grade 2 & $6.67 \%$ & $93.33 \%$ & $71.43 \%$ & $28.57 \%$ \\
\hline Grade $1 \mathrm{C}$ & 0 & 0 & 0 & 0 & 0 & Grade 3 & $8.11 \%$ & $91.89 \%$ & $58.82 \%$ & $41.18 \%$ \\
\hline Grade $2 \mathrm{~A}$ & 30 & 2 & 28 & 20 & 8 & Grade 4 & $16.00 \%$ & $84.00 \%$ & $52.38 \%$ & $47.62 \%$ \\
\hline Grade 2 B & 0 & 0 & 0 & 0 & 0 & Grade 5 & $25.00 \%$ & $75.00 \%$ & $50.00 \%$ & $50.00 \%$ \\
\hline Grade $3 \mathrm{~A}$. & 37 & 3 & 34 & 20 & 14 & Grade 6 & \#Num! & \#Num! & \#Num! & \#Num! \\
\hline Grade 3 B & 0 & 0 & 0 & 0 & 0 & & & & & \\
\hline Grade 4 & 25 & 4 & 21 & 11 & 10 & Remarks: & & & & \\
\hline Grade 5 & 16 & 4 & 12 & 6 & 6 & & & & & \\
\hline Grade 6 & 0 & 0 & 0 & 0 & 0 & & & & & \\
\hline School Total & 162 & 17 & 145 & 88 & 57 & School \%age & $10.49 \%$ & $89.51 \%$ & $60.69 \%$ & $39.31 \%$ \\
\hline
\end{tabular}

Note:

\#Numl = Enrollment in respective grade(s) is nill therefore calculation is not applicable 
Sub-Centre: Peshawar

\begin{tabular}{|c|c|c|c|c|c|c|c|c|c|c|}
\hline \multirow[b]{2}{*}{$\begin{array}{l}\text { Grades I } \\
\text { Sections }\end{array}$} & \multicolumn{5}{|c|}{ - Number of Students- } & \multirow[b]{2}{*}{ Grades } & \multicolumn{4}{|c|}{-Percent of Students } \\
\hline & Enrolled & Dropped & $\begin{array}{l}\text { Appeared } \\
\text { in Exam }\end{array}$ & $\begin{array}{l}\text { Passed in } \\
\text { Exams }\end{array}$ & $\begin{array}{c}\text { Failed in } \\
\text { Exams }\end{array}$ & & Dropped & $\begin{array}{l}\text { Appeared } \\
\text { in Exams }\end{array}$ & $\begin{array}{c}\text { Passed in } \\
\text { Exams }\end{array}$ & $\begin{array}{c}\text { Falled in } \\
\text { Exams }\end{array}$ \\
\hline Grade $1 \mathrm{~A}$ & 81 & 13 & 68 & 50 & 18 & Grade 1 & $18.01 \%$ & $81.99 \%$ & $76.52 \%$ & $23.48 \%$ \\
\hline Grade 1 B & 80 & 16 & 64 & 51 & 13 & Grade 2 & $1.92 \%$ & $98.08 \%$ & $74.51 \%$ & $25.49 \%$ \\
\hline Grade $1 \mathrm{C}$ & 0 & 0 & 0 & 0 & 0 & Grade 3 & $18.18 \%$ & $81.82 \%$ & $88.89 \%$ & $11.11 \%$ \\
\hline Grade 2 A & 52 & 1 & 51 & 38 & 13 & Grade 4 & $0.00 \%$ & $100.00 \%$ & $90.48 \%$ & $9.52 \%$ \\
\hline Grade 2 B & 0 & 0 & 0 & 0 & 0 & Grade 5 & $0.00 \%$ & $100.00 \%$ & $81.25 \%$ & $18.75 \%$ \\
\hline Grade $3 \mathrm{~A}$ & 44 & 8 & 36 & 32 & 4 & Grade 6 & $31.58 \%$ & $68.42 \%$ & $100.00 \%$ & $0.00 \%$ \\
\hline Grade 3 B & 0 & 0 & 0 & 0 & 0 & & & & & \\
\hline Grade 4 & 42 & 0 & 42 & 38 & 4 & Remarks: & & & & \\
\hline Grade 5 & 32 & 0 & 32 & 26 & 6 & & & & & \\
\hline Grade 6 & 19 & 6 & 13 & 13 & 0 & & & & & \\
\hline School Total & 350 & 44 & 306 & 248 & 58 & School \%age & $12.57 \%$ & $87.43 \%$ & $81.05 \%$ & $18.95 \%$ \\
\hline
\end{tabular}

\begin{tabular}{|c|c|c|c|c|c|c|c|c|c|c|}
\hline \multirow[b]{2}{*}{$\begin{array}{l}\text { Grades I } \\
\text { Sections }\end{array}$} & \multicolumn{5}{|c|}{ - Number of Students } & \multirow[b]{2}{*}{ Grades } & \multicolumn{4}{|c|}{ Percent of Students- } \\
\hline & Enrolled & Dropped & $\begin{array}{l}\text { Appeared } \\
\text { in Exam }\end{array}$ & $\begin{array}{l}\text { Passed in } \\
\text { Exams }\end{array}$ & $\begin{array}{l}\text { Failed in } \\
\text { Exams }\end{array}$ & & Dropped & $\begin{array}{c}\text { Appeared } \\
\text { in Exams }\end{array}$ & $\begin{array}{c}\text { Passed in } \\
\text { Exams }\end{array}$ & $\begin{array}{c}\text { Falled in } \\
\text { Exams }\end{array}$ \\
\hline Grade $1 \mathrm{~A}$ & 64 & 18 & 46 & 27 & 19 & Grade 1 & $28.13 \%$ & $71.88 \%$ & $58.70 \%$ & $41.30 \%$ \\
\hline Grade $1 \mathrm{~B}$ & 0 & 0 & 0 & 0 & 0 & Grade 2 & $5.63 \%$ & $94.37 \%$ & $47.76 \%$ & $52.24 \%$ \\
\hline Grade $1 \mathrm{C}$ & 0 & 0 & 0 & 0 & 0 & Grade 3 & $0.00 \%$ & $100.00 \%$ & $54.84 \%$ & $45.16 \%$ \\
\hline Grade 2 A & 35 & 3 & 32 & 16 & 16 & Grade 4 & $2.56 \%$ & $97.44 \%$ & $65.79 \%$ & $34.21 \%$ \\
\hline Grade 2 B & 36 & 1 & 35 & 16 & 19 & Grade 5 & $0.00 \%$ & $100.00 \%$ & $69.57 \%$ & $30.43 \%$ \\
\hline Grade $3 \mathrm{~A}$ & 31 & 0 & 31 & 17 & 14 & Grade 6 & $0.00 \%$ & $100.00 \%$ & $86.96 \%$ & $13.04 \%$ \\
\hline Grade 3 B & 0 & 0 & 0 & 0 & 0 & & & & & \\
\hline Grade 4 & 39 & 1 & 38 & 25 & 13 & Remarks: & & & & \\
\hline Grade 5 & 23 & 0 & 23 & 16 & 7 & & & & & \\
\hline Grade 6 & 23 & 0 & 23 & 20 & 3 & & & & & \\
\hline School Total & 251 & 23 & 228 & 137 & 91 & School \%age & $9.16 \%$ & $90.84 \%$ & $60.09 \%$ & $39.91 \%$ \\
\hline
\end{tabular}

\begin{tabular}{|c|c|c|c|c|c|c|c|c|c|c|}
\hline \multirow[b]{2}{*}{$\begin{array}{l}\text { Grades I } \\
\text { Sections }\end{array}$} & \multicolumn{5}{|c|}{-Number of Students } & \multirow[b]{2}{*}{ Grades } & \multicolumn{4}{|c|}{-Percent of Students } \\
\hline & Enrolled & Dropped & $\begin{array}{l}\text { Appeared } \\
\text { in Exam }\end{array}$ & $\begin{array}{l}\text { Passed in } \\
\text { Exams }\end{array}$ & $\begin{array}{l}\text { Failed in } \\
\text { Exams }\end{array}$ & & Dropped & $\begin{array}{l}\text { Appeared } \\
\text { in Exams }\end{array}$ & $\begin{array}{l}\text { Passed in } \\
\text { Exams }\end{array}$ & $\begin{array}{l}\text { Failed in } \\
\text { Exams }\end{array}$ \\
\hline Grade $1 \mathrm{~A}$ & 56 & 0 & 56 & 40 & 16 & Grade 1 & $0.00 \%$ & $100.00 \%$ & $75.22 \%$ & $24.78 \%$ \\
\hline Grade $1 \mathbf{B}$ & 57 & 0 & 57 & 45 & 12 & Grade 2 & $0.00 \%$ & $100.00 \%$ & $95.83 \%$ & $4.17 \%$ \\
\hline Grade $1 \mathrm{C}$ & 0 & 0 & 0 & 0 & 0 & Grade 3 & $0.00 \%$ & $100.00 \%$ & $96.00 \%$ & $4.00 \%$ \\
\hline Grade 2 A & 72 & 0 & 72 & 69 & 3 & Grade 4 & $3.85 \%$ & $96.15 \%$ & $92.00 \%$ & $8.00 \%$ \\
\hline Grade 2 B & 0 & 0 & 0 & 0 & 0 & Grade 5 & $3.33 \%$ & $96.67 \%$ & $96.55 \%$ & $3.45 \%$ \\
\hline Grade $3 \mathrm{~A}$ & 25 & 0 & 25 & 24 & 1 & Grade 6 & \#Num! & \#Num! & \#Num! & \#Num! \\
\hline Grade 3 B & 0 & 0 & 0 & 0 & 0 & & & & & \\
\hline Grade 4 & 26 & 1 & 25 & 23 & 2 & Remarks: & & & & \\
\hline Grade 5 & 30 & 1 & 29 & 28 & 1 & & & & & \\
\hline Grade 6 & 0 & 0 & 0 & 0 & 0 & & & & & \\
\hline School Total & 266 & 2 & 264 & 229 & 35 & School \%age & $0.75 \%$ & $99.25 \%$ & $86.74 \%$ & $13.26 \%$ \\
\hline
\end{tabular}


Sub-Centre: Peshawar

School Code: PS/MRD/PSB-126 School Title: ARPS 1 Gandaf Camp 1 - Peshawar

\begin{tabular}{|c|c|c|c|c|c|c|c|c|c|c|}
\hline \multirow[b]{2}{*}{$\begin{array}{l}\text { Grades / } \\
\text { Sections }\end{array}$} & \multicolumn{5}{|c|}{-Number of Students- } & \multirow[b]{2}{*}{ Grades } & \multicolumn{4}{|c|}{-Percent of Students- } \\
\hline & Enrolled & Dropped & $\begin{array}{l}\text { Appeared } \\
\text { in Exam }\end{array}$ & $\begin{array}{l}\text { Passed in } \\
\text { Exams }\end{array}$ & $\begin{array}{l}\text { Failed in } \\
\text { Exams }\end{array}$ & & Dropped & $\begin{array}{l}\text { Appeared } \\
\text { in Exams }\end{array}$ & $\begin{array}{l}\text { Passed in } \\
\text { Exams }\end{array}$ & $\begin{array}{l}\text { Faled in } \\
\text { Exams }\end{array}$ \\
\hline Grade $1 \mathrm{~A}$ & 60 & 0 & 60 & 48 & 12 & Grade 1 & $0.00 \%$ & $100.00 \%$ & $80.00 \%$ & $20.00 \%$ \\
\hline Grade 1 B & 0 & 0 & 0 & 0 & 0 & Grade 2 & $2.50 \%$ & $97.50 \%$ & $71.79 \%$ & $28.21 \%$ \\
\hline Grade $1 \mathrm{C}$ & 0 & 0 & 0 & 0 & 0 & Grade 3 & $0.00 \%$ & $100.00 \%$ & $88.24 \%$ & $11.76 \%$ \\
\hline Grade 2 A & 40 & 1 & 39 & 28 & 11 & Grade 4 & $0.00 \%$ & $100.00 \%$ & $87.50 \%$ & $12.50 \%$ \\
\hline Grade 2 B & 0 & 0 & 0 & 0 & 0 & Grade 5 & $0.00 \%$ & $100.00 \%$ & $64.29 \%$ & $35.71 \%$ \\
\hline Grade 3 A & 17 & 0 & 17 & 15 & 2 & Grade 6 & \#Num! & \#Num! & \#Num! & \#Num! \\
\hline Grade 3 B & 0 & 0 & 0 & 0 & 0 & & & & & \\
\hline Grade 4 & 24 & 0 & 24 & 21 & 3 & Remarks: & & & & \\
\hline Grade 5 & 14 & 0 & 14 & 9 & 5 & & & & & \\
\hline Grade 6 & 0 & 0 & 0 & 0 & 0 & & & & & \\
\hline School Total & 155 & 1 & 154 & 121 & 33 & School \%age & $0.65 \%$ & $99.35 \%$ & $78.57 \%$ & $21.43 \%$ \\
\hline
\end{tabular}

\begin{tabular}{|c|c|c|c|c|c|c|c|c|c|c|c|}
\hline \multirow[b]{2}{*}{$\begin{array}{l}\text { Grades I } \\
\text { Sections }\end{array}$} & \multicolumn{6}{|c|}{-Number of Students- } & \multirow[b]{2}{*}{ Grades } & \multicolumn{4}{|c|}{-Percent of Students } \\
\hline & ' & Enrolled & Dropped & $\begin{array}{l}\text { Appeared } \\
\text { in Exam }\end{array}$ & $\begin{array}{l}\text { Passed in } \\
\text { Exams }\end{array}$ & $\begin{array}{c}\text { Failed in } \\
\text { Exams }\end{array}$ & & Dropped & $\begin{array}{c}\text { Appeared } \\
\text { in Exams }\end{array}$ & $\begin{array}{c}\text { Passed in } \\
\text { Exams }\end{array}$ & $\begin{array}{c}\text { Failed in } \\
\text { Exams }\end{array}$ \\
\hline Grade $1 \mathrm{~A}$ & & 47 & 0 & 47 & 36 & 11 & Grade 1 & $0.00 \%$ & $100.00 \%$ & $88.17 \%$ & $11.83 \%$ \\
\hline Grade 1 B & & 46 & 0 & 46 & 46 & 0 & Grade 2 & $0.00 \%$ & $100.00 \%$ & $53.66 \%$ & $46.34 \%$ \\
\hline Grade $1 \mathrm{C}$ & & 0 & 0 & 0 & 0 & 0 & Grade 3 & $0.00 \%$ & $100.00 \%$ & $76.92 \%$ & $23.08 \%$ \\
\hline Grade 2 A & & 41 & 0 & 41 & 22 & 19 & Grade 4 & $0.00 \%$ & $100.00 \%$ & $80.00 \%$ & $20.00 \%$ \\
\hline Grade 2 B & & 0 & 0 & 0 & 0 & 0 & Grade 5 & $0.00 \%$ & $100.00 \%$ & $75.00 \%$ & $25.00 \%$ \\
\hline Grade $3 \mathrm{~A}$ & & 26 & 0 & 26 & 20 & 6 & Grade 6 & \#Num! & \#Num! & \#Num! & \#Num! \\
\hline Grade 3 B & & 0 & 0 & 0 & 0 & 0 & & & & & \\
\hline Grade 4 & & 25 & 0 & 25 & 20 & 5 & Remarks: & & & & \\
\hline Grade 5 & & 16 & 0 & 16 & 12 & 4 & & & & & \\
\hline Grade 6 & & 0 & 0 & 0 & 0 & 0 & & & & & \\
\hline School Total & & 201 & 0 & 201 & 156 & 45 & School \%age & $0.00 \%$ & $100.00 \%$ & $77.61 \%$ & $22.39 \%$ \\
\hline
\end{tabular}

\begin{tabular}{|c|c|c|c|c|c|c|c|c|c|c|}
\hline \multirow[b]{2}{*}{$\begin{array}{l}\text { Grades I } \\
\text { Sections }\end{array}$} & \multicolumn{5}{|c|}{-Number of Students- } & \multirow[b]{2}{*}{ Grades } & \multicolumn{4}{|c|}{-Percent of Students } \\
\hline & Enrolled & Dropped & $\begin{array}{c}\text { Appeared } \\
\text { in Exam }\end{array}$ & $\begin{array}{c}\text { Passed in } \\
\text { Exams }\end{array}$ & $\begin{array}{c}\text { Failed in } \\
\text { Exams }\end{array}$ & & Dropped & $\begin{array}{c}\text { Appeared } \\
\text { in Exams }\end{array}$ & $\begin{array}{c}\text { Passed in } \\
\text { Exams }\end{array}$ & $\begin{array}{c}\text { Falled in } \\
\text { Exams } \\
\end{array}$ \\
\hline Grade $1 \mathrm{~A}$ & 32 & 0 & 32 & 31 & 1 & Grade 1 & $0.00 \%$ & $100.00 \%$ & $93.22 \%$ & $6.78 \%$ \\
\hline Grade $1 \mathrm{~B}$ & 27 & 0 & 27 & 24 & 3 & Grade 2 & $0.00 \%$ & $100.00 \%$ & $63.89 \%$ & $36.11 \%$ \\
\hline Grade $1 \mathrm{C}$ & 0 & 0 & 0 & 0 & 0 & Grade 3 & $0.00 \%$ & $100.00 \%$ & $63.33 \%$ & $36.67 \%$ \\
\hline Grade 2 A & 36 & 0 & 36 & 23 & 13 & Grade 4 & $10.53 \%$ & $89.47 \%$ & $64.71 \%$ & $35.29 \%$ \\
\hline Grade 2 B & 0 & 0 & 0 & 0 & 0 & Grade 5 & $4.76 \%$ & $95.24 \%$ & $55.00 \%$ & $45.00 \%$ \\
\hline Grade $3 \mathrm{~A}$ & 30 & 0 & 30 & 19 & 11 & Grade 6 & \#Num! & \#Num! & \#Num! & \#Num! \\
\hline Grade 3 B & 0 & 0 & 0 & 0 & 0 & & & & & \\
\hline Grade 4 & 19 & 2 & 17 & 11 & 6 & Remarks: & & & & \\
\hline Grade 5 & 21 & 1 & 20 & 11 & 9 & & & & & \\
\hline Grade 6 & 0 & 0 & 0 & 0 & 0 & & & & & \\
\hline School Total & 165 & 3 & 162 & 119 & 43 & School \%age & $1.82 \%$ & $98.18 \%$ & $73.46 \%$ & $26.54 \%$ \\
\hline
\end{tabular}


Sub-Centre: Peshawar

\begin{tabular}{|c|c|c|c|c|c|c|c|c|c|c|}
\hline \multirow[b]{2}{*}{$\begin{array}{l}\text { Grades / } \\
\text { Sections }\end{array}$} & \multicolumn{5}{|c|}{$\begin{array}{l}\text { School Title: ARPS Purkh Jalala - Peshawa } \\
\text { - Number of Students- }\end{array}$} & \multicolumn{5}{|c|}{ Head Teacher: Hekmat Ullah } \\
\hline & Enrolled & Dropped & $\begin{array}{l}\text { Appeared } \\
\text { in Exam }\end{array}$ & $\begin{array}{l}\text { Passed in } \\
\text { Exams }\end{array}$ & $\begin{array}{l}\text { Failed in } \\
\text { Exams }\end{array}$ & Grades & Dropped & $\begin{array}{l}\text { Appeared } \\
\text { in Exams }\end{array}$ & $\begin{array}{l}\text { Passed in } \\
\text { Exams }\end{array}$ & $\begin{array}{l}\text { Failed in } \\
\text { Exams }\end{array}$ \\
\hline Grade $1 \mathrm{~A}$ & 37 & 0 & 37 & 17 & 20 & Grade 1 & $0.00 \%$ & $100.00 \%$ & $45.95 \%$ & $54.05 \%$ \\
\hline Grade 1 B & 0 & 0 & 0 & 0 & 0 & Grade 2 & $0.00 \%$ & $100.00 \%$ & $61.54 \%$ & $38.46 \%$ \\
\hline Grade $1 \mathrm{C}$ & 0 & 0 & 0 & 0 & 0 & Grade 3 & $13.33 \%$ & $86.67 \%$ & $92.31 \%$ & $7.69 \%$ \\
\hline Grade 2 A & 26 & 0 & 26 & 16 & 10 & Grade 4 & $0.00 \%$ & $100.00 \%$ & $100.00 \%$ & $0.00 \%$ \\
\hline Grade 2 B & 0 & 0 & 0 & 0 & 0 & Grade 5 & $0.00 \%$ & $100.00 \%$ & $100.00 \%$ & $0.00 \%$ \\
\hline Grade 3 A & 15 & 2 & 13 & 12 & 1 & Grade 6 & \#Num! & \#Num! & \#Num! & \#Num! \\
\hline Grade 3 B & 0 & 0 & 0 & 0 & 0 & & & & & \\
\hline Grade 4 & 12 & 0 & 12 & 12 & 0 & Remarks: & & & & \\
\hline Grade 5 & 12 & 0 & 12 & 12 & 0 & & & & & \\
\hline Grade 6 & 0 & 0 & 0 & 0 & 0 & & & & & \\
\hline School Total & 102 & 2 & 100 & 69 & 31 & School \%age & $1.96 \%$ & $98.04 \%$ & $69.00 \%$ & $31.00 \%$ \\
\hline
\end{tabular}

Head Teacher: Sartaj Khan

\begin{tabular}{|c|c|c|c|c|c|c|c|c|c|c|c|}
\hline \multirow[b]{2}{*}{$\begin{array}{l}\text { Grades I } \\
\text { Sections }\end{array}$} & \multicolumn{6}{|c|}{ - Number of Students___ } & \multirow[b]{2}{*}{ Grades } & \multicolumn{4}{|c|}{-Percent of Students } \\
\hline & ' & Enrolled & Dropped & $\begin{array}{l}\text { Appeared } \\
\text { in Exam }\end{array}$ & $\begin{array}{c}\text { Passed in } \\
\text { Exams }\end{array}$ & $\begin{array}{c}\text { Failed in } \\
\text { Exams }\end{array}$ & & Dropped & $\begin{array}{c}\text { Appeared } \\
\text { in Exams }\end{array}$ & $\begin{array}{c}\text { Passed in } \\
\text { Exams }\end{array}$ & $\begin{array}{c}\text { Falled in } \\
\text { Exams }\end{array}$ \\
\hline Grade $1 \mathrm{~A}$ & & 65 & 0 & 65 & 51 & 14 & Grade 1 & $0.00 \%$ & $100.00 \%$ & $78.46 \%$ & $21.54 \%$ \\
\hline Grade 1 B & & 0 & 0 & 0 & 0 & 0 & Grade 2 & $0.00 \%$ & $100.00 \%$ & $78.95 \%$ & $21.05 \%$ \\
\hline Grade $1 \mathrm{C}$ & & 0 & 0 & 0 & 0 & 0 & Grade 3 & $0.00 \%$ & $100.00 \%$ & $65.71 \%$ & $34.29 \%$ \\
\hline Grade $2 \mathrm{~A}$ & & 38 & 0 & 38 & 30 & 8 & Grade 4 & $0.00 \%$ & $100.00 \%$ & $100.00 \%$ & $0.00 \%$ \\
\hline Grade 2 B & & 0 & 0 & 0 & 0 & 0 & Grade 5 & $18.52 \%$ & $81.48 \%$ & $68.18 \%$ & $31.82 \%$ \\
\hline Grade $3 \mathrm{~A}$ & & 55 & 0 & 35 & 23 & 12 & Grade 6 & \#Num! & \#Num! & \#Num! & \#Num! \\
\hline Grade 3 B & & 0 & 0 & 0 & 0 & 0 & & & & & \\
\hline Grade 4 & & 23 & 0 & 23 & 23 & 0 & Remarks: & & & & \\
\hline Grade 5 & & 27 & 5 & 22 & 15 & 7 & & & & & \\
\hline Grade 6 & & 0 & 0 & 0 & 0 & 0 & & & & & \\
\hline School Total & & 188 & 5 & 183 & 142 & 41 & School \%age & $2.66 \%$ & $97.34 \%$ & $77.60 \%$ & $22.40 \%$ \\
\hline
\end{tabular}

\begin{tabular}{|c|c|c|c|c|c|c|c|c|c|c|}
\hline \multirow[b]{2}{*}{$\begin{array}{l}\text { Grades I } \\
\text { Sections }\end{array}$} & \multicolumn{5}{|c|}{ _Number of Students- } & \multirow[b]{2}{*}{ Grades } & \multicolumn{4}{|c|}{ Percent of Students } \\
\hline & Enrolled & Dropped & $\begin{array}{l}\text { Appeared } \\
\text { in Exam }\end{array}$ & $\begin{array}{l}\text { Passed in } \\
\text { Exams }\end{array}$ & $\begin{array}{l}\text { Failed in } \\
\text { Exams }\end{array}$ & & Dropped & $\begin{array}{l}\text { Appeared } \\
\text { in Exams }\end{array}$ & $\begin{array}{l}\text { Passed in } \\
\text { Exams }\end{array}$ & $\begin{array}{l}\text { Falled in } \\
\text { Exams }\end{array}$ \\
\hline Grade $1 \mathrm{~A}$ & 60 & 0 & 60 & 40 & 20 & Grade 1 & $0.00 \%$ & $100.00 \%$ & $55.00 \%$ & $45.00 \%$ \\
\hline Grade 1 B & 60 & 0 & 60 & 26 & 34 & Grade 2 & $0.00 \%$ & $100.00 \%$ & $53.33 \%$ & $46.67 \%$ \\
\hline Grade $1 \mathrm{C}$ & 0 & 0 & 0 & 0 & 0 & Grade 3 & $5.13 \%$ & $94.87 \%$ & $83.78 \%$ & $16.22 \%$ \\
\hline Grade $2 \mathrm{~A}$ & 75 & 0 & 75 & 40 & 35 & Grade 4 & $0.00 \%$ & $100.00 \%$ & $80.95 \%$ & $19.05 \%$ \\
\hline Grade 2 B & 0 & 0 & 0 & 0 & 0 & Grade 5 & $0.00 \%$ & $100.00 \%$ & $57.14 \%$ & $42.86 \%$ \\
\hline Grade $3 \mathrm{~A}$ & 39 & 2 & 37 & 31 & 6 & Grade 6 & $0.00 \%$ & $100.00 \%$ & $66.67 \%$ & $33.33 \%$ \\
\hline Grade 3 B & 0 & 0 & 0 & 0 & 0 & & & & & \\
\hline Grade 4 & 21 & 0 & 21 & 17 & 4 & Remarks: & & & & \\
\hline Grade 5 & 14 & 0 & 14 & 8 & 6 & & & & & \\
\hline Grade 6 & 9 & 0 & 9 & 6 & 3 & & & & & \\
\hline School Total & 278 & 2 & 276 & 168 & 108 & School \%age & $0.72 \%$ & $99.28 \%$ & $60.87 \%$ & $39.13 \%$ \\
\hline
\end{tabular}


Sub-Centre: Peshawar

\begin{tabular}{|c|c|c|c|c|c|c|c|c|c|c|}
\hline \multirow[b]{2}{*}{$\begin{array}{l}\text { Grades I } \\
\text { Sections }\end{array}$} & \multicolumn{5}{|c|}{ - Number of Students- } & \multirow[b]{2}{*}{ Grades } & \multicolumn{4}{|c|}{-Percent of Students- } \\
\hline & Enrolled & Dropped & $\begin{array}{l}\text { Appeared } \\
\text { in Exam }\end{array}$ & $\begin{array}{l}\text { Passed in } \\
\text { Exams }\end{array}$ & $\begin{array}{l}\text { Failed in } \\
\text { Exams }\end{array}$ & & Dropped & $\begin{array}{l}\text { Appeared } \\
\text { in Exams }\end{array}$ & $\begin{array}{l}\text { Passed in } \\
\text { Exams }\end{array}$ & $\begin{array}{l}\text { Failed in } \\
\text { Exams }\end{array}$ \\
\hline Grade $1 \mathrm{~A}$ & 105 & 11 & 94 & 47 & 47 & Grade 1 & $10.48 \%$ & $89.52 \%$ & $50.00 \%$ & $50.00 \%$ \\
\hline Grade 1 B & 0 & 0 & 0 & 0 & 0 & Grade 2 & $14.71 \%$ & $85.29 \%$ & $82.76 \%$ & $17.24 \%$ \\
\hline Grade $1 \mathrm{C}$ & 0 & 0 & 0 & 0 & 0 & Grade 3 & $7.14 \%$ & $92.86 \%$ & $100.00 \%$ & $0.00 \%$ \\
\hline Grade 2 A & 34 & 5 & 29 & 24 & 5 & Grade 4 & $0.00 \%$ & $100.00 \%$ & $100.00 \%$ & $0.00 \%$ \\
\hline Grade 2 B & 0 & 0 & 0 & 0 & 0 & Grade 5 & $10.00 \%$ & $90.00 \%$ & $100.00 \%$ & $0.00 \%$ \\
\hline Grade 3 A & 28 & 2 & 26 & 26 & 0 & Grade 6 & $7.14 \%$ & $92.86 \%$ & $100.00 \%$ & $0.00 \%$ \\
\hline Grade 3 B & 0 & 0 & 0 & 0 & 0 & & & & & \\
\hline Grade 4 & 15 & 0 & 15 & 15 & 0 & Remarks: & & & & \\
\hline Grade 5 & 20 & 2 & 18 & 18 & 0 & & & & & \\
\hline Grade 6 & 14 & 1 & 13 & 13 & 0 & & & & & \\
\hline School Total & 216 & 21 & 195 & 143 & 52 & School \%age & $9.72 \%$ & $90.28 \%$ & $73.33 \%$ & $26.67 \%$ \\
\hline
\end{tabular}

\begin{tabular}{|c|c|c|c|c|c|c|c|c|c|c|c|}
\hline \multirow[b]{2}{*}{$\begin{array}{l}\text { Grades I } \\
\text { Sections }\end{array}$} & \multicolumn{6}{|c|}{ - Number of Students- } & \multirow[b]{2}{*}{ Grades } & \multicolumn{4}{|c|}{-Percent of Students } \\
\hline & ' & Enrolled & Dropped & $\begin{array}{l}\text { Appeared } \\
\text { in Exam }\end{array}$ & $\begin{array}{l}\text { Passed in } \\
\text { Exams }\end{array}$ & $\begin{array}{l}\text { Failed in } \\
\text { Exams }\end{array}$ & & Dropped & $\begin{array}{l}\text { Appeared } \\
\text { in Exams }\end{array}$ & $\begin{array}{l}\text { Passed in } \\
\text { Exams }\end{array}$ & $\begin{array}{l}\text { Failed in } \\
\text { Exams }\end{array}$ \\
\hline Grade $1 \mathrm{~A}$ & & 59 & 9 & 50 & 29 & 21 & Grade 1 & $13.04 \%$ & $86.96 \%$ & $61.00 \%$ & $39.00 \%$ \\
\hline Grade 1 B & & 56 & 6 & 50 & 32 & 18 & Grade 2 & $14.81 \%$ & $85.19 \%$ & $60.87 \%$ & $39.13 \%$ \\
\hline Grade $1 \mathrm{C}$ & & 0 & 0 & 0 & 0 & 0 & Grade 3 & $18.75 \%$ & $81.25 \%$ & $92.31 \%$ & $7.69 \%$ \\
\hline Grade 2 A & & 54 & 8 & 46 & 28 & 18 & Grade 4 & $12.90 \%$ & $87.10 \%$ & $66.67 \%$ & $33.33 \%$ \\
\hline Grade 2 B & & 0 & 0 & 0 & 0 & 0 & Grade 5 & $16.67 \%$ & $83.33 \%$ & $100.00 \%$ & $0.00 \%$ \\
\hline Grade 3 A & & 32 & 6 & 26 & 24 & 2 & Grade 6 & \#Num! & \#Num! & \#Num! & \#Num! \\
\hline Grade 3 B & & 0 & 0 & 0 & 0 & 0 & & & & & \\
\hline Grade 4 & & 31 & 4 & 27 & 18 & 9 & Remarks: & & & & \\
\hline Grade 5 & & 12 & 2 & 10 & 10 & 0 & & & & & \\
\hline Grade 6 & & 0 & 0 & 0 & 0 & 0 & & & & & \\
\hline School Total & & 244 & 35 & 209 & 141 & 68 & School \%age & $14.34 \%$ & $85.66 \%$ & $67.46 \%$ & $32.54 \%$ \\
\hline
\end{tabular}

\begin{tabular}{|c|c|c|c|c|c|c|c|c|c|c|}
\hline \multirow[b]{2}{*}{$\begin{array}{l}\text { Grades I } \\
\text { Sections }\end{array}$} & \multicolumn{5}{|c|}{ _Number of Students__ } & \multirow[b]{2}{*}{ Grades } & \multicolumn{4}{|c|}{ Percent of Students } \\
\hline & Enrolled & Dropped & $\begin{array}{l}\text { Appeared } \\
\text { in Exam }\end{array}$ & $\begin{array}{l}\text { Passed in } \\
\text { Exams }\end{array}$ & $\begin{array}{c}\text { Failed in } \\
\text { Exams }\end{array}$ & & Dropped & $\begin{array}{l}\text { Appeared } \\
\text { in Exams }\end{array}$ & $\begin{array}{c}\text { Passed in } \\
\text { Exams } \\
\end{array}$ & $\begin{array}{c}\text { Failed in } \\
\text { Exams }\end{array}$ \\
\hline Grade $1 \mathrm{~A}$ & 50 & 0 & 50 & 32 & 18 & Grade 1 & $0.00 \%$ & $100.00 \%$ & $64.00 \%$ & $36.00 \%$ \\
\hline Grade 1 B & 0 & 0 & 0 & 0 & 0 & Grade 2 & $0.00 \%$ & $100.00 \%$ & $69.09 \%$ & $30.91 \%$ \\
\hline Grade $1 \mathrm{C}$ & 0 & 0 & 0 & 0 & 0 & Grade 3 & $0.00 \%$ & $100.00 \%$ & $54.29 \%$ & $45.71 \%$ \\
\hline Grade $2 \mathrm{~A}$ & 55 & 0 & 55 & 38 & 17 & Grade 4 & $0.00 \%$ & $100.00 \%$ & $60.00 \%$ & $40.00 \%$ \\
\hline Grade 2 B & 0 & 0 & 0 & 0 & 0 & Grade 5 & $0.00 \%$ & $100.00 \%$ & $92.86 \%$ & $7.14 \%$ \\
\hline Grade $3 \mathrm{~A}$ & 35 & 0 & 35 & 19 & 16 & Grade 6 & \#Num! & $\#$ Num! & \#Num! & \#Num! \\
\hline Grade 3 B & 0 & 0 & 0 & 0 & 0 & & & & & \\
\hline Grade 4 & 20 & 0 & 20 & 12 & 8 & Remarks: & & & & \\
\hline Grade 5 & 14 & 0 & 14 & 13 & 1 & & & & & \\
\hline Grade 6 & 0 & 0 & 0 & 0 & 0 & & & & & \\
\hline School Total & 174 & 0 & 174 & 114 & 60 & School \%age & $0.00 \%$ & $100.00 \%$ & $65.52 \%$ & $34.48 \%$ \\
\hline
\end{tabular}


Sub-Centre: Peshawar

\begin{tabular}{|c|c|c|c|c|c|c|c|c|c|c|}
\hline \multirow[b]{2}{*}{$\begin{array}{l}\text { Grades / } \\
\text { Sections }\end{array}$} & \multicolumn{5}{|c|}{ - Number of Students- } & \multirow[b]{2}{*}{ Grades } & \multicolumn{4}{|c|}{-Percent of Students } \\
\hline & Enrolled & Dropped & $\begin{array}{l}\text { Appeared } \\
\text { in Exam }\end{array}$ & $\begin{array}{l}\text { Passed in } \\
\text { Exams }\end{array}$ & $\begin{array}{l}\text { Failed in } \\
\text { Exams }\end{array}$ & & Dropped & $\begin{array}{l}\text { Appeared } \\
\text { in Exams }\end{array}$ & $\begin{array}{l}\text { Passed in } \\
\text { Exams }\end{array}$ & $\begin{array}{c}\text { Failed in } \\
\text { Exams }\end{array}$ \\
\hline Grade $1 \mathrm{~A}$ & 62 & 1 & 61 & 46 & 15 & Grade 1 & $2.59 \%$ & $97.41 \%$ & $76.99 \%$ & $23.01 \%$ \\
\hline Grade 1 B & 54 & 2 & 52 & 41 & 11 & Grade 2 & $2.56 \%$ & $97.44 \%$ & $84.21 \%$ & $15.79 \%$ \\
\hline Grade $1 \mathrm{C}$ & 0 & 0 & 0 & 0 & 0 & Grade 3 & $9.52 \%$ & $90.48 \%$ & $81.58 \%$ & $18.42 \%$ \\
\hline Grade 2 A & 41 & 0 & 41 & 32 & 9 & Grade 4 & $2.78 \%$ & $97.22 \%$ & $68.57 \%$ & $31.43 \%$ \\
\hline Grade 2 B & 37 & 2 & 35 & 32 & 3 & Grade 5 & $0.00 \%$ & $100.00 \%$ & $96.00 \%$ & $4.00 \%$ \\
\hline Grade 3 A & 42 & 4 & 38 & 31 & 7 & Grade 6 & $0.00 \%$ & $100.00 \%$ & $93.33 \%$ & $6.67 \%$ \\
\hline Grade 3 B & 0 & 0 & 0 & 0 & 0 & & & & & \\
\hline Grade 4 & 36 & 1 & 35 & 24 & 11 & Remarks: & & & & \\
\hline Grade 5 & 25 & 0 & 25 & 24 & 1 & & & & & \\
\hline Grade 6 & 15 & 0 & 15 & 14 & 1 & & & & & \\
\hline School Total & 312 & 10 & 302 & 244 & 58 & School \%age & $3.21 \%$ & $96.79 \%$ & $80.79 \%$ & $19.21 \%$ \\
\hline
\end{tabular}

Head Teacher: Abdul Sami

\begin{tabular}{|c|c|c|c|c|c|c|c|c|c|c|}
\hline \multirow[b]{2}{*}{$\begin{array}{l}\text { Grades / } \\
\text { Sections }\end{array}$} & \multicolumn{5}{|c|}{$\longrightarrow$ Number of Students } & \multirow[b]{2}{*}{ Grades } & \multicolumn{4}{|c|}{-Percent of Students } \\
\hline & Enrolled & Dropped & $\begin{array}{l}\text { Appeared } \\
\text { in Exam }\end{array}$ & $\begin{array}{l}\text { Passed in } \\
\text { Exams }\end{array}$ & $\begin{array}{l}\text { Failed in } \\
\text { Exams }\end{array}$ & & Dropped & $\begin{array}{l}\text { Appeared } \\
\text { in Exams }\end{array}$ & $\begin{array}{l}\text { Passed in } \\
\text { Exams }\end{array}$ & $\begin{array}{r}\text { Failed in } \\
\text { Exams }\end{array}$ \\
\hline Grade $1 \mathrm{~A}$ & 97 & 7 & 90 & 50 & 40 & Grade 1 & $7.22 \%$ & $92.78 \%$ & $55.56 \%$ & $44.44 \%$ \\
\hline Grade 1 B & 0 & 0 & 0 & 0 & 0 & Grade 2 & $4.00 \%$ & $96.00 \%$ & $73.61 \%$ & $26.39 \%$ \\
\hline Grade $1 \mathrm{C}$ & 0 & 0 & 0 & 0 & 0 & Grade 3 & $5.88 \%$ & $94.12 \%$ & $81.25 \%$ & $18.75 \%$ \\
\hline Grade 2 A & 75 & 3 & 72 & 53 & 19 & Grade 4 & $6.45 \%$ & $93.55 \%$ & $72.41 \%$ & $27.59 \%$ \\
\hline Grade 2 B & 0 & 0 & 0 & 0 & 0 & Grade 5 & $7.69 \%$ & $92.31 \%$ & $91.67 \%$ & $8.33 \%$ \\
\hline Grade 3 A & 34 & 2 & 32 & 26 & 6 & Grade 6 & $10.00 \%$ & $90.00 \%$ & $88.89 \%$ & $11.11 \%$ \\
\hline Grade 3 B & 0 & 0 & 0 & 0 & 0 & & & & & \\
\hline Grade 4 & 31 & 2 & 29 & 21 & 8 & Remarks: & & & & \\
\hline Grade 5 & 13 & 1 & 12 & 11 & 1 & & & & & \\
\hline Grade 6 & 10 & 1 & 9 & 8 & 1 & & & & & \\
\hline nool Total & 260 & 16 & 244 & 169 & 75 & School \%age & $6.15 \%$ & $93.85 \%$ & $69.26 \%$ & $30.74 \%$ \\
\hline
\end{tabular}

\begin{tabular}{|c|c|c|c|c|c|c|c|c|c|c|}
\hline \multirow[b]{2}{*}{$\begin{array}{l}\text { Grades I } \\
\text { Sections }\end{array}$} & \multicolumn{5}{|c|}{ - Number of Students- } & \multirow[b]{2}{*}{ Grades } & \multicolumn{4}{|c|}{ Percent of Students } \\
\hline & Enrolled & Dropped & $\begin{array}{l}\text { Appeared } \\
\text { in Exam }\end{array}$ & $\begin{array}{l}\text { Passed in } \\
\text { Exams }\end{array}$ & $\begin{array}{l}\text { Failed in } \\
\text { Exams }\end{array}$ & & Dropped & $\begin{array}{l}\text { Appeared } \\
\text { in Exams }\end{array}$ & $\begin{array}{l}\text { Passed in } \\
\text { Exams }\end{array}$ & $\begin{array}{c}\text { Fatied in } \\
\text { Exams }\end{array}$ \\
\hline Grade $1 \mathrm{~A}$ & 51 & 5 & 46 & 14 & 32 & Grade 1 & $4.81 \%$ & $95.19 \%$ & $47.47 \%$ & $52.53 \%$ \\
\hline Grade 1 B & 53 & 0 & 53 & 33 & 20 & Grade 2 & $27.50 \%$ & $72.50 \%$ & $62.07 \%$ & $37.93 \%$ \\
\hline Grade $1 \mathrm{C}$ & 0 & 0 & 0 & 0 & 0 & Grade 3 & $17.50 \%$ & $82.50 \%$ & $57.58 \%$ & $42.42 \%$ \\
\hline Grade 2 A & 40 & 11 & 29 & 18 & 11 & Grade 4 & $22.22 \%$ & $77.78 \%$ & $71.43 \%$ & $28.57 \%$ \\
\hline Grade 2 B & 0 & 0 & 0 & 0 & 0 & Grade 5 & $33.33 \%$ & $66.67 \%$ & $66.67 \%$ & $33.33 \%$ \\
\hline Grade 3 A & 40 & 7 & 33 & 19 & 14 & Grade 6 & $16.67 \%$ & $83.33 \%$ & $50.00 \%$ & $50.00 \%$ \\
\hline Grade 3 B & 0 & 0 & 0 & 0 & 0 & & & & & \\
\hline Grade 4 & 18 & 4 & 14 & 10 & 4 & Remarks: & & & & \\
\hline Grade 5 & 9 & 3 & 6 & 4 & 2 & & & & & \\
\hline Grade 6 & 12 & 2 & 10 & 5 & 5 & & & & & . \\
\hline School Total & 223 & 32 & 191 & 103 & 88 & School \%age & $14.35 \%$ & $85.65 \%$ & $53.93 \%$ & $46.07 \%$ \\
\hline
\end{tabular}


Sub-Centre: Peshawar

\begin{tabular}{|c|c|c|c|c|c|c|c|c|c|c|}
\hline \multirow[b]{2}{*}{$\begin{array}{l}\text { Grades I } \\
\text { Sections }\end{array}$} & \multicolumn{5}{|c|}{ - Number of Students } & \multirow[b]{2}{*}{ Grades } & \multicolumn{4}{|c|}{ Percent of Students } \\
\hline & Enrolled & Dropped & $\begin{array}{l}\text { Appeared } \\
\text { in Exam }\end{array}$ & $\begin{array}{l}\text { Passed in } \\
\text { Exams }\end{array}$ & $\begin{array}{c}\text { Failed in } \\
\text { Exams }\end{array}$ & & Dropped & $\begin{array}{l}\text { Appeared } \\
\text { in Exams }\end{array}$ & $\begin{array}{l}\text { Passed in } \\
\text { Exams }\end{array}$ & $\begin{array}{l}\text { Failed in } \\
\text { Exams }\end{array}$ \\
\hline Grade $1 \mathrm{~A}$ & 62 & 1 & 61 & 46 & 15 & Grade 1 & $2.59 \%$ & $97.41 \%$ & $76.99 \%$ & $23.01 \%$ \\
\hline Grade 1 B & 54 & 2 & 52 & 41 & 11 & Grade 2 & $2.56 \%$ & $97.44 \%$ & $84.21 \%$ & $15.79 \%$ \\
\hline Grade $1 \mathrm{C}$ & 0 & 0 & 0 & 0 & 0 & Grade 3 & $9.52 \%$ & $90.48 \%$ & $81.58 \%$ & $18.42 \%$ \\
\hline Grade 2 A & 41 & 0 & 41 & 32 & 9 & Grade 4 & $2.78 \%$ & $97.22 \%$ & $68.57 \%$ & $31.43 \%$ \\
\hline Grade 2 B & 37 & 2 & 35 & 32 & 3 & Grade 5 & $0.00 \%$ & $100.00 \%$ & $96.00 \%$ & $4.00 \%$ \\
\hline Grade $3 \mathrm{~A}$ & 42 & 4 & 38 & 31 & 7 & Grade 6 & $0.00 \%$ & $100.00 \%$ & $93.33 \%$ & $6.67 \%$ \\
\hline Grade 3 B & 0 & 0 & 0 & 0 & 0 & & & & & \\
\hline Grade 4 & 36 & 1 & 35 & 24 & 11 & Remarks: & & & & \\
\hline Grade 5 & 25 & 0 & 25 & 24 & 1 & & & & & \\
\hline Grade 6 & 15 & 0 & 15 & 14 & 1 & & & & & \\
\hline School Total & 312 & 10 & 302 & 244 & 58 & School \%age & $3.21 \%$ & $96.79 \%$ & $80.79 \%$ & $19.21 \%$ \\
\hline
\end{tabular}

School Code: PSIPSHIPSB-139 School Title: ARPS M Azakhil Bala - Peshawar

Head Teacher: Abdullah

\begin{tabular}{|c|c|c|c|c|c|c|c|c|c|c|c|}
\hline \multirow[b]{2}{*}{$\begin{array}{l}\text { Grades I } \\
\text { Sections }\end{array}$} & \multicolumn{6}{|c|}{ - Number of Students- } & \multirow[b]{2}{*}{ Grades } & \multicolumn{4}{|c|}{-Percent of Students } \\
\hline & $\prime$ & Enrolled & Dropped & $\begin{array}{l}\text { Appeared } \\
\text { in Exam }\end{array}$ & $\begin{array}{c}\text { Passed in } \\
\text { Exams }\end{array}$ & $\begin{array}{l}\text { Failed in } \\
\text { Exams }\end{array}$ & & Dropped & $\begin{array}{l}\text { Appeared } \\
\text { in Exams }\end{array}$ & $\begin{array}{c}\text { Passed in } \\
\text { Exams }\end{array}$ & $\begin{array}{l}\text { Falled in } \\
\text { Exams }\end{array}$ \\
\hline Grade $1 \mathrm{~A}$ & & 56 & 0 & 56 & 26 & 30 & Grade 1 & $0.00 \%$ & $100.00 \%$ & $40.18 \%$ & $59.82 \%$ \\
\hline Grade $1 \mathrm{~B}$ & & 56 & 0 & 56 & 19 & 37 & Grade 2 & $15.48 \%$ & $84.52 \%$ & $64.79 \%$ & $35.21 \%$ \\
\hline Grade $1 \mathrm{C}$ & & 0 & 0 & 0 & 0 & 0 & Grade 3 & $2.00 \%$ & $98.00 \%$ & $83.67 \%$ & $16.33 \%$ \\
\hline Grade 2 A & & 42 & 4 & 38 & 24 & 14 & Grade 4 & $0.00 \%$ & $100.00 \%$ & $95.65 \%$ & $4.35 \%$ \\
\hline Grade 2 B & & 42 & 9 & 33 & 22 & 11 & Grade 5 & $11.11 \%$ & $88.89 \%$ & $100.00 \%$ & $0.00 \%$ \\
\hline Grade $3 \mathrm{~A}$ & & 50 & 1 & 49 & 41 & 8 & Grade 6 & $0.00 \%$ & $100.00 \%$ & $100.00 \%$ & $0.00 \%$ \\
\hline Grade 3 B & & 0 & 0 & 0 & 0 & 0 & & & & & \\
\hline Grade 4 & & 23 & 0 & 23 & 22 & 1 & Remarks: & & & & \\
\hline Grade 5 & & 18 & 2 & 16 & 16 & 0 & & & & & \\
\hline Grade 6 & & 21 & 0 & 21 & 21 & 0 & & & & & \\
\hline School Total & & 308 & 16 & 292 & 191 & 101 & School \%age & $5.19 \%$ & $94.81 \%$ & $65.41 \%$ & $34.59 \%$ \\
\hline
\end{tabular}

\begin{tabular}{|c|c|c|c|c|c|c|c|c|c|c|}
\hline \multirow[b]{2}{*}{$\begin{array}{l}\text { Grades / } \\
\text { Sections }\end{array}$} & \multicolumn{5}{|c|}{-Number of Students- } & \multirow[b]{2}{*}{ Grades } & \multicolumn{4}{|c|}{-Percent of Students } \\
\hline & Enrolled & Dropped & $\begin{array}{l}\text { Appeared } \\
\text { in Exam }\end{array}$ & $\begin{array}{r}\text { Passed in } \\
\text { Exams }\end{array}$ & $\begin{array}{l}\text { Failed in } \\
\text { Exams }\end{array}$ & & Dropped & $\begin{array}{l}\text { Appeared } \\
\text { in Exams }\end{array}$ & $\begin{array}{l}\text { Passed in } \\
\text { Exams }\end{array}$ & $\begin{array}{l}\text { Faled in } \\
\text { Exams }\end{array}$ \\
\hline Grade $1 \mathrm{~A}$ & 50 & 6 & 44 & 40 & 4 & Grade 1 & $12.00 \%$ & $88.00 \%$ & $70.45 \%$ & $29.55 \%$ \\
\hline Grade 1 B & 50 & 6 & 44 & 22 & 22 & Grade 2 & $3.33 \%$ & $96.67 \%$ & $60.34 \%$ & $39.66 \%$ \\
\hline Grade $1 \mathrm{C}$ & 0 & 0 & 0 & 0 & 0 & Grade 3 & $12.50 \%$ & $87.50 \%$ & $51.43 \%$ & $48.57 \%$ \\
\hline Grade $2 \mathrm{~A}$ & 60 & 2 & 58 & 35 & 23 & Grade 4 & $23.08 \%$ & $76.92 \%$ & $75.00 \%$ & $25.00 \%$ \\
\hline Grade 2 B & 0 & 0 & 0 & 0 & 0 & Grade 5 & $25.81 \%$ & $74.19 \%$ & $73.91 \%$ & $26.09 \%$ \\
\hline Grade 3 A & 40 & 5 & 35 & 18 & 17 & Grade 6 & $11.76 \%$ & $88.24 \%$ & $100.00 \%$ & $0.00 \%$ \\
\hline Grade 3 B & 0 & 0 & 0 & 0 & 0 & & & & & \\
\hline Grade 4 & 26 & 6 & 20 & 15 & 5 & Remarks: & & & & \\
\hline Grade 5 & 31 & 8 & 23 & 17 & 6 & & & & & \\
\hline Grade 6 & 17 & 2 & 15 & 15 & 0 & & & & & \\
\hline School Total & 274 & 35 & 239 & 162 & 77 & School \%age & $12.77 \%$ & $87.23 \%$ & $67.78 \%$ & $32.22 \%$ \\
\hline
\end{tabular}


Sub-Centre: Peshawar

\begin{tabular}{|c|c|c|c|c|c|c|c|c|c|c|}
\hline \multirow[b]{2}{*}{$\begin{array}{l}\text { Grades / } \\
\text { Sections }\end{array}$} & \multicolumn{5}{|c|}{-Number of Students- } & \multirow[b]{2}{*}{ Grades } & \multicolumn{4}{|c|}{ Percent of Students } \\
\hline & Enrolled & Dropped & $\begin{array}{c}\text { Appeared } \\
\text { in Exam }\end{array}$ & $\begin{array}{c}\text { Passed in } \\
\text { Exams }\end{array}$ & $\begin{array}{c}\text { Failed in } \\
\text { Exams }\end{array}$ & & Dropped & $\begin{array}{c}\text { Appeared } \\
\text { in Exams }\end{array}$ & $\begin{array}{c}\text { Passed in } \\
\text { Exams }\end{array}$ & $\begin{array}{l}\text { Falled in } \\
\text { Exams }\end{array}$ \\
\hline Grade $1 \mathrm{~A}$ & 73 & 12 & 61 & 46 & 15 & Grade 1 & $14.06 \%$ & $85.94 \%$ & $73.33 \%$ & $26.67 \%$ \\
\hline Grade $1 \mathrm{~B}$ & 59 & 6 & 53 & 38 & 15 & Grade 2 & $14.29 \%$ & $85.71 \%$ & $79.63 \%$ & $20.37 \%$ \\
\hline Grade $1 \mathrm{C}$ & 60 & 9 & 51 & 37 & 14 & Grade 3 & $14.81 \%$ & $85.19 \%$ & $82.61 \%$ & $17.39 \%$ \\
\hline Grade $2 \mathrm{~A}$ & 70 & 10 & 60 & 48 & 12 & Grade 4 & $13.51 \%$ & $86.49 \%$ & $100.00 \%$ & $0.00 \%$ \\
\hline Grade 2 B & 56 & 8 & 48 & 38 & 10 & Grade 5 & $4.55 \%$ & $95.45 \%$ & $100.00 \%$ & $0.00 \%$ \\
\hline Grade $3 \mathrm{~A}$ & 78 & 9 & 69 & 57 & 12 & Grade 6 & \#Num! & \#Num! & \#Num! & \#Num! \\
\hline Grade 3 B & 3 & 3 & 0 & 0 & 0 & & & & & \\
\hline Grade 4 & 37 & 5 & 32 & 32 & 0 & Remarks: & & & & \\
\hline Grade 5 & 22 & 1 & 21 & 21 & 0 & & & & & \\
\hline Grade 6 & 0 & 0 & 0 & 0 & 0 & & & & & \\
\hline School Total & 458 & 63 & 395 & 317 & 78 & School \%age & $13.76 \%$ & $86.24 \%$ & $80.25 \%$ & $19.75 \%$ \\
\hline
\end{tabular}

\begin{tabular}{|c|c|c|c|c|c|c|c|c|c|c|}
\hline \multirow[b]{2}{*}{$\begin{array}{l}\text { Grades I } \\
\text { Sections }\end{array}$} & \multicolumn{5}{|c|}{-Number of Students- } & \multirow[b]{2}{*}{ Grades } & \multicolumn{4}{|c|}{ Percent of Students } \\
\hline & ' Enrolled & Dropped & $\begin{array}{l}\text { Appeared } \\
\text { in Exam }\end{array}$ & $\begin{array}{r}\text { Passed in } \\
\text { Exams }\end{array}$ & $\begin{array}{l}\text { Failed in } \\
\text { Exams }\end{array}$ & & Dropped & $\begin{array}{l}\text { Appeared } \\
\text { in Exams }\end{array}$ & $\begin{array}{r}\text { Passed in } \\
\text { Exams }\end{array}$ & $\begin{array}{c}\text { Failed in } \\
\text { Exams }\end{array}$ \\
\hline Grade $1 \mathrm{~A}$ & 60 & 2 & 58 & 55 & 3 & Grade 1 & $16.49 \%$ & $83.51 \%$ & $55.56 \%$ & $44.44 \%$ \\
\hline Grade 1 B & 64 & 5 & 59 & 30 & 29 & Grade 2 & $9.72 \%$ & $90.28 \%$ & $76.92 \%$ & $23.08 \%$ \\
\hline Grade $1 \mathrm{C}$ & 70 & 25 & 45 & 5 & 40 & Grade 3 & $0.00 \%$ & $100.00 \%$ & $85.19 \%$ & $14.81 \%$ \\
\hline Grade $2 \mathrm{~A}$ & 38 & 3 & 35 & 27 & 8 & Grade 4 & $8.33 \%$ & $91.67 \%$ & $95.45 \%$ & $4.55 \%$ \\
\hline Grade 2 B & 34 & 4 & 30 & 23 & 7 & Grade 5 & $30.00 \%$ & $70.00 \%$ & $100.00 \%$ & $0.00 \%$ \\
\hline Grade $3 \mathrm{~A}$ & 27 & 0 & 27 & 23 & 4 & Grade 6 & \#Num! & \#Num! & \#Num! & \#Num! \\
\hline Grade 3 B & 0 & 0 & 0 & 0 & 0 & & & & & \\
\hline Grade 4 & 24 & 2 & 22 & 21 & 1 & Remarks: & & & & \\
\hline Grade 5 & 10 & 3 & 7 & 7 & 0 & & & & & \\
\hline Grade 6 & 0 & 0 & 0 & 0 & 0 & & & & & \\
\hline School Total & 327 & 44 & 283 & 191 & 92 & School \%age & $13.46 \%$ & $86.54 \%$ & $67.49 \%$ & $32.51 \%$ \\
\hline
\end{tabular}

\begin{tabular}{|c|c|c|c|c|c|c|c|c|c|c|}
\hline \multirow[t]{2}{*}{ School Code: P } & \multicolumn{5}{|c|}{ — Number of Students_ } & \multirow[b]{2}{*}{ Grades } & \multirow{2}{*}{\multicolumn{3}{|c|}{$\begin{array}{l}\text {-Percent of Students } \\
\text { Appeared Passed in }\end{array}$}} & \multirow[b]{2}{*}{$\begin{array}{l}\text { Falied in } \\
\text { Exams }\end{array}$} \\
\hline & Enrolled & Dropped & $\begin{array}{l}\text { Appeared } \\
\text { in Exam }\end{array}$ & $\begin{array}{l}\text { Passed in } \\
\text { Exams }\end{array}$ & $\begin{array}{c}\text { Failed in } \\
\text { Exams }\end{array}$ & & & & & \\
\hline Grade $1 \mathrm{~A}$ & 64 & 3 & 61 & 37 & 24 & Grade 1 & $2.46 \%$ & $97.54 \%$ & $56.30 \%$ & $43.70 \%$ \\
\hline Grade 1 B & 58 & 0 & 58 & 30 & 28 & Grade 2 & $0.00 \%$ & $100.00 \%$ & $58.67 \%$ & $41.33 \%$ \\
\hline Grade $1 \mathrm{C}$ & 0 & 0 & 0 & 0 & 0 & Grade 3 & $0.00 \%$ & $100.00 \%$ & $67.31 \%$ & $32.69 \%$ \\
\hline Grade $2 \mathrm{~A}$ & 75 & 0 & 75 & 44 & 31 & Grade 4 & $0.00 \%$ & $100.00 \%$ & $48.98 \%$ & $51.02 \%$ \\
\hline Grade 2 B & 0 & 0 & 0 & 0 & 0 & Grade 5 & $0.00 \%$ & $100.00 \%$ & $50.00 \%$ & $50.00 \%$ \\
\hline Grade 3 A & 52 & 0 & 52 & 35 & 17 & Grade 6 & $3.85 \%$ & $96.15 \%$ & $68.00 \%$ & $32.00 \%$ \\
\hline Grade 3 B & 0 & 0 & 0 & 0 & 0 & & & & & \\
\hline Grade 4 & 49 & 0 & 49 & 24 & 25 & Remarks: & & & & \\
\hline Grade 5 & 28 & 0 & 28 & 14 & 14 & & & & & \\
\hline Grade 6 & 26 & 1 & 25 & 17 & 8 & & & & & \\
\hline School Total & 352 & 4 & 348 & 201 & 147 & School \%age & $1.14 \%$ & $98.86 \%$ & $57.76 \%$ & $42.24 \%$ \\
\hline
\end{tabular}


Sub-Centre: Peshawar

\begin{tabular}{|c|c|c|c|c|c|c|c|c|c|c|}
\hline \multirow[b]{2}{*}{$\begin{array}{l}\text { Grades/ } \\
\text { Sections }\end{array}$} & \multicolumn{5}{|c|}{ - Number of Students- } & \multirow[b]{2}{*}{ Grades } & \multicolumn{4}{|c|}{ Percent of Students } \\
\hline & Enrolled & Dropped & $\begin{array}{c}\text { Appeared } \\
\text { in Exam }\end{array}$ & $\begin{array}{c}\text { Passed in } \\
\text { Exams }\end{array}$ & $\begin{array}{c}\text { Failed in } \\
\text { Exams }\end{array}$ & & Dropped & $\begin{array}{l}\text { Appeared } \\
\text { in Exams }\end{array}$ & $\begin{array}{l}\text { Passed in } \\
\text { Exams }\end{array}$ & $\begin{array}{l}\text { Falled in } \\
\text { Exams }\end{array}$ \\
\hline Grade $1 \mathrm{~A}$ & 76 & 4 & 72 & 31 & 41 & Grade 1 & $5.26 \%$ & $94.74 \%$ & $43.06 \%$ & $56.94 \%$ \\
\hline Grade 1 B & 0 & 0 & 0 & 0 & 0 & Grade 2 & $0.00 \%$ & $100.00 \%$ & $61.29 \%$ & $38.71 \%$ \\
\hline Grade $1 \mathrm{C}$ & 0 & 0 & 0 & 0 & 0 & Grade 3 & $0.00 \%$ & $100.00 \%$ & $78.95 \%$ & $21.05 \%$ \\
\hline Grade $2 \mathrm{~A}$ & 31 & 0 & 31 & 19 & 12 & Grade 4 & $6.25 \%$ & $93.75 \%$ & $100.00 \%$ & $0.00 \%$ \\
\hline Grade 2 B & 0 & 0 & 0 & 0 & 0 & Grade 5 & $0.00 \%$ & $100.00 \%$ & $100.00 \%$ & $0.00 \%$ \\
\hline Grade $3 \mathrm{~A}$ & 19 & 0 & 19 & 15 & 4 & Grade 6 & \#Num! & \#Num! & \#Num! & \#Num! \\
\hline Grade 3 B & 0 & 0 & 0 & 0 & 0 & & & & & \\
\hline Grade 4 & 16 & 1 & 15 & 15 & 0 & Remarks: & & & & \\
\hline Grade 5 & 10 & 0 & 10 & 10 & 0 & & & & & \\
\hline Grade 6 & 0 & 0 & 0 & 0 & 0 & & & & & \\
\hline School Total & 152 & 5 & 147 & 90 & 57 & School \%age & $3.29 \%$ & $96.71 \%$ & $61.22 \%$ & $38.78 \%$ \\
\hline
\end{tabular}

\begin{tabular}{|c|c|c|c|c|c|c|c|c|c|c|}
\hline \multirow[b]{2}{*}{$\begin{array}{l}\text { Grades I } \\
\text { Sections }\end{array}$} & \multicolumn{5}{|c|}{-Number of Students- } & \multirow[b]{2}{*}{ Grades } & \multicolumn{4}{|c|}{ Percent of Students } \\
\hline & Enrolled & Dropped & $\begin{array}{l}\text { Appeared } \\
\text { in Exam }\end{array}$ & $\begin{array}{l}\text { Passed in } \\
\text { Exams }\end{array}$ & $\begin{array}{l}\text { Failed in } \\
\text { Exams }\end{array}$ & & Dropped & $\begin{array}{l}\text { Appeared } \\
\text { in Exams }\end{array}$ & $\begin{array}{c}\text { Passed in } \\
\text { Exams }\end{array}$ & $\begin{array}{l}\text { Failed in } \\
\text { Exams }\end{array}$ \\
\hline Grade $1 \mathrm{~A}$ & 40 & 0 & 40 & 22 & 18 & Grade 1 & $0.00 \%$ & $100.00 \%$ & $46.00 \%$ & $54.00 \%$ \\
\hline Grade 1 B & 60 & 0 & 60 & 24 & 36 & Grade 2 & $0.00 \%$ & $100.00 \%$ & $95.24 \%$ & $4.76 \%$ \\
\hline Grade $1 \mathrm{C}$ & 0 & 0 & 0 & 0 & 0 & Grade 3 & $0.00 \%$ & $100.00 \%$ & $72.41 \%$ & $27.59 \%$ \\
\hline Grade $2 \mathrm{~A}$ & 42 & 0 & 42 & 40 & 2 & Grade 4 & $0.00 \%$ & $100.00 \%$ & $65.52 \%$ & $34.48 \%$ \\
\hline Grade 2 B & 0 & 0 & 0 & 0 & 0 & Grade 5 & $0.00 \%$ & $100.00 \%$ & $69.23 \%$ & $30.77 \%$ \\
\hline Grade $3 \mathrm{~A}$ & 29 & 0 & 29 & 21 & 8 & Grade 6 & $0.00 \%$ & $100.00 \%$ & $85.71 \%$ & $14.29 \%$ \\
\hline Grade 3 B & 0 & 0 & 0 & 0 & 0 & & & & & \\
\hline Grade 4 & 29 & 0 & 29 & 19 & 10 & Remarks: & & & & \\
\hline Grade 5 & 26 & 0 & 26 & 18 & 8 & & & & & \\
\hline Grade 6 & 21 & 0 & 21 & 18 & 3 & & & & & \\
\hline School Total & 247 & 0 & 247 & 162 & 85 & School \%age & $0.00 \%$ & $100.00 \%$ & $65.59 \%$ & $34.41 \%$ \\
\hline
\end{tabular}

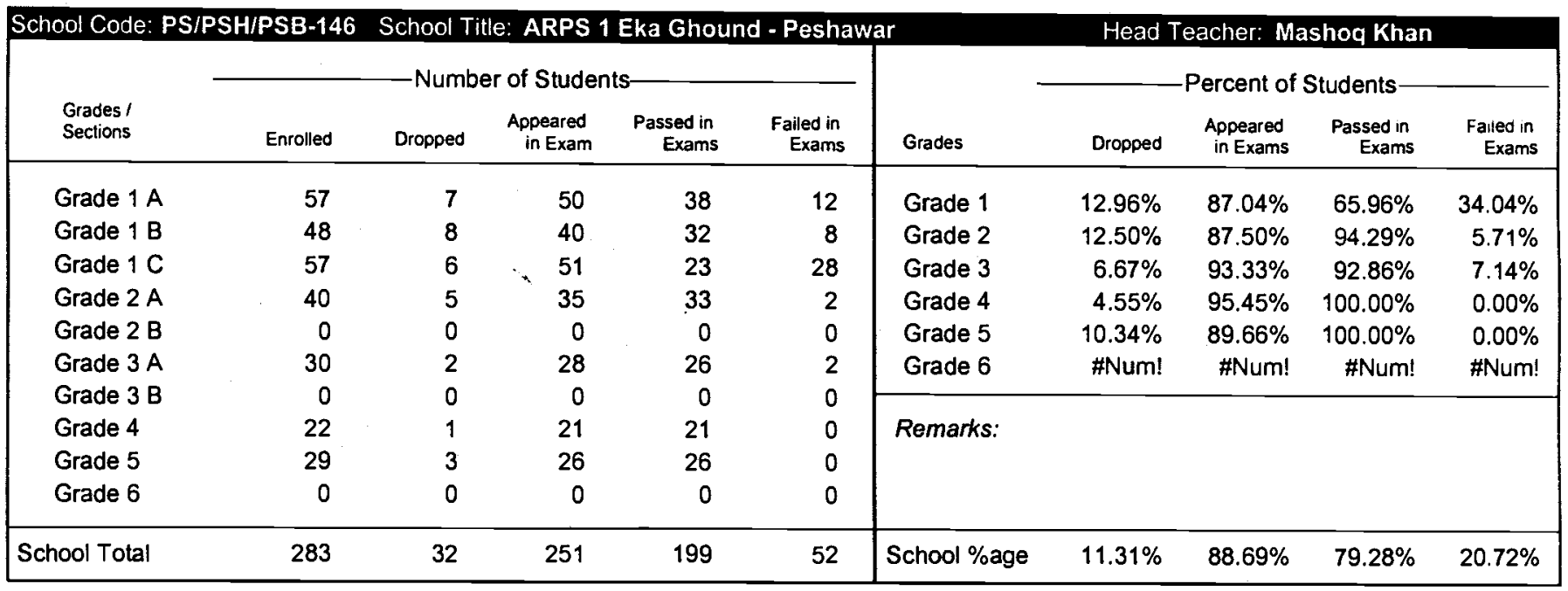


Sub-Centre: Peshawar

\begin{tabular}{|c|c|c|c|c|c|c|c|c|c|c|}
\hline \multirow[b]{2}{*}{$\begin{array}{l}\text { Grades I } \\
\text { Sections }\end{array}$} & \multicolumn{5}{|c|}{-Number of Students- } & \multirow[b]{2}{*}{ Grades } & \multicolumn{4}{|c|}{-Percent of Students } \\
\hline & Enrolied & Dropped & $\begin{array}{l}\text { Appeared } \\
\text { in Exam }\end{array}$ & $\begin{array}{c}\text { Passed in } \\
\text { Exams }\end{array}$ & $\begin{array}{l}\text { Failed in } \\
\text { Exams }\end{array}$ & & Dropped & $\begin{array}{l}\text { Appeared } \\
\text { in Exams }\end{array}$ & $\begin{array}{l}\text { Passed in } \\
\text { Exams }\end{array}$ & $\begin{array}{r}\text { Falled in } \\
\text { Exams }\end{array}$ \\
\hline Grade $1 \mathrm{~A}$ & 42 & 3 & 39 & 28 & 11 & Grade 1 & $17.05 \%$ & $82.95 \%$ & $55.14 \%$ & $44.86 \%$ \\
\hline Grade 1 B & 45 & 5 & 40 & 21 & 19 & Grade 2 & $11.76 \%$ & $88.24 \%$ & $46.67 \%$ & $53.33 \%$ \\
\hline Grade $1 \mathrm{C}$ & 42 & 14 & 28 & 10 & 18 & Grade 3 & $5.41 \%$ & $94.59 \%$ & $60.00 \%$ & $40.00 \%$ \\
\hline Grade $2 \mathrm{~A}$ & 51 & 6 & 45 & 21 & 24 & Grade 4 & $0.00 \%$ & $100.00 \%$ & $40.74 \%$ & $59.26 \%$ \\
\hline Grade 2 B & 0 & 0 & 0 & 0 & 0 & Grade 5 & $26.67 \%$ & $73.33 \%$ & $45.45 \%$ & $54.55 \%$ \\
\hline Grade $3 \mathrm{~A}$ & 37 & 2 & 35 & 21 & 14 & Grade 6 & $0.00 \%$ & $100.00 \%$ & $38.89 \%$ & $61.11 \%$ \\
\hline Grade 3 B & 0 & 0 & 0 & 0 & 0 & & & & & \\
\hline Grade 4 & 27 & 0 & 27 & 11 & 16 & Remarks: & & & & \\
\hline Grade 5 & 30 & 8 & 22 & 10 & 12 & & & & & \\
\hline Grade 6 & 18 & 0 & 18 & 7 & 11 & & & & & \\
\hline School Total & 292 & 38 & 254 & 129 & 125 & School \%age & $13.01 \%$ & $86.99 \%$ & $50.79 \%$ & $49.21 \%$ \\
\hline
\end{tabular}

\begin{tabular}{|c|c|c|c|c|c|c|c|c|c|c|c|}
\hline \multirow[b]{2}{*}{$\begin{array}{l}\text { Grades / } \\
\text { Sections }\end{array}$} & \multicolumn{6}{|c|}{-Number of Students- } & \multirow[b]{2}{*}{ Grades } & \multicolumn{4}{|c|}{-Percent of Students } \\
\hline & $'$ & Enrolled & Dropped & $\begin{array}{l}\text { Appeared } \\
\text { in Exam }\end{array}$ & $\begin{array}{c}\text { Passed in } \\
\text { Exams }\end{array}$ & $\begin{array}{c}\text { Failed in } \\
\text { Exams }\end{array}$ & & Dropped & $\begin{array}{l}\text { Appeared } \\
\text { in Exams }\end{array}$ & $\begin{array}{c}\text { Passed in } \\
\text { Exams }\end{array}$ & $\begin{array}{l}\text { Failed in } \\
\text { Exams }\end{array}$ \\
\hline Grade $1 \mathrm{~A}$ & & 63 & 0 & 63 & 32 & 31 & Grade 1 & $3.42 \%$ & $96.58 \%$ & $50.44 \%$ & $49.56 \%$ \\
\hline Grade 1 B & & 54 & 4 & 50 & 25 & 25 & Grade 2 & $0.00 \%$ & $100.00 \%$ & $60.32 \%$ & $39.68 \%$ \\
\hline Grade $1 \mathrm{C}$ & & 0 & 0 & 0 & 0 & 0 & Grade 3 & $2.94 \%$ & $97.06 \%$ & $63.64 \%$ & $36.36 \%$ \\
\hline Grade $2 \mathrm{~A}$ & & 63 & 0 & 63 & 38 & 25 & Grade 4 & $26.32 \%$ & $73.68 \%$ & $92.86 \%$ & $7.14 \%$ \\
\hline Grade 2 B & & 0 & 0 & 0 & 0 & 0 & Grade 5 & $31.25 \%$ & $68.75 \%$ & $100.00 \%$ & $0.00 \%$ \\
\hline Grade $3 \mathrm{~A}$ & & 34 & 1 & 33 & 21 & 12 & Grade 6 & $0.00 \%$ & $100.00 \%$ & $66.67 \%$ & $33.33 \%$ \\
\hline Grade 3 B & & 0 & 0 & 0 & 0 & 0 & & & & & \\
\hline Grade 4 & & 19 & 5 & 14 & 13 & 1 & Remarks: & & & & \\
\hline Grade 5 & & 16 & 5 & 11 & 11 & 0 & & & & & \\
\hline Grade 6 & & 12 & 0 & 12 & 8 & 4 & & & & & \\
\hline School Total & & 261 & 15 & 246 & 148 & 98 & School \%age & $5.75 \%$ & $94.25 \%$ & $60.16 \%$ & $39.84 \%$ \\
\hline
\end{tabular}

\begin{tabular}{|c|c|c|c|c|c|c|c|c|c|c|}
\hline \multirow[b]{2}{*}{$\begin{array}{l}\text { Grades I } \\
\text { Sections }\end{array}$} & \multicolumn{5}{|c|}{-Number of Students } & \multirow[b]{2}{*}{ Grades } & \multicolumn{4}{|c|}{-Percent of Students- } \\
\hline & Enrolled & Dropped & $\begin{array}{l}\text { Appeared } \\
\text { in Exam }\end{array}$ & $\begin{array}{c}\text { Passed in } \\
\text { Exams }\end{array}$ & $\begin{array}{l}\text { Failed in } \\
\text { Exams }\end{array}$ & & Dropped & $\begin{array}{l}\text { Appeared } \\
\text { in Exams }\end{array}$ & $\begin{array}{l}\text { Passed in } \\
\text { Exams }\end{array}$ & $\begin{array}{l}\text { Failed in } \\
\text { Exams }\end{array}$ \\
\hline Grade $1 \mathrm{~A}$ & 63 & 7 & 56 & 36 & 20 & Grade 1 & $13.66 \%$ & $86.34 \%$ & $59.49 \%$ & $40.51 \%$ \\
\hline Grade 1 B & 59 & 7 & 52 & 27 & 25 & Grade 2 & $3.76 \%$ & $96.24 \%$ & $62.50 \%$ & $37.50 \%$ \\
\hline Grade $1 \mathrm{C}$ & 61 & 11 & 50 & 31 & 19 & Grade 3 & $12.50 \%$ & $87.50 \%$ & $71.43 \%$ & $28.57 \%$ \\
\hline Grade 2 A & 66 & 4 & 62 & 39 & 23 & Grade 4 & $26.53 \%$ & $73.47 \%$ & $61.11 \%$ & $38.89 \%$ \\
\hline Grade 2 B & 67 & 1 & 66 & 41 & 25 & Grade 5 & $18.75 \%$ & $81.25 \%$ & $66.67 \%$ & $33.33 \%$ \\
\hline Grade 3 A & 47 & 6 & 41 & 34 & 7 & Grade 6 & $23.68 \%$ & $76.32 \%$ & $75.86 \%$ & $24.14 \%$ \\
\hline Grade 3 B & 41 & 5 & 36 & 21 & 15 & & & & & \\
\hline Grade 4 & 49 & 13 & 36 & 22 & 14 & Remarks: & & & & \\
\hline Grade 5 & 48 & 9 & 39 & 26 & 13 & & & & & \\
\hline Grade 6 & 38 & 9 & 29 & 22 & 7 & & & & & \\
\hline School Total & 539 & 72 & 467 & 299 & 168 & School \%age & $13.36 \%$ & $86.64 \%$ & $64.03 \%$ & $35.97 \%$ \\
\hline
\end{tabular}

Note: 
Sub-Centre: Peshawar

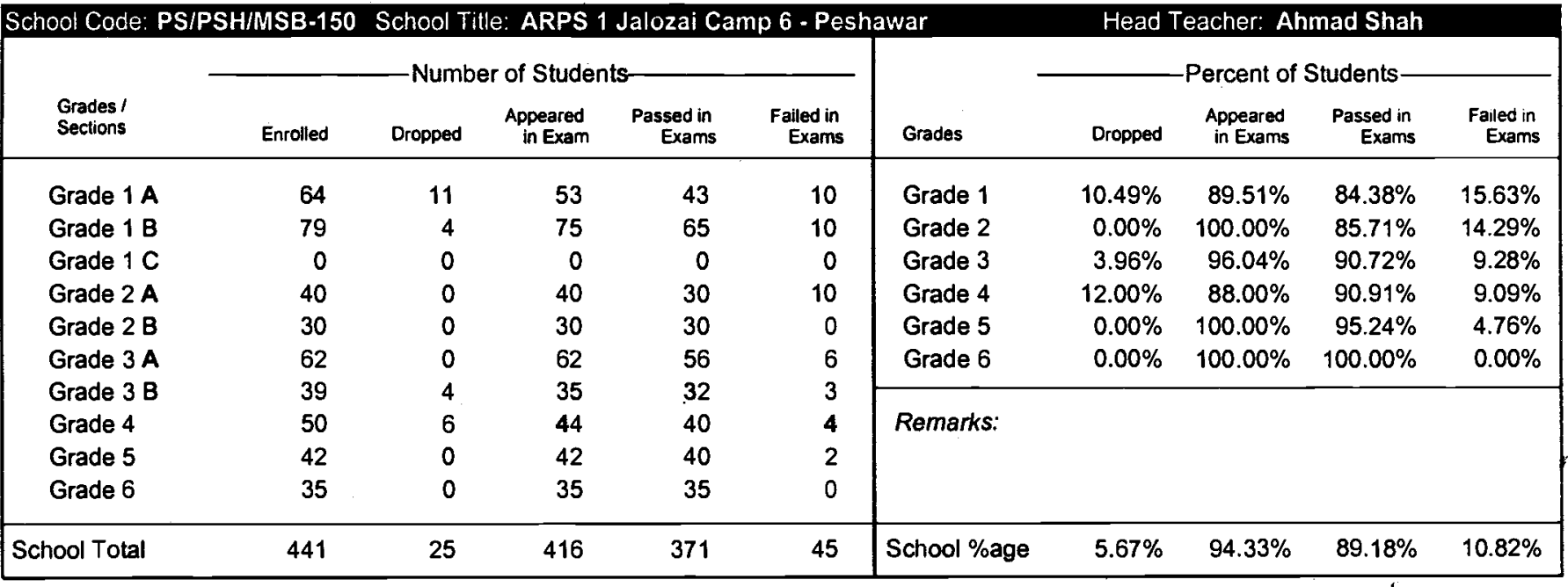

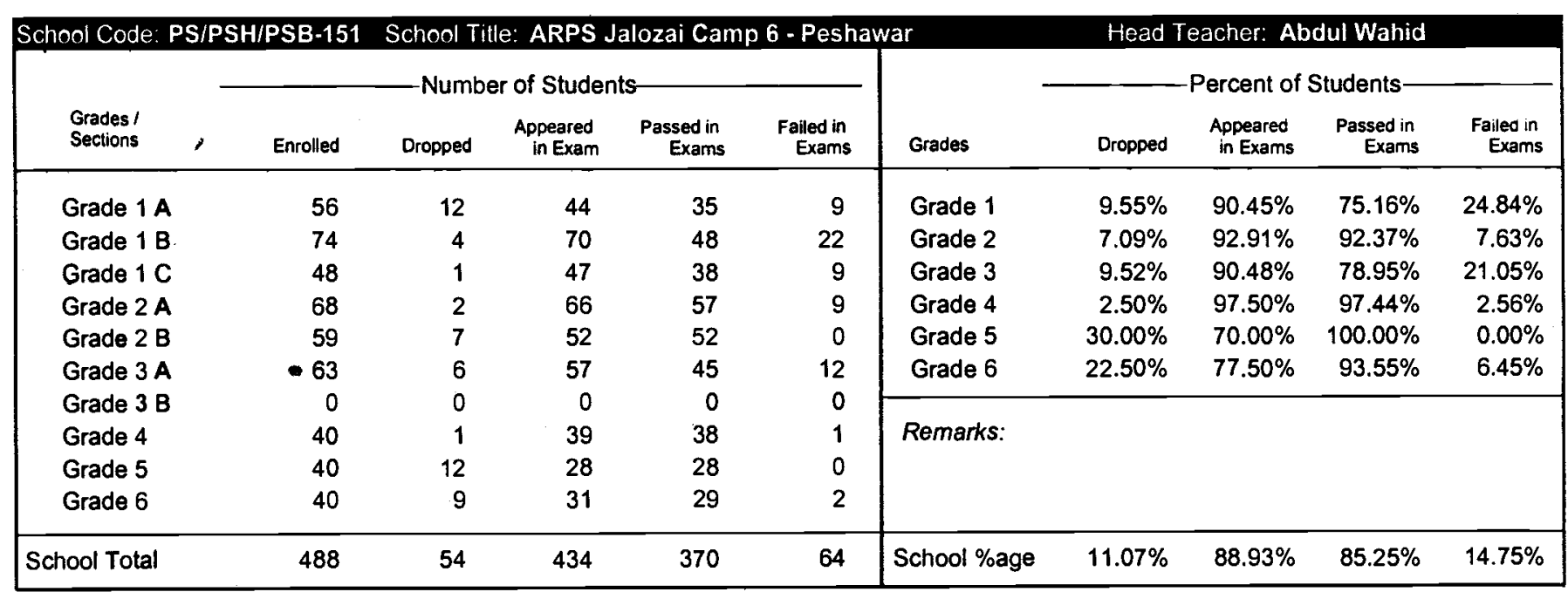

\begin{tabular}{|c|c|c|c|c|c|c|c|c|c|c|}
\hline \multirow[b]{2}{*}{$\begin{array}{l}\text { Grades I } \\
\text { Sections }\end{array}$} & \multicolumn{5}{|c|}{ - Number of Students } & \multirow[b]{2}{*}{ Grades } & \multicolumn{4}{|c|}{-Percent of Students } \\
\hline & Enrolled & Dropped & $\begin{array}{l}\text { Appeared } \\
\text { in Exam }\end{array}$ & $\begin{array}{l}\text { Passed in } \\
\text { Exams }\end{array}$ & $\begin{array}{l}\text { Failed in } \\
\text { Exams }\end{array}$ & & Dropped & $\begin{array}{l}\text { Appeared } \\
\text { in Exams }\end{array}$ & $\begin{array}{l}\text { Passed in } \\
\text { Exams }\end{array}$ & $\begin{array}{l}\text { Falled in } \\
\text { Exams }\end{array}$ \\
\hline Grade 1 A & 45 & 2 & 43 & 32 & 11 & Grade 1 & $3.96 \%$ & $96.04 \%$ & $54.64 \%$ & $45.36 \%$ \\
\hline Grade 1 B & 56 & 2 & 54 & 21 & 33 & Grade 2 & $4.82 \%$ & $95.18 \%$ & $70.89 \%$ & $29.11 \%$ \\
\hline Grade $1 \mathrm{C}$ & 0 & 0. & 0 & 0 & 0 & Grade 3 & $0.00 \%$ & $100.00 \%$ & $46.51 \%$ & $53.49 \%$ \\
\hline Grade 2 A & 40 & 1 & 39 & 28 & 11 & Grade 4 & $8.33 \%$ & $91.67 \%$ & $59.09 \%$ & $40.91 \%$ \\
\hline Grade 2 B & 43 & 3 & 40 & 28 & 12 & Grade 5 & $0.00 \%$ & $100.00 \%$ & $75.00 \%$ & $25.00 \%$ \\
\hline Grade 3 A & 43 & 0 & 43 & 20 & 23 & Grade 6 & $0.00 \%$ & $100.00 \%$ & $69.57 \%$ & $30.43 \%$ \\
\hline Grade 3 B & 0 & 0 & 0 & 0 & 0 & & & & & \\
\hline Grade 4 & 24 & 2 & 22 & 13 & 9 & Remarks: & & & & \\
\hline Grade 5 & 20 & 0 & 20 & 15 & 5 & & & & & \\
\hline Grade 6 & 23 & 0 & 23 & 16 & 7 & & & & & \\
\hline School Total & 294 & 10 & 284 & 173 & 111 & School \%age & $3.40 \%$ & $96.60 \%$ & $60.92 \%$ & $39.08 \%$ \\
\hline
\end{tabular}


Sub-Centre: Peshawar

\begin{tabular}{|c|c|c|c|c|c|c|c|c|c|c|}
\hline \multirow[b]{2}{*}{$\begin{array}{l}\text { Grades / } \\
\text { Sections }\end{array}$} & PSB-15 & $\begin{array}{l}\text { School T } \\
\text { - Numb }\end{array}$ & $\begin{array}{l}\text { ARPS } \\
\text { of Stude }\end{array}$ & ababya & $\mathrm{mp} 2$. & shawar & Head & $\begin{array}{l}\text { acher: N } \\
\text { ercent of }\end{array}$ & $\begin{array}{l}\text { qib Ullah } \\
\text { Students }\end{array}$ & $\ldots$ \\
\hline & Enrolled & Dropped & $\begin{array}{c}\text { Appeared } \\
\text { in Exam }\end{array}$ & $\begin{array}{l}\text { Passed in } \\
\text { Exams }\end{array}$ & $\begin{array}{l}\text { Failed in } \\
\text { Exams }\end{array}$ & Grades & Dropped & $\begin{array}{c}\text { Appeared } \\
\text { in Exams }\end{array}$ & $\begin{array}{l}\text { Passed in } \\
\text { Exams }\end{array}$ & $\begin{array}{l}\text { Failed in } \\
\text { Exams }\end{array}$ \\
\hline Grade 1 A & 70 & 5 & 65 & 50 & 15 & Grade 1 & $12.86 \%$ & $87.14 \%$ & $63.93 \%$ & $36.07 \%$ \\
\hline Grade 1 B & 70 & 13 & 57 & 28 & 29 & Grade 2 & $3.73 \%$ & $96.27 \%$ & $72.09 \%$ & $27.91 \%$ \\
\hline Grade $1 \mathrm{C}$ & 0 & 0 & 0 & 0 & 0 & Grade 3 & $6.02 \%$ & $93.98 \%$ & $80.77 \%$ & $19.23 \%$ \\
\hline Grade 2 A & 76 & 4 & 72 & 52 & 20 & Grade 4 & $6.67 \%$ & $93.33 \%$ & $87.50 \%$ & $12.50 \%$ \\
\hline Grade 2 B & 58 & 1 & 57 & 41 & 16 & Grade 5 & $6.06 \%$ & $93.94 \%$ & $100.00 \%$ & $0.00 \%$ \\
\hline Grade 3 A & 40 & 1 & 39 & 28 & 11 & Grade 6 & $17.65 \%$ & $82.35 \%$ & $100.00 \%$ & $0.00 \%$ \\
\hline Grade 3 B & 43 & 4 & 39 & 35 & 4 & & & & & \\
\hline Grade 4 & 60 & 4 & 56 & 49 & 7 & Remarks: & & & & \\
\hline Grade 5 & 33 & 2 & 31 & 31 & 0 & & & & & \\
\hline Grade 6 & 17 & 3 & 14 & 14 & 0 & & & & & \\
\hline School Total & 467 & 37 & 430 & 328 & 102 & School \%age & $7.92 \%$ & $92.08 \%$ & $76.28 \%$ & $23.72 \%$ \\
\hline
\end{tabular}

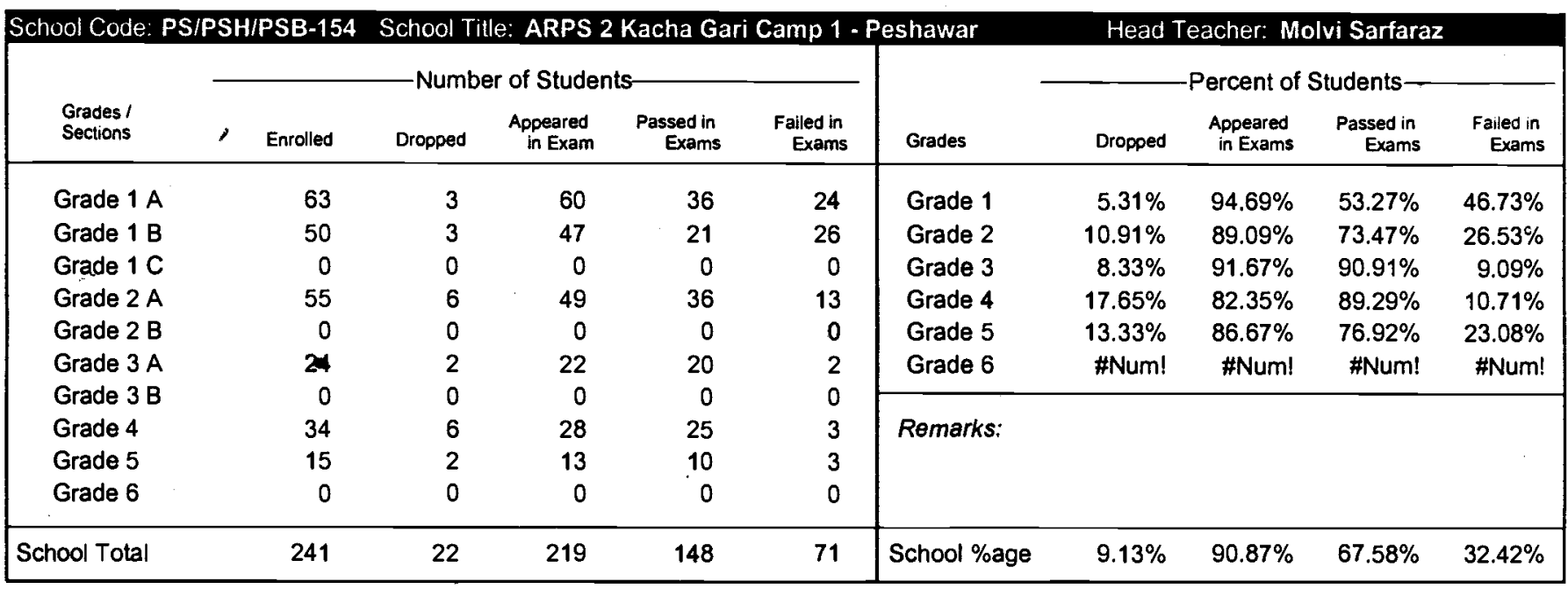

\begin{tabular}{|c|c|c|c|c|c|c|c|c|c|c|}
\hline \multirow[b]{2}{*}{$\begin{array}{l}\text { Grades / } \\
\text { Sections }\end{array}$} & \multicolumn{5}{|c|}{ Number of Students } & \multirow[b]{2}{*}{ Grades } & \multicolumn{4}{|c|}{-Percent of Students- } \\
\hline & Enrolled & Dropped & $\begin{array}{l}\text { Appeared } \\
\text { in Exam }\end{array}$ & $\begin{array}{l}\text { Passed in } \\
\text { Exams }\end{array}$ & $\begin{array}{l}\text { Failed in } \\
\text { Exams }\end{array}$ & & Dropped & $\begin{array}{l}\text { Appeared } \\
\text { in Exams }\end{array}$ & $\begin{array}{l}\text { Passed in } \\
\text { Exams }\end{array}$ & $\begin{array}{l}\text { Falled in } \\
\text { Exams }\end{array}$ \\
\hline Grade $1 \mathrm{~A}$ & 61 & 6 & 55 & 39 & 16 & Grade 1 & $8.40 \%$ & $91.60 \%$ & $63.30 \%$ & $36.70 \%$ \\
\hline Grade 1 B & 58 & 4 & 54 & 30 & 24 & Grade 2 & $12.20 \%$ & $87.80 \%$ & $69.44 \%$ & $30.56 \%$ \\
\hline Grade $1 \mathrm{C}$ & 0 & 0 & 0 & 0 & 0 & Grade 3 & $15.63 \%$ & $84.38 \%$ & $85.19 \%$ & $14.81 \%$ \\
\hline Grade $2 \mathrm{~A}$ & 41 & 5 & 36 & 25 & 11 & Grade 4 & $0.00 \%$ & $100.00 \%$ & $84.00 \%$ & $16.00 \%$ \\
\hline Grade 2 B & 0 & 0 & 0 & 0 & 0 & Grade 5 & $26.32 \%$ & $73.68 \%$ & $85.71 \%$ & $14.29 \%$ \\
\hline Grade $3 \mathrm{~A}$ & 32 & 5 & 27 & 23 & 4 & Grade 6 & \#Num! & \#Num! & \#Num! & \#Num! \\
\hline Grade 3 B & 0 & 0 & 0 & 0 & 0 & & & & & \\
\hline Grade 4 & 25 & 0 & 25 & 21 & 4 & Remarks: & & & & \\
\hline Grade 5 & 19 & 5 & 14 & 12 & 2 & & & & & \\
\hline Grade 6 & 0 & 0 & 0 & 0 & 0 & & & & & \\
\hline School Total & 236 & 25 & 211 & 150 & 61 & School \%age & $10.59 \%$ & $89.41 \%$ & $71.09 \%$ & $28.91 \%$ \\
\hline
\end{tabular}

Note:

\#Num! = Enrollment in respective grade(s) is nill therefore calculation is not applicable
Page 53 of 91

Produced by DP/MIS Section on 31.Jul-97 
Sub-Centre: Peshawar

\begin{tabular}{|c|c|c|c|c|c|c|c|c|c|c|}
\hline \multirow[b]{2}{*}{$\begin{array}{l}\text { Grades I } \\
\text { Sections }\end{array}$} & \multicolumn{5}{|c|}{-Number of Students } & \multirow[b]{2}{*}{ Grades } & \multicolumn{4}{|c|}{-Percent of Students- } \\
\hline & Enrolled & Dropped & $\begin{array}{l}\text { Appeared } \\
\text { in Exam }\end{array}$ & $\begin{array}{l}\text { Passed in } \\
\text { Exams }\end{array}$ & $\begin{array}{l}\text { Failed in } \\
\text { Exams }\end{array}$ & & Dropped & $\begin{array}{c}\text { Appeared } \\
\text { in Exams }\end{array}$ & $\begin{array}{c}\text { Passed in } \\
\text { Exams }\end{array}$ & $\begin{array}{l}\text { Falled in } \\
\text { Exams } \\
\end{array}$ \\
\hline Grade $1 \mathrm{~A}$ & 108 & 31 & 77 & 59 & 18 & Grade 1 & $23.78 \%$ & $76.22 \%$ & $56.03 \%$ & $43.97 \%$ \\
\hline Grade $1 \mathrm{~B}$ & 77 & 13 & 64 & 20 & 44 & Grade 2 & $5.32 \%$ & $94.68 \%$ & $52.81 \%$ & $47.19 \%$ \\
\hline Grade $1 \mathrm{C}$ & 0 & 0 & 0 & 0 & 0 & Grade 3 & $16.07 \%$ & $83.93 \%$ & $55.32 \%$ & $44.68 \%$ \\
\hline Grade $2 \mathrm{~A}$ & 52 & 2 & 50 & 25 & 25 & Grade 4 & $20.69 \%$ & $79.31 \%$ & $78.26 \%$ & $21.74 \%$ \\
\hline Grade 2 B & 42 & 3 & 39 & 22 & 17 & Grade 5 & $13.79 \%$ & $86.21 \%$ & $92.00 \%$ & $8.00 \%$ \\
\hline Grade $3 \mathrm{~A}$ & 56 & 9 & 47 & 26 & 21 & Grade 6 & \#Num! & \#Num! & \#Num! & \#Num! \\
\hline Grade 3 B & 0 & 0 & 0 & 0 & 0 & & & & & \\
\hline Grade 4 & 29 & 6 & 23 & 18 & 5 & Remarks: & & & & \\
\hline Grade 5 & 29 & 4 & 25 & 23 & 2 & & & & & \\
\hline Grade 6 & 0 & 0 & 0 & 0 & 0 & & & & & \\
\hline School Total & 393 & 68 & 325 & 193 & 132 & School \%age & $17.30 \%$ & $82.70 \%$ & $59.38 \%$ & $40.62 \%$ \\
\hline
\end{tabular}

\begin{tabular}{|c|c|c|c|c|c|c|c|c|c|c|c|}
\hline \multirow[b]{2}{*}{$\begin{array}{l}\text { Grades I } \\
\text { Sections }\end{array}$} & \multicolumn{6}{|c|}{ - Number of Students- } & \multirow[b]{2}{*}{ Grades } & \multicolumn{4}{|c|}{ Percent of Students- } \\
\hline & ' & Enrolled & Dropped & $\begin{array}{l}\text { Appeared } \\
\text { in Exam }\end{array}$ & $\begin{array}{c}\text { Passed in } \\
\text { Exams }\end{array}$ & $\begin{array}{l}\text { Failed in } \\
\text { Exams }\end{array}$ & & Dropped & $\begin{array}{l}\text { Appeared } \\
\text { in Exams }\end{array}$ & $\begin{array}{c}\text { Passed in } \\
\text { Exams }\end{array}$ & $\begin{array}{l}\text { Failed in } \\
\text { Exams }\end{array}$ \\
\hline Grade $1 \mathrm{~A}$ & & 70 & 4 & 66 & 50 & 16 & Grade 1 & $6.72 \%$ & $93.28 \%$ & $57.60 \%$ & $42.40 \%$ \\
\hline Grade 1 B & & 64 & 5 & 59 & 22 & 37 & Grade 2 & $0.00 \%$ & $100.00 \%$ & $76.09 \%$ & $23.91 \%$ \\
\hline Grade $1 \mathrm{C}$ & & 0 & 0 & 0 & 0 & 0 & Grade 3 & $0.00 \%$ & $100.00 \%$ & $87.23 \%$ & $12.77 \%$ \\
\hline Grade 2 A & & 53 & 0 & 53 & 41 & 12 & Grade 4 & $0.00 \%$ & $100.00 \%$ & $90.00 \%$ & $10.00 \%$ \\
\hline Grade 2 B & & 39 & 0 & 39 & 29 & 10 & Grade 5 & $8.33 \%$ & $91.67 \%$ & $95.45 \%$ & $4.55 \%$ \\
\hline Grade 3 A & & 47 & 0 & 47 & 41 & 6 & Grade 6 & \#Num! & \#Num! & \#Num! & \#Num! \\
\hline Grade 3 B & & 0 & 0 & 0 & 0 & 0 & & & & & \\
\hline Grade 4 & & 30 & 0 & 30 & 27 & 3 & Remarks: & & & & \\
\hline Grade 5 & & 24 & 2 & 22 & 21 & 1 & & & & & \\
\hline Grade 6 & & 0 & 0 & 0 & 0 & 0 & & & & & \\
\hline School Total & & 327 & 11 & 316 & 231 & 85 & School \%age & $3.36 \%$ & $96.64 \%$ & $73.10 \%$ & $26.90 \%$ \\
\hline
\end{tabular}

\begin{tabular}{|c|c|c|c|c|c|c|c|c|c|c|}
\hline \multirow[b]{2}{*}{$\begin{array}{l}\text { Grades / } \\
\text { Sections }\end{array}$} & \multicolumn{5}{|c|}{ - Number of Students } & \multirow[b]{2}{*}{ Grades } & \multicolumn{4}{|c|}{-Percent of Students } \\
\hline & Enrolled & Dropped & $\begin{array}{l}\text { Appeared } \\
\text { in Exam }\end{array}$ & $\begin{array}{c}\text { Passed in } \\
\text { Exams }\end{array}$ & $\begin{array}{r}\text { Failed in } \\
\text { Exams }\end{array}$ & & Dropped & $\begin{array}{l}\text { Appeared } \\
\text { in Exams }\end{array}$ & $\begin{array}{r}\text { Passed in } \\
\text { Exams }\end{array}$ & $\begin{array}{c}\text { Falled in } \\
\text { Exams }\end{array}$ \\
\hline Grade $1 \mathrm{~A}$ & 94 & 14 & 80 & 56 & 24 & Grade 1 & $10.94 \%$ & $89.06 \%$ & $67.84 \%$ & $32.16 \%$ \\
\hline Grade $1 \mathrm{~B}$ & 98 & 7 & 91 & 60 & 31 & Grade 2 & $18.95 \%$ & $81.05 \%$ & $70.13 \%$ & $29.87 \%$ \\
\hline Grade $1 \mathrm{C}$ & 0 & 0 & 0 & 0 & 0 & Grade 3 & $13.95 \%$ & $86.05 \%$ & $78.38 \%$ & $21.62 \%$ \\
\hline Grade 2 A & 50 & 14 & 36 & 33 & 3 & Grade 4 & $3.70 \%$ & $96.30 \%$ & $75.00 \%$ & $25.00 \%$ \\
\hline Grade 2 B & 45 & 4 & 41 & 21 & 20 & Grade 5 & $0.00 \%$ & $100.00 \%$ & $85.00 \%$ & $15.00 \%$ \\
\hline Grade $3 \mathrm{~A}$ & 86 & 12 & 74 & 58 & 16 & Grade 6 & $6.98 \%$ & $93.02 \%$ & $75.00 \%$ & $25.00 \%$ \\
\hline Grade 3 B & 0 & 0 & 0 & 0 & 0 & & & & & \\
\hline Grade 4 & 54 & 2 & 52 & 39 & 13 & Remarks: & & & & \\
\hline Grade 5 & 40 & 0 & 40 & 34 & 6 & & & & & \\
\hline Grade 6 & 43 & 3 & 40 & 30 & 10 & & & & & \\
\hline School Total & 510 & 56 & 454 & 331 & 123 & School \%age & $10.98 \%$ & $89.02 \%$ & $72.91 \%$ & $27.09 \%$ \\
\hline
\end{tabular}

Note:

\#Num! = Enrollment in respective grade(s) is nill therefore calculation is not applicable
Page 54 of 91

Produced by DPMIS Section on $31 \cdot$ Jul.97 
Sub-Centre: Peshawar

\begin{tabular}{|c|c|c|c|c|c|c|c|c|c|c|}
\hline \multirow[b]{2}{*}{$\begin{array}{l}\text { Grades I } \\
\text { Sections }\end{array}$} & \multicolumn{5}{|c|}{ - Number of Students- } & \multirow[b]{2}{*}{ Grades } & \multicolumn{4}{|c|}{-Percent of Students- } \\
\hline & Enrolled & Dropped & $\begin{array}{l}\text { Appeared } \\
\text { in Exam }\end{array}$ & $\begin{array}{l}\text { Passed in } \\
\text { Exams }\end{array}$ & $\begin{array}{l}\text { Failed in } \\
\text { Exams }\end{array}$ & & Dropped & $\begin{array}{l}\text { Appeared } \\
\text { in Exams }\end{array}$ & $\begin{array}{l}\text { Passed in } \\
\text { Exams }\end{array}$ & $\begin{array}{l}\text { Failed in } \\
\text { Exams }\end{array}$ \\
\hline Grade $1 \mathrm{~A}$ & 84 & 6 & 78 & 53 & 25 & Grade 1 & $10.99 \%$ & $89.01 \%$ & $59.88 \%$ & $40.12 \%$ \\
\hline Grade 1 B & 98 & 14 & 84 & 44 & 40 & Grade 2 & $9.35 \%$ & $90.65 \%$ & $61.86 \%$ & $38.14 \%$ \\
\hline Grade $1 \mathrm{C}$ & 0 & 0 & 0 & 0 & 0 & Grade 3 & $6.45 \%$ & $93.55 \%$ & $80.46 \%$ & $19.54 \%$ \\
\hline Grade 2 A & 53 & 9 & 44 & 31 & 13 & Grade 4 & $6.06 \%$ & $93.94 \%$ & $80.65 \%$ & $19.35 \%$ \\
\hline Grade 2 B & 54 & 1 & 53 & 29 & 24 & Grade 5 & $7.58 \%$ & $92.42 \%$ & $90.16 \%$ & $9.84 \%$ \\
\hline Grade $3 \mathrm{~A}$ & 93 & 6 & 87 & 70 & 17 & Grade 6 & $3.03 \%$ & $96.97 \%$ & $87.50 \%$ & $12.50 \%$ \\
\hline Grade 3 B & 0 & 0 & 0 & 0 & 0 & & & & & \\
\hline Grade 4 & 66 & 4 & 62 & 50 & 12 & Remarks: & & & & \\
\hline Grade 5 & 66 & 5 & 61 & 55 & 6 & & & & & \\
\hline Grade 6 & 66 & 2 & 64 & 56 & 8 & & & & & \\
\hline School Total & 580 & 47 & 533 & 388 & 145 & School \%age & $8.10 \%$ & $91.90 \%$ & $72.80 \%$ & $27.20 \%$ \\
\hline
\end{tabular}

\begin{tabular}{|c|c|c|c|c|c|c|c|c|c|c|c|}
\hline \multirow[b]{2}{*}{$\begin{array}{l}\text { Grades I } \\
\text { Sections }\end{array}$} & \multicolumn{6}{|c|}{ - Number of Students- } & \multirow[b]{2}{*}{ Grades } & \multicolumn{4}{|c|}{-Percent of Students- } \\
\hline & ' & Enrolled & Dropped & $\begin{array}{c}\text { Appeared } \\
\text { in Exam }\end{array}$ & $\begin{array}{l}\text { Passed in } \\
\text { Exams }\end{array}$ & $\begin{array}{c}\text { Failed in } \\
\text { Exams }\end{array}$ & & Dropped & $\begin{array}{l}\text { Appeared } \\
\text { in Exams }\end{array}$ & $\begin{array}{l}\text { Passed in } \\
\text { Exams }\end{array}$ & $\begin{array}{l}\text { Failed in } \\
\text { Exams }\end{array}$ \\
\hline Grade $1 \mathrm{~A}$ & & 59 & 2 & 57 & 41 & 16 & Grade 1 & $15.45 \%$ & $84.55 \%$ & $71.15 \%$ & $28.85 \%$ \\
\hline Grade 1 B & & 64 & 17 & 47. & 33 & 14 & Grade 2 & $9.52 \%$ & $90.48 \%$ & $89.47 \%$ & $10.53 \%$ \\
\hline Grade $1 \mathrm{C}$ & & 0 & 0 & 0 & 0 & 0 & Grade 3 & $0.00 \%$ & $100.00 \%$ & $97.37 \%$ & $2.63 \%$ \\
\hline Grade $2 \mathrm{~A}$ & & 45 & 4 & 41 & 37 & 4 & Grade 4 & $12.00 \%$ & $88.00 \%$ & $90.91 \%$ & $9.09 \%$ \\
\hline Grade 2 B & & 39 & 4 & 35 & 31 & 4 & Grade 5 & $8.00 \%$ & $92.00 \%$ & $86.96 \%$ & $13.04 \%$ \\
\hline Grade 3 A & & 30 & 0 & 38 & 37 & 1 & Grade 6 & \#Num! & \#Num! & \#Num! & \#Num! \\
\hline Grade 3 B & & 0 & 0 & 0 & 0 & 0 & & & & & \\
\hline Grade 4 & & 25 & 3 & 22 & 20 & 2 & Remarks: & & & & \\
\hline Grade 5 & & 25 & 2 & 23 & 20 & 3 & & & & & \\
\hline Grade 6 & & 0 & 0 & 0 & 0 & 0 & & & & & \\
\hline School Total & & 295 & 32 & 263 & 219 & 44 & School \%age & $10.85 \%$ & $89.15 \%$ & $83.27 \%$ & $16.73 \%$ \\
\hline
\end{tabular}

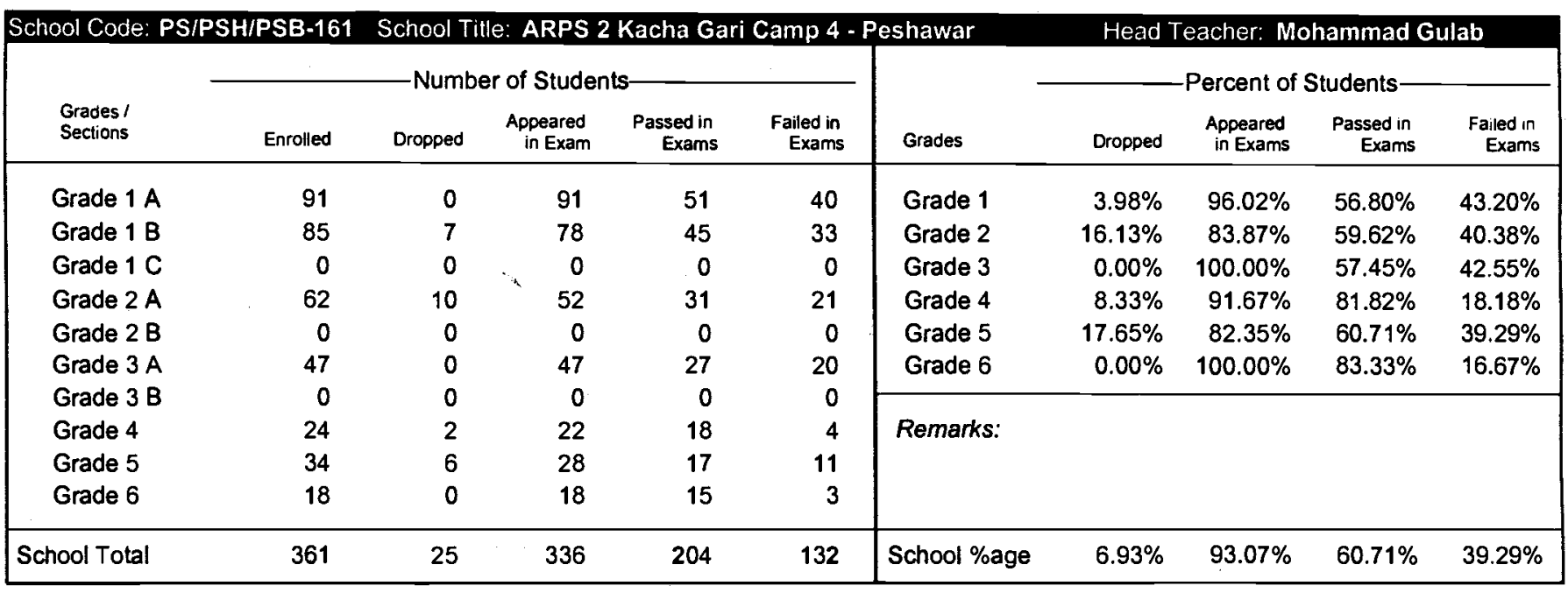


Sub-Centre: Peshawar

\begin{tabular}{|c|c|c|c|c|c|c|c|c|c|c|}
\hline \multirow[b]{2}{*}{$\begin{array}{l}\text { Grades / } \\
\text { Sections }\end{array}$} & \multicolumn{5}{|c|}{ — Number of Students } & \multirow[b]{2}{*}{ Grades } & \multicolumn{4}{|c|}{-Percent of Students- } \\
\hline & Enrolled & Dropped & $\begin{array}{l}\text { Appeared } \\
\text { in Exam }\end{array}$ & $\begin{array}{l}\text { Passed in } \\
\text { Exams }\end{array}$ & $\begin{array}{c}\text { Failed in } \\
\text { Exams }\end{array}$ & & Dropped & $\begin{array}{c}\text { Appeared } \\
\text { in Exams }\end{array}$ & $\begin{array}{c}\text { Passed in } \\
\text { Exams }\end{array}$ & $\begin{array}{c}\text { Falled in } \\
\text { Exams }\end{array}$ \\
\hline Grade $1 \mathrm{~A}$ & 78 & 7 & 71 & 43 & 28 & Grade 1 & $10.76 \%$ & $89.24 \%$ & $49.75 \%$ & $50.25 \%$ \\
\hline Grade 1 B & 74 & 9 & 65 & 25 & 40 & Grade 2 & $1.68 \%$ & $98.32 \%$ & $65.81 \%$ & $34.19 \%$ \\
\hline Grade $1 \mathrm{C}$ & 71 & 8 & 63 & 31 & 32 & Grade 3 & $11.51 \%$ & $88.49 \%$ & $69.11 \%$ & $30.89 \%$ \\
\hline Grade 2 A & 69 & 1 & 68 & 45 & 23 & Grade 4 & $7.14 \%$ & $92.86 \%$ & $92.31 \%$ & $7.69 \%$ \\
\hline Grade 2 B & 50 & 1 & 49 & 32 & 17 & Grade 5 & $15.79 \%$ & $84.21 \%$ & $71.88 \%$ & $28.13 \%$ \\
\hline Grade $3 \mathrm{~A}$ & 74 & 2 & 72 & 51 & 21 & Grade 6 & $3.45 \%$ & $96.55 \%$ & $100.00 \%$ & $0.00 \%$ \\
\hline Grade 3 B & 65 & 14 & 51 & 34 & 17 & & & & & \\
\hline Grade 4 & 42 & 3 & 39 & 36 & 3 & Remarks: & & & & \\
\hline Grade 5 & 38 & 6 & 32 & 23 & 9 & & & & & \\
\hline Grade 6 & 29 & 1 & 28 & 28 & 0 & & & & & \\
\hline School Total & 590 & 52 & 538 & 348 & 190 & School \%age & $8.81 \%$ & $91.19 \%$ & $64.68 \%$ & $35.32 \%$ \\
\hline
\end{tabular}

\begin{tabular}{|c|c|c|c|c|c|c|c|c|c|c|c|}
\hline \multirow[b]{2}{*}{$\begin{array}{l}\text { Grades I } \\
\text { Sections }\end{array}$} & \multicolumn{6}{|c|}{ - Number of Students } & \multirow[b]{2}{*}{ Grades } & \multicolumn{4}{|c|}{-Percent of Students- } \\
\hline & ' & Enrolled & Dropped & $\begin{array}{l}\text { Appeared } \\
\text { in Exam }\end{array}$ & $\begin{array}{l}\text { Passed in } \\
\text { Exams }\end{array}$ & $\begin{array}{l}\text { Failed in } \\
\text { Exams }\end{array}$ & & Dropped & $\begin{array}{l}\text { Appeared } \\
\text { in Exams }\end{array}$ & $\begin{array}{c}\text { Passed in } \\
\text { Exams }\end{array}$ & $\begin{array}{l}\text { Falled in } \\
\text { Exams }\end{array}$ \\
\hline Grade $1 \mathrm{~A}$ & & 103 & 6 & 97 & 72 & 25 & Grade 1 & $13.83 \%$ & $86.17 \%$ & $63.37 \%$ & $36.63 \%$ \\
\hline Grade 1 B & & 77 & 16 & 61 & 35 & 26 & Grade 2 & $2.75 \%$ & $97.25 \%$ & $64.41 \%$ & $35.59 \%$ \\
\hline Grade $1 \mathrm{C}$ & & 102 & 17 & 85 & 47 & 38 & Grade 3 & $15.38 \%$ & $84.62 \%$ & $80.68 \%$ & $19.32 \%$ \\
\hline Grade $2 \mathrm{~A}$ & & 103 & 5 & 98 & 52 & 46 & Grade 4 & $5.33 \%$ & $94.67 \%$ & $70.42 \%$ & $29.58 \%$ \\
\hline Grade 2 B & & 79 & 0 & 79 & 62 & 17 & Grade 5 & $7.25 \%$ & $92.75 \%$ & $84.38 \%$ & $15.63 \%$ \\
\hline Grade $3 \mathrm{~A}$ & & 50 & 8 & 44 & 35 & 9 & Grade 6 & $9.52 \%$ & $90.48 \%$ & $100.00 \%$ & $0.00 \%$ \\
\hline Grade 3 B & & 52 & 8 & 44 & 36 & 8 & & & & & \\
\hline Grade 4 & & 75 & 4 & 71 & 50 & 21 & Remarks: & & & & \\
\hline Grade 5 & & 69 & 5 & 64 & 54 & 10 & & & & & \\
\hline Grade 6 & & 42 & 4 & 38 & 38 & 0 & & & & & \\
\hline School Total & & 754 & 73 & 681 & 481 & 200 & School \%age & $9.68 \%$ & $90.32 \%$ & $70.63 \%$ & $29.37 \%$ \\
\hline
\end{tabular}

\begin{tabular}{|c|c|c|c|c|c|c|c|c|c|c|}
\hline \multirow[b]{2}{*}{$\begin{array}{l}\text { Grades / } \\
\text { Sections }\end{array}$} & \multicolumn{5}{|c|}{-Number of Students } & \multirow[b]{2}{*}{ Grades } & \multicolumn{4}{|c|}{-Percent of Students- } \\
\hline & Enrolled & Dropped & $\begin{array}{l}\text { Appeared } \\
\text { in Exam }\end{array}$ & $\begin{array}{c}\text { Passed in } \\
\text { Exams }\end{array}$ & $\begin{array}{l}\text { Falled in } \\
\text { Exams }\end{array}$ & & Dropped & $\begin{array}{l}\text { Appeared } \\
\text { in Exams }\end{array}$ & $\begin{array}{c}\text { Passed in } \\
\text { Exams }\end{array}$ & $\begin{array}{l}\text { Falled in } \\
\text { Exams }\end{array}$ \\
\hline Grade $1 \mathrm{~A}$ & 58 & 0 & 58 & 50 & 8 & Grade 1 & $2.59 \%$ & $97.41 \%$ & $88.50 \%$ & $11.50 \%$ \\
\hline Grade 1 B & 58 & 3 & 55 & 50 & 5 & Grade 2 & $4.76 \%$ & $95.24 \%$ & $82.50 \%$ & $17.50 \%$ \\
\hline Grade $1 \mathrm{C}$ & 0 & 0 & 0 & 0 & 0 & Grade 3 & $2.63 \%$ & $97.37 \%$ & $81.08 \%$ & $18.92 \%$ \\
\hline Grade $2 \mathrm{~A}$ & 42 & 2 & 40 & 33 & 7 & Grade 4 & $3.85 \%$ & $96.15 \%$ & $100.00 \%$ & $0.00 \%$ \\
\hline Grade 2 B & 0 & 0 & 0 & 0 & 0 & Grade 5 & $0.00 \%$ & $100.00 \%$ & $90.48 \%$ & $9.52 \%$ \\
\hline Grade 3 A & 38 & 1 & 37 & 30 & 7 & Grade 6 & $0.00 \%$ & $100.00 \%$ & $78.57 \%$ & $21.43 \%$ \\
\hline Grade 3 B & 0 & 0 & 0 & 0 & 0 & & & & & \\
\hline Grade 4 & 26 & 1 & 25 & 25 & 0 & Remarks: & & & & \\
\hline Grade 5 & 21 & 0 & 21 & 19 & 2 & & & & & \\
\hline Grade 6 & 14 & 0 & 14 & 11 & 3 & & & & & \\
\hline School Total & 257 & 7 & 250 & 218 & 32 & School \%age & $2.72 \%$ & $97.28 \%$ & $87.20 \%$ & $12.80 \%$ \\
\hline
\end{tabular}


Sub-Centre: Peshawar

\begin{tabular}{|c|c|c|c|c|c|c|c|c|c|c|}
\hline \multirow[b]{2}{*}{$\begin{array}{l}\text { Grades I } \\
\text { Sections }\end{array}$} & \multicolumn{5}{|c|}{ - Number of Students- } & \multirow[b]{2}{*}{ Grades } & \multicolumn{4}{|c|}{-Percent of Students } \\
\hline & Enrolled & Dropped & $\begin{array}{l}\text { Appeared } \\
\text { in Exam }\end{array}$ & $\begin{array}{c}\text { Passed in } \\
\text { Exams }\end{array}$ & $\begin{array}{l}\text { Failed In } \\
\text { Exams }\end{array}$ & & Dropped & $\begin{array}{l}\text { Appeared } \\
\text { in Exams }\end{array}$ & $\begin{array}{c}\text { Passed in } \\
\text { Exams }\end{array}$ & $\begin{array}{c}\text { Falled in } \\
\text { Exams }\end{array}$ \\
\hline Grade $1 \mathrm{~A}$ & 80 & 6 & 74 & 33 & 41 & Grade 1 & $7.50 \%$ & $92.50 \%$ & $44.59 \%$ & $55.41 \%$ \\
\hline Grade 1 B & 0 & 0 & 0 & 0 & 0 & Grade 2 & $10.00 \%$ & $90.00 \%$ & $86.67 \%$ & $13.33 \%$ \\
\hline Grade $1 \mathrm{C}$ & 0 & 0 & 0 & 0 & 0 & Grade 3 & $17.50 \%$ & $82.50 \%$ & $72.73 \%$ & $27.27 \%$ \\
\hline Grade 2 A & 50 & 5 & 45 & 39 & 6 & Grade 4 & $3.70 \%$ & $96.30 \%$ & $88.46 \%$ & $11.54 \%$ \\
\hline Grade 2 B & 0 & 0 & 0 & 0 & 0 & Grade 5 & $4.76 \%$ & $95.24 \%$ & $100.00 \%$ & $0.00 \%$ \\
\hline Grade $3 \mathrm{~A}$ & 40 & 7 & 33 & 24 & 9 & Grade 6 & \#Num! & \#Num! & \#Num! & \#Num! \\
\hline Grade 3 B & 0 & 0 & 0 & 0 & 0 & & & & & \\
\hline Grade 4 & 27 & 1 & 26 & 23 & 3 & Remarks: & & & & \\
\hline Grade 5 & 21 & 1 & 20 & 20 & 0 & & & & & \\
\hline Grade 6 & 0 & 0 & 0 & 0 & 0 & & & & & \\
\hline School Total & 218 & 20 & 198 & 139 & 59 & School \%age & $9.17 \%$ & $90.83 \%$ & $70.20 \%$ & $29.80 \%$ \\
\hline
\end{tabular}

\begin{tabular}{|c|c|c|c|c|c|c|c|c|c|c|}
\hline \multirow[b]{2}{*}{$\begin{array}{l}\text { Grades I } \\
\text { Sections }\end{array}$} & \multicolumn{5}{|c|}{ - Number of Students } & \multirow[b]{2}{*}{ Grades } & \multicolumn{4}{|c|}{-Percent of Students- } \\
\hline & Enrolled & Dropped & $\begin{array}{l}\text { Appeared } \\
\text { in Exam }\end{array}$ & $\begin{array}{l}\text { Passed in } \\
\text { Exams }\end{array}$ & $\begin{array}{l}\text { Failed in } \\
\text { Exams }\end{array}$ & & Dropped & $\begin{array}{l}\text { Appeared } \\
\text { in Exams }\end{array}$ & $\begin{array}{c}\text { Passed in } \\
\text { Exams }\end{array}$ & $\begin{array}{l}\text { Faled in } \\
\text { Exams }\end{array}$ \\
\hline Grade $1 \mathrm{~A}$ & 56 & 8 & 48 & 23 & 25 & Grade 1 & $9.91 \%$ & $90.09 \%$ & $52.00 \%$ & $48.00 \%$ \\
\hline Grade 1 B & 55 & 3 & 52 & 29 & 23 & Grade 2 & $8.51 \%$ & $91.49 \%$ & $81.40 \%$ & $18.60 \%$ \\
\hline Grade $1 \mathrm{C}$ & 0 & 0 & 0 & 0 & 0 & Grade 3 & $10.53 \%$ & $89.47 \%$ & $100.00 \%$ & $0.00 \%$ \\
\hline Grade $2 \mathrm{~A}$ & 47 & 4 & 43 & 35 & 8 & Grade 4 & $30.77 \%$ & $69.23 \%$ & $100.00 \%$ & $0.00 \%$ \\
\hline Grade 2 B & 0 & 0 & 0 & 0 & 0 & Grade 5 & $37.50 \%$ & $62.50 \%$ & $100.00 \%$ & $0.00 \%$ \\
\hline Grade $3 \mathrm{~A}$ & 19 & 2 & 17 & 17 & 0 & Grade 6 & \#Num! & \#Num! & \#Num! & \#Num! \\
\hline Grade 3 B & 0 & 0 & 0 & 0 & 0 & & & & & \\
\hline Grade 4 & 13 & 4 & 9 & 9 & 0 & Remarks: & & & & \\
\hline Grade 5 & 8 & 3 & 5 & 5 & 0 & & & & & \\
\hline Grade 6 & 0 & 0 & 0 & 0 & 0 & & & & & \\
\hline School Total & 198 & 24 & 174 & 118 & 56 & School \%age & $12.12 \%$ & $87.88 \%$ & $67.82 \%$ & $32.18 \%$ \\
\hline
\end{tabular}

\begin{tabular}{|c|c|c|c|c|c|c|c|c|c|c|}
\hline \multicolumn{6}{|c|}{ School Code: PS/PSH/PSC-167 } & \multicolumn{5}{|c|}{ Head Teacher: Zalma } \\
\hline $\begin{array}{l}\text { Grades I } \\
\text { Sections }\end{array}$ & Enrolled & Dropped & $\begin{array}{l}\text { Appeared } \\
\text { in Exam }\end{array}$ & $\begin{array}{l}\text { Passed in } \\
\text { Exams }\end{array}$ & $\begin{array}{c}\text { Failed in } \\
\text { Exams }\end{array}$ & Grades & Dropped & $\begin{array}{l}\text { Appeared } \\
\text { in Exams }\end{array}$ & $\begin{array}{l}\text { Passed in } \\
\text { Exams }\end{array}$ & $\begin{array}{l}\text { Failed in } \\
\text { Exams }\end{array}$ \\
\hline Grade $1 \mathrm{~A}$ & 46 & 0 & 46 & 36 & 10 & Grade 1 & $2.13 \%$ & $97.87 \%$ & $78.26 \%$ & $21.74 \%$ \\
\hline Grade 1 B & 48 & 2 & 46 & 36 & 10 & Grade 2 & $10.59 \%$ & $89.41 \%$ & $55.26 \%$ & $44.74 \%$ \\
\hline Grade $1 \mathrm{C}$ & 0 & 0 & 0 & 0 & 0 & Grade 3 & $10.00 \%$ & $90.00 \%$ & $88.89 \%$ & $11.11 \%$ \\
\hline Grade 2 A & 42 & 6 & 36 & 22 & 14 & Grade 4 & $11.11 \%$ & $88.89 \%$ & $95.83 \%$ & $4.17 \%$ \\
\hline Grade 2 B & 43 & 3 & 40 & 20 & 20 & Grade 5 & $10.00 \%$ & $90.00 \%$ & $72.22 \%$ & $27.78 \%$ \\
\hline Grade $3 \mathrm{~A}$ & 40 & 4 & 36 & 32 & 4 & Grade 6 & $5.00 \%$ & $95.00 \%$ & $94.74 \%$ & $5.26 \%$ \\
\hline Grade 3 B & 0 & 0 & 0 & 0 & 0 & & & & & \\
\hline Grade 4 & 27 & 3 & 24 & 23 & 1 & Remarks: & & & & \\
\hline Grade 5 & 20 & 2 & 18 & 13 & 5 & & & & & \\
\hline Grade 6 & 20 & 1 & 19 & 18 & 1 & & & & & \\
\hline School Total & 286 & 21 & 265 & 200 & 65 & School \%age & $7.34 \%$ & $92.66 \%$ & $75.47 \%$ & $24.53 \%$ \\
\hline
\end{tabular}

Note:

\#Numl = Enrollment in respective grade(s) is nill therefore calculation is not applicable
Page 57 of 91

Produced by DP/MIS Section on 31-Jul-97 
Sub-Centre: Peshawar

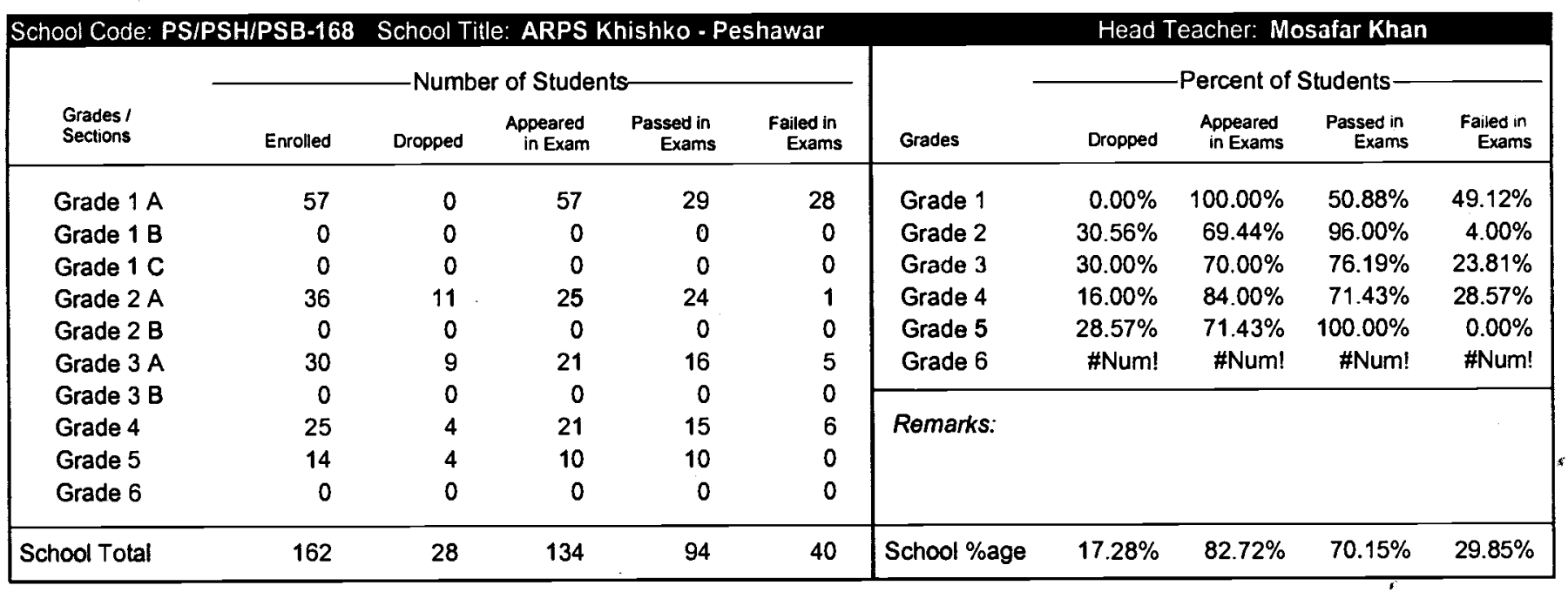

\begin{tabular}{|c|c|c|c|c|c|c|c|c|c|c|c|}
\hline \multicolumn{12}{|c|}{ School Code: PS/PSH/PSB-169 } \\
\hline \multirow[b]{2}{*}{$\begin{array}{l}\text { Grades I } \\
\text { Sections }\end{array}$} & \multicolumn{6}{|c|}{ - Number of Students- } & \multirow[b]{2}{*}{ Grades } & \multicolumn{4}{|c|}{ Percent of Students } \\
\hline & ' & Enrolled & Dropped & $\begin{array}{c}\text { Appeared } \\
\text { in Exam }\end{array}$ & $\begin{array}{l}\text { Passed in } \\
\text { Exams }\end{array}$ & $\begin{array}{c}\text { Failed in } \\
\text { Exams }\end{array}$ & & Dropped & $\begin{array}{l}\text { Appeared } \\
\text { in Exams }\end{array}$ & $\begin{array}{l}\text { Passed in } \\
\text { Exams }\end{array}$ & $\begin{array}{l}\text { Failed in } \\
\text { Exams }\end{array}$ \\
\hline Grade $1 \mathrm{~A}$ & & 71 & 0 & 71 & 38 & 33 & Grade 1 & $0.00 \%$ & $100.00 \%$ & $53.52 \%$ & $46.48 \%$ \\
\hline Grade 1 B & & 0 & 0 & 0 & 0 & 0 & Grade 2 & $8.89 \%$ & $91.11 \%$ & $63.41 \%$ & $36.59 \%$ \\
\hline Grade $1 \mathrm{C}$ & & 0 & 0 & 0 & 0 & 0 & Grade 3 & $0.00 \%$ & $100.00 \%$ & $72.73 \%$ & $27.27 \%$ \\
\hline Grade 2 A & & 45 & 4 & 41 & 26 & 15 & Grade 4 & $10.53 \%$ & $89.47 \%$ & $70.59 \%$ & $29.41 \%$ \\
\hline Grade 2 B & & 0 & 0 & 0 & 0 & 0 & Grade 5 & $0.00 \%$ & $100.00 \%$ & $65.00 \%$ & $35.00 \%$ \\
\hline Grade 3 A & & -22 & 0 & 22 & 16 & 6 & Grade 6 & \#Num! & \#Num! & \#Num! & \#Num! \\
\hline Grade 3 B & & 0 & 0 & 0 & 0 & 0 & & & & & \\
\hline Grade 4 & & 19 & 2 & 17 & 12 & 5 & Remarks: & & & & \\
\hline Grade 5 & & 20 & 0 & 20 & 13 & 7 & & & & & \\
\hline Grade 6 & & 0 & 0 & 0 & 0 & 0 & & & & & \\
\hline School Total & & 177 & 6 & 171 & 105 & 66 & School \%age & $3.39 \%$ & $96.61 \%$ & $61.40 \%$ & $38.60 \%$ \\
\hline
\end{tabular}

\begin{tabular}{|c|c|c|c|c|c|c|c|c|c|c|}
\hline \multirow[b]{2}{*}{$\begin{array}{l}\text { Grades I } \\
\text { Sections }\end{array}$} & \multicolumn{5}{|c|}{-Number of Students- } & \multirow[b]{2}{*}{ Grades } & \multicolumn{4}{|c|}{ Percent of Students } \\
\hline & Enrolled & Dropped & $\begin{array}{l}\text { Appeared } \\
\text { in Exam }\end{array}$ & $\begin{array}{c}\text { Passed in } \\
\text { Exams }\end{array}$ & $\begin{array}{l}\text { Failed in } \\
\text { Exams }\end{array}$ & & Dropped & $\begin{array}{l}\text { Appeared } \\
\text { in Exams }\end{array}$ & $\begin{array}{c}\text { Passed in } \\
\text { Exams }\end{array}$ & $\begin{array}{c}\text { Failed in } \\
\text { Exams }\end{array}$ \\
\hline Grade $1 \mathrm{~A}$ & 56 & 6 & 50 & 21 & 29 & Grade 1 & $5.93 \%$ & $94.07 \%$ & $52.25 \%$ & $47.75 \%$ \\
\hline Grade 1 B & 62 & 1 & 61 & 37 & 24 & Grade 2 & $0.00 \%$ & $100.00 \%$ & $48.08 \%$ & $51.92 \%$ \\
\hline Grade $1 \mathrm{C}$ & 0 & 0 & 0 & 0 & 0 & Grade 3 & $48.15 \%$ & $51.85 \%$ & $85.71 \%$ & $14.29 \%$ \\
\hline Grade $2 \mathrm{~A}$ & 52 & 0 & 52 & 25 & 27 & Grade 4 & $30.00 \%$ & $70.00 \%$ & $71.43 \%$ & $28.57 \%$ \\
\hline Grade 2 B & 0 & 0 & 0 & 0 & 0 & Grade 5 & $0.00 \%$ & $100.00 \%$ & $92.86 \%$ & $7.14 \%$ \\
\hline Grade 3 A & 27 & 13 & 14 & 12 & 2 & Grade 6 & \#Num! & \#Num! & \#um! & \#Num! \\
\hline Grade 3 B & 0 & 0 & 0 & 0 & 0 & & & & & \\
\hline Grade 4 & 20 & 6 & 14 & 10 & 4 & Remarks: & & & & \\
\hline Grade 5 & 14 & 0 & 14 & 13 & 1 & & & & & \\
\hline Grade 6 & 0 & 0 & 0 & 0 & 0 & & & & & \\
\hline School Total & 231 & 26 & 205 & 118 & 87 & School \%age & $11.26 \%$ & $88.74 \%$ & $57.56 \%$ & $42.44 \%$ \\
\hline
\end{tabular}

Note:

\#Numl = Enrollmenl in respective grade(s) is nill therefore calculation is not applicable 
Sub-Centre: Peshawar

\begin{tabular}{|c|c|c|c|c|c|c|c|c|c|c|}
\hline \multirow[b]{2}{*}{$\begin{array}{l}\text { Grades I } \\
\text { Sections }\end{array}$} & \multicolumn{5}{|c|}{-Number of Students- } & \multirow[b]{2}{*}{ Grades } & \multicolumn{4}{|c|}{-Percent of Students } \\
\hline & Enrolled & Dropped & $\begin{array}{l}\text { Appeared } \\
\text { in Exam }\end{array}$ & $\begin{array}{l}\text { Passed in } \\
\text { Exams }\end{array}$ & $\begin{array}{l}\text { Falled in } \\
\text { Exams }\end{array}$ & & Dropped & $\begin{array}{l}\text { Appeared } \\
\text { in Exams }\end{array}$ & $\begin{array}{c}\text { Passed in } \\
\text { Exams }\end{array}$ & $\begin{array}{c}\text { Failed in } \\
\text { Exams }\end{array}$ \\
\hline Grade $1 \mathrm{~A}$ & 77 & 0 & 77 & 39 & 38 & Grade 1 & $0.00 \%$ & $100.00 \%$ & $50.65 \%$ & $49.35 \%$ \\
\hline Grade 1 B & 0 & 0 & 0 & 0 & 0 & Grade 2 & $0.00 \%$ & $100.00 \%$ & $60.78 \%$ & $39.22 \%$ \\
\hline Grade $1 \mathrm{C}$ & 0 & 0 & 0 & 0 & 0 & Grade 3 & $0.00 \%$ & $100.00 \%$ & $63.64 \%$ & $36.36 \%$ \\
\hline Grade $2 \mathrm{~A}$ & 51 & 0 & 51 & 31 & 20 & Grade 4 & $0.00 \%$ & $100.00 \%$ & $68.18 \%$ & $31.82 \%$ \\
\hline Grade 2 B & 0 & 0 & 0 & 0 & 0 & Grade 5 & $0.00 \%$ & $100.00 \%$ & $28.57 \%$ & $71.43 \%$ \\
\hline Grade $3 \mathrm{~A}$ & 22 & 0 & 22 & 14 & 8 & Grade 6 & \#Num! & \#Num! & \#Num! & \#Num! \\
\hline Grade 3 B & 0 & 0 & 0 & 0 & 0 & & & & & \\
\hline Grade 4 & 22 & 0 & 22 & 15 & 7 & Remarks: & & & & \\
\hline Grade 5 & 21 & 0 & 21 & 6 & 15 & & & & & \\
\hline Grade 6 & 0 & 0 & 0 & 0 & 0 & & & & & \\
\hline School Total & 193 & 0 & 193 & 105 & 88 & School \%age & $0.00 \%$ & $100.00 \%$ & $54.40 \%$ & $45.60 \%$ \\
\hline
\end{tabular}

\begin{tabular}{|c|c|c|c|c|c|c|c|c|c|c|c|}
\hline \multirow[b]{2}{*}{$\begin{array}{l}\text { Grades / } \\
\text { Sections }\end{array}$} & \multicolumn{6}{|c|}{-Number of Students- } & \multirow[b]{2}{*}{ Grades } & \multicolumn{4}{|c|}{-Percent of Students } \\
\hline & , & Enrolled & Dropped & $\begin{array}{l}\text { Appeared } \\
\text { in Exam }\end{array}$ & $\begin{array}{l}\text { Passed in } \\
\text { Exams }\end{array}$ & $\begin{array}{l}\text { Failed in } \\
\text { Exams }\end{array}$ & & Dropped & $\begin{array}{l}\text { Appeared } \\
\text { in Exams }\end{array}$ & $\begin{array}{c}\text { Passed in } \\
\text { Exams }\end{array}$ & $\begin{array}{c}\text { Failed in } \\
\text { Exams } \\
\end{array}$ \\
\hline Grade $1 \mathrm{~A}$ & & 54 & 0 & 54 & 26 & 28 & Grade 1 & $0.00 \%$ & $100.00 \%$ & $50.00 \%$ & $50.00 \%$ \\
\hline Grade 1 B & & 52 & 0 & 52 & 25 & 27 & Grade 2 & $10.24 \%$ & $89.76 \%$ & $58.77 \%$ & $41.23 \%$ \\
\hline Grade $1 \mathrm{C}$ & & 50 & 0 & 50 & 27 & 23 & Grade 3 & $14.29 \%$ & $85.71 \%$ & $72.22 \%$ & $27.78 \%$ \\
\hline Grade 2 A & & 66 & 7 & 59 & 37 & 22 & Grade 4 & $14.52 \%$ & $85.48 \%$ & $64.15 \%$ & $35.85 \%$ \\
\hline Grade 2 B & & 61 & 6 & 55 & 30 & 25 & Grade 5 & $10.34 \%$ & $89.66 \%$ & $50.00 \%$ & $50.00 \%$ \\
\hline Grade $3 \mathrm{~A}$ & & 63 & 9 & 54 & 39 & 15 & Grade 6 & $6.25 \%$ & $93.75 \%$ & $60.00 \%$ & $40.00 \%$ \\
\hline Grade 3 B & & 0 & 0 & 0 & 0 & 0 & & & & & \\
\hline Grade 4 & & 62 & 9 & 53 & 34 & 19 & Remarks: & & & & \\
\hline Grade 5 & & 29 & 3 & 26 & 13 & 13 & & & & & \\
\hline Grade 6 & & 32 & 2 & 30 & 18 & 12 & & & & & \\
\hline School Total & & 469 & 36 & 433 & 249 & 184 & School \%age & $7.68 \%$ & $92.32 \%$ & $57.51 \%$ & $42.49 \%$ \\
\hline
\end{tabular}

\begin{tabular}{|c|c|c|c|c|c|c|c|c|c|c|}
\hline \multirow[b]{2}{*}{$\begin{array}{l}\text { Grades I } \\
\text { Sections }\end{array}$} & \multicolumn{5}{|c|}{ Number of Studente- } & \multirow[b]{2}{*}{ Grades } & \multicolumn{4}{|c|}{-Percent of Students } \\
\hline & Enrolled & Dropped & $\begin{array}{l}\text { Appeared } \\
\text { in Exam }\end{array}$ & $\begin{array}{l}\text { Passed in } \\
\text { Exams }\end{array}$ & $\begin{array}{l}\text { Failed in } \\
\text { Exams }\end{array}$ & & Dropped & $\begin{array}{c}\text { Appeared } \\
\text { in Exams }\end{array}$ & $\begin{array}{c}\text { Passed in } \\
\text { Exams }\end{array}$ & $\begin{array}{c}\text { Failed in } \\
\text { Exams }\end{array}$ \\
\hline Grade $1 \mathrm{~A}$ & 81 & 0 & 81 & 41 & 40 & Grade 1 & $0.00 \%$ & $100.00 \%$ & $50.62 \%$ & $49.38 \%$ \\
\hline Grade 1 B & 0 & 0 & 0 & 0 & 0 & Grade 2 & $0.00 \%$ & $100.00 \%$ & $54.55 \%$ & $45.45 \%$ \\
\hline Grade $1 \mathrm{C}$ & 0 & 0 & 0 & 0 & 0 & Grade 3 & $0.00 \%$ & $100.00 \%$ & $77.14 \%$ & $22.86 \%$ \\
\hline Grade 2 A & 55 & 0 & 55 & 30 & 25 & Grade 4 & $0.00 \%$ & $100.00 \%$ & $76.19 \%$ & $23.81 \%$ \\
\hline Grade 2 B & 0 & 0 & 0 & 0 & 0 & Grade 5 & $0.00 \%$ & $100.00 \%$ & $85.00 \%$ & $15.00 \%$ \\
\hline Grade 3 A & 35 & 0 & 35 & 27 & 8 & Grade 6 & \#Num! & \#Num! & \#Num! & \#Num! \\
\hline Grade 3 B & 0 & 0 & 0 & 0 & 0 & & & & & \\
\hline Grade 4 & 21 & 0 & 21 & 16 & 5 & Remarks: & & & & \\
\hline Grade 5 & 20 & 0 & 20 & 17 & 3 & & & & & \\
\hline Grade 6 & 0 & 0 & 0 & 0 & 0 & & & & & \\
\hline School Total & 212 & 0 & 212 & 131 & 81 & School \%age & $0.00 \%$ & $100.00 \%$ & $61.79 \%$ & $38.21 \%$ \\
\hline
\end{tabular}


Sub-Centre: Peshawar

\begin{tabular}{|c|c|c|c|c|c|c|c|c|c|c|}
\hline \multicolumn{11}{|c|}{ School Code: PS/PSH/MSB-174 School Title: ARPS Naguman - Peshawar } \\
\hline \multirow[b]{2}{*}{$\begin{array}{l}\text { Grades I } \\
\text { Sections }\end{array}$} & \multicolumn{5}{|c|}{-Number of Students- } & \multirow[b]{2}{*}{ Grades } & \multicolumn{4}{|c|}{ Percent of Students- } \\
\hline & Enrolled & Dropped & $\begin{array}{l}\text { Appeared } \\
\text { in Exam }\end{array}$ & $\begin{array}{c}\text { Passed in } \\
\text { Exams }\end{array}$ & $\begin{array}{c}\text { Failed in } \\
\text { Exams }\end{array}$ & & Dropped & $\begin{array}{c}\text { Appeared } \\
\text { in Exams }\end{array}$ & $\begin{array}{c}\text { Passed in } \\
\text { Exams }\end{array}$ & $\begin{array}{c}\text { Failed in } \\
\text { Exams }\end{array}$ \\
\hline Grade $1 \mathrm{~A}$ & 50 & 0 & 50 & 26 & 24 & Grade 1 & $0.00 \%$ & $100.00 \%$ & $59.76 \%$ & $40.24 \%$ \\
\hline Grade 1 B & 32 & 0 & 32 & 23 & 9 & Grade 2 & $8.33 \%$ & $91.67 \%$ & $70.91 \%$ & $29.09 \%$ \\
\hline Grade $1 \mathrm{C}$ & 0 & 0 & 0 & 0 & 0 & Grade 3 & $10.00 \%$ & $90.00 \%$ & $84.44 \%$ & $15.56 \%$ \\
\hline Grade 2 A & 60 & 5 & 55 & 39 & 16 & Grade 4 & $7.69 \%$ & $92.31 \%$ & $70.83 \%$ & $29.17 \%$ \\
\hline Grade 2 B & 0 & 0 & 0 & 0 & 0 & Grade 5 & $0.00 \%$ & $100.00 \%$ & $65.38 \%$ & $34.62 \%$ \\
\hline Grade 3 A & 50 & 5 & 45 & 38 & 7 & Grade 6 & $0.00 \%$ & $100.00 \%$ & $86.67 \%$ & $13.33 \%$ \\
\hline Grade 3 B & 0 & 0 & 0 & 0 & 0 & & & & & \\
\hline Grade 4 & 26 & 2 & 24 & 17 & 7 & Remarks: & & & & \\
\hline Grade 5 & 26 & 0 & 26 & 17 & 9 & & & & & \\
\hline Grade 6 & 15 & 0 & 15 & 13 & 2 & & & & & \\
\hline School Total & 259 & 12 & 247 & 173 & 74 & School \%age & $4.63 \%$ & $95.37 \%$ & $70.04 \%$ & $29.96 \%$ \\
\hline
\end{tabular}

\begin{tabular}{|c|c|c|c|c|c|c|c|c|c|c|c|}
\hline \multirow[b]{2}{*}{$\begin{array}{l}\text { Grades I } \\
\text { Sections }\end{array}$} & \multicolumn{6}{|c|}{-Number of Students- } & \multirow[b]{2}{*}{ Grades } & \multicolumn{4}{|c|}{-Percent of Students } \\
\hline & ' & Enrolled & Dropped & $\begin{array}{l}\text { Appeared } \\
\text { in Exam }\end{array}$ & $\begin{array}{l}\text { Passed in } \\
\text { Exams }\end{array}$ & $\begin{array}{l}\text { Failed in } \\
\text { Exams }\end{array}$ & & Dropped & $\begin{array}{l}\text { Appeared } \\
\text { in Exams }\end{array}$ & $\begin{array}{c}\text { Passed in } \\
\text { Exams }\end{array}$ & $\begin{array}{c}\text { Failed in } \\
\text { Exams } \\
\end{array}$ \\
\hline Grade $1 \mathrm{~A}$ & & 84 & 8 & 76 & 49 & 27 & Grade 1 & $4.88 \%$ & $95.12 \%$ & $58.33 \%$ & $41.67 \%$ \\
\hline Grade 1 B & & 80 & 0 & 80 & 42 & 38 & Grade 2 & $5.33 \%$ & $94.67 \%$ & $81.69 \%$ & $18.31 \%$ \\
\hline Grade $1 \mathrm{C}$ & & 0 & 0 & 0 & 0 & 0 & Grade 3 & $3.39 \%$ & $96.61 \%$ & $73.68 \%$ & $26.32 \%$ \\
\hline Grade $2 \mathrm{~A}$ & & 75 & 4 & 71 & 58 & 13 & Grade 4 & $13.89 \%$ & $86.11 \%$ & $74.19 \%$ & $25.81 \%$ \\
\hline Grade 2 B & & 0 & 0 & 0 & 0 & 0 & Grade 5 & $0.00 \%$ & $100.00 \%$ & $87.50 \%$ & $12.50 \%$ \\
\hline Grade $3 \mathrm{~A}$ & & 59 & 2 & 57 & 42 & 15 & Grade 6 & $0.00 \%$ & $100.00 \%$ & $100.00 \%$ & $0.00 \%$ \\
\hline Grade 3 B & & 0 & 0 & 0 & 0 & 0 & & & & & \\
\hline Grade 4 & & 36 & 5 & 31 & 23 & 8 & Remarks: & & & & \\
\hline Grade 5 & & 16 & 0 & 16 & 14 & 2 & & & & & \\
\hline Grade 6 & & 13 & 0 & 13 & 13 & 0 & & & & & \\
\hline School Total & & 363 & 19 & 344 & 241 & 103 & School \%age & $5.23 \%$ & $94.77 \%$ & $70.06 \%$ & $29.94 \%$ \\
\hline
\end{tabular}

\begin{tabular}{|c|c|c|c|c|c|c|c|c|c|c|}
\hline \multirow[b]{2}{*}{$\begin{array}{l}\text { Grades I } \\
\text { Sections }\end{array}$} & \multicolumn{5}{|c|}{ - Number of Students- } & \multirow[b]{2}{*}{ Grades } & \multicolumn{4}{|c|}{-Percent of Students- } \\
\hline & Enrolled & Dropped & $\begin{array}{l}\text { Appeared } \\
\text { in Exam }\end{array}$ & $\begin{array}{r}\text { Passed in } \\
\text { Exams }\end{array}$ & $\begin{array}{c}\text { Failed in } \\
\text { Exams }\end{array}$ & & Dropped & $\begin{array}{c}\text { Appeared } \\
\text { in Exams }\end{array}$ & $\begin{array}{c}\text { Passed in } \\
\text { Exams }\end{array}$ & $\begin{array}{l}\text { Failed in } \\
\text { Exams }\end{array}$ \\
\hline Grade $1 \mathrm{~A}$ & 66 & 12 & 54 & 37 & 17 & Grade 1 & $19.85 \%$ & $80.15 \%$ & $65.14 \%$ & $34.86 \%$ \\
\hline Grade 1 B & 70 & 15 & 55 & 34 & 21 & Grade 2 & $9.09 \%$ & $90.91 \%$ & $56.36 \%$ & $43.64 \%$ \\
\hline Grade $1 \mathrm{C}$ & 0 & 0 & 0 & 0 & 0 & Grade 3 & $5.81 \%$ & $94.19 \%$ & $79.01 \%$ & $20.99 \%$ \\
\hline Grade $2 \mathrm{~A}$ & 58 & 6 & 52 & 29 & 23 & Grade 4 & $9.09 \%$ & $90.91 \%$ & $64.00 \%$ & $36.00 \%$ \\
\hline Grade 2 B & 63 & 5 & 58 & 33 & 25 & Grade 5 & $3.03 \%$ & $96.97 \%$ & $68.75 \%$ & $31.25 \%$ \\
\hline Grade $3 \mathrm{~A}$ & 86 & 5 & 81 & 64 & 17 & Grade 6 & $13.89 \%$ & $86.11 \%$ & $83.87 \%$ & $16.13 \%$ \\
\hline Grade 3 B & 0 & 0 & 0 & 0 & 0 & & & & & \\
\hline Grade 4 & 55 & 5 & 50 & 32 & 18 & Remarks: & & & & \\
\hline Grade 5 & 33 & 1 & 32 & 22 & 10 & & & & & \\
\hline Grade 6 & 36 & 5 & 31 & 26 & 5 & & & & & \\
\hline School Total & 467 & 54 & 413 & 277 & 136 & School \%age & $11.56 \%$ & $88.44 \%$ & $67.07 \%$ & $32.93 \%$ \\
\hline
\end{tabular}

Note:

\#Num! = Enrollment in respective grade(s) is nill therefore calculation is not applicable
Page 60 of 91 Produced by DP/MIS Section on 31-Jul-97 
Sub-Centre: Peshawar

\begin{tabular}{|c|c|c|c|c|c|c|c|c|c|c|}
\hline \multirow[b]{2}{*}{$\begin{array}{l}\text { Grades I } \\
\text { Sections }\end{array}$} & \multicolumn{5}{|c|}{ - Number of Students } & \multirow[b]{2}{*}{ Grades } & \multicolumn{4}{|c|}{-Percent of Students } \\
\hline & Enrolled & Dropped & $\begin{array}{l}\text { Appeared } \\
\text { in Exam }\end{array}$ & $\begin{array}{l}\text { Passed in } \\
\text { Exams }\end{array}$ & $\begin{array}{l}\text { Failed in } \\
\text { Exams }\end{array}$ & & Dropped & $\begin{array}{l}\text { Appeared } \\
\text { in Exams }\end{array}$ & $\begin{array}{l}\text { Passed in } \\
\text { Exams }\end{array}$ & $\begin{array}{l}\text { Failed in } \\
\text { Exams }\end{array}$ \\
\hline Grade $1 \mathrm{~A}$ & 79 & 0 & 79 & 51 & 28 & Grade 1 & $0.00 \%$ & $100.00 \%$ & $64.56 \%$ & $35.44 \%$ \\
\hline Grade 1 B & 0 & 0 & 0 & 0 & 0 & Grade 2 & $0.00 \%$ & $100.00 \%$ & $80.00 \%$ & $20.00 \%$ \\
\hline Grade $1 \mathrm{C}$ & 0 & 0 & 0 & 0 & 0 & Grade 3 & $27.78 \%$ & $72.22 \%$ & $76.92 \%$ & $23.08 \%$ \\
\hline Grade 2 A & 35 & 0 & 35 & 28 & 7 & Grade 4 & $0.00 \%$ & $100.00 \%$ & $78.95 \%$ & $21.05 \%$ \\
\hline Grade 2 B & 0 & 0 & 0 & 0 & 0 & Grade 5 & $0.00 \%$ & $100.00 \%$ & $75.00 \%$ & $25.00 \%$ \\
\hline Grade 3 A & 36 & 10 & 26 & 20 & 6 & Grade 6 & \#Num! & \#Num! & \#Num! & \#Num! \\
\hline Grade 3 B & 0 & 0 & 0 & 0 & 0 & & & & & \\
\hline Grade 4 & 38 & 0 & 38 & 30 & 8 & Remarks: & & & & \\
\hline Grade 5 & 20 & 0 & 20 & 15 & 5 & & & & & \\
\hline Grade 6 & 0 & 0 & 0 & 0 & 0 & & & & & \\
\hline School Total & 208 & 10 & 198 & 144 & 54 & School \%age & $4.81 \%$ & $95.19 \%$ & $72.73 \%$ & $27.27 \%$ \\
\hline
\end{tabular}

\begin{tabular}{|c|c|c|c|c|c|c|c|c|c|c|}
\hline \multirow[b]{2}{*}{$\begin{array}{l}\text { Grades / } \\
\text { Sections }\end{array}$} & \multicolumn{5}{|c|}{ - Number of Students } & \multirow[b]{2}{*}{ Grades } & \multicolumn{4}{|c|}{-Percent of Students- } \\
\hline & 'Enrolled & Dropped & $\begin{array}{l}\text { Appeared } \\
\text { in Exam }\end{array}$ & $\begin{array}{r}\text { Passed in } \\
\text { Exams }\end{array}$ & $\begin{array}{l}\text { Failed in } \\
\text { Exams }\end{array}$ & & Dropped & $\begin{array}{l}\text { Appeared } \\
\text { in Exams }\end{array}$ & $\begin{array}{l}\text { Passed in } \\
\text { Exams }\end{array}$ & $\begin{array}{l}\text { Faled in } \\
\text { Exams }\end{array}$ \\
\hline Grade $1 \mathrm{~A}$ & 48 & 0 & 48 & 23 & 25 & Grade 1 & $0.00 \%$ & $100.00 \%$ & $43.75 \%$ & $56.25 \%$ \\
\hline Grade 1 B & 48 & 0 & 48 & 19 & 29 & Grade 2 & $0.00 \%$ & $100.00 \%$ & $43.75 \%$ & $56.25 \%$ \\
\hline Grade.1 C & 0 & 0 & 0 & 0 & 0 & Grade 3 & $0.00 \%$ & $100.00 \%$ & $54.05 \%$ & $45.95 \%$ \\
\hline Grade $2 \mathrm{~A}$ & 64 & 0 & 64 & 28 & 36 & Grade 4 & $0.00 \%$ & $100.00 \%$ & $100.00 \%$ & $0.00 \%$ \\
\hline Grade 2 B & 0 & 0 & 0 & 0 & 0 & Grade 5 & $12.50 \%$ & $87.50 \%$ & $100.00 \%$ & $0.00 \%$ \\
\hline Grade $3 \mathrm{~A}$ & $37=$ & 0 & 37 & 20 & 17 & Grade 6 & \#Num! & \#Num! & \#Num! & \#Num! \\
\hline Grade 3 B & 0 & 0 & 0 & 0 & 0 & & & & & \\
\hline Grade 4 & 19 & 0 & 19 & 19 & 0 & Remarks: & & & & \\
\hline Grade 5 & 16 & 2 & 14 & 14 & 0 & & & & & \\
\hline Grade 6 & 0 & 0 & 0 & 0 & 0 & & & & & \\
\hline School Total & 232 & 2 & 230 & 123 & 107 & School \%age & $0.86 \%$ & $99.14 \%$ & $53.48 \%$ & $46.52 \%$ \\
\hline
\end{tabular}

\begin{tabular}{|c|c|c|c|c|c|c|c|c|c|c|}
\hline \multirow[b]{2}{*}{$\begin{array}{l}\text { Grades I } \\
\text { Sections }\end{array}$} & \multicolumn{5}{|c|}{ —Number of Students- } & \multirow[b]{2}{*}{ Grades } & \multicolumn{4}{|c|}{-Percent of Students - } \\
\hline & Enrolled & Dropped & $\begin{array}{l}\text { Appeared } \\
\text { in Exam }\end{array}$ & $\begin{array}{c}\text { Passed in } \\
\text { Exams }\end{array}$ & $\begin{array}{c}\text { Failed in } \\
\text { Exams }\end{array}$ & & Dropped & $\begin{array}{c}\text { Appeared } \\
\text { in Exams }\end{array}$ & $\begin{array}{c}\text { Passed in } \\
\text { Exams }\end{array}$ & $\begin{array}{c}\text { Falled in } \\
\text { Exams }\end{array}$ \\
\hline Grade $1 \mathrm{~A}$ & 79 & 15 & 64 & 35 & 29 & Grade 1 & $14.18 \%$ & $85.82 \%$ & $53.04 \%$ & $46.96 \%$ \\
\hline Grade 1 B & 55 & 4 & 51 & 26 & 25 & Grade 2 & $7.32 \%$ & $92.68 \%$ & $65.79 \%$ & $34.21 \%$ \\
\hline Grade $1 \mathrm{C}$ & 0 & 0 & 0 & 0 & 0 & Grade 3 & $4.88 \%$ & $95.12 \%$ & $61.54 \%$ & $38.46 \%$ \\
\hline Grade $2 \mathrm{~A}$ & 41 & 3 & 38 & 25 & 13 & Grade 4 & $4.76 \%$ & $95.24 \%$ & $60.00 \%$ & $40.00 \%$ \\
\hline Grade 2 B & 0 & 0 & 0 & 0 & 0 & Grade 5 & $12.50 \%$ & $87.50 \%$ & $78.57 \%$ & $21.43 \%$ \\
\hline Grade 3 A & 41 & 2 & 39 & 24 & 15 & Grade 6 & $0.00 \%$ & $100.00 \%$ & $53.85 \%$ & $46.15 \%$ \\
\hline Grade 3 B & 0 & 0 & 0 & 0 & 0 & & & & & \\
\hline Grade 4 & 21 & 1 & 20 & 12 & 8 & Remarks: & & & & \\
\hline Grade 5 & 16 & 2 & 14 & 11 & 3 & & & & & \\
\hline Grade 6 & 26 & 0 & 26 & 14 & 12 & & & & & \\
\hline School Total & 279 & 27 & 252 & 147 & 105 & School \%age & $9.68 \%$ & $90.32 \%$ & $58.33 \%$ & $41.67 \%$ \\
\hline
\end{tabular}


Sub-Centre: Peshawar

\begin{tabular}{|c|c|c|c|c|c|c|c|c|c|c|}
\hline \multirow[b]{2}{*}{$\begin{array}{l}\text { Grades I } \\
\text { Sections }\end{array}$} & \multicolumn{5}{|c|}{ - Number of Students- } & \multirow[b]{2}{*}{ Grades } & \multicolumn{4}{|c|}{-Percent of Students- } \\
\hline & Enrolled & Dropped & $\begin{array}{l}\text { Appeared } \\
\text { in Exam }\end{array}$ & $\begin{array}{l}\text { Passed in } \\
\text { Exams }\end{array}$ & $\begin{array}{l}\text { Failed in } \\
\text { Exams }\end{array}$ & & Dropped & $\begin{array}{l}\text { Appeared } \\
\text { in Exams }\end{array}$ & $\begin{array}{l}\text { Passed in } \\
\text { Exams }\end{array}$ & $\begin{array}{l}\text { Failed in } \\
\text { Exams }\end{array}$ \\
\hline Grade $1 \mathrm{~A}$ & 76 & 8 & 68 & 54 & 14 & Grade 1 & $7.22 \%$ & $92.78 \%$ & $81.44 \%$ & $18.56 \%$ \\
\hline Grade 1 B & 54 & 2 & 52 & 43 & 9 & Grade 2 & $1.64 \%$ & $98.36 \%$ & $82.50 \%$ & $17.50 \%$ \\
\hline Grade $1 \mathrm{C}$ & 50 & 3 & 47 & 39 & 8 & Grade 3 & $0.00 \%$ & $100.00 \%$ & $90.91 \%$ & $9.09 \%$ \\
\hline Grade 2 A & 59 & 2 & 57 & 44 & 13 & Grade 4 & $10.00 \%$ & $90.00 \%$ & $88.89 \%$ & $11.11 \%$ \\
\hline Grade 2 B & 63 & 0 & 63 & 55 & 8 & Grade 5 & $1.45 \%$ & $98.55 \%$ & $89.71 \%$ & $10.29 \%$ \\
\hline Grade $3 \mathrm{~A}$ & 42 & 0 & 42 & 37 & 5 & Grade 6 & $3.57 \%$ & $96.43 \%$ & $96.30 \%$ & $3.70 \%$ \\
\hline Grade 3 B & 46 & 0 & 46 & 43 & 3 & & & & & \\
\hline Grade 4 & 90 & 9 & 81 & 72 & 9 & Remarks: & & & & \\
\hline Grade 5 & 69 & 1 & 68 & 61 & 7 & & & & & \\
\hline Grade 6 & 56 & 2 & 54 & 52 & 2 & & & & & \\
\hline School Total & 605 & 27 & 578 & 500 & 78 & School \%age & $4.46 \%$ & $95.54 \%$ & $86.51 \%$ & $13.49 \%$ \\
\hline
\end{tabular}

\begin{tabular}{|c|c|c|c|c|c|c|c|c|c|c|c|}
\hline \multirow[b]{2}{*}{$\begin{array}{l}\text { Grades ' } \\
\text { Sections }\end{array}$} & \multicolumn{6}{|c|}{ - Number of Students- } & \multirow[b]{2}{*}{ Grades } & \multicolumn{4}{|c|}{-Percent of Students } \\
\hline & ' & Enrolled & Dropped & $\begin{array}{l}\text { Appeared } \\
\text { in Exam }\end{array}$ & $\begin{array}{c}\text { Passed in } \\
\text { Exams }\end{array}$ & $\begin{array}{l}\text { Failed in } \\
\text { Exams }\end{array}$ & & Dropped & $\begin{array}{l}\text { Appeared } \\
\text { in Exams }\end{array}$ & $\begin{array}{l}\text { Passed in } \\
\text { Exams }\end{array}$ & $\begin{array}{l}\text { Falied in } \\
\text { Exams }\end{array}$ \\
\hline Grade $1 \mathrm{~A}$ & & 68 & 0 & 68 & 48 & 20 & Grade 1 & $0.00 \%$ & $100.00 \%$ & $62.50 \%$ & $37.50 \%$ \\
\hline Grade 1 B & & 76 & 0 & 76 & 42 & 34 & Grade 2 & $0.00 \%$ & $100.00 \%$ & $67.44 \%$ & $32.56 \%$ \\
\hline Grade $1 \mathrm{C}$ & & 0 & 0 & 0 & 0 & 0 & Grade 3 & $4.17 \%$ & $95.83 \%$ & $65.22 \%$ & $34.78 \%$ \\
\hline Grade $2 \mathrm{~A}$ & & 43 & 0 & 43 & 29 & 14 & Grade 4 & $0.00 \%$ & $100.00 \%$ & $85.00 \%$ & $15.00 \%$ \\
\hline Grade 2 B & & 0 & 0 & 0 & 0 & 0 & Grade 5 & $0.00 \%$ & $100.00 \%$ & $85.00 \%$ & $15.00 \%$ \\
\hline Grade $3 \mathrm{~A}$ & & 24 & 1 & 23 & 15 & 8 & Grade 6 & \#Num! & \#Num! & \#Num! & \#Num! \\
\hline Grade 3 B & & 0 & 0 & 0 & 0 & 0 & & & & & \\
\hline Grade 4 & & 20 & 0 & 20 & 17 & 3 & Remarks: & & & & \\
\hline Grade 5 & & 20 & 0 & 20 & 17 & 3 & & & & & \\
\hline Grade 6 & & 0 & 0 & 0 & 0 & 0 & & & & & \\
\hline School Total & & 251 & 1 & 250 & 168 & 82 & School \%age & $0.40 \%$ & $99.60 \%$ & $67.20 \%$ & $32.80 \%$ \\
\hline
\end{tabular}

\begin{tabular}{|c|c|c|c|c|c|c|c|c|c|c|}
\hline \multirow[b]{2}{*}{$\begin{array}{l}\text { Grades I } \\
\text { Sections }\end{array}$} & \multicolumn{5}{|c|}{ - Number of Students- } & \multirow[b]{2}{*}{ Grades } & \multicolumn{4}{|c|}{-Percent of Students } \\
\hline & Enrolled & Dropped & $\begin{array}{l}\text { Appeared } \\
\text { in Exam }\end{array}$ & $\begin{array}{l}\text { Passed in } \\
\text { Exams }\end{array}$ & $\begin{array}{c}\text { Failed in } \\
\text { Exams }\end{array}$ & & Dropped & $\begin{array}{c}\text { Appeared } \\
\text { in Exams }\end{array}$ & $\begin{array}{c}\text { Passed in } \\
\text { Exams }\end{array}$ & $\begin{array}{c}\text { Falled in } \\
\text { Exams }\end{array}$ \\
\hline Grade $1 \mathrm{~A}$ & 41 & 1 & 40 & 25 & 15 & Grade 1 & $9.09 \%$ & $90.91 \%$ & $55.00 \%$ & $45.00 \%$ \\
\hline Grade 1 B & 47 & 7 & 40 & 19 & 21 & Grade 2 & $0.00 \%$ & $100.00 \%$ & $70.00 \%$ & $30.00 \%$ \\
\hline Grade $1 \mathrm{C}$ & 0 & 0 & 0 & 0 & 0 & Grade 3 & $6.25 \%$ & $93.75 \%$ & $56.67 \%$ & $43.33 \%$ \\
\hline Grade $2 \mathrm{~A}$ & 40 & 0 & 40 & 28 & 12 & Grade 4 & $3.85 \%$ & $96.15 \%$ & $92.00 \%$ & $8.00 \%$ \\
\hline Grade 2 B & 0 & 0 & 0 & 0 & 0 & Grade 5 & $13.64 \%$ & $86.36 \%$ & $94.74 \%$ & $5.26 \%$ \\
\hline Grade $3 \mathrm{~A}$ & 32 & 2 & 30 & 17 & 13 & Grade 6 & $0.00 \%$ & $100.00 \%$ & $73.91 \%$ & $26.09 \%$ \\
\hline Grade 3 B & 0 & 0 & 0 & 0 & 0 & & & & & \\
\hline Grade 4 & 26 & 1 & 25 & 23 & 2 & Remarks: & & & & \\
\hline Grade 5 & 22 & 3 & 19 & 18 & 1 & & & & & \\
\hline Grade 6 & 23 & 0 & 23 & 17 & 6 & & & & & \\
\hline School Total & 231 & 14 & 217 & 147 & 70 & School \%age & $6.06 \%$ & $93.94 \%$ & $67.74 \%$ & $32.26 \%$ \\
\hline
\end{tabular}

Note:

\#Num! = Enrollment in respective grade(s) is nill therefore calculation is not applicable 
Sub-Centre: Peshawar

\begin{tabular}{|c|c|c|c|c|c|c|c|c|c|c|}
\hline \multirow[b]{2}{*}{$\begin{array}{l}\text { Grades I } \\
\text { Sections }\end{array}$} & \multicolumn{5}{|c|}{ - Number of Students- } & \multirow[b]{2}{*}{ Grades } & \multicolumn{4}{|c|}{-Percent of Students- } \\
\hline & Enrolled & Dropped & $\begin{array}{l}\text { Appeared } \\
\text { in Exam }\end{array}$ & $\begin{array}{r}\text { Passed in } \\
\text { Exams }\end{array}$ & $\begin{array}{l}\text { Failed in } \\
\text { Exams }\end{array}$ & & Dropped & $\begin{array}{c}\text { Appeared } \\
\text { in Exams }\end{array}$ & $\begin{array}{c}\text { Passed in } \\
\text { Exams }\end{array}$ & $\begin{array}{c}\text { Faled in } \\
\text { Exams }\end{array}$ \\
\hline Grade $1 \mathrm{~A}$ & 65 & 0 & 65 & 32 & 33 & Grade 1 & $0.00 \%$ & $100.00 \%$ & $49.23 \%$ & $50.77 \%$ \\
\hline Grade 1 B & 0 & 0 & 0 & 0 & 0 & Grade 2 & $0.00 \%$ & $100.00 \%$ & $64.29 \%$ & $35.71 \%$ \\
\hline Grade $1 \mathrm{C}$ & 0 & 0 & 0 & 0 & 0 & Grade 3 & $0.00 \%$ & $100.00 \%$ & $67.50 \%$ & $32.50 \%$ \\
\hline Grade 2 A & 56 & 0 & 56 & 36 & 20 & Grade 4 & $0.00 \%$ & $100.00 \%$ & $70.73 \%$ & $29.27 \%$ \\
\hline Grade 2 B & 0 & 0 & 0 & 0 & 0 & Grade 5 & $0.00 \%$ & $100.00 \%$ & $56.67 \%$ & $43.33 \%$ \\
\hline Grade $3 \mathrm{~A}$ & 40 & 0 & 40 & 27 & 13 & Grade 6 & $0.00 \%$ & $100.00 \%$ & $71.43 \%$ & $28.57 \%$ \\
\hline Grade 3 B & 0 & 0 & 0 & 0 & 0 & & & & & \\
\hline Grade 4 & 41 & 0 & 41 & 29 & 12 & Remarks: & & & & \\
\hline Grade 5 & 30 & 0 & 30 & 17 & 13 & & & & & \\
\hline Grade 6 & 14 & 0 & 14 & 10 & 4 & & & & & \\
\hline School Total & 246 & 0 & 246 & 151 & 95 & School \%age & $0.00 \%$ & $100.00 \%$ & $61.38 \%$ & $38.62 \%$ \\
\hline
\end{tabular}

\begin{tabular}{|c|c|c|c|c|c|c|c|c|c|c|}
\hline \multirow[b]{2}{*}{$\begin{array}{l}\text { Grades / } \\
\text { Sections }\end{array}$} & \multicolumn{5}{|c|}{ - Number of Students- } & \multirow[b]{2}{*}{ Grades } & \multicolumn{4}{|c|}{-Percent of Students - } \\
\hline & Enrolled & Dropped & $\begin{array}{l}\text { Appeared } \\
\text { in Exam }\end{array}$ & $\begin{array}{c}\text { Passed in } \\
\text { Exams }\end{array}$ & $\begin{array}{c}\text { Failed in } \\
\text { Exams }\end{array}$ & & Dropped & $\begin{array}{c}\text { Appeared } \\
\text { in Exams }\end{array}$ & $\begin{array}{c}\text { Passed in } \\
\text { Exams }\end{array}$ & $\begin{array}{l}\text { Falled in } \\
\text { Exams }\end{array}$ \\
\hline Grade $1 \mathrm{~A}$ & 68 & 20 & 48 & 31 & 17 & Grade 1 & $29.41 \%$ & $70.59 \%$ & $64.58 \%$ & $35.42 \%$ \\
\hline Grade 1 B & 0 & 0 & 0 & 0 & 0 & Grade 2 & $0.00 \%$ & $100.00 \%$ & $77.50 \%$ & $22.50 \%$ \\
\hline Grade $1 \mathrm{C}$ & 0 & 0 & 0 & 0 & 0 & Grade 3 & $0.00 \%$ & $100.00 \%$ & $69.57 \%$ & $30.43 \%$ \\
\hline Grade 2 A & 40 & 0 & 40 & 31 & 9 & Grade 4 & $4.55 \%$ & $95.45 \%$ & $71.43 \%$ & $28.57 \%$ \\
\hline Grade 2 B & 0 & 0 & 0 & 0 & 0 & Grade 5 & $0.00 \%$ & $100.00 \%$ & $93.75 \%$ & $6.25 \%$ \\
\hline Grade $3 \mathrm{~A}$ & - 23 & 0 & 23 & 16 & 7 & Grade 6 & $31.25 \%$ & $68.75 \%$ & $63.64 \%$ & $36.36 \%$ \\
\hline Grade 3 B & 0 & 0 & 0 & 0 & 0 & & & & & \\
\hline Grade 4 & 22 & 1 & 21 & 15 & 6 & Remarks: & & & & \\
\hline Grade 5 & 16 & 0 & 16 & 15 & 1 & & & & & \\
\hline Grade 6 & 16 & 5 & 11 & 7 & 4 & & & & & \\
\hline School Total & 185 & 26 & 159 & 115 & 44 & School \%age & $14.05 \%$ & $85.95 \%$ & $72.33 \%$ & $27.67 \%$ \\
\hline
\end{tabular}

\begin{tabular}{|c|c|c|c|c|c|c|c|c|c|c|}
\hline \multirow[b]{2}{*}{$\begin{array}{l}\text { Grades / } \\
\text { Sections }\end{array}$} & \multicolumn{5}{|c|}{-Number of Students- } & \multirow[b]{2}{*}{ Grades } & \multicolumn{4}{|c|}{ Percent of Students } \\
\hline & Enrolled & Dropped & $\begin{array}{l}\text { Appeared } \\
\text { in Exam }\end{array}$ & $\begin{array}{l}\text { Passed in } \\
\text { Exams }\end{array}$ & $\begin{array}{l}\text { Failed in } \\
\text { Exams }\end{array}$ & & Dropped & $\begin{array}{c}\text { Appeared } \\
\text { in Exams }\end{array}$ & $\begin{array}{c}\text { Passed in } \\
\text { Exams }\end{array}$ & $\begin{array}{c}\text { Falled in } \\
\text { Exams } \\
\end{array}$ \\
\hline Grade $1 \mathrm{~A}$ & 45 & 4 & 41 & 33 & 8 & Grade 1 & $3.05 \%$ & $96.95 \%$ & $76.38 \%$ & $23.62 \%$ \\
\hline Grade 1 B & 43 & 0 & 43 & 35 & 8 & Grade 2 & $0.00 \%$ & $100.00 \%$ & $71.67 \%$ & $28.33 \%$ \\
\hline Grade $1 \mathrm{C}$ & 43 & 0 & 43 & 29 & 14 & Grade 3 & $7.32 \%$ & $92.68 \%$ & $88.16 \%$ & $11.84 \%$ \\
\hline Grade 2 A & 62 & 0 & 62 & 42 & 20 & Grade 4 & $8.77 \%$ & $91.23 \%$ & $73.08 \%$ & $26.92 \%$ \\
\hline Grade 2 B & 58 & 0 & 58 & 44 & 14 & Grade 5 & $0.00 \%$ & $100.00 \%$ & $96.88 \%$ & $3.13 \%$ \\
\hline Grade 3 A & 40 & 3 & 37 & 30 & 7 & Grade 6 & $15.38 \%$ & $84.62 \%$ & $77.27 \%$ & $22.73 \%$ \\
\hline Grade 3 B & 42 & 3 & 39 & 37 & 2 & & & & & \\
\hline Grade 4 & 57 & 5 & 52 & 38 & 14 & Remarks: & & & & \\
\hline Grade 5 & 32 & 0 & 32 & 31 & 1 & & & & & \\
\hline Grade 6 & 26 & 4 & 22 & 17 & 5 & & & & & \\
\hline School Total & 448 & 19 & 429 & 336 & 93 & School \%age & $4.24 \%$ & $95.76 \%$ & $78.32 \%$ & $21.68 \%$ \\
\hline
\end{tabular}

Note:

\#Num! = Enrollment in respective grade(s) is nill therefore calculation is not applicable 
COPE Schools Based Annual Results for 1996-97 and Percentage Analysis

Sub-Centre: Peshawar

\begin{tabular}{|c|c|c|c|c|c|c|c|c|c|c|}
\hline \multirow[b]{2}{*}{$\begin{array}{l}\text { Grades / } \\
\text { Sections }\end{array}$} & \multicolumn{5}{|c|}{-Number of Students- } & \multirow[b]{2}{*}{ Grades } & \multicolumn{4}{|c|}{-Percent of Students- } \\
\hline & Enrolled & Dropped & $\begin{array}{l}\text { Appeared } \\
\text { in Exam }\end{array}$ & $\begin{array}{c}\text { Passed in } \\
\text { Exams }\end{array}$ & $\begin{array}{l}\text { Failed in } \\
\text { Exams }\end{array}$ & & Dropped & $\begin{array}{l}\text { Appeared } \\
\text { in Exams }\end{array}$ & $\begin{array}{c}\text { Passed in } \\
\text { Exams }\end{array}$ & $\begin{array}{l}\text { Failed in } \\
\text { Exams }\end{array}$ \\
\hline Grade $1 \mathrm{~A}$ & 48 & 0 & 48 & 20 & 28 & Grade 1 & $0.00 \%$ & $100.00 \%$ & $41.67 \%$ & $58.33 \%$ \\
\hline Grade 1 B & 0 & 0 & 0 & 0 & 0 & Grade 2 & $21.74 \%$ & $78.26 \%$ & $58.33 \%$ & $41.67 \%$ \\
\hline Grade $1 \mathrm{C}$ & 0 & 0 & 0 & 0 & 0 & Grade 3 & $23.81 \%$ & $76.19 \%$ & $65.63 \%$ & $34.38 \%$ \\
\hline Grade $2 \mathrm{~A}$ & 46 & 10 & 36 & 21 & 15 & Grade 4 & $27.27 \%$ & $72.73 \%$ & $70.83 \%$ & $29.17 \%$ \\
\hline Grade 2 B & 0 & 0 & 0 & 0 & 0 & Grade 5 & $22.58 \%$ & $77.42 \%$ & $62.50 \%$ & $37.50 \%$ \\
\hline Grade $3 \mathrm{~A}$ & 42 & 10 & 32 & 21 & 11 & Grade 6 & \#Num! & \#Num! & \#Num! & \#Num! \\
\hline Grade 3 B & 0 & 0 & 0 & 0 & 0 & & & & & \\
\hline Grade 4 & 33 & 9 & 24 & 17 & 7 & Remarks: & & & & \\
\hline Grade 5 & 31 & 7 & 24 & 15 & 9 & & & & & \\
\hline Grade 6 & 0 & 0 & 0 & 0 & 0 & & & & & \\
\hline School Total & 200 & 36 & 164 & 94 & 70 & School \%age & $18.00 \%$ & $82.00 \%$ & $57.32 \%$ & $42.68 \%$ \\
\hline
\end{tabular}

\begin{tabular}{|c|c|c|c|c|c|c|c|c|c|c|}
\hline Sub-Centre Total & 21,900 & 1,584 & 20,316 & 14,049 & 6,267 & Sub-Centre \%age & $7.23 \%$ & $92.77 \%$ & $69.15 \%$ & $30.85 \%$ \\
\hline
\end{tabular}


Sub-Centre: Thall

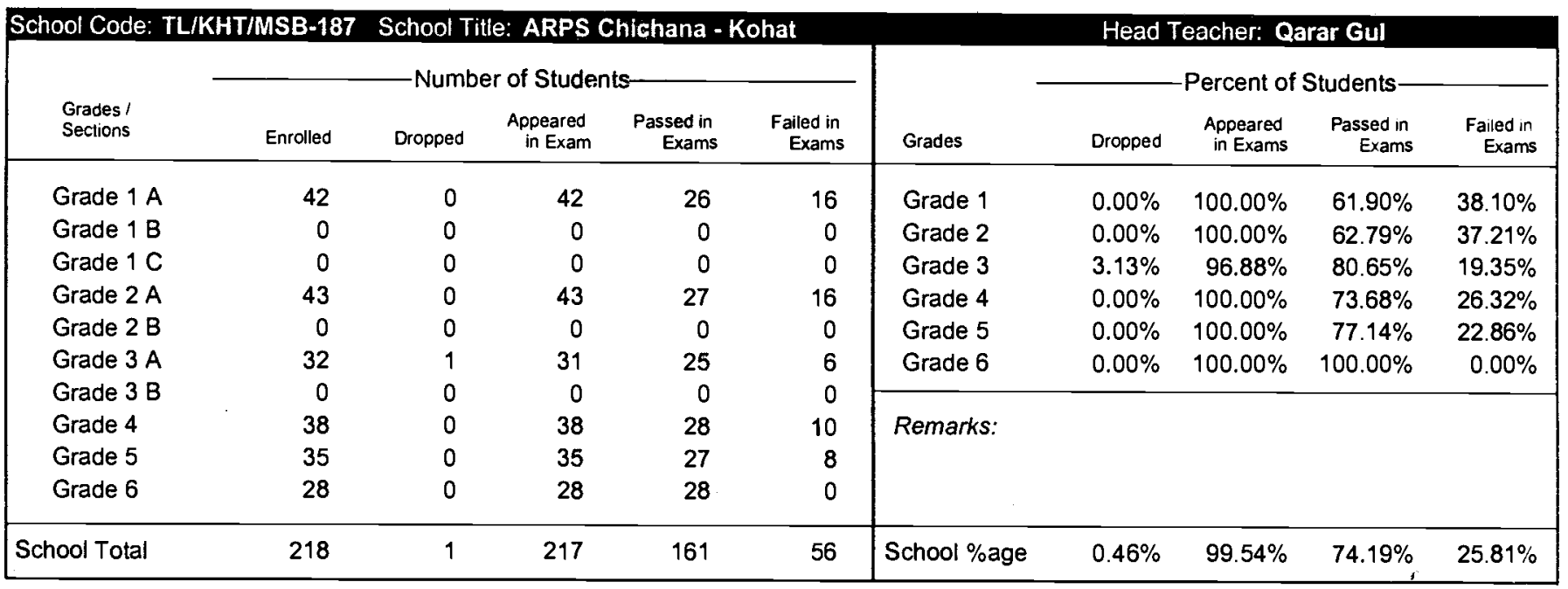

\begin{tabular}{|c|c|c|c|c|c|c|c|c|c|c|c|}
\hline \multirow[t]{2}{*}{ School Code: T } & \multicolumn{6}{|c|}{ School Title: ARPS Darsamand - Kohat } & \multicolumn{5}{|c|}{$\begin{array}{l}\text { Head Teacher: Mohammad Jamil } \\
\text { Percent of Students- }\end{array}$} \\
\hline & 1 & Enrolled & Dropped & $\begin{array}{l}\text { Appeared } \\
\text { in Exam }\end{array}$ & $\begin{array}{l}\text { Passed in } \\
\text { Exams }\end{array}$ & $\begin{array}{l}\text { Failed in } \\
\text { Exams }\end{array}$ & Grades & Dropped & $\begin{array}{l}\text { Appeared } \\
\text { in Exams }\end{array}$ & $\begin{array}{l}\text { Passed in } \\
\text { Exams }\end{array}$ & $\begin{array}{l}\text { Falied in } \\
\text { Exams }\end{array}$ \\
\hline Grade $1 \mathrm{~A}$ & & 48 & 5 & 43 & 29 & 14 & Grade 1 & $5.88 \%$ & $94.12 \%$ & $57.50 \%$ & $42.50 \%$ \\
\hline Grade 1 B & & 37 & 0 & 37 & 17 & 20 & Grade 2 & $13.25 \%$ & $86.75 \%$ & $56.94 \%$ & $43.06 \%$ \\
\hline Grade $1 \mathrm{C}$ & & 0 & 0 & 0 & 0 & 0 & Grade 3 & $10.53 \%$ & $89.47 \%$ & $73.53 \%$ & $26.47 \%$ \\
\hline Grade 2 A & & 39 & 5 & 34 & 21 & 13 & Grade 4 & $11.54 \%$ & $88.46 \%$ & $69.57 \%$ & $30.43 \%$ \\
\hline Grade 2 B & & 44 & 6 & 38 & 20 & 18 & Grade 5 & $0.00 \%$ & $100.00 \%$ & $100.00 \%$ & $0.00 \%$ \\
\hline Grade 3 A & & 38 & 4 & 34 & 25 & 9 & Grade 6 & $16.67 \%$ & $83.33 \%$ & $100.00 \%$ & $0.00 \%$ \\
\hline Grade 3 B & & 0 & 0 & 0 & 0 & 0 & & & & & \\
\hline Grade 4 & & 26 & 3 & 23 & 16 & 7 & Remarks: & & & & \\
\hline Grade 5 & & 17 & 0 & 17 & 17 & 0 & & & & & \\
\hline Grade 6 & & 18 & 3 & 15 & 15 & 0 & & & & & \\
\hline School Total & & 267 & 26 & 241 & 160 & 81 & School \%age & $9.74 \%$ & $90.26 \%$ & $66.39 \%$ & $33.61 \%$ \\
\hline
\end{tabular}

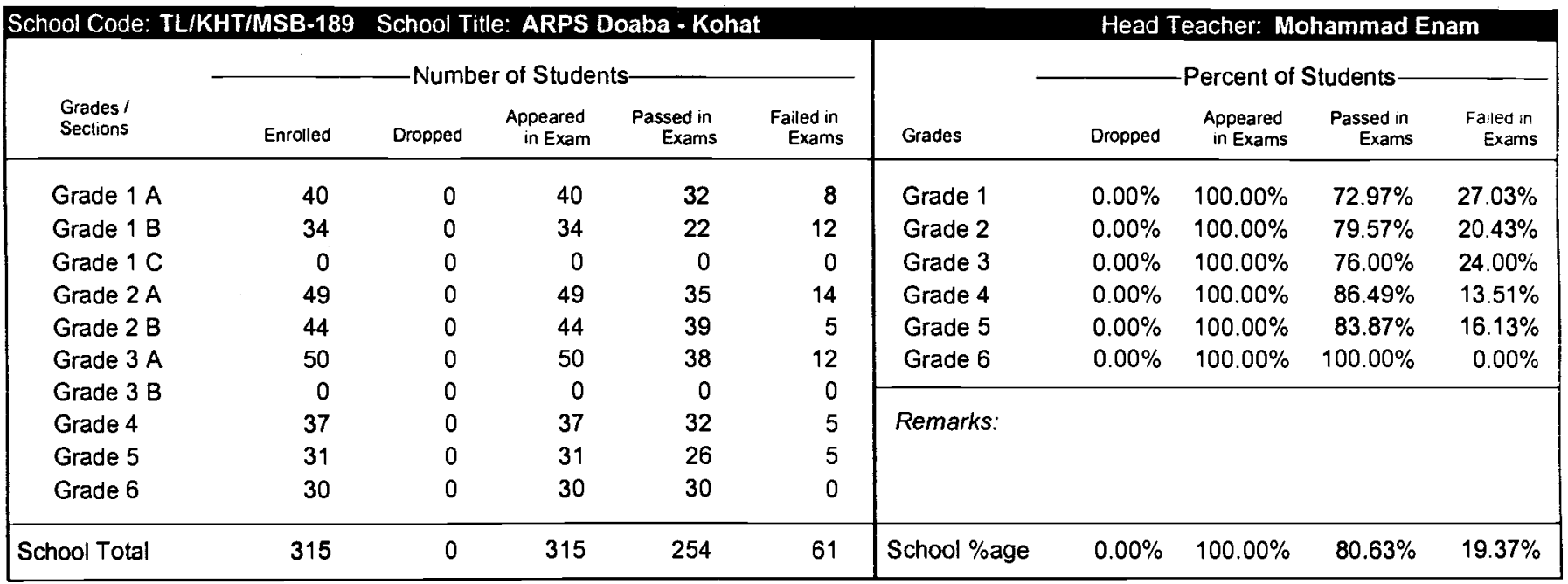


Sub-Centre: Thall

\begin{tabular}{|c|c|c|c|c|c|c|c|c|c|c|}
\hline \multirow[b]{2}{*}{$\begin{array}{l}\text { Grades I } \\
\text { Sections }\end{array}$} & \multicolumn{5}{|c|}{$\begin{array}{l}\text { School Tille: ARPS Ghamkol 2 - Kohat } \\
\text { Number of Students }\end{array}$} & \multicolumn{5}{|c|}{$\begin{array}{r}\text { Head Teacher: Syed Rafique } \\
\text { Percent of Students- }\end{array}$} \\
\hline & Enrolled & Dropped & $\begin{array}{l}\text { Appeared } \\
\text { in Exam }\end{array}$ & $\begin{array}{l}\text { Passed in } \\
\text { Exams }\end{array}$ & $\begin{array}{l}\text { Failed in } \\
\text { Exams }\end{array}$ & Grades & Dropped & $\begin{array}{l}\text { Appeared } \\
\text { in Exams }\end{array}$ & $\begin{array}{l}\text { Passed in } \\
\text { Exams }\end{array}$ & $\begin{array}{l}\text { Falled in } \\
\text { Exams }\end{array}$ \\
\hline Grade $1 \mathrm{~A}$ & 57 & 0 & 57 & 34 & 23 & Grade 1 & $0.00 \%$ & $100.00 \%$ & $58.30 \%$ & $41.70 \%$ \\
\hline Grade 1 B & 55 & 0 & 55 & 32 & 23 & Grade 2 & $0.00 \%$ & $100.00 \%$ & $69.57 \%$ & $30.43 \%$ \\
\hline Grade $1 \mathrm{C}$ & 111 & 0 & 111 & 64 & 47 & Grade 3 & $0.00 \%$ & $100.00 \%$ & $73.49 \%$ & $26.51 \%$ \\
\hline Grade $2 \mathrm{~A}$ & 81 & 0 & 81 & 57 & 24 & Grade 4 & $0.00 \%$ & $100.00 \%$ & $54.43 \%$ & $45.57 \%$ \\
\hline Grade 2 B & 57 & 0 & 57 & 39 & 18 & Grade 5 & $0.00 \%$ & $100.00 \%$ & $77.50 \%$ & $22.50 \%$ \\
\hline Grade $3 \mathrm{~A}$ & 38 & 0 & 38 & 30 & 8 & Grade 6 & $0.00 \%$ & $100.00 \%$ & $34.29 \%$ & $65.71 \%$ \\
\hline Grade 3 B & 45 & 0 & 45 & 31 & 14 & & & & & \\
\hline Grade 4 & 79 & 0 & 79 & 43 & 36 & Remarks: & & & & \\
\hline Grade 5 & 40 & 0 & 40 & 31 & 9 & & & & & \\
\hline Grade 6 & 35 & 0 & 35 & 12 & 23 & & & & & \\
\hline School Total & 598 & 0 & 598 & 373 & 225 & School \%age & $0.00 \%$ & $100.00 \%$ & $62.37 \%$ & $37.63 \%$ \\
\hline
\end{tabular}

\begin{tabular}{|c|c|c|c|c|c|c|c|c|c|c|c|}
\hline \multirow[b]{2}{*}{$\begin{array}{l}\text { Grades I } \\
\text { Sections }\end{array}$} & \multicolumn{6}{|c|}{ - Number of Students- } & \multirow[b]{2}{*}{ Grades } & \multicolumn{4}{|c|}{-Percent of Students } \\
\hline & 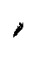 & Enrolled & Dropped & $\begin{array}{l}\text { Appeared } \\
\text { in Exam }\end{array}$ & $\begin{array}{l}\text { Passed in } \\
\text { Exams }\end{array}$ & $\begin{array}{l}\text { Failed in } \\
\text { Exams }\end{array}$ & & Dropped & $\begin{array}{l}\text { Appeared } \\
\text { in Exams }\end{array}$ & $\begin{array}{c}\text { Passed in } \\
\text { Exams }\end{array}$ & $\begin{array}{l}\text { Faled in } \\
\text { Exams }\end{array}$ \\
\hline Grade $1 \mathrm{~A}$ & & 50 & 5 & 45 & 38 & 7 & Grade 1 & $5.95 \%$ & $94.05 \%$ & $60.87 \%$ & $39.13 \%$ \\
\hline Grade 1 B & & 111 & 11 & 100 & 48 & 52 & Grade 2 & $1.55 \%$ & $98.45 \%$ & $66.93 \%$ & $33.07 \%$ \\
\hline Grade $1 \mathrm{C}$ & & 108 & 0 & 108 & 68 & 40 & Grade 3 & $2.63 \%$ & $97.37 \%$ & $73.87 \%$ & $26.13 \%$ \\
\hline Grade $2 \mathrm{~A}$ & & 62 & 1 & 61 & 42 & 19 & Grade 4 & $1.59 \%$ & $98.41 \%$ & $82.26 \%$ & $17.74 \%$ \\
\hline Grade 2 B & & 67 & 1 & 66 & 43 & 23 & Grade 5 & $0.00 \%$ & $100.00 \%$ & $100.00 \%$ & $0.00 \%$ \\
\hline Grade 3 A & & 55 & 0 & 55 & 43 & 12 & Grade 6 & $0.00 \%$ & $100.00 \%$ & $84.62 \%$ & $15.38 \%$ \\
\hline Grade 3 B & & 59 & 3 & 56 & 39 & 17 & & & & & \\
\hline Grade 4 & & 63 & 1 & 62 & 51 & 11 & Remarks: & & & & \\
\hline Grade 5 & & 32 & 0 & 32 & 32 & 0 & & & & & \\
\hline Grade 6 & & 26 & 0 & 26 & 22 & 4 & & & & & \\
\hline School Total & & 633 & 22 & 611 & 426 & 185 & School \%age & $3.48 \%$ & $96.52 \%$ & $69.72 \%$ & $30.28 \%$ \\
\hline
\end{tabular}

\begin{tabular}{|c|c|c|c|c|c|c|c|c|c|c|}
\hline \multicolumn{7}{|c|}{ School Code: TL/KHT/PSB-192 School Title: ARPS Ghiljo-Kohat } & \multicolumn{4}{|c|}{ Head Teacher: Agha Mohammad } \\
\hline \multirow[b]{2}{*}{$\begin{array}{l}\text { Grades / } \\
\text { Sections }\end{array}$} & & - Numb & of Stude & & & \multirow[b]{2}{*}{ Grades } & \multicolumn{4}{|c|}{-Percent of Students } \\
\hline & Enrolled & Dropped & $\begin{array}{l}\text { Appeared } \\
\text { in Examn }\end{array}$ & $\begin{array}{c}\text { Passed in } \\
\text { Exams }\end{array}$ & $\begin{array}{l}\text { Failed in } \\
\text { Exams }\end{array}$ & & Dropped & $\begin{array}{l}\text { Appeared } \\
\text { in Exams }\end{array}$ & $\begin{array}{c}\text { Passed in } \\
\text { Exams }\end{array}$ & $\begin{array}{c}\text { Failed in } \\
\text { Exams }\end{array}$ \\
\hline Grade $1 \mathrm{~A}$ & 48 & 0 & 48 & 38 & 10 & Grade 1 & $0.00 \%$ & $100.00 \%$ & $79.17 \%$ & $20.83 \%$ \\
\hline Grade $1 \mathrm{~B}$ & 0 & 0 & 0 & 0 & 0 & Grade 2 & $0.00 \%$ & $100.00 \%$ & $83.33 \%$ & $16.67 \%$ \\
\hline Grade $1 \mathrm{C}$ & 0 & 0 & 0 & 0 & 0 & Grade 3 & $0.00 \%$ & $100.00 \%$ & $96.77 \%$ & $3.23 \%$ \\
\hline Grade $2 \mathrm{~A}$ & 36 & 0 & 36 & 30 & 6 & Grade 4 & $15.00 \%$ & $85.00 \%$ & $70.59 \%$ & $29.41 \%$ \\
\hline Grade 2 B & 0 & 0 & 0 & 0 & 0 & Grade 5 & $0.00 \%$ & $100.00 \%$ & $81.25 \%$ & $18.75 \%$ \\
\hline Grade $3 \mathrm{~A}$ & 31 & 0 & 31 & 30 & 1 & Grade 6 & $0.00 \%$ & $100.00 \%$ & $70.00 \%$ & $30.00 \%$ \\
\hline Grade 3 B & 0 & 0 & 0 & 0 & 0 & & & & & \\
\hline Grade 4 & 20 & 3 & 17 & 12 & 5 & Remarks: & & & & \\
\hline Grade 5 & 16 & 0 & 16 & 13 & 3 & & & & & \\
\hline Grade 6 & 10 & 0 & 10 & 7 & 3 & & & & & \\
\hline School Total & 161 & 3 & 158 & 130 & 28 & School \%age & $1.86 \%$ & $98.14 \%$ & $82.28 \%$ & $17.72 \%$ \\
\hline
\end{tabular}


Sub-Centre: Thall

\begin{tabular}{|c|c|c|c|c|c|c|c|c|c|c|}
\hline \multirow[t]{2}{*}{ School Code: T } & \multicolumn{5}{|c|}{ School Title: ARPS Ghulam Banda - Kohat } & \multicolumn{5}{|c|}{$\begin{array}{l}\text { Head Teacher: Khan Doran } \\
\text { Percent of Students- }\end{array}$} \\
\hline & Enrolled & Dropped & $\begin{array}{l}\text { Appeared } \\
\text { in Exam }\end{array}$ & $\begin{array}{l}\text { Passed in } \\
\text { Exams }\end{array}$ & $\begin{array}{l}\text { Failed in } \\
\text { Exams }\end{array}$ & Grades & Dropped & $\begin{array}{l}\text { Appeared } \\
\text { in Exams }\end{array}$ & $\begin{array}{l}\text { Passed in } \\
\text { Exams }\end{array}$ & $\begin{array}{l}\text { Failed in } \\
\text { Exams }\end{array}$ \\
\hline Grade $1 \mathrm{~A}$ & 41 & 0 & 41 & 30 & 11 & Grade 1 & $0.00 \%$ & $100.00 \%$ & $64.52 \%$ & $35.48 \%$ \\
\hline Grade 1 B & 40 & 0 & 40 & 29 & 11 & Grade 2 & $2.00 \%$ & $98.00 \%$ & $59.18 \%$ & $40.82 \%$ \\
\hline Grade $1 \mathrm{C}$ & 43 & 0 & 43 & 21 & 22 & Grade 3 & $0.00 \%$ & $100.00 \%$ & $73.68 \%$ & $26.32 \%$ \\
\hline Grade 2 A & 50 & 1 & 49 & 29 & 20 & Grade 4 & $0.00 \%$ & $100.00 \%$ & $68.75 \%$ & $31.25 \%$ \\
\hline Grade 2 B & 0 & 0 & 0 & 0 & 0 & Grade 5 & $0.00 \%$ & $100.00 \%$ & $100.00 \%$ & $0.00 \%$ \\
\hline Grade $3 \mathrm{~A}$ & 38 & 0 & 38 & 28 & 10 & Grade 6 & $0.00 \%$ & $100.00 \%$ & $100.00 \%$ & $0.00 \%$ \\
\hline Grade 3 B & 0 & 0 & 0 & 0 & 0 & & & & & \\
\hline Grade 4 & 32 & 0 & 32 & 22 & 10 & Remarks: & & & & \\
\hline Grade 5 & 24 & 0 & 24 & 24 & 0 & & & & & \\
\hline Grade 6 & 12 & 0 & 12 & 12 & 0 & & & & & \\
\hline School Total & 280 & 1 & 279 & 195 & 84 & School \%age & $0.36 \%$ & $99.64 \%$ & $69.89 \%$ & $30.11 \%$ \\
\hline
\end{tabular}

\begin{tabular}{|c|c|c|c|c|c|c|c|c|c|c|c|}
\hline \multirow[b]{2}{*}{$\begin{array}{l}\text { Grades I } \\
\text { Sections }\end{array}$} & \multicolumn{6}{|c|}{ Number of Students- } & \multirow[b]{2}{*}{ Grades } & \multicolumn{4}{|c|}{ Percent of Students } \\
\hline & 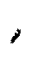 & Enrolled & Dropped & $\begin{array}{l}\text { Appeared } \\
\text { in Exam }\end{array}$ & $\begin{array}{c}\text { Passed in } \\
\text { Exams }\end{array}$ & $\begin{array}{l}\text { Failed in } \\
\text { Exams }\end{array}$ & & Dropped & $\begin{array}{l}\text { Appeared } \\
\text { in Exams }\end{array}$ & $\begin{array}{c}\text { Passed in } \\
\text { Exams }\end{array}$ & $\begin{array}{c}\text { Failed in } \\
\text { Exams }\end{array}$ \\
\hline Grade $1 \mathrm{~A}$ & & 45 & 0 & 45 & 25 & 20 & Grade 1 & $0.00 \%$ & $100.00 \%$ & $55.56 \%$ & $44.44 \%$ \\
\hline Grade $1 \mathrm{~B}$ & & 0 & 0 & 0 & 0 & 0 & Grade 2 & $0.00 \%$ & $100.00 \%$ & $62.50 \%$ & $37.50 \%$ \\
\hline Grade $1 \mathrm{C}$ & & 0 & 0 & 0 & 0 & 0 & Grade 3 & $0.00 \%$ & $100.00 \%$ & $66.67 \%$ & $33.33 \%$ \\
\hline Grade 2 A & & 40 & 0 & 40 & 25 & 15 & Grade 4 & $0.00 \%$ & $100.00 \%$ & $69.70 \%$ & $30.30 \%$ \\
\hline Grade 2 B & & 0 & 0 & 0 & 0 & 0 & Grade 5 & $0.00 \%$ & $100.00 \%$ & $70.59 \%$ & $29.41 \%$ \\
\hline Grade $3 \mathrm{~A}$ & & 30 & 0 & 30 & 20 & 10 & Grade 6 & $0.00 \%$ & $100.00 \%$ & $100.00 \%$ & $0.00 \%$ \\
\hline Grade 3 B & & 0 & 0 & 0 & 0 & 0 & & & & & \\
\hline Grade 4 & & 33 & 0 & 33 & 23 & 10 & Remarks: & & & & \\
\hline Grade 5 & & 17 & 0 & 17 & 12 & 5 & & & & & \\
\hline Grade 6 & & 15 & 0 & 15 & 15 & 0 & & & & & \\
\hline School Total & & $180^{\circ}$ & 0 & 180 & 120 & 60 & School \%age & $0.00 \%$ & $100.00 \%$ & $66.67 \%$ & $33.33 \%$ \\
\hline
\end{tabular}

\begin{tabular}{|c|c|c|c|c|c|c|c|c|c|c|}
\hline \multirow[b]{2}{*}{$\begin{array}{l}\text { Grades I } \\
\text { Sections }\end{array}$} & \multicolumn{5}{|c|}{ - Number of Students } & \multirow[b]{2}{*}{ Grades } & \multicolumn{4}{|c|}{ Percent of Students } \\
\hline & Enrolled & Dropped & $\begin{array}{l}\text { Appeared } \\
\text { in Exam }\end{array}$ & $\begin{array}{l}\text { Passed in } \\
\text { Exams }\end{array}$ & $\begin{array}{l}\text { Failed in } \\
\text { Exams }\end{array}$ & & Dropped & $\begin{array}{l}\text { Appeared } \\
\text { in Exams }\end{array}$ & $\begin{array}{l}\text { Passed in } \\
\text { Exams }\end{array}$ & $\begin{array}{c}\text { Falled in } \\
\text { Exams }\end{array}$ \\
\hline Grade $1 \mathrm{~A}$ & 41 & 0 & 41 & 36 & 5 & Grade 1 & $0.00 \%$ & $100.00 \%$ & $76.83 \%$ & $23.17 \%$ \\
\hline Grade 1 B & 41 & 0 & 41 & 27 & 14 & Grade 2 & $0.00 \%$ & $100.00 \%$ & $80.00 \%$ & $20.00 \%$ \\
\hline Grade $1 \mathrm{C}$ & 0 & 0 & 0 & 0 & 0 & Grade 3 & $5.26 \%$ & $94.74 \%$ & $79.63 \%$ & $20.37 \%$ \\
\hline Grade $2 \mathrm{~A}$ & 52 & 0 & 52 & 45 & 7 & Grade 4 & $0.00 \%$ & $100.00 \%$ & $84.38 \%$ & $15.63 \%$ \\
\hline Grade 2 B & 53 & 0 & 53 & 39 & 14 & Grade 5 & $0.00 \%$ & $100.00 \%$ & $79.41 \%$ & $20.59 \%$ \\
\hline Grade $3 \mathrm{~A}$ & 57 & 3 & 54 & 43 & 11 & Grade 6 & $0.00 \%$ & $100.00 \%$ & $77.78 \%$ & $22.22 \%$ \\
\hline Grade 3 B & 0 & 0 & 0 & 0 & 0 & & & & & \\
\hline Grade 4 & 32 & 0 & 32 & 27 & 5 & Remarks: & & & & \\
\hline Grade 5 & 34 & 0 & 34 & 27 & 7 & & & & & \\
\hline Grade 6 & 18 & 0 & 18 & 14 & 4 & & & & & \\
\hline School Total & 328 & 3 & 325 & 258 & 67 & School \%age & $0.91 \%$ & $99.09 \%$ & $79.38 \%$ & $20.62 \%$ \\
\hline
\end{tabular}


Sub-Centre: Thall

School Code: TL/KHT/PSB-196 School Title: ARPS 2 - Kahi - Kohat

Head Teacher: Abdur Rauf

\begin{tabular}{|c|c|c|c|c|c|c|c|c|c|c|}
\hline \multirow[b]{2}{*}{$\begin{array}{l}\text { Grades I } \\
\text { Sections }\end{array}$} & \multicolumn{5}{|c|}{ - Number of Students } & \multirow[b]{2}{*}{ Grades } & \multicolumn{4}{|c|}{-Percent of Students- } \\
\hline & Enrolled & Dropped & $\begin{array}{l}\text { Appeared } \\
\text { in Exam }\end{array}$ & $\begin{array}{l}\text { Passed in } \\
\text { Exams }\end{array}$ & $\begin{array}{c}\text { Failed in } \\
\text { Exams }\end{array}$ & & Dropped & $\begin{array}{l}\text { Appeared } \\
\text { in Exams }\end{array}$ & $\begin{array}{r}\text { Passed in } \\
\text { Exams }\end{array}$ & $\begin{array}{l}\text { Falleo in } \\
\text { Exams }\end{array}$ \\
\hline Grade $1 \mathrm{~A}$ & 72 & 0 & 72 & 40 & 32 & Grade 1 & $0.00 \%$ & $100.00 \%$ & $55.56 \%$ & $44.44 \%$ \\
\hline Grade $1 \mathrm{~B}$ & 0 & 0 & 0 & 0 & 0 & Grade 2 & $0.00 \%$ & $100.00 \%$ & $73.68 \%$ & $26.32 \%$ \\
\hline Grade $1 \mathrm{C}$ & 0 & 0 & 0 & 0 & 0 & Grade 3 & $0.00 \%$ & $100.00 \%$ & $92.11 \%$ & $7.89 \%$ \\
\hline Grade 2 A & 38 & 0 & 38 & 28 & 10 & Grade 4 & $0.00 \%$ & $100.00 \%$ & $94.29 \%$ & $5.71 \%$ \\
\hline Grade 2 B & 0 & 0 & 0 & 0 & 0 & Grade 5 & $0.00 \%$ & $100.00 \%$ & $76.92 \%$ & $23.08 \%$ \\
\hline Grade $3 \mathrm{~A}$ & 38 & 0 & 38 & 35 & 3 & Grade 6 & $0.00 \%$ & $100.00 \%$ & $100.00 \%$ & $0.00 \%$ \\
\hline Grade 3 B & 0 & 0 & 0 & 0 & 0 & & & & & \\
\hline Grade 4 & 35 & 0 & 35 & 33 & 2 & Remarks: & & & & \\
\hline Grade 5 & 26 & 0 & 26 & 20 & 6 & & & & & \\
\hline Grade 6 & 19 & 0 & 19 & 19 & 0 & & & & & \\
\hline School Total & 228 & 0 & 228 & 175 & 53 & School \%age & $0.00 \%$ & $100.00 \%$ & $76.75 \%$ & $23.25 \%$ \\
\hline
\end{tabular}

School Code: TL/KHT/PSB-197 School Title: ARPS 3 Kahi Kotat

Head Teacher: Khan Mohammad

\begin{tabular}{|c|c|c|c|c|c|c|c|c|c|c|}
\hline \multirow[b]{2}{*}{$\begin{array}{l}\text { Grades I } \\
\text { Sections }\end{array}$} & \multicolumn{5}{|c|}{-Number of Students- } & \multirow[b]{2}{*}{ Grades } & \multicolumn{4}{|c|}{ Percent of Students } \\
\hline & Enrolled & Dropped & $\begin{array}{l}\text { Appeared } \\
\text { in Exam }\end{array}$ & $\begin{array}{l}\text { Passed in } \\
\text { Exams }\end{array}$ & $\begin{array}{l}\text { Failed in } \\
\text { Exams }\end{array}$ & & Dropped & $\begin{array}{l}\text { Appeared } \\
\text { in Exams }\end{array}$ & $\begin{array}{l}\text { Passed in } \\
\text { Exams }\end{array}$ & $\begin{array}{l}\text { Falled in } \\
\text { Exams }\end{array}$ \\
\hline Grade $1 \mathrm{~A}$ & 60 & 0 & 60 & 25 & 35 & Grade 1 & $0.00 \%$ & $100.00 \%$ & $36.87 \%$ & $63.13 \%$ \\
\hline Grade $1 \mathrm{~B}$ & 60 & 0 & 60 & 28 & 32 & Grade 2 & $0.00 \%$ & $100.00 \%$ & $100.00 \%$ & $0.00 \%$ \\
\hline Grade $1 \mathrm{C}$ & 59 & 0 & 59 & 13 & 46 & Grade 3 & $0.00 \%$ & $100.00 \%$ & $100.00 \%$ & $0.00 \%$ \\
\hline Grade 2 A & 89 & 0 & 89 & 89 & 0 & Grade 4 & $0.00 \%$ & $100.00 \%$ & $100.00 \%$ & $0.00 \%$ \\
\hline Grade 2 B & 0 & 0 & 0 & 0 & 0 & Grade 5 & $0.00 \%$ & $100.00 \%$ & $100.00 \%$ & $0.00 \%$ \\
\hline Grade 3 A & $84^{\circ}$ & 0 & 84 & 84 & 0 & Grade 6 & $0.00 \%$ & $100.00 \%$ & $100.00 \%$ & $0.00 \%$ \\
\hline Grade 3 B & 0 & 0 & 0 & 0 & 0 & & & & & \\
\hline Grade 4 & 42 & 0 & 42 & 42 & 0 & Remarks: & & & & \\
\hline Grade 5 & 42 & 0 & 42 & 42 & 0 & & & & & \\
\hline Grade 6 & 17 & 0 & 17 & 17 & 0 & & & & & \\
\hline hool Total & 453 & 0 & 453 & 340 & 113 & School \%age & $0.00 \%$ & $100.00 \%$ & $75.06 \%$ & $24.94 \%$ \\
\hline
\end{tabular}

\begin{tabular}{|c|c|c|c|c|c|c|c|c|c|c|}
\hline \multirow[b]{2}{*}{$\begin{array}{l}\text { Grades I } \\
\text { Sections }\end{array}$} & \multicolumn{5}{|c|}{ - Number of Students- } & \multirow[b]{2}{*}{ Grades } & \multicolumn{4}{|c|}{-Percent of Students- } \\
\hline & Enrolled & Dropped & $\begin{array}{l}\text { Appeared } \\
\text { in Exam }\end{array}$ & $\begin{array}{l}\text { Passed in } \\
\text { Exams }\end{array}$ & $\begin{array}{l}\text { Failed in } \\
\text { Exams }\end{array}$ & & Dropped & $\begin{array}{c}\text { Appeared } \\
\text { in Exams }\end{array}$ & $\begin{array}{l}\text { Passed in } \\
\text { Exams }\end{array}$ & $\begin{array}{l}\text { Falied in } \\
\text { Exams }\end{array}$ \\
\hline Grade $1 \mathrm{~A}$ & 47 & 4 & 43 & 35 & 8 & Grade 1 & $10.53 \%$ & $89.47 \%$ & $82.35 \%$ & $17.65 \%$ \\
\hline Grade $1 \mathrm{~B}$ & 48 & 6 & 42 & 35 & 7 & Grade 2 & $9.89 \%$ & $90.11 \%$ & $78.05 \%$ & $21.95 \%$ \\
\hline Grade $1 \mathrm{C}$ & 0 & 0 & 0 & 0 & 0 & Grade 3 & $5.26 \%$ & $94.74 \%$ & $75.93 \%$ & $24.07 \%$ \\
\hline Grade 2 A & 45 & 7 & 38 & 31 & 7 & Grade 4 & $16.28 \%$ & $83.72 \%$ & $83.33 \%$ & $16.67 \%$ \\
\hline Grade 2 B & 46 & 2 & 44 & 33 & 11 & Grade 5 & $22.92 \%$ & $77.08 \%$ & $72.97 \%$ & $27.03 \%$ \\
\hline Grade 3 A & 57 & 3 & 54 & 41 & 13 & Grade 6 & $27.78 \%$ & $72.22 \%$ & $96.15 \%$ & $3.85 \%$ \\
\hline Grade 3 B & 0 & 0 & 0 & 0 & 0 & & & & & \\
\hline Grade 4 & 43 & 7 & 36 & 30 & 6 & Remarks: & & & & \\
\hline Grade 5 & 48 & 11 & 37 & 27 & 10 & & & & & \\
\hline Grade 6 & 36 & 10 & 26 & 25 & 1 & & & & & \\
\hline School Total & 370 & 50 & 320 & 257 & 63 & School \%age & $13.51 \%$ & $86.49 \%$ & $80.31 \%$ & $19.69 \%$ \\
\hline
\end{tabular}


Sub-Centre: Thall

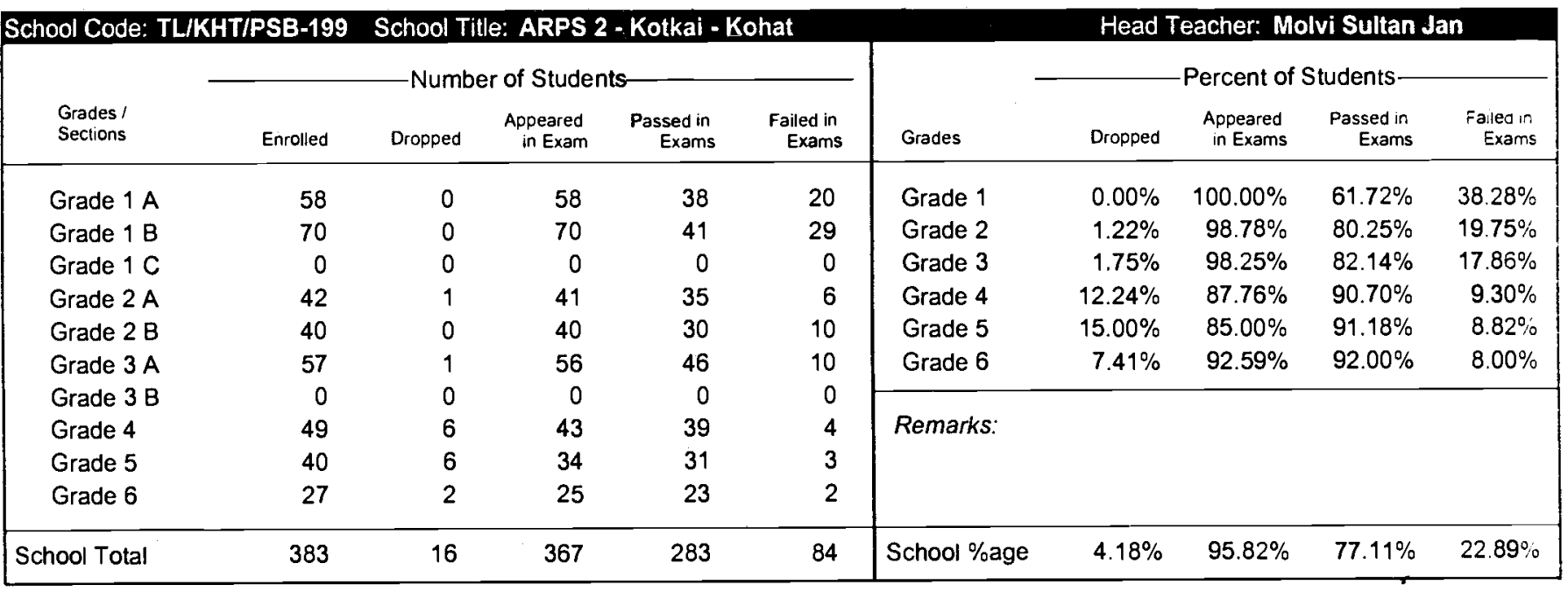

\begin{tabular}{|c|c|c|c|c|c|c|c|c|c|c|c|}
\hline \multirow[b]{2}{*}{$\begin{array}{l}\text { Grades / } \\
\text { Sections }\end{array}$} & \multirow[b]{2}{*}{ 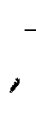 } & \multicolumn{5}{|c|}{ - Number of Students } & \multirow[b]{2}{*}{ Grades } & \multicolumn{4}{|c|}{-Percent of Students - } \\
\hline & & Enrolled & Dropped & $\begin{array}{l}\text { Appeared } \\
\text { in Exam }\end{array}$ & $\begin{array}{l}\text { Passed in } \\
\text { Exams }\end{array}$ & $\begin{array}{l}\text { Failed in } \\
\text { Exams }\end{array}$ & & Dropped & $\begin{array}{l}\text { Appeared } \\
\text { in Exams }\end{array}$ & $\begin{array}{l}\text { Passed in } \\
\text { Exams }\end{array}$ & $\begin{array}{c}\text { Falled in } \\
\text { Exams }\end{array}$ \\
\hline Grade 1 A & & 51 & 0 & 51 & 26 & 25 & Grade 1 & $0.00 \%$ & $100.00 \%$ & $48.60 \%$ & $51.40 \%$ \\
\hline Grade 1 B & & 56 & 0 & 56 & 26 & 30 & Grade 2 & $0.00 \%$ & $100.00 \%$ & $100.00 \%$ & $0.00 \%$ \\
\hline Grade $1 \mathrm{C}$ & & 0 & 0 & 0 & 0 & 0 & Grade 3 & $0.00 \%$ & $100.00 \%$ & $83.78 \%$ & $16.22 \%$ \\
\hline Grade 2 A & & 39 & 0 & 39 & 39 & 0 & Grade 4 & $0.00 \%$ & $100.00 \%$ & $76.19 \%$ & $23.81 \%$ \\
\hline Grade 2 B & & 0 & 0 & 0 & 0 & 0 & Grade 5 & $0.00 \%$ & $100.00 \%$ & $100.00 \%$ & $0.00 \%$ \\
\hline Grade 3 A & & -37 & 0 & 37 & 31 & 6 & Grade 6 & $0.00 \%$ & $100.00 \%$ & $100.00 \%$ & $0.00 \%$ \\
\hline Grade 3 B & & 0 & 0 & 0 & 0 & 0 & & & & & \\
\hline Grade 4 & & 42 & 0 & 42 & 32 & 10 & Remarks: & & & & \\
\hline Grade 5 & & 28 & 0 & 28 & 28 & 0 & & & & & \\
\hline Grade 6 & & 21 & 0 & 21 & 21 & 0 & & & & & \\
\hline School Total & & 274 & 0 & 274 & 203 & 71 & School \%age & $0.00 \%$ & $100.00 \%$ & $74.09 \%$ & $25.91 \%$ \\
\hline
\end{tabular}

\begin{tabular}{|c|c|c|c|c|c|c|c|c|c|c|}
\hline \multirow[b]{2}{*}{$\begin{array}{l}\text { Grades I } \\
\text { Sections }\end{array}$} & \multicolumn{5}{|c|}{ Number of Students } & \multirow[b]{2}{*}{ Grades } & \multicolumn{4}{|c|}{ Percent of Students } \\
\hline & Enrolled & Dropped & $\begin{array}{l}\text { Appeared } \\
\text { in Exam }\end{array}$ & $\begin{array}{l}\text { Passed in } \\
\text { Exams }\end{array}$ & $\begin{array}{c}\text { Failed in } \\
\text { Exams }\end{array}$ & & Dropped & $\begin{array}{l}\text { Appeared } \\
\text { in Exams }\end{array}$ & $\begin{array}{l}\text { Passed in } \\
\text { Exams }\end{array}$ & $\begin{array}{l}\text { Falled in } \\
\text { Exams }\end{array}$ \\
\hline Grade $1 \mathrm{~A}$ & 100 & 9 & 91 & 46 & 45 & Grade 1 & $9.00 \%$ & $91.00 \%$ & $50.55 \%$ & $49.45 \%$ \\
\hline Grade 1 B & 0 & 0 & 0 & 0 & 0 & Grade 2 & $2.63 \%$ & $97.37 \%$ & $64.86 \%$ & $35.14 \%$ \\
\hline Grade $1 \mathrm{C}$ & 0 & 0 & 0 & 0 & 0 & Grade 3 & $0.00 \%$ & $100.00 \%$ & $73.17 \%$ & $26.83 \%$ \\
\hline Grade 2 A & 38 & 1 & 37 & 24 & 13 & Grade 4 & $0.00 \%$ & $100.00 \%$ & $64.29 \%$ & $35.71 \%$ \\
\hline Grade 2 B & 0 & 0 & 0 & 0 & 0 & Grade 5 & $0.00 \%$ & $100.00 \%$ & $100.00 \%$ & $0.00 \%$ \\
\hline Grade 3 A & 41 & 0 & 41 & 30 & 11 & Grade 6 & $6.67 \%$ & $93.33 \%$ & $100.00 \%$ & $0.00 \%$ \\
\hline Grade 3 B & 0 & 0 & 0 & 0 & 0 & & & & & \\
\hline Grade 4 & 42 & 0 & 42 & 27 & 15 & Remarks: & & & & \\
\hline Grade 5 & 17 & 0 & 17 & 17 & 0 & & & & & \\
\hline Grade 6 & 15 & 1 & 14 & 14 & 0 & & & & & \\
\hline School Total & 253 & 11 & 242 & 158 & 84 & School \%age & $4.35 \%$ & $95.65 \%$ & $65.29 \%$ & $34.71 \%$ \\
\hline
\end{tabular}


Sub-Centre: Thall

\begin{tabular}{|c|c|c|c|c|c|c|c|c|c|c|}
\hline \multirow[b]{2}{*}{$\begin{array}{l}\text { Grades / } \\
\text { Sections }\end{array}$} & \multicolumn{5}{|c|}{-Number of Students- } & \multirow[b]{2}{*}{ Grades } & \multicolumn{4}{|c|}{-Percent of Students } \\
\hline & Enrolled & Dropped & $\begin{array}{l}\text { Appeared } \\
\text { in Exam }\end{array}$ & $\begin{array}{c}\text { Passed in } \\
\text { Exams }\end{array}$ & $\begin{array}{c}\text { Failed in } \\
\text { Exams }\end{array}$ & & Dropped & $\begin{array}{l}\text { Appeared } \\
\text { in Exams }\end{array}$ & $\begin{array}{c}\text { Passed in } \\
\text { Exams }\end{array}$ & $\begin{array}{l}\text { Failed in } \\
\text { Exams }\end{array}$ \\
\hline Grade $1 \mathrm{~A}$ & 50 & 0 & 50 & 31 & 19 & Grade 1 & $6.82 \%$ & $93.18 \%$ & $58.54 \%$ & $41.46 \%$ \\
\hline Grade $1 \mathrm{~B}$ & 38 & 6 & 32 & 17 & 15 & Grade 2 & $8.33 \%$ & $91.67 \%$ & $63.64 \%$ & $36.36 \%$ \\
\hline Grade $1 \mathrm{C}$ & 0 & 0 & 0 & 0 & 0 & Grade 3 & $8.16 \%$ & $91.84 \%$ & $62.22 \%$ & $37.78 \%$ \\
\hline Grade 2 A & 38 & 6 & 32 & 22 & 10 & Grade 4 & $0.00 \%$ & $100.00 \%$ & $78.13 \%$ & $21.88 \%$ \\
\hline Grade 2 B & 34 & 0 & 34 & 20 & 14 & Grade 5 & $7.14 \%$ & $92.86 \%$ & $76.92 \%$ & $23.08 \%$ \\
\hline Grade $3 \mathrm{~A}$ & 49 & 4 & 45 & 28 & 17 & Grade 6 & $0.00 \%$ & $100.00 \%$ & $84.21 \%$ & $15.79 \%$ \\
\hline Grade 3 B & 0 & 0 & 0 & 0 & 0 & & & & & \\
\hline Grade 4 & 32 & 0 & 32 & 25 & 7 & Remarks: & & & & \\
\hline Grade 5 & 28 & 2 & 26 & 20 & 6 & & & & & \\
\hline Grade 6 & 19 & 0 & 19 & 16 & 3 & & & & & \\
\hline School Total & 288 & 18 & 270 & 179 & 91 & School \%age & $6.25 \%$ & $93.75 \%$ & $66.30 \%$ & $33.70 \%$ \\
\hline
\end{tabular}

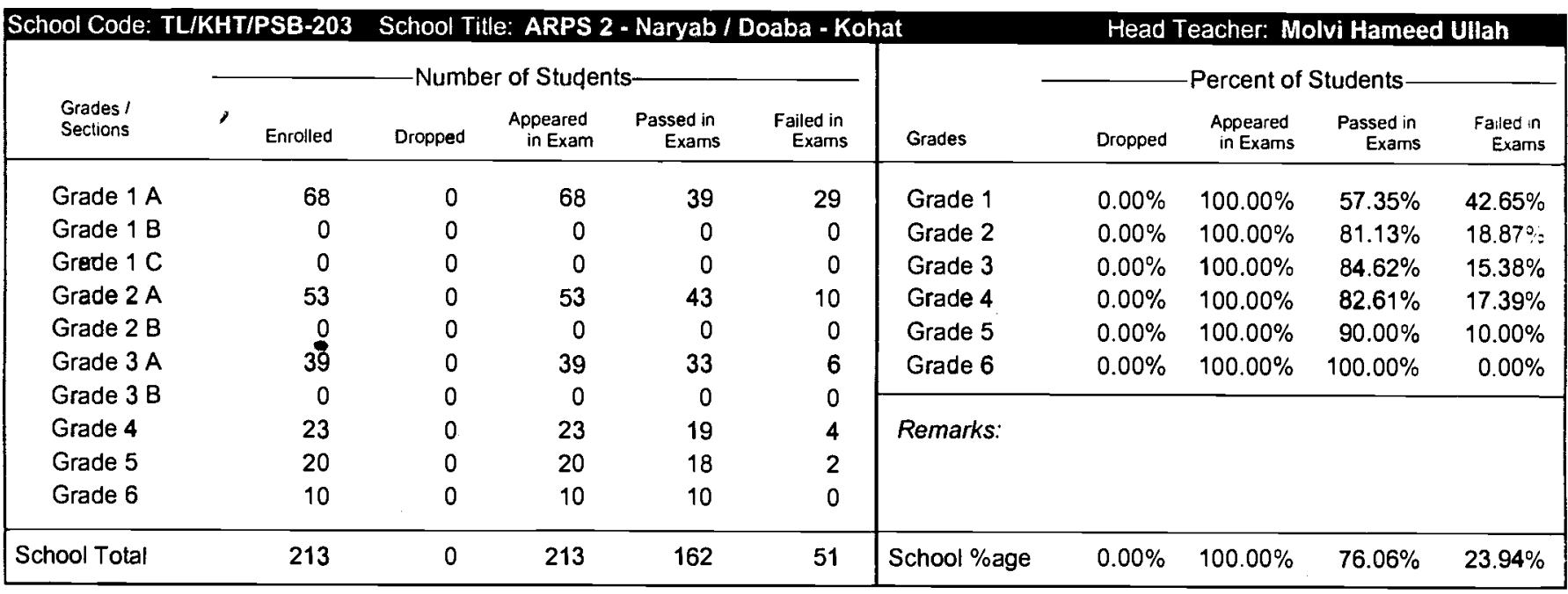

\begin{tabular}{|c|c|c|c|c|c|c|c|c|c|c|}
\hline \multirow[b]{2}{*}{$\begin{array}{l}\text { Grades } / \\
\text { Sections }\end{array}$} & \multicolumn{5}{|c|}{ School Tille: ARPS Oblan - Kohat } & \multicolumn{5}{|c|}{ Head Teacher: Shal Pach } \\
\hline & Enrolled & Dropped & $\begin{array}{l}\text { Appeared } \\
\text { in Exam }\end{array}$ & $\begin{array}{l}\text { Passed in } \\
\text { Exams }\end{array}$ & $\begin{array}{l}\text { Failed in } \\
\text { Exams }\end{array}$ & Grades & Dropped & $\begin{array}{l}\text { Appeared } \\
\text { in Exams }\end{array}$ & $\begin{array}{l}\text { Passed in } \\
\text { Exams }\end{array}$ & $\begin{array}{l}\text { Falied in } \\
\text { Exams }\end{array}$ \\
\hline Grade $1 \mathrm{~A}$ & 66 & 0 & 66 & 41 & 25 & Grade 1 & $0.00 \%$ & $100.00 \%$ & $58.52 \%$ & $41.48 \%$ \\
\hline Grade $1 \mathrm{~B}$ & 69 & 0 & 69 & 38 & 31 & Grade 2 & $0.00 \%$ & $100.00 \%$ & $61.90 \%$ & $38.10 \%$ \\
\hline Grade $1 \mathrm{C}$ & 0 & 0 & 0 & 0 & 0 & Grade 3 & $5.66 \%$ & $94.34 \%$ & $80.00 \%$ & $20.00 \%$ \\
\hline Grade $2 \mathrm{~A}$ & 42 & 0 & 42 & 26 & 16 & Grade 4 & $3.70 \%$ & $96.30 \%$ & $73.08 \%$ & $26.92 \%$ \\
\hline Grade 2 B & 42 & 0 & 42 & 26 & 16 & Grade 5 & $0.00 \%$ & $100.00 \%$ & $100.00 \%$ & $0.00 \%$ \\
\hline Grade $3 \mathrm{~A}$ & 53 & 3 & 50 & 40 & 10 & Grade 6 & $0.00 \%$ & $100.00 \%$ & $100.00 \%$ & $0.00 \%$ \\
\hline Grade 3 B & 0 & 0 & 0 & 0 & 0 & & & & & \\
\hline Grade 4 & 27 & 1 & 26 & 19 & 7 & Remarks: & & & & \\
\hline Grade 5 & 10 & 0 & 10 & 10 & 0 & & & & & \\
\hline Grade 6 & 13 & 0 & 13 & 13 & 0 & & & & & \\
\hline School Total & 322 & 4 & 318 & 213 & 105 & School \%age & $1.24 \%$ & $98.76 \%$ & $66.98 \%$ & $33.02 \%$ \\
\hline
\end{tabular}


Sub-Centre: Thall

\begin{tabular}{|c|c|c|c|c|c|c|c|c|c|c|}
\hline \multirow[b]{2}{*}{$\begin{array}{l}\text { Grades I } \\
\text { Sections }\end{array}$} & \multicolumn{5}{|c|}{ Number of Students- } & \multirow[b]{2}{*}{ Grades } & \multicolumn{4}{|c|}{-Percent of Students } \\
\hline & Enrolled & Dropped & $\begin{array}{l}\text { Appeared } \\
\text { in Exam }\end{array}$ & $\begin{array}{r}\text { Passed in } \\
\text { Exams }\end{array}$ & $\begin{array}{l}\text { Failed in } \\
\text { Exams }\end{array}$ & & Dropped & $\begin{array}{c}\text { Appeared } \\
\text { in Exams }\end{array}$ & $\begin{array}{r}\text { Passed in } \\
\text { Exams }\end{array}$ & $\begin{array}{c}\text { Faited in } \\
\text { Exams }\end{array}$ \\
\hline Grade $1 \mathrm{~A}$ & 71 & 14 & 57 & 36 & 21 & Grade 1 & $19.72 \%$ & $80.28 \%$ & $63.16 \%$ & $36.84 \%$ \\
\hline Grade 1 B & 0 & 0 & 0 & 0 & 0 & Grade 2 & $17.50 \%$ & $82.50 \%$ & $78.79 \%$ & $21.21 \%$ \\
\hline Grade $1 \mathrm{C}$ & 0 & 0 & 0 & 0 & 0 & Grade 3 & $17.86 \%$ & $82.14 \%$ & $65.22 \%$ & $34.78 \%$ \\
\hline Grade $2 \mathrm{~A}$ & 40 & 7 & 33 & 26 & 7 & Grade 4 & $10.53 \%$ & $89.47 \%$ & $64.71 \%$ & $35.29 \%$ \\
\hline Grade 2 B & 0 & 0 & 0 & 0 & 0 & Grade 5 & $25.00 \%$ & $75.00 \%$ & $50.00 \%$ & $50.00 \%$ \\
\hline Grade 3 A & 28 & 5 & 23 & 15 & 8 & Grade 6 & \#Num! & \#Num! & \#Num! & \#Num! \\
\hline Grade 3 B & 0 & 0 & 0 & 0 & 0 & & & & & \\
\hline Grade 4 & 19 & 2 & 17 & 11 & 6 & Remarks: & & & & \\
\hline Grade 5 & 16 & 4 & 12 & 6 & 6 & & & & & \\
\hline Grade 6 & 0 & 0 & 0 & 0 & 0 & & & & & \\
\hline School Total & 174 & 32 & 142 & 94 & 48 & School \%age & $18.39 \%$ & $81.61 \%$ & $66.20 \%$ & $33.80 \%$ \\
\hline
\end{tabular}

\begin{tabular}{|c|c|c|c|c|c|c|c|c|c|c|c|}
\hline \multicolumn{12}{|c|}{ School Code: TL/KHT/MSB-206 School Title: ARPS 1 - Tall 2 - Kohat } \\
\hline \multirow[b]{2}{*}{$\begin{array}{l}\text { Grades / } \\
\text { Sections }\end{array}$} & \multicolumn{6}{|c|}{ Number of Students- } & \multirow[b]{2}{*}{ Grades } & \multicolumn{4}{|c|}{-Percent of Students- } \\
\hline & ' & Enrolled & Dropped & $\begin{array}{l}\text { Appeared } \\
\text { in Exam }\end{array}$ & $\begin{array}{l}\text { Passed in } \\
\text { Exams }\end{array}$ & $\begin{array}{c}\text { Failed in } \\
\text { Exams }\end{array}$ & & Dropped & $\begin{array}{l}\text { Appeared } \\
\text { in Exams }\end{array}$ & $\begin{array}{c}\text { Passed in } \\
\text { Exams }\end{array}$ & $\begin{array}{c}\text { Failed in } \\
\text { Exams }\end{array}$ \\
\hline Grade $1 \mathrm{~A}$ & & 52 & 1 & 51 & 41 & 10 & Grade 1 & $1.92 \%$ & $98.08 \%$ & $80.39 \%$ & $19.61 \%$ \\
\hline Grade 1 B & & 0 & 0 & 0 & 0 & 0 & Grade 2 & $2.90 \%$ & $97.10 \%$ & $62.69 \%$ & $37.31 \%$ \\
\hline Grade $1 \mathrm{C}$ & & 0 & 0 & 0 & 0 & 0 & Grade 3 & $2.13 \%$ & $97.87 \%$ & $65.22 \%$ & $34.78^{c} \%$ \\
\hline Grade $2 \mathrm{~A}$ & & 32 & 1 & 31 & 20 & 11 & Grade 4 & $0.00 \%$ & $100.00 \%$ & $78.13 \%$ & $21.88 \%$ \\
\hline Grade 2 B & & 37 & 1 & 36 & 22 & 14 & Grade 5 & $0.00 \%$ & $100.00 \%$ & $83.78 \%$ & $16.22 \%$ \\
\hline Grade 3 A & & 47 & 1 & 46 & 30 & 16 & Grade 6 & $3.03 \%$ & $96.97 \%$ & $65.63 \%$ & $34.38 \%$ \\
\hline Grade 3 B & & 0 & 0 & 0 & 0 & 0 & & & & & \\
\hline Grade 4 & & 32 & 0 & 32 & 25 & 7 & Remarks: & & & & \\
\hline Grade 5 & & 37 & 0 & 37 & 31 & 6 & & & & & \\
\hline Grade 6 & & 33 & 1 & 32 & 21 & 11 & & & & & \\
\hline School Total & & 270 & 5 & 265 & 190 & 75 & School \%age & $1.85 \%$ & $98.15 \%$ & $71.70 \%$ & $28.30 \%$ \\
\hline
\end{tabular}

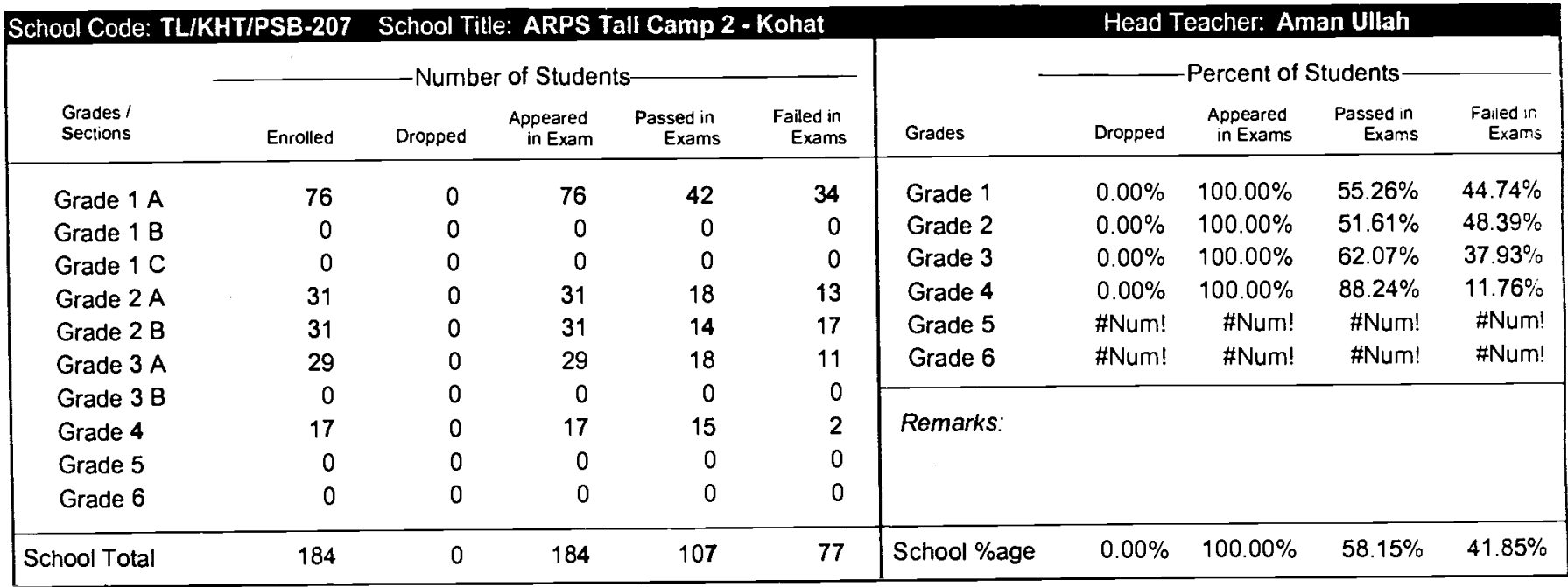

Note: 
Sub-Centre: Thall

School Code: TL/KRMIPSB-208 School Title: ARPS Baggan - Kurrum

Head Teacher: Mohammad Qasim Jan

\begin{tabular}{|c|c|c|c|c|c|c|c|c|c|c|}
\hline \multirow[b]{2}{*}{$\begin{array}{l}\text { Grades / } \\
\text { Sections }\end{array}$} & \multicolumn{5}{|c|}{ - Number of Students- } & \multirow[b]{2}{*}{ Grades } & \multicolumn{4}{|c|}{-Percent of Students } \\
\hline & Enrolled & Dropped & $\begin{array}{l}\text { Appeared } \\
\text { in Exam }\end{array}$ & $\begin{array}{c}\text { Passed in } \\
\text { Exams }\end{array}$ & $\begin{array}{c}\text { Failed in } \\
\text { Exams }\end{array}$ & & Dropped & $\begin{array}{l}\text { Appeared } \\
\text { in Exams }\end{array}$ & $\begin{array}{c}\text { Passed in } \\
\text { Exams }\end{array}$ & $\begin{array}{l}\text { Falled in } \\
\text { Exams }\end{array}$ \\
\hline Grade $1 \mathrm{~A}$ & 106 & 0 & 106 & 59 & 47 & Grade 1 & $0.00 \%$ & $100.00 \%$ & $55.66 \%$ & $44.34 \%$ \\
\hline Grade $1 \mathrm{~B}$ & 0 & 0 & 0 & 0 & 0 & Grade 2 & $0.00 \%$ & $100.00 \%$ & $75.61 \%$ & $24.39 \%$ \\
\hline Grade $1 \mathrm{C}$ & 0 & 0 & 0 & 0 & 0 & Grade 3 & $0.00 \%$ & $100.00 \%$ & $78.57 \%$ & $21.43 \%$ \\
\hline Grade 2 A & 41 & 0 & 41 & 31 & 10 & Grade 4 & $0.00 \%$ & $100.00 \%$ & $85.71 \%$ & $14.29 \%$ \\
\hline Grade 2 B & 0 & 0 & 0 & 0 & 0 & Grade 5 & $0.00 \%$ & $100.00 \%$ & $75.00 \%$ & $25.00 \%$ \\
\hline Grade $3 \mathrm{~A}$ & 28 & 0 & 28 & 22 & 6 & Grade 6 & $0.00 \%$ & $100.00 \%$ & $85.71 \%$ & $14.29 \%$ \\
\hline Grade 3 B & 0 & 0 & 0 & 0 & 0 & & & & & \\
\hline Grade 4 & 14 & 0 & 14 & 12 & 2 & Remarks: & & & & \\
\hline Grade 5 & 8 & 0 & 8 & 6 & 2 & & & & & \\
\hline Grade 6 & 14 & 0 & 14 & 12 & 2 & & & & & \\
\hline School Total & 211 & 0 & 211 & 142 & 69 & School \%age & $0.00 \%$ & $100.00 \%$ & $67.30 \%$ & $32.70 \%$ \\
\hline
\end{tabular}

\begin{tabular}{|c|c|c|c|c|c|c|c|c|c|c|c|}
\hline \multirow[b]{2}{*}{$\begin{array}{l}\text { Grades I } \\
\text { Sections }\end{array}$} & \multicolumn{6}{|c|}{ - Number of Students- } & \multirow[b]{2}{*}{ Grades } & \multicolumn{4}{|c|}{-Percent of Students - } \\
\hline & ' & Enrolled & Dropped & $\begin{array}{l}\text { Appeared } \\
\text { in Exam }\end{array}$ & $\begin{array}{c}\text { Passed in } \\
\text { Exams }\end{array}$ & $\begin{array}{l}\text { Failed in } \\
\text { Exams }\end{array}$ & & Dropped & $\begin{array}{l}\text { Appeared } \\
\text { in Exams }\end{array}$ & $\begin{array}{l}\text { Passed in } \\
\text { Exams }\end{array}$ & $\begin{array}{l}\text { Falled in } \\
\text { Exams }\end{array}$ \\
\hline Grade $1 \mathrm{~A}$ & & 45 & 7 & 38 & 21 & 17 & Grade 1 & $10.47 \%$ & $89.53 \%$ & $77.92 \%$ & $22.08 \%$ \\
\hline Grade $1 \mathrm{~B}$ & & 41 & 2 & 39 & 39 & 0 & Grade 2 & $9.72 \%$ & $90.28 \%$ & $76.92 \%$ & $23.08 \%$ \\
\hline Grade $1 \mathrm{C}$ & & 0 & 0 & 0 & 0 & 0 & Grade 3 & $10.00 \%$ & $90.00 \%$ & $91.67 \%$ & $8.33 \%$ \\
\hline Grade 2 A & & 72 & 7 & 65 & 50 & 15 & Grade 4 & $13.04 \%$ & $86.96 \%$ & $85.00 \%$ & $15.00 \%$ \\
\hline Grade 2 B & & 0 & 0 & 0 & 0 & 0 & Grade 5 & $39.29 \%$ & $60.71 \%$ & $94.12 \%$ & $5.88 \%$ \\
\hline Grade $3 \mathrm{~A}$ & & 40 & 4 & 36 & 33 & 3 & Grade 6 & $0.00 \%$ & $100.00 \%$ & $83.33 \%$ & $16.67 \%$ \\
\hline Grade 3 B & & 0 & 0 & 0 & 0 & 0 & & & & & \\
\hline Grade 4 & & 23 & 3 & 20 & 17 & 3 & Remarks: & & & & \\
\hline Grade 5 & & 28 & 11 & 17 & 16 & 1 & & & & & \\
\hline Grade 6 & & 12 & 0 & 12 & 10 & 2 & & & & & \\
\hline School Total & & 261 & 34 & 227 & 186 & 41 & School \%age & $13.03 \%$ & $86.97 \%$ & $81.94 \%$ & $18.06 \%$ \\
\hline
\end{tabular}

\begin{tabular}{|c|c|c|c|c|c|c|c|c|c|c|}
\hline \multirow[b]{2}{*}{$\begin{array}{l}\text { Grades I } \\
\text { Sections }\end{array}$} & MSB-21 & $\begin{array}{l}\text { ichool T } \\
\text {-Numb }\end{array}$ & $\begin{array}{l}\text { ARPS } \\
\text { of Studer }\end{array}$ & Chap & rrum & \multirow[b]{2}{*}{ Grades } & \multicolumn{4}{|c|}{ Percent of Students } \\
\hline & Enrolled & Dropped & $\begin{array}{l}\text { Appeared } \\
\text { in Exam }\end{array}$ & $\begin{array}{c}\text { Passed in } \\
\text { Exams }\end{array}$ & $\begin{array}{l}\text { Failed in } \\
\text { Exams }\end{array}$ & & Dropped & $\begin{array}{l}\text { Appeared } \\
\text { in Exams }\end{array}$ & $\begin{array}{l}\text { Passed in } \\
\text { Exams }\end{array}$ & $\begin{array}{l}\text { Falled in } \\
\text { Exams }\end{array}$ \\
\hline Grade $1 \mathrm{~A}$ & 53 & 0 & 53 & 30 & 23 & Grade 1 & $0.00 \%$ & $100.00 \%$ & $56.60 \%$ & $43.40 \%$ \\
\hline Grade 1 B & 0 & 0 & 0 & 0 & 0 & Grade 2 & $0.00 \%$ & $100.00 \%$ & $74.29 \%$ & $25.71 \%$ \\
\hline Grade $1 \mathrm{C}$ & 0 & 0 & 0 & 0 & 0 & Grade 3 & $0.00 \%$ & $100.00 \%$ & $68.97 \%$ & $31.03 \%$ \\
\hline Grade 2 A & 35 & 0 & 35 & 26 & 9 & Grade 4 & $0.00 \%$ & $100.00 \%$ & $77.78 \%$ & $22.22 \%$ \\
\hline Grade 2 B & 0 & 0 & 0 & 0 & 0 & Grade 5 & $0.00 \%$ & $100.00 \%$ & $93.33 \%$ & $6.67 \%$ \\
\hline Grade $3 \mathrm{~A}$ & 29 & 0 & 29 & 20 & 9 & Grade 6 & $7.69 \%$ & $92.31 \%$ & $100.00 \%$ & $0.00 \%$ \\
\hline Grade 3 B & 0 & 0 & 0 & 0 & 0 & & & & & \\
\hline Grade 4 & 27 & 0 & 27 & 21 & 6 & Remarks: & & & & \\
\hline Grade 5 & 15 & 0 & 15 & 14 & 1 & & & & & \\
\hline Grade 6 & 13 & 1 & 12 & 12 & 0 & & & & & \\
\hline School Total & 172 & 1 & 171 & 123 & 48 & School \%age & $0.58 \%$ & $99.42 \%$ & $71.93 \%$ & $28.07 \%$ \\
\hline
\end{tabular}


Sub-Centre: Thall

\begin{tabular}{|c|c|c|c|c|c|c|c|c|c|c|}
\hline \multicolumn{11}{|c|}{ School Code: TL/KRMIPSB-211 School Title: ARPS 2 - Chappari - Kurrum } \\
\hline \multirow[b]{2}{*}{$\begin{array}{l}\text { Grades I } \\
\text { Sections }\end{array}$} & \multicolumn{5}{|c|}{-Number of Students- } & \multirow[b]{2}{*}{ Grades } & \multicolumn{4}{|c|}{-Percent of Students } \\
\hline & Enrolled & Dropped & $\begin{array}{l}\text { Appeared } \\
\text { in Exam }\end{array}$ & $\begin{array}{l}\text { Passed in } \\
\text { Exams }\end{array}$ & $\begin{array}{l}\text { Failed in } \\
\text { Exams }\end{array}$ & & Dropped & $\begin{array}{l}\text { Appeared } \\
\text { in Exams }\end{array}$ & $\begin{array}{l}\text { Passed in } \\
\text { Exams }\end{array}$ & $\begin{array}{l}\text { Fallec in } \\
\text { Exams }\end{array}$ \\
\hline Grade $1 \mathrm{~A}$ & 54 & 2 & 52 & 32 & 20 & Grade 1 & $3.70 \%$ & $96.30 \%$ & $61.54 \%$ & $38.46 \%$ \\
\hline Grade $1 \mathrm{~B}$ & 0 & 0 & 0 & 0 & 0 & Grade 2 & $3.13 \%$ & $96.88 \%$ & $77.42 \%$ & $22.58 \%$ \\
\hline Grade $1 \mathrm{C}$ & 0 & 0 & 0 & 0 & 0 & Grade 3 & $8.33 \%$ & $91.67 \%$ & $81.82 \%$ & $18.18 \%$ \\
\hline Grade $2 \mathrm{~A}$ & 32 & 1 & 31 & 24 & 7 & Grade 4 & $10.00 \%$ & $90.00 \%$ & $77.78 \%$ & $22.22 \%$ \\
\hline Grade 2 B & 0 & 0 & 0 & 0 & 0 & Grade 5 & $6.67 \%$ & $93.33 \%$ & $100.00 \%$ & $0.00 \%$ \\
\hline Grade $3 \mathrm{~A}$ & 24 & 2 & 22 & 18 & 4 & Grade 6 & $6.67 \%$ & $93.33 \%$ & $100.00 \%$ & $0.00 \%$ \\
\hline Grade 3 B & 0 & 0 & 0 & 0 & 0 & & & & & \\
\hline Grade 4 & 20 & 2 & 18 & 14 & 4 & Remarks: & & & & \\
\hline Grade 5 & 15 & 1 & 14 & 14 & 0 & & & & & \\
\hline Grade 6 & 15 & 1 & 14 & 14 & 0 & & & & & \\
\hline School Total & 160 & 9 & 151 & 116 & 35 & School \%age & $5.63 \%$ & $94.37 \%$ & $76.82 \%$ & $23.18 \%$ \\
\hline
\end{tabular}

\begin{tabular}{|c|c|c|c|c|c|c|c|c|c|c|c|}
\hline \multirow[b]{2}{*}{$\begin{array}{l}\text { Grades / } \\
\text { Sections }\end{array}$} & \multicolumn{6}{|c|}{ _ Number of Students- } & \multirow[b]{2}{*}{ Grades } & \multicolumn{4}{|c|}{-Percent of Students- } \\
\hline & , & Enrolled & Dropped & $\begin{array}{l}\text { Appeared } \\
\text { in Exam }\end{array}$ & $\begin{array}{l}\text { Passed in } \\
\text { Exams }\end{array}$ & $\begin{array}{c}\text { Failed in } \\
\text { Exams }\end{array}$ & & Dropped & $\begin{array}{l}\text { Appeared } \\
\text { in Exams }\end{array}$ & $\begin{array}{c}\text { Passed in } \\
\text { Exams }\end{array}$ & $\begin{array}{c}\text { Failed in } \\
\text { Exams } \\
\end{array}$ \\
\hline Grade $1 \mathrm{~A}$ & & 112 & 10 & 102 & 88 & 14 & Grade 1 & $8.93 \%$ & $91.07 \%$ & $86.27 \%$ & $13.73 \%$ \\
\hline Grade $1 \mathrm{~B}$ & & 0 & 0 & 0 & 0 & 0 & Grade 2 & $5.17 \%$ & $94.83 \%$ & $92.73 \%$ & $7.27 \%$ \\
\hline Grade $1 \mathrm{C}$ & & 0 & 0 & 0 & 0 & 0 & Grade 3 & $8.51 \%$ & $91.49 \%$ & $100.00 \%$ & $0.00 \%$ \\
\hline Grade $2 \mathrm{~A}$ & & 58 & 3 & 55 & 51 & 4 & Grade 4 & $23.53 \%$ & $76.47 \%$ & $100.00 \%$ & $0.00 \%$ \\
\hline Grade 2 B & & 0 & 0 & 0 & 0 & 0 & Grade 5 & $18.75 \%$ & $81.25 \%$ & $100.00 \%$ & $0.00 \%$ \\
\hline Grade $3 \mathrm{~A}$ & & 47 & 4 & 43 & 43 & 0 & Grade 6 & $0.00 \%$ & $100.00 \%$ & $100.00 \%$ & $0.00 \%$ \\
\hline Grade 3 B & & 0 & 0 & 0 & 0 & 0 & & & & & \\
\hline Grade 4 & & 17 & 4 & 13 & 13 & 0 & Remarks: & & & & \\
\hline Grade 5 & & 16 & 3 & 13 & 13 & 0 & & & & & \\
\hline Grade 6 & & 8 & 0 & 8 & 8 & 0 & & & & & \\
\hline School Total & & 258 & 24 & 234 & 216 & 18 & School \%age & $9.30 \%$ & $90.70 \%$ & $92.31 \%$ & $7.69 \%$ \\
\hline
\end{tabular}

\begin{tabular}{|c|c|c|c|c|c|c|c|c|c|c|}
\hline \multirow[b]{2}{*}{$\begin{array}{l}\text { Grades I } \\
\text { Sections }\end{array}$} & \multicolumn{5}{|c|}{ Number of Students______ } & \multirow[b]{2}{*}{ Grades } & \multicolumn{4}{|c|}{ Percent of Students- } \\
\hline & Enrolled & Dropped & $\begin{array}{l}\text { Appeared } \\
\text { in Exam }\end{array}$ & $\begin{array}{l}\text { Passed in } \\
\text { Exams }\end{array}$ & $\begin{array}{l}\text { Failed in } \\
\text { Exams }\end{array}$ & & Dropped & $\begin{array}{l}\text { Appeared } \\
\text { in Exams }\end{array}$ & $\begin{array}{l}\text { Passed in } \\
\text { Exams }\end{array}$ & $\begin{array}{l}\text { Failed in } \\
\text { Exams }\end{array}$ \\
\hline Grade $1 \mathrm{~A}$ & 107 & 33 & 74 & 62 & 12 & Grade 1 & $30.84 \%$ & $69.16 \%$ & $83.78 \%$ & $16.22 \%$ \\
\hline Grade $1 \mathrm{~B}$ & 0 & 0 & 0 & 0 & 0 & Grade 2 & $10.17 \%$ & $89.83 \%$ & $92.45 \%$ & $7.55 \%$ \\
\hline Grade $1 \mathrm{C}$ & 0 & 0 & 0 & 0 & 0 & Grade 3 & $11.36 \%$ & $88.64 \%$ & $89.74 \%$ & $10.26 \%$ \\
\hline Grade 2 A & 59 & 6 & 53 & 49 & 4 & Grade 4 & $11.11 \%$ & $88.89 \%$ & $87.50 \%$ & $12.50 \%$ \\
\hline Grade 2 B & 0 & 0 & 0 & 0 & 0. & Grade 5 & $26.67 \%$ & $73.33 \%$ & $90.91 \%$ & $9.09 \%$ \\
\hline Grade 3 A & 44 & 5 & 39 & 35 & 4 & Grade 6 & $11.11 \%$ & $88.89 \%$ & $100.00 \%$ & $0.00 \%$ \\
\hline Grade 3 B & 0 & 0 & 0 & 0 & 0 & & & & & \\
\hline Grade 4 & 27 & 3 & 24 & 21 & 3 & Remarks: & & & & \\
\hline Grade 5 & 30 & 8 & 22 & 20 & 2 & & & & & \\
\hline Grade 6 & 18 & 2 & 16 & 16 & 0 & & & & & \\
\hline School Total & 285 & 57 & 228 & 203 & 25 & School \%age & $20.00 \%$ & $80.00 \%$ & $89.04 \%$ & $10.96 \%$ \\
\hline
\end{tabular}

Note:

\#Num! = Enrollment in respective grade(s) is nill therefore calculation is not applicable
Page 73 of 91

Produced by DP/MIS Section on 31-Jul-97 
Sub-Centre: Thall

\begin{tabular}{|c|c|c|c|c|c|c|c|c|c|c|}
\hline \multicolumn{11}{|c|}{ School Code: TL/KRM/PSB-214 School Title: ARPS 1 - Khapyanga - Kurrum } \\
\hline \multirow[b]{2}{*}{$\begin{array}{l}\text { Grades I } \\
\text { Sections }\end{array}$} & \multicolumn{5}{|c|}{ Number of Students- } & \multirow[b]{2}{*}{ Grades } & \multicolumn{4}{|c|}{-Percent of Students } \\
\hline & Enrolled & Dropped & $\begin{array}{l}\text { Appeared } \\
\text { in Exam }\end{array}$ & $\begin{array}{r}\text { Passed in } \\
\text { Exams }\end{array}$ & $\begin{array}{c}\text { Failed in } \\
\text { Exams }\end{array}$ & & Dropped & $\begin{array}{c}\text { Appeared } \\
\text { in Exams }\end{array}$ & $\begin{array}{c}\text { Passed in } \\
\text { Exams }\end{array}$ & $\begin{array}{l}\text { Farled in } \\
\text { Exams }\end{array}$ \\
\hline Grade $1 \mathrm{~A}$ & 51 & 5 & 46 & 42 & 4 & Grade 1 & $9.80 \%$ & $90.20 \%$ & $91.30 \%$ & $8.70 \%$ \\
\hline Grade $1 \mathrm{~B}$ & 0 & 0 & 0 & 0 & 0 & Grade 2 & $7.41 \%$ & $92.59 \%$ & $88.00 \%$ & $12.00 \%$ \\
\hline Grade $1 \mathrm{C}$ & 0 & 0 & 0 & 0 & 0 & Grade 3 & $0.00 \%$ & $100.00 \%$ & $81.25 \%$ & $18.75 \%$ \\
\hline Grade $2 \mathrm{~A}$ & 27 & 2 & 25 & 22 & 3 & Grade 4 & $9.09 \%$ & $90.91 \%$ & $80.00 \%$ & $20.00 \%$ \\
\hline Grade 2 B & 0 & 0 & 0 & 0 & 0 & Grade 5 & $9.09 \%$ & $90.91 \%$ & $100.00 \%$ & $0.00 \%$ \\
\hline Grade 3 A & 16 & 0 & 16 & 13 & 3 & Grade 6 & \#Num! & \#Num! & \#Num! & \#Num! \\
\hline Grade 3 B & 0 & 0 & 0 & 0 & 0 & & & & & \\
\hline Grade 4 & 11 & 1 & 10 & 8 & 2 & Remarks: & & & & \\
\hline Grade 5 & 11 & 1 & 10 & 10 & 0 & & & & & \\
\hline Grade 6 & 0 & 0 & 0 & 0 & 0 & & & & & \\
\hline School Total & 116 & 9 & 107 & 95 & 12 & School \%age & $7.76 \%$ & $92.24 \%$ & $88.79 \%$ & $11.21 \%$ \\
\hline
\end{tabular}

\begin{tabular}{|c|c|c|c|c|c|c|c|c|c|c|c|}
\hline \multirow[b]{2}{*}{$\begin{array}{l}\text { Grades / } \\
\text { Sections }\end{array}$} & \multicolumn{6}{|c|}{ - Number of Students } & \multirow[b]{2}{*}{ Grades } & \multicolumn{4}{|c|}{ Percent of Students } \\
\hline & $y$ & Enrolled & Dropped & $\begin{array}{c}\text { Appeared } \\
\text { in Exam }\end{array}$ & $\begin{array}{c}\text { Passed in } \\
\text { Exams }\end{array}$ & $\begin{array}{c}\text { Failed in } \\
\text { Exams }\end{array}$ & & Dropped & $\begin{array}{c}\text { Appeared } \\
\text { in Exams }\end{array}$ & $\begin{array}{c}\text { Passed in } \\
\text { Exams }\end{array}$ & $\begin{array}{c}\text { Failed in } \\
\text { Exams }\end{array}$ \\
\hline Grade $1 \mathrm{~A}$ & & 49 & 6 & 43 & 25 & 18 & Grade 1 & $12.24 \%$ & $87.76 \%$ & $58.14 \%$ & $41.86 \%$ \\
\hline Grade 1 B & & 0 & 0 & 0 & 0 & 0 & Grade 2 & $3.23 \%$ & $96.77 \%$ & $83.33 \%$ & $16.67 \%$ \\
\hline Grade $1 \mathrm{C}$ & & 0 & 0 & 0 & 0 & 0 & Grade 3 & $19.23 \%$ & $80.77 \%$ & $80.95 \%$ & $19.05^{\circ} \%$ \\
\hline Grade $2 \mathrm{~A}$ & & 31 & 1 & 30 & 25 & 5 & Grade 4 & $0.00 \%$ & $100.00 \%$ & $90.91 \%$ & $9.09 \%$ \\
\hline Grade 2 B & & 0 & 0 & 0 & 0 & 0 & Grade 5 & $33.33 \%$ & $66.67 \%$ & $100.00 \%$ & $0.00 \%$ \\
\hline Grade $3 \mathrm{~A}$ & & 26 & 5 & 21 & 17 & 4 & Grade 6 & \#Num! & \#Num! & \#Num! & \#Num! \\
\hline Grade 3 B & & 0 & 0 & 0 & 0 & 0 & & & & & \\
\hline Grade 4 & & 11 & 0 & 11 & 10 & 1 & Remarks: & & & & \\
\hline Grade 5 & & 6 & 2 & 4 & 4 & 0 & & & & & \\
\hline Grade 6 & & 0 & 0 & 0 & 0 & 0 & & & & & \\
\hline School Total & & 123 & 14 & 109 & 81 & 28 & School \%age & $11.38 \%$ & $88.62 \%$ & $74.31 \%$ & $25.69 \%$ \\
\hline
\end{tabular}

\begin{tabular}{|c|c|c|c|c|c|c|c|c|c|c|}
\hline \multirow[b]{2}{*}{$\begin{array}{l}\text { Grades I } \\
\text { Sections }\end{array}$} & \multicolumn{5}{|c|}{ - Number of Students } & \multirow[b]{2}{*}{ Grades } & \multicolumn{4}{|c|}{ Percent of Students } \\
\hline & Enrolled & Dropped & $\begin{array}{l}\text { Appeared } \\
\text { in Exam }\end{array}$ & $\begin{array}{l}\text { Passed in } \\
\text { Exams }\end{array}$ & $\begin{array}{c}\text { Failed in } \\
\text { Exams }\end{array}$ & & Dropped & $\begin{array}{c}\text { Appeared } \\
\text { in Exams }\end{array}$ & $\begin{array}{c}\text { Passed in } \\
\text { Exams }\end{array}$ & $\begin{array}{c}\text { Fallec in } \\
\text { Exams } \\
\end{array}$ \\
\hline Grade $1 \mathrm{~A}$ & 24 & 0 & 24 & 18 & 6 & Grade 1 & $0.00 \%$ & $100.00 \%$ & $75.00 \%$ & $25.00 \%$ \\
\hline Grade $1 \mathrm{~B}$ & 0 & 0 & 0 & 0 & 0 & Grade 2 & $0.00 \%$ & $100.00 \%$ & $93.75 \%$ & $6.25 \%$ \\
\hline Grade $1 \mathrm{C}$ & 0 & 0 & 0 & 0 & 0 & Grade 3 & $0.00 \%$ & $100.00 \%$ & $87.50 \%$ & $12.50 \%$ \\
\hline Grade $2 \mathrm{~A}$ & 16 & 0 & 16 & 15 & 1 & Grade 4 & $0.00 \%$ & $100.00 \%$ & $100.00 \%$ & $0.00 \%$ \\
\hline Grade 2 B & 0 & 0 & 0 & 0 & 0 & Grade 5 & \#Num! & \#um! & \#Num! & \#Num! \\
\hline Grade $3 \mathrm{~A}$ & 16 & 0 & 16 & 14 & 2 & Grade 6 & \#Num! & \#Num! & \#Num! & \#Num! \\
\hline Grade 3 B & 0 & 0 & 0 & 0 & 0 & & & & & \\
\hline Grade 4 & 13 & 0 & 13 & 13 & 0 & Remarks: & & & & \\
\hline Grade 5 & 0 & 0 & 0 & 0 & 0 & & & & & \\
\hline Grade 6 & 0 & 0 & 0 & 0 & 0 & & & & & \\
\hline School Total & 69 & 0 & 69 & 60 & 9 & School \%age & $0.00 \%$ & $100.00 \%$ & $86.96 \%$ & $13.04 \%$ \\
\hline
\end{tabular}


Sub-Centre: Thall

\begin{tabular}{|c|c|c|c|c|c|c|c|c|c|c|}
\hline \multirow[b]{2}{*}{$\begin{array}{l}\text { Grades / } \\
\text { Sections }\end{array}$} & \multicolumn{5}{|c|}{ - Number of Students } & \multirow[b]{2}{*}{ Grades } & \multicolumn{4}{|c|}{-Percent of Students } \\
\hline & Enrolled & Dropped & $\begin{array}{l}\text { Appeareo } \\
\text { in Exam }\end{array}$ & $\begin{array}{l}\text { Passed in } \\
\text { Exams }\end{array}$ & $\begin{array}{l}\text { Failed in } \\
\text { Exams }\end{array}$ & & Dropped & $\begin{array}{l}\text { Appeared } \\
\text { in Exams }\end{array}$ & $\begin{array}{l}\text { Passed in } \\
\text { Exams }\end{array}$ & $\begin{array}{l}\text { Faled in } \\
\text { Exams }\end{array}$ \\
\hline Grade $1 \mathrm{~A}$ & 63 & 2 & 61 & 59 & 2 & Grade 1 & $3.17 \%$ & $96.83 \%$ & $96.72 \%$ & $3.28 \%$ \\
\hline Grade 1 B & 0 & 0 & 0 & 0 & 0 & Grade 2 & $0.00 \%$ & $100.00 \%$ & $92.50 \%$ & $7.50 \%$ \\
\hline Grade $1 \mathrm{C}$ & 0 & 0 & 0 & 0 & 0 & Grade 3 & $2.86 \%$ & $97.14 \%$ & $97.06 \%$ & $2.94 \%$ \\
\hline Grade 2 A & 40 & 0 & 40 & 37 & 3 & Grade 4 & $0.00 \%$ & $100.00 \%$ & $92.59 \%$ & $7.41 \%$ \\
\hline Grade 2 B & 0 & 0 & 0 & 0 & 0 & Grade 5 & $0.00 \%$ & $100.00 \%$ & $100.00 \%$ & $0.00 \%$ \\
\hline Grade $3 \mathrm{~A}$ & 35 & 1 & 34 & 33 & 1 & Grade 6 & $0.00 \%$ & $100.00 \%$ & $100.00 \%$ & $0.00 \%$ \\
\hline Grade 3 B & 0 & 0 & 0 & 0 & 0 & & & & & \\
\hline Grade 4 & 27 & 0 & 27 & 25 & 2 & Remarks: & & & & \\
\hline Grade 5 & 12 & 0 & 12 & 12 & 0 & & & & & \\
\hline Grade 6 & 8 & 0 & 8 & 8 & 0 & & & & & \\
\hline School Total & 185 & 3 & 182 & 174 & 8 & School \%age & $1.62 \%$ & $98.38 \%$ & $95.60 \%$ & $4.40 \%$ \\
\hline
\end{tabular}

\begin{tabular}{|c|c|c|c|c|c|c|c|c|c|c|}
\hline \multirow[b]{2}{*}{$\begin{array}{l}\text { Grades I } \\
\text { Sections }\end{array}$} & \multicolumn{5}{|c|}{-Number of Students- } & \multirow[b]{2}{*}{ Grades } & \multicolumn{4}{|c|}{ Percent of Students } \\
\hline & Enrolled & Dropped & $\begin{array}{l}\text { Appeared } \\
\text { in Exam }\end{array}$ & $\begin{array}{l}\text { Passed in } \\
\text { Exams }\end{array}$ & $\begin{array}{c}\text { Failed in } \\
\text { Exarns }\end{array}$ & & Dropped & $\begin{array}{l}\text { Appeared } \\
\text { in Exams }\end{array}$ & $\begin{array}{l}\text { Passed in } \\
\text { Exams }\end{array}$ & $\begin{array}{c}\text { Falled in } \\
\text { Exams }\end{array}$ \\
\hline Grade $1 \mathrm{~A}$ & 63 & 12 & 51 & 43 & 8 & Grade 1 & $19.05 \%$ & $80.95 \%$ & $84.31 \%$ & $15.69 \%$ \\
\hline Grade 1 B & 0 & 0 & 0 & 0 & 0 & Grade 2 & $7.14 \%$ & $92.86 \%$ & $76.92 \%$ & $23.08 \%$ \\
\hline Grade $1 \mathrm{C}$ & 0 & 0 & 0 & 0 & 0 & Grade 3 & $11.54 \%$ & $88.46 \%$ & $91.30 \%$ & $8.70 \%$ \\
\hline Grade 2 A & 28 & 2 & 26 & 20 & 6 & Grade 4 & $7.14 \%$ & $92.86 \%$ & $92.31 \%$ & $7.69 \%$ \\
\hline Grade 2 B & 0 & 0 & 0 & 0 & 0 & Grade 5 & $0.00 \%$ & $100.00 \%$ & $100.00 \%$ & $0.00 \%$ \\
\hline Grade $3 \mathrm{~A}$ & $26^{\circ}$ & 3 & 23 & 21 & 2 & Grade 6 & \#Num! & \#Num! & \#Num! & \#Num! \\
\hline Grade 3 B & 0 & 0 & 0 & 0 & 0 & & & & & \\
\hline Grade 4 & 14 & 1 & 13 & 12 & 1 & Remarks: & & & & \\
\hline Grade 5 & 8 & 0 & 8 & 8 & 0 & & & & & \\
\hline Grade 6 & 0 & 0 & 0 & 0 & 0 & & & & & \\
\hline School Total & 139 & 18 & 121 & 104 & 17 & School \%age & $12.95 \%$ & $87.05 \%$ & $85.95 \%$ & $14.05 \%$ \\
\hline
\end{tabular}

\begin{tabular}{|c|c|c|c|c|c|c|c|c|c|c|}
\hline \multirow[b]{2}{*}{$\begin{array}{l}\text { Grades I } \\
\text { Sections }\end{array}$} & \multicolumn{5}{|c|}{ Number of Students } & \multirow[b]{2}{*}{ Grades } & \multicolumn{4}{|c|}{-Percent of Students } \\
\hline & Enrolled & Dropped & $\begin{array}{l}\text { Appeared } \\
\text { in Exam }\end{array}$ & $\begin{array}{l}\text { Passed in } \\
\text { Exams }\end{array}$ & $\begin{array}{l}\text { Failed in } \\
\text { Exams }\end{array}$ & & Dropped & $\begin{array}{l}\text { Appeared } \\
\text { in Exams }\end{array}$ & $\begin{array}{c}\text { Passed in } \\
\text { Exams }\end{array}$ & $\begin{array}{c}\text { Falteo in } \\
\text { Exams }\end{array}$ \\
\hline Grade $1 \mathrm{~A}$ & 30 & 7 & 23 & 23 & 0 & Grade 1 & $23.33 \%$ & $76.67 \%$ & $100.00 \%$ & $0.00 \%$ \\
\hline Grade $1 \mathrm{~B}$ & 0 & 0 & 0 & 0 & 0 & Grade 2 & $12.90 \%$ & $87.10 \%$ & $92.59 \%$ & $7.41 \%$ \\
\hline Grade $1 \mathrm{C}$ & 0 & 0 & 0 & 0 & 0 & Grade 3 & $25.00 \%$ & $75.00 \%$ & $80.95 \%$ & $19.05 \%$ \\
\hline Grade $2 \mathrm{~A}$ & 31 & 4 & 27 & 25 & 2 & Grade 4 & $18.75 \%$ & $81.25 \%$ & $100.00 \%$ & $0.00 \%$ \\
\hline Grade 2 B & 0 & 0 & 0 & 0 & 0 & Grade 5 & \#Num! & \#Num! & \#Num! & \#Num! \\
\hline Grade $3 \mathrm{~A}$ & 28 & 7 & 21 & 17 & 4 & Grade 6 & \#Num! & \#Num! & \#Num! & \#Num! \\
\hline Grade 3 B & 0 & 0 & 0 & 0 & 0 & & & & & \\
\hline Grade 4 & 16 & 3 & 13 & 13 & 0 & Remarks: & & & & \\
\hline Grade 5 & 0 & 0 & 0 & 0 & 0 & & & & & \\
\hline Grade 6 & 0 & 0 & 0 & 0 & 0 & & & & & \\
\hline School Total & 105 & 21 & 84 & 78 & 6 & School \%age & $20.00 \%$ & $80.00 \%$ & $92.86 \%$ & $7.14 \%$ \\
\hline
\end{tabular}


Sub-Centre: Thall

\begin{tabular}{|c|c|c|c|c|c|c|c|c|c|c|}
\hline \multirow[b]{2}{*}{$\begin{array}{l}\text { Grades I } \\
\text { Sections }\end{array}$} & \multicolumn{5}{|c|}{ - Number of Students- } & \multirow[b]{2}{*}{ Grades } & \multicolumn{4}{|c|}{-Percent of Students- } \\
\hline & Enrolled & Dropped & $\begin{array}{c}\text { Appeared } \\
\text { in Exam }\end{array}$ & $\begin{array}{c}\text { Passed in } \\
\text { Exams }\end{array}$ & $\begin{array}{c}\text { Failed in } \\
\text { Exams }\end{array}$ & & Dropped & $\begin{array}{l}\text { Appeared } \\
\text { in Exams }\end{array}$ & $\begin{array}{l}\text { Passed in } \\
\text { Exams }\end{array}$ & $\begin{array}{l}\text { Falled in } \\
\text { Exams }\end{array}$ \\
\hline Grade $1 \mathrm{~A}$ & 36 & 12 & 24 & 24 & 0 & Grade 1 & $33.33 \%$ & $66.67 \%$ & $100.00 \%$ & $0.00 \%$ \\
\hline Grade 1 B & 0 & 0 & 0 & 0 & 0 & Grade 2 & $0.00 \%$ & $100.00 \%$ & $100.00 \%$ & $0.00 \%$ \\
\hline Grade $1 \mathrm{C}$ & 0 & 0 & 0 & 0 & 0 & Grade 3 & $6.67 \%$ & $93.33 \%$ & $100.00 \%$ & $0.00 \%$ \\
\hline Grade $2 \mathrm{~A}$ & 26 & 0 & 26 & 26 & 0 & Grade 4 & $47.06 \%$ & $52.94 \%$ & $100.00 \%$ & $0.00 \%$ \\
\hline Grade 2 B & 0 & 0 & 0 & 0 & 0 & Grade 5 & \#Num! & \#Num! & \#Num! & \#Num! \\
\hline Grade 3 A & 15 & 1 & 14 & 14 & 0 & Grade 6 & \#Num! & $\#$ Num! & \#Num! & \#Num! \\
\hline Grade 3 B & 0 & 0 & 0 & 0 & 0 & & & & & \\
\hline Grade 4 & 17 & 8 & 9 & 9 & 0 & Remarks: & & & & \\
\hline Grade 5 & 0 & 0 & 0 & 0 & 0 & & & & & \\
\hline Grade 6 & 0 & 0 & 0 & 0 & 0 & & & & & \\
\hline School Total & 94 & 21 & 73 & 73 & 0 & School \%age & $22.34 \%$ & $77.66 \%$ & $100.00 \%$ & $0.00 \%$ \\
\hline
\end{tabular}

\begin{tabular}{|c|c|c|c|c|c|c|c|c|c|c|c|}
\hline \multirow[b]{2}{*}{$\begin{array}{l}\text { Grades / } \\
\text { Sections }\end{array}$} & \multicolumn{6}{|c|}{ - Number of Students- } & \multirow[b]{2}{*}{ Grades } & \multicolumn{4}{|c|}{ Percent of Students } \\
\hline & ' & Enrolled & Dropped & $\begin{array}{l}\text { Appeared } \\
\text { in Exam }\end{array}$ & $\begin{array}{c}\text { Passed in } \\
\text { Exams }\end{array}$ & $\begin{array}{l}\text { Failed in } \\
\text { Exams }\end{array}$ & & Dropped & $\begin{array}{l}\text { Appeared } \\
\text { in Exams }\end{array}$ & $\begin{array}{c}\text { Passed in } \\
\text { Exams }\end{array}$ & $\begin{array}{l}\text { Falled in } \\
\text { Exams }\end{array}$ \\
\hline Grade $1 \mathrm{~A}$ & & 68 & 18 & 50 & 50 & 0 & Grade 1 & $26.40 \%$ & $73.60 \%$ & $100.00 \%$ & $0.00 \%$ \\
\hline Grade $1 \mathrm{~B}$ & & 57 & 15 & 42 & 42 & 0 & Grade 2 & $13.33 \%$ & $86.67 \%$ & $91.35 \%$ & $8.65 \%$ \\
\hline Grade $1 \mathrm{C}$ & & 0 & 0 & 0 & 0 & 0 & Grade 3 & $7.50 \%$ & $92.50 \%$ & $95.95 \%$ & $4.05 \%$ \\
\hline Grade $2 \mathrm{~A}$ & & 64 & 5 & 59 & 54 & 5 & Grade 4 & $14.00 \%$ & $86.00 \%$ & $90.70 \%$ & $9.30 \%$ \\
\hline Grade 2 B & & 56 & 11 & 45 & 41 & 4 & Grade 5 & $10.00 \%$ & $90.00 \%$ & $96.30 \%$ & $3.70 \%$ \\
\hline Grade 3 A & & $80^{\circ}$ & 6 & 74 & 71 & 3 & Grade 6 & $37.93 \%$ & $62.07 \%$ & $100.00 \%$ & $0.00 \%$ \\
\hline Grade $3 \mathrm{~B}$ & & 0 & 0 & 0 & 0 & 0 & & & & & \\
\hline Grade 4 & & 50 & 7 & 43 & 39 & 4 & Remarks: & & & & \\
\hline Grade 5 & & 30 & 3 & 27 & 26 & 1 & & & & & \\
\hline Grade 6 & & 29 & 11 & 18 & 18 & 0 & & & & & \\
\hline School Total & & 434 & 76 & 358 & 341 & 17 & School \%age & $17.51 \%$ & $82.49 \%$ & $95.25 \%$ & $4.75 \%$ \\
\hline
\end{tabular}

\section{School Code: TLIKRMIPSB-222 School Title: ARPS 2 Satheen Hilly Area Kurrum}

\begin{tabular}{|c|c|c|c|c|c|c|c|c|c|c|}
\hline \multirow[b]{2}{*}{$\begin{array}{l}\text { Grades I } \\
\text { Sections }\end{array}$} & \multicolumn{5}{|c|}{-Number of Students- } & \multirow[b]{2}{*}{ Grades } & \multicolumn{4}{|c|}{-Percent of Students } \\
\hline & Enrolled & Dropped & $\begin{array}{l}\text { Appeared } \\
\text { in Exam }\end{array}$ & $\begin{array}{l}\text { Passed in } \\
\text { Exams }\end{array}$ & $\begin{array}{l}\text { Failed in } \\
\text { Exams }\end{array}$ & & Dropped & $\begin{array}{c}\text { Appeared } \\
\text { in Exarns }\end{array}$ & $\begin{array}{l}\text { Passed in } \\
\text { Exams }\end{array}$ & $\begin{array}{l}\text { Faled in } \\
\text { Exams }\end{array}$ \\
\hline Grade $1 \mathrm{~A}$ & 61 & 0 & 61 & 55 & 6 & Grade 1 & $0.00 \%$ & $100.00 \%$ & $90.16 \%$ & $9.84 \%$ \\
\hline Grade $1 \mathrm{~B}$ & 0 & 0 & 0 & 0 & 0 & Grade 2 & $0.00 \%$ & $100.00 \%$ & $88.24 \%$ & $11.76 \%$ \\
\hline Grade $1 \mathrm{C}$ & 0 & 0 & 0 & 0 & 0 & Grade 3 & $0.00 \%$ & $100.00 \%$ & $88.89 \%$ & $11.11 \%$ \\
\hline Grade $2 \mathrm{~A}$ & 34 & 0 & 34 & 30 & 4 & Grade 4 & $0.00 \%$ & $100.00 \%$ & $81.82 \%$ & $18.18 \%$ \\
\hline Grade 2 B & 0 & 0 & 0 & 0 & 0 & Grade 5 & $0.00 \%$ & $100.00 \%$ & $100.00 \%$ & $0.00 \%$ \\
\hline Grade $3 \mathrm{~A}$ & 18 & 0 & 18 & 16 & 2 & Grade 6 & \#Num! & \#Num! & \#Num! & \#um! \\
\hline Grade 3 B & 0 & 0 & 0 & 0 & 0 & & & & & \\
\hline Grade 4 & 22 & 0 & 22 & 18 & 4 & Remarks: & & & & \\
\hline Grade 5 & 22 & 0 & 22 & 22 & 0 & & & & & \\
\hline Grade 6 & 0 & 0 & 0 & 0 & 0 & & & & & \\
\hline School Total & 157 & 0 & 157 & 141 & 16 & School \%age & $0.00 \%$ & $100.00 \%$ & $89.81 \%$ & $10.19 \%$ \\
\hline
\end{tabular}

Note:

\#Num! = Enrollment in respective grade(s) is nill therefore calculation is nol applicable
Page 76 of 91

Produced by DP/MIS Section on 31-Jul-97 
Sub-Centre: Thall School Code: TL/KRM/MSB-223 School Title: ARPS 1 Shasho Hilly Area Kurrum Head Teacher. Inayat Ullah

\begin{tabular}{|c|c|c|c|c|c|c|c|c|c|c|}
\hline \multirow[b]{2}{*}{$\begin{array}{l}\text { Grades ! } \\
\text { Sections }\end{array}$} & \multicolumn{5}{|c|}{-Number of Students- } & \multirow[b]{2}{*}{ Grades } & \multicolumn{4}{|c|}{ Percent of Students- } \\
\hline & Enrolled & Dropped & $\begin{array}{l}\text { Appeared } \\
\text { in Exam }\end{array}$ & $\begin{array}{r}\text { Passed in } \\
\text { Exams }\end{array}$ & $\begin{array}{c}\text { Faited in } \\
\text { Exams }\end{array}$ & & Dropped & $\begin{array}{l}\text { Appeareo } \\
\text { in Exams }\end{array}$ & $\begin{array}{l}\text { Passec in } \\
\text { Exams }\end{array}$ & $\begin{array}{c}\text { Fa:ez:- } \\
\text { Exams }\end{array}$ \\
\hline Grade $1 \mathrm{~A}$ & 60 & 21 & 39 & 34 & 5 & Grade 1 & $35.00 \%$ & $65.00 \%$ & $87.18 \%$ & $12.82^{\circ} \mathrm{o}$ \\
\hline Grade $1 \mathrm{~B}$ & 0 & 0 & 0 & 0 & 0 & Grade 2 & $17.54 \%$ & $82.46 \%$ & $97.87 \%$ & $2.13 \%$ \\
\hline Grade $1 \mathrm{C}$ & 0 & 0 & 0 & 0 & 0 & Grade 3 & $35.00 \%$ & $65.00 \%$ & $100.00 \%$ & $0.00^{\circ}=$ \\
\hline Grade 2 A & 57 & 10 & 47 & 46 & 1 & Grade 4 & $10.00 \%$ & $90.00 \%$ & $80.56 \%$ & $19.44 \%$ \\
\hline Grade 2 B & 0 & 0 & 0 & 0 & 0 & Grade 5 & $19.05 \%$ & $80.95 \%$ & $82.35 \%$ & $17.05^{\circ}=$ \\
\hline Grade $3 \mathrm{~A}$ & 40 & 14 & 26 & 26 & 0 & Grade 6 & $19.23 \%$ & $80.77 \%$ & $100.00^{\circ} \%$ & $0.00^{\circ}=$ \\
\hline Grade 3 B & 0 & 0 & 0 & 0 & 0 & & & & & \\
\hline Grade 4 & 40 & 4 & 36 & 29 & 7 & Remarks: & & & & \\
\hline Grade 5 & 21 & 4 & 17 & 14 & 3 & & & & & \\
\hline Grade 6 & 26 & 5 & 21 & 21 & 0 & & & & & \\
\hline School Total & 244 & 58 & 186 & 170 & 16 & School \%age & $23.77 \%$ & $76.23 \%$ & $91.40 \%$ & $8.00^{\circ}$ \\
\hline
\end{tabular}

\begin{tabular}{|c|c|c|c|c|c|c|c|c|c|c|c|}
\hline \multirow[b]{2}{*}{$\begin{array}{l}\text { Grades / } \\
\text { Sections }\end{array}$} & \multicolumn{6}{|c|}{ - Number of Students } & \multirow[b]{2}{*}{ Grades } & \multicolumn{4}{|c|}{-Percent of Students - } \\
\hline & ' & Enrolled & Dropped & $\begin{array}{l}\text { Appeared } \\
\text { in Exam }\end{array}$ & $\begin{array}{l}\text { Passed in } \\
\text { Exams }\end{array}$ & $\begin{array}{l}\text { Failed in } \\
\text { Exams }\end{array}$ & & Dropped & $\begin{array}{l}\text { Appeared } \\
\text { in Exams }\end{array}$ & $\begin{array}{l}\text { Passed ir. } \\
\text { Exams }\end{array}$ & 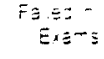 \\
\hline Grade $1 \mathrm{~A}$ & & 41 & 3 & 38 & 30 & 8 & Grade 1 & $7.32 \%$ & $92.68 \%$ & $78.95 \%$ & $21.05^{\circ}=$ \\
\hline Grade 1 B & & 0 & 0 & 0 & 0 & 0 & Grade 2 & $27.91 \%$ & $72.09 \%$ & $96.77 \%$ & 3230 \\
\hline Grade $1 \mathrm{C}$ & & 0 & 0 & 0 & 0 & 0 & Grade 3 & $20.00 \%$ & $80.00 \%$ & $95.00 \%$ & $E: 2 \div 0$ \\
\hline Grade 2 A & & 43 & 12 & 31 & 30 & 1 & Grade 4 & $6.67 \%$ & $93.33 \%$ & $100.00 \%$ & $0.00^{\circ} \%$ \\
\hline Grade 2 B & & 0 & 0 & 0 & 0 & 0 & Grade 5 & $6.67 \%$ & $93.33 \%$ & $92.86 \%$ & $7.14^{\circ} 0$ \\
\hline Grade $3 \mathrm{~A}$ & & -25 & 5 & 20 & 19 & 1 & Grade 6 & $11.11 \%$ & $88.89 \%$ & $87.50 \%$ & $12.50 \%$ \\
\hline Grade 3 B & & 0 & 0 & 0 & 0 & 0 & & & & & \\
\hline Grade 4 & & 15 & 1 & 14 & 14 & 0 & Remarks: & & & & \\
\hline Grade 5 & & 15 & 1 & 14 & 13 & 1 & & & & & \\
\hline Grade 6 & & 9 & 1 & 8 & 7 & 1 & & & & & \\
\hline School Total & & 148 & 23 & 125 & 113 & 12 & School \%age & $15.54 \%$ & $84.46 \%$ & $90.40 \%$ & $9.60^{\circ}$ \\
\hline
\end{tabular}

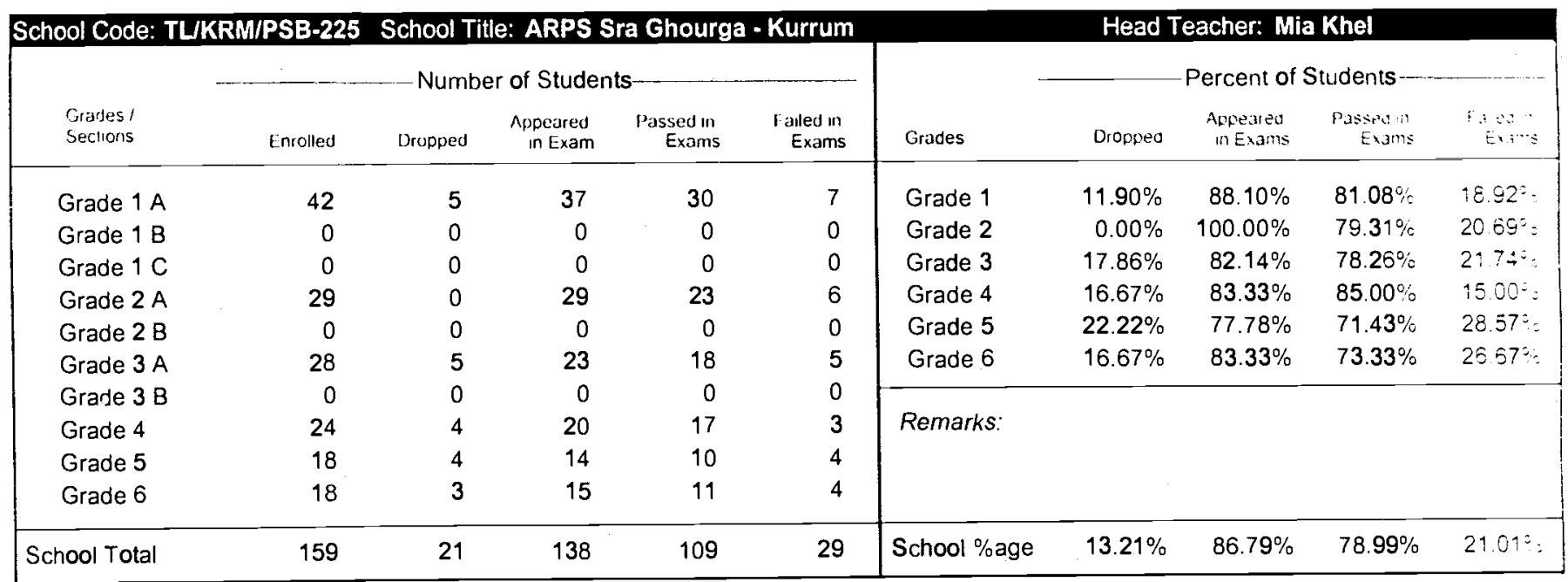


Sub-Centre: Thall

\begin{tabular}{|c|c|c|c|c|c|c|c|c|c|c|}
\hline \multirow[b]{2}{*}{$\begin{array}{l}\text { Grades I } \\
\text { Sections }\end{array}$} & \multicolumn{5}{|c|}{ - Number of Students- } & \multirow[b]{2}{*}{ Grades } & \multicolumn{4}{|c|}{-Percent of Students- } \\
\hline & Enrolled & Oropped & $\begin{array}{l}\text { Appeared } \\
\text { in Exam }\end{array}$ & $\begin{array}{l}\text { Passed in } \\
\text { Exams }\end{array}$ & $\begin{array}{l}\text { Failed in } \\
\text { Exams }\end{array}$ & & Oropped & $\begin{array}{l}\text { Appeared } \\
\text { in Exams }\end{array}$ & $\begin{array}{l}\text { Passed in } \\
\text { Exams }\end{array}$ & $\begin{array}{l}\text { Falled in } \\
\text { Exams }\end{array}$ \\
\hline Grade $1 \mathrm{~A}$ & 69 & 1 & 68 & 60 & 8 & Grade 1 & $0.83 \%$ & $99.17 \%$ & $89.08 \%$ & $10.92 \%$ \\
\hline Grade 1 B & 51 & 0 & 51 & 46 & 5 & Grade 2 & $8.89 \%$ & $91.11 \%$ & $92.68 \%$ & $7.32 \%$ \\
\hline Grade $1 \mathrm{C}$ & 0 & 0 & 0 & 0 & 0 & Grade 3 & $3.77 \%$ & $96.23 \%$ & $90.20 \%$ & $9.80 \%$ \\
\hline Grade $2 \mathrm{~A}$ & 45 & 4 & 41 & 38 & 3 & Grade 4 & $8.70 \%$ & $91.30 \%$ & $100.00 \%$ & $0.00 \%$ \\
\hline Grade 2 B & 0 & 0 & 0 & 0 & 0 & Grade 5 & \#Num! & \#Num! & \#Num! & \#Num! \\
\hline Grade $3 \mathrm{~A}$ & 53 & 2 & 51 & 46 & 5 & Grade 6 & \#Num! & \#Num! & \#Num! & \#Num! \\
\hline Grade 3 B & 0 & 0 & 0 & 0 & 0 & & & & & \\
\hline Grade 4 & 23 & 2 & 21 & 21 & 0 & Remarks: & & & & \\
\hline Grade 5 & 0 & 0 & 0 & 0 & 0 & & & & & \\
\hline Grade 6 & 0 & 0 & 0 & 0 & 0 & & & & & \\
\hline School Total & 241 & 9 & 232 & 211 & 21 & School \%age & $3.73 \%$ & $96.27 \%$ & $90.95 \%$ & $9.05 \%$ \\
\hline
\end{tabular}

\begin{tabular}{|c|c|c|c|c|c|c|c|c|c|c|c|}
\hline \multirow[b]{2}{*}{$\begin{array}{l}\text { Grades I } \\
\text { Sections }\end{array}$} & \multicolumn{6}{|c|}{ - Number of Students } & \multirow[b]{2}{*}{ Grades } & \multicolumn{4}{|c|}{-Percent of Students- } \\
\hline & ' & Enrolled & Oropped & $\begin{array}{l}\text { Appeared } \\
\text { in Exam }\end{array}$ & $\begin{array}{r}\text { Passed in } \\
\text { Exams }\end{array}$ & $\begin{array}{l}\text { Failed in } \\
\text { Exams }\end{array}$ & & Oropped & $\begin{array}{l}\text { Appeared } \\
\text { in Exams }\end{array}$ & $\begin{array}{l}\text { Passed in } \\
\text { Exams }\end{array}$ & $\begin{array}{l}\text { Falled in } \\
\text { Exams }\end{array}$ \\
\hline Grade $1 \mathrm{~A}$ & & 62 & 15 & 47 & 43 & 4 & Grade 1 & $15.08 \%$ & $84.92 \%$ & $91.59 \%$ & $8.41 \%$ \\
\hline Grade 1 B & & 64 & 4 & 60 & 55 & 5 & Grade 2 & $22.22 \%$ & $77.78 \%$ & $90.48 \%$ & $9.52 \%$ \\
\hline Grade $1 \mathrm{C}$ & & 0 & 0 & 0 & 0 & 0 & Grade 3 & $23.40 \%$ & $76.60 \%$ & $100.00 \%$ & $0.00 \%$ \\
\hline Grade 2 A & & 41 & 10 & 31 & 25 & 6 & Grade 4 & $20.75 \%$ & $79.25 \%$ & $85.71 \%$ & $14.29 \%$ \\
\hline Grade 2 B & & 40 & 8 & 32 & 32 & 0 & Grade 5 & $17.02 \%$ & $82.98 \%$ & $97.44 \%$ & $2.56 \%$ \\
\hline Grade $3 \mathrm{~A}$ & & 47 & 11 & 36 & 36 & 0 & Grade 6 & $35.14 \%$ & $64.86 \%$ & $100.00 \%$ & $0.00 \%$ \\
\hline Grade 3 B & & 0 & 0 & 0 & 0 & 0 & & & & & \\
\hline Grade 4 & & 53 & 11 & 42 & 36 & 6 & Remarks: & . & & & \\
\hline Grade 5 & & 47 & 8 & 39 & 38 & 1 & & & & & \\
\hline Grade 6 & & 37 & 13 & 24 & 24 & 0 & & & & & \\
\hline School Total & & 391 & 80 & 311 & 289 & 22 & School \%age & $20.46 \%$ & $79.54 \%$ & $92.93 \%$ & $7.07 \%$ \\
\hline
\end{tabular}

\begin{tabular}{|c|c|c|c|c|c|c|c|c|c|c|}
\hline Sub-Centre Total & 10,344 & 670 & 9,674 & 7,463 & 2,211 & Sub-Centre \%age & $6.48 \%$ & $93.52 \%$ & $77.14 \%$ & $22.86 \%$ \\
\hline
\end{tabular}


Sub-Centre: Timargara

\begin{tabular}{|c|c|c|c|c|c|c|c|c|c|c|}
\hline \multicolumn{7}{|c|}{ School Code: TM/BJR/PSB-228 School Title: ARPS Nawababad - Timergara } & \multicolumn{4}{|c|}{ Head Teacher: Khan Mohammad } \\
\hline \multirow[b]{2}{*}{$\begin{array}{l}\text { Grades I } \\
\text { Sections }\end{array}$} & & -Numb & of Stude & & & \multirow[b]{2}{*}{ Grades } & \multicolumn{4}{|c|}{ Percent of Students- } \\
\hline & Enrolied & Dropped & $\begin{array}{l}\text { Appeared } \\
\text { in Exam }\end{array}$ & $\begin{array}{c}\text { Passed in } \\
\text { Exams }\end{array}$ & $\begin{array}{l}\text { Failed in } \\
\text { Exams }\end{array}$ & & Dropped & $\begin{array}{l}\text { Appeared } \\
\text { in Exams }\end{array}$ & $\begin{array}{c}\text { Passed in } \\
\text { Exams }\end{array}$ & $\begin{array}{l}\text { Failed in } \\
\text { Exams }\end{array}$ \\
\hline Grade $1 \mathrm{~A}$ & 63 & 11 & 52 & 40 & 12 & Grade 1 & $17.46 \%$ & $82.54 \%$ & $76.92 \%$ & $23.08 \%$ \\
\hline Grade $1 \mathrm{~B}$ & 0 & 0 & 0 & 0 & 0 & Grade 2 & $0.00 \%$ & $100.00 \%$ & $82.86 \%$ & $17.14 \%$ \\
\hline Grade $1 \mathrm{C}$ & 0 & 0 & 0 & 0 & 0 & Grade 3 & $0.00 \%$ & $100.00 \%$ & $67.74 \%$ & $32.26 \%$ \\
\hline Grade $2 \mathrm{~A}$ & 35 & 0 & 35 & 29 & 6 & Grade 4 & $0.00 \%$ & $100.00 \%$ & $83.87 \%$ & $16.13 \%$ \\
\hline Grade 2 B & 0 & 0 & 0 & 0 & 0 & Grade 5 & $0.00 \%$ & $100.00 \%$ & $83.33 \%$ & $16.67 \%$ \\
\hline Grade $3 \mathrm{~A}$ & 31 & 0 & 31 & 21 & 10 & Grade 6 & $14.29 \%$ & $85.71 \%$ & $83.33 \%$ & $16.67 \%$ \\
\hline Grade 3 B & 0 & 0 & 0 & 0 & 0 & & & & & \\
\hline Grade 4 & 31 & 0 & 31 & 26 & 5 & Remarks: & & & & \\
\hline Grade 5 & 24 & 0 & 24 & 20 & 4 & & & & & \\
\hline Grade 6 & 21 & 3 & 18 & 15 & 3 & & & & & \\
\hline School Total & 205 & 14 & 191 & 151 & 40. & School \%age & $6.83 \%$ & $93.17 \%$ & $79.06 \%$ & $20.94 \%$ \\
\hline
\end{tabular}

\begin{tabular}{|c|c|c|c|c|c|c|c|c|c|c|c|}
\hline \multirow[b]{2}{*}{$\begin{array}{l}\text { Grades I } \\
\text { Sections }\end{array}$} & \multicolumn{6}{|c|}{ - Number of Students- } & \multirow[b]{2}{*}{ Grades } & \multicolumn{4}{|c|}{ Percent of Students- } \\
\hline & , & Enrolled & Dropped & $\begin{array}{l}\text { Appeared } \\
\text { in Exam }\end{array}$ & $\begin{array}{l}\text { Passed in } \\
\text { Exams }\end{array}$ & $\begin{array}{c}\text { Failed in } \\
\text { Exams }\end{array}$ & & Dropped & $\begin{array}{l}\text { Appeared } \\
\text { in Exams }\end{array}$ & $\begin{array}{l}\text { Passed in } \\
\text { Exams }\end{array}$ & $\begin{array}{c}\text { Failed in } \\
\text { Exams }\end{array}$ \\
\hline Grade $1 \mathrm{~A}$ & & 57 & 12 & 45 & 36 & 9 & Grade 1 & $21.05 \%$ & $78.95 \%$ & $80.00 \%$ & $20.00 \%$ \\
\hline Grade $1 \mathrm{~B}$ & & 0 & 0 & 0 & 0 & 0 & Grade 2 & $4.55 \%$ & $95.45 \%$ & $83.33 \%$ & $16.67 \%$ \\
\hline Grade $1 \mathrm{C}$ & & 0 & 0 & 0 & 0 & 0 & Grade 3 & $0.00 \%$ & $100.00 \%$ & $84.31 \%$ & $15.69 \%$ \\
\hline Grade 2 A & & 44 & 2 & 42 & 35 & 7 & Grade 4 & $9.62 \%$ & $90.38 \%$ & $78.72 \%$ & $21.28 \%$ \\
\hline Grade 2 B & & 0 & 0 & 0 & 0 & 0 & Grade 5 & $35.90 \%$ & $64.10 \%$ & $100.00 \%$ & $0.00 \%$ \\
\hline Grade $3 \mathrm{~A}$ & & 5 th & 0 & 51 & 43 & 8 & Grade 6 & $0.00 \%$ & $100.00 \%$ & $84.62 \%$ & $15.38 \%$ \\
\hline Grade 3 B & & 0 & 0 & 0 & 0 & 0 & & & & & \\
\hline Grade 4 & & 52 & 5 & 47 & 37 & 10 & Remarks: & & & & \\
\hline Grade 5 & & 39 & 14 & 25 & 25 & 0 & & & & & \\
\hline Grade 6 & & 26 & 0 & 26 & 22 & 4 & & & & & \\
\hline School Total & & 269 & 33 & 236 & 198 & 38 & School \%age & $12.27 \%$ & $87.73 \%$ & $83.90 \%$ & $16.10 \%$ \\
\hline
\end{tabular}

\begin{tabular}{|c|c|c|c|c|c|c|c|c|c|c|}
\hline \multirow[b]{2}{*}{$\begin{array}{l}\text { Grades I } \\
\text { Sections }\end{array}$} & \multicolumn{5}{|c|}{ - Number of Students- } & \multirow[b]{2}{*}{ Grades } & \multicolumn{4}{|c|}{ Percent of Students } \\
\hline & Enrolled & Dropped & $\begin{array}{l}\text { Appeared } \\
\text { in Exam }\end{array}$ & $\begin{array}{c}\text { Passed in } \\
\text { Exams }\end{array}$ & $\begin{array}{c}\text { Failed in } \\
\text { Exams }\end{array}$ & & Dropped & $\begin{array}{l}\text { Appeared } \\
\text { in Exams }\end{array}$ & $\begin{array}{l}\text { Passed in } \\
\text { Exams }\end{array}$ & $\begin{array}{c}\text { Failed in } \\
\text { Exams }\end{array}$ \\
\hline Grade $1 \mathrm{~A}$ & 58 & 17 & 41 & 32 & 9 & Grade 1 & $29.31 \%$ & $70.69 \%$ & $78.05 \%$ & $21.95 \%$ \\
\hline Grade 1 B & 0 & 0 & 0 & 0 & 0 & Grade 2 & $23.08 \%$ & $76.92 \%$ & $85.00 \%$ & $15.00 \%$ \\
\hline Grade $1 \mathrm{C}$ & 0 & 0 & 0 & 0 & 0 & Grade 3 & $11.54 \%$ & $88.46 \%$ & $91.30 \%$ & $8.70 \%$ \\
\hline Grade $2 \mathrm{~A}$ & 26 & 6 & 20 & 17 & 3 & Grade 4 & $16.67 \%$ & $83.33 \%$ & $86.67 \%$ & $13.33 \%$ \\
\hline Grade 2 B & 0 & 0 & 0 & 0 & 0 & Grade 5 & \#Num! & \#Num! & \#Num! & \#Num! \\
\hline Grade 3 A & 26 & 3 & 23 & 21 & 2 & Grade 6 & \#Num! & \#Num! & \#Num! & \#Num! \\
\hline Grade 3 B & 0 & 0 & 0 & 0 & 0 & & & & & \\
\hline Grade 4 & 18 & 3 & 15 & 13 & 2 & Remarks: & & & & \\
\hline Grade 5 & 0 & 0 & 0 & 0 & 0 & & & & & \\
\hline Grade 6 & 0 & 0 & 0 & 0 & 0 & & & & & \\
\hline School Total & 128 & 29 & 99 & 83 & 16 & School \%age & $22.66 \%$ & $77.34 \%$ & $83.84 \%$ & $16.16 \%$ \\
\hline
\end{tabular}

Note:

\#Um! = Enrollment in respective grade(s) is nill therefore calculation is not applicable
Page 79 of 91 Produced by DP/MIS Section on 31-Jul-97 
Sub-Centre: Timargara

\begin{tabular}{|c|c|c|c|c|c|c|c|c|c|c|}
\hline \multirow[b]{2}{*}{$\begin{array}{l}\text { Grades / } \\
\text { Sections }\end{array}$} & \multicolumn{5}{|c|}{-Number of Students- } & \multirow[b]{2}{*}{ Grades } & \multicolumn{4}{|c|}{ Percent of Students } \\
\hline & Enrolled & Dropped & $\begin{array}{l}\text { Appeared } \\
\text { in Exam }\end{array}$ & $\begin{array}{l}\text { Passed in } \\
\text { Exams }\end{array}$ & $\begin{array}{l}\text { Failed in } \\
\text { Exams }\end{array}$ & & Dropped & $\begin{array}{l}\text { Appeared } \\
\text { in Exams }\end{array}$ & $\begin{array}{l}\text { Passed in } \\
\text { Exams }\end{array}$ & $\begin{array}{r}\text { Failed in } \\
\text { Exams }\end{array}$ \\
\hline Grade $1 \mathrm{~A}$ & 55 & 0 & 55 & 40 & 15 & Grade 1 & $0.00 \%$ & $100.00 \%$ & $60.00 \%$ & $40.00 \%$ \\
\hline Grade $1 \mathrm{~B}$ & 52 & 0 & 52 & 21 & 31 & Grade 2 & $0.00 \%$ & $100.00 \%$ & $80.00 \%$ & $20.00 \%$ \\
\hline Grade $1 \mathrm{C}$ & 48 & 0 & 48 & 32 & 16 & Grade 3 & $19.05 \%$ & $80.95 \%$ & $91.18 \%$ & $8.82 \%$ \\
\hline Grade $2 \mathrm{~A}$ & 60 & 0 & 60 & 48 & 12 & Grade 4 & $0.00 \%$ & $100.00 \%$ & $73.81 \%$ & $26.19 \%$ \\
\hline Grade 2 B & 0 & 0 & 0 & 0 & 0 & Grade 5 & $0.00 \%$ & $100.00 \%$ & $65.91 \%$ & $34.09 \%$ \\
\hline Grade 3 A & 42 & 8 & 34 & 31 & 3 & Grade 6 & $30.00 \%$ & $70.00 \%$ & $100.00 \%$ & $0.00 \%$ \\
\hline Grade 3 B & 0 & 0 & 0 & 0 & 0 & & & & & \\
\hline Grade 4 & 42 & 0 & 42 & 31 & 11 & Remarks: & & & & \\
\hline Grade 5 & 44 & 0 & 44 & 29 & 15 & & & & & \\
\hline Grade 6 & 20 & 6 & 14 & 14 & 0 & & & & & \\
\hline School Total & 363 & 14 & 349 & 246 & 103 & School \%age & $3.86 \%$ & $96.14 \%$ & $70.49 \%$ & $29.51 \%$ \\
\hline
\end{tabular}

\begin{tabular}{|c|c|c|c|c|c|c|c|c|c|c|c|}
\hline \multirow[b]{2}{*}{$\begin{array}{l}\text { Grades / } \\
\text { Sections }\end{array}$} & \multicolumn{6}{|c|}{-Number of Students- } & \multirow[b]{2}{*}{ Grades } & \multicolumn{4}{|c|}{ Percent of Students } \\
\hline & ' & Enrolled & Dropped & $\begin{array}{c}\text { Appeared } \\
\text { in Exam }\end{array}$ & $\begin{array}{l}\text { Passed in } \\
\text { Exams }\end{array}$ & $\begin{array}{c}\text { Failed in } \\
\text { Exams }\end{array}$ & & Dropped & $\begin{array}{l}\text { Appeared } \\
\text { in Exams }\end{array}$ & $\begin{array}{c}\text { Passed in } \\
\text { Exams }\end{array}$ & $\begin{array}{l}\text { Failed in } \\
\text { Exams }\end{array}$ \\
\hline Grade $1 \mathrm{~A}$ & & 50 & 7 & 43 & 31 & 12 & Grade 1 & $10.09 \%$ & $89.91 \%$ & $63.27 \%$ & $36.73 \%$ \\
\hline Grade $1 \mathrm{~B}$ & & 59 & 4 & 55 & 31 & 24 & Grade 2 & $0.00 \%$ & $100.00 \%$ & $53.16 \%$ & $46.84 \%$ \\
\hline Grade 1 C & & 0 & 0 & 0 & 0 & 0 & Grade 3 & $0.00 \%$ & $100.00 \%$ & $48.57 \%$ & $51.43 \%$ \\
\hline Grade $2 \mathrm{~A}$ & & 39 & 0 & 39 & 22 & 17 & Grade 4 & $9.52 \%$ & $90.48 \%$ & $84.21 \%$ & $15.79 \%$ \\
\hline Grade 2 B & & 40 & 0 & 40 & 20 & 20 & Grade 5 & $25.00 \%$ & $75.00 \%$ & $66.67 \%$ & $33.33 \%$ \\
\hline Grade 3 A & & 85 & 0 & 35 & 17 & 18 & Grade 6 & $0.00 \%$ & $100.00 \%$ & $58.82 \%$ & $41.18 \%$ \\
\hline Grade 3 B & & 0 & 0 & 0 & 0 & 0 & & & & & \\
\hline Grade 4 & & 21 & 2 & 19 & 16 & 3 & Remarks: & & & & \\
\hline Grade 5 & & 28 & 7 & 21 & 14 & 7 & & & & & \\
\hline Grade 6 & & 17 & 0 & 17 & 10 & 7 & & & & & \\
\hline School Total & & 289 & 20 & 269 & 161 & 108 & School \%age & $6.92 \%$ & $93.08 \%$ & $59.85 \%$ & $40.15 \%$ \\
\hline
\end{tabular}

\begin{tabular}{|c|c|c|c|c|c|c|c|c|c|c|}
\hline \multirow[b]{2}{*}{$\begin{array}{l}\text { Grades I } \\
\text { Sections }\end{array}$} & \multicolumn{5}{|c|}{-Number of Students- } & \multirow[b]{2}{*}{ Grades } & \multicolumn{4}{|c|}{ Percent of Students- } \\
\hline & Enrolled & Dropped & $\begin{array}{l}\text { Appeared } \\
\text { in Exam }\end{array}$ & $\begin{array}{c}\text { Passed in } \\
\text { Exams }\end{array}$ & $\begin{array}{l}\text { Failed in } \\
\text { Exams }\end{array}$ & & Dropped & $\begin{array}{l}\text { Appeared } \\
\text { in Exams }\end{array}$ & $\begin{array}{l}\text { Passed in } \\
\text { Exams }\end{array}$ & $\begin{array}{l}\text { Failed in } \\
\text { Exams }\end{array}$ \\
\hline Grade $1 \mathrm{~A}$ & 55 & 0 & 55 & 36 & 19 & Grade 1 & $0.00 \%$ & $100.00 \%$ & $65.45 \%$ & $34.55 \%$ \\
\hline Grade 1 B & 0 & 0 & 0 & 0 & 0 & Grade 2 & $0.00 \%$ & $100.00 \%$ & $59.52 \%$ & $40.48 \%$ \\
\hline Grade $1 \mathrm{C}$ & 0 & 0 & 0 & 0 & 0 & Grade 3 & $0.00 \%$ & $100.00 \%$ & $57.14 \%$ & $42.86 \%$ \\
\hline Grade 2 A & 42 & 0 & 42 & 25 & 17 & Grade 4 & $0.00 \%$ & $100.00 \%$ & $50.00 \%$ & $50.00 \%$ \\
\hline Grade 2 B & 0 & 0 & 0 & 0 & 0 & Grade 5 & $0.00 \%$ & $100.00 \%$ & $50.00 \%$ & $50.00 \%$ \\
\hline Grade $3 \mathrm{~A}$ & 42 & 0 & 42 & 24 & 18 & Grade 6 & $0.00 \%$ & $100.00 \%$ & $42.86 \%$ & $57.14 \%$ \\
\hline Grade 3 B & 0 & 0 & 0 & 0 & 0 & & & & & \\
\hline Grade 4 & 36 & 0 & 36 & 18 & 18 & Remarks: & & & & \\
\hline Grade 5 & 32 & 0 & 32 & 16 & 16 & & & & & \\
\hline Grade 6 & 21 & 0 & 21 & 9 & 12 & & & & & \\
\hline School Total & 228 & 0 & 228 & 128 & 100 & School \%age & $0.00 \%$ & $100.00 \%$ & $56.14 \%$ & $43.86 \%$ \\
\hline
\end{tabular}


Sub-Centre: Timargara

\begin{tabular}{|c|c|c|c|c|c|c|c|c|c|c|}
\hline \multirow[b]{2}{*}{$\begin{array}{l}\text { Grades / } \\
\text { Sections }\end{array}$} & \multicolumn{5}{|c|}{ - Number of Students- } & \multirow[b]{2}{*}{ Grades } & \multicolumn{4}{|c|}{-Percent of Students } \\
\hline & Enrolled & Dropped & $\begin{array}{c}\text { Appeared } \\
\text { in Exam }\end{array}$ & $\begin{array}{r}\text { Passed in } \\
\text { Exams }\end{array}$ & $\begin{array}{l}\text { Failed in } \\
\text { Exams }\end{array}$ & & Dropped & $\begin{array}{l}\text { Appeared } \\
\text { in Exams }\end{array}$ & $\begin{array}{l}\text { Passed in } \\
\text { Exams }\end{array}$ & $\begin{array}{c}\text { Failed in } \\
\text { Exams }\end{array}$ \\
\hline Grade $1 \mathrm{~A}$ & 42 & 4 & 38 & 18 & 20 & Grade 1 & $3.70 \%$ & $96.30 \%$ & $36.54 \%$ & $63.46 \%$ \\
\hline Grade 1 B & 66 & 0 & 66 & 20 & 46 & Grade 2 & $10.00 \%$ & $90.00 \%$ & $44.44 \%$ & $55.56 \%$ \\
\hline Grade $1 \mathrm{C}$ & 0 & 0 & 0 & 0 & 0 & Grade 3 & $18.75 \%$ & $81.25 \%$ & $84.62 \%$ & $15.38 \%$ \\
\hline Grade $2 \mathrm{~A}$ & 20 & 2 & 18 & 8 & 10 & Grade 4 & \#Num! & \#Num! & \#Num! & \#Num! \\
\hline Grade 2 B & 0 & 0 & 0 & 0 & 0 & Grade 5 & \#Num! & \#Num! & \#Num! & \#Num! \\
\hline Grade $3 \mathrm{~A}$ & 16 & 3 & 13 & 11 & 2 & Grade 6 & \#Num! & \#Num! & \#Num! & \#Num! \\
\hline Grade 3 B & 0 & 0 & 0 & 0 & 0 & & & & & \\
\hline Grade 4 & 0 & 0 & 0 & 0 & 0 & Remarks: & & & & \\
\hline Grade 5 & 0 & 0 & 0 & 0 & 0 & & & & & \\
\hline Grade 6 & 0 & 0 & 0 & 0 & 0 & & & & & \\
\hline School Total & 144 & 9 & 135 & 57 & 78 & School \%age & $6.25 \%$ & $93.75 \%$ & $42.22 \%$ & $57.78 \%$ \\
\hline
\end{tabular}

\begin{tabular}{|c|c|c|c|c|c|c|c|c|c|c|}
\hline \multirow[b]{2}{*}{$\begin{array}{l}\text { Grades I } \\
\text { Sections }\end{array}$} & \multicolumn{5}{|c|}{-Number of Students- } & \multirow[b]{2}{*}{ Grades } & \multicolumn{4}{|c|}{-Percent of Students } \\
\hline & Enrolled & Dropped & $\begin{array}{c}\text { Appeared } \\
\text { in Exam }\end{array}$ & $\begin{array}{c}\text { Passed in } \\
\text { Exams }\end{array}$ & $\begin{array}{l}\text { Failed in } \\
\text { Exams }\end{array}$ & & Dropped & $\begin{array}{l}\text { Appeared } \\
\text { in Exams }\end{array}$ & $\begin{array}{c}\text { Passed in } \\
\text { Exams }\end{array}$ & $\begin{array}{l}\text { Failed in } \\
\text { Exams }\end{array}$ \\
\hline Grade $1 \mathrm{~A}$ & 75 & 15 & 60 & 32 & 28 & Grade 1 & $20.00 \%$ & $80.00 \%$ & $53.33 \%$ & $46.67 \%$ \\
\hline Grade 1 B & 0 & 0 & 0 & 0 & 0 & Grade 2 & $10.42 \%$ & $89.58 \%$ & $74.42 \%$ & $25.58 \%$ \\
\hline Grade $1 \mathrm{C}$ & 0 & 0 & 0 & 0 & 0 & Grade 3 & $0.00 \%$ & $100.00 \%$ & $71.43 \%$ & $28.57 \%$ \\
\hline Grade 2 A & 48 & 5 & 43 & 32 & 11 & Grade 4 & $18.75 \%$ & $81.25 \%$ & $73.08 \%$ & $26.92 \%$ \\
\hline Grade 2 B & 0 & 0 & 0 & 0 & 0 & Grade 5 & $36.36 \%$ & $63.64 \%$ & $57.14 \%$ & $42.86 \%$ \\
\hline Grade $3 \mathrm{~A}$ & $35^{\circ}$ & 0 & 35 & 25 & 10 & Grade 6 & $19.05 \%$ & $80.95 \%$ & $52.94 \%$ & $47.06 \%$ \\
\hline Grade 3 B & 0 & 0 & 0 & 0 & 0 & & & & & \\
\hline Grade 4 & 32 & 6 & 26 & 19 & 7 & Remarks: & & & & \\
\hline Grade 5 & 22 & 8 & 14 & 8 & 6 & & & & & \\
\hline Grade 6 & 21 & 4 & 17 & 9 & 8 & & & & & \\
\hline School Total & 233 & 38 & 195 & 125 & 70 & School \%age & $16.31 \%$ & $83.69 \%$ & $64.10 \%$ & $35.90 \%$ \\
\hline
\end{tabular}

\begin{tabular}{|c|c|c|c|c|c|c|c|c|c|c|}
\hline \multirow[b]{2}{*}{$\begin{array}{l}\text { Grades / } \\
\text { Sections }\end{array}$} & \multicolumn{5}{|c|}{ - Number of Students- } & \multirow[b]{2}{*}{ Grades } & \multicolumn{4}{|c|}{-Percent of Students } \\
\hline & Enrolled & Dropped & $\begin{array}{l}\text { Appeared } \\
\text { in Exam }\end{array}$ & $\begin{array}{c}\text { Passed in } \\
\text { Exams }\end{array}$ & $\begin{array}{l}\text { Failed in } \\
\text { Exams }\end{array}$ & & Dropped & $\begin{array}{l}\text { Appeared } \\
\text { in Exams }\end{array}$ & $\begin{array}{l}\text { Passed in } \\
\text { Exams }\end{array}$ & $\begin{array}{l}\text { Falled in } \\
\text { Exams }\end{array}$ \\
\hline Grade $1 \mathrm{~A}$ & 80 & 20 & 60 & 37 & 23 & Grade 1 & $25.00 \%$ & $75.00 \%$ & $61.67 \%$ & $38.33 \%$ \\
\hline Grade 1 B & 0 & 0 & 0 & 0 & 0 & Grade 2 & $10.42 \%$ & $89.58 \%$ & $72.09 \%$ & $27.91 \%$ \\
\hline Grade $1 \mathrm{C}$ & 0 & 0 & 0 & 0 & 0 & Grade 3 & $9.38 \%$ & $90.63 \%$ & $65.52 \%$ & $34.48 \%$ \\
\hline Grade 2 A & 48 & 5 & 43 & 31 & 12 & Grade 4 & $11.11 \%$ & $88.89 \%$ & $81.25 \%$ & $18.75 \%$ \\
\hline Grade 2 B & 0 & 0 & 0 & 0 & 0 & Grade 5 & $31.25 \%$ & $68.75 \%$ & $54.55 \%$ & $45.45 \%$ \\
\hline Grade 3 A & 32 & 3 & 29 & 19 & 10 & Grade 6 & $27.27 \%$ & $72.73 \%$ & $62.50 \%$ & $37.50 \%$ \\
\hline Grade 3 B & 0 & 0 & 0 & 0 & 0 & & & & & \\
\hline Grade 4 & 18 & 2 & 16 & 13 & 3 & Remarks: & & & & \\
\hline Grade 5 & 16 & 5 & 11 & 6 & 5 & & & & & \\
\hline Grade 6 & 11 & 3 & 8 & 5 & 3 & & & & & \\
\hline School Total & 205 & 38 & 167 & 111 & 56 & School \%age & $18.54 \%$ & $81.46 \%$ & $66.47 \%$ & $33.53 \%$ \\
\hline
\end{tabular}

Note:

\#Num! = Enrollment in respective grade(s) is nill therefore calculation is not applicable
Page 81 of 91

Produced by DPIMIS Section on 31-Jul-97 
Sub-Centre: Timargara

\begin{tabular}{|c|c|c|c|c|c|c|c|c|c|c|}
\hline \multirow[b]{2}{*}{$\begin{array}{l}\text { Grades / } \\
\text { Sections }\end{array}$} & \multicolumn{5}{|c|}{ - Number of Students- } & \multirow[b]{2}{*}{ Grades } & \multicolumn{4}{|c|}{-Percent of Students- } \\
\hline & Enrolled & Dropped & $\begin{array}{l}\text { Appeared } \\
\text { in Exam }\end{array}$ & $\begin{array}{l}\text { Passed in } \\
\text { Exams }\end{array}$ & $\begin{array}{c}\text { Failed in } \\
\text { Exams }\end{array}$ & & Dropped & $\begin{array}{l}\text { Appeared } \\
\text { in Exams }\end{array}$ & $\begin{array}{l}\text { Passed in } \\
\text { Exams }\end{array}$ & $\begin{array}{l}\text { Failed in } \\
\text { Exams }\end{array}$ \\
\hline Grade $1 \mathrm{~A}$ & 75 & 5 & 70 & 41 & 29 & Grade 1 & $3.94 \%$ & $96.06 \%$ & $63.93 \%$ & $36.07 \%$ \\
\hline Grade $1 \mathrm{~B}$ & 52 & 0 & 52 & 37 & 15 & Grade 2 & $10.17 \%$ & $89.83 \%$ & $60.38 \%$ & $39.62 \%$ \\
\hline Grade $1 \mathrm{C}$ & 0 & 0 & 0 & 0 & 0 & Grade 3 & $12.50 \%$ & $87.50 \%$ & $65.71 \%$ & $34.29 \%$ \\
\hline Grade $2 \mathrm{~A}$ & 59 & 6 & 53 & 32 & 21 & Grade 4 & $0.00 \%$ & $100.00 \%$ & $62.50 \%$ & $37.50 \%$ \\
\hline Grade 2 B & 0 & 0 & 0 & 0 & 0 & Grade 5 & $21.05 \%$ & $78.95 \%$ & $60.00 \%$ & $40.00 \%$ \\
\hline Grade $3 \mathrm{~A}$ & 40 & 5 & 35 & 23 & 12 & Grade 6 & $15.79 \%$ & $84.21 \%$ & $68.75 \%$ & $31.25 \%$ \\
\hline Grade 3 B & 0 & 0 & 0 & 0 & 0 & & & & & \\
\hline Grade 4 & 24 & 0 & 24 & 15 & 9 & Remarks: & & & & \\
\hline Grade 5 & 19 & 4 & 15 & 9 & 6 & & & & & \\
\hline Grade 6 & 19 & 3 & 16 & 11 & 5 & & & & & \\
\hline School Total & 288 & 23 & 265 & 168 & 97 & School \%age & $7.99 \%$ & $92.01 \%$ & $63.40 \%$ & $36.60 \%$ \\
\hline
\end{tabular}

\begin{tabular}{|c|c|c|c|c|c|c|c|c|c|c|c|}
\hline \multirow[b]{2}{*}{$\begin{array}{l}\text { Grades I } \\
\text { Sections }\end{array}$} & \multicolumn{6}{|c|}{-Number of Students- } & \multirow[b]{2}{*}{ Grades } & \multicolumn{4}{|c|}{-Percent of Students } \\
\hline & ' & Enrolled & Dropped & $\begin{array}{l}\text { Appeared } \\
\text { in Exam }\end{array}$ & $\begin{array}{l}\text { Passed in } \\
\text { Exams }\end{array}$ & $\begin{array}{c}\text { Failed in } \\
\text { Exams }\end{array}$ & & Dropped & $\begin{array}{l}\text { Appeared } \\
\text { in Exams }\end{array}$ & $\begin{array}{l}\text { Passed in } \\
\text { Exams }\end{array}$ & $\begin{array}{l}\text { Failed in } \\
\text { Exams }\end{array}$ \\
\hline Grade $1 \mathrm{~A}$ & & 52 & 0 & 52 & 47 & 5 & Grade 1 & $16.00 \%$ & $84.00 \%$ & $94.29 \%$ & $5.71 \%$ \\
\hline Grade $1 \mathrm{~B}$ & & 73 & 20 & 53 & 52 & 1 & Grade 2 & $0.00 \%$ & $100.00 \%$ & $83.33 \%$ & $16.67 \%$ \\
\hline Grade $1 \mathrm{C}$ & & 0 & 0 & 0 & 0 & 0 & Grade 3 & $0.00 \%$ & $100.00 \%$ & $85.71 \%$ & $14.29 \%$ \\
\hline Grade $2 \mathrm{~A}$ & & 42 & 0 & 42 & 35 & 7 & Grade 4 & $25.00 \%$ & $75.00 \%$ & $95.24 \%$ & $4.76 \%$ \\
\hline Grade 2 B & & 0 & 0 & 0 & 0 & 0 & Grade 5 & $0.00 \%$ & $100.00 \%$ & $100.00 \%$ & $0.00 \%$ \\
\hline Grade $3 \mathrm{~A}$ & & 35 & 0 & 35 & 30 & 5 & Grade 6 & $57.14 \%$ & $42.86 \%$ & $100.00 \%$ & $0.00 \%$ \\
\hline Grade 3 B & & 0 & 0 & 0 & 0 & 0 & & & & & \\
\hline Grade 4 & & 28 & 7 & 21 & 20 & 1 & Remarks: & & & & \\
\hline Grade 5 & & 14 & 0 & 14 & 14 & 0 & & & & & \\
\hline Grade 6 & & 21 & 12 & 9 & 9 & 0 & & & & & \\
\hline School Total & & 265 & 39 & 226 & 207 & 19 & School \%age & $14.72 \%$ & $85.28 \%$ & $91.59 \%$ & $8.41 \%$ \\
\hline
\end{tabular}

\begin{tabular}{|c|c|c|c|c|c|c|c|c|c|c|}
\hline \multirow[b]{2}{*}{$\begin{array}{l}\text { Grades I } \\
\text { Sections }\end{array}$} & \multicolumn{5}{|c|}{ - Number of Students- } & \multirow[b]{2}{*}{ Grades } & \multicolumn{4}{|c|}{-Percent of Students- } \\
\hline & Enrolled & Dropped & $\begin{array}{c}\text { Appeared } \\
\text { in Exam }\end{array}$ & $\begin{array}{l}\text { Passed in } \\
\text { Exams }\end{array}$ & $\begin{array}{c}\text { Failed in } \\
\text { Exams }\end{array}$ & & Dropped & $\begin{array}{l}\text { Appeared } \\
\text { in Exams }\end{array}$ & $\begin{array}{l}\text { Passed in } \\
\text { Exams }\end{array}$ & $\begin{array}{c}\text { Failed in } \\
\text { Exams }\end{array}$ \\
\hline Grade $1 \mathrm{~A}$ & 36 & 0 & 36 & 20 & 16 & Grade 1 & $10.26 \%$ & $89.74 \%$ & $61.43 \%$ & $38.57 \%$ \\
\hline Grade $1 \mathrm{~B}$ & 42 & 8 & 34 & 23 & 11 & Grade 2 & $0.00 \%$ & $100.00 \%$ & $75.00 \%$ & $25.00 \%$ \\
\hline Grade $1 \mathrm{C}$ & 0 & 0 & 0 & 0 & 0 & Grade 3 & $0.00 \%$ & $100.00 \%$ & $93.75 \%$ & $6.25 \%$ \\
\hline Grade 2 A & 24 & 0 & 24 & 18 & 6 & Grade 4 & $0.00 \%$ & $100.00 \%$ & $86.67 \%$ & $13.33 \%$ \\
\hline Grade 2 B & 0 & 0 & 0 & 0 & 0 & Grade 5 & $0.00 \%$ & $100.00 \%$ & $80.00 \%$ & $20.00 \%$ \\
\hline Grade $3 \mathrm{~A}$ & 16 & 0 & 16 & 15 & 1 & Grade 6 & \#Num! & \#Num! & \#Num! & \#Num! \\
\hline Grade 3 B & 0 & 0 & 0 & 0 & 0 & & & & & \\
\hline Grade 4 & 15 & 0 & 15 & 13 & 2 & Remarks: & & & & \\
\hline Grade 5 & 10 & 0 & 10 & 8 & 2 & & & & & \\
\hline Grade 6 & 0 & 0 & 0 & 0 & 0 & & & & & \\
\hline School Total & 143 & 8 & 135 & 97 & 38 & School \%age & $5.59 \%$ & $94.41 \%$ & $71.85 \%$ & $28.15 \%$ \\
\hline
\end{tabular}


Sub-Centre: Timargara

\begin{tabular}{|c|c|c|c|c|c|c|c|c|c|c|}
\hline \multirow[b]{2}{*}{$\begin{array}{l}\text { Grades / } \\
\text { Sections }\end{array}$} & \multicolumn{5}{|c|}{-Number of Students- } & \multirow[b]{2}{*}{ Grades } & \multicolumn{4}{|c|}{-Percent of Students } \\
\hline & Enrolled & Dropped & $\begin{array}{l}\text { Appeared } \\
\text { in Exam }\end{array}$ & $\begin{array}{l}\text { Passed in } \\
\text { Exams }\end{array}$ & $\begin{array}{c}\text { Failed in } \\
\text { Exams }\end{array}$ & & Dropped & $\begin{array}{l}\text { Appeared } \\
\text { in Exams }\end{array}$ & $\begin{array}{l}\text { Passed in } \\
\text { Exams }\end{array}$ & $\begin{array}{l}\text { Failed in } \\
\text { Exams }\end{array}$ \\
\hline Grade $1 \mathrm{~A}$ & 75 & 5 & 70 & 40 & 30 & Grade 1 & $3.13 \%$ & $96.88 \%$ & $76.13 \%$ & $23.87 \%$ \\
\hline Grade $1 \mathrm{~B}$ & 40 & 0 & 40 & 38 & 2 & Grade 2 & $7.69 \%$ & $92.31 \%$ & $83.33 \%$ & $16.67 \%$ \\
\hline Grade $1 \mathrm{C}$ & 45 & 0 & 45 & 40 & 5 & Grade 3 & $16.67 \%$ & $83.33 \%$ & $80.00 \%$ & $20.00 \%$ \\
\hline Grade 2 A & 39 & 3 & 36 & 30 & 6 & Grade 4 & $20.00 \%$ & $80.00 \%$ & $75.00 \%$ & $25.00 \%$ \\
\hline Grade 2 B & 0 & 0 & 0 & 0 & 0 & Grade 5 & $33.33 \%$ & $66.67 \%$ & $80.00 \%$ & $20.00 \%$ \\
\hline Grade $3 \mathrm{~A}$ & 30 & 5 & 25 & 20 & 5 & Grade 6 & $33.33 \%$ & $66.67 \%$ & $90.00 \%$ & $10.00 \%$ \\
\hline Grade 3 B & 0 & 0 & 0 & 0 & 0 & & & & & \\
\hline Grade 4 & 20 & 4 & 16 & 12 & 4 & Remarks: & & & & \\
\hline Grade 5 & 15 & 5 & 10 & 8 & 2 & & & & & \\
\hline Grade 6 & 15 & 5 & 10 & 9 & 1 & & & & & \\
\hline School Total & 279 & 27 & 252 & 197 & 55 & School \%age & $9.68 \%$ & $90.32 \%$ & $78.17 \%$ & $21.83 \%$ \\
\hline
\end{tabular}

\begin{tabular}{|c|c|c|c|c|c|c|c|c|c|c|}
\hline \multirow[b]{2}{*}{$\begin{array}{l}\text { Grades I } \\
\text { Sections }\end{array}$} & \multicolumn{5}{|c|}{ - Number of Students- } & \multirow[b]{2}{*}{ Grades } & \multicolumn{4}{|c|}{ Percent of Students } \\
\hline & Enrolled & Dropped & $\begin{array}{l}\text { Appeared } \\
\text { in Exam }\end{array}$ & $\begin{array}{c}\text { Passed in } \\
\text { Exams }\end{array}$ & $\begin{array}{c}\text { Failed in } \\
\text { Exams }\end{array}$ & & Dropped & $\begin{array}{c}\text { Appeared } \\
\text { in Exams }\end{array}$ & $\begin{array}{l}\text { Passed in } \\
\text { Exams }\end{array}$ & $\begin{array}{l}\text { Failed in } \\
\text { Exams }\end{array}$ \\
\hline Grade $1 \mathrm{~A}$ & 92 & 0 & 92 & 31 & 61 & Grade 1 & $0.00 \%$ & $100.00 \%$ & $33.70 \%$ & $66.30 \%$ \\
\hline Grade 1 B & 0 & 0 & 0 & 0 & 0 & Grade 2 & $14.63 \%$ & $85.37 \%$ & $88.57 \%$ & $11.43 \%$ \\
\hline Grade $1 \mathrm{C}$ & 0 & 0 & 0 & 0 & 0 & Grade 3 & $41.18 \%$ & $58.82 \%$ & $80.00 \%$ & $20.00 \%$ \\
\hline Grade $2 \mathrm{~A}$ & 41 & 6 & 35 & 31 & 4 & Grade 4 & $0.00 \%$ & $100.00 \%$ & $100.00 \%$ & $0.00 \%$ \\
\hline Grade 2 B & 0 & 0 & 0 & 0 & 0 & Grade 5 & $0.00 \%$ & $100.00 \%$ & $66.67 \%$ & $33.33 \%$ \\
\hline Grade $3 \mathrm{~A}$ & $34^{-\infty}$ & 14 & 20 & 16 & 4 & Grade 6 & $0.00 \%$ & $100.00 \%$ & $78.57 \%$ & $21.43 \%$ \\
\hline Grade 3 B & 0 & 0 & 0 & 0 & 0 & & & & & \\
\hline Grade 4 & 17 & 0 & 17 & 17 & 0 & Remarks: & & & & \\
\hline Grade 5 & 15 & 0 & 15 & 10 & 5 & & & & & \\
\hline Grade 6 & 28 & 0 & 28 & 22 & 6 & & & & & \\
\hline School Total & 227 & 20 & 207 & 127 & 80 & School \%age & $8.81 \%$ & $91.19 \%$ & $61.35 \%$ & $38.65 \%$ \\
\hline
\end{tabular}

\begin{tabular}{|c|c|c|c|c|c|c|c|c|c|c|}
\hline \multirow[b]{2}{*}{$\begin{array}{l}\text { Grades I } \\
\text { Sections }\end{array}$} & \multicolumn{5}{|c|}{ - Number of Students- } & \multirow[b]{2}{*}{ Grades } & \multicolumn{4}{|c|}{ Percent of Students } \\
\hline & Enrolled & Dropped & $\begin{array}{c}\text { Appeared } \\
\text { in Exam }\end{array}$ & $\begin{array}{c}\text { Passed in } \\
\text { Exams }\end{array}$ & $\begin{array}{l}\text { Failed in } \\
\text { Exams }\end{array}$ & & Dropped & $\begin{array}{c}\text { Appeared } \\
\text { in Exams }\end{array}$ & $\begin{array}{c}\text { Passed in } \\
\text { Exams } \\
\end{array}$ & $\begin{array}{l}\text { Falled in } \\
\text { Exams }\end{array}$ \\
\hline Grade $1 \mathrm{~A}$ & 73 & 6 & 67 & 40 & 27 & Grade 1 & $26.32 \%$ & $73.68 \%$ & $70.78 \%$ & $29.22 \%$ \\
\hline Grade 1 B & 49 & 14 & 35 & 17 & 18 & Grade 2 & $10.00 \%$ & $90.00 \%$ & $57.14 \%$ & $42.86 \%$ \\
\hline Grade $1 \mathrm{C}$ & 87 & 35 & 52 & 52 & 0 & Grade 3 & $11.67 \%$ & $88.33 \%$ & $69.81 \%$ & $30.19 \%$ \\
\hline Grade 2 A & 70 & 7 & 63 & 36 & 27 & Grade 4 & $0.00 \%$ & $100.00 \%$ & $93.33 \%$ & $6.67 \%$ \\
\hline Grade 2 B & 0 & 0 & 0 & 0 & 0 & Grade 5 & $0.00 \%$ & $100.00 \%$ & $100.00 \%$ & $0.00 \%$ \\
\hline Grade $3 \mathrm{~A}$ & 60 & 7 & 53 & 37 & 16 & Grade 6 & \#Num! & \#Num! & \#Num! & \#Num! \\
\hline Grade 3 B & 0 & 0 & 0 & 0 & 0 & & & & & \\
\hline Grade 4 & 30 & 0 & 30 & 28 & 2 & Remarks: & & & & \\
\hline Grade 5 & 22 & 0 & 22 & 22 & 0 & & & & & \\
\hline Grade 6 & 0 & 0 & 0 & 0 & 0 & & & & & \\
\hline School Total & 391 & 69 & 322 & 232 & 90 & School \%age & $17.65 \%$ & $82.35 \%$ & $72.05 \%$ & $27.95 \%$ \\
\hline
\end{tabular}

Note:

\#Num! = Enrollment in respective grade(s) is nill therefore calculation is not applicable
Page 83 of 91

Produced by DP/MIS Section on 31-Jul-97 
Sub-Centre: Timargara

\begin{tabular}{|c|c|c|c|c|c|c|c|c|c|c|}
\hline \multicolumn{11}{|c|}{ School Code: TM/BJR/PSB-243 School Title: ARPS Umari - Timergara } \\
\hline $\begin{array}{l}\text { Grades / } \\
\text { Sections }\end{array}$ & Enrolled & Dropped & $\begin{array}{c}\text { Appeared } \\
\text { in Exam }\end{array}$ & $\begin{array}{c}\text { Passed in } \\
\text { Exams }\end{array}$ & $\begin{array}{c}\text { Failed in } \\
\text { Exams }\end{array}$ & Grades & Dropped & $\begin{array}{c}\text { Appeared } \\
\text { in Exams }\end{array}$ & $\begin{array}{l}\text { Passed in } \\
\text { Exams }\end{array}$ & $\begin{array}{l}\text { Faleo in } \\
\text { Exams }\end{array}$ \\
\hline Grade $1 \mathrm{~A}$ & 97 & 0 & 97 & 31 & 66 & Grade 1 & $0.00 \%$ & $100.00 \%$ & $31.96 \%$ & $68.04 \%$ \\
\hline Grade 1 B & 0 & 0 & 0 & 0 & 0 & Grade 2 & $0.00 \%$ & $100.00 \%$ & $88.89 \%$ & $11.11 \%$ \\
\hline Grade $1 \mathrm{C}$ & 0 & 0 & 0 & 0 & 0 & Grade 3 & $0.00 \%$ & $100.00 \%$ & $19.05 \%$ & $80.95 \%$ \\
\hline Grade 2 A & 27 & 0 & 27 & 24 & 3 & Grade 4 & $40.74 \%$ & $59.26 \%$ & $56.25 \%$ & $43.75 \%$ \\
\hline Grade 2 B & 0 & 0 & 0 & 0 & 0 & Grade 5 & $0.00 \%$ & $100.00 \%$ & $38.89 \%$ & $61.11 \%$ \\
\hline Grade 3 A & 42 & 0 & 42 & 8 & 34 & Grade 6 & \#Num! & \#Num! & \#Num! & \#Num! \\
\hline Grade 3 B & 0 & 0 & 0 & 0 & 0 & & & & & \\
\hline Grade 4 & 27 & 11 & 16 & 9 & 7 & Remarks: & & & & \\
\hline Grade 5 & 18 & 0 & 18 & 7 & 11 & & & & & \\
\hline Grade 6 & 0 & 0 & 0 & 0 & 0 & & & & & \\
\hline School Total & 211 & 11 & 200 & 79 & 121 & School \%age & $5.21 \%$ & $94.79 \%$ & $39.50 \%$ & $60.50 \%$ \\
\hline
\end{tabular}

\begin{tabular}{|c|c|c|c|c|c|c|c|c|c|c|c|}
\hline \multirow[b]{2}{*}{$\begin{array}{l}\text { Grades / } \\
\text { Sections }\end{array}$} & \multicolumn{6}{|c|}{-Number of Students- } & \multirow[b]{2}{*}{ Grades } & \multicolumn{4}{|c|}{-Percent of Students } \\
\hline & 1 & Enrolled & Dropped & $\begin{array}{c}\text { Appeared } \\
\text { in Exam }\end{array}$ & $\begin{array}{c}\text { Passed in } \\
\text { Exams }\end{array}$ & $\begin{array}{l}\text { Failed in } \\
\text { Exams }\end{array}$ & & Oropped & $\begin{array}{l}\text { Appeared } \\
\text { in Exams }\end{array}$ & $\begin{array}{c}\text { Passed in } \\
\text { Exams }\end{array}$ & $\begin{array}{l}\text { Faled in } \\
\text { Exams }\end{array}$ \\
\hline Grade $1 \mathrm{~A}$ & & 46 & 6 & 40 & 25 & 15 & Grade 1 & $16.41 \%$ & $83.59 \%$ & $66.36 \%$ & $33.64 \%$ \\
\hline Grade 1 B & & 40 & 5 & 35 & 22 & 13 & Grade 2 & $11.32 \%$ & $88.68 \%$ & $76.60 \%$ & $23.40 \%$ \\
\hline Grade $1 \mathrm{C}$ & & 42 & 10 & 32 & 24 & 8 & Grade 3 & $19.15 \%$ & $80.85 \%$ & $78.95 \%$ & $21.05 \%$ \\
\hline Grade 2 A & & 53 & 6 & 47 & 36 & 11 & Grade 4 & $14.29 \%$ & $85.71 \%$ & $100.00 \%$ & $0.00 \%$ \\
\hline Grade 2 B & & 0 & 0 & 0 & 0 & 0 & Grade 5 & $33.33 \%$ & $66.67 \%$ & $100.00 \%$ & $0.00 \%$ \\
\hline Grade $3 \mathrm{~A}$ & & 47 & 9 & 38 & 30 & 8 & Grade 6 & \#Num! & \#Num! & \#Num! & \#Num! \\
\hline Grade 3 B & & 0 & 0 & 0 & 0 & 0 & & & & & \\
\hline Grade 4 & & 21 & 3 & 18 & 18 & 0 & Remarks: & & & & \\
\hline Grade 5 & & 12 & 4 & 8 & 8 & 0 & & & & & \\
\hline Grade 6 & & 0 & 0 & 0 & 0 & 0 & & & & & \\
\hline School Total & & 261 & 43 & 218 & 163 & 55 & School \%age & $16.48 \%$ & $83.52 \%$ & $74.77 \%$ & $25.23 \%$ \\
\hline
\end{tabular}

\begin{tabular}{|c|c|c|c|c|c|c|c|c|c|c|}
\hline \multirow[b]{2}{*}{$\begin{array}{l}\text { Grades ! } \\
\text { Sections }\end{array}$} & \multicolumn{5}{|c|}{ Number of Students } & \multirow[b]{2}{*}{ Grades } & \multicolumn{4}{|c|}{-Percent of Students- } \\
\hline & Enrolled & Dropped & $\begin{array}{l}\text { Appeared } \\
\text { in Exam }\end{array}$ & $\begin{array}{r}\text { Passed in } \\
\text { Exams }\end{array}$ & $\begin{array}{l}\text { Failed in } \\
\text { Exams }\end{array}$ & & Dropped & $\begin{array}{l}\text { Appeared } \\
\text { in Exams }\end{array}$ & $\begin{array}{l}\text { Passed in } \\
\text { Exams }\end{array}$ & $\begin{array}{l}\text { Falled in } \\
\text { Exams }\end{array}$ \\
\hline Grade $1 \mathrm{~A}$ & 51 & 8 & 43 & 33 & 10 & Grade 1 & $22.60 \%$ & $77.40 \%$ & $67.26 \%$ & $32.74 \%$ \\
\hline Grade 1 B & 60 & 23 & 37 & 24 & 13 & Grade 2 & $23.21 \%$ & $76.79 \%$ & $58.14 \%$ & $41.86 \%$ \\
\hline Grade $1 \mathrm{C}$ & 35 & 2 & 33 & 19 & 14 & Grade 3 & $9.09 \%$ & $90.91 \%$ & $56.67 \%$ & $43.33 \%$ \\
\hline Grade 2 A & 56 & 13 & 43 & 25 & 18 & Grade 4 & $0.00 \%$ & $100.00 \%$ & $71,43 \%$ & $28.57 \%$ \\
\hline Grade 2 B & 0 & 0 & 0 & 0 & 0 & Grade 5 & $0.00 \%$ & $100.00 \%$ & $90.48 \%$ & $9.52 \%$ \\
\hline Grade 3 A & 33 & 3 & 30 & 17 & 13 & Grade 6 & $20.00 \%$ & $80.00 \%$ & $75.00 \%$ & $25.00 \%$ \\
\hline Grade 3 B & 0 & 0 & 0 & 0 & 0 & & & & & \\
\hline Grade 4 & 21 & 0 & 21 & 15 & 6 & Remarks: & & & & \\
\hline Grade 5 & 21 & 0 & 21 & 19 & 2 & & & & & \\
\hline Grade 6 & 15 & 3 & 12 & 9 & 3 & & & & & \\
\hline School Total & 292 & 52 & 240 & 161 & 79 & School \%age & $17.81 \%$ & $82.19 \%$ & $67.08 \%$ & $32.92 \%$ \\
\hline
\end{tabular}


Sub-Centre: Timargara

\begin{tabular}{|c|c|c|c|c|c|c|c|c|c|c|}
\hline \multirow[b]{2}{*}{$\begin{array}{l}\text { Grades / } \\
\text { Sections }\end{array}$} & \multicolumn{5}{|c|}{-Number of Students- } & \multirow[b]{2}{*}{ Grades } & \multicolumn{4}{|c|}{-Percent of Students- } \\
\hline & Enrolled & Dropped & $\begin{array}{l}\text { Appeared } \\
\text { in Exam }\end{array}$ & $\begin{array}{c}\text { Passed in } \\
\text { Exams }\end{array}$ & $\begin{array}{l}\text { Failed in } \\
\text { Exams }\end{array}$ & & Dropped & $\begin{array}{l}\text { Appeared } \\
\text { in Exams }\end{array}$ & $\begin{array}{c}\text { Passed in } \\
\text { Exams }\end{array}$ & $\begin{array}{c}\text { Failed in } \\
\text { Exams }\end{array}$ \\
\hline Grade $1 \mathrm{~A}$ & 25 & 0 & 25 & 25 & 0 & Grade 1 & $0.00 \%$ & $100.00 \%$ & $100.00 \%$ & $0.00 \%$ \\
\hline Grade $1 \mathrm{~B}$ & 0 & 0 & 0 & 0 & 0 & Grade 2 & $0.00 \%$ & $100.00 \%$ & $80.00 \%$ & $20.00 \%$ \\
\hline Grade $1 \mathrm{C}$ & 0 & 0 & 0 & 0 & 0 & Grade 3 & $0.00 \%$ & $100.00 \%$ & $92.31 \%$ & $7.69 \%$ \\
\hline Grade $2 \mathrm{~A}$ & 15 & 0 & 15 & 12 & 3 & Grade 4 & $0.00 \%$ & $100.00 \%$ & $100.00 \%$ & $0.00 \%$ \\
\hline Grade 2 B & 0 & 0 & 0 & 0 & 0 & Grade 5 & $0.00 \%$ & $100.00 \%$ & $83.33 \%$ & $16.67 \%$ \\
\hline Grade $3 \mathrm{~A}$ & 13 & 0 & 13 & 12 & 1 & Grade 6 & \#Num! & \#Num! & \#Num! & \#Num! \\
\hline Grade 3 B & 0 & 0 & 0 & 0 & 0 & & & & & \\
\hline Grade 4 & 7 & 0 & 7 & 7 & 0 & Remarks: & & & & \\
\hline Grade 5 & 6 & 0 & 6 & 5 & 1 & & & & & \\
\hline Grade 6 & 0 & 0 & 0 & 0 & 0 & & & & & \\
\hline School Total & 66 & 0 & 66 & 61 & 5 & School \%age & $0.00 \%$ & $100.00 \%$ & $92.42 \%$ & $7.58 \%$ \\
\hline
\end{tabular}

\begin{tabular}{|c|c|c|c|c|c|c|c|c|c|c|c|}
\hline \multirow[b]{2}{*}{$\begin{array}{l}\text { Grades / } \\
\text { Sections }\end{array}$} & \multicolumn{6}{|c|}{ Number of Students- } & \multirow[b]{2}{*}{ Grades } & \multirow[b]{2}{*}{ Dropped } & \multicolumn{3}{|c|}{ Percent of Students } \\
\hline & , & Enrolled & Dropped & $\begin{array}{l}\text { Appeared } \\
\text { in Exam }\end{array}$ & $\begin{array}{l}\text { Passed in } \\
\text { Exams }\end{array}$ & $\begin{array}{c}\text { Failed in } \\
\text { Exams }\end{array}$ & & & $\begin{array}{l}\text { Appeared } \\
\text { in Exams }\end{array}$ & $\begin{array}{r}\text { Passed in } \\
\text { Exams }\end{array}$ & $\begin{array}{l}\text { Failed in } \\
\text { Exams }\end{array}$ \\
\hline Grade $1 \mathrm{~A}$ & & 52 & 0 & 52 & 28 & 24 & Grade 1 & $0.00 \%$ & $100.00 \%$ & $53.85 \%$ & $46.15 \%$ \\
\hline Grade 1 B & & 0 & 0 & 0 & 0 & 0 & Grade 2 & $0.00 \%$ & $100.00 \%$ & $76.00 \%$ & $24.00 \%$ \\
\hline Grade $1 \mathrm{C}$ & & 0 & 0 & 0 & 0 & 0 & Grade 3 & $5.00 \%$ & $95.00 \%$ & $73.68 \%$ & $26.32 \%$ \\
\hline Grade $2 \mathrm{~A}$ & & 25 & 0 & 25 & 19 & 6 & Grade 4 & $0.00 \%$ & $100.00 \%$ & $73.33 \%$ & $26.67 \%$ \\
\hline Grade 2 B & & 0 & 0 & 0 & 0 & 0 & Grade 5 & $0.00 \%$ & $100.00 \%$ & $60.00 \%$ & $40.00 \%$ \\
\hline Grade 3 A & & 80 & 1 & 19 & 14 & 5 & Grade 6 & $0.00 \%$ & $100.00 \%$ & $70.59 \%$ & $29.41 \%$ \\
\hline Grade 3 B & & 0 & 0 & 0 & 0 & 0 & & & & & \\
\hline Grade 4 & & 15 & 0 & 15 & 11 & 4 & Remarks: & & & & \\
\hline Grade 5 & & 15 & 0 & 15 & 9 & 6 & & & & & \\
\hline Grade 6 & & 17 & 0 & 17 & 12 & 5 & & & & & \\
\hline School Total & & 144 & 1 & 143 & 93 & 50 & School \%age & $0.69 \%$ & $99.31 \%$ & $65.03 \%$ & $34.97 \%$ \\
\hline
\end{tabular}

\begin{tabular}{|c|c|c|c|c|c|c|c|c|c|c|}
\hline \multicolumn{11}{|c|}{ School Code: TM/CHTIPSB-248 School Title: ARPS Kalkatak - Timergara } \\
\hline \multirow[b]{2}{*}{$\begin{array}{l}\text { Grades I } \\
\text { Sections }\end{array}$} & & - Numb & r of Stude & & & \multirow[b]{2}{*}{ Grades } & \multicolumn{4}{|c|}{-Percent of Students- } \\
\hline & Enrolled & Dropped & $\begin{array}{l}\text { Appeared } \\
\text { in Exam }\end{array}$ & $\begin{array}{c}\text { Passed in } \\
\text { Exams }\end{array}$ & $\begin{array}{c}\text { Failed in } \\
\text { Exams }\end{array}$ & & Dropped & $\begin{array}{l}\text { Appeared } \\
\text { in Exams }\end{array}$ & $\begin{array}{c}\text { Passed in } \\
\text { Exams }\end{array}$ & $\begin{array}{c}\text { Failed in } \\
\text { Exams }\end{array}$ \\
\hline Grade $1 \mathrm{~A}$ & 34 & 0 & 34 & 21 & 13 & Grade 1 & $0.00 \%$ & $100.00 \%$ & $61.76 \%$ & $38.24 \%$ \\
\hline Grade 1 B & 0 & 0 & 0 & 0 & 0 & Grade 2 & $0.00 \%$ & $100.00 \%$ & $80.00 \%$ & $20.00 \%$ \\
\hline Grade $1 \mathrm{C}$ & 0 & 0 & 0 & 0 & 0 & Grade 3 & $0.00 \%$ & $100.00 \%$ & $78.95 \%$ & $21.05 \%$ \\
\hline Grade $2 \mathrm{~A}$ & 20 & 0 & 20 & 16 & 4 & Grade 4 & $0.00 \%$ & $100.00 \%$ & $0.00 \%$ & $100.00 \%$ \\
\hline Grade 2 B & 0 & 0 & 0 & 0 & 0 & Grade 5 & \#Num! & \#Num! & \#Num! & \#Num! \\
\hline Grade $3 \mathrm{~A}$ & 19 & 0 & 19 & 15 & 4 & Grade 6 & \#Num! & \#Num! & \#Num! & \#Num! \\
\hline Grade 3 B & 0 & 0 & 0 & 0 & 0 & & & & & \\
\hline Grade 4 & 10 & 0 & 10 & 0 & 10 & Remarks: & & & & \\
\hline Grade 5 & 0 & 0 & 0 & 0 & 0 & & & & & \\
\hline Grade 6 & 0 & 0 & 0 & 0 & 0 & & & & & \\
\hline School Total & 83 & 0 & 83 & 52 & 31 & School \%age & $0.00 \%$ & $100.00 \%$ & $62.65 \%$ & $37.35 \%$ \\
\hline
\end{tabular}

Note:

\#Um! = Enroliment in respective grade(s) is nill therefore calculation is not applicable
Page 85 of 91

Produced by DP/MIS Section on 31-Jul-97 
Sub-Centre: Timargara

\begin{tabular}{|c|c|c|c|c|c|c|c|c|c|c|}
\hline \multirow[b]{2}{*}{$\begin{array}{l}\text { Grades I } \\
\text { Seclions }\end{array}$} & \multicolumn{5}{|c|}{-Number of Students- } & \multirow[b]{2}{*}{ Grades } & \multicolumn{4}{|c|}{-Percent of Students } \\
\hline & Enrolled & Dropped & $\begin{array}{l}\text { Appeared } \\
\text { in Exam }\end{array}$ & $\begin{array}{c}\text { Passed in } \\
\text { Exams }\end{array}$ & $\begin{array}{c}\text { Failed in } \\
\text { Exams }\end{array}$ & & Dropped & $\begin{array}{l}\text { Appeared } \\
\text { in Exams }\end{array}$ & $\begin{array}{c}\text { Passed in } \\
\text { Exams }\end{array}$ & $\begin{array}{l}\text { Fai:ed a } \\
\text { Exams }\end{array}$ \\
\hline Grade $1 \mathrm{~A}$ & 32 & 0 & 32 & 18 & 14 & Grade 1 & $0.00 \%$ & $100.00 \%$ & $56.25 \%$ & $+3.75 \%$ \\
\hline Grade 1 B & 0 & 0 & 0 & 0 & 0 & Grade 2 & $10.00 \%$ & $90.00 \%$ & $66.67 \%$ & $33.33 \%$ \\
\hline Grade $1 \mathrm{C}$ & 0 & 0 & 0 & 0 & 0 & Grade 3 & $16.67 \%$ & $83.33 \%$ & $40.00 \%$ & $60.00 \%$ \\
\hline Grade 2 A & 10 & 1 & 9 & 6 & 3 & Grade 4 & $12.50 \%$ & $87.50 \%$ & $57.14 \%$ & $42.80 \%$ \\
\hline Grade 2 B & 0 & 0 & 0 & 0 & 0 & Grade 5 & $0.00 \%$ & $100.00 \%$ & $100.00 \%$ & $0.00 \%$ \\
\hline Grade $3 \mathrm{~A}$ & 6 & 1 & 5 & 2 & 3 & Grade 6 & \#Num! & \#Num! & \#Num! & \#Num! \\
\hline Grade 3 B & 0 & 0 & 0 & 0 & 0 & & & & & \\
\hline Grade 4 & 8 & 1 & 7 & 4 & 3 & Remarks: & & & & \\
\hline Grade 5 & 5 & 0 & 5 & 5 & 0 & & & & & \\
\hline Grade 6 & 0 & 0 & 0 . & 0 & 0 & & & & & \\
\hline School Total & 61 & 3 & 58 & 35 & 23 & School \%age & $4.92 \%$ & $95.08 \%$ & $60.34 \%$ & $3960 \%=$ \\
\hline
\end{tabular}

\begin{tabular}{|c|c|c|c|c|c|c|c|c|c|c|}
\hline School Code: T & PSC-25 & ichool T & ARPS & so - Tim & & & Head & eacher: $\mathbf{G}$ & ylam Mol & mad \\
\hline$\cdot$ & & -Numb & of Studer & & & & & Percent of & tudents- & $\square$ \\
\hline $\begin{array}{l}\text { Grades I } \\
\text { Seclions }\end{array}$ & Enrolled & Dropped & $\begin{array}{l}\text { Appeared } \\
\text { in Exam }\end{array}$ & $\begin{array}{c}\text { Passed in } \\
\text { Exams }\end{array}$ & $\begin{array}{l}\text { Failed in } \\
\text { Exams }\end{array}$ & Grades & Dropped & $\begin{array}{l}\text { Appeared } \\
\text { in Exams }\end{array}$ & $\begin{array}{c}\text { Passed in } \\
\text { Exams }\end{array}$ & $\begin{array}{c}F a_{a}=0 ; \\
\text { Exa-s }\end{array}$ \\
\hline Grade $1 \mathrm{~A}$ & 33 & 1 & 32 & 25 & 7 & Grade 1 & $3.03 \%$ & $96.97 \%$ & $78.13 \%$ & $21.88 \%$ \\
\hline Grade 1 B & 0 & 0 & 0 & 0 & 0 & Grade 2 & $0.00 \%$ & $100.00 \%$ & $89.47 \%$ & $10.53^{\circ}=$ \\
\hline Grade $1 \mathrm{C}$ & 0 & 0 & 0 & 0 & 0 & Grade 3 & $7.14 \%$ & $92.86 \%$ & $100.00 \%$ & $0.20:=$ \\
\hline Grade $2 \mathrm{~A}$ & 19 & 0 & 19 & 17 & 2 & Grade 4 & $20.00 \%$ & $80.00 \%$ & $87.50 \%$ & $12.50 \%$ \\
\hline Grade 2 B & 0 & 0 & 0 & 0 & 0 & Grade 5 & $0.00 \%$ & $100.00 \%$ & $83.33 \%$ & $16.67 \%$ \\
\hline Grade 3 A & 14 & 1 & 13 & 13 & 0 & Grade 6 & \#Num! & \#Num! & \#Num! & \#Num! \\
\hline Grade 3 B & $\theta$ & 0 & 0 & 0 & 0 & & & & & \\
\hline Grade 4 & 10 & 2 & 8 & 7 & 1 & Remarks: & & & & \\
\hline Grade 5 & 6 & 0 & 6 & 5 & 1 & & & & & \\
\hline Grade 6 & 0 & 0 & 0 & 0 & 0 & & & & & \\
\hline School Total & 82 & 4 & 78 & 67 & 11 & School \%age & $4.88 \%$ & $95.12 \%$ & $85.90 \%$ & $14.10 \%$ \\
\hline
\end{tabular}

\begin{tabular}{|c|c|c|c|c|c|c|c|c|c|c|}
\hline \multirow[b]{2}{*}{$\begin{array}{l}\text { Grades / } \\
\text { Sections }\end{array}$} & PSC-25 & \multicolumn{4}{|c|}{ Number of Students- } & \multirow[b]{2}{*}{ Grades } & \multicolumn{4}{|c|}{-Percent of Students } \\
\hline & Enrolled & Dropped & $\begin{array}{l}\text { Appeared } \\
\text { in Exam }\end{array}$ & $\begin{array}{r}\text { Passed in } \\
\text { Exams }\end{array}$ & $\begin{array}{l}\text { Failed in } \\
\text { Exams }\end{array}$ & & Dropped & $\begin{array}{l}\text { Appeared } \\
\text { in Exams }\end{array}$ & $\begin{array}{l}\text { Passed in } \\
\text { Exams }\end{array}$ & 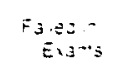 \\
\hline Grade $1 \mathrm{~A}$ & 37 & 0 & 37 & 30 & 7 & Grade 1 & $0.00 \%$ & $100.00 \%$ & $81.08 \%$ & $18.92 \%$ \\
\hline Grade 1 B & 0 & 0 & 0 & 0 & 0 & Grade 2 & $0.00 \%$ & $100.00 \%$ & $100.00 \%$ & $0.00 \%$ \\
\hline Grade $1 \mathrm{C}$ & 0 & 0 & 0 & 0 & 0 & Grade 3 & $0.00 \%$ & $100.00 \%$ & $100.00 \%$ & $0.00 \div=$ \\
\hline Grade $2 \mathrm{~A}$ & 20 & 0 & 20 & 20 & 0 & Grade 4 & $0.00 \%$ & $100.00 \%$ & $100.00 \%$ & $0.00 \div=$ \\
\hline Grade 2 B & 0 & 0 & 0 & 0 & 0 & Grade 5 & \#Num! & \#Num! & \#Num! & \#Num! \\
\hline Grade $3 \mathrm{~A}$ & 15 & 0 & 15 & 15 & 0 & Grade 6 & \#Num! & \#Num! & $\#$ Num! & \#Num! \\
\hline Grade 3 B & 0 & 0 & 0 & 0 & 0 & & & & & \\
\hline Grade 4 & 12 & 0 & 12 & 12 & 0 & Remarks: & & & & \\
\hline Grade 5 & 0 & 0 & 0 & 0 & 0 & & & & & \\
\hline Grade 6 & 0 & 0 & 0 & 0 & 0 & & & & & \\
\hline School Total & 84 & 0 & 84 & 77 & 7 & School \%age & $0.00 \%$ & $100.00 \%$ & $91.67 \%$ & $8.33 \%$ \\
\hline
\end{tabular}

Note: 
Sub-Centre: Timargara

\begin{tabular}{|c|c|c|c|c|c|c|c|c|c|c|}
\hline \multirow[b]{2}{*}{$\begin{array}{l}\text { Grades I } \\
\text { Sections }\end{array}$} & \multicolumn{5}{|c|}{-Number of Students- } & \multirow[b]{2}{*}{ Grades } & \multicolumn{4}{|c|}{-Percent of Students } \\
\hline & Enrolled & Dropped & $\begin{array}{l}\text { Appeared } \\
\text { in Exam }\end{array}$ & $\begin{array}{l}\text { Passed in } \\
\text { Exams }\end{array}$ & $\begin{array}{l}\text { Failed in } \\
\text { Exams }\end{array}$ & & Dropped & $\begin{array}{c}\text { Appeared } \\
\text { in Exams }\end{array}$ & $\begin{array}{r}\text { Passed in } \\
\text { Exams }\end{array}$ & $\begin{array}{c}\text { Failed in } \\
\text { Exams }\end{array}$ \\
\hline Grade $1 \mathrm{~A}$ & 35 & 0 & 35 & 23 & 12 & Grade 1 & $0.00 \%$ & $100.00 \%$ & $65.71 \%$ & $34.29 \%$ \\
\hline Grade 1 B & 0 & 0 & 0 & 0 & 0 & Grade 2 & $0.00 \%$ & $100.00 \%$ & $52.38 \%$ & $47.62 \%$ \\
\hline Grade $1 \mathrm{C}$ & 0 & 0 & 0 & 0 & 0 & Grade 3 & $18.18 \%$ & $81.82 \%$ & $44.44 \%$ & $55.56 \%$ \\
\hline Grade $2 \mathrm{~A}$ & 21 & 0 & 21 & 11 & 10 & Grade 4 & $16.67 \%$ & $83.33 \%$ & $80.00 \%$ & $20.00 \%$ \\
\hline Grade 2 B & 0 & 0 & 0 & 0 & 0 & Grade 5 & $0.00 \%$ & $100.00 \%$ & $85.71 \%$ & $14.29 \%$ \\
\hline Grade 3 A & 11 & 2 & 9 & 4 & 5 & Grade 6 & \#Num! & \#Num! & \#Num! & \#Num! \\
\hline Grade 3 B & 0 & 0 & 0 & 0 & 0 & & & & & \\
\hline Grade 4 & 6 & 1 & 5 & 4 & 1 & Remarks: & & & & \\
\hline Grade 5 & 7 & 0 & 7 & 6 & 1 & & & & & \\
\hline Grade 6 & 0 & 0 & 0 & 0 & 0 & & & & & \\
\hline School Total & 80 & 3 & 77 & 48 & 29 & School \%age & $3.75 \%$ & $96.25 \%$ & $62.34 \%$ & $37.66 \%$ \\
\hline
\end{tabular}

\begin{tabular}{|c|c|c|c|c|c|c|c|c|c|c|c|}
\hline \multirow[b]{2}{*}{$\begin{array}{l}\text { Grades I } \\
\text { Sections }\end{array}$} & \multicolumn{6}{|c|}{ - Number of Students- } & \multirow[b]{2}{*}{ Grades } & \multicolumn{4}{|c|}{-Percent of Students } \\
\hline & , & Enrolled & Dropped & $\begin{array}{l}\text { Appeared } \\
\text { in Exam }\end{array}$ & $\begin{array}{l}\text { Passed in } \\
\text { Exams }\end{array}$ & $\begin{array}{c}\text { Failed in } \\
\text { Exams }\end{array}$ & & Dropped & $\begin{array}{l}\text { Appeared } \\
\text { in Exams }\end{array}$ & $\begin{array}{l}\text { Passed in } \\
\text { Exams }\end{array}$ & $\begin{array}{l}\text { Failed in } \\
\text { Exams }\end{array}$ \\
\hline Grade $1 \mathrm{~A}$ & & 32 & 6 & 26 & 24 & 2 & Grade 1 & $18.75 \%$ & $81.25 \%$ & $92.31 \%$ & $7.69 \%$ \\
\hline Grade 1 B & & 0 & 0 & 0 & 0 & 0 & Grade 2 & $0.00 \%$ & $100.00 \%$ & $93.33 \%$ & $6.67 \%$ \\
\hline Grade $1 \mathrm{C}$ & & 0 & 0 & 0 & 0 & 0 & Grade 3 & $11.11 \%$ & $88.89 \%$ & $93.75 \%$ & $6.25 \%$ \\
\hline Grade $2 \mathrm{~A}$ & & 30 & 0 & 30 & 28 & 2 & Grade 4 & $31.25 \%$ & $68.75 \%$ & $90.91 \%$ & $9.09 \%$ \\
\hline Grade 2 B & & 0 & 0 & 0 & 0 & 0 & Grade 5 & $0.00 \%$ & $100.00 \%$ & $75.00 \%$ & $25.00 \%$ \\
\hline Grade $3 \mathrm{~A}$ & & 18 & 2 & 16 & 15 & 1 & Grade 6 & $20.00 \%$ & $80.00 \%$ & $100.00 \%$ & $0.00 \%$ \\
\hline Grade 3 B & & 0 & 0 & 0 & 0 & 0 & & & & & \\
\hline Grade 4 & & 16 & 5 & 11 & 10 & 1 & Remarks: & & & & \\
\hline Grade 5 & & 16 & 0 & 16 & 12 & 4 & & & & & \\
\hline Grade 6 & & 10 & 2 & 8 & 8 & 0 & & & & & \\
\hline School Total & & 122 & 15 & 107 & 97 & 10 & School \%age & $12.30 \%$ & $87.70 \%$ & $90.65 \%$ & $9.35 \%$ \\
\hline
\end{tabular}

\begin{tabular}{|c|c|c|c|c|c|c|c|c|c|c|}
\hline \multicolumn{6}{|c|}{ School Code: TM/DIR/PSC-254 School Title: ARPS Barawal - Timergara } & \multicolumn{5}{|c|}{ Head Teacher: Gul Zaman } \\
\hline \multirow[b]{2}{*}{$\begin{array}{l}\text { Grades I } \\
\text { Sections }\end{array}$} & \multicolumn{5}{|c|}{ Number of Students } & \multirow[b]{2}{*}{ Grades } & \multicolumn{4}{|c|}{ Percent of Students- } \\
\hline & Enrolled & Dropped & $\begin{array}{l}\text { Appeared } \\
\text { in Exam }\end{array}$ & $\begin{array}{r}\text { Passed in } \\
\text { Exams }\end{array}$ & $\begin{array}{l}\text { Failed in } \\
\text { Exams }\end{array}$ & & Dropped & $\begin{array}{l}\text { Appeared } \\
\text { in Exams }\end{array}$ & $\begin{array}{l}\text { Passed in } \\
\text { Exams }\end{array}$ & $\begin{array}{c}\text { Failed in } \\
\text { Exams }\end{array}$ \\
\hline Grade $1 \mathrm{~A}$ & 72 & 0 & 72 & 68 & 4 & Grade 1 & $0.00 \%$ & $100.00 \%$ & $94.44 \%$ & $5.56 \%$ \\
\hline Grade 1 B & 0 & 0 & 0 & 0 & 0 & Grade 2 & $0.00 \%$ & $100.00 \%$ & $80.00 \%$ & $20.00 \%$ \\
\hline Grade $1 \mathrm{C}$ & 0 & 0 & 0 & 0 & 0 & Grade 3 & $0.00 \%$ & $100.00 \%$ & $100.00 \%$ & $0.00 \%$ \\
\hline Grade $2 \mathrm{~A}$ & 30 & 0 & 30 & 24 & 6 & Grade 4 & $0.00 \%$ & $100.00 \%$ & $75.00 \%$ & $25.00 \%$ \\
\hline Grade 2 B & 0 & 0 & 0 & 0 & 0 & Grade 5 & $0.00 \%$ & $100.00 \%$ & $61.54 \%$ & $38.46 \%$ \\
\hline Grade $3 \mathrm{~A}$ & 20 & 0 & 20 & 20 & 0 & Grade 6 & $0.00 \%$ & $100.00 \%$ & $100.00 \%$ & $0.00 \%$ \\
\hline Grade 3 B & 0 & 0 & 0 & 0 & 0 & & & & & \\
\hline Grade 4 & 24 & 0 & 24 & 18 & 6 & Remarks: & & & & \\
\hline Grade 5 & 13 & 0 & 13 & 8 & 5 & & & & & \\
\hline Grade 6 & 8 & 0 & 8 & 8 & 0 & & & & & \\
\hline School Total & 167 & 0 & 167 & 146 & 21 & School \%age & $0.00 \%$ & $100.00 \%$ & $87.43 \%$ & $12.57 \%$ \\
\hline
\end{tabular}


Sub-Centre: Timargara

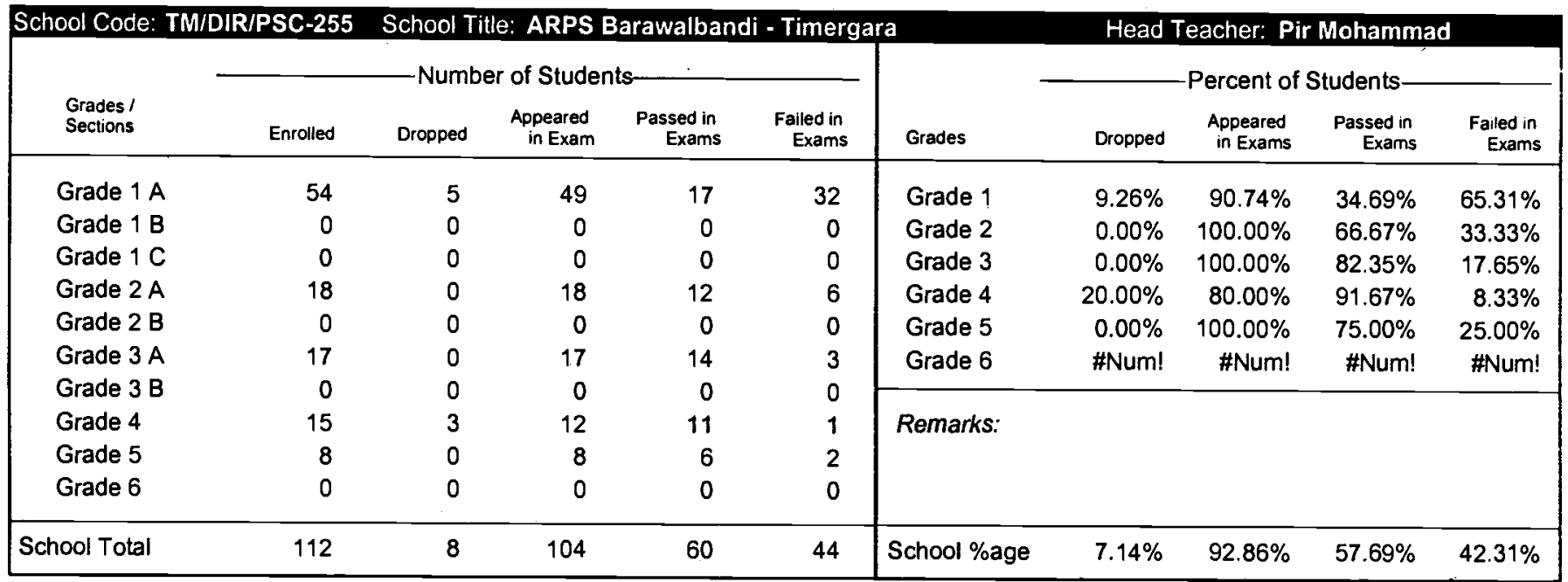

\begin{tabular}{|c|c|c|c|c|c|c|c|c|c|c|}
\hline \multirow[b]{2}{*}{$\begin{array}{l}\text { Grades I } \\
\text { Sections }\end{array}$} & \multicolumn{5}{|c|}{ - Number of Students } & \multirow[b]{2}{*}{ Grades } & \multicolumn{4}{|c|}{ Percent of Students - } \\
\hline & I Enrolled & Dropped & $\begin{array}{l}\text { Appeared } \\
\text { in Exam }\end{array}$ & $\begin{array}{l}\text { Passed in } \\
\text { Exams }\end{array}$ & $\begin{array}{l}\text { Failed in } \\
\text { Exams }\end{array}$ & & Dropped & $\begin{array}{l}\text { Appeared } \\
\text { in Exams }\end{array}$ & $\begin{array}{l}\text { Passed in } \\
\text { Exams }\end{array}$ & $\begin{array}{l}\text { Failed in } \\
\text { Exams }\end{array}$ \\
\hline Grade $1 \mathrm{~A}$ & 53 & 8 & 45 & 20 & 25 & Grade 1 & $20.21 \%$ & $79.79 \%$ & $52.00 \%$ & $48.00 \%$ \\
\hline Grade 1 B & 41 & 11 & 30 & 19 & 11 & Grade 2 & $18.00 \%$ & $82.00 \%$ & $56.10 \%$ & $43.90 \%$ \\
\hline Grade $1 \mathrm{C}$ & 0 & 0 & 0 & 0 & 0 & Grade 3 & $36.36 \%$ & $63.64 \%$ & $85.71 \%$ & $14.29 \%$ \\
\hline Grade 2 A & 50 & 9 & 41 & 23 & 18 & Grade 4 & $42.31 \%$ & $57.69 \%$ & $86.67 \%$ & $13.33 \%$ \\
\hline Grade 2 B & 0 & 0 & 0 & 0 & 0 & Grade 5 & $16.67 \%$ & $83.33 \%$ & $70.00 \%$ & $30.00 \%$ \\
\hline Grade 3 A & $44-$ & 16 & 28 & 24 & 4 & Grade 6 & \#Num! & \#Num! & \#Num! & \#Num! \\
\hline Grade 3 B & 0 & 0 & 0 & 0 & 0 & & & & & \\
\hline Grade 4 & 26 & 11 & 15 & 13 & 2 & Remarks: & & & & \\
\hline Grade 5 & 12 & 2 & 10 & 7 & 3 & & & & & \\
\hline Grade 6 & 0 & 0 & 0 & 0 & 0 & & & & & \\
\hline School Total & 226 & 57 & 169 & 106 & 63 & School \%age & $25.22 \%$ & $74.78 \%$ & $62.72 \%$ & $37.28 \%$ \\
\hline
\end{tabular}

\begin{tabular}{|c|c|c|c|c|c|c|c|c|c|c|}
\hline \multirow[t]{2}{*}{ School Code: T } & \multicolumn{5}{|c|}{ School Title: ARPS 2 Chakdara 2} & \multicolumn{5}{|c|}{$\begin{array}{l}\text { Head Teacher: Haji Sami Ullah } \\
\text { _Percent of Students }\end{array}$} \\
\hline & Enrolled & Dropped & $\begin{array}{l}\text { Appeared } \\
\text { in Exam }\end{array}$ & $\begin{array}{l}\text { Passed in } \\
\text { Exams }\end{array}$ & $\begin{array}{l}\text { Failed in } \\
\text { Exams }\end{array}$ & Grades & Dropped & $\begin{array}{l}\text { Appeared } \\
\text { in Exams }\end{array}$ & $\begin{array}{l}\text { Passed in } \\
\text { Exams }\end{array}$ & $\begin{array}{l}\text { Failed in } \\
\text { Exams }\end{array}$ \\
\hline Grade $1 \mathrm{~A}$ & 96 & 0 & 96 & 32 & 64 & Grade 1 & $0.00 \%$ & $100.00 \%$ & $33.33 \%$ & $66.67 \%$ \\
\hline Grade 1 B & 0 & 0 & 0 & 0 & 0 & Grade 2 & $45.00 \%$ & $55.00 \%$ & $95.45 \%$ & $4.55 \%$ \\
\hline Grade $1 \mathrm{C}$ & 0 & 0 & 0 & 0 & 0 & Grade 3 & $32.14 \%$ & $67.86 \%$ & $89.47 \%$ & $10.53 \%$ \\
\hline Grade $2 \mathrm{~A}$ & 40 & 18 & 22 & 21 & 1 & Grade 4 & $0.00 \%$ & $100.00 \%$ & $70.00 \%$ & $30.00 \%$ \\
\hline Grade 2 B & 0 & 0 & 0 & 0 & 0 & Grade 5 & $0.00 \%$ & $100.00 \%$ & $86.67 \%$ & $13.33 \%$ \\
\hline Grade 3 A & 28 & 9 & 19 & 17 & 2 & Grade 6 & $0.00 \%$ & $100.00 \%$ & $76.92 \%$ & $23.08 \%$ \\
\hline Grade 3 B & 0 & 0 & 0 & 0 & 0 & & & & & \\
\hline Grade 4 & 20 & 0 & 20 & 14 & 6 & Remarks: & & & & \\
\hline Grade 5 & 15 & 0 & 15 & 13 & 2 & & & & & \\
\hline Grade 6 & 13 & 0 & 13 & 10 & 3 & & & & & \\
\hline School Total & 212 & 27 & 185 & 107 & 78 & School \%age & $12.74 \%$ & $87.26 \%$ & $57.84 \%$ & $42.16 \%$ \\
\hline
\end{tabular}


Sub-Centre: Timargara

\begin{tabular}{|c|c|c|c|c|c|c|c|c|c|c|}
\hline \multirow[b]{2}{*}{$\begin{array}{l}\text { Grades I } \\
\text { Sections }\end{array}$} & \multicolumn{5}{|c|}{ - Number of Students } & \multirow[b]{2}{*}{ Grades } & \multicolumn{4}{|c|}{-Percent of Students } \\
\hline & Enrolled & Dropped & $\begin{array}{l}\text { Appeared } \\
\text { in Exam }\end{array}$ & $\begin{array}{c}\text { Passed in } \\
\text { Exams }\end{array}$ & $\begin{array}{c}\text { Failed in } \\
\text { Exams }\end{array}$ & & Dropped & $\begin{array}{l}\text { Appeared } \\
\text { in Exams }\end{array}$ & $\begin{array}{c}\text { Passed in } \\
\text { Exams }\end{array}$ & $\begin{array}{l}\text { Falled in } \\
\text { Exams }\end{array}$ \\
\hline Grade $1 \mathrm{~A}$ & 76 & 0 & 76 & 37 & 39 & Grade 1 & $0.00 \%$ & $100.00 \%$ & $48.68 \%$ & $51.32 \%$ \\
\hline Grade 1 B & 0 & 0 & 0 & 0 & 0 & Grade 2 & $0.00 \%$ & $100.00 \%$ & $89.29 \%$ & $10.71 \%$ \\
\hline Grade $1 \mathrm{C}$ & 0 & 0 & 0 & 0 & 0 & Grade 3 & $0.00 \%$ & $100.00 \%$ & $95.45 \%$ & $4.55 \%$ \\
\hline Grade 2 A & 28 & 0 & 28 & 25 & 3 & Grade 4 & $0.00 \%$ & $100.00 \%$ & $85.71 \%$ & $14.29 \%$ \\
\hline Grade 2 B & 0 & 0 & 0 & 0 & 0 & Grade 5 & $0.00 \%$ & $100.00 \%$ & $84.62 \%$ & $15.38 \%$ \\
\hline Grade $3 \mathrm{~A}$ & 22 & 0 & 22 & 21 & 1 & Grade 6 & $0.00 \%$ & $100.00 \%$ & $75.00 \%$ & $25.00 \%$ \\
\hline Grade 3 B & 0 & 0 & 0 & 0 & 0 & & & & & \\
\hline Grade 4 & 21 & 0 & 21 & 18 & 3 & Remarks: & & & & \\
\hline Grade 5 & 13 & 0 & 13 & 11 & 2 & & & & & \\
\hline Grade 6 & 20 & 0 & 20 & 15 & 5 & & & & & \\
\hline School Total & 180 & 0 & 180 & 127 & 53 & School \%age & $0.00 \%$ & $100.00 \%$ & $70.56 \%$ & $29.44 \%$ \\
\hline
\end{tabular}

\begin{tabular}{|c|c|c|c|c|c|c|c|c|c|c|}
\hline \multirow[b]{2}{*}{$\begin{array}{l}\text { Grades I } \\
\text { Sections }\end{array}$} & \multicolumn{5}{|c|}{ - Number of Students } & \multirow[b]{2}{*}{ Grades } & \multicolumn{4}{|c|}{ - Percent of Students- } \\
\hline & Enrolled & Dropped & $\begin{array}{l}\text { Appeared } \\
\text { in Exam }\end{array}$ & $\begin{array}{l}\text { Passed in } \\
\text { Exams }\end{array}$ & $\begin{array}{l}\text { Failed in } \\
\text { Exams }\end{array}$ & & Dropped & $\begin{array}{c}\text { Appeared } \\
\text { in Exams }\end{array}$ & $\begin{array}{l}\text { Passed in } \\
\text { Exams }\end{array}$ & $\begin{array}{l}\text { Failed in } \\
\text { Exams }\end{array}$ \\
\hline Grade $1 \mathrm{~A}$ & 41 & 0 & 41 & 25 & 16 & Grade 1 & $0.00 \%$ & $100.00 \%$ & $60.98 \%$ & $39.02 \%$ \\
\hline Grade 1 B & 0 & 0 & 0 & 0 & 0 & Grade 2 & $0.00 \%$ & $100.00 \%$ & $87.50 \%$ & $12.50 \%$ \\
\hline Grade $1 \mathrm{C}$ & 0 & 0 & 0 & 0 & 0 & Grade 3 & $11.11 \%$ & $88.89 \%$ & $100.00 \%$ & $0.00 \%$ \\
\hline Grade 2 A & 16 & 0 & 16 & 14 & 2 & Grade 4 & $25.00 \%$ & $75.00 \%$ & $100.00 \%$ & $0.00 \%$ \\
\hline Grade 2 B & 0 & 0 & 0 & 0 & 0 & Grade 5 & \#Num! & \#Num! & \#Num! & \#Num! \\
\hline Grade 3 A & 9 & 1 & 8 & 8 & 0 & Grade 6 & \#Num! & \#Num! & \#Num! & \#Num! \\
\hline Grade 3 B & 0 & 0 & 0 & 0 & 0 & & & & & \\
\hline Grade 4 & 8 & 2 & 6 & 6 & 0 & Remarks: & & & & \\
\hline Grade 5 & 0 & 0 & 0 & 0 & 0 & & & & & \\
\hline Grade 6 & 0 & 0 & 0 & 0 & 0 & & & & & \\
\hline School Total & 74 & 3 & 71 & 53 & 18 & School \%age & $4.05 \%$ & $95.95 \%$ & $74.65 \%$ & $25.35 \%$ \\
\hline
\end{tabular}

\begin{tabular}{|c|c|c|c|c|c|c|c|c|c|c|}
\hline \multirow[b]{2}{*}{$\begin{array}{l}\text { Grades I } \\
\text { Sections }\end{array}$} & \multicolumn{5}{|c|}{-Number of Students- } & \multirow[b]{2}{*}{ Grades } & \multicolumn{4}{|c|}{-Percent of Students - } \\
\hline & Enrolled & Dropped & $\begin{array}{l}\text { Appeared } \\
\text { in Exam }\end{array}$ & $\begin{array}{l}\text { Passed in } \\
\text { Exams }\end{array}$ & $\begin{array}{l}\text { Failed in } \\
\text { Exams }\end{array}$ & & Dropped & $\begin{array}{l}\text { Appeared } \\
\text { in Exams }\end{array}$ & $\begin{array}{c}\text { Passed in } \\
\text { Exams }\end{array}$ & $\begin{array}{l}\text { Failed in } \\
\text { Exams }\end{array}$ \\
\hline Grade $1 \mathrm{~A}$ & 0 & 0 & 0 & 0 & 0 & Grade 1 & \#Num! & \#Num! & \#Num! & \#Num! \\
\hline Grade $1 \mathrm{~B}$ & 0 & 0 & 0 & 0 & 0 & Grade 2 & \#Num! & \#um! & \#Num! & \#Num! \\
\hline Grade $1 \mathrm{C}$ & 0 & 0 & 0 & 0 & 0 & Grade 3 & \#Num! & \#Num! & \#Num! & \#Num! \\
\hline Grade 2 A & 0 & 0 & 0 & 0 & 0 & Grade 4 & \#Num! & \#Num! & \#Num! & \#Num! \\
\hline Grade 2 B & 0 & 0 & 0 & 0 & 0 & Grade 5 & \#Num! & \#Num! & \#Num! & \#Num! \\
\hline Grade $3 \mathrm{~A}$ & 0 & 0 & 0 & 0 & 0 & Grade 6 & \#Num! & \#Num! & \#Num! & \#Num! \\
\hline Grade 3 B & 0 & 0 & 0 & 0 & 0 & & \multirow{4}{*}{\multicolumn{4}{|c|}{$\begin{array}{l}\text { This school was started in March, } 1997 . \\
\text { Therfore Annual Results for 1996-97 } \\
\text { are not be available. }\end{array}$}} \\
\hline Grade 4 & 0 & 0 & 0 & 0 & 0 & Remarks: & & & & \\
\hline Grade 5 & 0 & 0 & 0 & 0 & 0 & & & & & \\
\hline Grade 6 & 0 & 0 & 0 & 0 & 0 & & & & & \\
\hline School Total & 0 & 0 & 0 & 0 & 0 & School \%age & \#Num! & \#Num! & \#Num! & \#Num! \\
\hline
\end{tabular}


Sub-Centre: Timargara

\begin{tabular}{|c|c|c|c|c|c|c|c|c|c|c|}
\hline \multirow[b]{2}{*}{$\begin{array}{l}\text { Grades I } \\
\text { Sections }\end{array}$} & \multicolumn{5}{|c|}{-Number of Students } & \multirow[b]{2}{*}{ Grades } & \multicolumn{4}{|c|}{-Percent of Students- } \\
\hline & Enrolled & Dropped & $\begin{array}{l}\text { Appeared } \\
\text { in Exam }\end{array}$ & $\begin{array}{l}\text { Passed in } \\
\text { Exams }\end{array}$ & $\begin{array}{c}\text { Failed in } \\
\text { Exams }\end{array}$ & & Dropped & $\begin{array}{c}\text { Appeared } \\
\text { in Exams }\end{array}$ & $\begin{array}{c}\text { Passed in } \\
\text { Exams }\end{array}$ & $\begin{array}{l}\text { Failed in } \\
\text { Exams }\end{array}$ \\
\hline Grade $1 \mathrm{~A}$ & 61 & 12 & 49 & 35 & 14 & Grade 1 & $21.43 \%$ & $78.57 \%$ & $70.71 \%$ & $29.29 \%$ \\
\hline Grade 1 B & 65 & 15 & 50 & 35 & 15 & Grade 2 & $16.67 \%$ & $83.33 \%$ & $92.00 \%$ & $8.00 \%$ \\
\hline Grade $1 \mathrm{C}$ & 0 & 0 & 0 & 0 & 0 & Grade 3 & $12.00 \%$ & $88.00 \%$ & $86.36 \%$ & $13.64 \%$ \\
\hline Grade $2 \mathrm{~A}$ & 30 & 5 & 25 & 23 & 2 & Grade 4 & $17.86 \%$ & $82.14 \%$ & $78.26 \%$ & $21.74 \%$ \\
\hline Grade 2 B & 0 & 0 & 0 & 0 & 0 & Grade 5 & $23.53 \%$ & $76.47 \%$ & $100.00 \%$ & $0.00 \%$ \\
\hline Grade $3 \mathrm{~A}$ & 25 & 3 & 22 & 19 & 3 & Grade 6 & $14.29 \%$ & $85.71 \%$ & $88.89 \%$ & $11.11 \%$ \\
\hline Grade 3 B & 0 & 0 & 0 & 0 & 0 & & & & & \\
\hline Grade 4 & 28 & 5 & 23 & 18 & 5 & Remarks: & & & & \\
\hline Grade 5 & 17 & 4 & 13 & 13 & 0 & & & & & \\
\hline Grade 6 & 21 & 3 & 18 & 16 & 2 & & & & & \\
\hline School Total & 247 & 47 & 200 & 159 & 41 & School \%age & $19.03 \%$ & $80.97 \%$ & $79.50 \%$ & $20.50 \%$ \\
\hline
\end{tabular}

\begin{tabular}{|c|c|c|c|c|c|c|c|c|c|c|c|}
\hline \multirow[b]{2}{*}{$\begin{array}{l}\text { Grades / } \\
\text { Sections }\end{array}$} & \multicolumn{6}{|c|}{ - Number of Students- } & \multirow[b]{2}{*}{ Grades } & \multicolumn{4}{|c|}{ Percent of Students } \\
\hline & ' & Enrolled & Dropped & $\begin{array}{l}\text { Appeared } \\
\text { in Exam }\end{array}$ & $\begin{array}{c}\text { Passed in } \\
\text { Exams }\end{array}$ & $\begin{array}{l}\text { Failed in } \\
\text { Exams }\end{array}$ & & Dropped & $\begin{array}{l}\text { Appeared } \\
\text { in Exams }\end{array}$ & $\begin{array}{c}\text { Passed in } \\
\text { Exams }\end{array}$ & $\begin{array}{l}\text { Failed in } \\
\text { Exams }\end{array}$ \\
\hline Grade $1 \mathrm{~A}$ & & 40 & 0 & 40 & 20 & 20 & Grade 1 & $0.00 \%$ & $100.00 \%$ & $50.00 \%$ & $50.00 \%$ \\
\hline Grade 1 B & & 0 & 0 & 0 & 0 & 0 & Grade 2 & $9.52 \%$ & $90.48 \%$ & $78.95 \%$ & $21.05 \%$ \\
\hline Grade $1 \mathrm{C}$ & & 0 & 0 & 0 & 0 & 0 & Grade 3 & $20.00 \%$ & $80.00 \%$ & $75.00 \%$ & $25.00 \%$ \\
\hline Grade 2 A & & 21 & 2 & 19 & 15 & 4 & Grade 4 & $26.67 \%$ & $73.33 \%$ & $100.00 \%$ & $0.00 \%$ \\
\hline Grade 2 B & & 0 & 0 & 0 & .0 & 0 & Grade 5 & $30.00 \%$ & $70.00 \%$ & $100.00 \%$ & $0.00 \%$ \\
\hline Grade $3 \mathrm{~A}$ & & 15 & 3 & 12 & 9 & 3 & Grade 6 & \#Num! & \#Num! & \#Num! & \#Num! \\
\hline Grade 3 B & & 0 & 0 & 0 & 0 & 0 & & & & & \\
\hline Grade 4 & & 15 & 4 & 11 & 11 & 0 & Remarks: & & & & \\
\hline Grade 5 & & 10 & 3 & 7 & 7 & 0 & & & & & \\
\hline Grade 6 & & 0 & 0 & 0 & 0 & 0 & & & & & \\
\hline School Total & & 101 & 12 & 89 & 62 & 27 & School \%age & $11.88 \%$ & $88.12 \%$ & $69.66 \%$ & $30.34 \%$ \\
\hline
\end{tabular}

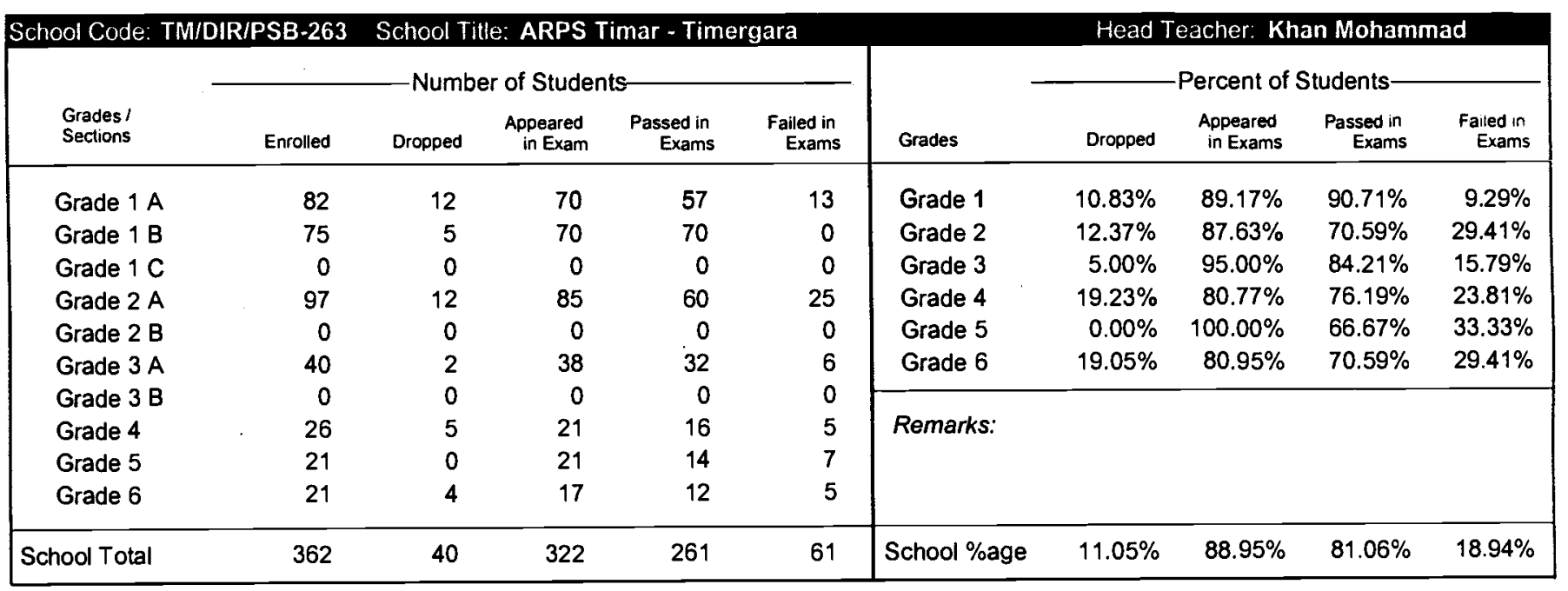


Sub-Centre: Timargara

\begin{tabular}{|c|c|c|c|c|c|c|c|c|c|c|}
\hline \multirow[b]{2}{*}{$\begin{array}{l}\text { Grades I } \\
\text { Sections }\end{array}$} & \multicolumn{5}{|c|}{ - Number of Students- } & \multirow[b]{2}{*}{ Grades } & \multicolumn{4}{|c|}{-Percent of Students } \\
\hline & Enrolled & Dropped & $\begin{array}{l}\text { Appeared } \\
\text { in Exam }\end{array}$ & $\begin{array}{l}\text { Passed in } \\
\text { Exams }\end{array}$ & $\begin{array}{l}\text { Failed in } \\
\text { Exams }\end{array}$ & & Dropped & $\begin{array}{l}\text { Appeared } \\
\text { in Exams }\end{array}$ & $\begin{array}{l}\text { Passed in } \\
\text { Exams }\end{array}$ & $\begin{array}{c}\text { Failed in } \\
\text { Exams }\end{array}$ \\
\hline Grade $1 \mathrm{~A}$ & 69 & 8 & 61 & 55 & 6 & Grade 1 & $11.59 \%$ & $88.41 \%$ & $90.16 \%$ & $9.84 \%$ \\
\hline Grade 1 B & 0 & 0 & 0 & 0 & 0 & Grade 2 & $10.53 \%$ & $89.47 \%$ & $91.18 \%$ & $8.82 \%$ \\
\hline Grade $1 \mathrm{C}$ & 0 & 0 & 0 & 0 & 0 & Grade 3 & $24.24 \%$ & $75.76 \%$ & $84.00 \%$ & $16.00 \%$ \\
\hline Grade $2 \mathrm{~A}$ & 38 & 4 & 34 & 31 & 3 & Grade 4 & $6.67 \%$ & $93.33 \%$ & $100.00 \%$ & $0.00 \%$ \\
\hline Grade 2 B & 0 & 0 & 0 & 0 & 0 & Grade 5 & $10.00 \%$ & $90.00 \%$ & $88.89 \%$ & $11.11 \%$ \\
\hline Grade $3 \mathrm{~A}$ & 33 & 8 & 25 & 21 & 4 & Grade 6 & $0.00 \%$ & $100.00 \%$ & $83.33 \%$ & $16.67 \%$ \\
\hline Grade 3 B & 0 & 0 & 0 & 0 & 0 & & & & & \\
\hline Grade 4 & 15 & 1 & 14 & 14 & 0 & Remarks: & & & & \\
\hline Grade 5 & 10 & 1 & 9 & 8 & 1 & & & & & \\
\hline Grade 6 & 12 & 0 & 12 & 10 & 2 & & & & & \\
\hline School Total & 177 & 22 & 155 & 139 & 16 & School \%age & $12.43 \%$ & $87.57 \%$ & $89.68 \%$ & $10.32 \%$ \\
\hline
\end{tabular}

\begin{tabular}{|c|c|c|c|c|c|c|c|c|c|c|c|}
\hline \multicolumn{12}{|c|}{ School Code: TM/DIR/PSB-265 School Title: ARPS Toor 2 - Timergara } \\
\hline \multirow[b]{2}{*}{$\begin{array}{l}\text { Grades I } \\
\text { Sections }\end{array}$} & \multirow[b]{2}{*}{ ' } & \multicolumn{5}{|c|}{-Number of Students } & \multirow[b]{2}{*}{ Grades } & \multicolumn{4}{|c|}{-Percent of Students- } \\
\hline & & Enrolled & Dropped & $\begin{array}{l}\text { Appeared } \\
\text { in Exam }\end{array}$ & $\begin{array}{c}\text { Passed in } \\
\text { Exams }\end{array}$ & $\begin{array}{l}\text { Failed in } \\
\text { Exams }\end{array}$ & & Dropped & $\begin{array}{l}\text { Appeared } \\
\text { in Exams }\end{array}$ & $\begin{array}{l}\text { Passed in } \\
\text { Exams }\end{array}$ & $\begin{array}{l}\text { Failed in } \\
\text { Exams }\end{array}$ \\
\hline Grade $1 \mathrm{~A}$ & & 74 & 14 & 60 & 20 & 40 & Grade 1 & $18.92 \%$ & $81.08 \%$ & $33.33 \%$ & $66.67 \%$ \\
\hline Grade 1 B & & 0 & 0 & 0 & 0 & 0 & Grade 2 & $9.09 \%$ & $90.91 \%$ & $66.67 \%$ & $33.33 \%$ \\
\hline Grade $1 \mathrm{C}$ & & 0 & 0 & 0 & 0 & 0 & Grade 3 & $21.43 \%$ & $78.57 \%$ & $68.18 \%$ & $31.82 \%$ \\
\hline Grade 2 A & & 33 & 3 & 30 & 20 & 10 & Grade 4 & $28.57 \%$ & $71.43 \%$ & $70.00 \%$ & $30.00 \%$ \\
\hline Grade 2 B & & 0 & 0 & 0 & 0 & 0 & Grade 5 & $33.33 \%$ & $66.67 \%$ & $75.00 \%$ & $25.00 \%$ \\
\hline Grade 3 A & & - 28 & 6 & 22 & 15 & 7 & Grade 6 & $0.00 \%$ & $100.00 \%$ & $77.78 \%$ & $22.22 \%$ \\
\hline Grade 3 B & & 0 & 0 & 0 & 0 & 0 & & & & & \\
\hline Grade 4 & & 14 & 4 & 10 & 7 & 3 & Remarks: & & & & \\
\hline Grade 5 & & 18 & 6 & 12 & 9 & 3 & & & & & \\
\hline Grade 6 & & 9 & 0 & 9 & 7 & 2 & & & & & \\
\hline School Total & & 176 & 33 & 143 & 78 & 65 & School \%age & $18.75 \%$ & $81.25 \%$ & $54.55 \%$ & $45.45 \%$ \\
\hline
\end{tabular}

\begin{tabular}{llllll|lllll}
\hline Sub-Centre Total & 7,177 & 762 & 6,415 & 4,519 & 1,896 & Sub-Centre \%age & $10.62 \%$ & $89.38 \%$ & $70.44 \%$ & $29.56 \%$ \\
\hline \hline Grand Total & 60,804 & 4,739 & 56,065 & 40,431 & 15,634 & Grand \%age & $7.79 \%$ & $92.21 \%$ & $72.11 \%$ & $27.89 \%$ \\
\hline \hline
\end{tabular}

